\title{
1998 Comprehensive TNX Area Annual Groundwater and Effectiveness Monitoring Report
}

\section{Part I}

by

J. Chase

Westinghouse Savannah River Company

Savannah River Site

Aiken, South Carolina 29808
RECORDS ADMINISTRATION

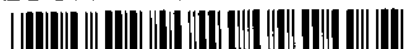

$\mathrm{R} 0125206$

\section{DOE Contract No. DE-AC09-96SR18500}

This paper was prepared in connection with work done under the above contract number with the U. S.

Department of Energy. By acceptance of this paper, the publisher and/or recipjent acknowledges the U. S. Government's right to retain a nonexclusive, royalty-free license in and to any copyright covering this paper, along with the right to reproduce and to authorize others to reproduce all or part of the copyrighted paper. 


\section{United States Department of Energy}

Savannah River Site

\section{Comprehensive TNX Area Annual Groundwater and Effectiveness Monitoring Report (U)}

WSRC-RP-99-4003

May 1999

Authorized Derivative Classifier and Reviewing Official:

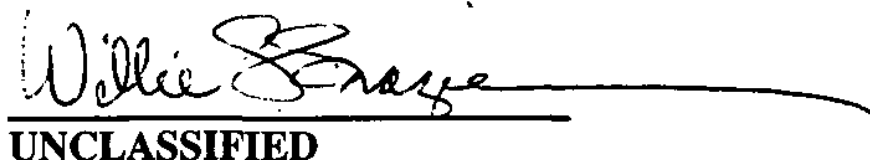

Does Not Contain Unclassified Controlled Nuclear Information

Prepared By:

Westinghouse Savannah River Company

Savannah River Company

Aiken, SC 29808

Prepared for the U. S. Department of Energy under Contract No. DE-AC09-96-SR18500

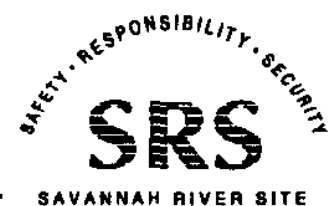




\section{United States Department of Energy}

Savannah River Site

\section{Comprehensive TNX Area Annual Groundwater and Effectiveness Monitoring Report (U)}

WSRC-RP-99-4003

May 1999

Authorized Derivative Classifier and Reviewing Official:

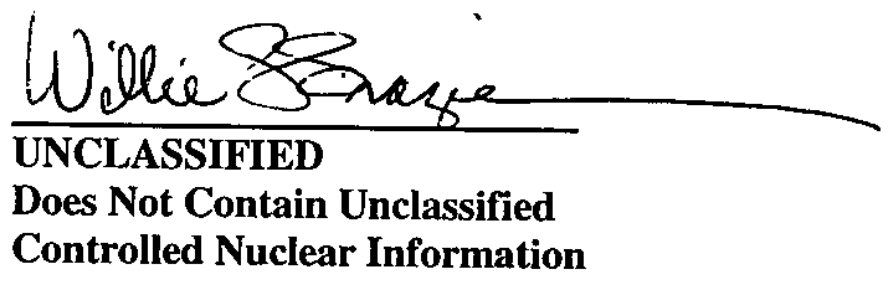

Prepared By:

Westinghouse Savannah River Company Savannah River Company Aiken, SC 29808

Prepared for the U. S. Department of Energy under Contract No. DE-AC09-96-SR18500

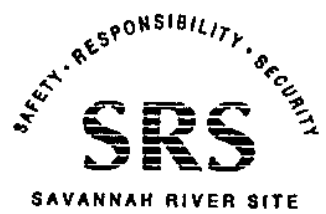




\section{DISCLAIMER}

This report was prepared as an account of work sponsored by an agency of the United States Government. Neither the United States Government nor any agency thereof, nor any of their employees, makes any warranty, express or implied, or assumes any legal liability or responsibility for the accuracy, completeness, or usefulness of any information, apparatus, product, or process disclosed, or represents that its use would not infringe privately owned rights. Reference herein to any specific commercial product, process, or service by trade name, trademark, manufacturer, or otherwise does not necessarily constitute or imply its endorsement, recommendation, or favoring by the United States Government or any agency thereof. The views and opinions of authors expressed herein do not necessarily state or reflect those of the United States Government or any agency thereof.

This report has been reproduced directly from the best available copy.

Available to DOE and DOE contractors from the Office of Scientific and Technical Information, P.O. Box 62, Oak Ridge, TN 37831; prices available from (615) 576-8401.

Available to the public from the National Technical Information Service, U.S. Department of Commerce; 5285 Port Royal Road, Springfield, VA 22161. 


\section{United States Department of Energy}

Savannah River Site

\section{Comprehensive TNX Area Annual Groundwater and Effectiveness Monitoring Report (U)}

WSRC-RP-99-4003

May 1999

Authorized Derivative Classifier and Reviewing Official:

UNCLASSIFIED

Does Not Contain Unclassified

Controlled Nuclear Information

Prepared By:

Westinghouse Savannah River Company

Savannah River Company

Aiken, SC 29808

Prepared for the U. S. Department of Energy under Contract No. DE-AC09-96-SR18500

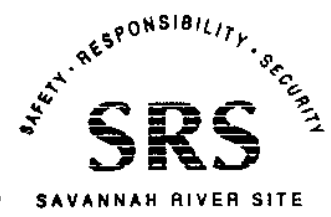




\section{$\therefore \quad$ DISCLAIMER}

This report was prepared by Westinghouse Savannah River Company (WSRC for the United States Department of Energy under Contract No. DE-AC09-96SR18500) and is an account of work performed under that contract. Reference herein to any specific commercial product, process, or service by trademark, name, manufacturer, or otherwise does not necessarily constitute or imply endorsement, recommendation, or favoring of same by WSRC or by the United States Government or any agency thereof. 


\section{TABLE OF CONTENTS}

SECTION

$\underline{\text { PaGE }}$

LIST OF FIGURES .............................................................................................................. iv

LIST OF TABLES .................................................................................................................... v

LIST OF APPENDICES ....................................................................................... vi

EXECUTIVE SUMMARY ….....................................................................................

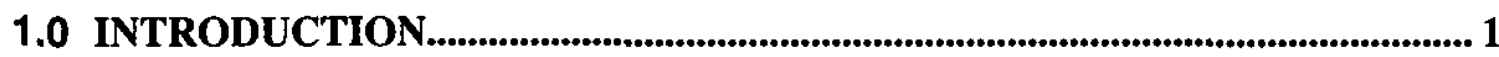

1.1 Organization of This Report................................................................................... 3

2.0 OBJECTIVES OF THE INTERIM ACTION .......................................................... 5

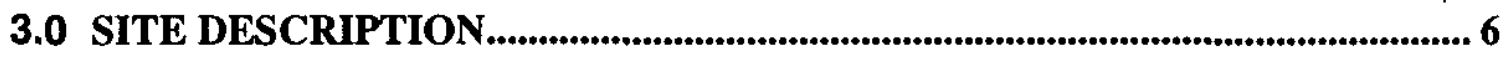

3.1 Operating History of TNX Waste Units...................................................................... 6

3.2 Regulatory History.................................................................................................................. 8

3.3 Site Hydrogeology ........................................................................................................... 10

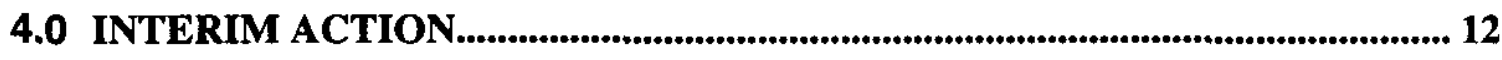

4.1 Recovery Well Network................................................................................................. 12

4.2 GeoSiphon Technology Demonstration ................................................................. 13

4.3 Monitoring Well Installations...................................................................................... 13

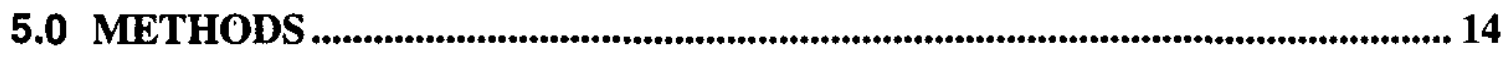

5.1 Sampling Procedures ..................................................................................................... 14

5.2 Data Validation and Verification ............................................................................. 18

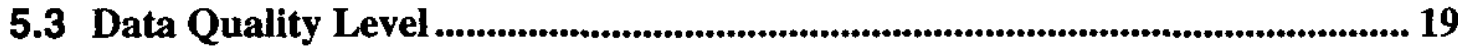

5.4 Process and Documentation ................................................................................. 20

5.5 Quality Control Samples ............................................................................................ 21

5.6 Conclusions....................................................................................................................... 21

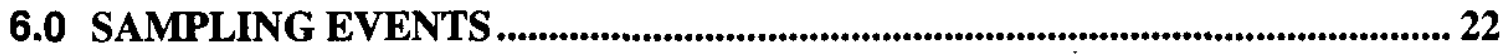

6.1 Analyses Scheduled........................................................................................................ 22

6.2 The Well Network ............................................................................................................ 23

6.3 Synchronous Water Level Measurements ................................................................. 25

6.4 Groundwater Flow Directions and Rates .................................................................. 31

6.5 Precipitation Measurements ....................................................................................... 31

6.6 Purging and Sampling Problems ........................................................................... 32

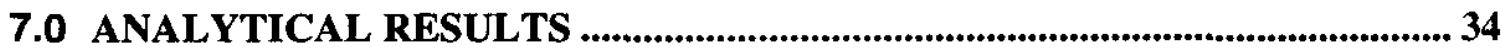


7.1 Results for Primary and Recovery Wells.............................................................. 35

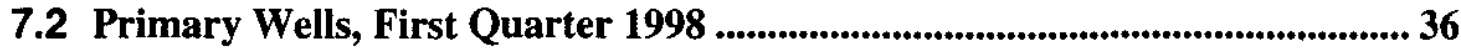

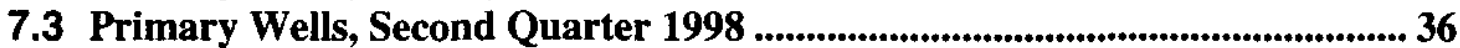

7.4 Primary Wells, Third Quarter 1998................................................................ 37

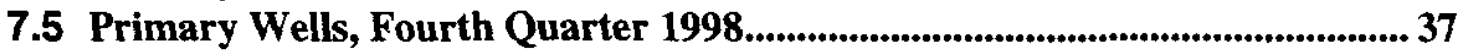

7.6 Analytical Results for Recovery Wells................................................................ 40

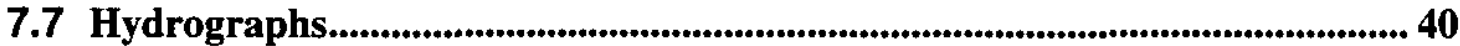

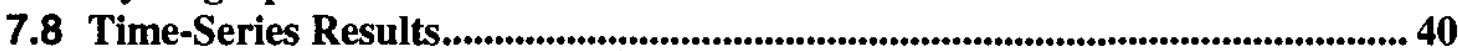

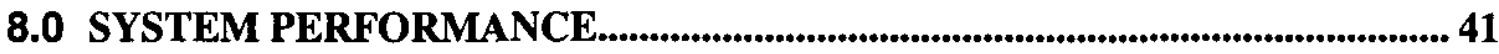

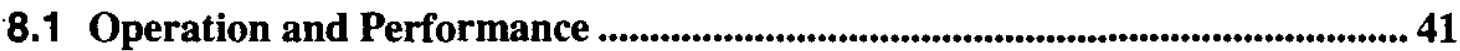

8.2 Removal of Groundwater Contaminated with Trichloroethylene .................. 43

8.3 Removal of Groundwater Contaminated with Carbon Tetrachloride .......... 44

8.4 Containment of Groundwater Contamination................................................. 44

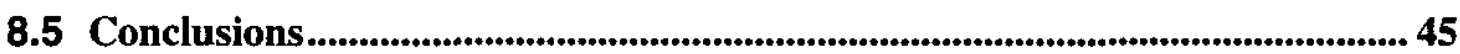

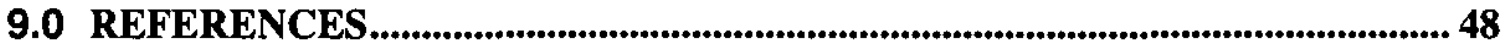

\section{LIST OF FIGURES}

Figure 1. - Location of the TNX AREA At The Savannah River Site .............. 2

FIGURE 2. LOGS FROM SOIL BORING SB-1 AT TNX .............................................. 11

Figure 3. LOCATION OF TNX AREa MONITORING Wells FOR STARTUP AND

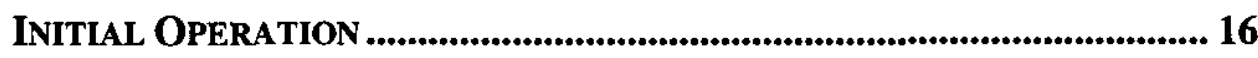

Figure 4. WATER TABle MAP OF The UNCONFINEd AQUifer at THE TNX AREA, THIRD QUARTER 1996.................................................................. 26

Figure 5. WATER-TABLE MAP Of THE UNCONFINED AQUIFER AT THE TNX AREA, NOVEMBER 1998 ....................................................................................... 28

Figure 6. SUMMARY OF HiStORIC (1973-1997) RAINFALl DATA FOR D-AREA AND 1998 RAINFALL DATA FOR D-AREA ................................................ 30

Figure 7. TIME SERIES FOR TNX AIR STRIPPER AND RECOVERY WELL NETWORK 42

FIGURE 8. TNX AREA TCE CONCENTRATION IN GROUNDWATER, FOURTH QUARTER 1998 WITH PROJECTED 4 Q96 500PPB PLUME SUPERIMPOSED ..............................................................46 


\section{LIST OF TABLES}

TABLE 1. RAINFALL (INCH) AT 400-D, 1994 - 1998 ............................................ 32

TABLE 2. WELLS NOT SAMPLED DURING 1998 ......................................................... 33

Table 3. Constituents Exceeding Applicable Standards in Primary

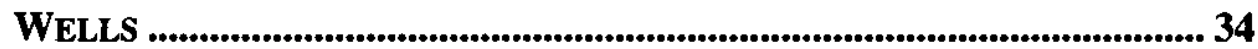

Table 4. Monthily Constituents Above APPlicable Limits in APPENDIX A FOR THE RECOVERY WELLS DURING 1998.......................................... 39

Table 5. Gallons Treated and INFLUENT Concentrations of Trichloroethylene (TCE) AND CARbon Tetrachloride (CCI 4 ) 43 


\section{LIST OF APPENDICES}

APPENDIX A DATA REVIEW KEY AND PRIMARY DRINKING WATER STANDARDS............ A-1 APPENDIX B GROUNDWATER MONITORING RESULTS TABLES ................................... B-1

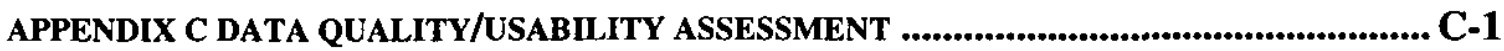

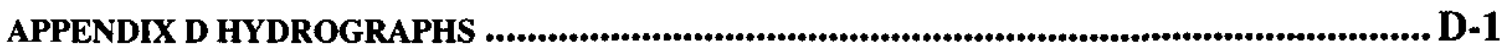

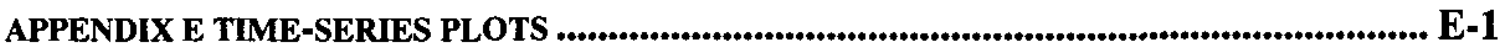

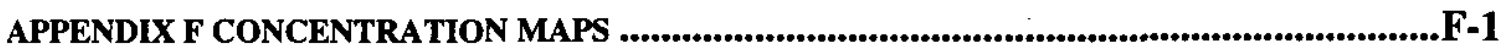

TNX ARea Trichloroethylene ConCENTration in GroundWater, First QUARTER 1998 ..... Trichloroethylene Concentrations in Groundwater at THE TNX AREa, FourTh QUARTER

Carbon Tetrachlorme Concentrations in Groundwater at the TNX Area, First QUARTER 1998

Carbon Tetrachloride Concentrations in Groundwater at the TNX AREa, Fourth QUARTER 1998

Tetrachloroethylene Concentrations IN Groundwater at THE TNX AREA, First QUARTER.

Tetrachloroethylene Concentrations in Groundwater at the TNX ARea, Fourth QUARTER 1998

ChLOROFORM CONCENTRATIONS IN GROUNDWATER AT THE TNX AREA, FIRST QUARTER 1998 ....

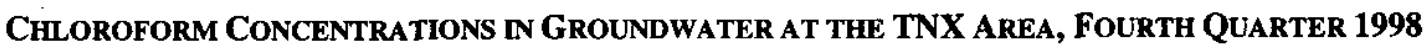
Mercury Concentrations in GroundWater at the TNX AREA, First QUARTER 1998 Mercury Concentrations in Groundwater at the TNX AREA, Fourth QUARTER 1998 ........ Lead Concentrations in Groundwater aT the TNX AREa, First Quarter 1998 LEAD CONCENTRATIONS IN GROUNDWATER AT THE TNX AREA, FouRTH QUARTER 1998. NItrate Concentrations in GroundWater at THE TNX AREA, First Quarter 1998 Nitrate ConCENTRations In Groundwater at THE TNX AREA, FourTh QUARTER 1998........... Grośs AlPha Concentrations in Groundwater at the TNX AREA, FirSt Quarter 1998..... Gross AlPHa Concentrations in Groundwater at THE AREA, FOURTH QUarter 1998. 


\section{LIST OF ACRONYMS AND ABBREVIATIONS}

BRA

CERCLA

$\mathrm{ft}^{3} / \mathrm{min}$

CMS/FS

CVOC

DWPF

EMS

ERD

EPD/EMS

GIMS

gpm

IROD

LDRR

$\mu \mathrm{g} / \mathrm{L}$

MRD

NPDES

NTU

PDWS

PVC

QA/QC

RCRA

RDR/RAA

RFI/RI

ROD

RPD
Baseline Risk Assessment

Comprehensive Environmental Response, And Liability Act

Cubic Foot Per Minute

Corrective Measures Study/Feasibility Study

Chlorinated Volatile Organic Compound

Defense Waste Processing Facility

Addendum To The Environmental Monitoring Strategy (For TNX Area)

Environmental Restoration Division

Environmental Protection Department/Environmental Monitoring

Section

Geochemical Information Management System

Gallons Per Minute

Interim Record Of Decision

Laboratory Data Records Review

Micrograms Per Liter

Mean Relative Difference

National Pollutant Discharge Elimination System

Nephelolometric Turbidity Units

Primary Drinking Water Standard(S)

Polyvinyl Chloride

Quality Assurance/Quality Control

Resource Conservation And Recovery Act

Remedial Design Report/Remedial Action

Rcra Facility Investigation/Remedial Investigation

Record Of Decision

Relative Percent Difference 
1998 Comprehensive TNX Area Annual Groundwater and Effectiveness Monitoring Report (U)

Savannah River Site

May 1999

SB/PP

SCDHEC

SRS

SRTC

TCE

US DOE

US EPA

UTM

WSRC
Statement Of Basis/Proposed Plan

South Carolina Department Of Health And Environmental Control

Savannah River Site

Savannah River Technology Center

Trichloroethylene

United States Department Of Energy

United States Environmental Protection Agency

Universal Transverse Mercator

Westinghouse Savannah River Company
Page viii of ix

-RP-99-4003
Unclassified 


\section{EXECUTIVE SUMMARY}

Shallow groundwater beneath the TNX Area at the Savannah River Site (SRS) has been contaminated with chlorinated volatile organic compounds (CVOCs) such as trichloroethylene (TCE) and carbon tetrachloride (CCLA). In November 1994, an Interim Record of Decision (IROD) was agreed to and signed by the U.S. Department of Energy (US DOE), the Environmental Protection Agency (US EPA), and the South Carolina Department of Health and Environmental Control (SCDHEC). The IROD requires installation of a hybrid groundwater corrective action (HGCA) to stabilize the plume of groundwater contamination and remove CVOCS dissolved in the groundwater. The hybrid groundwater corrective action included a recovery well network, an air stripper, and an airlift recirculation well. The recirculation well was dropped pursuant to a test that indicated the well to be ineffective at the TNX Area. Consequently, the groundwater corrective action was changed from a hybrid to a single action, pump-and-treat approach.

The Interim Action (IA) T-1 Air Stripper System began operation on September 16, 1996. A comprehensive groundwater monitoring program was initiated to measure the effectiveness of the system. During calendar year 1998, 30.6-million gallons were treated. In addition the system removed 21 pounds of TCE and 2.52 pounds of $\mathrm{CCI}_{4}$ during 1998. The recovery well network created a "capture zone" that stabilized the plume of contaminated groundwater.

The IA is meeting its objectives and is capable of continuing to do so until the final groundwater remedial action is in place. 


\subsection{INTRODUCTION}

The TNX Area, located in the southwest part of Savannah River Site (SRS) (Figure 1), is operated by the Savannah River Technology Center (SRTC), formerly known as the Savannah River Laboratory. The Environmental Restoration Division (ERD) manages the Interim Action (IA) in the area. Under the Federal Facility Agreement for SRS, the TNX Area Operable Unit includes the TNX Burying Ground, the Old TNX Seepage Basin, the New TNX Seepage Basin, and the groundwater beneath the area.

Groundwater beneath the TNX Area is contaminated with chlorinated volatile organic compounds (CVOCs) such as trichloroethylene, carbon tetrachloride, and tetrachloroethylene (perchloroethylene). The IA for the TNX Area is designed to capture or contain groundwater with contaminant concentrations in excess of 500 $\mu \mathrm{g} / \mathrm{L}$ and reduce the mass of the contamination. The IA includes a recovery well network and an air stripper.

Analyses of depth-discrete sediment samples collected in the central portion of TNX indicate CVOC contamination in a zone 30 feet thick, extending 15 feet above and below the water table. Cis-dichloroethylene in modest concentrations indicates biodegradation of CVOCs in the unsaturated zone.

The IA to contain the plume of trichloroethylene began operations September 16, 1996. The effectiveness of the IA can be assessed by examination of the results of the monitoring program conducted since that time to evaluate the capture zone and measure identifiable changes in contaminant concentrations in the monitoring and recovery wells throughout the area. These changes are reported as specific concentrations in designated wells, as trends in time-series plots for indicator constituents, and as changes in the areal extent of the $500-\mu \mathrm{g} / \mathrm{L}$ plume of 


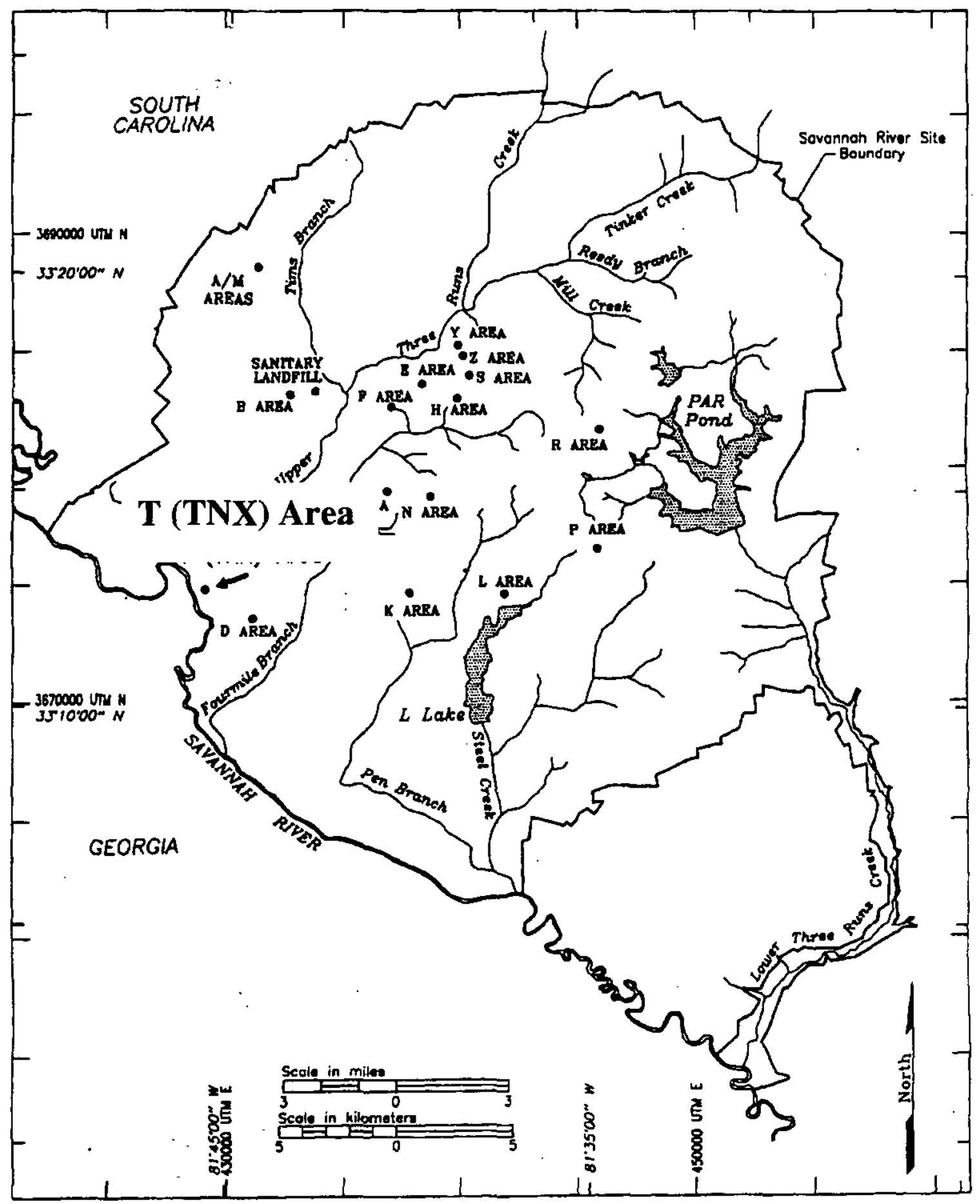

Figure 1. Location of the TNX Area at the Savannah River Site 
trichloroethylene. In addition, changes in water elevations in monitoring wells and piezometers throughout the site are examined as indicators of plume containment.

\subsection{Organization of This Report}

This report presents the data collected during Calendar Year 1998 in support of the IA and illustrates the effectiveness of the action in plume containment and contaminant reduction.

The text of the report includes the objectives of the IA; a description of the site, including the operating and regulatory history of TNX-Area waste units and a brief description of the site hydrogeology; discussion of the methods selected for site remediation; discussion of sampling events and analytical results; and a summary of system operation and maintenance during 1998.

Maps in this report illustrate the location of TNX within SRS (Figure 1), the location of the groundwater monitoring wells in TNX Area, the groundwater elevation of wells screened in the water table during Third Quarter 1996 (immediately prior to the start of remediation activities) and Fourth Quarter 1998, and the horizontal extent of trichloroethylene during Fourth Quarter 1998 with the projected plume for October 1996 superimposed over the current data postings.

Maximum results for analytes that exceeded the Safe Drinking Water Act final primary drinking water standards (PDWS) (Appendix A) in sampled primary . wells during 1998 are provided in the Analytical Results section of this report, as are the analyses from the TRW wells (the recovery wells) that exceeded the PDWS. The introduction to Appendix A provides definitions of the abbreviations and modifiers used in the results tables as well as descriptions of holding times, data rounding, and data qualification practices. Field data and analytical results of 
each well used for monitoring the effectiveness of the IA appear in Appendix B, Tables B-1 through B-6.

Table B-1 presents results for each analyte for the primary wells during each quarter of the year. Table B-1 also identifies the analytical laboratories that conducted the analyses and the analyses that received modifiers (which help identify laboratory accuracy and precision) or that exceeded the US EPAapproved holding times during Fourth Quarter 1998. Table B-2 presents the Appendix IX results taken annually for primary wells. The TRW well series was sampled monthly, beginning in September 1996. Table B-3 presents results for each analysis in TRW wells during each month of 1998. For these wells, the analyses that received modifiers or that exceeded the US EPA-approved holding times are listed in Table B-3. Table B-4 presents the field data results for the secondary wells for each quarter during 1998. Table B-5 presents groundwatermonitoring results for other TNX Area wells.

Table B-6 presents the water elevations for TNX Area wells as measured by SRTC that are not present in the Geochemical Information Management System (GIMS) database. GIMS data is the official data from the derived samples. Additional data was provided by SRTC. Appendix C gives a general assessment of the quality and usability of the data provided by Environmental Protection Department/Environmental Monitoring Section (EPD/EMS). Appendix D contains hydrographs for TNX Area wells. The hydrographs contain the SRTC measurements from Table B-6 as well as those recorded by Westinghouse Savannah River Company (WSRC) EPD/EMS. Appendix E contains time-series plots showing time versus concentration for carbon tetrachloride, nitrate, tetrachloroethylene, trichloroethylene, chloroform, gross alpha, mercury, and lead. Additionally, concentration maps are included for 1998 First and Fourth Quarter data in Appendix F. Concentration maps were developed only for those contaminants identified as constituents of interest in the Effectiveness Monitoring 
Strategy Addendum for the TNX Groundwater Operable Unit RDR/RA Work Plan (WSRC 1996).

\subsection{OBJECTIVES OF THE INTERIM ACTION}

The initial proposal in the Interim Record of Decision (IROD)(WSRC 1994b) set the objectives and goals of the IA at TNX for a hybrid groundwater corrective action consisting of a recovery well network, low-profile air stripper, and airlift recirculation well (WSRC 1994a). However, the recirculation well system has been determined to be ineffective in TNX Area because of both geological factors and the nature of the contamination. The decision to remove the in situ portion of the remediation strategy was issued as an Explanation of Significant Differences (WSRC 1997b) in accordance with Comprehensive Environmental Response, Compensation, and Liability Act (CERCLA) regulations. Evidence to date indicates that the recovery well network alone will meet the overall intent of the IROD.

The major objectives and goals of the IA at TNX are to

- stabilize the plume of 500- $\mu \mathrm{g} / \mathrm{L}$ trichloroethylene contamination and

- remove trichloroethylene contamination in the groundwater near the plume core.

Successful achievement of these objectives will

- prevent further aquifer degradation,

- maintain risk at acceptable levels to the onsite worker at the seep line, and

- reduce potential risk to human health and the environment in general. 
The monitoring program assesses the effectiveness of the IA by measuring changes in contaminant concentrations in the primary monitoring and recovery wells identified in the Addendum to the Environmental Monitoring Strategy (for TNX Area) (WSRC 1997b). These changes are reported here as specific concentrations in designated wells and as time-series plots for the primary wells. In addition, water-elevation changes for TNX Area are illustrated in the maps in this report and the hydrography in Appendix D.

Primary wells monitor the extent and severity of groundwater contamination and, with secondary wells, are used to determine groundwater flow and direction. Recovery wells draw in contaminated groundwater and direct it to the air stripper, where CVOCs are removed by contacting the contaminated groundwater with air. Clean water discharged by the air stripper is directed to the National Pollutant Discharge Elimination System (NPDES) permitted outfall X-008 for release to the Savannah River.

\subsection{SITE DESCRIPTION}

This section outlines important historical and regulatory events in TNX Area.

\subsection{Operating History of TNX Waste Units}

- The TNX Burying Ground was built in 1953 to dispose of debris resulting from the explosion of an experimental evaporator in TNX Area earlier that year. The debris included conduits, drums, tin, and structural steel contaminated with uranyl nitrate and depleted uranium. No additional wastes were disposed of in the TNX Burying Ground after the Old Burial Ground (643-E) was opened later in 1953.

- Between 1980 and 1984, most of the waste buried at the TNX Burying Ground was excavated and sent to the Radioactive Waste Burial Ground (643- 
7E). The _remaining waste lies beneath asphalt, buildings, and transformer pads. An estimated 60 pounds of uranyl nitrate remains buried at the TNX Burying Ground, constituting 5 percent of the initial inventory.

- The Old TNX Seepage Basin was an unlined excavation designed to contain wastewater until it could seep into the underlying sediments that were believed to act as a natural ion-exchange media. A 6-inch pipe connected the inlet section of the basin to the seepage section, and a similar pipe carried overflow to the adjacent floodplain during periods of unusually high flow and precipitation to the basin. The Old TNX Seepage Basin received process wastewater between 1958 and 1980 from pilot scale tests conducted in the TNX Area for the Defense Waste Processing Facility (DWPF) and the General Separations Area. The waste discharged to the basin included large quantities of mercury, primarily in the form of mercuric nitrate, other heavy metals, sodium compounds, depleted uranium and other radionuclides.

- Discharge of wastewater to the Old TNX Seepage Basin ceased in 1980, and the basin was closed in 1981. During closure, the basin wall was breached, and the impounded water was drained into the adjacent wetlands. The basin was then backed with a sand and clay mixture and covered with a clay cap. Part of the capped Old TNX Seepage Basin was revegetated, and the remainder was covered with asphalt and used for equipment storage.

- The New TNX Seepage Basin, an unlined excavation constructed to replace the Old TNX Seepage Basin, operated between 1980 and 1988. While in operation, the basin received waste from pilot scale chemical processing tests at DWPF and the chemical separation areas. The waste included simulated non-radioactive DWPF sludge; șimulated, non-radioactive salt supernate; glass frit; other processing chemicals; and laboratory sink wastewater. Strict institutional controls were put in place for the use of the New TNX Seepage 
Basin to ensure that no hazardous waste was discharged to the basin. During periods of unusually high discharge, the seepage section of the basin overflowed to. Outfall $\mathrm{X}-013 \mathrm{~A}$, which in turn discharged to a local surface depression.

- On August 13,1988, discharges to the New TNX Seepage Basin ceased and were rerouted to the TNX Effluent Treatment Facility.

\subsection{Regulatory History}

- A closure plan for the New TNX Seepage Basin was submitted to SCDHEC in March 1992.

- Currently the basin is inactive and constantly filled with $2+$ feet of accumulated rainwater.

- The SRS Federal Facility Agreement of November 24, 1992, which directs the comprehensive remediation of SRS, identified the TNX Burying Ground, the Old TNX Seepage Basin, the New TNX Seepage Basin, and the TNX groundwater as units regulated under the Resource Conservation and Recovery Act (RCRA)/CERCLA program.

- An IA Proposed Plan for the TNX-Area Operable Unit was submitted to United States Environmental Protection Agency (US EPA) and South Carolina Department of Health and Environmental Control (SCDHEC) in 1994. The plan proposed the evaluation of a hybrid treatment strategy for remediating the groundwater contamination at TNX Area. The strategy proposed a combination of pump-and-treat and in situ groundwater treatments. The method was to employ four recovery wells with an air stripper and an airlift recirculation well (WSRC 1994a). 
- In November 1994, an IROD for the TNX Groundwater Operable Unit was authorized by US EPA, SCDHEC, and United States Department of Energy (US DOE) (WSRC 1994b). IA operations began on September 16, 1996. This - report discusses groundwater monitoring undertaken to demonstrate the effectiveness of this strategy.

- The air stripper system is permitted as an industrial wastewater treatment facility with both NPDES and Air Quality Control permits.

- The RCRA Facility Investigation/Remedial Investigation (RFI/RI) work plan for the TNX-Area Operable Unit was submitted in November 1995. The objectives of the investigation were to 1) determine the nature and extent of hazardous substance releases from the unit; 2) prepare human health and ecological risk assessments; 3) collect data to aid in refining the extent of hazardous substance migration in the groundwater beneath the TNX Area; and 4) prepare a Corrective Measures Study/Feasibility Study (CMS/FS) report.

- The TNX-Area Operable Unit field characterization report was submitted in 1996. Sampling for the RFI/RI field characterization was performed between March 4 and September 26, 1996. The RFI/RI report with Baseline Risk Assessment (BRA) for the TNX-Area Operable Unit was submitted in 1997.

- An Explanation of Significant Differences was issued on September 22, 1997, to announce the change in the interim remediation strategy for the TNX Groundwater Operable Unit from a hybrid action to a single pump-and-treat approach (WSRC 1997b).

- The Rev. 0 RFI/RI/BRA report for the TNX Area June 27, 1997 Operable Unit was submitted to US EPA and SCDHEC. The RFI/RI/BRA (Rev. 1.2) was approved by SCDHEC on December 9, 1998, and by US EPA on January $5,1999$. 
- The Rev..0 combined CMS/FS/Statement of Basis/Proposed Plan (SB/PP) and draft Record of Decision (ROD) for the TNX Area Operable Unit was submitted to US EPA and SCDHEC on July 9, 1998.

\subsection{Site Hydrogeology}

The Floridan Aquifer System is the aquifer system of concern within the TNX area between Upper Three Runs Creek and the southern boundary of SRS. The Floridan Aquifer System is divided into two aquifer units separated by a confining unit. From bottom to top, they are known as the Gordon Aquifer Unit, the Gordon Confining Unit, and the Upper Three Runs Aquifer Unit (Figure 2).

CVOC contamination at TNX is found only in the unconfined aquifer, which receives recharge from above due to infiltrating precipitation. The unconfined aquifer also receives recharge from the underlying semiconfined aquifer, which is at a higher pressure. The hydraulic conductivity of the unconfined aquifer varies from 20-60 feet/day (WSRC 1997c).

The Savannah River is the major discharge point for the Floridan Aquifer System and thus controls the direction and rate of lateral and vertical groundwater movement. 
1998 Comprehensive TNX Area Annual Groundwater and Effectiveness Monitoring Report (U)

Savannah River Site

May 1999
WSRC-RP-99-4003

Unclassified

Page 11 of 49

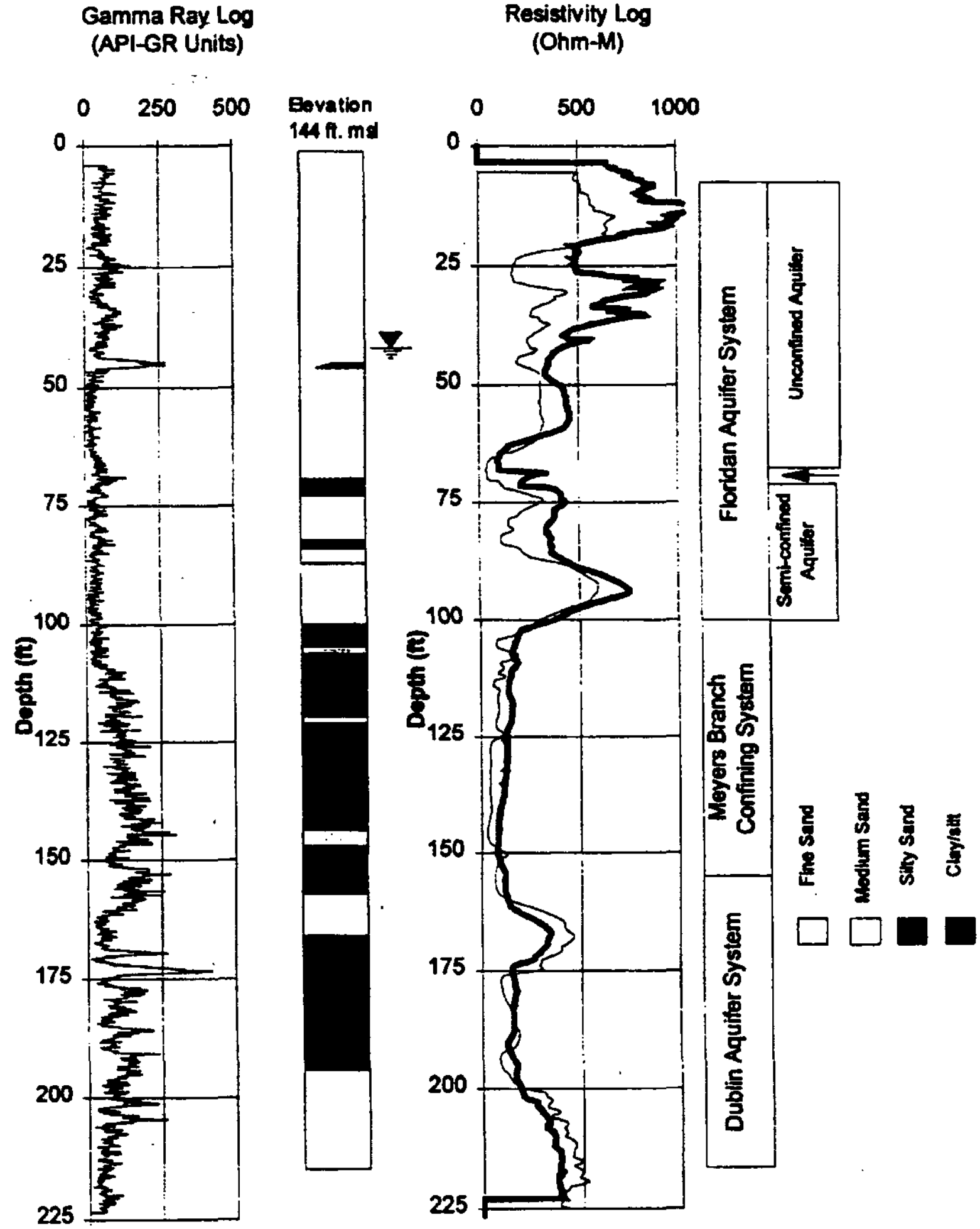

Figure 2. Logs from Soil Boring SB-1 at TNX 


\subsection{INTERIM ACTION}

The TNX-Area IA consists of an ex situ system of four recovery wells pumping groundwater from the downgradient edge of the initial $500-\mu \mathrm{g} / \mathrm{L}$ trichloroethylene plume to a low profile air stripper for removal of volatile organic constituents. The stripper is also able to treat purge water generated during sampling of TNX Area wells.

An in situ treatment system using airlift recirculation well TRW 1 was planned as part of a hybrid corrective action but was dropped from the action following field testing.

SRTC performed the pump test and the computer modeling of the water-table aquifer that were used in the design of the IA.

\subsection{Recovery Well Network}

The recovery well network is intended to stop the migration of contaminated groundwater containing more than $500 \mu \mathrm{g} / \mathrm{L}$ trichloroethylene and to remove dissolved trichloroethylene from the plume core. Recovery wells TRW 1, 2, 3, and 4 are used to pump contaminated water from the unconfined aquifer (PostConstruction Report WSRC-RP-96-826, Rev. 2) (Figure 3). This pumping produces cones of depression on the water table that surround each recovery well. The recovery wells are located in such a way that the cones of depression overlap and create a capture zone that prevents contaminated groundwater (greater than $500 \mu \mathrm{g} / \mathrm{L}$ trichloroethylene) from migrating any farther downgradient and into the Savannah River floodplain. An air stripper is used to treat the contaminated groundwater collected during operation of the recovery well network and purge water generated during sampling of monitoring wells. 


\subsection{GeoSiphon Technology Demonstration}

From June 18, 1998, to November 13, 1998, SRTC conducted Phase II testing of the GeoSiphon technology for remediation of groundwater that contains low concentrations (5 - $200 \mu \mathrm{g} / \mathrm{L}$ ) of CVOC (WSRC 1999). The GeoSiphon cell is located in the Savannah River floodplain west of TNX Area, next to well TNX 11 (See Figure 3). The cell is 8 feet in diameter and constructed with granular cast iron as the filter pack and 12-inch well screen and casing. Additional construction details of the cell can be found in WSRC 1998. Results of Phase II testing show the GeoSiphon cell is capable of producing and treating a steady sustained flow of groundwater contaminated with CVOCs at up to $4.5 \mathrm{gpm}$ using a siphon equipped with an air chamber. Optimized siphon line routes and air chamber configurations suggest the system at TNX is capable of operating at approximately $15 \mathrm{gpm}$. In September 1998, a second GeoSiphon cell was installed for use in the next phase of testing.

\subsection{Monitoring Well Installations}

Several monitoring wells were installed during 1998 in support of different projects. All of the wells were installed by SCDHEC-licensed drillers using an auger and were constructed of 2-inch polyvinyl chloride (PVC). A list of these wells well as the purpose of installation is provided below. The wells were installed by SRTC in 1998. All well installation records have been submitted to SCDHEC in accordance with terms and conditions of the well installation permits. 
List of wells installed at TNX Area during 1998

Well ID Purpose

SCDHEC Well

Installation Permit

\#

TGSC2 Second GeoSiphon cell for use in dual cell test

SF-97-002

TCM4 - 8 Monitor dual cell test of GeoSiphon Technology

HW-98-037

TNX28 - Characterize extent of CVOC contamination in

HW-98-082

37 groundwater beneath Savannah River floodplain west of TNX Area

TNX38 - Monitor water table elevation north of TNX around

HW-98-082

40 natural surface depression.

\subsection{METHODS}

\subsection{Sampling Procedures}

The sampling procedure (WSRC Hydrogeologic Data Collection Procedures and Specifications Manual) for pumped wells requires evacuation of a minimum of two well volumes and stabilization of $\mathrm{pH}$, specific conductance, and turbidity prior to sample collection. Stability is established when a minimum of three consecutive measurements, taken within a given time period, are within a specified tolerance range. If a well pumps dry before two well volumes have been purged or before stabilization has been achieved, it must be revisited within 24 hours for the data to be considered as a single sampling event. On the second visit within 24 hours, samples are taken without purging or stability measurements; thus these samples may not be representative of groundwater quality.

Most wells at SRS have dedicated pumps. Dedicated variable-speed pumps have been installed in wells TBG 4 and TNX 23D, 24D, and 27D. Samples from wells with variable-speed pumps are collected at a slower rate to minimize turbidity, which has been associated with artificially elevated metals levels. Decreased aluminum and iron concentrations, as well as lower turbidity values, have been 
This page intentionally left blank. 

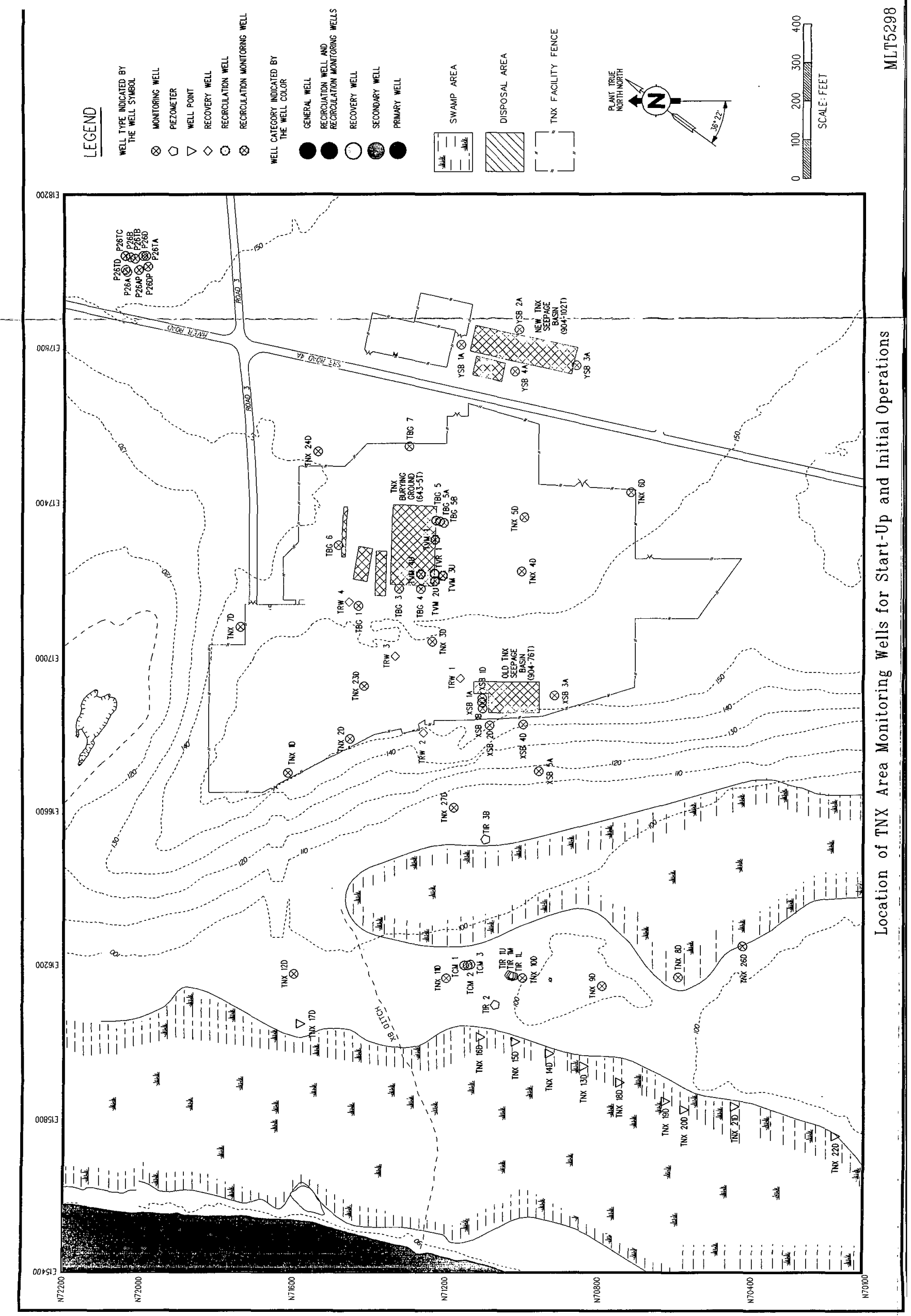
1998 Comprehensive TNX Area Annual Groundwater and Effectiveness Monitoring Report (U) Savannah River Site

May 1999
WSRC-RP-99-4003

Unclassified

Page 16 of 49

Figure 3. Location of TNX Area Monitoring Wells for Startup and Initial Operation 
This page intentionally left blank. 
observed in samples from wells with variable-speed pumps. A few wells were sampled with hand-held submersible pumps during 1998.

The recovery well pumps run continuously except during system shutdowns. EPD/EMS samplers take depth-to-water measurements at each sampling event, and SRTC personnel collect synchronous depth-to-water measurements monthly. SRTC synchronous measurements were used for the Fourth Quarter 1998 water table map. Hydrographs in Appendix D contain both sets of measurements. The SRTC measurements for 1998 may be found in Table B-6 of this report. Purge water from TNX-Area wells containing elevated levels of contaminants is disposed of in the purge water station on the stripper.

\subsection{Data Validation and Verification}

Sample collection, shipping, and analytical information compiled for the project is validated and verified. The information reviewed includes the following:

- Sample Documentation in the Field Logs and Chain-of-Custody (COC) Forms

- Sample Holding Times

- Initial and Continuing Instrument Calibration

- Analyte Identification

- Analyte Quantitation

- Analytical Error

- Analysis of Blanks

- Laboratory Performance Evaluations 
- Quantitation Limits

The validation process begins with sample scheduling and continues through data reporting. Appendix $\mathrm{C}$ gives a general assessment of the quality and usability of the data provided by EPD/EMS.

During validation and verification of the analytical data, qualifiers are checked and applied to provide additional information about data quality. The laboratories provide qualifiers in reporting the analytical data (Appendix C).

During 1998 the qualifiers in the computer data records were changed. Prior to October 1998, each analytical record in the computer data files contained a result and an analysis qualifier. The result qualifier described the analytical result. The analysis qualifier described issues arising during the analytical process. After October 1998, the result qualifier had been changed to the US EPA Functional Guidelines Codes and the analysis qualifier changed to the US EPA STORET Codes (see Appendix A, Data Modifiers).

\subsection{Data Quality Level}

The data for this project were validated as definitive data. Definitive-level data are used for data collection activities that require a high degree of qualitative and quantitative accuracy for all findings. Rigorous methods of analysis and quality assurance are used for those samples considered essential in making a decision. This data level is intended to give the decision maker a level of confidence to make decisions regarding the following:

- Treatment

- Disposal

- Site remediation and/or removal of pollutants 
- Health risk or environmental impact

- Cleanup verification, pollutant source identification, delineation of contaminants

- Other significant decisions where an action level is of concern

\subsection{Process and Documentation}

Sample documentation and maintenance of chain of custody (COC) were examined by reviewing the field logs and $\mathrm{COC}$ forms. Sample-holding times were checked by comparing the time between sample collection and analysis with US EPA-published maximum holding times. Samples that exceeded holding time are marked in the data tables (Appendix B) with a " $Q$ " in the modifier columns. Analytical instrument calibration was reviewed as part of the quarterly laboratory data records review (LDRR), in which the laboratory's records for a percentage of the samples analyzed each quarter are reviewed for adherence to method-specific quality assurance requirements and accuracy in reporting.

Analyte identification and quantitation were verified as part of the computerized checking of the electronic data deliverables, during review of the analytical narratives provided by the laboratory and as part of the LDRR. Anomalies were clarified with the laboratories wherever possible, and records not meeting criteria were qualified. In evaluating analytical error, percent recoveries for qualitycontrol samples were reviewed, and the sample data were qualified where necessary. Field-generated blanks (field and trip blanks) and laboratory-generated blanks were examined. Results of these reviews are located in the quality control samples section of the EPD/EMS quarterly groundwater monitoring reports. Laboratory performance evaluations conducted by EPD/EMS, US EPA, and US DOE are detailed in the EPD/EMS quarterly groundwater monitoring reports. 


\subsection{Quality Control Samples}

EPD/EMS selected approximately 5 percent of the sampled TNX-Area wells each quarter to receive split and blind replicate samples as part of the EPD/EMS quality assurance. As part of their quality assurance procedures, the laboratories also duplicated certain analyses.

The results. of these analyses are used for both intra-laboratory and interlaboratory comparisons. The EPD/EMS quarterly groundwater monitoring reports provide full replicate results and statistical comparisons of blind replicate and duplicate results. The highest result is provided in the Appendix B data tables of this report.

\subsection{Conclusions}

Except for certain radiological parameters, analytical results are generated using SCDHEC-certified laboratories and follow US EPA SW-846 methodologies. There were 10,388 sample results; each result represents an individually measured parameter, compound, element, or isotope. Of the 10,388 samples, $43(0.4$ percent) were rejected; 40 radiological results from one well and one method account for the majority of the rejects.

Overall analytical data quality for 1998 at TNX is very good except for one laboratory problem that developed during the fourth quarter. A GC-MS instrument performing semivolatile analyses for method EPA8270C went down and did not run again until hold times had expired for several samples. This accounted for 1,408 of the 1,488 (14 percent) out of hold records and was from a one-time isolated event. Only 11 ( 0.1 percent $)$ sample results were affected by detection of an analyte in the laboratory blank. 


\subsection{SAMPLING_EVENTS}

\subsection{Analyses Scheduled}

During 1998, groundwater samples from TNX-Area wells were analyzed according to the sampling and analysis plan in the Effectiveness Monitoring Strategy Addendum for the TNX Groundwater Operable Unit RDR/RA Work Plan (WSRC 1996), which identifies the following wells as primary, secondary, or recovery wells:

- Primary: P 26A; TBG 1, 3,4,5, 5A, 5B, and 6; TNX 1D, 2D, 3D, 4D, 7D, 8D, 9D, 10D, 11D, 12D, and 27D; XSB 1A, 1B, 1D, 2D, 3A, 4D, and 5A

- Secondary: P 26B and 26D; TBG 7; TNX 5D and 6D; YSB 1A, 2A, 3A, and $4 \mathrm{~A}$

- Recovery: TRW 1,2, 3, and 4

Samples for constituents listed in the Table 3 of the Effectiveness Monitoring Strategy Addendum for the TNX Groundwater Operable Unit RDR/RA Work Plan (WSRC 1996) were collected quarterly from the primary wells and monthly from the recovery wells. A full Appendix IX set of analyses was collected during the third quarter from the primary wells. The secondary wells were sampled quarterly for field parameters. In addition, the wells were monitored, as requested, for other constituents as part of the SRS Groundwater Monitoring Program and for other investigations conducted at the site. These results may be found in the EPD/EMS quarterly comprehensive groundwater monitoring reports. The groundwater samples are unfiltered; therefore, the results for metals are for total recoverable metals. 


\section{May 1999}

Page 23 of 49

\subsection{The Well Network}

The background, assessment, recovery, and groundwater monitoring well network at the TNX Area (Figure 3) have been developed over the past decade and a half as described chronologically below:

- Monitoring wells YSB 1A, 2A, 3A, and 4A were installed during late 1983.

- Monitoring wells XSB 1A, 1B, 1D, 2D, 3A, and 4D were installed between 1984 and 1989.

The installation date for well XSB 5A is uncertain.

- Background wells P 26A, 26B, and 26D were installed during the second half of 1986.

- Monitoring wells TBG $1,3,4,5,5 \mathrm{~A}, 5 \mathrm{~B}, 6$, and 7 were installed between 1988 and 1989.

- Assessment wells TNX 1D, 2D, 3D, 4D, 5D, 6D, 7D, 8D, 9D, 10D, 11D, and 12D were installed during late 1989.

- According to SRTC records, TNX clusters $61,65,66$, and 72 were installed in April 1991. These wells are not discussed in this report.

- Seep line monitoring wells TNX 13D, 14D, 15D, 16D, 17D, 18D, 19D, 20D, 21D, and 22D were installed during 1994.

- Recovery wells TRW 1, 2, 3, and 4 were installed between 1994 and 1995.

- Recirculation well TVR 1A and its monitoring well clusters TVM 1, 2, 3, and 4 were installed in October and November 1995. 
1998 Comprehensive TNX Area Annual Groundwater

and Effectiveness Monitoring Report (U)

Savannah River Site

May 1999
WSRC-RP-99-4003

Unclassified

Page 24 of 49

- Monitoring wells TNX 23D, 24D, 26D, and 27D were installed during 1996.

- Special monitoring wells TIR $1 \mathrm{~L}, 1 \mathrm{M}, 1 \mathrm{U}, 2$, and 3B were installed during the second half of 1996 to assist in assessing intrinsic remediation. These wells are not discussed in this report.

The TNX-Area network used to assess the IA includes the 39 wells identified as primary, secondary, and recovery wells in the Analyses Scheduled section above.

Screen zone assignments for the wells in the TNX-Area network are described in the Effectiveness Monitoring Strategy Addendum for the TNX Groundwater Operable Unit RDR/RA Work Plan (WSRC 1996) as follows

- Unconfined Aquifer: wells P 26B and 26D; TBG 1, 3, 4, 5, 5A, 6, and 7; TNX 1D, 2D, 3D, 4D, 5D, 6D, 7D, 8D, 9D, 10D, 11D, 12D, 13D, 14D, 15D, 16D, 17D, 18D, 19D, 20D, 21D, 22D, 23D, 24D, 26D, and 27D; TRW 1, 2, 3, and 4; XSB 1A, 1D, 2D, 3A, 4D, and 5A and YSB 1A, 2A, 3A, and 4A as well as the TVM and TVR wells

- Semiconfined Aquifer: wells P 26A, TBG 5B, and XSB 1B

The following wells, used for hydrographs (Appendix D), are screened across the water table:

- P 26D; TBG 1, 3, 4, 5, 6, and 7; TNX 1D, 2D, 3D, 4D, 5D, 6D, 7D, 8D, 9D, 10D, 11D, 12D, 13D, and 27D; TRW 1, 2, 3, and 4; TVM 1U, 2U, 3U, and $4 \mathrm{U} ; \mathrm{XSB} 1 \mathrm{D}, 2 \mathrm{D}, 3 \mathrm{~A}, 4 \mathrm{D}$, and $5 \mathrm{~A}$; YSB $\mathrm{U} \sim 3 \mathrm{~A}$ and $4 \mathrm{~A}$. 


\subsection{Synchronous Water Level Measurements}

Water level data have been used to prepare water-table maps (Figures 4 and 5) and hydrographs (Appendix D), as described under "Sampling Procedures" on page 13 of this report. This information will be used to evaluate the effectiveness of the recovery well network. The hydrogeology in TNX Area is evaluated using Figures 4 and 5 to determine groundwater flow rates and directions and using precipitation data (Figure 6), and the water elevation difference to determine the effects of the recovery well system. The water table maps were constructed for Third Quarter 1996 using water elevations measured by EPD/EMS samplers prior to September 14, 1996, and for Fourth Quarter 1998 using synchronous water elevations measured by SRTC in November 1998. The EPD/EMS measurements are recorded in the GIMS database, as are all the analytical data. The SRTC measurements for Fourth Quarter 1998 may be found in Table B-6 of this report. 


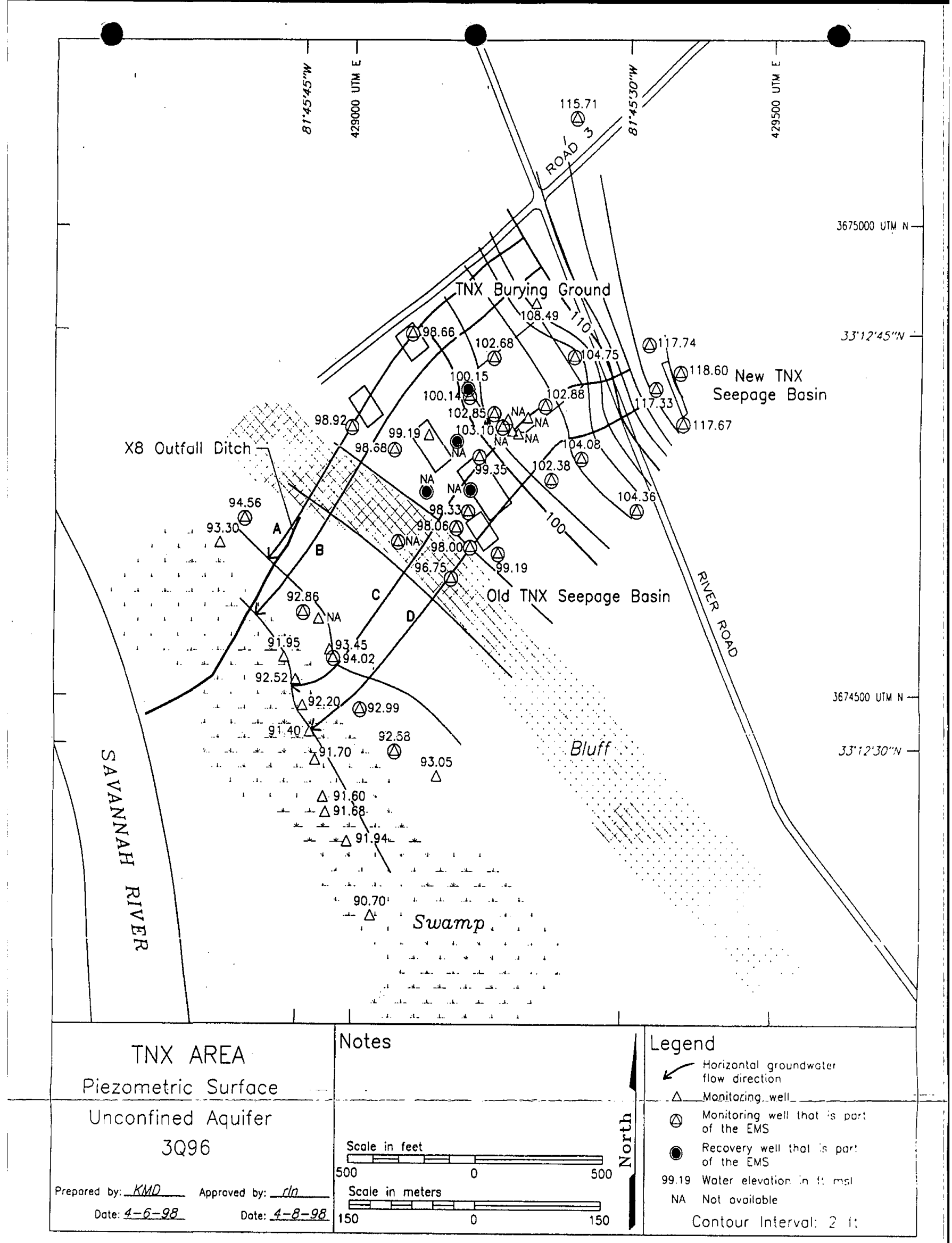

Figure 4 Water-Table Map of the Unconfined Aquifer at the TNX Area, Third Quarter 1996 
1998 Comprehensive TNX Area Annual Groundwater

and Effectiveness Monitoring Report (U)

Savannah River Site

May 1999
WSRC-RP-99-4003

Unclassified

Page 26 of 49

Figure 4. Water Table Map of the Unconfined Aquifer at the TNX Area, Third Quarter 1996 
1998 Comprehensive TNX Area Annual Groundwater and Effectiveness Monitoring Report (U) Savannah River Site

May 1999
WSRC-RP-99-4003

Unclassified

Page 27 of 49

This page intentionally left blank. 
$\begin{array}{lll}\text { Savannah River Site } & \text { Prepared in Cooperation with TNX Area Annual Groundwater }\end{array}$ Site Geotechnical Services Environmental Restoration and Savannah River Technology Center and Effectiveness Monitoring Report

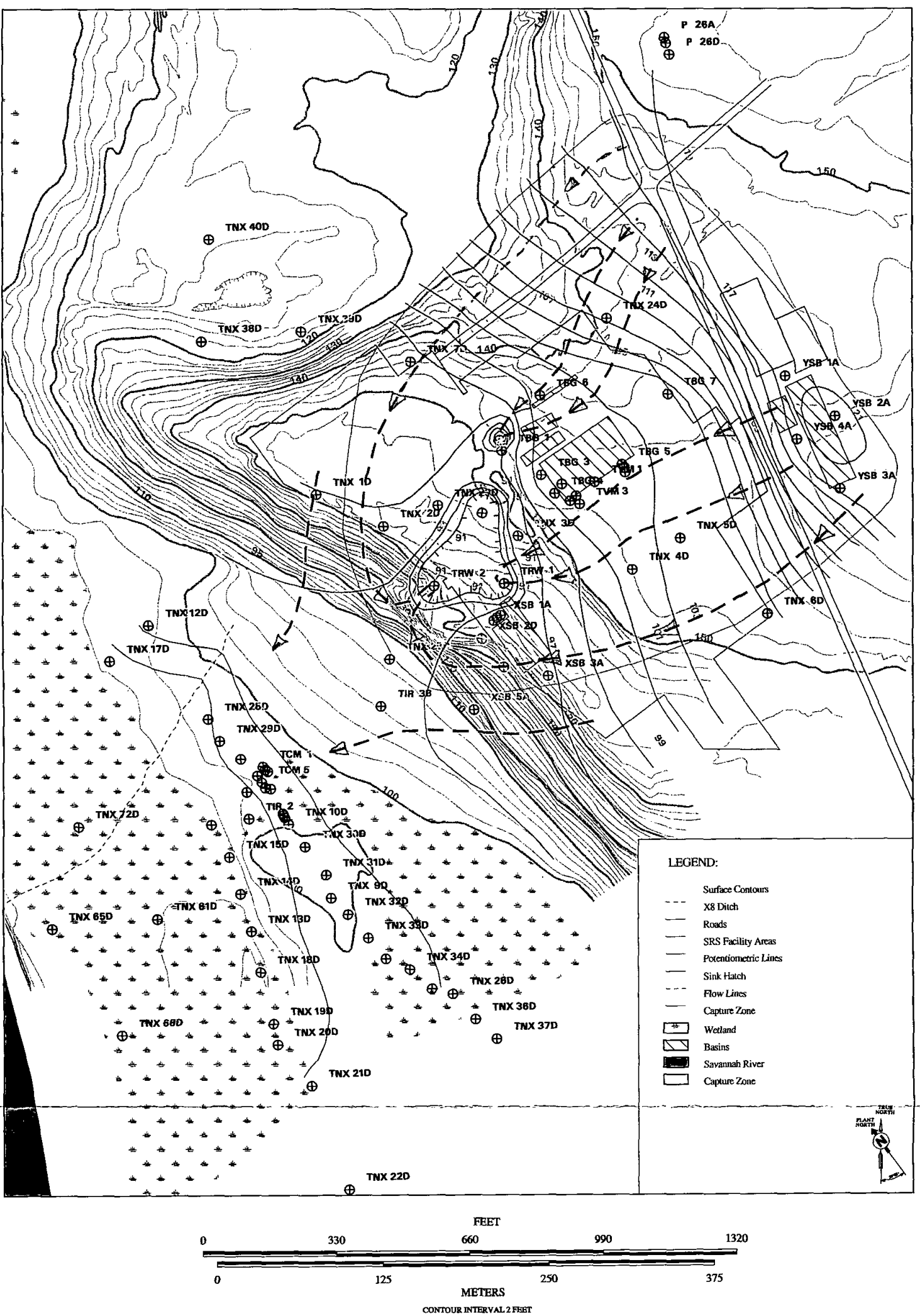

TNX AREA WATER TABLE MAP OF THE UNCONFI NED AQUI FI ER, FOURTH QUARTER 1998

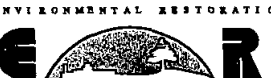

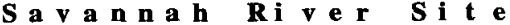

Ai ken, So ut h Caroli na

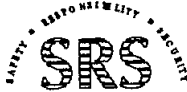


Figure 5. Water-Table Map of the Unconfined Aquifer at the TNX Area, Fourth 1998 
This page intentionally left blank. 


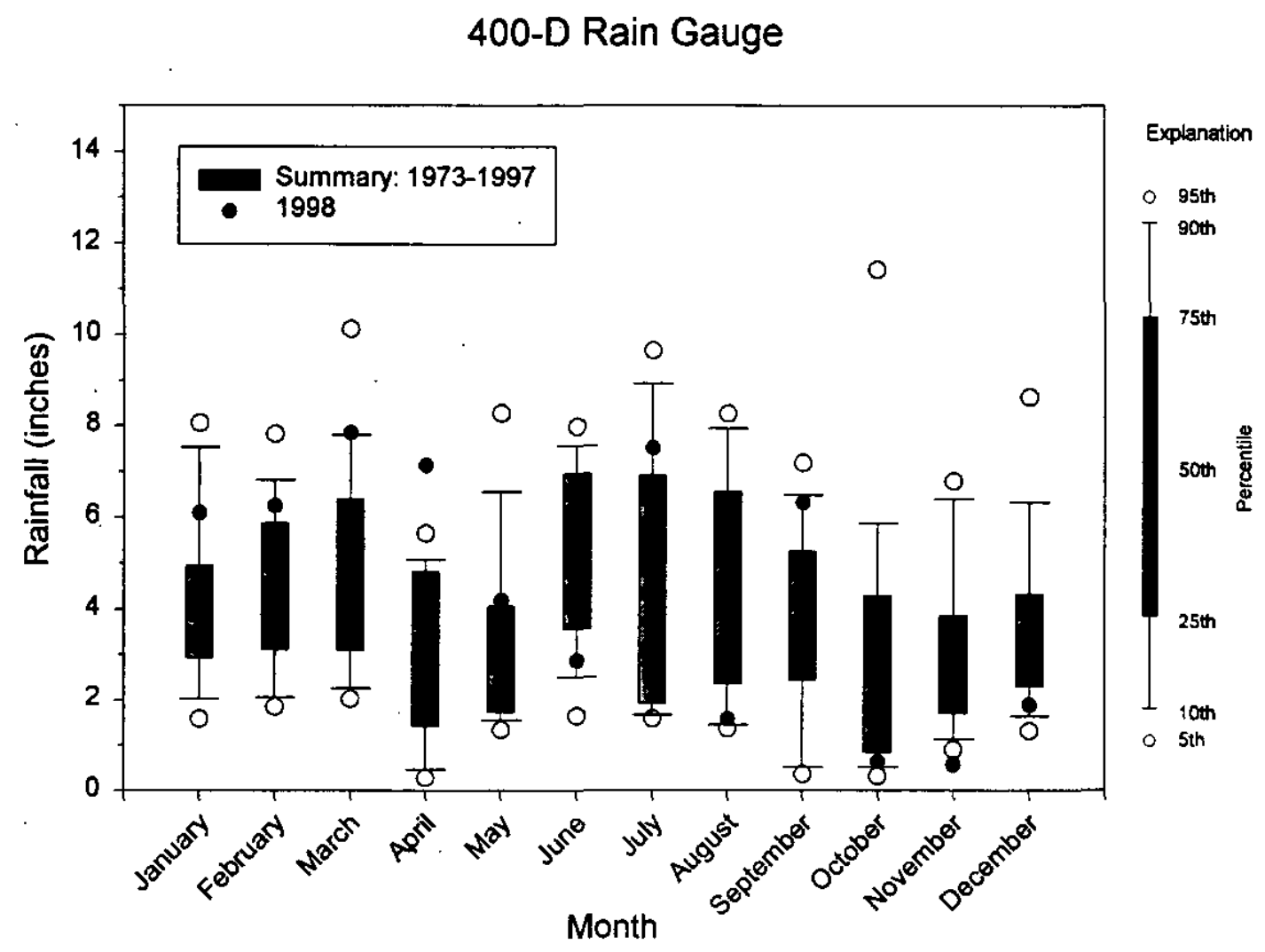

Figure 6. Summary of Historic (1973-1997) Rainfall Data for D-Area and 1998 Rainfall Data for D-Area 


\subsection{Groundwater Flow Directions and Rates}

Figures 4 and 5 provide water elevation maps for the uppermost aquifers beneath TNX Area during Third Quarter 1996 and November 1998, respectively. The maps are oriented to true north using Universal Transverse Mercator (UTM) coordinates.

During 1998 the horizontal groundwater flow in the unconfined aquifer beneath the TNX Area changed compared to previous years. In 1998, as previously, the horizontal flow in the eastern section of the area was to the southwest. The water continued to the southwest toward the Savannah River Swamp. In 1998, the horizontal flow in the western portion of the area was toward the pumping wells. The potentiometric surface in the semiconfined aquifer could not be contoured because of the near-linear distribution of the three wells in this aquifer.

The groundwater flow velocity is directly related to the hydraulic gradient. The pumping wells are drawing down the potentiometric surface and increasing the hydraulic gradient. Groundwater flow velocities are increasing in the area, with the greatest increases near the pumping wells.

\subsection{Precipitation Measurements}

Daily precipitation measurements were made at a meteorological station located approximately 2.5 miles southeast of TNX Area at D Area (see Figure 1). The measurements represent the total precipitation for a 24-hour period. Table 1 is a monthly summary for rainfall at D Area for the last 5 years. Monthly rainfall was within the normal range for most of 1997. Rainfall 1998 was below normal, as indicated in Figure 6. See also the hydrographs in Appendix D. 
Table 1. Rainfall (inch) at 400-D, $1994-1998$

\begin{tabular}{|l|l|l|l|l|l|}
\hline Month & 1994 & 1995 & 1996 & 1997 & 1998 \\
\hline January & 4.71 & 5.28 & 2.6 & 4.14 & 6.10 \\
\hline February & 4.49 & 6.06 & 2.05 & 5.1 & 6.26 \\
\hline March & 6.72 & 2.47 & 6.26 & 1.98 & 7.86 \\
\hline April & 1.29 & 0.17 & 1.69 & 3.42 & 4.19 \\
\hline May & 1.66 & 2.28 & 1.6 & 1.69 & 7.14 \\
\hline June & 7.33 & 7.24 & 3.65 & 6.82 & 2.86 \\
\hline July & 6.08 & 4.2 & 4.89 & 6.54 & 7.52 \\
\hline August & 3.62 & 6.86 & 7.93 & 1.37 & 1.58 \\
\hline September & 2.33 & 3.95 & 3.72 & 5.41 & 6.33 \\
\hline October & 8.98 & 2.11 & 1.98 & 4.74 & 0.65 \\
\hline November & 2.86 & 2.49 & 1.5 & 4.29 & 0.57 \\
\hline December & 4.71 & 4.47 & 2.74 & 7.93 & 1.89 \\
\hline $\begin{array}{l}\text { Annual } \\
\text { Totals }\end{array}$ & 54.78 & 47.58 & 40.61 & 53.43 & 52.95 \\
\hline
\end{tabular}

\subsection{Purging and Sampling Problems}

From January to February wells TRW3 and 4 were out of service for pipe replacement. Piping was switched from galvanized pipe to PVC. During March the air stripper was dismantled to clean lower trays and sump level probe, consequently the recovery wells were not sampled. April the stripper was shut down for to replace a switch, approximate downtime was 1 day. During April the System was restarted with new piping in place. TRW 1 and 4 were not operated in April due to drawdown test. All TRW wells were returned to service on May 20, 1998. At well TNX 27D on June 3, 1998, three gallons were purged through the - sample port. P 26D was sampled on August 10, 1998, but samples were not shipped because of historical total activity results. The well was re-sampled on September 8, 1998, and the samples were shipped as non-radioactive. Well TNX 21D had a turbidity reading of 38.9 NTU on December 7, 1998. Water level measurements were not taken at well TRW 3 on October 15, 1998, November 30, 
1998 Comprehensive TNX Area Annual Groundwater

and Effectiveness Monitoring Report (U)

Savannah River Site

May 1999
WSRC-RP-99-4003

Unclassified

Page 33 of 49

1998, or December 16, 1998; the well did not have water in the standpipe. These problems are illustrated in Table 2.

Table 2. Wells not Sampled During 1998

\begin{tabular}{|l|ll|}
\hline Well & Sample Date & Reason \\
\hline TRW 1 & $03 / 31 / 98$ & Inoperable \\
TRW 2 & $03 / 31 / 98$ & Inoperable \\
TRW 3 & $01 / 01-01 / 31 / 98$ & Not running \\
& $02 / 27 / 98$ & Not running \\
& $03 / 31 / 98$ & Inoperable. A scheduled replicate and field blank also \\
& & were not taken \\
TRW 4 & $01 / 01-01 / 31 / 98$ & Not running \\
& $02 / 01-02 / 28 / 98$ & Not running \\
& $03 / 31 / 98$ & Inoperable \\
TRW 1 & No date given - April & Pump inoperable \\
& COC & \\
TRW 2 & No date given - April & Pump inoperable \\
& COC & \\
TRW 3 & No date given - April & Pump inoperable \\
& COC & \\
TRW 4 & No date given - April & Pump inoperable \\
& COC & \\
TNX 11D & $08 / 06 / 98$ & 10 gallons purged through sample port to lower turbidity \\
TNX 27D & $09 / 30 / 98$ & 1 gallon purged through sample port \\
TBG 4 & $12 / 04 / 98$ & Cap cannot be removed \\
TCM 2 & $12 / 04 / 98$ & Water level probe in well \\
TNX 9D & 12/03/98 & Water level probe in well \\
TNX 10D & $12 / 03 / 98$ & Water level probe in well \\
TNX 11D & $12 / 03 / 98$ & Water level probe in well \\
TNX 23D & $12 / 03 / 98$ & Water level probe in well \\
\hline & &
\end{tabular}

Monitoring wells TBG4, TNX9D, TNX10D, and TNX11D are designated primary wells in the Environmental Monitoring Strategy Addendum for the TNX Groundwater Operable Unit RDR/RA Work Plan. The regulators were notified of the missed sampling event, and a make-up sampling event was performed in January/Febuary 1999. The missed sampling event did not compromise the 
1998 Comprehensive TNX Area Annual Groundwater

WSRC-RP-99-4003

and Effectiveness Monitoring Report $(U)$

Unclassified

Savannah River Site

May 1999

Page 34 of 49

ability to ascertain the effectiveness of the T-1 Air Stripper System. This data will be reported in the semi-annual report due in the September 1999 timeframe.

\subsection{ANALYTICAL RESULTS}

Maximum results for analytes that equaled or were above the Final PDWS or groundwater maximum constituent level (MCL) protection standard in sampled wells during 1998 are provided in Table 3 for Primary Wells and Table 4 for Recovery Wells.

Table 3. Constituents Exceeding Applicable Standards in Primary Wells

\begin{tabular}{|c|c|c|c|c|c|c|c|}
\hline Well Name & Constituent & $\mathrm{MCL}$ & Unit & 1Q98 & $2 Q 98$ & $3 Q 98$ & 4Q 98 \\
\hline \multirow[t]{4}{*}{ TBG 1} & Carbon Tetrachloride & 5 & $\mu \mathrm{g} / \mathrm{L}$ & 15 & 19.8 & 34.3 & 26 \\
\hline & Gross Alpha & 15 & $\mathrm{PCi} / \mathrm{mL}$ & -- & 31.68 & 31.96 & 22.14 \\
\hline & Nitrate as Nitrogen & 10000 & $\mu \mathrm{g} / \mathrm{L}$ & -- & --- & 12200 & 13600 \\
\hline & Trichloroethylene & 5 & $\mu \mathrm{g} / \mathrm{L}$ & 3.6 & 6.59 & 8.16 & 14.2 \\
\hline \multirow[t]{6}{*}{ TBG 3} & Carbon Tetrachloride & 5 & $\mu \mathrm{g} / \mathrm{L}$ & 222 & 336 & 408 & 275 \\
\hline & Cis-1,2-Dichloroethylene & 70 & $\mu g / L$ & NA & NA & 111 & 98.6 \\
\hline & Gross Alpha & 15 & $\mathrm{PCi} / \mathrm{mL}$ & -- & 35.8 & 23.48 & 23.58 \\
\hline & Nitrate as Nitrogen & 10000 & $\mu g / L$ & --- & $-\cdots$ & 15400 & 18300 \\
\hline & Tetrachloroethylene & 5 & $\mu \mathrm{g} / \mathrm{L}$ & --- & 8.05 & 12.6 & 14 \\
\hline & Trichloroethylene & 5 & $\mu \mathrm{g} / \mathrm{L}$ & 394 & 1040 & 875 & 488 \\
\hline \multirow[t]{2}{*}{ TBG 5} & Total Organic Halogens & 50 & $\mu \mathrm{g} / \mathrm{L}$ & NA & NA & NA & 747 \\
\hline & Trichloroethylene & 5 & $\mu \mathrm{g} / \mathrm{L}$ & 1710 & 1510 & 844 & 1460 \\
\hline \multirow[t]{3}{*}{ TBG 6} & Carbon Tetrachloride & 5 & $\mu \mathrm{g} / \mathrm{L}$ & 19.7 & 7.07 & $-\cdots$ & --- \\
\hline & Nitrate as Nitrogen & 10000 & $\mu g / L$ & --- & --- & 13400 & 10700 \\
\hline & Trichloroethylene & 5 & $\mu g / L$ & 428 & 800 & 465 & 1450 \\
\hline \multirow[t]{4}{*}{ TNX 3D } & Carbon Tetrachloride & 5 & $\mu \mathrm{g} / \mathrm{L}$ & 28.4 & 31.8 & 26.7 & 74.2 \\
\hline & Cis-1,2-Dichloroethylene & 70 & $\mu \mathrm{g} / \mathrm{L}$ & NA & NA & 22.1 & 192 \\
\hline & Tetrachloroethylene & 5 & $\mu \mathrm{g} / \mathrm{L}$ & 22 & 12.8 & $\cdots$ & 13.5 \\
\hline & Trichloroethylene & 5 & $\mu g / L$ & 176 & 230 & 293 & 442 \\
\hline TNX 4D & $\begin{array}{l}\text { Bis(2-ethylhexyl) } \\
\text { phthalate }\end{array}$ & 6 & $\mu \mathrm{g} / \mathrm{L}$ & NA & NA & -- & 7.74 \\
\hline TNX 8D & Trichloroethylene & 5 & $\mu \mathrm{g} / \mathrm{L}$ & 7.12 & 8.53 & 7.18 & 7.1 \\
\hline TNX 16D & Trichloroethylene & 5 & $\mu \mathrm{g} / \mathrm{L}$ & NA & 45.1 & 49 & 48.4 \\
\hline \multirow[t]{2}{*}{ TNX 27D } & Nitrate as Nitrogen & 10000 & $\mu \mathrm{g} / \mathrm{L}$ & --- & $\therefore$ & 4100 & 21900 \\
\hline & Trichloroethylene & 5 & $\mu \mathrm{g} / \mathrm{L}$ & --- & --- & 7.89 & 99.2 \\
\hline
\end{tabular}




\begin{tabular}{|c|c|c|c|c|c|c|c|}
\hline Well Name & Constituent & MCL & Unit & $1 Q 98$ & $2 Q 98$ & $3 Q 98$ & $4 Q 98$ \\
\hline \multirow[t]{2}{*}{ XSB ID } & $\begin{array}{l}\text { Bis(2-ethylhexyl) } \\
\text { phthalate* }\end{array}$ & 6 & $\mu \mathrm{g} / \mathrm{L}$ & NA & $\mathrm{NA}$ & -- & 8.68 \\
\hline & Trichloroethylene & 5 & $\mu \mathrm{g} / \mathrm{L}$ & 12.4 & 282 & 19 & 21.6 \\
\hline \multirow[t]{2}{*}{ XSB 2D } & $\begin{array}{l}\text { Bis(2-ethylhexyl) } \\
\text { phthalate* }\end{array}$ & 6 & $\mu \mathrm{g} / \mathrm{L}$ & NA & NA & -- & 30.3 \\
\hline & Trichloroethylene & 5 & $\mu \mathrm{g} / \mathrm{L}$ & 21.5 & 31.4 & 15.2 & 22.5 \\
\hline \multirow[t]{2}{*}{$\mathrm{XSB} 3 \mathrm{~A}$} & Total Organic Halogens & 50 & $\mu \mathrm{g} / \mathrm{L}$ & NA & NA & NA & 54.7 \\
\hline & Trichloroethylene & 5 & $\mu \mathrm{g} / \mathrm{L}$ & 7.09 & -- & 12.3 & 63.1 \\
\hline XSB 4D & $\begin{array}{l}\text { Bis(2-ethylhexyl) } \\
\text { phthalate* }\end{array}$ & 6 & $\mu \mathrm{g} / \mathrm{L}$ & NA & NA & --- & 7.54 \\
\hline
\end{tabular}

NOTES: $\quad$ This table presents the highest value for duplicate/replicate results. See Tables B-1 and B-2 for modifiers that may have been applied to these results.

$\mathrm{NA}=$ not analyzed

-- = analyzed but not above PDWS

* = Appendix IX constituent (analyzed Third and Fourth Quarter only).

Results that equaled or exceeded final PDWS may be described as exceeding standards, above standards, or elevated. The final PDWS are used as guidelines in this compliance report to meet regulatory requirements. Constituent results in Tables B-1, B-2, and B-3 that appear to equal the final PDWS but are not marked in the $S T$ column (exceeded standard) are below the final PDWS in the database. Database results, which are the results that are compared to the final PDWS, have more significant digits than the results given in this report. Apparent discrepancies are due to the rounding of reported results.

\subsection{Results for Primary and Recovery Wells}

Among wells identified in the Effectiveness Monitoring Strategy Addendum for the TNX Groundwater Operable Unit RDR/RA Work Plan (WSRC 1996) as monitoring the semi-confined aquifer zone, only the Appendix IX metal Bis(2ethylhexyl) phthalate in wells XSB 1D, 2D, and 4D during the Fourth Quarter exceeded its standard. Discussion of other results above standards for the primary wells follows. Note that for most of the constituents of concern, there is a general 
reduction in frequency of wells containing constituents above standards and in the maximum concentrations of these constituents.

\subsection{Primary Wells, First Quarter 1998}

- Trichloroethylene was elevated in eight wells; The maximum concentration occurred in well TBG $5 \mathrm{~A}$ at $1,710 \mu \mathrm{g} / \mathrm{L}$.

- Carbon tetrachloride was elevated in four wells, with a maximum concentration of $222 \mu \mathrm{g} / \mathrm{L}$ in well TBG 3.

- Gross alpha was elevated in two wells, with a maximum concentration of 14.5 $\mathrm{pCi} / \mathrm{L}$ in well TBG 3.

- Tetrachloroethylene was elevated in well TBG 3D with a concentration of 22 $\mu \mathrm{g} / \mathrm{L}$.

\subsection{Primary Wells, Second Quarter 1998}

- Trichloroethylene was elevated in nine wells. The maximum concentration occurred in well TBG $5 \mathrm{~A}$ at $1,510 \mu \mathrm{g} / \mathrm{L}$.

- Carbon tetrachloride, was elevated in four wells, with a maximum concentration of $336 \mu \mathrm{g} / \mathrm{L}$ in well TBG 3 .

- Gross alpha was elevated in two wells, with a maximum concentration of 35.8 $\mathrm{pCi} / \mathrm{L}$, in well TBG 3.

- tetrachloroethylene was elevated in two wells. TBG 3D has the maximum concentration of $22 \mu \mathrm{g} / \mathrm{L}$. 


\subsection{Primary Wells, Third Quarter 1998}

- Trichloroethylene was elevated in eleven wells. The maximum concentration occurred in well TBG 3 at $875 \mu \mathrm{g} / \mathrm{L}$.

- Nitrate as nitrogen was elevated in four wells, with a maximum concentration at well TBG 3 of $15,400 \mu \mathrm{g} / \mathrm{L}$.

- Carbon tetrachloride was elevated in three wells, with a maximum concentration of $408 \mu \mathrm{g} / \mathrm{L}$ in well TBG 3 .

- Gross alpha was elevated in two wells, with a maximum concentration of $31.96 \mathrm{pCi} / \mathrm{L}$ in well TBG 1.

- Tetrachloroethylene was elevated in well TBG 3 with a concentration of 12.6 $\mu \mathrm{g} / \mathrm{L}$.

\subsection{Primary Wells, Fourth Quarter 1998}

- Trichloroethylene was elevated in eleven wells. The maximum concentration occurred in well TBG 5A at $1,460 \mu \mathrm{g} / \mathrm{L}$.

- Nitrate as nitrogen was elevated in four wells, with a maximum concentration at well TBG 3 of $15,400 \mu \mathrm{g} / \mathrm{L}$.

- Bis(2-ethylhexyl) phthalate was elevated in four wells, with a maximum concentration at XSB 2D with a reading of $30.2 \mu \mathrm{g} / \mathrm{L}$.

- Carbon tetrachloride was elevated in four wells, with a maximum concentration of $275 \mu \mathrm{g} / \mathrm{L}$ in well TBG 3 .

- Gross alpha was elevated in two wells, with a maximum concentration of $23.58 \mathrm{pCi} / \mathrm{L}$ in well TBG 3.

- Mercury total recoverable was elevated in two wells, with a maximum concentration at well XSB $1 \mathrm{~A}$ of $3.44 \mu \mathrm{g} / \mathrm{L}$. 
- Tetrachloroethylene was elevated in two wells; TBG 3 has a maximum concentration of $14 \mu \mathrm{g} / \mathrm{L}$. 


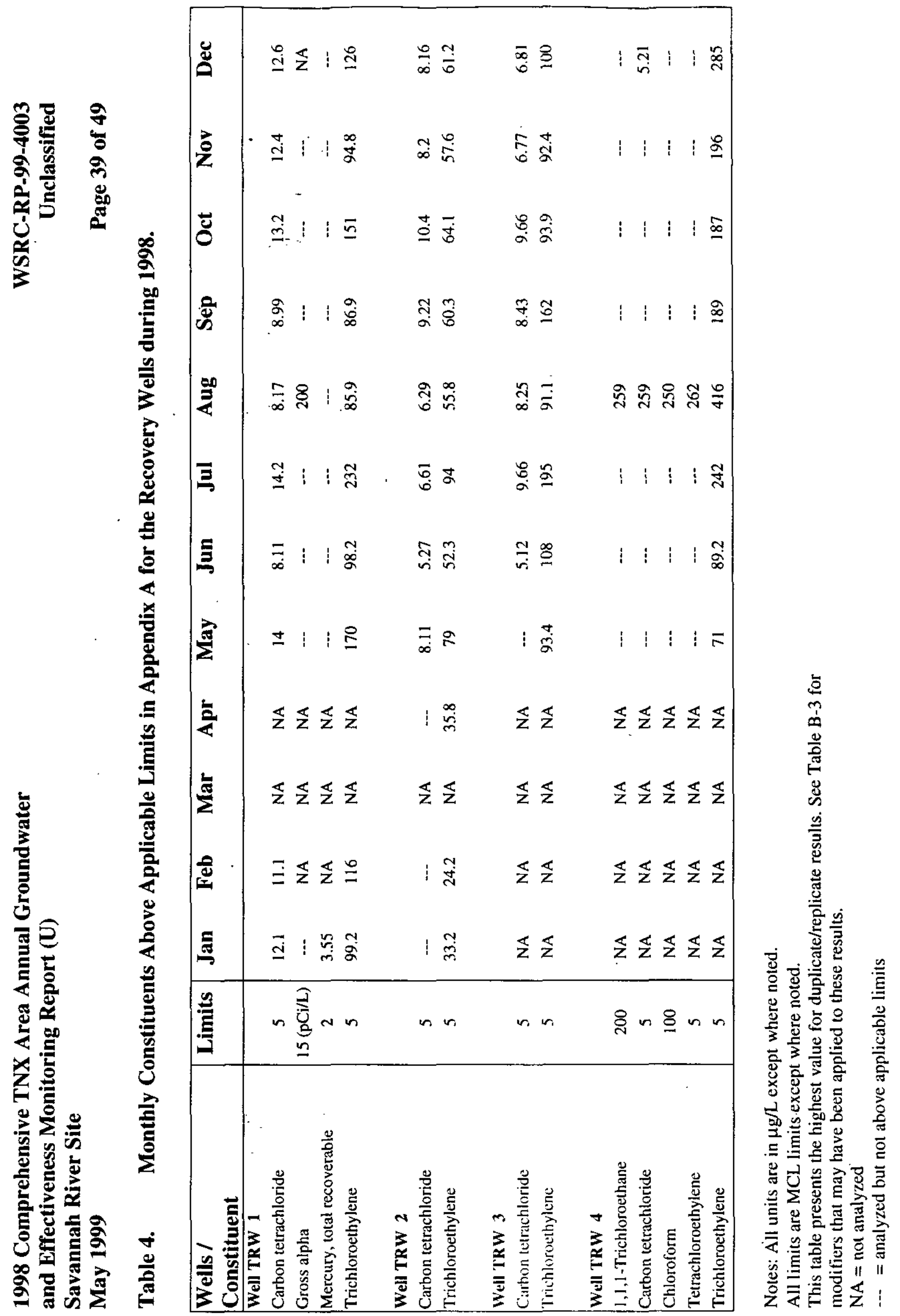




\subsection{Analytical Results for Recovery Wells}

All of the recovery wells exceeded the PDWS for trichloroethylene at every sampling event. The highest value observed was $285 \mu \mathrm{g} / \mathrm{L}$ in well TRW 4 during the Fourth Quarter 1998. Elevated levels of carbon tetrachloride also were seen frequently, with a maximum value for carbon tetrachloride of $10.4 \mu \mathrm{g} / \mathrm{L}$ in well TRW 2 during Fourth Quarter 1998.

\subsection{Hydrographs}

Hydrographs showing water elevation changes over time for TNX area wells in the Addendum to the Effectveness Monitoring Strategy are provided in Appendix $\mathrm{D}$ of this report. The date that pumping started is indicated by a vertical line in each hydrograph, labeled September 16, 1996. The hydrographs were constructed from water level measurements made by both EPD/EMS samplers and SRTC personnel. The EPD/EMS measurements may be found in previous groundwater monitoring reports for TNX Area, EPD/EMS quarterly site-wide groundwater monitoring reports, and the GIMS database. The SRTC measurements are located in Table B-6 of this report:

\subsection{Time-Series Results}

Time-series plots of carbon tetrachloride, nitrate-nitrite, tetrachloroethylene, and trichloroethylene levels for the primary wells from First Quarter 1991 through Fourth Quarter 1998 are in Appendix E. Results for both nitrate and nitrate-nitrite as nitrogen analyses are included in the nitrate-nitrite plots. 


\subsection{SYSTEM PERFORMANCE}

\subsection{Operation and Performance}

The operating history of the T-1 air stripper is summarized in Figure 7. The air stripper operated from the start of pumping in the recovery wells in September 1996 to the end of 1998 with only one unplanned shutdown. The system shut down because of a low air flow alarm. Alarm malfunction was found to be the cause of the problem.

No significant modifications were made to the system. Some minor improvements were made during the outage.

The system treated 62.6 million gallons between September 16, 1996, and January 1, 1999. During calendar year 1998, 30.6 million gallons were treated, representing almost half of the entire production. In 1998, approximately 775 gallons were processed through the purge water tank to the stripper. Performance has continued to be good.

Following initial start-up activities in September 1996, the stripper was operated at approximately $68 \mathrm{gpm}$. With the exception of controls at wells TRW 3 and 4 to prevent these wells from running dry, flow was not restricted. The airflow at this time was approximately $900 \mathrm{ft}^{3} / \mathrm{min}$. During 1998 the stripper operated at an average of approximately $64.6 \mathrm{gpm}$. The airflow during 1998 was approximately $800 \mathrm{ft}^{3} / \mathrm{min}$. 
TNX Air Stripper

and Recovery Well Network

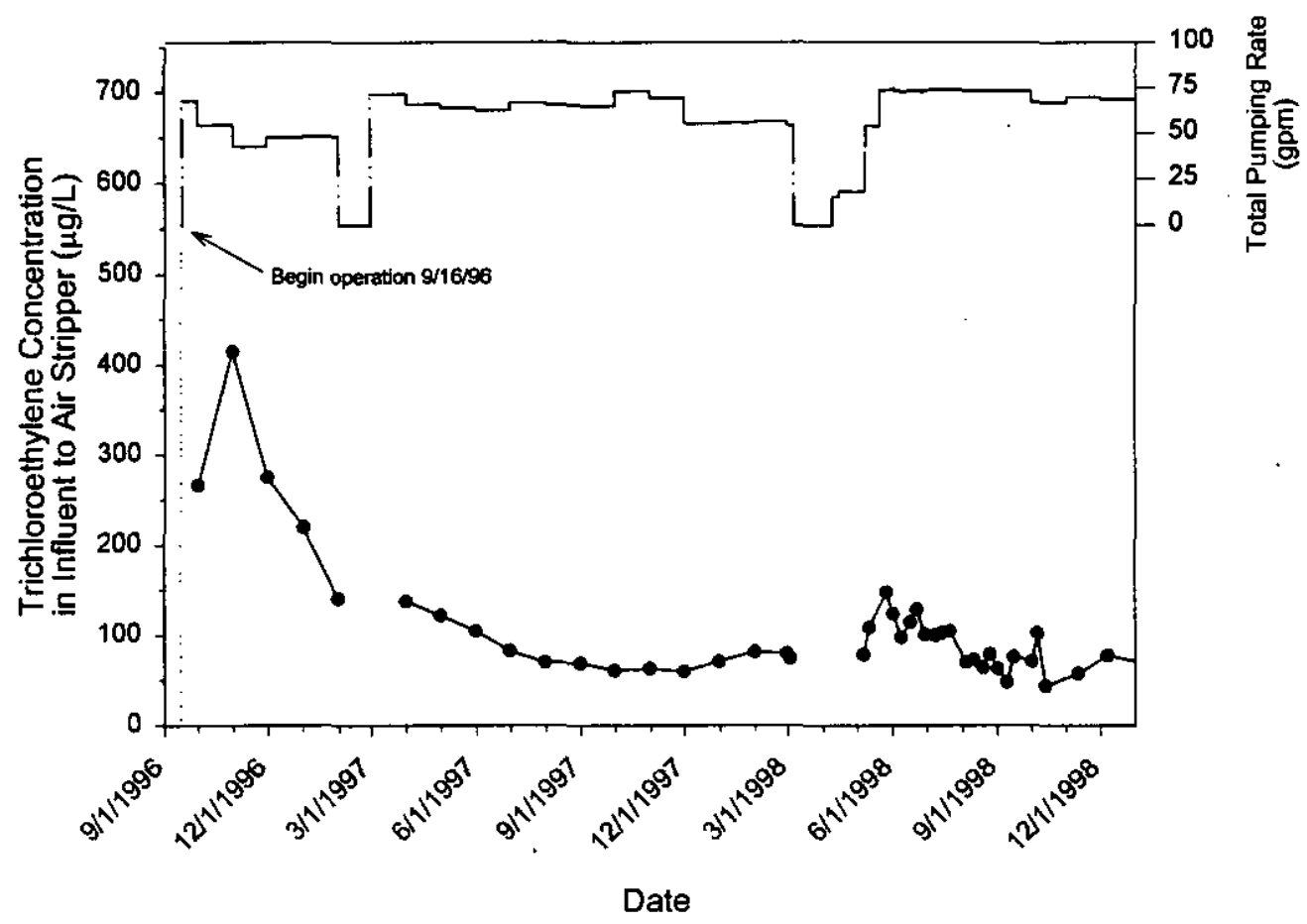

Figure 7. Time series for TNX Air Stripper and Recovery Well Network

The system treated 30.6 million gallons of water during the 1998 timeframe as summarized in Table 5 below. Performance of the system is good with the exception of the minor shutdown described above. On average, approximately 2.6 million gallons were treated in the system per month. 
1998 Comprehensive TNX Area Annual Groundwater and Effectiveness Monitoring Report (U)

Savannah River Site

May 1999
WSRC-RP-99-4003

Unclassified

Page 43 of 49

Table 5. Gallons Treated and Influent Concentrations of Trichloroethylene (TCE) and Carbon Tetrachloride $\left(\mathrm{CCI}_{4}\right)$

\begin{tabular}{|l|c|c|c|c|c|c|}
\hline Month & $\begin{array}{c}\text { Gallons } \\
\text { Treated } \\
\text { (Monthly in } \\
\text { Millions) }\end{array}$ & $\begin{array}{c}\text { Gallons treated } \\
\text { (Cumulative, in } \\
\text { millions) }\end{array}$ & $\begin{array}{c}\text { Influent } \\
\text { Concentration } \\
\text { of TCE }(\mu \mathrm{g} / \mathbf{L})\end{array}$ & $\begin{array}{c}\text { Pounds of } \\
\text { TCE } \\
\text { Removed }\end{array}$ & $\begin{array}{c}\text { Influent } \\
\text { Concentrati } \\
\text { on of CCL } \mathbf{4} \\
(\mu \mathrm{g} / \mathrm{L})\end{array}$ & $\begin{array}{c}\text { Pounds } \\
\text { of CCL } \\
\text { Removed }\end{array}$ \\
\hline Jan-98 & 2.48 & 2.48 & 82 & 1.70 & 13 & 0.27 \\
\hline Feb-98 & 1.97 & 4.45 & 80 & 1.32 & 7 & 0.12 \\
\hline Mar-98 & 0.34 & 4.78 & 74 & 0.21 & 2.5 & 0.01 \\
\hline Apr-98 & 0.61 & 5.38 & 48 & 0.24 & 4 & 0.02 \\
\hline May-98 & 2.56 & 7.95 & 114 & 2.44 & 15 & 0.32 \\
\hline Jun-98 & 3.44 & 11.4 & 113 & 3.24 & 12 & 0.34 \\
\hline Jul-98 & 3.00 & 14.4 & 103 & 2.58 & 11 & 0.28 \\
\hline Aug-98 & 3.62 & 18.0 & 72 & 2.18 & 10 & 0.30 \\
\hline Sep-98 & 2.90 & 20.9 & 62 & 1.50 & 9 & 0.22 \\
\hline Oct-98 & 3.04 & 24.0 & 73 & 1.85 & 8 & 0.20 \\
\hline Nov-98 & 3.32 & 27.3 & 58 & 1.61 & 7 & 0.19 \\
\hline Dec-98 & 3.38 & 30.6 & 78 & 2.20 & 8.9 & 0.25 \\
\hline $\begin{array}{c}\text { Average } \\
\text { Reading for }\end{array}$ & 2.555 & & 79.75 & 1.754 & 8.95 & 0.209 \\
1998 & & & & & & \\
\hline
\end{tabular}

Concentrations in bold are estimated

Concentrations in italics are averages of two or more samples

\subsection{Removal of Groundwater Contaminated with Trichloroethylene}

Approximately 21.1 pounds of trichloroethylene was removed from the groundwater between January 1998 and December 31, 1998, using the recovery well network and air stripper. Currently the average removal of trichloroethylene from the recovery well system is approximately 1.75 pounds per month. This is an increase from last year's monthly average of 1.4 pounds, an increase of $25 \%$ over the previous year.

The trichloroethylene concentration in water collected by the recovery wells initially contained approximately $82 \mu \mathrm{g} / \mathrm{L}$ in January 1998 and decreased to 78 $\mu \mathrm{g} / \mathrm{L}$ in December (see Table 5 above). Table 5 shows that the concentration of 
trichloroethylene averaged $79.75 \mu \mathrm{g} / \mathrm{L}$ for the year. The average of the year is within 5 percent of the concentration reported in January and December of 1998 (see Table 5 above).

\subsection{Removal of Groundwater Contaminated with Carbon Tetrachloride}

Approximately 2.52. pounds of carbon tetrachloride was removed from the groundwater between January 1, 1998, and December 12, 1998, using the recovery well network and air stripper. Currently the average removal of carbon tetrachloride from the recovery well system is approximately 0.21 pounds per month.

The carbon tetrachloride concentration in water collected by the recovery wells initially contained approximately $13 \mu \mathrm{g} / \mathrm{L}$ in January 1998 and decreased to 8.9 $\mu \mathrm{g} / \mathrm{L}$ in December (a drop of approximately 46 percent). Table 5 shows that the concentration of carbon tetrachloride averaged $8.95 \mu \mathrm{g} / \mathrm{L}$ for the year.

\subsection{Containment of Groundwater Contamination}

Operation of the TNX recovery well network significantly altered groundwater flow patterns in the unconfined aquifer beneath the TNX Area. Alterations of the flow patterns are illustrated by comparing Figures 4 and 5 . Figure 4 shows the configuration of the water table before operation of the recovery well system, and Figure 5 shows the configuration of the water table during the operation of the recovery well system in 1998 . The recovery well system produced significant drawdown beneath the TNX Area. The drawdown produced a capture zone that entirely encompasses the groundwater beneath TNX that contains $>500 \mu \mathrm{g} / \mathrm{L}$ of trichloroethylene. The capture zone is illustrated in Figure 5 by the shaded area.

The effect of the IA recovery well pumping may be seen in the changes in water elevation in monitoring wells close to the recovery well since September 1996. 
Water elevations in those monitoring wells prior to September 1998 and in monitoring wells away from the influence of the recovery well system show variations related to rainfall, proximity to streams, outfalls, other physical features, and other factors.

Figure 8 depicts Fourth Quarter 1998 TCE concentrations with the projected Fourth Quarter $1996500 \mathrm{ppb}$ plume superimposed. Analysis indicates that the footprint of the $500 \mathrm{ppb}$ TCE plume has been substantially reduced since operation of the IA.

\subsection{Conclusions}

The T-1 Air Stripper System is working. Table 5 indicates that removal for trichloroethylene and carbon tetrachloride from the groundwater in TNX is proceeding at a rate of 1.75 pounds per month and 0.21 pounds per month, respectively. 


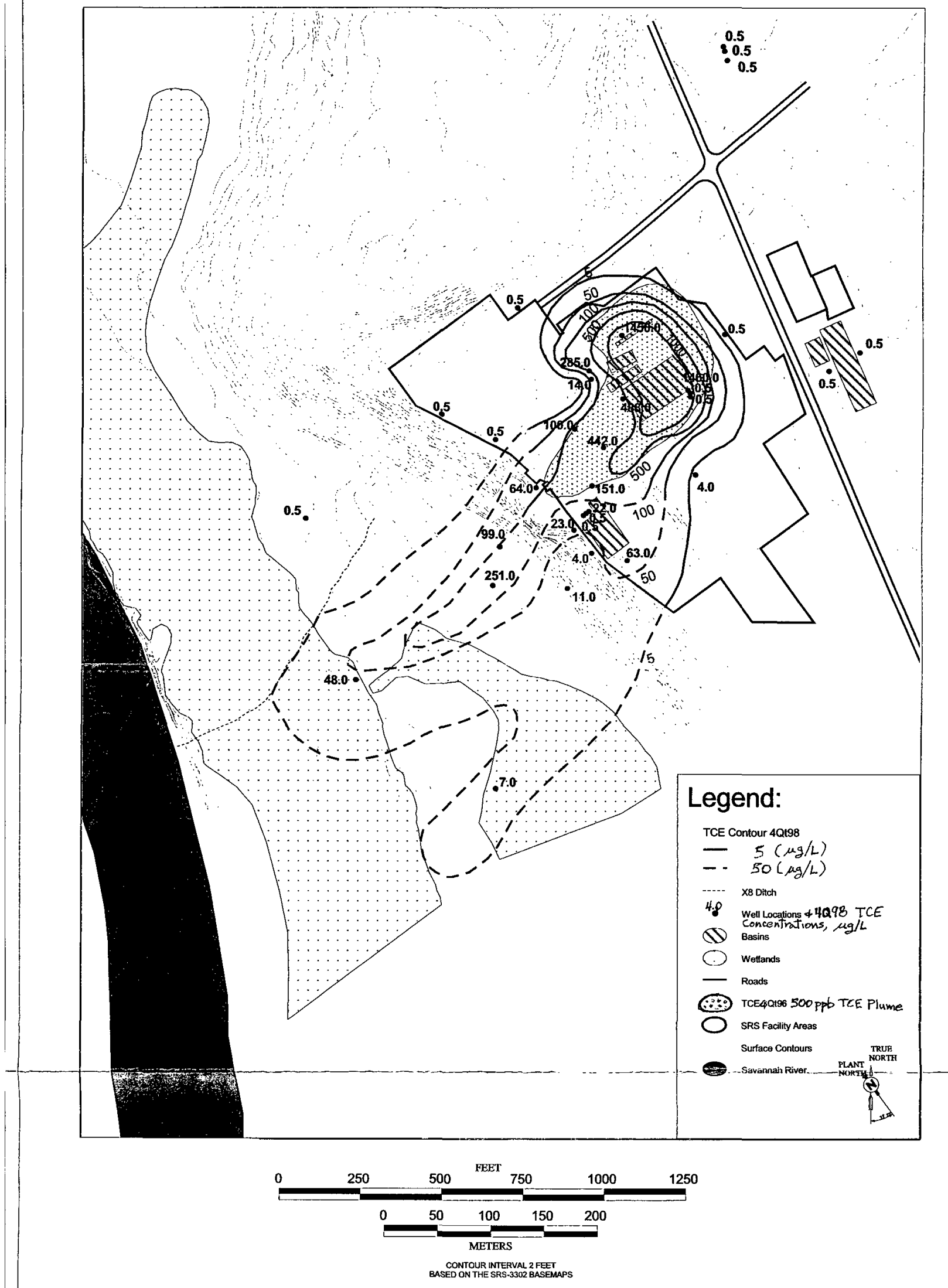

TNX AREA TCE CONCENTRATIONS IN GROUNDWATER WITH PROJECTED 4Q96 500 PPB PLUME SUPERIMPOSED 
Figure 8. TNX Area TCE Concentration in Groundwater, FOURTH . Quarter 1998 WITH Projected 4Q96 500PPB Plume Superimposed 
This page intentionally left blank. 


\section{REFERENCES}

EPA 1996. National Primary Drinking Water Regulations, Code of Federal Regulations, Section 40, Part 141, pp. 592-732, U.S. Environmental Protection Agency, Washington, DC.

Nichols 1993: Characterization of Shallow Groundwater at TNX (U) WSRC-TR92-508. Westinghouse Savannah River Company, Aiken, SC 29802

WSRC, 1994a. 1994 Interim Action Proposed Plan for the TNX Area Operable Unit $(U)$, WSRC-TR-92-229, Rev. 2, August. Westinghouse Savannah River Company, Aiken, SC

WSRC, 1994b. Interim Action Record of Decision, Remedial Alternative Selection

- TNX Area Groundwater Operable Unit (U), WSRC-TR-94-0375, Rev. 1, October. Westinghouse Savannah River Company, Aiken, SC

WSRC 1996 TNX Groundwater Operable Unit Remedial Design Report / Remedial Action Work Plan, $(U)$ WSRC-TR-95-0284 Rev. 1.3, September 24, 1996 Westinghouse Savannah River Company, Aiken, SC

WSRC, 1997a. Post-Construction Report for the TNX Groundwater Operable Unit Interim Remedial Action (U), WSRC-RP-96-0826, Rev. 1, January. Westinghouse Savannah River Company, Aiken, SC

WSRC, 1997b. Explanation of Significant Differences for the TNX Area Groundwater Operable Unit (U), WSRC-RP-97-169, Rev. 1, September Westinghouse Savannah River Company, Aiken, SC 29802 
WSRC, 1997c. Addendum to the TNX Shallow Groundwater Characterization Report (U), WSRC-TR-97-0337, Rev. 0, October. Westinghouse Savannah River Company, Aiken, SC

WSRC, 1998: Phifer, M. A., F. C. Sappington, and M. E. Denham. TNX GeoSiphon Cell (TGSC-1) Phase I Deployment / Demonstration Final Report (U). WSRC-TR-98-00032, Rev. 0. Westinghouse Savannah River Company, Aiken, $\mathrm{SC}$

WSRC, 1999. Phifer, M. A., F. C. Sappington, R. L. Nichols, and K. L. Dixon. TNX GeoSiphon Cell (TGSC-1) Phase II Deployment / Demonstration Final Report $(U)$. WSRC-TR-98-000432, Rev. 0. Westinghouse Savannah River Company, Aiken, SC 
1998 Comprehensive TNX Area Annual Groundwater and Effectiveness Monitoring Report $(U)$

Savannah River Site

May 1999
WSRC-RP-99-4003

Unclassified

\section{APPENDIX A}

Data Review Key and Primary Drinking Water Standards 
This page intentionally left blank.

A - 2 


\section{Data Review Key}

This report contains analytical data for samples taken during 1998 from locations at TNX Area at SRS. The report presents monitoring results that equaled or exceeded the Safe Drinking Water Act final PDWS or screening levels established by US EPA (Appendix A), the South Carolina final PDWS for lead (Appendix A, Table A-1).

\section{Key to Reading the Tables}

The following abbreviations may appear in the data tables:

\section{Constituents}

\section{$1,2,3,4,6,7,8-\mathrm{HPCDD}$ 1,2,3,4,6,7,8-HPCDF $1,2,3,4,7,8-H X C D D$ 1,2,3,4,7,8-HXCDF Lindane PCB}

1,2,3,7,8-PCDD 1,2,3,7,8-PCDF Sp. Conductance TCDD

TCDF

\section{Laboratories}

ES

EX

GE and GP

TM

WA

Sampling Codes

B

$\mathrm{C}$

D

E 1,2,3,4,6,7,8-heptachlorodibenzo-p-dioxin $1,2,3,4,6,7,8$-heptachlorodibenzo-p-furan $1,2,3,4,7,8$-hexachlorodibenzo-p-dioxin 1,2,3,4,7,8-hexachlorodibenzo-p-furan gamma-benzene hexachloride polychlorinated biphenyl 1,2,3,7,8-pentachlorodibenzo-p-dioxin 1,2,3,7,8-pentachlorodibenzo-p-furan specific conductance tetrachlorodibenzo-p-dioxin tetrachlorodibenzo-p-furan
QST Environmental, Inc. EMAX laboratory Services General Engineering Laboratories TMA/Eberline Recra LabNet [formally Roy F. Weston, Inc.] blank sample was collected well was pumping continuously well was dry . equipment blank was collected 


\section{Savannah River Site}

May 1999

L

$\mathbf{P}$

S

$\mathbf{X}$

\section{Sampling Methods}

B

$\mathrm{P}$

$S$

V

\section{Units}

E

$\mathrm{mg} / \mathrm{L}$

$\mathrm{msl}$

MSL

NTU

$\mathrm{pCi} / \mathrm{L}$

$\mathrm{pCi} / \mathrm{mL}$

$\mathrm{pH}$

$\mu \mathrm{g} / \mathrm{L}$

$\mu \mathrm{S} / \mathrm{cm}$ well went dry during sampling; insufficient water to collect all samples

well went dry before sampling began; only depth to water can be determined inaccessibility or mechanical failure prevented sample collection and field analysis of the water no water in standpipe; for water level events only well went dry during purging; samples collected after well recovered sample collected using an open-bucket bailer sample collected using a bladder pump sample collected using a single-speed centrifugal downhole pump sample collected using a variable-speed pump

exponential notation (e.g., $1.1 \mathrm{E}-09=1.1 \times 10^{-9}=$ 0.0000000011 )

milligrams per liter

mean sea level

million structures per liter

turbidity unit

picocuries per liter

picocuries per milliliter

$\mathrm{pH}$ unit

micrograms per liter

microsiemens per centimeter

Other

CS

$\mathrm{D}$

GS .

$\mathrm{H}$

Mod

PDWS

PVC

TOC
Carbon steel

Primary drinking water standard (PDWS) column in data tables

Groundwater protection standard column in data tables

Holding-time column in data tables

Modifier column in data tables

Primary drinking water standard

Polyvinyl chloride

Top of casing 


\section{Holding Times}

Standard analytical methods include a limit, called holding time, on the maximum elapsed time between sample collection and extraction or analysis by the laboratory. In the data tables, a $Q$ on the right side of the modifier column indicates that holding time was exceeded. Analyses performed beyond holding times may not yield valid results.

SCDHEC allows only 15 minutes to elapse between sampling and analysis for $\mathrm{pH}$. Thus only field $\mathrm{pH}$ measurements can meet the holding time criterion; laboratory $\mathrm{pH}$ analyses always will exceed it.

The laboratory procedure used to determine specific conductance allows one day to elapse between sampling and analysis. Thus laboratory specific conductance measurements may exceed the holding time criterion.

\section{Data Modifiers}

The contract laboratories continually assess their own accuracy and precision according to US EPA guidelines. They submit sample- or batch-specific quality assurance/quality control information either at the same time as they submit analytical results or they submit them in a quarterly summary. Properly defined and used modifiers (also referred to as qualifiers) can be a key component in assessing data usability. Modifiers designed by the EPD/EMS and used by the primary laboratories up to October 1, 1998, are defined below. After October 1, 1998, the US EPA Functional Guidelines Codes and EPA STORET Codes definitions were adopted. These modifiers appear in the data tables under the column Mod. 
Codes for modifiers on left side of column:

Result Qualifiers .

\begin{tabular}{|c|c|}
\hline Modifie & Qualifiers before 10/1/98 \\
\hline (blank) & Data not remarked. Number should be interpreted exactly as reported. \\
\hline I & $\begin{array}{l}\text { The value in the result field is the instrument reading, not the sample quantification } \\
\text { limit. Always used with the result qualifier } U \text {. }\end{array}$ \\
\hline $\mathbf{J}$ & The analytical result is an estimated quantity. \\
\hline L & $\begin{array}{l}\text { Off-scale high. The actual value is not known but is known to be greater than } \\
\text { value shown. }\end{array}$ \\
\hline $\mathbf{R}$ & $\begin{array}{l}\text { Rejected because performance requirements in the sample or associated quality } \\
\text { control analyses were not met. The analyte may or may not be present. }\end{array}$ \\
\hline $\mathrm{U}$ & $\begin{array}{l}\text { Material analyzed for but not detected. Analytical result reported is less than the } \\
\text { sample quantitation limit. }\end{array}$ \\
\hline
\end{tabular}

EPA Functional Guidelines Codes [formerly Result Qualifiers] (EPA 1994b and 1994c)

\begin{tabular}{|l|l|}
\hline Modifier & Qualifiers after 10/198 \\
\hline (Blank) & Data not remarked. The analytical result is acceptable for use as reported. \\
\hline$J$ & $\begin{array}{l}\text { The analyte was positively identified; the associated numerical value is an estimated } \\
\text { concentration of the analyte in the sample. }\end{array}$ \\
\hline$N$ & $\begin{array}{l}\text { The analysis indicates the presence of an analyte for which there is presumptive evidence to make } \\
\text { a tentative identification. Use for all TIC results. }\end{array}$ \\
\hline$R$ & $\begin{array}{l}\text { The sample results are rejected due to serious deficiencies in the ability to analyze the sample and } \\
\text { meet quality control criteria. The presence or absence of the analyte cannot be verified. }\end{array}$ \\
\hline$U$ & $\begin{array}{l}\text { Material analyzed for but not detected. The analyte concentration is <ssEQL. } \\
\text { The analysis indicates the presence of an analyte that has been tentatively identified and the } \\
\text { associated numerical value represents its approximate concentration. }\end{array}$ \\
\hline$U J$ & $\begin{array}{l}\text { The analyte was not detected above the reported sample quantitation limit. The reported } \\
\text { quantitation limit is approximate and may not represent the actual limit of quantitation necessary } \\
\text { to accurately and precisely measure the analyte in the sample. }\end{array}$ \\
\hline
\end{tabular}


Codes for modifiers on right side of column;

\begin{tabular}{|c|c|}
\hline $\begin{array}{l}\text { Analysis } \\
\text { Qualifiers } \\
\text { Modifier }\end{array}$ & Qualifiers before 10/1/98 \\
\hline $\mathrm{E}$ & $\begin{array}{l}\text { The detected result is between the sample-specific EQL and the method } \\
\text { detection limit. Report the actual result detected. }\end{array}$ \\
\hline I & $\begin{array}{l}\text { Matrix Spike/Matrix Spike Duplicate (VI, Organics FG; VI, Pesticides) } \\
\text { Matrix Spike Sample Analysis (VII, Inorganics FG) } \\
\text { Spike recovery not within control limits. Use alone or with } J \text { or } R \text {. }\end{array}$ \\
\hline $\mathrm{K}$ & $\begin{array}{l}\text { Tentatively Identified Compounds (TICs) (IX, Organics FG) } \\
\text { A tentatively identified compound is a suspected aldol-condensation product. } \\
\text { Use with both } J \text { and } N \text {. }\end{array}$ \\
\hline $\mathrm{L}$ & $\begin{array}{l}\text { Calibration Criteria Not Met (III, Organics FG; III, Pesticides FG; II, } \\
\text { Inorganics FG) } \\
\text { Calibration criteria (initial or continuing) were not met. Use with } J \text { or } R \text {. See } \\
\text { also } Z \text { for inorganics. }\end{array}$ \\
\hline Q & $\begin{array}{l}\text { Holding Time (I, Organics FG; I, Pesticides FG; I, Inorganics FG) } \\
\text { Sample held beyond normal holding time. In addition, if the holding time is } \\
\text { exceeded by less than } 30 \text { days, use a } J \text {; if the holding time is exceeded by } 30 \\
\text { days or more, use an } R \text {. }\end{array}$ \\
\hline $\mathrm{V}$ & $\begin{array}{l}\text { Laboratory Blanks Contaminated (IV, Organics FG; IV, Pesticides FG; III, } \\
\text { Inorganics FG) } \\
\text { Indicates the analyte was detected in both the sample and associated method } \\
\text { blank. Use with the result qualifier } V \text { for organics or pesticides if the sample } \\
\text { falls within the } 5 \text { times and } 10 \text { times rule for organics or the } 5 \text { times rule for } \\
\text { pesticides and inorganics. Report the actual result detected. }\end{array}$ \\
\hline
\end{tabular}


Savannah River Site

May 1999

EPA STORET Codes [formerly Analysis Qualifiers] (EPA 1992)

\begin{tabular}{|l|l|}
\hline Modifier & Qualifiers after 10/1/98 \\
\hline$($ Blank $)$ & Data not remarked. \\
\hline$I$ & The result is less than the ssEQL, but equal to or greater than the MDL. \\
\hline$J$ & The result is estimated. \\
\hline$K$ & The actual concentration is known to be less than the reported result. \\
\hline$L$ & The actual concentration is known to be greater than the reported result. \\
\hline$Q$ & The sample was held beyond the normal holding time prior to analysis. \\
\hline$V$ & The analyte was detected in both the method blank and the sample. \\
\hline
\end{tabular}


Table A-1. Drinking Water Standards or Maximum Concentration Levels

\begin{tabular}{|c|c|c|c|c|c|}
\hline Analyte & & Unit & Level & Status & Source \\
\hline Alachlor & & $\mu \mathrm{g} / \mathrm{L}$ & 2 & Final & EPA 1996 \\
\hline Aldicarb ${ }^{a}$ & & $\mu g / L$ & 3 & Final & EPA 1996 \\
\hline Aldicarb sulfone & & $\mu \mathrm{g} / \mathrm{L}$ & 2 & Final & EPA 1996 \\
\hline Aldicarb sulfoxide " & & $\mu \mathrm{g} / \mathrm{L}$ & 4 & Final & EPA 1996 \\
\hline Antimony & & $\mu \mathrm{g} / \mathrm{L}$ & 6 & Final & EPA 1996 \\
\hline Arsenic & & $\mu g / L$ & 50 & Final & EPA 1996 \\
\hline Asbestos & & Fibers/L & $7,000,000$ & Final & EPA 1996 \\
\hline Atrazine & $\cdot$ & $\mu \mathrm{g} / \mathrm{L}$ & 3 & Final & EPA 1996 \\
\hline Barium & & $\mu g / L$ & 2,000 & Final & EPA 1996 \\
\hline Benzene & & $\mu \mathrm{g} / \mathrm{L}$ & 5 & Final & EPA 1996 \\
\hline Benzo[a]pyrene & & $\mu \mathrm{g} / \mathrm{L}$ & 0.2 & Final & EPA 1996 \\
\hline Beryllium & & $\mu \mathrm{g} / \mathrm{L}$ & 4 & Final & EPA 1996 \\
\hline Bis(2-ethylhexyl) phthalate & & $\mu \mathrm{g} / \mathrm{L}$ & 6 & Final & EPA 1996 \\
\hline Bromodichloromethane & & $\mu \mathrm{g} / \mathrm{L}$ & 100 & Final & EPA 1996 \\
\hline Bromoform & & $\mu \mathrm{g} / \mathrm{L}$ & 100 & Final & EPA 1996 \\
\hline 2-sec-Butyl-4,6-dinitrophenol & & $\mu \mathrm{g} / \mathrm{L}$ & 7 & Final & EPA 1996 \\
\hline Cadmium & & $\mu \mathrm{g} / \mathrm{L}$ & 5 & Final & EPA 1996 \\
\hline Carbofuran & & $\mu g / L$ & 40 & Final & EPA 1996 \\
\hline Carbon tetrachloride & & $\mu \mathrm{g} / \mathrm{L}$ & 5 & Final & EPA 1996 \\
\hline Chlordane & & $\mu \mathrm{g} / \mathrm{L}$ & 2 & Final & EPA 1996 \\
\hline Chlorobenzene & & $\mu \mathrm{g} / \mathrm{L}$ & 100 & Final & EPA 1996 \\
\hline Chloroethene (Vinyl chloride) & & $\mu g / L$ & 2 & Final & EPA 1996 \\
\hline Chloroform & & $\mu \mathrm{g} / \mathrm{L}$ & 100 & Final & EPA 1996 \\
\hline Chromium & & $\mu \mathrm{g} / \mathrm{L}$ & 100 & Final & EPA 1996 \\
\hline Copper & & $\mu \mathrm{g} / \mathrm{L}$ & 1,000 & Final & SCDHEC 1981 \\
\hline Cyanide & & $\mu \mathrm{g} / \mathrm{L}$ & 200 & Final & EPA 1996 \\
\hline Dalapon $^{a}$ & & $\mu \mathrm{g} / \mathrm{L}$ & 200 & Final & EPA 1996 \\
\hline Dibromochloromethane & & $\mu g / L$ & 100 & Final & EPA 1996 \\
\hline 1,2-Dibromo-3-chloropropane & & $\mu \mathrm{g} / \mathrm{L}$ & 0.2 & Final & EPA 1996 \\
\hline 1,2-Dibromoethane & & $\mu g / L$ & 0.05 & Final & EPA 1996 \\
\hline 1,2-Dichlorobenzene & & $\mu \mathrm{g} / \mathrm{L}$ & 600 & Final & EPA 1996 \\
\hline 1,4-Dichlorobenzene & & $\mu \mathrm{g} / \mathrm{L}$ & 75 & Final & EPA 1996 \\
\hline 1,2-Dichloroethane & & $\mu \mathrm{g} / \mathrm{L}$ & 5 & Final & EPA 1996 \\
\hline 1,1-Dichloroethylene & & $\mu \mathrm{g} / \mathrm{L}$ & 7 & Final & EPA 1996 \\
\hline 1,2-Dichloroethylene & & $\mu \mathrm{g} / \mathrm{L}$ & 50 & Final & EPA 1996 \\
\hline cis-1,2-Dichloroethylene & & $\mu \mathrm{g} / \mathrm{L}$ & 70 & Final & EPA 1996 \\
\hline trans-1,2-Dichloroethylene & & $\mu \mathrm{g} / \mathrm{L}$ & 100 & Final & EPA 1996 \\
\hline Dichloromethane (Methylene chloride) & & $\mu \mathrm{g} / \mathrm{L}$ & 5 & Final & EPA 1996 \\
\hline 2,4-Dichlorophenoxyacetic acid & & $\mu \mathrm{g} / \mathrm{L}$ & 70 & Final & EPA 1996 \\
\hline 1,2-Dichloropropane - & & $\mu \mathrm{g} / \mathrm{L}$ & 5 & Final & EPA 1996 \\
\hline Di(2-ethylhexyl) adipate ${ }^{a}$ & & $\mu \mathrm{g} / \mathrm{L}$ & 400 & Final & EPA 1996 \\
\hline Diquat dibromide & & $\mu \mathrm{g} / \mathrm{L}$ & 20 & Final & EPA 1996 \\
\hline Endothall ${ }^{a}$ & & $\mu \mathrm{g} / \mathrm{L}$ & 100 & Final & EPA 1996 \\
\hline Endrin & ${ }^{\circ}$ & $\mu \mathrm{g} / \mathrm{L}$ & 2 & Final & EPA 1996 \\
\hline Ethylbenzene & & $\mu \mathrm{g} / \mathrm{L}$ & 700 & Final & EPA 1996 \\
\hline Fluoride & & $\mu \mathrm{g} / \mathrm{L}$ & 4,000 & Final & EPA 1996 \\
\hline
\end{tabular}


1998 Comprehensive TNX Area Annual Groundwater

WSRC-RP-99-4003

and Effectiveness Monitoring Report (U)

Unclassified

Savannah River Site

May 1999

\begin{tabular}{|c|c|c|c|c|}
\hline Analyte & Unit & Level & Status & Source \\
\hline Glyphosate & $\mu \mathrm{g} / \mathrm{L}$ & 700 & Final & EPA 1996 \\
\hline Gross alpha ${ }^{b}$ & $\mathrm{pCi} / \mathrm{L}$ & $1.5 \mathrm{E}+01$ & Final & EPA 1996 \\
\hline Heptachlor & $\mu g / L$ & 0.4 & Final & EPA 1996 \\
\hline Heptachlor epoxide & $\mu \mathrm{g} / \mathrm{L}$ & 0.2 & Final & EPA 1996 \\
\hline Hexachlorobenzene & $\mu \mathrm{g} / \mathrm{L}$ & 1 & Final & EPA 1996 \\
\hline Hexachlorocyclopentadiene & $\mu \mathrm{g} / \mathrm{L}$ & 50 & Final & EPA 1996 \\
\hline Lead & $\mu \mathrm{g} / \mathrm{L}$ & 50 & Final & SCDHEC 1981 \\
\hline Lindane & $\mu \mathrm{g} / \mathrm{L}$ & 0.2 & Final & EPA 1996 \\
\hline Mercury & $\mu \mathrm{g} / \mathrm{L}$ & 2 & Final & EPA 1996 \\
\hline Methoxychlor & $\mu \mathrm{g} / \mathrm{L}$ & .40 & Final & EPA 1996 \\
\hline Nickel & $\mu \mathrm{g} / \mathrm{L}$ & 100 & Final & EPA 1996 \\
\hline Nitrate as nitrogen & $\mu \mathrm{g} / \mathrm{L}$ & 10,000 & Final & EPA 1996 \\
\hline Nitrate-nitrite as nitrogen & $\mu \mathrm{g} / \mathrm{L}$ & 10,000 & Final & EPA 1996 \\
\hline Nitrite as nitrogen & $\mu g / L$ & 1,000 & Final & EPA 1996 \\
\hline Nonvolatile beta & $\mathrm{pCi} / \mathrm{L}$ & $5 \mathrm{E}+01$ & Interim Final & EPA 1977 \\
\hline Oxamyl $^{a}$ & $\mu g / L$ & 200 & Final & EPA 1996 \\
\hline PCB 1016 & $\mu \mathrm{g} / \mathrm{L}$ & 0.5 & Final & EPA 1996 \\
\hline PCB I 221 & $\mu \mathrm{g} / \mathrm{L}$ & 0.5 & Final & EPA 1996 \\
\hline PCB 1232 & $\mu \mathrm{g} / \mathrm{L}$ & 0.5 & Final & EPA 1996 \\
\hline PCB 1242 & $\mu \mathrm{g} / \mathrm{L}$ & 0.5 & Final & EPA 1996 \\
\hline РCB 1248 & $\mu g / L$ & 0.5 & Final & EPA 1996 \\
\hline PCB 1254 & $\mu g / L$ & 0.5 & Final & EPA 1996 \\
\hline PCB 1260 & $\mu \mathrm{g} / \mathrm{L}$ & 0.5 & Final & EPA 1996 \\
\hline PCB 1262 & $\mu \mathrm{g} / \mathrm{L}$ & 0.5 & Final & EPA 1996 \\
\hline Pentachlorophenol & $\mu \mathrm{g} / \mathrm{L}$ & 1 & Final & EPA 1996 \\
\hline Picloram $^{2}$ & $\mu \mathrm{g} / \mathrm{L}$ & 500 & Final & EPA 1996 \\
\hline Selenium & $\mu \mathrm{g} / \mathrm{L}$ & 50 & Final & EPA 1996 \\
\hline Simazine ${ }^{2}$ & $\mu \mathrm{g} / \mathrm{L}$ & 4 & Final & EPA 1996 \\
\hline Strontium-89/90 ${ }^{\mathrm{c}}$ & $\mathrm{pCi} / \mathrm{L}$ & $8 \mathrm{E}+00$ & Final & EPA 1996 \\
\hline Strontium-90 & $\mathrm{pCi} / \mathrm{L}$ & $8 \mathrm{E}+00$ & Final & EPA 1996 \\
\hline Styrene & $\mu \mathrm{g} / \mathrm{L}$ & 100 & Final & EPA 1996 \\
\hline $2,3,7,8-\mathrm{TCDD}$ & $\mu \mathrm{g} / \mathrm{L}$ & 0.00003 & Final & EPA 1996 \\
\hline Tetrachloroethylene & $\mu \mathrm{g} / \mathrm{L}$ & 5 & Final & EPA 1996 \\
\hline Thallium & $\mu \mathrm{g} / \mathrm{L}$ & 2 & Final & EPA 1996 \\
\hline Toluene & $\mu \mathrm{g} / \mathrm{L}$ & 1,000 & Final & EPA 1996 \\
\hline Toxaphene & $\mu \mathrm{g} / \mathrm{L}$ & 3 & Final & EPA 1996 \\
\hline 2,4,5-TP (Silvex) & $\mu \mathrm{g} / \mathrm{L}$ & 50 & Final & EPA 1996 \\
\hline 1,2,4-Trichlorobenzene & $\mu \mathrm{g} / \mathrm{L}$ & 70 & Final & EPA 1996 \\
\hline 1,1,1-Trichloroethane & $\mu \mathrm{g} / \mathrm{L}$ & 200 & Final & EPA 1996 \\
\hline 1,1,2-Trichloroethane & $\mu \mathrm{g} / \mathrm{L}$ & 5 & Final & EPA 1996 \\
\hline Trichloroethylene & $\mu \mathrm{g} / \mathrm{L}$ & 5 & Final & EPA 1996 \\
\hline Tritium & $\mathrm{pCi} / \mathrm{mL}$ & $2 \mathrm{E}+01$ & Final & EPA' 1996 \\
\hline Xylenes & $\mu g / L$ & 10,000 & Final & EPA 1996 \\
\hline
\end{tabular}

Note: Final PDWS were assigned to alachlor, aldicarb, aldicarb sulfone, aldicarb sulfoxide, atrazine, carbofuran, dalapon, di(2-ethylhexyl) adipate, diquat dibromide, endothall, glyphosate, oxamyl, picloram, and simazine in the SRS Groundwater Monitoring Program for the first time beginning First Quarter 1994.

at present, EMS does not perform this analysis because the constituent is not in the current contract.

b The standard given is for gross alpha including radium-226 but excluding radon and uranium.

$$
\text { A }-8
$$




\section{Savannah River Site}

May 1999

c For double radionuclide analyses where each separate radionuclide has its own standard, the more stringent standard is used.

\section{Flagging Criteria}

The Savannah River Site EPD/EMS flagging criteria are as follows:

- Flag 2 criteria for constituents equal the Safe Drinking Water Act (SDWA) final Primary Drinking Water Standards (PDWS), the SDWA proposed PDWS, or the SDWA Secondary Drinking Water Standards (SDWS). If a constituent does not have a drinking water standard, the Flag 2 criterion equals 10 times the method detection limit (MDL) calculated as the 90th percentile detection limit obtained recently by one of the primary analytical laboratories.

- Flag 1 criteria for constituents equal one-half of the final PDWS, one-half the proposed PDWS, or one-half the SDWS. If a constituent does not have a drinking water standard, the Flag 1 criterion equals 5 times the MDL calculated as the 90th percentile detection limit obtained recently by one of the primary analytical laboratories.

- Flag 0 criteria are assigned to constituent levels below Flag 1 criteria, constituent levels below the sample detection limits, or constituents having no flagging criteria.

The following parameters are exceptions to the flagging rules:

- EPD/EMS sets flagging criteria for specific conductance and $\mathrm{pH}$. No flags are set for alkalinity, calcium, carbonate, magnesium, potassium, silica, sodium, total dissolved solids, total phosphates (as P), and total phosphorus. Analyses for these parameters are conducted as part of the biennial comprehensive analyses or by special request.

- Aesthetic parameters such as color, corrosivity, $\mathrm{pH}$, odor, surfactants, and turbidity are not assigned flagging criteria but are analyzed by special request.

- Common laboratory contaminants and cleaners such as dichloromethane (methylene chloride), ketones, phthalates, and toluene are not assigned flagging criteria unless they have primary drinking water standards. These constituents are analyzed by special request. 


\section{Analyte}

Acenaphthene

Acenaphthylene

Acetone

Acetonitrile (Methyl cyanide)

Acetophenone

2-Acetylaminofluorene

Acrolein

Acrylonitrile

Actinium-228

Alachlor

Aldicarb $^{b}$

Aldicarb sulfone ${ }^{b}$

Aldicarb sulfoxide ${ }^{b}$

Aldrin

Alkalinity (as $\mathrm{CaCO}_{3}$ )

Allyl chloride

Aluminum

Aluminum, dissolved

Aluminum, total recoverable

Americium-241

Americium-243

4-Aminobiphenyl

Ammonia

Ammonia nitrogen

Aniline

Anthracene

Antimony

Antimony, dissolved

Antimony, total recoverable

Antimony-125

Aramite

Arsenic

Assenic, dissolved

Arsenic, total recoverable

Asbestos

Atrazine

Azobenzene

Barium

Barium, dissolved

Barium, total recoverable

Barium- $140^{\circ}$

Benzene

alpha-Benzene hexachloride

beta-Benzene hexachloride

delta-Benzene hexachloride

Benzidine

Benzo[ $a]$ anthracene

Benzo[b]fluoranthene

Benzo[ $k]$ fluoranthene

Benzoic acid

Benzo $[g, h, i]$ perylene

Benzo[a]pyrene

\begin{tabular}{|c|c|c|c|}
\hline$\underline{\text { Unit }}$ & Flag 1 & Flag 2 & $\underline{\text { Source }}^{2}$ \\
\hline$\mu \mathrm{g} / \mathrm{L}$ & 50 & 100 & EPA Method 8270 \\
\hline$\mu \mathrm{g} / \mathrm{L}$ & 50 & 100 & EPA Method 8270 \\
\hline$\mu \mathrm{g} / \mathrm{L}$ & 500 & 1,000 & EPA Method 8240 \\
\hline$\mu \mathrm{g} / \mathrm{L}$ & 500 & 1,000 & EPA Method 8240 \\
\hline$\mu \mathrm{g} / \mathrm{L}$ & 50 & 100 & EPA Method 8270 \\
\hline$\mu \mathrm{g} / \mathrm{L}$ & 50 & 100 & EPA Method 8270 \\
\hline$\mu \mathrm{g} / \mathrm{L}$ & 100 & 200 & EPA Method 8240 \\
\hline$\mu \mathrm{g} / \mathrm{L}$ & 100 & 200 & EPA Method 8240 \\
\hline $\mathrm{pCi} / \mathrm{L}$ & $1.64 \mathrm{E}+03$ & $3.27 \mathrm{E}+03$ & Proposed PDWS (EPA 1991) \\
\hline$\mu g / L$ & 1 & 2 & Final PDWS (EPA 1993a) \\
\hline$\mu \mathrm{g} / \mathrm{L}$ & 1.5 & 3 & Final PDWS (EPA 1993a) \\
\hline$\mu \mathrm{g} / \mathrm{L}$ & 1 & 2 & Final PDWS (EPA 1993a) \\
\hline$\mu \mathrm{g} / \mathrm{L}$ & 2 & 4 & Final PDWS (EPA 1993a) \\
\hline \multirow[t]{2}{*}{$\mu \mathrm{g} / \mathrm{L}$} & 0.25 & 0.5 & EPA Method 8080 \\
\hline & No flag & No flag & Set by EPD/EMS \\
\hline$\mu g / L$ & 250 & 500 & EPA Method 8240 \\
\hline$\mu \mathrm{g} / \mathrm{L}$ & 25 & 50 & SDWS (EPA, 1993b) \\
\hline$\mu \mathrm{g} / \mathrm{L}$ & 25 & 50 & SDWS (EPA, 1993b) \\
\hline$\mu g / L$ & 25 & 50 & SDWS (EPA, 1993b) \\
\hline $\mathrm{pCi} / \mathrm{L}$ & $3.17 \mathrm{E}+00$ & $6.34 \mathrm{E}+00$ & Proposed PDWS (EPA 1991) \\
\hline $\mathrm{pCi} / \mathrm{L}$ & $3.19 \mathrm{E}+00$ & $6.37 \mathrm{E}+00$ & Proposed PDWS (EPA 1991) \\
\hline$\mu \mathrm{g} / \mathrm{L}$ & 50 & 100 & EPA Method 8270 \\
\hline$\mu \mathrm{g} / \mathrm{L}$ & 500 & 1,000 & APHA Method 417B \\
\hline$\mu \mathrm{g} / \mathrm{L}$ & 500 & 1,000 & EPA Method 350.1 \\
\hline$\mu \mathrm{g} / \mathrm{L}$ & 50 & 100 & EPA Method 8270 \\
\hline$\mu \mathrm{g} / \mathrm{L}$ & 50 & 100 & EPA Method 8270 \\
\hline$\mu \mathrm{g} / \mathrm{L}$ & 3 & 6 & Final PDWS (EPA 1993a) \\
\hline$\mu \mathrm{g} / \mathrm{L}$ & 3 & 6 & Final PDWS (EPA 1993a) \\
\hline$\mu g / L$ & 3 & 6 & Final PDWS (EPA 1993a) \\
\hline $\mathrm{pCi} / \mathrm{L}$ & $1.5 \mathrm{E}+02$ & $3 E+02$ & Interim Final PDWS (EPA, 1977) \\
\hline$\mu \mathrm{g} / \mathrm{L}$ & 50 & 100 & EPA Method 8270 \\
\hline$\mu \mathrm{g} / \mathrm{L}$ & 25 & 50 & Final PDWS (EPA 1993a) \\
\hline$\mu \mathrm{g} / \mathrm{L}$ & 25 & 50 & Final PDWS (EPA 1993a) \\
\hline$\mu \mathrm{g} / \mathrm{L}$ & 25 & 50 & Final PDWS (EPA 1993a) \\
\hline Fibers/L & $3,500,000$ & $7,000,000$ & Final PDWS (EPA 1993a) \\
\hline$\mu \mathrm{g} / \mathrm{L}$ & 1.5 & 3 & Final PDWS (EPA 1993a) \\
\hline$\mu \mathrm{g} / \mathrm{L}$ & 50 & 100 & EPA Method 625 \\
\hline$\mu g / L$ & 1,000 & 2,000 & Final PDWS (EPA 1993a) \\
\hline$\mu \mathrm{g} / \mathrm{L}$ & 1,000 & 2,000 & Final PDWS (EPA 1993a) \\
\hline$\mu \mathrm{g} / \mathrm{L}$ & 1,000 & 2,000 & Final PDWS (EPA 1993a) \\
\hline $\mathrm{pCi} / \mathrm{L}$ & $4.5 E+01$ & $9 \mathrm{E}+01$ & Interim Final PDWS (EPA 1977) \\
\hline$\mu \mathrm{g} / \mathrm{L}$ & 2.5 & 5 & Final PDWS (EPA 1993a) \\
\hline$\mu \mathrm{g} / \mathrm{L}$ & 0.25 & 0.5 & EPA Method 8080 \\
\hline$\mu \mathrm{g} / \mathrm{L}$ & 0.25 & 0.5 & EPA Method 8080 \\
\hline$\mu \mathrm{g} / \mathrm{L}$ & 0.25 & 0.5 & EPA Method 8080 \\
\hline$\mu \mathrm{g} / \mathrm{L}$ & 250 & 500 & EPA Method 8270 \\
\hline$\mu \mathrm{g} / \mathrm{L}$ & 0.05 & 0.1 & Proposed PDWS (EPA 1990) \\
\hline$\mu \mathrm{g} / \mathrm{L}$ & 0.1 & 0.2 & Proposed PDWS (EPA 1990) \\
\hline$\mu \mathrm{g} / \mathrm{L}$ & 0.1 & 0.2 & Proposed PDWS (EPA 1990) \\
\hline$\mu \mathrm{g} / \mathrm{L}$ & 250 & 500 & EPA Method 8270 \\
\hline$\mu \mathrm{g} / \mathrm{L}$ & 50 & 100 & EPA Method 8270 \\
\hline$\mu g / L$ & 0.1 & 0.2 & Final PDWS (EPA 1993a) \\
\hline
\end{tabular}

Source ${ }^{2}$

EPA Method 8270

EPA Method 8270

PA Method 8270

Proposed PDWS (EPA 1991)

Final PDWS

EPA Method 8080

EPD/EM

WS (EPA, 1993b)

Proposed PDWS (EPA 1991)

Final PDWS (EPA 1993a)

Interim Final PDWS (EPA, 1977)

EPA Method 8270

Final PDWS (EPA 1993a)

Final PDWS (EPA 1993a)

Final PDWS (EPA 1993a)

Final PDWS (EPA 1993a)

EPA Method 8080

Proposed PDWS (EPA 1990)

Proposed PDWS (EPA 1990)

EPA Method 8270

Final PDWS (EPA 1993a) 


\section{Savannah River Site}

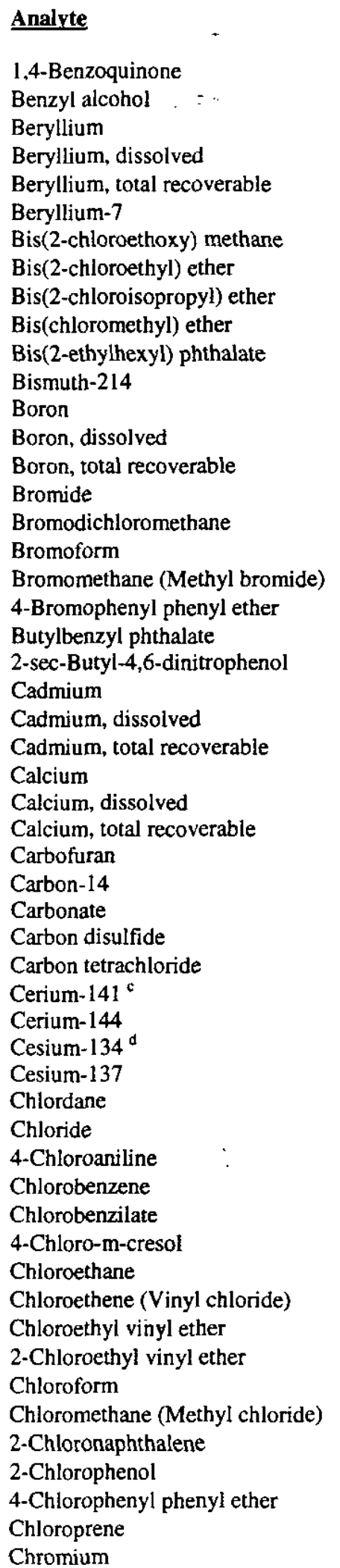

\begin{tabular}{|c|c|c|c|}
\hline Unit & Flag 1 & Flag 2 & Source" \\
\hline$\mu \mathrm{g} / \mathrm{L}$ & 50 & 100 & EPA Method 8270 \\
\hline$\mu \mathrm{g} / \mathrm{L}$ & 50 & 100 & EPA Method 8270 \\
\hline$\mu g / L$ & 2 & 4 & Final PDWS (EPA 1993a) \\
\hline$\mu \mathrm{g} / \mathrm{L}$ & 2 & 4 & Final PDWS (EPA 1993a) \\
\hline$\mu g / L$ & 2 & 4 & Final PDWS (EPA 1993a) \\
\hline $\mathrm{pCi} / \mathrm{L}$ & $3 E+03$ & $6 \mathrm{E}+03$ & Interim Final PDWS (EPA 1977) \\
\hline$\mu \mathrm{g} / \mathrm{L}$ & 50 & 100 & EPA Method 8270 \\
\hline$\mu \mathrm{g} / \mathrm{L}$ & 50 & 100 & EPA Method 8270 \\
\hline$\mu g / L$ & 50 & 100 & EPA Method 8270 \\
\hline$\mu \mathrm{g} / \mathrm{L}$ & 50 & 100 & EPA Method 8270 \\
\hline$\mu \mathrm{g} / \mathrm{L}$ & 3 & 6 & Final PDWS (EPA 1993a) \\
\hline $\mathrm{pCi} / \mathrm{L}$ & $9.4 \mathrm{E}+03$ & $1.89 \mathrm{E}+04$ & Proposed PDWS (EPA 1991) \\
\hline$\mu \mathrm{g} / \mathrm{L}$ & 150 & 300 & EPA Method 6010 \\
\hline$\mu g / L$ & 150 & 300 & EPA Method 6010 \\
\hline$\mu \mathrm{g} / \mathrm{L}$ & 150 & 300 & EPA Method 6010 \\
\hline$\mu \mathrm{g} / \mathrm{L}$ & 5,000 & 10,000 & EPA Method 300.0 \\
\hline$\mu \mathrm{g} / \mathrm{L}$ & 50 & 100 & Final PDWS (EPA 1993a) \\
\hline$\mu \mathrm{g} / \mathrm{L}$ & 50 & 100 & Final PDWS (EPA 1993a) \\
\hline$\mu \mathrm{g} / \mathrm{L}$ & 5 & 10 & EPA Method 8240 \\
\hline$\mu \mathrm{g} / \mathrm{L}$ & 50 & 100 & EPA Method 8270 \\
\hline & No flag & No flag & Set by EPD/EMS \\
\hline$\mu \mathrm{g} / \mathrm{L}$ & 3.5 & 7 & Final PDWS (EPA 1993a) \\
\hline$\mu \mathrm{g} / \mathrm{L}$ & 2.5 & 5 & Final PDWS (EPA 1993a) \\
\hline$\mu g / L$ & 2.5 & 5 & Final PDWS (EPA 1993a) \\
\hline$\mu \mathrm{g} / \mathrm{L}$ & 2.5 & 5 & Final PDWS (EPA 1993a) \\
\hline & No flag & No flag & Set by EPD/EMS \\
\hline & No flag & No flag & Set by EPD/EMS \\
\hline & No tlag & No flag & Set by EPD/EMS \\
\hline$\mu g / L$ & 20 & 40 & Final PDWS (EPA 1993a) \\
\hline $\mathrm{pCi} / \mathrm{L}$ & $\begin{array}{l}1 \mathrm{E}+03 \\
\text { No flag }\end{array}$ & $\begin{array}{l}2 \mathrm{E}+03 \\
\text { No flag }\end{array}$ & $\begin{array}{l}\text { Interim Final PDWS (EPA 1977) } \\
\text { Set by EPD/EMS }\end{array}$ \\
\hline$\mu g / L$ & 5 & 10 & EPA Method 8240 \\
\hline$\mu \mathrm{g} / \mathrm{L}$ & 2.5 & 5 & Final PDWS (EPA 1993a) \\
\hline $\mathrm{pCi} / \mathrm{L}$ & $1.5 E+02$ & $3 \mathrm{E}+02$ & Interim Final PDWS (EPA 1977) \\
\hline $\mathrm{pCi} / \mathrm{L}$ & $1.31 \mathrm{E}+02$ & $2.61 \mathrm{E}+02$ & Proposed PDWS (EPA 1991) \\
\hline $\mathrm{pCi} / \mathrm{L}$ & $4.07 \mathrm{E}+01$ & $8.13 E+01$ & Proposed PDWS (EPA 1991) \\
\hline $\mathrm{pCi} / \mathrm{L}$ & $1 \mathrm{E}+02$ & $2 \mathrm{E}+02$ & Interim Final PDWS (EPA 1977) \\
\hline$\mu \mathrm{g} / \mathrm{L}$ & 1 & 2 & Final PDWS (EPA 1993a) \\
\hline$\mu g / L$ & 125,000 & 250,000 & SDWS (EPA 1993b) \\
\hline$\mu \mathrm{g} / \mathrm{L}$ & 50 & 100 & EPA Method 8270 \\
\hline$\mu \mathrm{g} / \mathrm{L}$ & 50 & 100 & Final PDWS (EPA 1993a) \\
\hline$\mu \mathrm{g} / \mathrm{L}$ & 50 & 100 & EPA Method 8270 \\
\hline$\mu \mathrm{g} / \mathrm{L}$ & 50 & 100 & EPA Method 8270 \\
\hline$\mu \mathrm{g} / \mathrm{L}$ & 5 & 10 & EPA Method 8240 \\
\hline$\mu \mathrm{g} / \mathrm{L}$ & 1 & 2 & Final PDWS (EPA 1993a) \\
\hline$\mu \mathrm{g} / \mathrm{L}$ & 5 & 10 & EPA Method 8240 \\
\hline$\mu \mathrm{g} / \mathrm{L}$ & 5 & 10 & EPA Method 8240 \\
\hline$\mu \mathrm{g} / \mathrm{L}$ & 50 & 100 & Final PDWS (EPA 1993a) \\
\hline$\mu \mathrm{g} / \mathrm{L}$ & 5 & 10 & EPA Method 8240 \\
\hline$\mu \mathrm{g} / \mathrm{L}$ & 50 & 100 & EPA Method 8240 \\
\hline$\mu \mathrm{g} / \mathrm{L}$ & 50 & 100 & EPA Method 8270 \\
\hline$\mu \mathrm{g} / \mathrm{L}$ & 50 & 100 & EPA Method 8270 \\
\hline$\mu \mathrm{g} / \mathrm{L}$ & 1,000 & 2,000 & EPA Method 8240 \\
\hline$\mu g / L$ & 50 & 100 & Final PDWS (EPA 1993a) \\
\hline
\end{tabular}




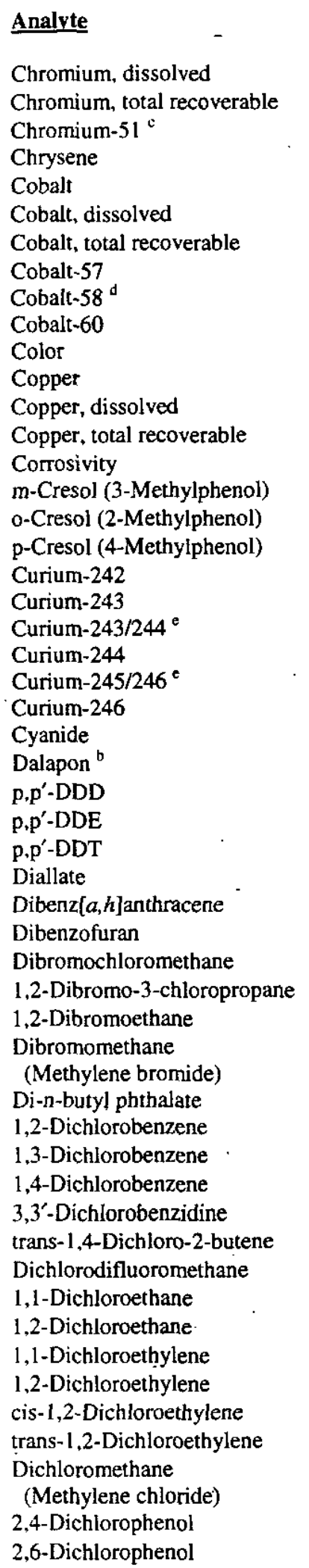

\begin{tabular}{|c|c|c|}
\hline Unit & Flag 1 & Flag 2 \\
\hline$\mu \mathrm{g} / \mathrm{L}$ & 50 & 100 \\
\hline$\mu \mathrm{g} / \mathrm{L}$ & 50 & 100 \\
\hline $\mathrm{pCi} / \mathrm{L}$ & $3 E+03$ & $6 E+03$ \\
\hline$\mu \mathrm{g} / \mathrm{L}$ & 0.1 & 0.2 \\
\hline$\mu \mathrm{g} / \mathrm{L}$ & 20 & 40 \\
\hline$\mu \mathrm{g} / \mathrm{L}$ & 20 & 40 \\
\hline$\mu \mathrm{g} / \mathrm{L}$ & 20 & 40 \\
\hline $\mathrm{pCi} / \mathrm{L}$ & $5 E+02$ & $1 E+03$ \\
\hline $\mathrm{pCi} / \mathrm{L}$ & $4.5 E+03$ & $9 E+03$ \\
\hline $\mathrm{pCi} / \mathrm{L}$ & $\begin{array}{l}5 E+01 \\
\text { No flag }\end{array}$ & $\begin{array}{l}1 \mathrm{E}+02 \\
\text { No flag }\end{array}$ \\
\hline$\mu \mathrm{g} / \mathrm{L}$ & 500 & 1,000 \\
\hline$\mu \mathrm{g} / \mathrm{L}$ & 500 & 1,000 \\
\hline$\mu \mathrm{g} / \mathrm{L}$ & 500 & 1,000 \\
\hline & No flag & No flag \\
\hline$\mu \mathrm{g} / \mathrm{L}$ & 50 & 100 \\
\hline$\mu \mathrm{g} / \mathrm{L}$ & 50 & 100 \\
\hline$\mu \mathrm{g} / \mathrm{L}$ & 50 & 100 \\
\hline $\mathrm{pCi} / \mathrm{L}$ & $6.65 \mathrm{E}+01$ & $1.33 \mathrm{E}+02$ \\
\hline $\mathrm{pCi} / \mathrm{L}$ & $4.15 E+00$ & $8.3 E+00$ \\
\hline $\mathrm{pCi} / \mathrm{L}$ & $4.15 \mathrm{E}+00$ & $8.3 \mathrm{E}+00$ \\
\hline $\mathrm{pCi} / \mathrm{L}$ & $4.92 \mathrm{E}+00$ & $9.84 \mathrm{E}+00$ \\
\hline $\mathrm{pCi} / \mathrm{L}$ & $3.12 \mathrm{E}+00$ & $6.23 \mathrm{E}+00$ \\
\hline $\mathrm{pCi} / \mathrm{L}$ & $3.14 E+00$ & $6.27 \mathrm{E}+00$ \\
\hline$\mu \mathrm{g} / \mathrm{L}$ & 100 & 200 \\
\hline$\mu \mathrm{g} / \mathrm{L}$ & 100 & 200 \\
\hline$\mu \mathrm{g} / \mathrm{L}$ & 0.5 & 1 \\
\hline$\mu \mathrm{g} / \mathrm{L}$ & 0.5 & 1 \\
\hline$\mu \mathrm{g} / \mathrm{L}$ & 0.5 & 1 \\
\hline$\mu \mathrm{g} / \mathrm{L}$ & 50 & 100 \\
\hline$\mu g / L$ & 0.15 & 0.3 \\
\hline$\mu \mathrm{g} / \mathrm{L}$ & 50 & 100 \\
\hline$\mu \mathrm{g} / \mathrm{L}$ & 50 & 100 \\
\hline$\mu \mathrm{g} / \mathrm{L}$ & 0.1 & 0.2 \\
\hline$\mu \mathrm{g} / \mathrm{L}$ & 0.025 & 0.05 \\
\hline$\mu \mathrm{g} / \mathrm{L}$ & 5 & 10 \\
\hline & No flag & No flag \\
\hline$\mu \mathrm{g} / \mathrm{L}$ & 300 & 600 \\
\hline$\mu \mathrm{g} / \mathrm{L}$ & 50 & 100 \\
\hline$\mu \mathrm{g} / \mathrm{L}$ & 37.5 & 75 \\
\hline$\mu \mathrm{g} / \mathrm{L}$ & 50 & 100 \\
\hline$\mu \mathrm{g} / \mathrm{L}$ & 150 & 300 \\
\hline$\mu \mathrm{g} / \mathrm{L}$ & 5 & 10 \\
\hline$\mu \mathrm{g} / \mathrm{L}$ & 5 & 10 \\
\hline$\mu \mathrm{g} / \mathrm{L}$ & 2.5 & 5 \\
\hline$\mu \mathrm{g} / \mathrm{L}$ & 3.5 & 7 \\
\hline$\mu \mathrm{g} / \mathrm{L}$ & 25 & 50 \\
\hline$\mu g / L$ & 35 & 70 \\
\hline$\mu \mathrm{g} / \mathrm{L}$ & 50 & 100 \\
\hline$\mu \mathrm{g} / \mathrm{L}$ & 2.5 & 5 \\
\hline$\mu \mathrm{g} / \mathrm{L}$ & 50 & 100 \\
\hline$\mu \mathrm{g} / \mathrm{L}$ & 50 & 100 \\
\hline
\end{tabular}

Source $^{2}$

Final PDWS (EPA 1993a)

Final PDWS (EPA 1993a)

Interim Final PDWS (EPA, 1977)

Proposed PDWS (EPA 1990)

EPA Method 6010

EPA Method 6010

EPA Method 6010

Interim Final PDWS (EPA 1977)

Interim Final PDWS (EPA 1977)

Interim Final PDWS (EPA 1977)

Set by EPD/EMS

Final PDWS (SCDHEC 1981)

Final PDWS (SCDHEC 1981)

Final PDWS (SCDHEC 1981)

Set by EPD/EMS

EPA Method 8270

EPA Method 8270

EPA Method 8270

Proposed PDWS (EPA 1991)

Proposed PDWS (EPA 1991)

Proposed PDWS (EPA 1991)

Proposed PDWS (EPA 1991)

Proposed PDWS (EPA 1991)

Proposed PDWS (EPA 1991)

Final PDWS (EPA 1993a)

Final PDWS (EPA 1993a)

EPA Method 8080

EPA Method 8080

EPA Method 8080

EPA Method 8270

Proposed PDWS (EPA, 1990)

EPA Method 8270

Final PDWS (EPA 1993a)

Final PDWS (EPA 1993a)

Final PDWS (EPA 1993a)

EPA Method 8240

Set by EPD/EMS

Final PDWS (EPA 1993a)

EPA Method 8270

Final PDWS (EPA 1993a)

EPA Method 8270

EPA Method 8240

EPA Method 8240

EPA Method 8240

Final PDWS (EPA 1993a)

Final PDWS (EPA 1993a)

Final PDWS (EPA 1993a)

Final PDWS (EPA 1993a)

Final PDWS (EPA 1993a)

Final PDWS (EPA 1993a)

EPA Method 8270

EPA Method 8270 


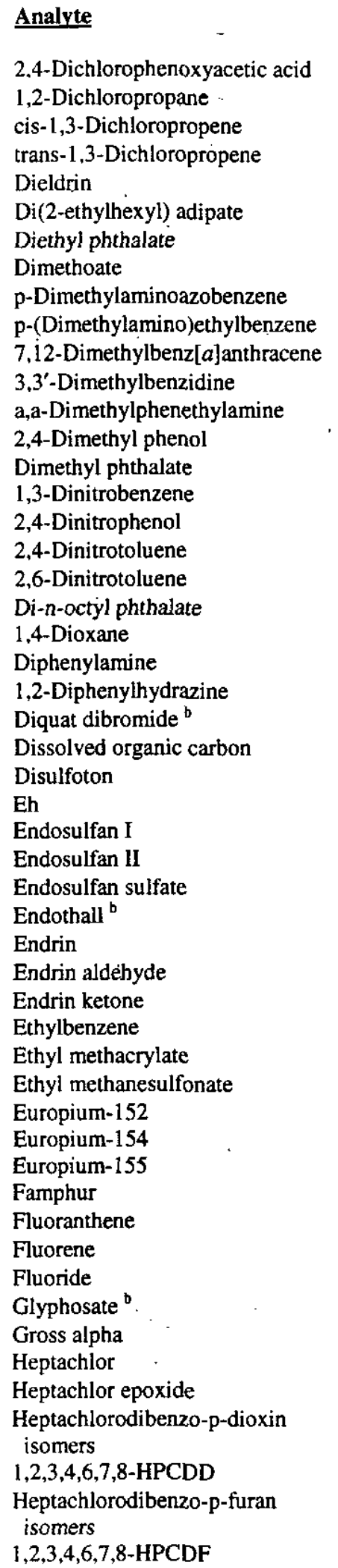

\begin{tabular}{|c|c|c|c|}
\hline Unit & Flag 1 & Flag 2 & Source \\
\hline$\mu \mathrm{g} / \mathrm{L}$ & 35 & 70 & Final PDWS (EPA 1993a) \\
\hline$\mu \mathrm{g} / \mathrm{L}$ & 2.5 & 5 & Final PDWS (EPA 1993a) \\
\hline$\mu \mathrm{g} / \mathrm{L}$ & 5 & 10 & EPA Method 8240 \\
\hline$\mu \mathrm{g} / \mathrm{L}$ & 5 & 10 & EPA Method 8240 \\
\hline$\mu \mathrm{g} / \mathrm{L}$ & 2.5 & 5 & EPA Method 8080 \\
\hline \multirow{2}{*}{$\mu \mathrm{g} / \mathrm{L}$} & 200 & 400 & Final PDWS (EPA 1993a) \\
\hline & No flag & No flag & Set by EPD/EMS \\
\hline$\mu \mathrm{g} / \mathrm{L}$ & 50 & 100 & EPA Method 8270 \\
\hline$\mu \mathrm{g} / \mathrm{L}$ & 50 & 100 & EPA Method 8270 \\
\hline$\mu \mathrm{g} / \mathrm{L}$ & 50 & 100 & EPA Method 8270 \\
\hline$\mu \mathrm{g} / \mathrm{L}$ & 50 & 100 & EPA Method 8270 \\
\hline$\mu \mathrm{g} / \mathrm{L}$ & 50 & 100 & EPA Method 8270 \\
\hline$\mu \mathrm{g} / \mathrm{L}$ & 50 & 100 & EPA Method 8270 \\
\hline \multirow[t]{2}{*}{$\mu \mathrm{g} / \mathrm{L}$} & 50 & 100 & EPA Method 8270 \\
\hline & No flag & No flag & Set by EPD/EMS \\
\hline$\mu \mathrm{g} / \mathrm{L}$ & 50 & 100 & EPA Method 8270 \\
\hline$\mu \mathrm{g} / \mathrm{L}$ & 250 & 500 & EPA Method 8270 \\
\hline$\mu \mathrm{g} / \mathrm{L}$ & 50 & 100 & EPA Method 8270 \\
\hline \multirow[t]{2}{*}{$\mu \mathrm{g} / \mathrm{L}$} & 50 & 100 & EPA Method 8270 \\
\hline & No flag & No flag & Set by EPD/EMS \\
\hline$\mu \mathrm{g} / \mathrm{L}$ & 50 & 100 & EPA Method 8270 \\
\hline$\mu \mathrm{g} / \mathrm{L}$ & 50 & 100 & EPA Method 8270 \\
\hline$\mu \mathrm{g} / \mathrm{L}$ & 50 & 100 & EPA Method 8270 \\
\hline$\mu \mathrm{g} / \mathrm{L}$ & 10 & 20 & Final PDWS (EPA 1993a) \\
\hline$\mu \mathrm{g} / \mathrm{L}$ & 5,000 & 10,000 & EPA Method 9060 \\
\hline \multirow[t]{2}{*}{$\mu \mathrm{g} / \mathrm{L}$} & 50 & 100 & EPA Method 8270 \\
\hline & No flag & No flag & Set by EPD/EMS \\
\hline$\mu \mathrm{g} / \mathrm{L}$ & 0.5 & 1 & EPA Method 8080 \\
\hline$\mu \mathrm{g} / \mathrm{L}$ & 0.5 & 1 & EPA Method 8080 \\
\hline$\mu \mathrm{g} / \mathrm{L}$ & 0.5 & 1 & EPA Method 8080 \\
\hline$\mu \mathrm{g} / \mathrm{L}$ & 50 & 100 & Final PDWS (EPA 1993a) \\
\hline$\mu \mathrm{g} / \mathrm{L}$ & 1 & 2 & Final PDWS (EPA 1993a) \\
\hline \multirow[t]{2}{*}{$\mu \mathrm{g} / \mathrm{L}$} & 0.5 & 1 & EPA Method 8080 \\
\hline & No flag & No flag & Set by EPD/EMS \\
\hline$\mu \mathrm{g} / \mathrm{L}$ & 350 & 700 & Final PDWS (EPA 1993a) \\
\hline$\mu \mathrm{g} / \mathrm{L}$ & 50 & 100 & EPA Method 8270 \\
\hline$\mu \mathrm{g} / \mathrm{L}$ & 50 & 100 & EPA Method 8270 \\
\hline $\mathrm{pCi} / \mathrm{L}$ & $3 E+01$ & $6 \mathrm{E}+01$ & Interim Final PDWS (EPA 1977) \\
\hline $\mathrm{pCi} / \mathrm{L}$ & $1 \mathrm{E}+02$ & $2 \mathrm{E}+02$ & Interim Final PDWS (EPA 1977) \\
\hline $\mathrm{pCi} / \mathrm{L}$ & $3 E+02$ & $6 E+02$ & Interim Final PDWS (EPA 1977) \\
\hline$\mu \mathrm{g} / \mathrm{L}$ & 50 & 100 & EPA Method 8270 \\
\hline$\mu \mathrm{g} / \mathrm{L}$ & 50 & 100 & EPA Method 8270 \\
\hline$\mu \mathrm{g} / \mathrm{L}$ & 50 & 100 & EPA Method 8270 \\
\hline$\mu \mathrm{g} / \mathrm{L}$ & 2,000 & 4,000 & Final PDWS (EPA 1993a) \\
\hline$\mu \mathrm{g} / \mathrm{L}$ & 350 & 700 & Final PDWS (EPA 1993a) \\
\hline $\mathrm{pCi} / \mathrm{L}$ & $7.5 \mathrm{E}+00$ & $1.5 E+01$ & Final PDWS (EPA 1993a) \\
\hline$\mu g / L$ & 0.2 & 0.4 & Final PDWS (EPA 1993a) \\
\hline$\mu \mathrm{g} / \mathrm{L}$ & 0.1 & 0.2 & Final PDWS (EPA 1993a) \\
\hline$\mu \mathrm{g} / \mathrm{L}$ & 0.00325 & 0.0065 & EPA Method 8280 \\
\hline$\mu \mathrm{g} / \mathrm{L}$ & 0.00325 & 0.0065 & EPA Method 8280 \\
\hline$\mu \mathrm{g} / \mathrm{L}$ & 0.00225 & 0.0045 & EPA Method 8280 \\
\hline$\mu \mathrm{g} / \mathrm{L}$ & 0.00225 & 0.0045 & EPA Method 8280 \\
\hline
\end{tabular}


Analyte

Hexachlorobenzene

Hexachlorobutadienē

Hexachlorocyclopentadiene

Hexachlorodibenzo-p-dioxin

isomers

1,2,3,4,7,8-HXCDD

Hexachlorodibenzo-p-furan

isomers

1,2,3,4,7,8-HXCDF

Hexachloroethane

Hexachlorophene

Hexachloropropene

2-Hexanone

Indeno $[1,2,3-c, d]$ pyrene

lodine

Iodine- 129

Iodine- $131^{c}$

Iodomethane (Methyl iodide)

Iron

Iron, dissolved

Iron, total recoverable

Iron-55 ${ }^{\mathrm{c}}$

Iron- $59^{\mathrm{c}}$

Isobutyl alcohol

Isodrin

Isophorone

Isosafrole

Kepone

Lanthanum- $140^{\circ}$

Lead

Lead, dissolved

Lead, total recoverable

Lead-212

Lindane

Lithium

Lithium, dissolved

Lithium, total recoverable

Magnesium

Magnesium, dissolved

Magnesium, total recoverable

Manganese

Manganese, dissolved

Manganese, total recoverable

Manganese-54

Mercury

Mercury, dissolved

Mercury, total recoverable

Methacrylonitrile

Methapyrilene

Methoxychlor

3-Methylcholanthrene

2-Methyl-4,6-dinitrophenol

Methyl ethyl ketone

Methyl isobutyl ketone

\begin{tabular}{|c|c|c|c|}
\hline Unit & Flag 1 & Flag 2 & Source \\
\hline$\mu \mathrm{g} / \mathrm{L}$ & 0.5 & 1 & Final PDWS (EPA 1993a) \\
\hline$\mu \mathrm{g} / \mathrm{L}$ & 50 & 100 & EPA Method 8270 \\
\hline$\mu \mathrm{g} / \mathrm{L}$ & 25 & 50 & Final PDWS (EPA 1993a) \\
\hline$\mu \mathrm{g} / \mathrm{L}$ & 0.00225 & 0.0045 & EPA Method 8280 \\
\hline$\mu \mathrm{g} / \mathrm{L}$ & 0.00225 & 0.0045 & EPA Method 8280 \\
\hline$\mu \mathrm{g} / \mathrm{L}$ & 0.002 & 0.004 & EPA Method 8280 \\
\hline$\mu \mathrm{g} / \mathrm{L}$ & 0.002 & 0.004 & EPA Method 8280 \\
\hline$\mu \mathrm{g} / \mathrm{L}$ & 50 & 100 & EPA Method 8270 \\
\hline$\mu \mathrm{g} / \mathrm{L}$ & 250 & 500 & EPA Method 8270 \\
\hline$\mu \mathrm{g} / \mathrm{L}$ & 50 & 100 & EPA Method 8270 \\
\hline$\mu \mathrm{g} / \mathrm{L}$ & 50 & 100 & EPA Method 8240 \\
\hline$\mu \mathrm{g} / \mathrm{L}$ & 50 & 100 & EPA Method 8270 \\
\hline$\mu \mathrm{g} / \mathrm{L}$ & 250 & 500 & APHA Method 415A \\
\hline $\mathrm{pCi} / \mathrm{L}$ & $5 \mathrm{E}-01$ & $1 E+00$ & Interim Final PDWS (EPA 1977) \\
\hline $\mathrm{pCi} / \mathrm{L}$ & $1.5 E+00$ & $3 \mathrm{E}+\infty$ & Interim Final PDWS (EPA 1977) \\
\hline$\mu \mathrm{g} / \mathrm{L}$ & 75 & 150 & EPA Method 8240 \\
\hline$\mu \mathrm{g} / \mathrm{L}$ & 150 & 300 & SDWS (EPA, 1993b) \\
\hline$\mu \mathrm{g} / \mathrm{L}$ & 150 & 300 & SDWS (EPA, 1993b) \\
\hline$\mu \mathrm{g} / \mathrm{L}$ & 150 & 300 & SDWS (EPA, 1993b) \\
\hline $\mathrm{pCi} / \mathrm{L}$ & $1 E+03$ & $2 \mathrm{E}+03$ & Interim Final PDWS (EPA 1977) \\
\hline $\mathrm{pCi} / \mathrm{L}$ & $1 E+02$ & $2 \mathrm{E}+02$ & Interim Final PDWS (EPA 1977) \\
\hline$\mu \mathrm{g} / \mathrm{L}$ & 500 & 1,000 & EPA Method 8240 \\
\hline$\mu g / L$ & 50 & 100 & EPA Method 8270 \\
\hline$\mu g / L$ & 50 & 100 & EPA Method 8270 \\
\hline$\mu \mathrm{g} / \mathrm{L}$ & 50 & 100 & EPA Method 8270 \\
\hline$\mu \mathrm{g} / \mathrm{L}$ & 50 & 100 & EPA Method 8270 \\
\hline $\mathrm{pCi} / \mathrm{L}$ & $3 E+01$ & $6 \mathrm{E}+01$ & Interim Final PDWS (EPA 1977) \\
\hline$\mu \mathrm{g} / \mathrm{L}$ & 25 & 50 & Final PDWS (SCDHEC 1981) \\
\hline$\mu \mathrm{g} / \mathrm{L}$ & 25 & 50 & Final PDWS (SCDHEC 1981) \\
\hline$\mu \mathrm{g} / \mathrm{L}$ & 25 & 50 & Final PDWS (SCDHEC 1981) \\
\hline $\mathrm{pCi} / \mathrm{L}$ & $6.2 E+01$ & $1.23 \mathrm{E}+02$ & Proposed PDWS (EPA 1991) \\
\hline$\mu \mathrm{g} / \mathrm{L}$ & 0.1 & 0.2 & Final PDWS (EPA 1993a) \\
\hline$\mu \mathrm{g} / \mathrm{L}$ & 25 & 50 & EPA Method 6010 \\
\hline$\mu \mathrm{g} / \mathrm{L}$ & 25 & 50 & EPA Method 6010 \\
\hline \multirow[t]{4}{*}{$\mu \mathrm{g} / \mathrm{L}$} & 25 & 50 & EPA Method 6010 \\
\hline & No flag & No flag & Set by EPD/EMS \\
\hline & No flag & No flag & Set by EPD/EMS \\
\hline & No flag & No flag & Set by EPD/EMS \\
\hline$\mu \mathrm{g} / \mathrm{L}$ & 25 & 50 & SDWS (EPA, 1993b) \\
\hline$\mu \mathrm{g} / \mathrm{L}$ & 25 & 50 & SDWS (EPA, 1993b) \\
\hline$\mu \mathrm{g} / \mathrm{L}$ & 25 & 50 & SDWS (EPA, 1993b) \\
\hline $\mathrm{pCi} / \mathrm{L}$ & $1.5 \mathrm{E}+02$ & $3 \mathrm{E}+02$ & Interim Final PDWS (EPA, 1977) \\
\hline$\mu \mathrm{g} / \mathrm{L}$ & 1 & 2 & Final PDWS (EPA 1993a) \\
\hline$\mu \mathrm{g} / \mathrm{L}$ & 1 & 2 & Final PDWS (EPA 1993a) \\
\hline$\mu \mathrm{g} / \mathrm{L}$ & 1 & 2 & Final PDWS (EPA 1993a) \\
\hline$\mu \mathrm{g} / \mathrm{L}$ & 250 & 500 & EPA Method 8240 \\
\hline$\mu \mathrm{g} / \mathrm{L}$ & 50 & 100 & EPA Method 8270 \\
\hline$\mu \mathrm{g} / \mathrm{L}$ & 20 & 40 & Final PDWS (EPA 1993a) \\
\hline$\mu \mathrm{g} / \mathrm{L}$ & 50 & 100 & EPA Method 8270 \\
\hline \multirow[t]{3}{*}{$\mu \mathrm{g} / \mathrm{L}$} & 250 & 500 & EPA Method 8270 \\
\hline & No flag & No flag & Set by EPD/EMS \\
\hline & No flag & No flag & Set by EPD/EMS \\
\hline
\end{tabular}




Analyte
Methyl methacrylate
Methyl methanesulfonate
2-Methylnaphthalene
Molybdenum
Molybdenum, dissolved
Molybdenum, total recoverable
Naphthalene
1,4-Naphthoquinone
l-Naphthylamine
2-Naphthylamine
Neptunium-237
Nickel
Nickel, dissolved
Nickel, total recoverable
Nickel-59
Nickel-63
Niobium-95
Nitrate as nitrogen
Nitrate-nitrite as nitrogen
Nitrite as nitrogen
m-Nitroaniline
O-Nitroaniline
p-Nitroaniline
Nitrobenzene
Nitrogen by Kjeldahl method
2-Nitrophenol
4-Nitrophenol
4-Nitroquinoline-1-oxide
N-Nitrosodi-n-butylamine
N-Nitrosodiethylamine
N-Nitrosodimethylamine
N-Nitrosodiphenylamine
N-Nitrosodipropylamine
N-Nitrosomethylethylamine
N-Nitrosomorpholine
N-Nitrosopiperidine
N-Nitrosopyrrolidine
5-Nitro-o-toluidine
Nonvolatile beta
Octachlorodibenzo-p-dioxin
isomers
Octachlorodibenzo-p-furan
isomers
Odor
Oil \& Grease
Oxamyl
Parathion
PCB 1221616 1248
PCB 1254

\begin{tabular}{|c|c|c|}
\hline Unit & Flag 1 & Flag 2 \\
\hline$\mu \mathrm{g} / \mathrm{L}$ & 50 & 100 \\
\hline$\mu \mathrm{g} / \mathrm{L}$ & 50 & 100 \\
\hline$\mu \mathrm{g} / \mathrm{L}$ & 50 & 100 \\
\hline$\mu \mathrm{g} / \mathrm{L}$ & 250 & 500 \\
\hline$\mu \mathrm{g} / \mathrm{L}$ & 250 & 500 \\
\hline$\mu \mathrm{g} / \mathrm{L}$ & 250 & 500 \\
\hline$\mu \mathrm{g} / \mathrm{L}$ & 50 & 100 \\
\hline$\mu \mathrm{g} / \mathrm{L}$ & 50 & 100 \\
\hline$\mu \mathrm{g} / \mathrm{L}$ & 50 & 100 \\
\hline$\mu \mathrm{g} / \mathrm{L}$ & 50 & 100 \\
\hline $\mathrm{pCi} / \mathrm{L}$ & $3.53 E+00$ & $7.06 \mathrm{E}+00$ \\
\hline$\mu \mathrm{g} / \mathrm{L}$ & 50 & 100 \\
\hline$\mu \mathrm{g} / \mathrm{L}$ & 50 & 100 \\
\hline$\mu \mathrm{g} / \mathrm{L}$ & 50 & 100 \\
\hline $\mathrm{pCi} / \mathrm{L}$ & $1.5 E+02$ & $3 E+02$ \\
\hline $\mathrm{pCi} / \mathrm{L}$ & $2.5 \mathrm{E}+01$ & $5 E+01$ \\
\hline $\mathrm{pCi} / \mathrm{L}$ & $1.5 E+02$ & 3. $E+02$ \\
\hline$\mu \mathrm{g} / \mathrm{L}$ & 5,000 & 10,000 \\
\hline$\mu \mathrm{g} / \mathrm{L}$ & 5,000 & 10,000 \\
\hline$\mu \mathrm{g} / \mathrm{L}$ & 500 & 1,000 \\
\hline$\mu \mathrm{g} / \mathrm{L}$ & 50 & 100 \\
\hline$\mu \mathrm{g} / \mathrm{L}$ & 50 & 100 \\
\hline$\mu \mathrm{g} / \mathrm{L}$ & 50 & 100 \\
\hline$\mu \mathrm{g} / \mathrm{L}$ & 50 & 100 \\
\hline$\mu \mathrm{g} / \mathrm{L}$ & 500 & 1,000 \\
\hline$\mu \mathrm{g} / \mathrm{L}$ & 50 & 100 \\
\hline$\mu \mathrm{g} / \mathrm{L}$ & 50 & 100 \\
\hline$\mu g / L$ & 50 & 100 \\
\hline$\mu \mathrm{g} / \mathrm{L}$ & 50 & 100 \\
\hline$\mu \mathrm{g} / \mathrm{L}$ & 50 & 100 \\
\hline$\mu \mathrm{g} / \mathrm{L}$ & 50 & 100 \\
\hline$\mu \mathrm{g} / \mathrm{L}$ & 50 & 100 \\
\hline$\mu \mathrm{g} / \mathrm{L}$ & 50 & 100 \\
\hline$\mu \mathrm{g} / \mathrm{L}$ & 50 & 100 \\
\hline$\mu \mathrm{g} / \mathrm{L}$ & 50 & 100 \\
\hline$\mu \mathrm{g} / \mathrm{L}$ & 50 & 100 \\
\hline$\mu \mathrm{g} / \mathrm{L}$ & 50 & 100 \\
\hline$\mu \mathrm{g} / \mathrm{L}$ & 50 & 100 \\
\hline $\mathrm{pCi} / \mathrm{L}$ & $2.5 \mathrm{E}+01$ & $5 E+01$ \\
\hline$\mu g / L$ & 0.005 & 0.01 \\
\hline \multirow[t]{2}{*}{$\mu g / L$} & 0.005 & 0.01 \\
\hline & No flag & No flag \\
\hline$\mu \mathrm{g} / \mathrm{L}$ & 5,000 & 10,000 \\
\hline$\mu \mathrm{g} / \mathrm{L}$ & 100 & 200 \\
\hline$\mu \mathrm{g} / \mathrm{L}$ & 0.25 & 0.5 \\
\hline$\mu \mathrm{g} / \mathrm{L}$ & 0.25 & 0.5 \\
\hline$\mu \mathrm{g} / \mathrm{L}$ & 0.25 & 0.5 \\
\hline$\mu \mathrm{g} / \mathrm{L}$ & 0.25 & 0.5 \\
\hline$\mu \mathrm{g} / \mathrm{L}$ & 0.25 & 0.5 \\
\hline$\mu \mathrm{g} / \mathrm{L}$ & 0.25 & 0.5 \\
\hline$\mu \mathrm{g} / \mathrm{L}$ & 0.25 & 0.5 \\
\hline$\mu \mathrm{g} / \mathrm{L}$ & 0.25 & 0.5 \\
\hline
\end{tabular}

Source $^{2}$

EPA Method 8270

EPA Method 8270

EPA Method 8270

EPA Method 6010

EPA Method 6010

EPA Method 6010

EPA Method 8270

EPA Method 8270

EPA Method 8270

EPA Method 8270

Proposed PDWS (EPA 1991)

Final PDWS (EPA 1993a)

Final PDWS (EPA 1993a)

Final PDWS (EPA 1993a)

Interim Final PDWS (EPA, 1977)

Interim Final PDWS (EPA, 1977)

Interim Final PDWS (EPA, 1977)

Final PDWS (EPA 1993a)

Final PDWS (EPA 1993a)

Final PDWS (EPA 1993a)

EPA Method 8270

EPA Method 8270

EPA Method 8270

EPA Method 8270

EPA Method 351.2

EPA Method 8270

EPA Method 8270

EPA Method 8270

EPA Method 8270

EPA Method 8270

EPA Method 8270

EPA Method 8270

EPA Method 8270

EPA Method 8270

EPA Method 8270

EPA Method 8270

EPA Method 8270

EPA Method 8270

Interim Final PDWS (EPA, 1977)

EPA Method 8280

EPA Method 8280

Set by EPD/EMS

EPA Method 413.1

Final PDWS (EPA 1993a)

EPA Method 8080

EPA Method 8080

Final PDWS (EPA 1993a)

Final PDWS (EPA 1993a)

Final PDWS (EPA 1993a)

Final PDWS (EPA 1993a)

Final PDWS (EPA 1993a)

Final PDWS (EPA 1993a) 


Analyte
PCB 1260
PCB 1262
Pentachlorobenzene
Pentachlorodibenzo-p-dioxin
isomers
1,2,3,7,8-PCDD
Pentachlorodibenzo-p-furan
isomers
1,2,3,7,8-PCDF
Pentachloroethane
Pentachloronitrobenzene
Pentachlorophenol
pH
pH
Phenacetin
Phenanthrene
Phenol
Phenols
p-Phenylenediamine
Phorate
Picloram
2-Picoline
Plutonium-238
Plutonium-239
Plutonium-239/240
Plutonium-240
Plutonium-241
Plutonium-242
Potassium
Potassium, dissolved
Potassium, total recoverable
Potassium-40
Promethium-144
Promethium-146
Promethium-147
Pronamid
Propionitrile
Pyrene
Pyridine
Radium (alpha-emitting)
Radium-226
Radium-228
Radon-222
Ruthenium-103 c
Ruthenium-106
Safrole
Selenium
Selenium, dissolved
Silica
Silica, total recoverable
Silver, dissolved
Silver, total recoverable

\begin{tabular}{|c|c|c|c|}
\hline Unit & Flag 1 & Flag 2 & Source \\
\hline$\mu \mathrm{g} / \mathrm{L}$ & 0.25 & 0.5 & Final PDWS (EPA 1993a) \\
\hline$\mu \mathrm{g} / \mathrm{L}$ & 0.25 & 0.5 & Final PDWS (EPA 1993a) \\
\hline$\mu \mathrm{g} / \mathrm{L}$ & 50 & 100 & EPA Method 8270 \\
\hline$\mu \mathrm{g} / \mathrm{L}$ & 0.00275 & 0.0055 & EPA Method 8280 \\
\hline$\mu \mathrm{g} / \mathrm{L}$ & 0.00275 & 0.0055 & EPA Method 8280 \\
\hline$\mu \mathrm{g} / \mathrm{L}$ & 0.00275 & 0.0055 & EPA Method 8280 \\
\hline$\mu \mathrm{g} / \mathrm{L}$ & 0.00275 & 0.0055 & EPA Method 8280 \\
\hline$\mu \mathrm{g} / \mathrm{L}$ & 50 & 100 & EPA Method 8270 \\
\hline$\mu \mathrm{g} / \mathrm{L}$ & 50 & 100 & EPA Method 8270 \\
\hline$\mu \mathrm{g} / \mathrm{L}$ & 0.5 & 1 & Final PDWS (EPA 1993a) \\
\hline $\mathrm{pH}$ & 8 & 10 & Set by EPD/EMS \\
\hline $\mathrm{pH}$ & 4 & 3 & Set by EPD/EMS \\
\hline$\mu \mathrm{g} / \mathrm{L}$ & 50 & 100 & EPA Method 8270 \\
\hline$\mu \mathrm{g} / \mathrm{L}$ & 50 & 100 & EPA Method 8270 \\
\hline$\mu \mathrm{g} / \mathrm{L}$ & 50 & 100 & EPA Method 8270 \\
\hline$\mu g / L$ & 25 & 50 & EPA Method 420.1 \\
\hline$\mu \mathrm{g} / \mathrm{L}$ & 50 & 100 & EPA Method 8270 \\
\hline$\mu \mathrm{g} / \mathrm{L}$ & 0.5 & 1 & EPA Method 8080 \\
\hline$\mu \mathrm{g} / \mathrm{L}$ & 250 & 500 & Final PDWS (EPA 1993a) \\
\hline$\mu \mathrm{g} / \mathrm{L}$ & 50 & 100 & EPA Method 8270 \\
\hline $\mathrm{pCi} / \mathrm{L}$ & $3.51 E+00$ & $7.02 \mathrm{E}+00$ & Proposed PDWS (EPA 1991) \\
\hline $\mathrm{pCi} / \mathrm{L}$ & $3.11 \mathrm{E}+01$ & $6.21 E+01$ & Proposed PDWS (EPA 1991) \\
\hline $\mathrm{pCi} / \mathrm{L}$ & $3.11 \mathrm{E}+01$ & $6.21 \mathrm{E}+01$ & Proposed PDWS (EPA 1991) \\
\hline $\mathrm{pCi} / \mathrm{L}$ & $3.11 \mathrm{E}+01$ & $6.22 \mathrm{E}+01$ & Proposed PDWS (EPA 1991) \\
\hline $\mathrm{pCi} / \mathrm{L}$ & $3.13 E+01$ & $6.26 \mathrm{E}+01$ & Proposed PDWS (EPA 1991) \\
\hline \multirow[t]{4}{*}{$\mathrm{pCi} / \mathrm{L}$} & $3.27 \mathrm{E}+01$ & $6.54 \mathrm{E}+01$ & Proposed PDWS (EPA 1991) \\
\hline & No flag & No flag & Set by EPD/EMS \\
\hline & No flag & No flag & Set by EPD/EMS \\
\hline & No flag & No flag & Set by EPD/EMS \\
\hline $\mathrm{pCi} / \mathrm{L}$ & $1.5 \mathrm{E}+02$ & $3 E+02$ & Proposed PDWS (EPA 1986) \\
\hline $\mathrm{pCi} / \mathrm{L}$ & $5 E+01$ & $1 E+02$ & EPA Method 901.1 \\
\hline $\mathrm{pCi} / \mathrm{L}$ & $5 \mathrm{E}+01$ & $1 \mathrm{E}+02$ & EPA Method 901.1 \\
\hline $\mathrm{pCi} / \mathrm{L}$ & $2.62 \mathrm{E}+03$ & $5.24 \mathrm{E}+03$ & Proposed PDWS (EPA 1991) \\
\hline$\mu \mathrm{g} / \mathrm{L}$ & 50 & 100 & EPA Method 8270 \\
\hline$\mu \mathrm{g} / \mathrm{L}$ & 1,000 & 2,000 & EPA Method 8240 \\
\hline$\mu \mathrm{g} / \mathrm{L}$ & 50 & 100 & EPA Method 8270 \\
\hline$\mu g / L$ & 50 & 100 & EPA Method 8270 \\
\hline $\mathrm{pCi} / \mathrm{L}$ & $1 \mathrm{E}+0 \mathrm{I}$ & $2 \mathrm{E}+01$ & Proposed PDWS (EPA 1991) \\
\hline $\mathrm{pCi} / \mathrm{L}$ & $1 E+01$ & $2 \mathrm{E}+01$ & Proposed PDWS (EPA 1991) \\
\hline $\mathrm{pCi} / \mathrm{L}$ & $1 E+01$ & $2 \mathrm{E}+0 \mathrm{I}$ & Proposed PDWS (EPA 1991) \\
\hline $\mathrm{pCi} / \mathrm{L}$ & $1.5 E+02$ & $3 E+02$ & Proposed PDWS (EPA 1991) \\
\hline $\mathrm{pCi} / \mathrm{L}$ & $1 E+02$ & $2 \mathrm{E}+02$ & Interim Final PDWS (EPA, 1977) \\
\hline $\mathrm{pCi} / \mathrm{L}$ & $1.5 \mathrm{E}+01$ & $3 \mathrm{E}+0 \mathrm{t}$ & Interim Final PDWS (EPA, 1977) \\
\hline$\mu \mathrm{g} / \mathrm{L}$ & 50 & 100 & EPA Method 8270 \\
\hline$\mu \mathrm{g} / \mathrm{L}$ & 25 & 50 & Final PDWS (EPA 1993a) \\
\hline$\mu \mathrm{g} / \mathrm{L}$ & 25 & 50 & Final PDWS (EPA 1993a) \\
\hline \multirow[t]{4}{*}{$\mu \mathrm{g} / \mathrm{L}$} & 25 & 50 & Final PDWS (EPA 1993a) \\
\hline & No flag & No flag & Set by EPD/EMS \\
\hline & No flag & No flag & Set by EPD/EMS \\
\hline & No flag & No flag & Set by EPD/EMS \\
\hline$\mu \mathrm{g} / \mathrm{L}$ & 50 & 100 & SDWS (EPA 1993b) \\
\hline$\mu \mathrm{g} / \mathrm{L}$ & 50 & 100 & SDWS (EPA 1993b) \\
\hline$\mu \mathrm{g} / \mathrm{L}$ & 50 & 100 & SDWS (EPA 1993b) \\
\hline
\end{tabular}


Analyte

Simazine ${ }^{b}$

Sodium

Sodium, dissolved

Sodium, total recoverable

Sodium-22

Specific conductance

Strontium-89

Strontium-89/90

Strontium-90

Styrene

Sulfate

Sulfide

Sulfotepp

Surfactants

2,3,7,8-TCDD

2,3,7,8-TCDF

Technetium-99

1,2,4,5-Tetrachlorobenzene

Tetrachlorodibenzo-p-dioxin isomers

Tetrachlorodibenzo-p-furan isomers

1,1,1,2-Tetrachloroethane

1,1,2,2-Tetrachloroethane

Tetrachloroethylene

2,3,4,6-Tetrachlorophenol

Thallium

Thallium, dissolved

Thallium, total recoverable

Thionazin

Thorium-228

Thorium-230

Thorium-232

Thorium-234

Tin

Tin, dissolved

Tin, total recoverable

Tin- $113^{c}$

Toluene

o-Toluidine

Total carbon

Total coliform

Total dissolved solids

Total hydrocarbons

Total inorganic carbon

Total organic carbon

Total organic halogens

Total organic nitrogen

Total petroleum hydrocarbons

Total phosphates (as P)

Total phosphorus

Toxaphene

2,4,5-TP (Silvex)

Tributyl phosphate

1,2,4-Trichlorobenzene $\underline{\text { Unit }}$

Flag 1

$\mu \mathrm{g} /$

2

No flag

No flag

No tlag

$\mathrm{pCi} / \mathrm{L} \quad 2.33 \mathrm{E}+02$

$\mu \mathrm{S} / \mathrm{cm} \quad 250$

$\mathrm{pCi} / \mathrm{L} \quad 1 \mathrm{E}+01$

$\mathrm{pCi} / \mathrm{L} \quad 4 \mathrm{E}+00$

$\mathrm{pCi} / \mathrm{L} \quad 4 \mathrm{E}+00$

$\mu \mathrm{g} / \mathrm{L}$

50

$\mu \mathrm{g} / \mathrm{L} \quad 200,000$

$\mu \mathrm{g} / \mathrm{L} \quad 5,000$

$\mu g / L$

50

No flag

$\mu \mathrm{g} / \mathrm{L} \quad 0.000015$

$\mu \mathrm{g} / \mathrm{L} \quad 0.002$

$\mathrm{pCi} / \mathrm{L} \quad 4.5 \mathrm{E}+02$

$\mu \mathrm{g} / \mathrm{L} \quad 50$

$\mu \mathrm{g} / \mathrm{L} \quad 0.00225$

$\mu g / L \quad 0.002$

$\mu \mathrm{g} / \mathrm{L} \quad 5$

$\mu \mathrm{g} / \mathrm{L}$

$\mu g / L$

$\mu \mathrm{g} / \mathrm{L}$

$\mu g / L$

$\mu \mathrm{g} / \mathrm{L}$

$\mu g / L$

$\mu \mathrm{g} / \mathrm{L}$

$\mathrm{pCi} / \mathrm{L}$

$\mathrm{pCi} /$

4.4E+0

$\mathrm{pCi} / \mathrm{L} \quad 2 \mathrm{E}+02$

$\mu \mathrm{g} / \mathrm{L} \quad 10$

$\mu \mathrm{g} / \mathrm{L} \quad 10$

$\mu \mathrm{g} / \mathrm{L} \quad 10$

$\mathrm{pCi} / \mathrm{L}$

$\mu \mathrm{g} / \mathrm{L}$

$\mu \mathrm{g} / \mathrm{L}$

$\mu \mathrm{g} / \mathrm{L}$

$1.5 \mathrm{E}+02$

500

$$
50
$$

5,000

0

No flag

$\mu \mathrm{g} / \mathrm{L}$

5,000

$\mu \mathrm{g} / \mathrm{L}$

5,000

$\mu \mathrm{g} / \mathrm{L}$

5,000

$\mu \mathrm{g} / \mathrm{L}$

25

$\mu \mathrm{g} / \mathrm{L} \quad 500$

$\mu \mathrm{g} / \mathrm{L} \quad 5,000$

No flag

No flag

$\mu g / L$

$\mu \mathrm{g} / \mathrm{L}$

1.5

1.5

$\mu \mathrm{g} / \mathrm{L} \quad 50$

$\mu \mathrm{g} / \mathrm{L} \quad 35$
Flag 2

4

No flag

No flag

No flag

$4.66 \mathrm{E}+02$

500

$2 \mathrm{E}+01$

$8 \mathrm{E}+00$

$8 \mathrm{E}+00$

100

400,000

10,000

100

No flag

0.00003

0.004

$9 \mathrm{E}+02$

100

0.0045

0.004

10

10

5

100

2

2

2

100

$1.25 \mathrm{E}+02$

$7.92 \mathrm{E}+01$

$8.8 \mathrm{E}+01$

$4.01 \mathrm{E}+02$

20

20

20

$3 \mathrm{E}+02$

1,000

100

10,000

0

No flag

10,000

10,000

10,000

50

1,000

10,000

No flag

No flag

3

50

100

70
Source

Final PDWS (EPA 1993a)

Set by EPD/EMS

Set by EPD/EMS

Set by EPD/EMS

Proposed PDWS (EPA 1991)

Set by EPD/EMS

Interim Final PDWS (EPA 1977)

Final PDWS (EPA 1993a)

Final PDWS (EPA 1993a)

Final PDWS (EPA 1993a)

Proposed PDWS (EPA, 1990)

EPA Method 9030

EPA Method 8270

Set by EPD/EMS

Final PDWS (EPA 1993a)

EPA Method 8280

Interim Final PDWS (EPA 1977)

EPA Method 8270

EPA Method 8280

EPA Method 8280

EPA Method 8240

EPA Method 8240

Final PDWS (EPA 1993a)

EPA Method 8270

Final PDWS (EPA 1993a)

Final PDWS (EPA 1993a)

Final PDWS (EPA 1993a)

EPA Method 8270

Proposed PDWS (EPA 1991)

Proposed PDWS (EPA 1991)

Proposed PDWS (EPA 1991)

Proposed PDWS (EPA 1991)

EPA Method 282.2

EPA Method 282.2

EPA Method 282.2

Interim Final PDWS (EPA 1977)

Final PDWS (EPA 1993a)

EPA Method 8270

EPA Method 9060

Final PDWS (EPA 1993a)

Set by EPD/EMS

EPA Method 418.1

EPA Method 9060

EPA Method 9060

EPA Method 9020

APHA Method 420

EPA Method 418.1

Set by EPD/EMS

Set by EPD/EMS

Final PDWS (EPA 1993a)

Final PDWS (EPA 1993a)

EPA Method 8270

Final PDWS (EPA 1993a) 
Analyte

1,1,1-Trichloroethane

1,1,2-Trichloroethane.

Trichloroethylene

Trichlorofluoromethane

2,4,5-Trichlorophenol

2,4,6-Trichlorophenol

2,4,5-Trichlorophenoxyacetic acid

1,2,3-Trichloropropane

$0,0,0-T r i e t h y l$ phosphorothioate

1,3,5-Trinitrobenzene

Tritium

Turbidity ${ }^{\mathrm{g}}$

Uranium

Uranium, dissolved

Uranium, total recoverable

Uranium alpha activity

Uranium-233/234 ${ }^{\mathrm{e}}$

Uranium-234

Uranium-235

Uranium-238

Vanadium

Vanadium, dissolved

Vanadium, total recoverable

Vinyl acetate

Xylenes

Yttrium-88

Zinc

Zinc, dissolved

Zinc, total recoverable

Zinc-65

Zirconium- $95^{c}$

Zirconium/Niobium-95 ${ }^{\mathrm{c}}$

\begin{tabular}{|c|c|c|}
\hline Unit & Flag 1 & Flag 2 \\
\hline$\mu \mathrm{g} / \mathrm{L}$ & 100 & 200 \\
\hline$\mu \mathrm{g} / \mathrm{L}$ & 2.5 & 5 \\
\hline$\mu \mathrm{g} / \mathrm{L}$ & 2.5 & 5 \\
\hline$\mu \mathrm{g} / \mathrm{L}$ & 5 & 10 \\
\hline$\mu \mathrm{g} / \mathrm{L}$ & 50 & 100 \\
\hline$\mu \mathrm{g} / \mathrm{L}$ & 50 & 100 \\
\hline$\mu \mathrm{g} / \mathrm{L}$ & 2.5 & 5 \\
\hline$\mu \mathrm{g} / \mathrm{L}$ & 5 & 10 \\
\hline$\mu g / L$ & 50 & 100 \\
\hline$\mu \mathrm{g} / \mathrm{L}$ & 50 & 100 \\
\hline $\mathrm{pCi} / \mathrm{mL}$ & $\begin{array}{l}\text { 1E+01 } \\
\text { No flag }\end{array}$ & $\begin{array}{l}2 E+01 \\
\text { No flag }\end{array}$ \\
\hline$\mu \mathrm{g} / \mathrm{L}$ & 10 & 20 \\
\hline$\mu \mathrm{g} / \mathrm{L}$ & 10 & 20 \\
\hline$\mu \mathrm{g} / \mathrm{L}$ & 10 & 20 \\
\hline $\mathrm{pCi} / \mathrm{L}$ & $1.5 \mathrm{E}+01$ & $3 E+0 t$ \\
\hline $\mathrm{pCi} / \mathrm{L}$ & $6.9 \mathrm{E}+00$ & $1.38 \mathrm{E}+01$ \\
\hline $\mathrm{pCi} / \mathrm{L}$ & $6.95 \mathrm{E}+00$ & $1.39 E+01$ \\
\hline $\mathrm{pCi} / \mathrm{L}$ & $7.25 \mathrm{E}+00$ & $1.45 E+01$ \\
\hline $\mathrm{pCi} / \mathrm{L}$ & $7.3 E+00$ & $1.46 \mathrm{E}+01$ \\
\hline$\mu \mathrm{g} / \mathrm{L}$ & 40 & 80 \\
\hline$\mu \mathrm{g} / \mathrm{L}$ & 40 & 80 \\
\hline$\mu \mathrm{g} / \mathrm{L}$ & 40 & 80 \\
\hline$\mu \mathrm{g} / \mathrm{L}$ & 5 & 10 \\
\hline$\mu \mathrm{g} / \mathrm{L}$ & 5,000 & 10,000 \\
\hline $\mathrm{pCi} / \mathrm{L}$ & $5 E+01$ & $1 \mathrm{E}+02$ \\
\hline$\mu \mathrm{g} / \mathrm{L}$ & 2,500 & 5,000 \\
\hline$\mu \mathrm{g} / \mathrm{L}$ & 2,500 & 5,000 \\
\hline$\mu \mathrm{g} / \mathrm{L}$ & 2,500 & 5,000 \\
\hline $\mathrm{pCi} / \mathrm{L}$ & $1.5 E+02$ & $3 E+02$ \\
\hline $\mathrm{pCi} / \mathrm{L}$ & $1 E+02$ & $2 \mathbf{E}+02$ \\
\hline $\mathrm{pCi} / \mathrm{L}$ & $1 \mathrm{E}+02$ & $2 \mathrm{E}+02$ \\
\hline
\end{tabular}

Source

Final PDWS (EPA 1993a)

Final PDWS (EPA 1993a)

Final PDWS (EPA 1993a)

EPA Method 8240

EPA Method 8270

EPA Method 8270

EPA Method 8150

EPA Method 8240

EPA Method 8270

EPA Method 8270

Final PDWS (EPA 1993a)

Set by EPD/EMS

Proposed PDWS (EPA 1991)

Proposed PDWS (EPA 1991)

Proposed PDWS (EPA 1991)

Proposed PDWS (EPA 1991)

Proposed PDWS (EPA 1991)

Proposed PDWS (EPA 1991)

Proposed PDWS (EPA 1991)

Proposed PDWS (EPA 1991)

EPA Method 6010

EPA Method 6010

EPA Method 6010

EPA Method 8240

Final PDWS (EPA 1993a)

EPA Method 901.1

SDWS (EPA, 1993b)

SDWS (EPA, 1993b)

SDWS (EPA, 1993b)

Interim Final PDWS (EPA 1977)

Interim Final PDWS (EPA 1977)

Interim Final PDWS (EPA 1977)

a References for dated sources are at the end of this appendix.

b EMS is currently unable to perform this analysis.

E EMS discontinued monitoring this radionuclide because it is inappropriate for the SRS Groundwater Monitoring Program.

d EPD/EMS set this flagging criterion using the 1991 proposed PDWS because the final PDWS in 1977 may have been in error.

e For double radionuclide analyses where each separate radionuclide has its own standard, the more stringent standard is used.

f The applied standard is for radium-226.

q The primary maximum contaminant level range for turbidity is 1-5 NTU, which is inappropriate for the SRS Groundwater Monitoring Program.

\section{References}

EPA (U.S. Environmental Protection Agency), 1977. National Interim Primary Drinking Water Regulations, EPA-570/9-76-003. Washington, DC.

EPA (U.S. Environmental Protection Agency), 1986. Water Pollution Control; National Primary Drinking Water Regulations, Radionuclides (Proposed). Federal Register. September 30, 1986, pp. 34835-34862. Washington, DC.

$$
\text { A }-18
$$


EPA (U.S. Environmental Protection Agency), 1990. National Primary and Secondary Drinking Water Regulations; Synthetic Organic Chemicals and Inorganic Chemicals (Proposed Rule). Federal Register, July 25, 1990, pp. 30369-30448. Washington, DC.

EPA (U.S. Environmental Protection Agency), 1991. National Primary Drinking Water Regulations; Radionuclides; Proposed Rule. Federal Register, July 18, 1991, pp. 33052-33127. Washington, DC.

EPA (U.S. Environmental Protection Agency), 1993a. National Primary Drinking Water Regulations. Code of Federal Regulations, Section 40, Part 141, pp. 592-732. Washington, DC.

EPA (U.S. Environmental Protection Agency), 1993b. National Secondary Drinking Water Regulations. Code of Federal Regulations, Section 40, Part 143, pp. 774-777. Washington, DC.

SCDHEC (South Carolina Department of Health and Environmental Control), 1981. State Primary Drinking Water Regulations, R.61-58.5. Columbia, SC.

This page intentionally left blank. 


\section{APPENDIX B \\ Groundwater Monitoring Results Tables}


List Of Tables

\begin{tabular}{|c|c|c|}
\hline able B-1 & $\begin{array}{l}\text { Description } \\
\text { Groundwater Monitoring Results For Individual } \\
\text { Primary Wells }\end{array}$ & $\begin{array}{l}\text { Page \# } \\
\text { B-3 }\end{array}$ \\
\hline 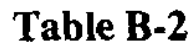 & Appendix IX Results for Primary Wells & B-29 \\
\hline e B. & $\begin{array}{l}\text { Groundwater Monitoring Results for Individual } \\
\text { Recovery Wells, Monthly Sampling }\end{array}$ & B-181 \\
\hline$a x$ & Field Data For Secondary Wells & B-193 \\
\hline 1 & $\begin{array}{l}\text { Groundwater Monitoring Results For Other TNX } \\
\text { Area Wells }\end{array}$ & B-202 \\
\hline & $\begin{array}{l}\text { Water Elevations for TNX-Area Wells From SRTC } \\
\text { Measurements in } 1998 .\end{array}$ & B-215 \\
\hline
\end{tabular}

Page \#

B-193

B-202

B-215

B - 2 
Table B-1. Groundwater Monitoring Results for Individual Primary Wells

WELL: P 26A

SRS Coord Lat/Longitude Screen Zone Elevation Top of Standpipe Top of Casing Casing Pump Screen Zone N 72010.4 33.214 Deg N $32.0-22.0 \mathrm{ft} \mathrm{msl} 154.5 \mathrm{ftmsl} 154.0 \mathrm{ftmsl} 4$ 4" PVC $\mathrm{S}$ Serniconfined E 18055.981 .759 Deg W

$\begin{array}{lllll}\text { SAMPLE DATE } & 03 / 02 / 98 & 05 / 12 / 98 & 08 / 05 / 98 & 12 / 01 / 98\end{array}$

FIELD DATA

\begin{tabular}{|c|c|c|c|c|c|}
\hline Analyte & 101998 & 201998 & 301998 & 401998 & Unit \\
\hline Depth to water & 32.43 & 32 & 35.3 & 36.98 & At BTOS \\
\hline $\mathrm{pH}$ & 6.5 & 5.4 & 5.5 & 4.9 & \\
\hline Sp. Conductance & 37 & 34 & 38 & 36 & $\mathrm{uS} / \mathrm{cm}$ \\
\hline Water temperature & 20.1 & 16 & 20.1 & 18.9 & deg. C \\
\hline Alkalinity as $\mathrm{CaCO} 3$ & 5 & 2 & 4 & 4 & $\mathrm{mg} / \mathrm{L}$ \\
\hline Phenolpthalein Alkalinity & 0. & 0 & 0 & 0 & $\mathrm{mg} / \mathrm{L}$ \\
\hline Turbidity & .4 & 1.6 & .4 & .6 & NTU \\
\hline Volumes purged & 2.14336 & 2.28659 & 2.04934 & 2.31017 & gallons \\
\hline
\end{tabular}

\section{ANALYTICAL DATA}

Analvte

Boron, total recoverable

Carbon tetrachloride

Chloroform

Gross alpha

Lead, total recoverable

Manganese, total recoverable

Mercury, total recoverable

Nitrate as nitrogen

Tetrachloroethylene

Trichloroethylene

\begin{tabular}{|c|c|c|c|c|c|c|c|c|c|}
\hline 101998 & Mod & 201998 & Mod & 301998 & Mod & 401998 & $\underline{\mathbf{M}}$ & ad & Unit \\
\hline & & & & & & 8.93 & J & I & $\mathrm{ug} / \mathrm{L}$ \\
\hline$<.405$ & U & $<.405$ & $\mathrm{U}$ & $<5$ & $\mathrm{U}$ & $<1$ & $\mathrm{U}$ & & $\mathrm{ug} / \mathrm{L}$ \\
\hline$<.428$ & U & $<.428$ & U & $<5$ & U & $<1$ & $U$ & & $\mathrm{ug} / \mathrm{L}$ \\
\hline .53 & UI & 2.96 & & .641 & UI & $<.49$ & $\mathrm{U}$ & & $\mathrm{pCi} / \mathrm{L}$ \\
\hline$<5$ & $\mathbf{U}$ & $<5$ & $\mathbf{U}$ & $<10$ & $\mathrm{U}$ & $\begin{array}{c}<10 \\
16.5\end{array}$ & $\mathrm{U}$ & - & $\begin{array}{l}\mathrm{ug} / \mathrm{L} \\
\mathrm{ug} / \mathrm{L}\end{array}$ \\
\hline$<.2$ & $U$ & $<.2$ & $\mathrm{U}$ & $<.5$ & U & $<.5$ & $\mathrm{U}$ & & $u g / L$ \\
\hline$<100$ & $\mathrm{U}$ & $<60$ & U. V & $<100$ & U & $<100$ & $\pi$ & $Q$ & ug/L \\
\hline$<.569$ & $\mathrm{U}$ & $<.569$ & $\mathrm{U}$ & $<5$ & U & $<1$ & $U$ & & ug $/ \mathrm{L}$ \\
\hline$<.39$ & $U$ & $<.39$ & $\mathrm{U}$ & $<5$ & $\mathrm{U}$ & $<1$ & $\mathrm{U}$ & & $\mathrm{ug} / \mathrm{L}$ \\
\hline
\end{tabular}

Note: Concentrations in bold exceed the Drinking Water Standards listed in Appendix A. Units are for all four quarters. 
WELL: TBG 1 $\frac{\text { SRS Coord, Lat/Longitude }}{\text { N 71429.5 }} \frac{\text { Screen Zone Elevation Top of Standpipe Top of Casing }}{33.212 \mathrm{Deg} \mathrm{N}} \frac{\text { Casing }}{109.1-89.1 \mathrm{ft} \mathrm{msl}} \frac{\text { Pump }}{151.4 \mathrm{ft} \mathrm{msl}} \frac{\text { Screen Zone }}{151.2 \mathrm{ft} \mathrm{msl}}$ E 17134.7 81.760 Deg W

SAMPLE DATE

03/02/98

$05 / 13 / 98$

08/06/98

$12 / 04 / 98$

\section{FIELD DATA}

Analyte

Depth to water

$\mathrm{pH}$

Sp. Conductance

Water temperature

Alkalinity as $\mathrm{CaCO} 3$

Phenolpthalein Alkalinity

Turbidity

Volumes purged

Sampling codes

$\begin{array}{ll}\frac{101998}{49.26} & 201998 \\ 4.3 & 49 \\ 160 & 4.2 \\ 21.9 & 140 \\ 0 & 17 \\ 0 & 0 \\ .4 & 0 \\ 3.68038 & 1.2 \\ & 6.28375\end{array}$

301998

52.35

4

150

27.8

0

0

1.3

0

NX
401998 Unit

53.91 ft BTOS

4.2

$140 \quad \mathrm{uS} / \mathrm{cm}$

22.3 deg. C

$0 \quad \mathrm{mg} / \mathrm{L}$

$0 \quad \mathrm{mg} / \mathrm{L}$

$.6 \quad$ NTU

3.90869 gallons

\section{ANALYTICAL DATA}

\section{Analyte}

Boron, total recoverable

Carbon tetrachloride

Chloroform

Gross alpha

Lead, total recoverable

Manganese, total recoverable

Mercury, total recoverable

Nitrate as nitrogen

Tetrachloroethylene

Trichloroethylene
101998 Mod 201998 Mod 301998 Mod 401998 Mod Unit

\begin{tabular}{|c|c|c|c|c|c|c|c|c|c|c|c|}
\hline 15 & & 19.8 & & & 34.3 & & & $\begin{array}{l}29.8 \\
26\end{array}$ & $\begin{array}{l}J \\
J\end{array}$ & $\begin{array}{l}1 \\
\mathrm{~L}\end{array}$ & $\begin{array}{l}u g / L \\
u g / L\end{array}$ \\
\hline$<.428$ & $\mathbf{U}$ & $<.428$ & $\mathbf{U}$ & & $<5$ & $\mathbf{U}$ & & .644 & $\mathbf{J}$ & I & $\mathrm{ug} / \mathrm{L}$ \\
\hline 8.34 & & 31.68 & & & 30.82 & & V & 22.14 & & & $\mathrm{pCi} / \mathrm{L}$ \\
\hline$<5$ & $\mathbf{U}$ & $<5$ & $\mathrm{U}$ & & $<10$ & U & & $\begin{array}{l}<10 \\
99.8\end{array}$ & $\mathrm{U}$ & & $\begin{array}{l}\mathrm{ug} / \mathrm{L} \\
\mathrm{ug} / \mathrm{L}\end{array}$ \\
\hline $\begin{array}{l}<.2 \\
11650\end{array}$ & $\mathrm{U}$ & $\begin{array}{l}.17 \\
13290\end{array}$ & $\mathbf{J}$ & $E$ & $\begin{array}{l}<.5 \\
12200\end{array}$ & $\mathrm{U}$ & & $\begin{array}{l}<.5 \\
13600\end{array}$ & $\begin{array}{l}\mathrm{U} \\
\mathrm{J}\end{array}$ & $\mathrm{Q}$ & $\begin{array}{l}\mathrm{ug} / \mathrm{L} \\
\mathrm{ug} / \mathrm{L}\end{array}$ \\
\hline$<.569$ & U & .962 & & & 1.66 & J & $\mathrm{E}$ & 4.27 & & & $\mathrm{ug} / \mathrm{L}$ \\
\hline 3.6 & & 6.59 & & & 8.16 & & & 14.2 & & & $\mathrm{ug} / \mathrm{L}$ \\
\hline
\end{tabular}


WELL: TBG 3

SRS Coord. Lat/Longitude Screen Zone Eleyation Top of Standpipe Top of Casing Casing Pump Screen Zone N 71324.1 33.212 Deg N $\quad 108.9-88.9 \mathrm{ft} \mathrm{msl} \quad 151.4 \mathrm{ft} \mathrm{msl} 151.2 \mathrm{ftmsl} 4 " \mathrm{STL} \quad \mathrm{S}$ Unconfined E 17177.781 .760 Deg W

$\begin{array}{lllll}\text { SAMPLE DATE } & 03 / 05 / 98 & 05 / 11 / 98 & 08 / 04 / 98 & 12 / 04 / 98\end{array}$

\section{FIELD DATA}

$\begin{array}{ll}\text { Analyte } & \mathbf{1 0 1 9 9 8} \\ \text { Depth to water } & 46.35 \\ \text { pH } & 4 \\ \text { Sp. Conductance } & 200 \\ \text { Water temperature } & 18 \\ \text { Alkalinity as CaCO3 } & 0 \\ \text { Phenolpthalein Alkalinity } & 0 \\ \text { Turbidity } & 2.7 \\ \text { Volumes purged } & 3.91850\end{array}$

201998
44.7
4.4
140
17
0
0
1.7
7.36211

301998
47.85
4.4
140
25.3
0
0
1.4
4.21977

$\begin{array}{ll}\frac{401998}{50.1} & \text { Unit } \\ 4.1 & \text { ft BTOS } \\ 170 & \\ 24.1 & \text { uS/cm } \\ 0 & \text { deg. C } \\ 0 & \mathrm{mg} / \mathrm{L} \\ .7 & \mathrm{mg} / \mathrm{L} \\ 2.12415 & \text { NTU } \\ & \text { gallons }\end{array}$

\section{ANALYTICAL DATA}

\section{Analve}

Boron, total recoverable

Carbon tetrachloride

Chloroform

Gross alpha

Lead, total recoverable

Manganese, total recoverable

Mercury, total recoverable

Nitrate as nitrogen

Tetrachloroethylene

Trichloroethylene

\section{Mod 201998 Mod 301998 Mod}

401998 Mod Unit

\begin{tabular}{|c|c|c|c|c|c|c|c|c|c|c|c|c|}
\hline 222 & & & 336 & & & 408 & & & 150 & $\mathbf{J}$ & $\mathrm{L}$ & $\mathrm{ug} / \mathrm{L}$ \\
\hline 12.1 & & & 20.5 & & & 25.3 & $\mathrm{~J}$ & $E$ & 16.6 & & & $\mathrm{ug} / \mathrm{L}$ \\
\hline 14.5 & & & 35.8 & & & 26.42 & & & 23.58 & & & $\mathrm{pCi} / \mathrm{L}$ \\
\hline$<5$ & $U$ & & $<5$ & $U$ & & $<10$ & $U$ & & $\begin{array}{r}<10 \\
314\end{array}$ & $U$ & & $\begin{array}{l}\mathrm{ug} / \mathrm{L} \\
\mathrm{ug} / \mathrm{L}\end{array}$ \\
\hline .5 & & & .34 & & & $<.5$ & $\mathrm{U}$ & & $.871^{\circ}$ & & & $\mathrm{ug} / \mathrm{L}$ \\
\hline 18270 & $\mathrm{~J}$ & QV & 14190 & $\mathrm{~J}$ & QV & 15400 & & & 18300 & $\mathbf{J}$ & $Q$ & $\mathrm{ug} / \mathrm{L}$ \\
\hline$<5.69$ & $\mathrm{U}$ & & 8.05 & & & 12.6 & $\mathrm{~J}$ & $\mathrm{E}$ & 14 & & & $\mathrm{ug} / \mathrm{L}$ \\
\hline 394 & & & 1040 & & & 875 & & & 488 & & & $\mathrm{ug} / \mathrm{L}$ \\
\hline
\end{tabular}

Note: Concentrations in bold exceed the Drinking Water Standards listed in Appendix A. Units are for all four quarters. 
WELL: TBG 4

SRS Coord, Lat/hongitude Screen Zone Eleyation Top of Standpipe Top of Casing Casing Pump Screen Zone N 71267.1 33.211 Deg N $109.3-89.3 \mathrm{ft} \mathrm{msl} 151.6 \mathrm{ftmsl} 151.3 \mathrm{ftmsl} 4 " \mathrm{sTL} V$ Unconfined E 17177.781 .760 Deg W
SAMPLE DATE
$03 / 05 / 98$
$05 / 11 / 98$
$08 / 04 / 98$

\section{FIELD DATA}

$\begin{array}{ll}\text { Analyte } & \mathbf{1 0 1 9 9 8} \\ \text { Depth to water } & 46.2 \\ \text { pH } & 3.8 \\ \text { Sp. Conductance } & 260 \\ \text { Water temperature } & 17 \\ \text { Alkalinity as CaCO3 } & 0 \\ \text { Phenolpthalein Alkalinity } & 0 \\ \text { Turbidity } & 3.4 \\ \text { Volumes purged } & 2.31553 \\ \text { Sampling codes } & \end{array}$

\begin{tabular}{ll}
$\mathbf{2 0 1 9 9 8}$ & $\mathbf{3 0 1 9 9 8}$ \\
\hline 44.4 & 47 \\
4.2 & 4.3 \\
220 & 220 \\
18 & 24.1 \\
0 & 0 \\
0 & 0 \\
1.5 & 1.7 \\
2.42517 & 3.35366
\end{tabular}

\begin{tabular}{ll}
401998 & Unit \\
\cline { 2 - 3 } & ft BTOS \\
& uS/cm \\
deg. C \\
$\mathrm{mg} / \mathrm{L}$ \\
$\mathrm{mg} / \mathrm{L}$ \\
$\mathrm{NTU}$ \\
gallons
\end{tabular}

\section{ANALYTICAL DATA}

\section{Analyte}

Carbon tetrachloride

Chloroform

Gross alpha

Lead, total recoverable

Mercury, total recoverable

Nitrate as nitrogen

Tetrachloroethylene

Trichloroethylene

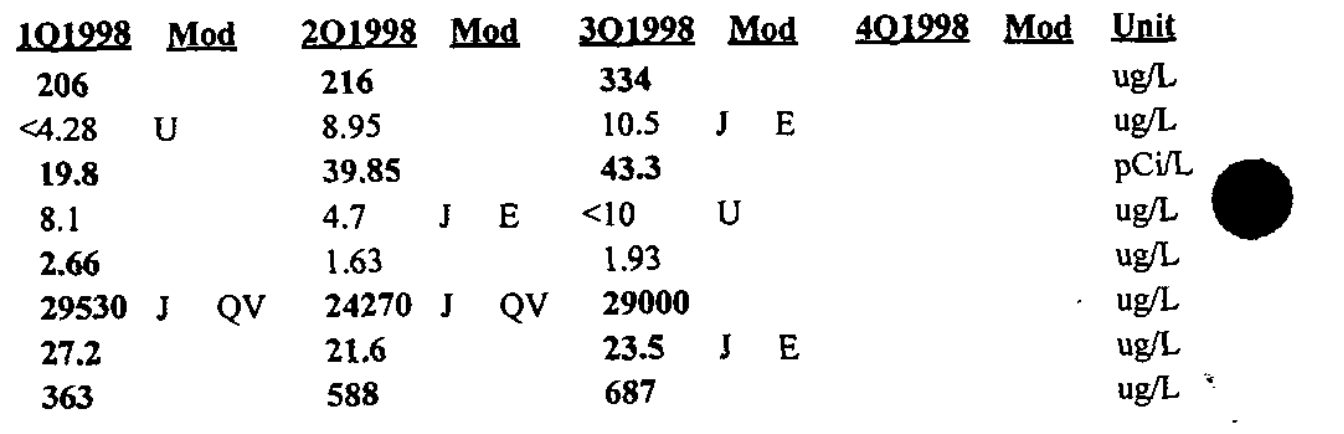


WELL: TBG 5

SRS Coord, Lat/Longitude Screen Zone Elevation Top ofStandpipe Top of Casing Casing Pump Screen Zone N 71226.5 33.212 Deg N E 17354.5 81.759 Deg W $-112.4-92.4 \mathrm{ft} \mathrm{msl}$ $149.6 \mathrm{ft} \mathrm{msl}$ $149.4 \mathrm{ft} \mathrm{msl}$

4 " STL

B

Unconfined

SAMPLE DATE

03/05/98

$05 / 11 / 98$

08/04/98

$12 / 04 / 98$

\section{FIELD DATA}

Analyte

Depth to water

$\mathrm{pH}$

Sp. Conductance

Water temperature

Alkalinity as $\mathrm{CaCO} 3$

Phenolpthalein Alkalinity

Turbidity

Volumes purged

Sampling codes

$\begin{array}{lll}101998 & 201998 & 301998 \\ 44.15 & 41.8 & 45.1 \\ 5 & 5 & 5.1 \\ 76 . & 60 & 64 \\ 17 & 17 & 26.4 \\ 3 & 1 & 1 \\ 0 & 0 & 0 \\ 4.1 & 1.8 & 1.1 \\ 1.42356 & 2.70780 & 2.43390\end{array}$

$\begin{array}{ll}\frac{401998}{47.8} & \text { Unit } \\ 4.8 & \text { ft BTOS } \\ 61 & \\ 23.4 & \text { uS/cm } \\ 0 & \text { deg. C } \\ 0 & \text { mg/L } \\ 2.1 & \text { mg/L } \\ 4.30806 & \text { NTU } \\ & \text { gallons }\end{array}$

\section{ANALYTICAL DATA}

\section{Analvte}

Boron, total recoverable

Carbon tetrachloride

Chloroform

Gross alpha

Lead, total recoverable

Manganese, total recoverable

Mercury, total recoverable

Nitrate as nitrogen

Tetrachloroethylene

Trichloroethylene

\section{Mod 201998 Mod 301998 Mod 401998 Mod Unit}

\begin{tabular}{|c|c|c|c|c|c|c|}
\hline$<40.5$ & $\mathrm{U}$ & & $<.405$ & $\mathrm{U}$ & & $<50$ \\
\hline$<42.8$ & $\mathrm{U}$ & & $<.428$ & U & & $<50$ \\
\hline-.09 & UI & & 2.44 & & & 1.51 \\
\hline 3.3 & $\mathbf{J}$ & $\mathrm{E}$ & $<5$ & $\mathrm{U}$ & & $<10$ \\
\hline$<.2$ & $\mathrm{U}$ & & $<.2$ & $\mathrm{U}$ & & $<.5$ \\
\hline 5010 & $\mathbf{J}$ & QV & 3510 & J & QV & 3520 \\
\hline 106 & & & $<.569$ & $U$ & & $<50$ \\
\hline 1710 & & & 1510 & & & 844 \\
\hline
\end{tabular}

$<1 \quad$ U L $\quad$ ug/L

.627 J $\quad \mathrm{I} \quad \mathrm{ug} / \mathrm{L}$

$2.63 \quad \mathrm{~J} \quad \mathrm{I} \quad \mathrm{pCi} / \mathrm{L}$

$<5.66$ JU I ug/L

$8.86 \quad J \quad I^{-}$ug/L

$<.5 \mathrm{U} \quad \mathrm{ug} / \mathrm{L}$

$3090 \mathrm{~J} \quad \mathrm{Q} \quad \mathrm{ug} / \mathrm{L}$

$<1 \quad$ U $\quad$ ug/L

$232 \quad u g / L$
15.3 J I ug/L

Note: Concentrations in bold exceed the Drinking Water Standards listed in Appendix A. Units are for all four quarters. 
WELL: TBG 5A

SRS Coord. Lat/Longitude Screen Zone Elevation Top of Standpipe Top of Casing Casing Pump Screen Zone N 71206.8 33.212 Deg N $80.0-70.0 \mathrm{ftmsl} 150.2 \mathrm{ftmsl} 150.0 \mathrm{ftmsl} 4$ " STL S Unconfined E $17348.8 \quad 81.759$ Deg W

\section{SAMPLE DATE}

FIELD DATA

Analyte

Depth to water

$\mathrm{pH}$

Sp. Conductance

Water temperature

Alkalinity as $\mathrm{CaCO} 3$

Phenolpthalein Alkalinity

Turbidity

Volumes purged

Sampling codes
$03 / 02 / 98$

101998

44.83

5.5

29

22

4

0 .

.3

2.55727

$05 / 13 / 98$

08/05/98

$12 / 04 / 98$

\begin{tabular}{|c|c|c|c|}
\hline 201998 & $\underline{301998}$ & 401998 & Unit \\
\hline $\begin{array}{l}43.45 \\
5 ?\end{array}$ & $\begin{array}{l}46.7 \\
5.4\end{array}$ & $\begin{array}{l}48.9 \\
49\end{array}$ & ft BTOS \\
\hline $\begin{array}{l}5.2 \\
28\end{array}$ & $\begin{array}{l}3.4 \\
28\end{array}$ & $\begin{array}{l}4.9 \\
29\end{array}$ & $\mathrm{uS} / \mathrm{cm}$ \\
\hline 17 & 22 & 22 & deg. C \\
\hline 3 & 5 & 2 & $\mathrm{mg} / \mathrm{L}$ \\
\hline 0 & 0 & 0 & $\mathrm{mg} / \mathrm{L}$ \\
\hline 2.5 & .8 & .9 & NTU \\
\hline 4.04558 & 5.72219 & 3.48012 & gallons \\
\hline
\end{tabular}

\section{ANALYTICAL DATA}

Analvte

Boron, total recoverable

Carbon tetrachloride

Chloroform

Gross alpha

Lead, total recoverable

Manganese, total recoverable

Mercury, total recoverable

Nitrate as nitrogen

Tetrachloroethylene

Trichloroethylene
101998 Mod

\section{Mod}

$\underline{301998 \text { Mod }}$

401998 Mod Unit $<266$ U ug/L

$\begin{array}{llllll}<.405 & \text { UJ } O & <.405 & \mathrm{U} & <5 & \mathrm{U}\end{array}$

$<.428$ UJ $\mathrm{O} \quad<.428 \quad \mathrm{U}$

. .41 UI

1.22

$<5 \quad \mathrm{U}$

$<5 \quad \mathrm{U}$

$<5$

U

.98 UI

$<10 \quad \mathrm{U}$

$<.2$

650

$<.2$

750

U

$<.569$

$<.569$

$\mathrm{U}$

UJ $\mathrm{O}$

$<.569$

$<.39$

UJ $\mathrm{O}$

$<.39$

$<.5$

590

U

$<5$

$<5$

$<1$ JU Q ug/L

$<1 \quad$ JU $Q \quad$ ug/L

$1.74 \mathrm{~J} \quad \mathrm{I} \quad \mathrm{pCi} / \mathrm{L}$

$<47$ U ug/L

3 J I - ug/L

$<.7$ U $\quad$ ug/L

722 J $\quad$ Q ug/L

$<1$ JU Q ug/L

$<1$ JU $Q$ ug/L

Note: Concentrations in bold exceed the Drinking Water Standards listed in Appendix A. Units are for all four quarters. 
WELL: TBG 5B

SRS Coord. Latalongitude Screen Zone Elevation Top of Standpipe Top of Casing Casing Pump ScreenZone N 71216.8 33.212 Deg N $56.2-46.2 \mathrm{ft} \mathrm{msl} 149.6 \mathrm{ft} \mathrm{msl} 149.4 \mathrm{ft} \mathrm{msl} 4 " \mathrm{STL}$ S Semiconfined E 17354.8 81.759 Deg W

SAMPLE DATE

FIELD DATA

Analyte
Depth to water
pH
Sp. Conductance
Water temperature
Alkalinity as CaCO3
Phenolpthalein Alkalinity
Turbidity
Volumes purged
Sampling codes

\section{ANALYTICAL DATA}

Analyte

Boron, total recoverable

Carbon tetrachloride

Chloroform

Gross alpha

Lead, total recoverable

Manganese, total recoverable

Mercury, total recoverable

Nitrate as nitrogen

Tetrachloroethylene

Trichioroethylene $\begin{array}{lll}03 / 03 / 98 & 05 / 15 / 98 & 08 / 05 / 98\end{array}$

$12 / 04 / 98$

\begin{tabular}{|c|c|c|c|c|}
\hline 101998 & 201998 & 301998 & 401998 & Unit \\
\hline $\begin{array}{l}32.58 \\
6.4\end{array}$ & $\begin{array}{l}32.5 \\
4.9\end{array}$ & $\begin{array}{l}38.2 \\
5\end{array}$ & $\begin{array}{l}37.55 \\
4.6\end{array}$ & $\overline{\mathrm{At} \text { BTOS }}$ \\
\hline $\begin{array}{l}32 \\
20.8\end{array}$ & $\begin{array}{l}35 \\
18.9\end{array}$ & $\begin{array}{l}32 \\
22.9\end{array}$ & $\begin{array}{l}32 \\
22\end{array}$ & $\begin{array}{l}\mathrm{uS} / \mathrm{cm} \\
\mathrm{deg} . \mathrm{C}\end{array}$ \\
\hline 4 & 0 & 3 & 1 & $\mathrm{mg} / \mathrm{L}$ \\
\hline 0 & 0 & 0 & 0 & $\mathrm{mg} / \mathrm{L}$ \\
\hline $\begin{array}{l}.8 \\
2.50395\end{array}$ & $\begin{array}{l}2.5 \\
2.75986\end{array}$ & $\begin{array}{l}1.3 \\
3.37711\end{array}$ & $\begin{array}{l}2.2 \\
2.64708\end{array}$ & $\begin{array}{l}\text { NTU } \\
\text { gallons }\end{array}$ \\
\hline
\end{tabular}

\begin{tabular}{|c|c|c|c|c|c|c|c|c|c|}
\hline 101998 & Mod & 201998 & Mod & 301998 & Mod & 401998 & $\mathbf{M}$ & & Unit \\
\hline & & & & & & 13.5 & J & I & ug/L \\
\hline$<1$ & U & $<1$ & $\mathbf{U}$ & $<5$ & $\mathbf{U}$ & $<1$ & $\mathrm{JU}$ & L & $\mathrm{ug} / \mathrm{L}$ \\
\hline$<1$ & $\mathrm{U}$ & $<1$ & $\mathrm{U}$ & $<5$ & U & $<1$ & $\mathrm{U}$ & & $\mathrm{ug} / \mathrm{L}$ \\
\hline 1.82 & & $<1.15$ & U V & .16 & UI & $<.73$ & $\mathrm{U}$ & & $\mathrm{pCi} / \mathrm{L}$ \\
\hline$<5$ & U & $<5$ & $\mathrm{U}$ & $<10$ & U & $\begin{array}{l}<10 \\
16.6\end{array}$ & $\mathrm{U}$ & & $\begin{array}{l}\mathrm{ug} / \mathrm{L} \\
\mathrm{ug} / \mathrm{L}\end{array}$ \\
\hline$<.2$ & U & $<.2$ & $\mathrm{U}$ & $<.5$ & U & $<.5$ & $\mathrm{U}$ & & $\mathrm{ug} / \mathrm{L}$ \\
\hline$<100$ & $U$ & $<100$ & UJ Q & $<200$ & $U$ & 114 & $\mathbf{J}$ & $Q$ & ug/L " \\
\hline$<1$ & $\mathrm{U}$ & $<1$ & U & $<5$ & U & $<1$ & $\mathrm{U}$ & & $\mathrm{ug} / \mathrm{L}$ \\
\hline$<1$ & $\mathrm{U}$ & $<1$ & U & $<5$ & $U$ & $<1$ & $U$ & & $\mathrm{ug} / \mathrm{L}$ \\
\hline
\end{tabular}

Note: Concentrations in bold exceed the Drinking Water Standards listed in Appendix A. Units are for all four quarters. 
WELL: TBG 6

SRS Coord Lat/Longitude ScreenZone Elevation Top of Standpipe Top of Casing Casing Pump ScreenZone N $71482.3 \quad 33.212$ Deg N 109.1 - 89.1 ft ms

$148.3 \mathrm{ft} \mathrm{msl}$

$148.1 \mathrm{ft} \mathrm{msl} \quad 4^{\prime \prime S T L}$

$\mathrm{S}$

Unconfined

E $17290.5 \quad 81.760 \mathrm{Deg} \mathrm{W}$

$\begin{array}{llll}03 / 05 / 98 & 05 / 11 / 98 & 08 / 04 / 98 & 12 / 04 / 98\end{array}$

SAMPLE DATE

FIELD DATA

$\begin{array}{ll}\text { Analyte } & \mathbf{1 0 1 9 9 8} \\ \text { Depth to water } & 43.45 \\ \text { pH } & 4.2 \\ \text { Sp. Conductance } & 180 \\ \text { Water temperature } & 16 \\ \text { Alkalinity as CaCO3 } & 0 \\ \text { Phenolpthalein Alkalinity } & 0 \\ \text { Turbidity } & 5 \\ \text { Volumes purged } & 2.35276 \\ \text { Sampling codes } & \end{array}$

$\begin{array}{ll}201998 & \frac{301998}{44.75} \\ 42 & 4.7 \\ 4.8 & 100 \\ 120 & 23.6 \\ 17 & 0 \\ 0 & 0 \\ 0 & 5.3 \\ 4.2 & 3.74412 \\ 3.13845 & \end{array}$

\begin{tabular}{ll}
$\frac{401998}{46.96}$ & Unit \\
4.4 & ft BTOS \\
110 & \\
21.3 & uS/cm \\
0 & deg. C \\
0 & $\mathrm{mg} / \mathrm{L}$ \\
4.1 & $\mathrm{mg} / \mathrm{L}$ \\
.253221 & NTU \\
$\mathrm{NX}$ & gallons \\
\hline
\end{tabular}

\section{ANALYTICAL DATA}

\section{Analyte}

Boron, total recoverable

Carbon tetrachloride

Chloroform

Gross alpha

Lead, total recoverable

Manganese, total recoverable

Mercury, total recoverable

Nitrate as nitrogen

Tetrachloroethylene

Trichloroethylene

\begin{tabular}{|c|c|c|c|c|c|c|c|c|c|c|}
\hline 101998 & $\mathbf{M}$ & od & 201998 & Mod & 301998 & Mod & 401998 & M & od & Unit \\
\hline & & & & & & & 20.7 & $\mathbf{J}$ & I & $\mathrm{ug} / \mathrm{L}$ \\
\hline 19.7 & & 0 & 7.07 & & $<50$ & $\mathrm{U}$ & $<\mathbf{5 0}$ & $\mathrm{U}$ & L & $\mathrm{ug} / \mathrm{L}$ \\
\hline$<4.28$ & UJ & $\mathrm{O}$ & .899 & & $<50$ & U & 1.18 & & & $\mathrm{ug} / \mathrm{L}$ \\
\hline 3.36 & & & 20.49 & & 10.31 & & 9.38 & & & $\mathrm{pCi} / \mathrm{L}$ \\
\hline$<5$ & $\mathbf{U}$ & & $<5$ & U & $<10$ & $\mathrm{U}$ & $\begin{array}{c}<10 \\
102\end{array}$ & $\mathrm{U}$ & & $\begin{array}{l}u g / L \\
u g / L\end{array}$ \\
\hline .63 & & & .56 & & .275 & E & .435 & $\mathbf{J}$ & I & $\mathrm{ug} / \mathrm{L}$ \\
\hline 17740 & $J$ & QV & 11590 & QV & 13400 & & 10700 & J & $Q$ & $\mathrm{ug} / \mathrm{L}$ \\
\hline$<5.69$ & UJ & o & $<.569$ & U & $<50$ & U & $<1$ & $\mathrm{U}$ & & $u g / L$ \\
\hline 428 & & 0 & 800 & & 465 & & 252 & & & $u g / L$ \\
\hline
\end{tabular}


WELL: TNX 10

SRS Coord, Lat/Longitude Screen Zone Elevation Top of Standpipe Top of Casing Casing Pump Screen Zone $\begin{array}{ll}\text { N } 71613.5 & 33.211 \mathrm{Deg} \mathrm{N} \\ \text { E } 16699.6 & 81.762 \mathrm{Deg} \mathrm{W}\end{array}$ $99.6-79.6 \mathrm{ft} \mathrm{msl}$ $156.7 \mathrm{ft} \mathrm{msl}$ $156.5 \mathrm{ft} \mathrm{msl}$ 4 " STL $\mathrm{S}$ Unconfined

SAMPLE DATE

FIELD DATA

Analyte
Depth to water
pH
Sp. Conductance.
Water temperature
Alkalinity as CaCO3
Phenolpthalein Alkalinity
Turbidity
Volumes purged
Sampling codes

\section{ANALYTICAL DATA}

\section{Analyte}

Boron, total recoverable

Carbon tetrachloride

Chloroform

Gross alpha

Lead, total recoverable

Manganese, total recoverable

Mercury, total recoverable

Nitrate as nitrogen

Tetrachloroethylene

Trichloroethylene
$03 / 02 / 98$

$05 / 13 / 98$

$\underline{201998}$

55.99

4.5

41

22.1

5

0

.5

3.54303
.6

19.4

4

6

.

\begin{tabular}{l}
101998 \\
\hline 59.69 \\
5.8 \\
41 \\
19.4 \\
4 \\
0 \\
.6 \\
3.54303
\end{tabular}

$08 / 05 / 98$

$12 / 02 / 98$ 
WELL: TNX 2D

SRS Coord Lat/Longitude Screen Zone Elevation Top of Standpipe Top of Casing Casing Pump Screen Zone

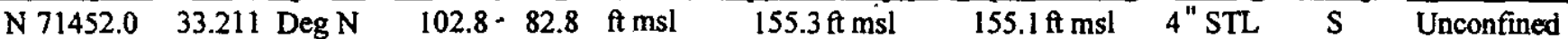
E 16788.281 .761 Deg W

$\begin{array}{llll}03 / 02 / 98 & 05 / 13 / 98 & 08 / 05 / 98 & 12 / 02 / 98\end{array}$

SAMPLE DATE

FIELD DATA

Analyte
Depth to water
pH
Sp. Conductance
Water temperature
Alkalinity as CaCO3
Phenolpthalein Alkalinity
Turbidity
Volumes purged
Sampling codes

\section{ANALYTICAL DATA}

\section{Analyte}

Boron, total recoverable

Carbon tetrachloride

Chloroform

Gross alpha

Lead, total recoverable

Manganese, total recoverable

Mercury, total recoverable

Nitrate as nitrogen

Tetrachloroethylene

Trichloroethylene

101998
54.93
6
49
20.3
6
0
3.4
4.56352

101998 Mod

$$
<.405 \mathrm{U}
$$

.882

-.02 UI

$<5 \quad \mathrm{U}$

$<.2 \quad \mathrm{U}$

1250

$<.569 \quad \mathrm{U}$

$<.39 \quad \mathrm{U}$
201998

54.97

5.7

41

22.9

4

0

1.9

4.39813
301998

57.6

5.6

50

21.8

6

0

1.9

4.77020
$401998 \quad$ Unit

58.97 At BTOS

5.6

$48 \quad \mathrm{uS} / \mathrm{cm}$

21.8

5

0

.7

3.65945 deg. C

$\mathrm{mg} / \mathrm{L}$

$\mathrm{mg} / \mathrm{L}$

NTU

gallons

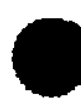


WELL: TNX 3D

SRSCoord Lat/ongitude Screen Zone Elevation Top of Standpipe Top of Casing Casing Pump Screen Zone N 71236.7 33.211 Deg N E 17043.1 81.760 Deg W $104.9-84.9 \mathrm{ft} \mathrm{msl}$ $154.5 \mathrm{ft} \mathrm{msl}$ $154.3 \mathrm{ft} \mathrm{msl}$ 4 "STL $\mathrm{S}$ Unconfined

SAMPLE DATE 03/05/98

$05 / 11 / 98$

08/04/98

$12 / 04 / 98$

\section{FIELD DATA}

Analyte
Depth to water
pH
Sp. Conductance
Water temperature
Alkalinity as CaCO3
Phenolpthalein Alkalinity
Turbidity
Volumes purged
Sampling codes

ANALYTICAL DATA

\section{Analvte}

Boron, total recoverable

Carbon tetrachloride

Chloroform

Gross alpha

Lead, total recoverable

Manganese, total recoverable

Mercury, total recoverable

Nitrate as nitrogen

Tetrachloroethylene

Trichloroethylene

$\begin{array}{ll}\frac{101998}{53.95} & \frac{201998}{53.2} \\ & \\ .690662 & .752785 \\ & \text { LNS }\end{array}$

$\underline{301998}$

56.45

401998

58.02

Unit

4.9

110

23.4

4

0

.706281

LNS

NX

$\mathrm{uS} / \mathrm{cm}$ deg. C $\mathrm{mg} / \mathrm{L}$

$\mathrm{mg} / \mathrm{L}$

NTU gallons

ft BTOS

101998 Mod 201998 Mod 301998 Mod 401998 Mod Unit

\begin{tabular}{|c|c|c|c|c|c|c|c|c|c|c|c|c|}
\hline & & & & & & & & & 94.5 & $\mathrm{~J}$ & I & $\mathrm{ug} / \mathrm{L}$ \\
\hline 28.4 & & & 31.8 & & & 29.1 & & & 51.1 & $\mathrm{~J}$ & L & $\mathrm{ug} / \mathrm{L}$ \\
\hline 1.86 & & & 2.24 & & & 1.35 & $\mathbf{J}$ & $E$ & 9.39 & & & $\mathrm{ug} / \mathrm{L}$ \\
\hline$-2: 22$ & U & & 19.31 & & & 12.74 & & & 5.88 & & & $\mathrm{pCi} / \mathrm{L}$ \\
\hline 12.6 & & & 3.6 & $\mathbf{J}$ & E & $<10$ & $\mathbf{U}$ & & $\begin{array}{l}<10 \\
56.3\end{array}$ & $\mathbf{U}$ & & $\begin{array}{l}\mathrm{ug} / \mathrm{L} \\
\mathrm{ug} / \mathrm{h}\end{array}$ \\
\hline 3.24 & & & 1.23 & & & .399 & $\mathbf{J}$ & $\mathrm{E}$ & .333 & $\mathrm{~J}$ & I & $\mathrm{ug} / \mathrm{L}$ \\
\hline 34090 & $\mathbf{J}$ & QV & 19830 & & & 8630 & & & 9000 & $\mathrm{~J}$ & $\mathrm{Q}$ & ug/L \\
\hline 22 & & & 12.8 & & & $<25$ & $\mathrm{U}$ & & 11.1 & & & $\mathrm{ug} / \mathrm{L}$ \\
\hline 176 & & & 230 & & & 303 & $\mathrm{~L}$ & & 442 & & & $\mathrm{ug} / \mathrm{L}$ \\
\hline
\end{tabular}

Note: Concentrations in bold exceed the Drinking Water Standards listed in Appendix A. Units are for all four quarters. 
WELL: TNX 4D

SRS Coord, Lat/Longitude ScreenZone Elevation TopofStandpipe Top of Casing Casing Pump Screen Zone

N $71002.7 \frac{13.211 \mathrm{Deg} \mathrm{N}}{105.5-85.5 \mathrm{ft} \mathrm{msl} 150.0 \mathrm{ftmsl}} 149.8 \mathrm{ft} \mathrm{msl} 4$ " STL S Unconfined E $17223.0 \quad 81.759$ Deg W

SAMPLE DATE

FIELD DATA

Analvte

Depth to water

$\mathrm{pH}$

Sp. Conductance

Water temperature

Alkalinity as $\mathrm{CaCO} 3$

Phenolpthalein Alkalinity

Turbidity

Volumes purged

Sampling codes
$03 / 02 / 98$

101998

45.02

4.9

67

19.8

1

0

1.8

3.08357
$05 / 13 / 98$

201998

42.9

783565

LNS

08/06/98

$12 / 02 / 98$

301998
45.88
4.9
45
21.4
0
0
3.8
2.64823

401998

48.57

5.2

55

21.9

0

0

12.8

.387639

NX
Unit ft BTOS

$\mathrm{uS} / \mathrm{cm}$

deg. C $\mathrm{mg} / \mathrm{L}$ $\mathrm{mg} / \mathrm{L}$ NTU gallons

\section{ANALYTICAL DATA}

\section{Analvte}

Boron, total recoverable

Carbon tetrachloride

Chloroform

Gross alpha

Lead, total recoverable

Manganese, total recoverable

Mercury, total recoverable

Nitrate as nitrogen

Tetrachloroethylene

Trichloroethylene
101998 Mod 201998 Mod 301998 Mod 401998 Mod Unit

$\begin{array}{clcccccccc}5.5 & & 1.5 & & <5 & \mathrm{U} & 2 & & \mathrm{ug} / \mathrm{L} \\ <.428 & \mathrm{U} & <.428 & \mathrm{U} & <5 & \mathrm{U} & <1 & \mathrm{U} & \mathrm{ug} / \mathrm{L} \\ .97 & \mathrm{UI} & 4.55 & & 4.8 & & \mathrm{~V} & 8.52 & & \mathrm{pC} / \mathrm{L} \\ <5 & \mathrm{U} & <5 & \mathrm{U} & <10 & \mathrm{U} & <10 & \mathrm{U} & \mathrm{ug} / \mathrm{L} \\ & & & & & & 19.5 & & \mathrm{ug} / \mathrm{L} \\ <.2 & \mathrm{U} & <.2 & \mathrm{U} & <.5 & \mathrm{U} & <.5 & \mathrm{U} & \mathrm{ug} / \mathrm{L} \\ 3860 & & 2070 & & 2100 & & 3320 & \mathrm{~J} & \mathrm{Q} & \mathrm{ug} / \mathrm{L} \\ 3.98 & & .858 & & <5 & \mathrm{U} & & 2.55 & & \mathrm{ug} / \mathrm{L} \\ 5.24 & & 2.27 & & 1.79 & \mathrm{~J} & \mathrm{E} & 3.97 & & \mathrm{ug} / \mathrm{L}\end{array}$


WELL: TNX 7D

SRS Coord. Lat/Longitude Screen Zone Elevation Top of Standpipe Top of Casing Casing Pump Screen Zone $\mathrm{N} 71738.133 .212 \mathrm{Deg} \mathrm{N} 103.6-83.6 \mathrm{ft} \mathrm{msl} 151.1 \mathrm{ft} \mathrm{msl} 150.9 \mathrm{ftmsl} 4$ " STL $\mathrm{S}$ Unconfined E 17080.6 81.761 Deg W

$03 / 02 / 98 \quad 05 / 13 / 98 \quad 08 / 05 / 98 \quad 12 / 02 / 98$

SAMPLE DATE

FIELD DATA

Analyte

Depth to water

$\mathrm{pH}$

Sp. Conductance

Water temperature

Alkalinity as $\mathrm{CaCO} 3$

Phenolpthalein Alkalinity

Turbidity

Volumes purged

Sampling codes

101998
48.13
7.3
25
19.7
10
0
1.4
2.86270

101998
201998

48.5

$\begin{array}{ll} & 32 \\ & 20.6 \\ & 3 \\ & 0 \\ 1.05410 & 1.2 \\ \text { LNS } & 2.45261\end{array}$

301998

51.14

5.4

LNS

\begin{tabular}{|c|c|c|c|c|c|c|c|c|c|}
\hline 101998 & Mod & 201998 & Mod & 301998 & Mod & 401998 & Mo & & Unit \\
\hline & & & & & & 22.3 & $\mathbf{J}$ & I & $\mathrm{ug} / \mathrm{L}$ \\
\hline$<.405$ & $\mathrm{U}$ & $<.405$ & $U$ & $<5$ & $\mathrm{U}$ & $<1$ & $\mathrm{U}$ & & $\mathrm{ug} / \mathrm{L}$ \\
\hline$<.428$ & U & $<.428$ & U & $<5$ & U & $<1$ & $\mathrm{U}$ & & $\mathrm{ug} / \mathrm{L}$ \\
\hline-.4 & UI & 2.47 & & .05 & UI & 3.27 & J & I & $\mathrm{pCi} / \mathrm{L}$ \\
\hline$<5$ & $\mathrm{U}$ & 4.1 & E & $<10$ & $\mathrm{U}$ & $<4.4$ & $\mathrm{JU}$ & I & $\mathrm{ug} / \mathrm{L}$ \\
\hline & & & & & & 7.91 & $J$ & I & ug/L \\
\hline$<.2$ & $\mathbf{U}$ & $<.2$ & $\mathrm{U}$ & $<.5$ & U & $<.5$ & $\mathrm{U}$ & & $\mathrm{ug} / \mathrm{L}$ \\
\hline 50 & J $\quad$ E & $<100$ & $\mathrm{U}$ & $<100$ & U & 641 & J & $Q$ & $\mathrm{ug} / \mathrm{L}$ \\
\hline$<.569$ & $\mathbf{U}$ & $<.569$ & U & $<5$ & $\mathrm{U}$ & $<1$ & $\mathrm{U}$ & & $\mathrm{ug} / \mathrm{L}$ \\
\hline$<.39$ & $\mathrm{U}$ & $<.39$ & $\mathrm{U}$ & $<5$ & U & $<1$ & U & & $\mathrm{ug} / \mathrm{L}$ \\
\hline
\end{tabular}

\section{ANALYTICAL DATA}

\section{Analvte}

Boron, total recoverable

Carbon tetrachloride

Chloroform

Gross alpha

Lead, total recoverable

Manganese, total recoverable

Mercury, total recoverable

Nitrate as nitrogen

Tetrachloroethylene

Trichloroethylene

\begin{tabular}{ll} 
401998 & Unit \\
\hline 52.57 & ft BTOS \\
5.4 & \\
50 & uS/cm \\
20.2 & deg. C \\
6 & mg/L \\
0 & mg/L \\
5.1 & NTU \\
.310466 & gallons \\
NX &
\end{tabular}


WELL: TNX 8D

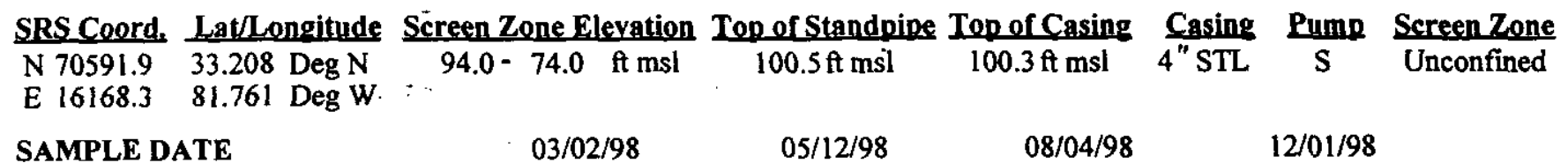

$\begin{array}{lllll}\text { SAMPLE DATE } & 03 / 02 / 98 & 05 / 12 / 98 & 08 / 04 / 98 & 12 / 01 / 98\end{array}$

\section{FIELD DATA}

Analvte

Depth to water

$\mathrm{pH}$

Sp. Conductance

Water temperature

Alkalinity as $\mathrm{CaCO} 3$

Phenolpthalein Alkalinity

Turbidity

Volumes purged

Sampling codes

101998
4.18
5.3
140
16
10
0
3.4
3.37681

201998

5.66

4.6

100

19

5

0

2

3.17581

\section{8}

6.85

5

110

21.7

7

0

1.2

5.17274
$401998 \quad$ Unit

7.74

5.1

110

19.1

7

0

1.4

4.27092 ft BTOS

$\mathrm{uS} / \mathrm{cm}$

deg. C

$\mathrm{mg} / \mathrm{L}$

$\mathrm{mg} / \mathrm{L}$

NTU

gallons

\section{ANALYTICAL DATA}

\section{Analyte}

Boron, total recoverable

Carbon tetrachloride

Chloroform

Gross alpha

Lead, total recoverable

Manganese, total recoverable

Mercury, total recoverable

Nitrate as nitrogen

Tetrachloroethylene

Trichloroethylene
101998 Mod 201998 Mod 301998 Mod 401998 Mod Unit

\begin{tabular}{|c|c|c|c|c|c|c|c|c|c|}
\hline & & & & & & 582 & & & $u g / L$ \\
\hline$<.405$ & $\mathrm{U}$ & $<.405$ & $\mathrm{U}$ & $<5$ & $\mathrm{U}$ & $<1$ & U & & $\mathrm{ug} / \mathrm{L}$ \\
\hline$<.428$ & $\mathrm{U}$ & .596 & & $<5$ & $\mathrm{U}$ & $<1$ & $\mathrm{U}$ & & $\mathrm{ug} / \mathrm{L}$ \\
\hline-2.35 & UI & .68 & UI & .58 & UI & $<.31$ & $\mathrm{U}$ & & $\mathrm{pCi} / \mathrm{L}$ \\
\hline$<5$ & $\mathrm{U}$ & $<5$ & $\mathrm{U}$ & $<10$ & $\mathbf{U}$ & $\begin{array}{c}<10 \\
14.1\end{array}$ & $\mathbf{U}$ & & $\begin{array}{l}\text { ug/L } \\
\text { ug/L }\end{array}$ \\
\hline$<.2$ & $\mathrm{U}$ & $<, 2$ & U & $<.5$ & $\mathbf{U}$ & $<.5$ & $\mathbf{U}$ & & ug/L \\
\hline 4470 & & $\$ 340$ & V & 4920 & & 3780 & $\mathrm{~J}$ & $Q$ & $\lg / \mathrm{L}$ \\
\hline $\begin{array}{r}<.569 \\
7.12\end{array}$ & $U$ & $\begin{array}{r}<.569 \\
8.53\end{array}$ & U & $\begin{array}{l}<5 \\
7.18\end{array}$ & $\mathrm{U}$ & $\begin{array}{l}<1 \\
7.1\end{array}$ & $\mathrm{U}$ & & $\begin{array}{l}\mathrm{ug} / \mathrm{L} \\
\mathrm{ug} / \mathrm{L}\end{array}$ \\
\hline
\end{tabular}


WSRC-RP-99-4003

Unclassified

WELL: TNX 9D

$\frac{\text { SRS Coord. }}{\text { N 70791.4 }} \frac{\text { Lat/Longitude }}{33.209 \mathrm{Deg} \mathrm{N}} \frac{\text { Screen Zone Elevation Top of Standpipe Top of Casing }}{95.4-75.4 \mathrm{ft} \mathrm{msl}} \frac{\text { Casing }}{101.9 \mathrm{ft} \mathrm{msl}} \frac{\text { Pump }}{101.7 \mathrm{ftmsl}} \frac{\text { Screen Zone }}{4 " \mathrm{STL}}$ E 16145.8

$$
95.4-75.4 \mathrm{ft} \mathrm{msl} \quad 101.9 \mathrm{ft} \mathrm{msl}
$$

03/03/98

$05 / 12 / 98$

08/04/98
101998

5.72

5.9

90

16.8

10

0

1.8

6.59236
201998

6.25

5.8

96

19

11

0

1.3

Turbidity

Sampling codes
7.07074
301998

8.75

5.2

170

20.5

3

0

3

2.69266 $\underline{401998}$

Unit

ft BTOS

$\mathrm{uS} / \mathrm{cm}$

deg. C

$\mathrm{mg} / \mathrm{L}$

$\mathrm{mg} / \mathrm{L}$

NTU

gallons

\section{ANALYTICAL DATA}

\section{Analvte}

Carbon tetrachloride

Chloroform

Gross alpha

Lead, total recoverable

Mercury, total recoverable

Nitrate as nitrogen

Tetrachloroethylene

Trichloroethylene

\section{Mod}

$<1 \quad \mathrm{U}$

$<1 \quad$ U

1.5

$<5 \quad \mathrm{U}$

$<2 \quad \mathrm{U}$

$<100$ U

$<1 \quad$ U

$<1 \quad$ U
201998 Mod

$<.405$

$<.428$

1.18

$<5$

$<.2$

$<240$

$<.569$

.399
$301998 \mathrm{Mo}$

$<5$ U

$<5$

1.94

$<10 \quad \mathrm{U}$

$<.5 \quad \mathrm{U}$

811

$<5$

U

$<5 \quad \mathrm{U}$
401998 Mod Unit $\mathrm{ug} / \mathrm{L}$ ug/L $\mathrm{pCi} / \mathrm{L}$ ug/L ug/L ug/L ug/L ug/L

Note: Concentrations in bold exceed the Drinking Water Standards listed in Appendix A. Units are for all four quarters. 
WELL: TNX 10D

SRS Coord. Lat/Longitude Screen Zone Elevation Top of Standoipe Top of Casing Casing Pump Screen Zone N 70999.3 33.209 Deg N 97.0-77.0 ft msl $102.5 \mathrm{ft} \mathrm{msl} 102.3 \mathrm{ft} \mathrm{msl} 4{ }^{\text {" PVC }} \mathrm{S}$ Unconfined E 16166.781 .762 Deg W

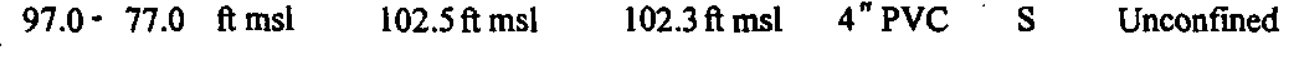

SAMPLE DATE

\section{FIELD DATA}

\section{Anaivte}

Depth to water

$\mathrm{pH}$

Sp. Conductance

Water temperature

Alkalinity as $\mathrm{CaCO} 3$

Phenolpthalein Alkalinity

Turbidity

Volumes purged

Sampling codes
03/05/98

$101998 \quad 201998$

6

140

13

14

0

14.4

3.94920

$05 / 12 / 98$

6.75
9.56

5.2

140

20.6

2

0

14.5

3.00229

NPS
401998 Unit

ft BTOS

$\mathrm{uS} / \mathrm{cm}$

deg. C

$\mathrm{mg} / \mathrm{L}$

$\mathrm{mg} / \mathrm{L}$

NTU

gallons

\section{ANALYTICAL DATA}

\section{Analvte}

Carbon tetrachloride

Chloroform

Gross alpha

Lead, total recoverable

Mercury, total recoverable

Nitrate as nitrogen

Tetrachloroethylene

Trichloroethylene
101998 Mod

$<.405$

$<.428$

$$
U
$$

$\mathrm{U}$

20192

-.64 UI

$1<.405$

$<.428$

$U$

$\mathrm{U}$

1.23

$<5 \quad \mathrm{U}$

$<5$

$<.2 \quad \mathrm{U}$

$<230$

$<.569$

$<.2$

U

U

900

$<.569$

$<.39$

2.28
301998 Mod

$<5$

$<5$

2.24

$<10$

$<.5$

6230

$<5$

19.5
401998 Mod Unit $\mathrm{ug} / \mathrm{L}$ $u g / L$ $\mathrm{pCi} / \mathrm{L}$ $\mathrm{ug} / \mathrm{L}$ $u g / L$ ug/L ug/L $\mathrm{ug} / \mathrm{L}$

Note: Concentrations in bold exceed the Drinking Water Standards listed in Appendix A. Units are for all four quarters. 
WELL: TNX 11D

SRS Coord Lat/Longitude Screen Zone Elevation Top of Standpipe Ton of Casing Casing Pump Screen Zone N 71199.3 33.210 Deg N

E $16165.5 \quad 81.762$ Deg W $93.2-73.2 \mathrm{ft} \mathrm{msl},{ }^{\circ} 100.3 \mathrm{ft} \mathrm{msl}$ $99.8 \mathrm{ft} \mathrm{msl}$ 4 " PVC $\mathrm{S}$

Unconfined

SAMPLE DATE

03/03/98

$05 / 12 / 98$

08/06/98

\section{FIELD DATA}

\section{Analyte}

Depth to water

$\mathrm{pH}$

Sp. Conductance

Water temperature

Alkalinity as $\mathrm{CaCO} 3$

Phenolpthalein Alkalinity

Turbidity

Volumes purged

Sampling codes

$\mathbf{1 0 1 9 9 8}$
4.06
7.1
53
19.6
3
0
2.4
3.99020

$\frac{201998}{5.5}$
5
56
23
2
0
2.9
3.25107

301998

7.45

5

50

21.2

1

0

10.2

4.05973

\section{ANALYTICAL DATA}

\section{Analyte}

Carbon tetrachloride

Chloroform

Gross alpha

Lead, total recoverable

Mercury, total recoverable

Nitrate as nitrogen

Tetrachloroethylene

Trichloroethylene

\section{Mod}

$<.405 \mathrm{U}$

$<.428 \quad \mathrm{U}$

-.69 UI

$<5 \quad \mathrm{U}$

$<.2$ U

$<100 \quad \mathrm{U}$

$<.569$ U

.998

\section{Mod}

$<.405 \mathrm{U}$

$<.428 \quad \mathrm{U}$

.94 UI

$<5 \quad U$

$<.2 \quad U$

23390

$<.569 \quad \mathrm{U}$

1.54
301998 Mod 401998 Mod Unit

$<5 \quad \mathrm{U}$

$<5 \quad \mathrm{U}$

$<1.82$ U V

$<10 \quad \mathrm{U}$

$<.5 \quad \mathrm{U}$

$<100 \quad U$

$<5 \quad \mathrm{U}$

$2.22 \mathrm{~J} \quad \mathrm{E}$ $\mathrm{ug} / \mathrm{L}$

ug $/ \mathrm{L}$

$\mathrm{pCi} / \mathrm{L}$

ug/L

$u g / L$

$u g / L$

$\mathrm{ug} / \mathrm{L}$

ug/L"

Note: Concentrations in bold exceed the Drinking Water Standards listed in Appendix A. Units are for all four quarters. 
WELL: TNX 12D

SRSCoord, Lat/Longitude Screen Zone Elevation Top of Standpipe Top of Casing Casing Bump Screen Zone

N 71598.3 33.210 Deg N

$93.1-73.1 \mathrm{ft} \mathrm{msl}$

$99.4 \mathrm{ft} \mathrm{msl}$

$99.2 \mathrm{ft} \mathrm{msl}$

4" PVC

$\mathrm{S}$

Unconfined

E 16176.3 81.763 Deg W.

SAMPLE DATE

$03 / 02 / 98$

$05 / 12 / 98$

08/06/98

12/01/98

\section{FIELD DATA}

\section{Analyte}

Depth to water

$\mathrm{pH}$

Sp. Conductance

Water temperature

Alkalinity as $\mathrm{CaCO} 3$

Phenolpthalein Alkalinity

Turbidity

Volumes purged

Sampling codes

\section{ANALYTICAL DATA}

\section{Analyte}

Boron, total recoverable

Carbon tetrachloride

Chloroform

Gross alpha

Lead, total recoverable

Manganese, total recoverable

Mercury, total recoverable

Nitrate as nitrogen

Tetrachloroethylene

Trichloroethylene

\section{8}

2.7

7.2

59

18.5

15

0

.4

2.93152

201998
3.93
6.3
55
19.6
16
0
.3
3.02540

301998
5.34
6
58
20.2
14
0
.4
2.42316

401998

5.71

5.7

57

20.2

12

0

.7

4.41094

Unit
ft BTOS
uS/cm
deg. C
$\mathrm{mg} / \mathrm{L}$
$\mathrm{mg} / \mathrm{L}$
$\mathrm{NTU}$
gallons

101998 Mod 201998 Mod 301998 Mod 401998 Mod Unit

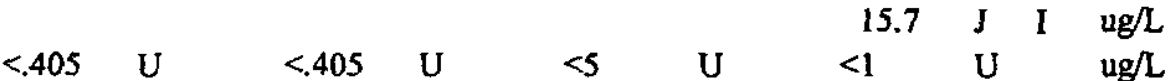

$<428 \quad U$

$<.405$

U

-.75 UI

$<.428$

$<5$

U

$<1$

1.42

$<5$

U

$<1$

$<1.75$

U

$<5 \quad U$

$<5 \quad U$

$<10$

U

$<.94$

$<10$

U

$\mathrm{ug} / \mathrm{L}$

$\begin{array}{lllll}<.2 & \mathrm{U} & <.2 & \mathrm{U} & .614\end{array}$

$<100$

$<60$

.614
$<100$

$<.569$

U V

$<.569 \quad \mathrm{U}$

$<.39$

$<5$

$<.39$

$\mathrm{U}$

U

U

$<5$

753

$<100$ JU Q

$<1 \quad$ U

$<1$

U

ug/L

$\mathrm{pCi} / \mathrm{L}$

$\mathrm{ug} / \mathrm{L}$

ug/L

$\mathrm{ug} / \mathrm{L}$

$\mathrm{ug} / \mathrm{L}$

$\mathrm{ug} / \mathrm{L}$

$\mathrm{ug} / \mathrm{L}$

Note: Concentrations in bold exceed the Drinking Water Standards listed in Appendix A. Units are for all four quarters. 
SRS Coord, Lat/Longitude Screen Zone Elevation Top of Standpipe Top of Casing Casing Pump Screen Zone N 71180.1 33.210 Deg N E 16609.1 81.761 Deg W $101.3-81.3 \mathrm{ft} \mathrm{msl} \quad 110.6 \mathrm{fmsl} 110.6 \mathrm{ftmsl} 2 " \mathrm{PVC}$ Unconfined

SAMPLE DATE

FIELD DATA

\section{Analyte}

Depth to water

$\mathrm{pH}$

Sp. Conductance

Water temperature

Alkalinity as $\mathrm{CaCO} 3$

Phenolpthalein Alkalinity

Turbidity

Volumes purged

Sampling codes
03/04/98

101998

12.55

8.1

89

13.6

15

0

.5

4.71835

$05 / 15 / 98$

08/05/98

$12 / 04 / 98$

$\begin{array}{lll}\frac{301998}{14.39} & \frac{401998}{15.8} & \text { Unit } \\ 6 & 5.4 & \text { ft BTOS } \\ 140 & 220 & \\ 20.7 & 20.7 & \text { uS } / \mathrm{cm} \\ 24 & 4 & \text { deg. C } \\ 0 & 0 & \mathrm{mg} / \mathrm{L} \\ 1.9 & 3.2 & \mathrm{mg} / \mathrm{L} \\ 3.66832 & 4.05004 & \mathrm{NTU} \\ & & \text { gallons }\end{array}$

\section{ANALYTICAL DATA}

\section{Analvte}

Boron, total recoverable

Carbon tetrachloride

Chloroform

201998

12.69

5.9

100

20.1

23

0

1.9

8.05200

1.9

4.05004

\section{Mod}

\section{Mod}

301998 Mod

401998 Mod Unit

$<.405$

$<.428 \quad U$

$29.1 \mathrm{~J}$

$<.405$

$40.9 \mathrm{~J} \quad \mathrm{I}$

$\mathrm{ug} / \mathrm{L}$

Gross alpha

$<.428$

U

$<5$

$<5$

U

16 J L

L $u g / L$

Lead, total recoverable

Manganese, total recoverable

Mercury, total recoverable

Nitrate as nitrogen

Tetrachloroethylene

Trichloroethylene

. .65

5.5

UI $<.67$

$<5$

$<.5 \quad \mathrm{U}$

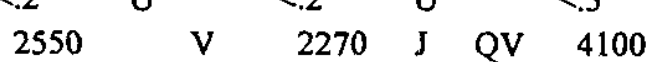

$\begin{array}{llll}<.569 & \mathrm{U} & <.569 \quad \mathrm{U}\end{array}$

$<5$

7.89
1.59

5.39

$<10$

369

.425

21900

2.96

99.2
ug/L

$\mathrm{pCi} / \mathrm{L}$

ug/L

ug/L

$\mathrm{ug} / \mathrm{L}$

$\mathrm{ug} / \mathrm{L}$

ug/L

ug $/$

Note: Concentrations in bold exceed the Drinking Water Standards listed in Appendix A. Units are for all four quarters. 
WELL: XSB IA

SRS Coord Lat/Longitude Screen Zone Elevation Top of Standpipe Top of Casing

N 71 105.4 33.211 Deg N

E 16883.0 81.760 Deg W -

$53.6-43.6 \mathrm{ft} \mathrm{msl}$

03/03/98

$156.2 \mathrm{ft} \mathrm{msl}$

$05 / 15 / 98$

$156.0 \mathrm{ft} \mathrm{msl}$

Casing

4 "STL

$\frac{\text { Pump }}{\mathrm{S}} \frac{\text { Screen Zone }}{\text { Unconfined }}$

SAMPLE DATE

FIELD DATA

Analyte

Depth to water

$\mathrm{pH}$

Sp. Conductance

Water temperature

Alkalinity as $\mathrm{CaCO} 3$

Phenolpthalein Alkalinity

Turbidity

Volumes purged

Sampling codes

101998
56.97
5.2
84
20.9
5
0.
.3
2.39260

201998

56.75

5.2

70

19.2

1

0

1.7

2.87621
08/06/98

$12 / 03 / 98$
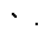

ANALYTICAL DATA

\section{Analyte}

Boron, total recoverable

Carbon tetrachloride

Chloroform

Gross alpha

Lead, total recoverable

Manganese, total recoverable

Mercury, total recoverable

Nitrate as nitrogen

Tetrachloroethylene

Trichloroethylene
$101998 \mathrm{Mad} 201998$ Mod $301998 \mathrm{Mod}$ 401998 Mod Unit

$<.405 \quad U \quad 687 \quad \mathrm{ug} / \mathrm{L}$

$\begin{array}{lllllllll}<.405 & \mathrm{U} & <1 & \mathrm{U} & <5 & \mathrm{U} & <1 & \mathrm{U} & \mathrm{ug} / \mathrm{L}\end{array}$

$\begin{array}{lllllllll}-428 & \mathrm{U} & <1 & \mathrm{U} & <5 & \mathrm{U} & <1 & \mathrm{U} & \mathrm{ug} / \mathrm{L}\end{array}$

$\begin{array}{llllllllllll}-.86 & \text { UI } & <1.67 & \mathrm{U} & \mathrm{V} & <.91 & \mathrm{U} & \mathrm{V} & 1.03 & \mathrm{~J} & \mathrm{I} & \mathrm{pCi} / \mathrm{L}\end{array}$

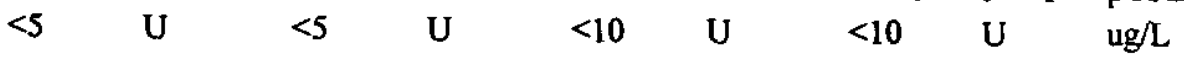

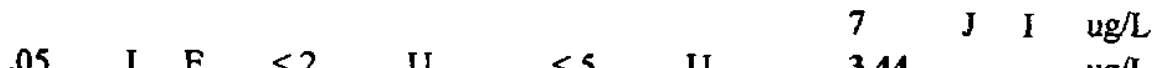

$\begin{array}{lllllllll}.05 & \mathrm{~J} & \mathrm{E} & <.2 & \mathrm{U} & <.5 & \mathrm{U} & \mathbf{3 . 4 4} & \mathrm{ug} / \mathrm{L}\end{array}$

$\begin{array}{lllllllllll}2590 & V & 1810 & \text { J } & \text { Q } & 1990 & 1590 & \text { J } & \text { Q } & \text { ug/L }\end{array}$

$\begin{array}{lllllllll}<.569 & \mathrm{U} & <1 & \mathrm{U} & <5 & \mathrm{U} & <1 & \mathrm{U} & \mathrm{ug} / \mathrm{L}\end{array}$

$\begin{array}{lllllllll}<.39 & \mathrm{U} & <1 & \mathrm{U} & <5 & \mathrm{U} & <1 & \mathrm{U} & \mathrm{ug} / \mathrm{L}\end{array}$

Note: Concentrations in bold exceed the Driaking Water Standards listed in Appendix A. Units are for all four quarters. 
WELL: XSB 1B

SRS Coord. Lat/Longitude Screen Zone Elevation Top of Standpipe Top of Casing Casing Pump Screen Zone

N 71105.0 33.211 Deg N $74.6-64.6 \mathrm{ft} \mathrm{msl}$

$156.2 \mathrm{ft} \mathrm{msl}$

$155.9 \mathrm{ft} \mathrm{ms}$ $4 "$ STL

S Semiconfined

E 16872.9 81.760 Deg W

SAMPLE DATE

03/03/98

$05 / 13 / 98$

08/06/98

$12 / 02 / 98$

FIELD DATA

$\begin{array}{ll}\text { Analvte } & \mathbf{1 0 1 9 9 8} \\ \text { Depth to water } & \mathbf{4 9 . 2 4} \\ \text { pH } & 5.3 \\ \text { Sp. Conductance } & 35 \\ \text { Water temperature } & 20.4 \\ \text { Alkalinity as CaCO3 } & 4 \\ \text { Phenolpthalein Alkalinity } & 0 \\ \text { Turbidity } & 5.4 \\ \text { Volumes purged } & 2.71824 \\ \text { Sampling codes } & \end{array}$

201998

50.4

5.2

34

16

4

0

4.2

8.05057
301998

54.58

4.9

33

23.2

2

0

4.3

4.69107
$401998 \quad$ Unit

55.35 ft BTOS

5.2

$31 \quad \mathrm{uS} / \mathrm{cm}$

21.6 deg. C

$2 \mathrm{mg} / \mathrm{L}$

$0 \mathrm{mg} / \mathrm{L}$

7.4 NTU

6.02124 gallons

\section{ANALYTICAL DATA}

\section{Analyte}

Boron, total recoverable

Carbon tetrachloride

Chloroform

Gross alpha

Lead, total recoverable

Manganese, total recoverable

Mercury, total recoverable

Nitrate as nitrogen

Tetrachloroethylene

Trichloroethylene

\begin{tabular}{|c|c|c|c|c|c|c|c|c|}
\hline 101998 & Mod & 201998 & Mod & 301998 & Mod & $\frac{401998}{<266}$ & $\underset{U}{\text { Mod }}$ & $\frac{\text { Unit }}{\mathrm{ug} / \mathrm{L}}$ \\
\hline$<.405$ & UJ $O$ & $<.405$ & UJ $\mathrm{O}$ & $<5$ & $U$ & $<1$ & $\mathrm{JU} Q$ & ug/L \\
\hline$<.428$ & UJ $O$ & $<.428$ & UJ $\mathrm{O}$ & $<5$ & U & $<1$ & $\mathrm{JU} \mathrm{Q}$ & $\mathrm{ug} / \mathrm{L}$ \\
\hline-.21 & UI & 1.29 & & $<1.53$ & U V & $<1.49$ & $\mathbf{U}$ & $\mathrm{pCi} / \mathrm{L}$ \\
\hline$<5$ & $\mathrm{U}$ & $<5$ & $\mathrm{U}$ & $<10$ & $\mathrm{U}$ & $\begin{array}{c}<47 \\
17.8\end{array}$ & $U$ & $\begin{array}{l}\mathrm{ug} / \mathrm{L} \\
\mathrm{ug} / \mathrm{L}\end{array}$ \\
\hline$<.2$ & U & $<.2$ & $\mathrm{U}$ & $<.5$ & U & $<.7$ & $\mathrm{U}$ & $\mathrm{ug} / \mathrm{L}$ \\
\hline$<50$ & UJ EV & 130 & & $<100$ & $U$ & 11 & J IQ & $\mathrm{ug} / \mathrm{L}$ " \\
\hline$<.569$ & UJ 0 & $<.569$ & UJ $\mathrm{O}$ & $<5$ & $U$ & $<1$ & $J U$ & $\mathrm{ug} / \mathrm{L}$ \\
\hline$<.39$ & UJ $O$ & $<.39$ & UJ $O$ & $<5$ & U & $<1$ & JU Q & ug/L \\
\hline
\end{tabular}

Note: Concentrations in bold exceed the Drinking Water Standards listed in Appendix A. Units are for all four quarters. 
WELL: XSB 1D

\begin{tabular}{|c|c|c|c|c|c|c|c|}
\hline SRS Coord. & Lat/Longitude & Screen Zone Elevation & Top of Standpine & Ten of Casing & Casing & Pumn & Screen Zone \\
\hline $\begin{array}{l}\text { N } 71104.8 \\
\text { E } 16893.5\end{array}$ & $\begin{array}{l}33.211 \text { Deg N } \\
81.760 \text { Deg W. }\end{array}$ & $107.9-87.9 \mathrm{ft} \mathrm{msl}$ & $156.2 \mathrm{ft} \mathrm{msl}$ & $156.0 \mathrm{ft} \mathrm{msl}$ & STL & S & Unconfined \\
\hline
\end{tabular}

SAMPLE DATE

03/05/98

$05 / 13 / 98$

$08 / 06 / 98$

$12 / 03 / 98$

FIELD DATA

Analvte

Depth to water

$\mathrm{pH}$

Sp. Conductance

Water temperature

Alkalinity as $\mathrm{CaCO} 3$

Phenolpthalein Alkalinity

Turbidity

Volumes purged

Sampling codes

101998
57
4.6
62
17
0
0
.7
9.61327

9.61327

201998
56.45
4.2
660
16
0
0
.9
18.8422

18.8422
301998

59.1

4.6

80

23.1

0

0

.3

7.96070
401998

60.57

4.3

74

21.1

0

0

1

6.27571
Unit ft BTOS

uS $/ \mathrm{cm}$

deg. C

$\mathrm{mg} / \mathrm{L}$

$\mathrm{mg} / \mathrm{L}$

NTU

gallons

\section{ANALYTICAL DATA}

\section{Analyte}

Boron, total recoverable

Carbon tetrachloride

Chloroform

Gross alpha

Lead, total recoverable

Manganese, total recoverable

Mercury, total recoverable

Nitrate as nitrogen

Tetrachloroethylene

Trichloroethylene
101998 Mod 201998 Mod 301998 Mod 401998 Mod Unit

\begin{tabular}{|c|c|c|c|c|c|c|c|c|c|c|c|c|}
\hline$<.405$ & $\mathbf{U}$ & & 5.77 & & & $<5$ & $U$ & & $<1$ & $\mathbf{U}$ & & $\mathrm{ug} / \mathrm{L}$ \\
\hline$<.428$ & $\mathrm{U}$ & & 1.58 & & & $<5$ & $\mathrm{U}$ & & $<1$ & $\mathbf{U}$ & & $\mathrm{ug} / \mathrm{L}$ \\
\hline .11 & UI & & 36.75 & & & 3.48 & & V & $<2.75$ & $\mathbf{U}$ & & $\mathrm{Ci} / \mathrm{L}$ \\
\hline 3.4 & J & E & $<5$ & U & & $<10$ & $U$ & & $\begin{array}{l}<10 \\
21.4\end{array}$ & $U$ & & $\begin{array}{l}u g / L \\
u g / L\end{array}$ \\
\hline .05 & $\mathbf{J}$ & $\mathrm{E}$ & 5.74 & & & .449 & $\mathbf{J}$ & E & $<.5$ & $\mathrm{U}$ & & $\mathrm{ug} / \mathrm{L}$ \\
\hline 1310 & $\mathrm{~J}$ & QV & 83280 & $\mathbf{J}$ & $Q$ & 4570 & & & 3930 & $\mathbf{J}$ & $Q$ & $\mathrm{ug} / \mathrm{h}$ \\
\hline$<.569$ & $U$ & & 1.13 & & & $<5$ & $\mathrm{U}$ & & $<1$ & $\mathrm{U}$ & & $1 \mathrm{~g} / \mathrm{L}$ \\
\hline 12.4 & & & 282 & & & 19 & & & 21.6 & & & $/ \mathrm{L}$ \\
\hline
\end{tabular}

Note: Concentrations in bold exceed the Drinking Water Standards listed in Appendix A. Units are for all four quarters. 
WELL: XSB 2D

SRS Coord. Lat/Longitude Screen Zone Elevation Top of Standpipe Top of Casing Casing Pump Screen Zone

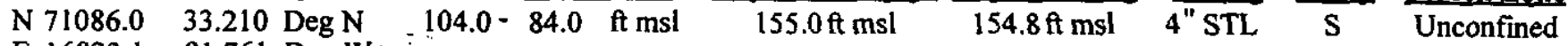
E 16823.1 81.761 Deg W
SAMPLE DATE
03/05/98
$05 / 11 / 98$
$08 / 04 / 98$
$12 / 04 / 98$

FIELD DATA

Analyte

Depth to water

$\mathrm{pH}$

Sp. Conductance

Water temperature

Alkalinity as $\mathrm{CaCO} 3$

Phenolpthalein Alkalinity

Turbidity

Volumes purged

Sampling codes

$\begin{array}{ll}\mathbf{1 0 1 9 9 8} & \mathbf{2 0 1 9 9 8} \\ 55.8 & 55.15 \\ 5.6 & 6 \\ 160 & 145 \\ 17 & 18 \\ 16 & 23 \\ 0 & 0 \\ .7 & 1.4 \\ 10.4675 & 4.48064\end{array}$

3ف்1998

58

5.8

120

24.6

12

0

.6

11.1947

$\begin{array}{ll}\frac{401998}{59.44} & \text { Unit } \\ 4.8 & \text { ft BTOS } \\ 120 & \\ 20.2 & \text { uS/cm } \\ 9 & \text { deg. C } \\ 0 & \mathrm{mg} / \mathrm{L} \\ .7 & \mathrm{mg} / \mathrm{L} \\ 3.62311 & \text { NTU } \\ & \text { gallons }\end{array}$

\section{ANALYTICAL DATA}

\section{Analyte}

Boron, total recoverable

Carbon tetrachloride

Chloroform

Gross alpha

Lead, total recoverable

Manganese, total recoverable

Mercury, total recoverable

Nitrate as nitrogen

Tetrachloroethylene

Trichloroethylene
101998 Mod 201998 Mod 301998 Mod 401998 Mod Unit

557

$<1 \quad$ U L $\quad$ ug/L

$1.61 \quad \mathrm{ug} / \mathrm{L}$

2.27 J I $\quad \mathrm{pCi} / \mathrm{L}$

$<10 \quad \mathrm{U} \quad \mathrm{ug} / \mathrm{L}$

$14 \quad \operatorname{ug} / \mathrm{L}$

$\begin{array}{lllllllll}<.2 & \mathrm{U} & <.2 & \mathrm{U} & <.5 & \mathrm{U} & <.5 & \mathrm{U} & \mathrm{ug} / \mathrm{L}\end{array}$

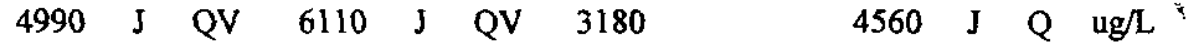

$<.569$ U $\quad<.569 \quad \mathrm{U}$

21.5
$<5$

14.5
$<1$

22.5 ug/L $\mathrm{ug} / \mathrm{L}$

Note: Concentrations in bold exceed the Drinking Water Standards listed in Appendix A. Units are for all four quarters. 
WELL: XSB 3A

$\frac{\text { SRS Coord, }}{\mathrm{N} 70915.3} \frac{\text { Lat/Longitude }}{33.210 \mathrm{Deg} \mathrm{N}} \frac{\text { Screen Zone Elevation Top of Standpioe Topof Casing }}{103.2-87.4 \mathrm{ftmsl}} \frac{\text { Casing }}{157.3 \mathrm{ft} \mathrm{msl}} \frac{\text { Pump }}{157.0 \mathrm{ft} \mathrm{msl}} \frac{\text { Screen Zone }}{4 \text { "STL }}$

E 16901.3 81.760 Deg W

SAMPLE DATE

$03 / 03 / 98$

FIELD DATA

Analyte

Depth to water

$\mathrm{pH}$

Sp. Conductance

Water temperature

Alkalinity as $\mathrm{CaCO} 3$

Phenolpthalein Alkalinity

Turbidity

Volumes purged

Sampling codes

\section{ANALYTICAL DATA}

\section{Analvte}

Boron, total recoverable

Carbon tetrachloride

Chloroform

Gross alpha

Lead, total recoverable

Manganese, total recoverable

Mercury, total recoverable

Nitrate as nitrogen

Tetrachloroethylene

Trichloroethylene

$<.405$

4.46

101998
56.54
4.7
180
21.4
4
0
.4
3.73511

201998

55.9

5

120

16

1

0

1.1

7.56632
$08 / 06 / 98$

$12 / 03 / 98$

$\begin{array}{lll}\frac{301998}{58.57} & \frac{401998}{59.9} & \text { Unit } \\ 4.8 & 4.9 & \text { ft BTOS } \\ 190 & 170 & \text { uS } / \mathrm{cm} \\ 21.9 & 21.2 & \text { deg. C } \\ 0 & 0 & \mathrm{mg} / \mathrm{L} \\ 0 & 0 & \mathrm{mg} / \mathrm{L} \\ .7 & .8 & \mathrm{NTU} \\ 2.62588 & 5.81468 & \text { gallons }\end{array}$

101998 Mod

201998 Mod

$301998 \mathrm{Mod}$

401998 Mod Unit

787

U

$<.405$

U

$<25$

$<25$

6.93

16.06

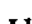

4.5

J E

49.7

$<1$

.765

7.72

$<7.11$

39.6

$<.5 \quad \mathrm{U}$

8730

$<25$

.346

$<200$

$<1$

$12.3 \mathrm{~J} \quad \mathrm{E}$

63.1
ug/L

$\mathrm{ug} / \mathrm{L}$

ug/L

$\mathrm{pCi} / \mathrm{L}$

U I ug/L

ug/L

J I ug/L

$\pi Q \quad$ ug $/$ ?

$U$

$\mathrm{ug} / \mathrm{L}$

ug/L

Note: Concentrations in bold exceed the Drinking Water Standards listed in Appendix A. Units are for all four quarters. 
WELL: XSB 4D

SRS Coord. Lat/Longitude Screen Zone Elevation Topof Standpipe Top of Casing Casing Pump Screen Zone N 70997.9 33.210 Deg N $103.9-83.9 \mathrm{ft} \mathrm{msl}$ $155.2 \mathrm{ft} \mathrm{msl}$ $154.9 \mathrm{ft} \mathrm{msl}$ 4 "STL $\mathrm{S}$ Unconfined E 16826.2 81.760 Deg W.

03/03/98

$05 / 12 / 98$

08/05/98

$12 / 03 / 98$

FIELD DATA

Analvte

Depth to water

$\mathrm{pH}$

Sp. Conductance

Water temperature

Alkalinity as $\mathrm{CaCO} 3$

Phenolpthalein Alkalinity

Turbidity

Volumes purged

Sampling codes

$\begin{array}{lllll}101998 & 201998 & \frac{301998}{401998} & \text { Unit } \\ 55.67 & 55 & 57.72 & 59.19 & \text { ft BTOS } \\ 5.4 & 5.4 & 5.2 & 5.2 & \\ 160 & 220 & 140 & 130 & \text { uS/cm } \\ 21 & 18 & 22.3 & 21.1 & \text { deg. C } \\ 10 & 4 & 6 & 11 & \mathrm{mg} / \mathrm{L} \\ 0 & 0 & 0 & 0 & \mathrm{mg} / \mathrm{L} \\ 1 & 2.7 & .7 & 1.2 & \mathrm{NTU} \\ 3.28147 & 6.09756 & 9.52744 & 6.84104 & \text { gallons }\end{array}$

\section{ANALYTICAL DATA}

\section{Analyte}

Boron, total recoverable

\section{Mod 201998 Mod}

Carbon tetrachloride

Chloroform

$<.405 \mathrm{U} \quad .811$

$1.32 \quad 1.11$

Gross alpha

Lead, total recoverable

.02 UI

10.25

$<5 \quad \mathrm{U}$

$<5$

Manganese, total recoverable

Mercury, total recoverable

Nitrate as nitrogen

Tetrachloroethylene

Trichloroethylene

$.15 \mathrm{~J} \quad \mathrm{E}$

$5140 \quad \mathrm{~V}$

$<.569 \quad \mathrm{U}$

2.14

v

$\mathbf{5 . 5 8}$
$<60$
$<.569$
$\mathbf{2 8 8}$

$\mathrm{U}$

301998 Mod

401998 Mod Unit

811 ug/L

$<1 \quad$ U $\quad$ ug/L

$1.41 \quad \mathrm{ug} / \mathrm{L}$

$2.83 \quad \mathrm{~J} \quad \mathrm{I} \quad \mathrm{pCi} / \mathrm{L}$

$<10$ U ug/L

$4.28 \quad \mathrm{~J} \quad \mathrm{I} \quad \mathrm{ug} / \mathrm{L}$

$<.5$ U $\quad$ ug/L

$4340 \mathrm{~J} \quad \mathrm{Q} \quad \mathrm{ug} / \mathrm{L}$ *

7090

$<5$

9.29

$<1$

4.04

$\mathrm{ug} / \mathrm{L}$

ug/L

Note: Concentrations in bold exceed the Drinking Water Standards listed in Appendix A. Units are for all four quarters. 
WELL: XSB 5A

SRS Coord. Lat/Longitude ScreenZone Elevation Top of Standpipe Tep of Casing Casing Pump Screen Zone N 70956.3 33.210 Deg N $108.9-108.9 \mathrm{ft} \mathrm{msl} \quad 112.2 \mathrm{ft} \mathrm{msl}$

$112.0 \mathrm{ft} \mathrm{msl}$ $4^{\prime \prime S T L}$ $\mathrm{S}$ Unconfined E 16703.7 81.761 Deg W.

03/04/98

$05 / 15 / 98$

$08 / 10 / 98$

$12 / 07 / 98$

\section{FIELD DATA}

\section{Analvte}

Depth to water

$\mathrm{pH}$

Sp. Conductance

Water temperature

Alkalinity as $\mathrm{CaCO} 3$

Phenolpthalein Alkalinity

Turbidity

Volumes purged

Sampling codes

\begin{tabular}{|c|c|}
\hline 101998 & 201998 \\
\hline 13.06 & 13.24 \\
\hline 5.7 & 5.3 \\
\hline 230 & 190 \\
\hline 19.3 & 17.8 \\
\hline 7 & 4 \\
\hline 0 & 0 \\
\hline 2.7 & 2 \\
\hline-5.5098 & -6.0134 \\
\hline
\end{tabular}

$\frac{301998}{14.92}$
5.3
200
20.9
11
0
1.6
-6.1904

401998

16.7

5.1

170

20.2

6

0

1.9

$-5.8286$
Unit ft BTOS

$\mathrm{uS} / \mathrm{cm}$

deg. C

$\mathrm{mg} / \mathrm{L}$

$\mathrm{mg} / \mathrm{L}$

NTU

gallons

\section{ANALYTICAL DATA}

\section{Analyte}

Boron, total recoverable

Carbon tetrachloride

Chloroform

Gross alpha

Lead, total recoverable

Manganese, total recoverable

Mercury, total recoverable

Nitrate as nitrogen

Tetrachloroethylene

Trichloroethylene

\section{Mod 201998 Mod 301998 Mod}

\begin{tabular}{|c|c|c|c|}
\hline$<.405$ & $<.405$ & UJ $\mathrm{C}$ & $<5$ \\
\hline .96 & $<.428$ & UJ & $<5$ \\
\hline 2.11 & 3.94 & $V$ & 2.88 \\
\hline 46 & 43.8 & & 28.9 \\
\hline
\end{tabular}

2.6

1897

1.43

1.95

$<.569 \quad \mathrm{U}$

34.5
11320 J QV 10700

$<.569$ UJ $\mathrm{O}<5$

$\begin{array}{llll}5.13 & \mathrm{~J} & \mathrm{O} & \mathbf{2 0 . 6}\end{array}$
401998 Mod Unit

$<100 \quad \mathrm{u} \quad \mathrm{ug} / \mathrm{L}$

$<1 \quad$ J $\mathrm{L} \quad$ ug/L

$.846 \mathrm{~J} \quad \mathrm{I} \quad \mathrm{ug} / \mathrm{L}$

$4.27 \quad \mathrm{~J} \quad \mathrm{I} \quad \mathrm{pCi} / \mathrm{L}$

$17.2 \cdot \mathrm{ug} / \mathrm{L}$

$\begin{array}{llll}3.7 & \mathrm{~J} & \mathrm{I} & \mathrm{ug} / \mathrm{L}\end{array}$

$1.06 \quad$ ug/L

$8640 \mathrm{~J} Q \mathrm{ug} / \mathrm{L}$

$<1 \quad$ U $\quad$ ug/L

10.6 ug/L 
Table B-2. Appendix IX Results for Primary Wells

WELL: P 26A

SRS Coord. Lat/Longitude Screen Zone Elevation Top of Standpipe Top of Casing Casing Pump Screen Zone N 72010.4 33.214 Deg N $32.0-22.0 \mathrm{ftmsl} 154.5 \mathrm{ft}$ msl $154.0 \mathrm{ft} \mathrm{msl} 4$ "PVC S Semiconfined E 18055.981 .759 Deg W

SAMPLE DATE

FIELD DATA

Analvte

Depth to water

$\mathrm{pH}$

Sp. Conductance

Water temperature

Alkalinity as $\mathrm{CaCO} 3$

Phenolpthalein Alkalinity

Turbidity

Volumes purged

Sampling codes
$03 / 02 / 98$

101998

32.43

6.5

37

20.1

5

0

.4

2.14336

$05 / 12 / 98$

$08 / 05 / 98$

$12 / 01 / 98$

201998

32

5.4

34

16

2

0

1.6

2.28659

301998

401998

Unit

36.98

4.9

5.5

38

20.1

4

0

.4

2.04934

36

18.9

4

0

.6

2.31017

ft BTOS

$\mathrm{uS} / \mathrm{cm}$

deg. C

$\mathrm{mg} / \mathrm{L}$

$\mathrm{mg} / \mathrm{L}$

NTU

gallons

\section{ANALYTICAL' DATA}

\section{Analyte}

1,1,1,2-Tetrachloroethane

1,1,1-Trichloroethane

1,1,2,2-Tetrachloroethane

1,1,2-Trichloroethane

1,1-Dichloroethane

1,1-Dichloroethylene

1,2,3-Trichloropropane

1,2,4,5-Tetrachlorobenzene

1,2,4-Trichlorobenzene

1,2-Dibromo-3-chloropropane

1,2-Dibromoethane

1,2-Dichlorobenzene

1,2-Dichloroethane

1,2-Dichloropropane

1,3,5-Trinitrobenzene

1,3-Dichlorobenzene

1,3-Dinitrobenzene

1,4-Dichlorobenzene

1,4-Dioxane

1,4-Naphthoquinone

1-Naphthylamine

2,2-Oxybis(1-chloropropane)

2,3,4,6-Tetrachlorophenol

2,3,7,8-TCDD

2,4,5-T

2,4,5-TP (Silvex)

2,4,5-Trichlorophenol
101998 Mod 201998 Mod 301998 Mod 401998 Mod Unit

$<5 \quad U$

$<5 \quad U$

$<5 \quad \mathrm{U}$

$<5 \quad \mathrm{U}$

$<5 \quad \mathrm{U}$

$<S \quad U$

$<5 \quad$ U

$<10$ UJ Q

$<10$

$<5$

UJ

$<5 \quad U$

$<10$ UJ Q

$<5 \quad \mathrm{U}$

$<5 \quad \mathrm{U}$

$<10$ UJ Q $<10$

$<10$

UJ $Q$

$<$

ug/L

ug/L

ug/L

$\mathrm{ug} / \mathrm{L}$.

$\mathrm{ug} / \mathrm{L}$

ug/L

ug/L

$\mathrm{ug} / \mathrm{L}$

ug/L

$\mathrm{ug} / \mathrm{L}$

ug/L

$\mathrm{ug} / \mathrm{L}$

$\mathrm{ug} / \mathrm{L}$

ug/L

$\mathrm{ug} / \mathrm{L}$

U $\cdot u g / L$

$<10$ UJ CQ ug/L

$<10$ UJ Q ug/L

$<10$ UJ $Q<10 \quad$ U $u g / L$

$<10$ UJ $Q<10 \quad U \quad$ ug/L

$<10$ UJ $Q \quad<10 \quad$ JU $Q \quad u g / L$

$<10$ UJ $Q \quad<10 \quad$ U $\quad$ ug/L

$<.0014 \mathrm{U}$

$<$ UJ IO

$\mathrm{ug} / \mathrm{L}$

$<1$ UJ IO

ug/L

$\operatorname{ug} / 2$

$<25$ UJ Q $<10$ J $Q \quad$ ug/L

Note: Concentrations in bold exceed the Drinking Water Standards listed in Appendix A. Units are for all four quarters. 
WELL: P 26A

\section{ANALYTICAL DATA}

\section{Analyte}

2,4,6-Trichlorophenol

2,4-Dichlorophenol

2,4-Dichlorophenoxyacetic acid

2,4-Dimethyl phenol

2,4-Dinitrophenol

2,4-Dinitrotoluene

2,6-Dichlorophenol

2,6-Dinitrotoluene

2-Acetylaminofluorene

2-Chloronaphthaiene

2-Chlorophenol

2-Hexanone

2-Methyl-4,6-dinitrophenol

2-Methylnaphthalene

2-Naphthylamine

2-Nitrophenol

2-Picoline

2-sec-Butyl-4,6-dinitrophenol

3,3"-Dichlorobenzidine

3,3"-Dimethylbenzidine

3-Methylcholanthrene

4-Aminobiphenyl

4-Bromophenyl phenyl ether

4-Chloro-m-cresol

4-Chloroaniline

4-Chlorophenyl phenyl ether

4-Nitrophenol

4-Nitroquinoline-l-oxide

5-Nitro-o-toluidine

7,12-Dimethylbenz(a)anthracene

Acenaphthene

Acenaphthylene

Acetone

Acetonitrile (Methyl cyanide)

Acetophenone

Acrolein

Acrylonitrile

Aldrin

Allyl chloride

Aluminum, total recoverable

Aniline

Anthracene

Antimony, total recoverable
101998 Mod 201998 Mod 301998 Mod 401998 Mod Unit

$<10$ UJ Q $<25$ JU Q ug/L

$<10$ UJ $Q \quad<10 \quad$ JU $Q \quad$ ug/L

$<1.09$ U $\mathrm{ug} / \mathrm{L}$

$<10$ UJ Q $<10$ JU $Q \quad$ ug/L

$<25$ UJ Q $<25 \quad$ JU $Q \quad$ ug/L

$<10$ UJ $Q \quad<10$ JU $Q \quad$ ug/L

$<10$ UJ $Q<10 \quad$ U $\quad$ ug/L

$<10$ UJ $Q<10$ JU $Q \quad u g / L$

$<10$ UJ Q $<10 \quad$ U $\quad$ ug/L

$<10$ UJ $Q<10$ JU $Q \quad u g / L$

$<10$ UJ $Q \quad<10$ JU $Q \quad u g / L$

$<10$ U ug/L

$<25$ UJ Q : $<25$ JU Q ug/L

$<10$ UJ $Q \quad<10 \quad$.JU $Q \quad u g / L$

$<10$ UJ Q $<10 \quad$ U $\quad \mathrm{ug} / \mathrm{L}$

$<10$ U $<10 \quad J U Q \quad$ ug/L

$<10$ UJ $Q \quad<10 \quad$ U $\quad$ ug/L

$<50$ UJ $Q \quad<10 \quad$ U $\quad u g / L$

$<10$ UJ $Q \quad<10$ J $Q \quad$ ug/L

$<10$ UJ $Q \quad<20 \quad$ U $\quad$ ug/L

$<10$ UJ Q $<10 \quad$ U $\quad$ ug/L

$<10$ UJ Q $<10 \quad$ U $\quad$ ug/L

$<10$ UJ Q $\quad<10 \quad$ JU $Q \quad$ ug/L

$<10$ UJ $Q<10$ JU $Q \quad u g / L$

$<10$ UJ $Q \quad<10 \quad$ JU $Q \quad$ ug/L

$<10$ UJ $Q<10$ JU $Q \quad$ ug/L

$<25$ UJ Q $<25$ JU $Q \quad$ ug/L

$<20$ UJ $Q \quad<50 \quad$ U $\quad$ ug/L

$<10$ UJ Q $<10 \quad$ U $\quad$ ug/L

$<10$ UJ $Q \quad<10 \quad$ U $\quad \mathrm{ug} / \mathrm{L}$

$<10 \quad \mathrm{U} \quad<10 \quad$ JU $Q \quad$ ug/L

$<10$ UJ Q $<10$ JU $Q \quad$ ug/L

$<10$ U ug/L

$<20$ U ug/L

$<10$ UJ $Q \quad<10 \quad$ U $\quad$ ug/L

$<20$ U ug/L

$<5$ U ug/L

$<.026$ UJ C ug/L

$<10$ U ug/L

$<146 \quad \mathrm{U} \quad<200 \mathrm{U} \cdot \mathrm{ug} / \mathrm{L}$

$<10$ UJ Q $<25$ JU Q ug/L

$<10$ UJ Q $<10$ JU $Q \quad$ ug/L

$<27$ U ug/L

Note: Concentrations in bold exceed the Drinking Water Standards listed in Appendix A. Units are for all four quarters. 
WELL: P 26A

\section{ANALYTICAL DATA}

Analyte

Aramite

Arsenic, total recoverable

Barium, total recoverable

Benzene

Benzo(a)anthracene

Benzo(a)pyrene

Benzo(b)fluoranthene

Benzo(g,h,i)perylene

Benzo(k)fluoranthene

Benzoic acid

Benzyl alcohol

Beryllium, total recoverable

Bis(2-chloroethoxy) methane

Bis(2-chloroethyl) ether

Bis(2-ethylhexyl) phthalate

Bromodichloromethane

Bromoform

Bromomethane (Methyl bromide)

Butylbenzyl phthalate

Cadmium, total recoverable

Carbazole

Carbon disulfide

Chlordane

Chlorobenzene

Chlorobenzilate

Chloroethane

Chloroethene (Vinyl chloride)

Chloromethane (Methyl chloride)

Chloroprene

Chromium, total recoverable

Chrysene

Cobalt, total recoverable

Copper, total recoverable

Cyanide

Di-n-butyl phthalate

Di-n-octyl phthalate

Diallate

Dibenz(a,h)anthracene

Dibenzofuran

Dibromochloromethane

Dibromomethane (Methylene bromide'

Dichlorodifluoromethane

Dichloromethane (Methylene chloride)
101998 Mod 201998 Mod 301998 Mod 401998 Mod Unit

$<20$ UJ $Q \quad<10 \quad U \quad$ ug/L

$<10 \quad U \quad u g / L$

$\begin{array}{llll}26.6 & \mathrm{~V} & 24.5 & \mathrm{ug} / \mathrm{L}\end{array}$

$<5 \quad \mathrm{U} \quad \mathrm{ug} / \mathrm{L}$

$<10$ UJ $Q \quad<10 \quad$ JU $Q \quad$ ug/L

$<10$ UJ $Q \quad<10 \quad$ JU $Q \quad$ ug/L

$<10$ UJ $Q \quad<10 \quad$ JU $Q \quad u g / L$

$<10$ UJ $Q \quad<10$ JU $Q \quad$ ug/L

$<10$ UJ $Q \quad<10 \quad$ JU $Q \quad$ ug/L

25 UJ Q ug/L

$<10$ UJ Q $<10$ JU Q ug/L

$1.1 \mathrm{~J} \quad \mathrm{E} \quad \mathrm{ug} / \mathrm{L}$

$<10$ UJ $Q,<10 \quad$ JU $Q \quad$ ug/L

$<10$ UJ $Q \quad<10$ JU $Q \quad$ ug/L

$<4.12$ UJ QV $<10$ JU $Q \quad$ ug/L

$<\mathrm{U} \quad \mathrm{ug} / \mathrm{L}$

$<5 \quad \mathrm{u} \quad \mathrm{ug} / \mathrm{L}$

$<10 \quad \mathrm{U} \quad \mathrm{ug} / \mathrm{L}$

$<10 \quad$ UJ Q $\quad<10 \quad \pi \quad$ Q $\quad$ ug $/ \mathrm{L}$

$<4.7 \quad \mathrm{U} \quad \mathrm{ug} / \mathrm{L}$

$<10 \quad \mathrm{U} \quad \mathrm{ug} / \mathrm{L}$

$<$ U ug/L

$<.521 \quad \mathrm{U} \quad \mathrm{ug} / \mathrm{L}$

$<5$ U $u g / L$;

$<10 \quad$ UJ $Q \quad<10 \quad$ U $\quad$ ug/L

$<10 \quad U \quad$ ug/L

$<5 \quad \mathrm{u} \quad \mathrm{ug} / \mathrm{L}$

$<10$ U ug/L

$<5 \quad \mathrm{U} \quad \mathrm{ug} / \mathrm{L}$

$1.3 \mathrm{~J}$ EV ug/L

$\begin{array}{llllll}<10 & \text { UJ } Q & <10 \quad \mathrm{JU} & \mathrm{Q} & \mathrm{ug} / \mathrm{L}\end{array}$

$<4.5 \quad U \quad \mathrm{ug} / \mathrm{L}$

$<15 \quad \mathrm{U} \quad \mathrm{ug} / \mathrm{L}$

$<50$ U ug/L

$<10 \quad \mathrm{U} \quad<10 \quad \pi \quad \mathrm{Q} \quad \mathrm{ug} / \mathrm{L}$

$<10$ UJ $Q \quad<10$ jU $Q \quad$ ug $/$

$<10$ UJ $Q \quad<10 \quad$ U $\quad$ ug/L

$<10$ UJ $Q \quad<10 \quad \pi \quad Q \quad u g / L$

$<10$ UJ $Q \quad<10 \quad$ JU $Q \quad$ ug/L

$<5 \quad \mathrm{U} \quad$ ug/L

$<5 \quad \mathrm{U} \quad \mathrm{ug} / \mathrm{L}$

$<10 \quad \mathrm{U} \quad \mathrm{ug} / \mathrm{L}$

$<4.86$ U V ug/L

Note: Concentrations in bold exceed the Drinking Water Standards tisted in Appendix A. Units are for all four quarters. 
WELL: P 26A

\section{ANALYTICAL DATA}

Analyte

Dieldrin

Diethyl phthalate

Dimethoate

Dimethyl phthalate

Diphenylamine

Disulfoton

Endosulfan I

Endosulfan II

Endosulfan sulfate

Endrin

Endrin aldehyde

Ethyl methacrylate

Ethyl methanesulfonate

Ethylbenzene

Famphur

Fluoranthene

Fluorene

Heptachlor

Heptachlor epoxide

Hexachlorobenzene

Hexachlorobutadiene

Hexachlorocyclopentadiene

Hexachlorodibenzo-p-dioxins

Hexachlorodibenzo-p-furans

Hexachloroethane

Hexachlorophene

Hexachloropropene

Indeno(1,2,3-c,d)pyrene

Iodomethane (Methyl iodide)

Iron, total recoverable

Isobutyl alcohol

Isodrin

Isophorone

Isosafrole

Kepone

Lindane

Methacrylonitrile

Methapyrilene

Methoxychlor

Methyl ethyl ketone

Methyl isobutyl ketone

Methyl methacrylate

Methyl methanesulfonate
101998 Mod 201998 Mod 301998 Mod 401998 Mod Unit

$<.052 \quad \mathrm{U}$

$<10 \quad$ UJ $Q \quad<10 \quad$ JU $Q \quad$ ug/L

$<1.06 \quad \mathrm{U} \quad<10 \quad \mathrm{U} \quad \mathrm{ug} / \mathrm{L}$

$<10$ UJ $Q \quad<10$ JU $Q \quad$ ug/L

$<10$ UI $Q \quad<10$ U ug/L

$<1.06 \quad \mathrm{U} \quad<10 \quad \mathrm{U} \quad \mathrm{ug} / \mathrm{L}$

$<.026 \mathrm{U} \quad \mathrm{ug} / \mathrm{L}$

$<.052 \quad \mathrm{U}$

$<.052 \mathrm{U}$

$<.052 \quad \mathrm{U}$

$\mathrm{ug} / \mathrm{L}$

$\mathrm{ug} / \mathrm{L}$

$\mathrm{ug} / \mathrm{L}$

$\mathrm{ug} / \mathrm{L}$

$<.052 \quad U$

$<10$ UJ Q

$<10$ UJ

$\mathrm{ug} / \mathrm{L}$

$<5 \quad$ U

$<2.74 \quad \mathrm{U}$

$\begin{array}{llllll}<10 & \text { UJ } Q & <10 \quad J U & \mathrm{ug} / \mathrm{L}\end{array}$

$\begin{array}{llllllll}<10 & \mathrm{U} & <10 & \mathrm{JU} & \mathrm{Q} & \mathrm{ug} / \mathrm{L}\end{array}$

$<.026 \quad \mathrm{U}$

$<.026 \quad \mathrm{U}$

$<10 \quad$ UJ $Q \quad<10 \quad$ JU $Q \quad u g / L$

$<10 \quad$ UJ $Q \quad<10 \quad$ JU $Q$

$\begin{array}{llllll}<10 & \text { UJ } Q & <10 & \mathrm{JU} & \mathrm{Q} & \mathrm{ug} / \mathrm{L}\end{array}$

$<.0013$ U

$<.0009$ U

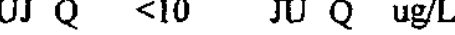

$<100$ UJ $Q \quad$ ug/L

$<10$ UJ Q ug/L

$<10 \quad$ UJ $Q \quad<10 \quad$ JU $Q \quad$ ug/L

$<5 \quad \mathrm{u} \quad \mathrm{ug} / \mathrm{L}$

937

1270

847

$\mathrm{ug} / \mathrm{L}$

$\begin{array}{llllll}<100 & \mathrm{U} & & & \mathrm{ug} / \mathrm{L} \\ <.052 & \mathrm{U} & <10 & \mathrm{U} & \mathrm{ug} / \mathrm{L}\end{array}$

$<10$ UJ $Q \quad<10$ JU $Q \quad$ ug/L

$<10$ UJ $Q \quad<10 \quad$ U $\quad u g / L$

$<.309 \quad \mathrm{U} \quad<10 \quad \mathrm{U} \quad \mathrm{ug} / \mathrm{L}$

$<.026$ U $u g / L$

$<500 \quad \mathrm{U} \quad \mathrm{ug} / \mathrm{L}$

$<10$ UJ $Q \quad<10 \quad$ U ug/L

$<.26$ U ug/L

$<10 \quad \mathrm{U} \quad \mathrm{ug} / \mathrm{L}$

$<5 \quad$ U $\quad u g / L$

$<10 \quad$ UJ $Q \quad$ ug/L

$<10$ UJ $Q \quad<10 \quad$ U $\quad u g / L$

Note: Concentrations in bold exceed the Drinking Water Stendards listed in Appendix A. Units are for all four quarters. 
WELL: P 26A

\section{ANALYTICAL DATA}

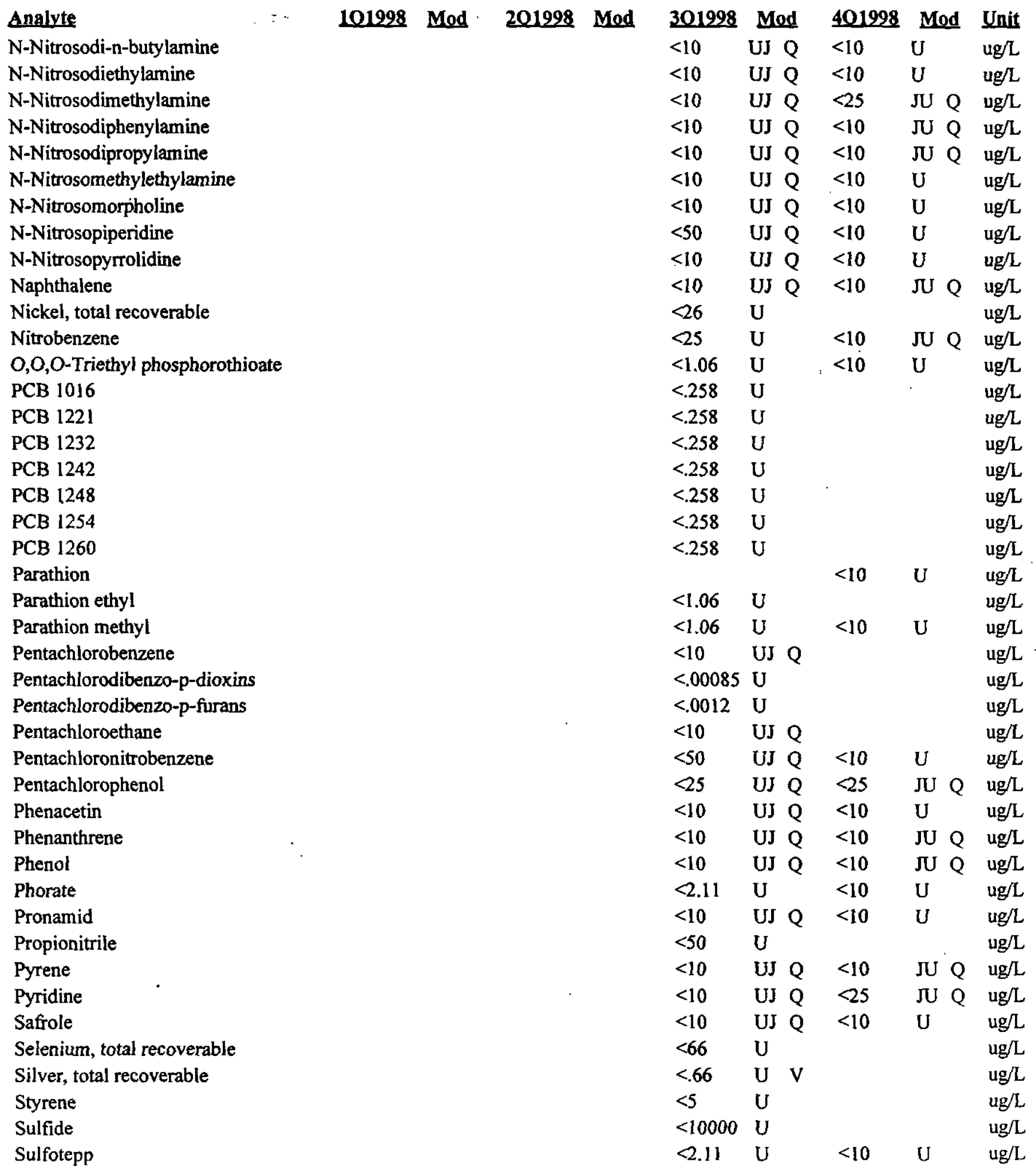

Note: Concentrations in bold exceed the Drinking Water Standards listed in Appendix A. Units are for all four quarters. 
WELL: P 26A

\section{ANALYTICAL DATA}

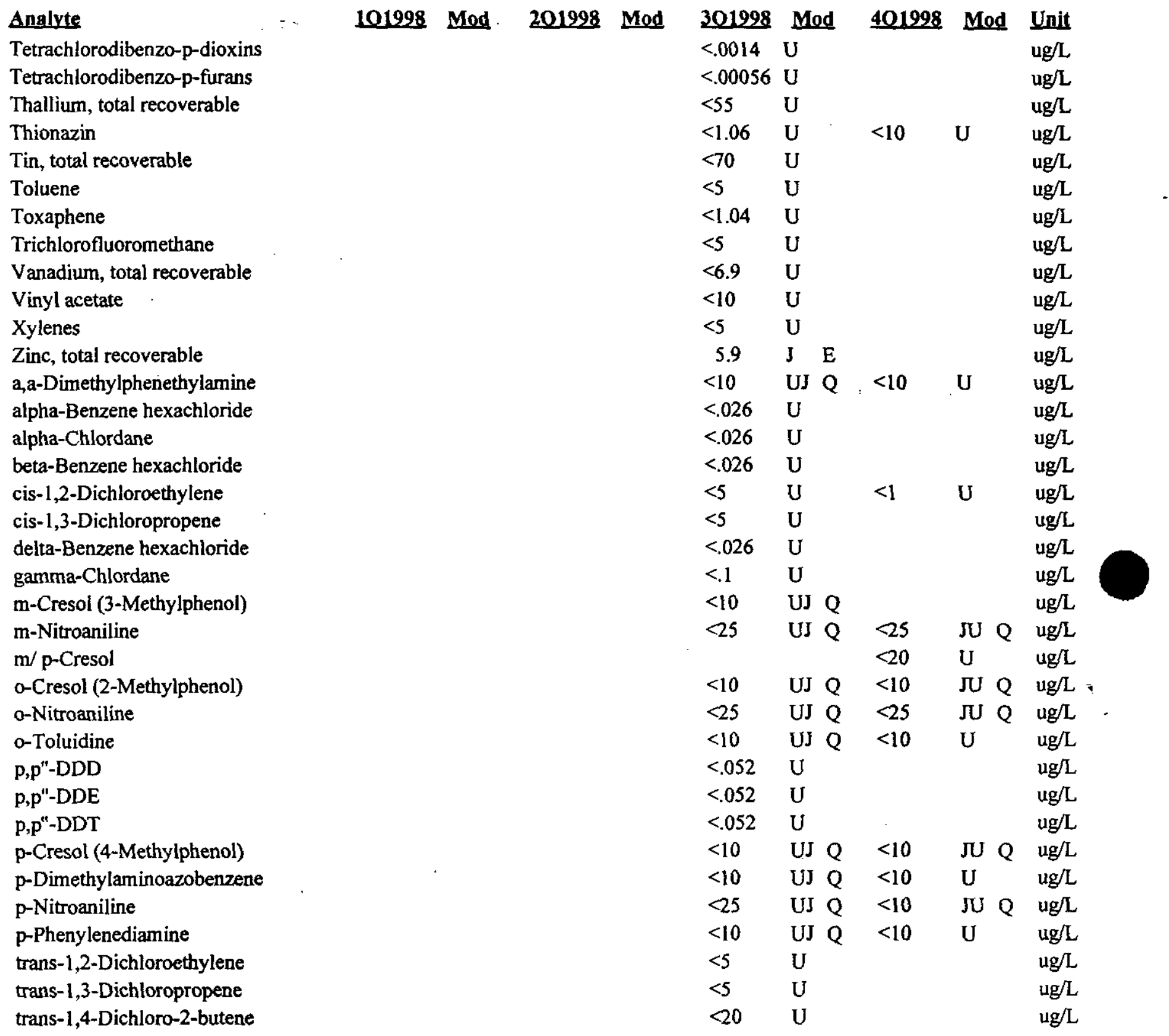


WSRC-RP-99-4003

Unclassified

WELL: TBG 1

SRS Coord, Lat/Longitude Screen Zone Elevation Top of Standpipe Top of Casing Casing Pump Screen Zone N 71429.5 33.212 Deg N $109.1-89.1 \mathrm{ftmsl} 151.4 \mathrm{ft} \mathrm{msl} 151.2 \mathrm{ftmsl} 4$ "STL $\mathrm{S}$ Unconfined E 17134.7 81.760 Deg W

SAMPLE DATE

$03 / 02 / 98$

$05 / 13 / 98$

$08 / 06 / 98$

$12 / 04 / 98$

FIELD DATA

Analyte

Depth to water

$\mathrm{pH}$

Sp. Conductance

Water temperature

Alkalinity as $\mathrm{CaCO} 3$

Phenolpthalein Alkalinity

Turbidity

Volumes purged

Sampling codes

\section{ANALYTICAL DATA}

Analyte

1,1,1,2-Tetrachloroethane

1,1,1-Trichloroethane

1,1,2,2-Tetrachloroethane

1,1,2-Trichloroethane

1,1-Dichloroethane

1,1-Dichloroethylene

1,2,3-Trichloropropane

1,2,4-Trichlorobenzene

1,2-Dibromo-3-chloropropane

1,2-Dibromoethane

1,2-Dichlorobenzene

1,2-Dichloroethane

1,2-Dichloropropane

1,3,5-Trinitrobenzene

1,3-Dichlorobenzene

1,3-Dinitrobenzene

1,4-Dichlorobenzene

1,4-Dioxane

1,4-Naphthoquinone

1-Naphthylamine

2,2-Oxybis(1-chloropropane)

2,3,4,6-Tetrachlorophenol

2,3,7,8-TCDD

$2,4,5-\mathrm{T}$

2,4,5-TP (Silvex)

2,4,5-Trichlorophenol

2,4,6-Trichlorophenol

\begin{tabular}{lll}
$\mathbf{1 0 1 9 9 8}$ & $\mathbf{2 0 1 9 9 8}$ & $\underline{\mathbf{3 0 1 9 9 8}}$ \\
\hline 49.26 & 49 & 52.35 \\
4.3 & 4.2 & 4 \\
160 & 140 & 150 \\
21.9 & 17 & 27.8 \\
0 & 0 & 0 \\
0 & 0 & 0 \\
.4 & 1.2 & 1.3 \\
3.68038 & 6.28375 & 0 \\
& & $\mathrm{NX}$
\end{tabular}

401998

Unit

53.91

ft BTOS

4.2

$140 \quad$ uS $/ \mathrm{cm}$

22.3 deg. $\mathrm{C}$

$0 \quad \mathrm{mg} / \mathrm{L}$

$0 \quad \mathrm{mg} / \mathrm{L}$

.6

3.90869

NTU

gallons

\section{Mod 201998 Mod}

$<.462 \quad \mathrm{U} \quad<.462$

$<.462 \quad U$

\section{1}

$<5 \quad \mathrm{U}$

$<5 \quad U$

$<5 \quad$ U

$<5 \quad \mathrm{U}$

$<5 \quad$ U

$<5 \quad \mathrm{U}$

$<5 \quad \mathrm{U}$

$<10 \quad U$

$<5 \quad U$

$<5 \quad \mathrm{U}$

$<5 \quad \mathrm{U}$

$<5 \quad \mathrm{U}$

$<5 \quad U$

$<5 \quad U$

$<5 \quad$ U

$<1000$

U

$<10$

401998 Mad Unit

ug $/$

$<\quad$ U ug/L

$\mathrm{ug} / \mathrm{L}$

$\mathrm{ug} / \mathrm{L}$

ug/L

ug/L

$\mathrm{ug} / \mathrm{L}$

$<10$

JU

ug $/ \mathrm{L}$

$\mathrm{ug} / \mathrm{L}$

$\mathrm{ug} / \mathrm{L}$

$\mathrm{ug} / \mathrm{L}$

$\mathrm{ug} / \mathrm{L}$

ugh

$\mathrm{ug} / \mathrm{L}$

ug/L

$<10 \quad \mathrm{U} \quad \mathrm{ug} / \mathrm{L}$

$\mathrm{ug} / \mathrm{L}$

$\mathrm{ug} / \mathrm{L}$

$<10 \quad \mathrm{U} \quad \mathrm{ug} / \mathrm{L}$

$<10$ U ug/L

$<10 \quad \mathrm{U} \quad<10 \quad$ JU $Q \quad \mathrm{ug} / \mathrm{L}$

$<10$ U ug/L

$<.00037 \mathrm{U}$

$<.2$ US $\mathrm{O}$

$\mathrm{ug} / \mathrm{L}$

$<.2$ UJ 0

$\mathrm{ug} / \mathrm{L}$

$<10 \quad U$

ug/L

$<25$ U

$<10$

JU $Q \quad u g / L$

$<25$

JU Q ug/L

Note: Concentrations in bold exceed the Drinking Water Standards listed in Appendix A. Units are for all four quarters. 
WELL: TBG 1

ANALYTICAL DATA

\section{Analyte}

2,4-Dichlorophenol

2,4-Dichlorophenoxyacetic acid

2,4-Dimethyl phenol

2,4-Dinitrophenol

2,4-Dinitrotoluene

2,6-Dichlorophenol

2,6-Dinitrotoluene

2-Acetylaminofluorene

2-Chloronaphthalene

2-Chlorophenol

2-Hexanone

2-Methyl-4,6-dinitrophenol

2-Methylnaphthalene

2-Naphthylamine

2-Nitrophenol

2-Picoline

2-sec-Butyl-4,6-dinitrophenol

3,3"-Dichlorobenzidine

3,3"-Dimethylbenzidine

3-Methylcholanthrene

4-Aminobiphenyl

4-Bromophenyl phenyl ether

4-Chloro-m-cresol

4-Chloroaniline

4-Chlorophenyl phenyl ether

4-Nitrophenol

4-Nitroquinoline-1-oxide

5-Nitro-o-toluidine

7,12-Dimethylbenz(a)anthracene

Acenaphthene

Acenaphthylene

Acetone

Acetonitrile (Methyl cyanide)

Acetophenone

Acrolein

Acrylonitrile

Aldrin

Allyl chloride

Aluminum, total recoverable

Aniline

Anthracene

Antimony, total recoverable

Aramite
101998 Mod 201998 Mod 301998 Mod 401998 Mod Unit

$\begin{array}{llllll}<10 & \mathrm{U} & <10 & \mathrm{JU} & \mathrm{Q} & \mathrm{ug} / \mathrm{L}\end{array}$

$<.2$ UJ $\mathrm{O} \quad$ ug/L

$<10 \quad \mathrm{U} \quad<10 \quad$ JU $\mathrm{Q} \quad \mathrm{ug} / \mathrm{L}$

$<25 \quad \mathrm{U} \quad<25 \quad$ JU $\mathrm{Q} \quad \mathrm{ug} / \mathrm{L}$

$<10 \quad \mathrm{U} \quad<10 \quad$ JU $Q \quad$ ug/L

$<10 \quad \mathrm{U} \quad \mathrm{ug} / \mathrm{L}$

$\begin{array}{llllll}<10 & \mathrm{U} & <10 & \mathrm{JU} & \mathrm{Q} & \mathrm{ug} / \mathrm{L}\end{array}$

$<10 \quad \mathrm{U} \quad \mathrm{ug} / \mathrm{L}$

$<10 \quad \mathrm{U} \quad<10 \quad \mathrm{~J} Q \mathrm{Q} \quad \mathrm{ug} / \mathrm{L}$

$<10 \quad \mathrm{U} \quad<10 \quad \pi \quad \mathrm{Q} \quad \mathrm{ug} / \mathrm{L}$

$<5 \quad \mathrm{U} \quad \mathrm{ug} / \mathrm{L}$

$<25 \quad \mathrm{U} \quad<25 \quad$ JU Q $\quad$ ug/L

$<10 \quad \mathrm{U}:<10 \quad$ JU $Q \quad$ ug/L

$<10$ U ug/L

$<10 \quad \mathrm{U} \quad<10 \quad$ JU $Q \quad \mathrm{ug} / \mathrm{L}$

$<10$ U ug/L

$<10$ U ug/L

$<10 \quad \mathrm{U} \quad<10 \quad \mathrm{JU} \quad \mathrm{Q} \quad \mathrm{ug} / \mathrm{L}$

$<20$ U ug/L

$<10 \quad \mathrm{U} \quad \mathrm{ug} / \mathrm{L}$

$<10$ U ug/L

$<10 \quad \mathrm{U} \quad<10 \quad$ JU $Q \quad$ ug/L

$<10 \quad \mathrm{U} \quad<10 \quad$ JU Q $\quad$ ug/L

$<10$ U $\quad<10$ JU Q ug/L :

$<10 \quad \mathrm{U} \quad<10 \quad$ JU $\mathrm{Q} \quad \mathrm{ug} / \mathrm{L}$

$<25$ U $<25$ JU Q ug/L

$<50$ U ug/L

$<10 \quad$ U ug/L

$<10$ U ug/L

$<10 \quad \mathrm{U} \quad<10 \quad$ JU $Q \quad$ ug $/ \mathrm{L}$

$<10$ U $\quad<10$ JU $Q \quad$ ug/L

$<20$ U ug/L

$<500 \quad \mathrm{u}, \mathrm{ug} / \mathrm{L}$

$<10 \quad \mathrm{U} \quad \mathrm{ug} / \mathrm{L}$

$<50 \quad \mathrm{U} \quad \mathrm{ug} / \mathrm{L}$

$<50$ U ug/L

$<, 1 \quad \mathrm{U}$

$<10 \quad \mathrm{U}$

$214 \quad 255$

$<25$ U $\quad<25$ jU $Q \quad$ ug $/ \mathrm{L}$

$<10 \quad$ U $\quad<10 \quad$ JU $Q \quad$ ug/

$<100 \quad \mathrm{u} \quad \mathrm{ug} / \mathrm{L}$

$<10 \quad \mathrm{U} \quad \mathrm{ug} / \mathrm{L}$

Note: Concentrations is bold exceed the Drinking Water Standards listed in Appendix A. Units are for all four quarters. 
WELL: TBG 1

\section{ANALYTICAL DATA}

\section{Analyte}

101998 Mod 201998 Mod 301998 Mod 401998 Mod Unit

Arsenic, total recoverable

$<10 \quad \mathrm{U}$

ug/L

Barium, total recoverable

143

Benzene

Benzo(a)anthracene

Benzo(a)pyrene

Benzo(b)fluoranthene

Benzo(g,h,i)perylene

Benzo(k)fluoranthene

Benzoic acid

$$
<5
$$

Benzyl alcohol

Beryllium, total recoverable

Bis(2-chloroethoxy) methane

Bis(2-chloroethyl) ether

Bis(2-ethylhexyl) phthalate

Bromodichloromethane

Bromoform

Bromomethane (Methyl bromide)

Butylbenzyl phthalate

Cadmium, total recoverable

Carbazole

Carbon disulfide

$<5 \quad \mathrm{U}$

$<10 \quad \mathrm{U}$

$<10 \quad \mathrm{U}$

$<10 \quad \mathrm{U}$

$<10 \quad \mathrm{U}$

$<25 \quad \mathrm{U}$

$\mathrm{ug} / \mathrm{L}$

$<10 \quad \mathrm{U}$

$<10 \quad U$

$<10 \quad \mathrm{U}$

$<10 \quad \mathrm{U}$

$<5 \quad \mathrm{U}$

$<5 \quad \mathrm{U}$

$<5 \quad \mathrm{U}$

$<10 \quad \mathrm{U}$.

$<10 \quad \mathrm{U}$

$<0 \quad \mathrm{U}$

$<5 \quad U$

Chlorobenzene

Chlorobenzilate

Chloroethane

Chloroethene (Vinyl chloride)

Chloromethane (Methyl chloride)

Chloroprene

Chromium, total recoverable

Chrysene

Cobalt, total recoverable

Copper, total recoverable

Cyanide

Di-n-butyl phthalate

Di-n-octyl phthalate

Diallate

Dibenz(a,h)anthracene

Dibenzofuran

$<5$

Dibromochloromethane

Dibromomethane (Methylene bromide;

Dichlorodifluoromethane

Dichloromethane (Methylene chloride)

Dieldrin

Diethyl phthalate

$<5 \quad \mathrm{U}$

$<10 \quad \mathrm{U}$

$<5 \quad \mathrm{U}$

$<5 \quad \mathrm{U}$

$<50 \quad \mathrm{U}$

$<10 \quad \mathrm{U}$

$<10 \quad \mathrm{U}$

$5.19 \mathrm{~J}$

$<20$ U

$<10 \quad \mathrm{U}$

$<10 \quad \mathrm{U}$

$<10 \quad \mathrm{U}$

$<20 \quad$ U

$<10$

$<5$

$<5$

$<5$

$<10$

$<.2$

$<10$
$<10$

$<10$

$<10$

$<10$

$<10$

$<10$

$<10$

$<10$

$<10$

$\mathrm{Q} \quad u g / L$

JU $Q \quad$ ug/L

JU Q ug/L

$\pi \mathrm{U} \quad$ ug/L

JU $Q \quad u g / L$

ug/L

JU $Q \quad u g / L$

$\mathrm{ug} / \mathrm{L}$

$\pi \quad Q \quad u g /$

JU Q ug/L

JU Q ug/L

$\mathrm{ug} / \mathrm{L}$

ug/L

$\mathrm{ug} / \mathrm{L}$

$<10$ JU Q ug/L

$\mathrm{ug} / \mathrm{L}$

$u g / L$

$\mathrm{ug} / \mathrm{L}$

$\mathrm{ug} / \mathrm{L}$

$<10$ U ug/L

$\mathrm{ug} / \mathrm{L}$.

$u g / L$

ug/L

$\mathrm{ug} / \mathrm{L}$

$\mathrm{ug} / \mathrm{L}$

$<10 \quad$ JU Q ug/L

ug/L

ug/L

ug/L

$<10 \quad$ JU Q ug/L

$<10$ JU Q ug/L

$<10 \quad$ U ug/L

$<10$ JU Q ug/L

$<10$

$\pi \mathrm{Q} \quad \mathrm{ug} / \mathrm{L}$

ug/L

ug/L

ug/L

$\mathrm{ug} / \mathrm{L}$

$\mathrm{ug} / \mathrm{L}$

$<10 \quad J U$ Q $\quad$ ug/L

Note: Concentrations in bold exceed the Drinking Water Standards listed in Appendix A. Units are for all four quarters. 
WELL: TBG 1

\section{ANALYTICAL DATA}

\section{Analvte}

Dimethoate

Dimethyl phthalate

Diphenylamine

Disulfoton

Endosulfan I

Endosulfan II

Endosulfan sulfate

Endrin

Endrin aldehyde

Ethyl methacrylate

Ethyl methanesulfonate

Ethylbenzene

Fluoranthene

Fluorene

Heptachlor

Heptachlor epoxide

Hexachlorobenzene

Hexachlorobutadiene

Hexachlorocyclopentadiene

Hexachlorodibenzo-p-dioxins

Hexachlorodibenzo-p-furans

Hexachloroethane

Indeno(1,2,3-c,d)pyrene

Iodomethane (Methyl iodide)

Iron, total recoverable

Isobutyl alcohol

Isodrin

Isophorone

Isosafrole

Kepone

Lindane

Methacrylonitrile

Methapyrilene

Methoxychlor

Methyl ethyl ketone

Methyl isobutyl ketone

Methyl methacrylate

Methyl methanesulfonate

N-Nitrosodi-n-butylamine

N-Nitrosodiethylamine

$\mathrm{N}$-Nitrosodimethylamine

N-Nitrosodiphenylamine

$\mathrm{N}$-Nitrosodipropylamine

\section{Mod 201998 Mod 301998 Mod}

$<10 \quad \mathrm{U}$

401998 Mod Unit

$<10 \quad$ U ug/L

$<10 \quad$ JU $Q \quad$ ug/L

$<10$ U ug/L

$<10 \quad \mathrm{U} \quad \mathrm{ug} / \mathrm{L}$

$<.1 \quad$ U ug/L

$<.2$ U $u g / L$

$<.2$ U $\quad$ ug/L

$<.2 \quad \mathrm{U}$

$<.2 \quad \mathrm{U}$

$<5 \quad \mathrm{U}$

$<5 \quad \mathrm{U}$

$<10 \quad \mathrm{U}$

$<10 \quad \mathrm{U}$

$<.1 \quad \mathrm{U}$

$<.1 \quad \mathrm{U}$

$<10 \quad \mathrm{U}$

$<20 \quad \mathrm{U}$

$<10 \quad \mathrm{U}$

$<.00076 \mathrm{U}$

$<.00075 \mathrm{U}$

$<10 \quad \mathrm{U}$

$<10 \quad \mathrm{U}$

$<5 \quad \mathrm{U}$

60.3

79.9

$<1500 \quad \mathrm{U}$

$<20 \quad$ U

$<10 \quad \mathrm{U} \quad$ ug/L

$<10 \quad \mathrm{U} \quad$ ug/L

$\mathrm{ug} / \mathrm{L}$

$\mathrm{ug} / \mathrm{L}$

ug/L

$<10 \quad$ JU Q $\quad$ ug/L

$<10 \quad$ JU $Q \quad$ ug/L

ug/L

$\mathrm{ug} / \mathrm{L}$

$<10 \quad$ JU Q ug/L

$<10 \quad \pi \mathrm{Q} \quad$ ug/L

$<10$ JU Q ug/L

$\mathrm{ug} / \mathrm{L}$

$u g / \mathrm{L}$

$<10 \quad$ JU $Q \quad$ ug/L

$<10$ JU Q ug/L

$\mathrm{ug} / \mathrm{L}$

$9.7 \mathrm{~J} \quad \mathrm{I} \quad \mathrm{ug} / \mathrm{L}$

$\mathrm{ug} / \mathrm{L}$

$<10 \quad \mathrm{U} \quad \mathrm{ug} / \mathrm{L}$

$<10 \quad$ JU Q ug/L

$<10 \cdot \mathrm{U} \quad \mathrm{ug} / \mathrm{L}$

$<10$ U ug/L

$.02 \quad \mathrm{~J} \quad \mathrm{E} \quad \mathrm{ug} / \mathrm{L}$

$<500$ U $u g / L$

$<10 \quad U \quad$ ug/L

$<1 \quad$ U

$<10 \quad \mathrm{U}$

$<5$. U

$<50 \quad \mathrm{U}$

$\mathrm{ug} / \mathrm{L}$

$\mathrm{ug} / \mathrm{L}$

ug/

$\mathrm{ug} / \mathrm{L}$

$<10 \quad \mathrm{U} \quad \mathrm{ug} / \mathrm{L}$

$<10 \quad \mathrm{U} \quad \mathrm{ug} / \mathrm{L}$

$<10$ U ug/L

$<20 \quad$ U $\quad<25 \quad$ J $Q \quad$ ug $/ \mathrm{L}$

$<10 \quad \mathrm{U} \quad<10 \quad$ JU $Q \quad$ ug/L

$<10 \quad \mathrm{U} \quad<10 \quad \mathrm{JU} Q \quad \mathrm{Qg} / \mathrm{L}$

Note: Concentrations in bold exceed the Drinking Water Standards listed in Appendix A. Units are for all four quarters. 
WELL: TBG 1

ANALYTICAL DATA

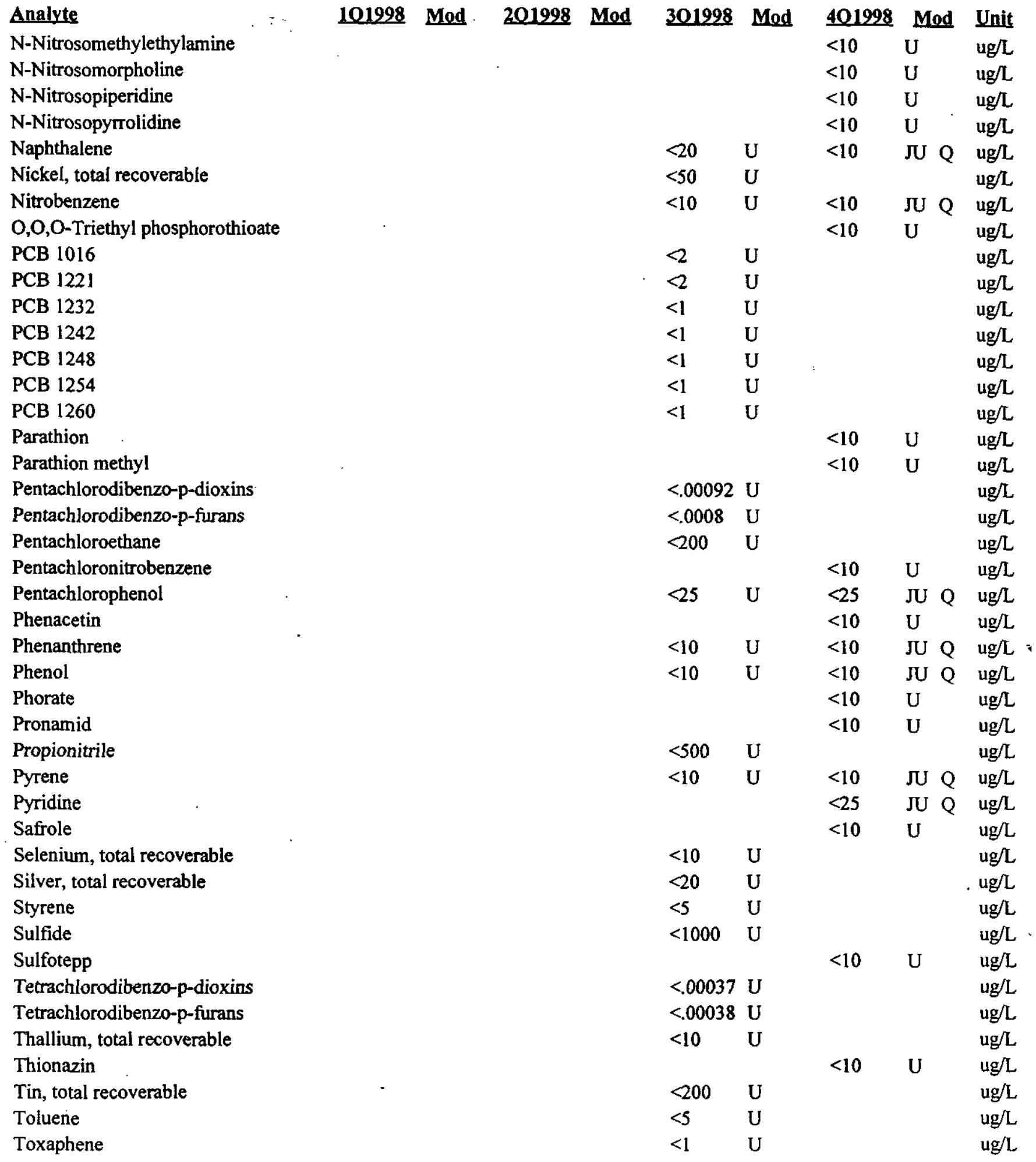

Note: Concentrations in bold exceed the Drinking Water Standards listed in Appendix A. Units are for all four quarters. 
WELL: TBG 1

ANALYTICAL DATA

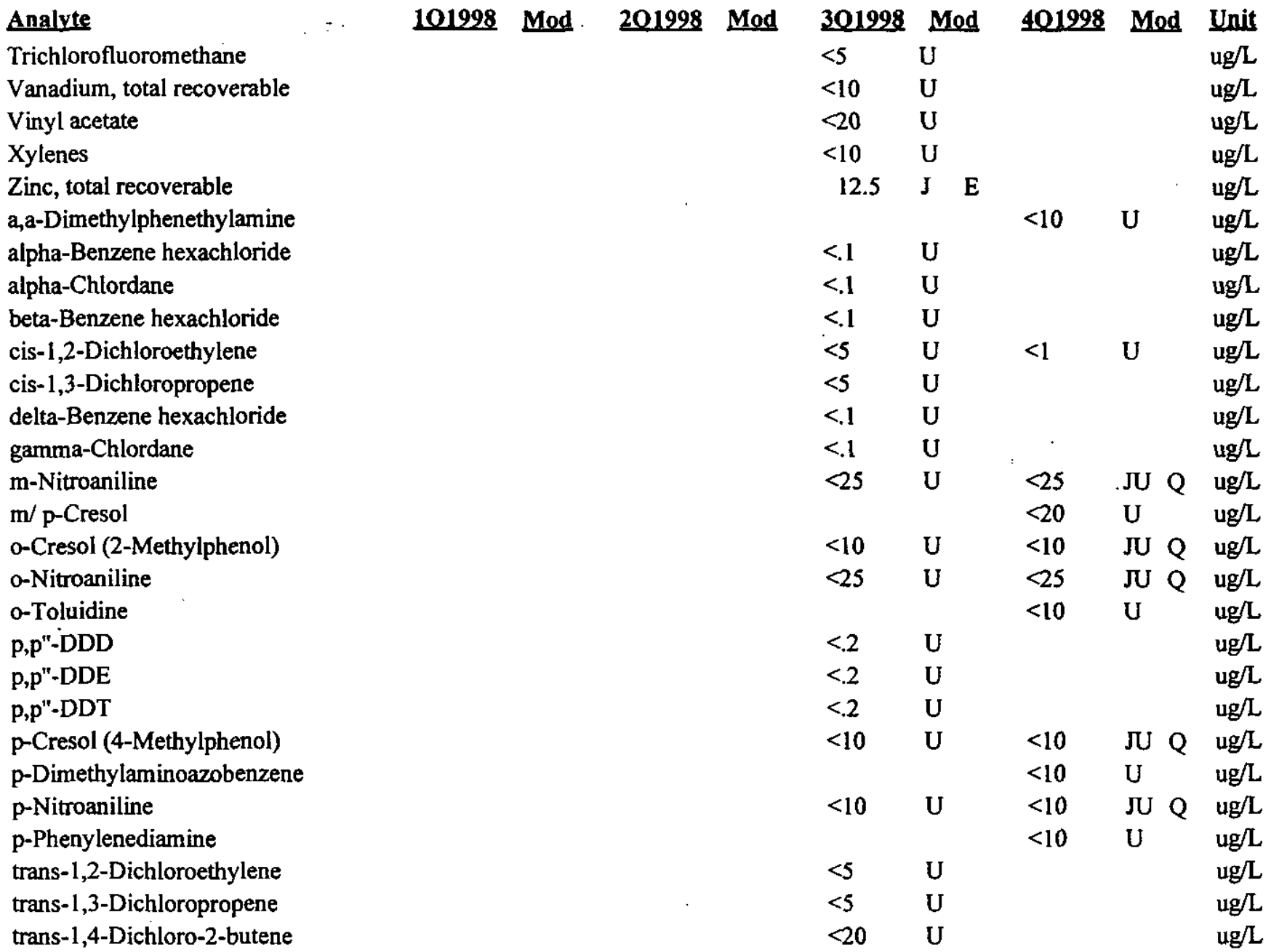


WELL: TBG 3

SRS Coord, Lat/Longitude Screen Zone Elevation Top of Standpipe Top of Casing Casing Pump Screen Zone N 71324.1 $33.212 \mathrm{Deg} N \quad 108.9-88.9 \mathrm{ftmsl} 151.4 \mathrm{ft} \mathrm{msl} 151.2 \mathrm{ftmsl} 4$ " STL $\mathrm{S}$ Unconfined E 17177.781 .760 Deg W

$108.9-88.9 \mathrm{ftmsl} 151.4 \mathrm{ftmsl} 151.2 \mathrm{ftmsl} 4 " \mathrm{STL}$ S Unconfined

SAMPLE DATE

$03 / 05 / 98$

$05 / 11 / 98$

$08 / 04 / 98$

$12 / 04 / 98$

\section{FIELD DATA}

\begin{tabular}{|c|c|c|c|c|c|}
\hline Analyte & 101998 & 201998 & 301998 & 401998 & Unit \\
\hline $\begin{array}{l}\text { Depth to water } \\
\mathrm{pH}\end{array}$ & $\begin{array}{l}46.35 \\
4\end{array}$ & $\begin{array}{l}44.7 \\
4.4\end{array}$ & $\begin{array}{l}47.85 \\
4.4\end{array}$ & $\begin{array}{l}50.1 \\
4.1\end{array}$ & ft BTOS \\
\hline Sp. Conductance & 200 & 140 & 140 & 170 & $\mathrm{uS} / \mathrm{cm}$ \\
\hline Water temperature & 18 & 17 & 25.3 & 24.1 & deg. $C$ \\
\hline Alkalinity as $\mathrm{CaCO} 3$ & 0 & 0 & 0 & 0 & $\mathrm{mg} / \mathrm{L}$ \\
\hline Phenolpthalein Alkalinity & 0 & 0 & 0 & 0 & $\mathrm{mg} / \mathrm{L}$ \\
\hline Turbidity & 2.7 & 1.7 & 1.4 & .7 & NTU \\
\hline Volumes purged & 3.91850 & 7.36211 & 4.21977 & 2.12415 & gallons \\
\hline
\end{tabular}

\section{ANALYTICAL DATA}

\section{Analyte}

1,1,1,2-Tetrachloroethane

1,1,1-Trichloroethane

1,1,2,2-Tetrachloroethane

1,1,2-Trichloroethane

1,1-Dichloroethane

1,1-Dichloroethylene

1,2,3-Trichloropropane

1,2,4-Trichlorobenzene

1,2-Dibromo-3-chloropropane

1,2-Dibromoethane

1,2-Dichlorobenzene

1,2-Dichloroethane

1,2-Dichloropropane

1,3,5-Trinitrobenzene

1,3-Dichlorobenzene

1,3-Dinitrobenzene

1,4-Dichlorobenzene

1,4-Dioxane

1,4-Naphthoquinone

1-Naphthylamine

.2,2-Oxybis(1-chloropropane)

2,3,4,6-Tetrachlorophenol

$2,3,7,8$-TCDD

2,4,5-T

2,4,5-TP (Silvex)

2,4,5-Trichlorophenol

2,4,6-Trichlorophenol
101998 Mod

201998 Mod

301998 Mod

$<50 \quad \mathrm{U}$

$<4.62 \quad U$

$<.462 \quad \mathrm{U}$

$<50$

$<50$

$<50$

$<50$

$<50$

$<50$

$<10$

$<50$

$<50$

$<50$

$<50$

$<50$

$<50$

$<50 \quad \mathrm{U}$

$<10000$

$<10$
$<.00048$
$<.2$
.55
$<10$
$<25$
401998 Mod Unit

$\mathrm{ug} / \mathrm{L}$

ug/L

ug/L

ug $/ \mathrm{L}$

$\mathrm{ug} / \mathrm{L}$

ug/L

$\mathrm{ug} / \mathrm{L}$

$\mathrm{ug} / \mathrm{L}$

ug $/$

$\mathrm{ug} / \mathrm{L}$

$\mathrm{ug} / \mathrm{L}$

$\mathrm{ug} / \mathrm{L}$

$u g / L$

$<10 \quad \mathrm{U} \quad$ ug/L

$\mathrm{ug} / \mathrm{L}$

$<10 \quad \mathrm{U} \quad \mathrm{ug} / \mathrm{L}$

$\mathrm{ug} / \mathrm{L}$

$u g / L$

$\begin{array}{lll}<10 & \mathrm{U} & \mathrm{ug} / \mathrm{L} \\ <10 & \mathrm{U} & \mathrm{ug} / \mathrm{L}\end{array}$

$<10$ JU Q ug/L

$<10 \quad \mathrm{U} \quad \mathrm{ug} / \mathrm{L}$

uǵl

$\mathrm{ug} / \mathrm{L}$

$\mathrm{ug} / \mathrm{L}$

$<10$ JU Q ug/L

$<25 \quad$ JU $Q \quad$ ug/L

Note: Concentrations in bold exceed the Drinking Water Standards listed in Appendix A. Units are for all four quarters. 
WELL: TBG 3

\section{ANALYTICAL DATA}

\section{Analvte}

2,4-Dichlorophenol

2,4-Dichlorophenoxyacetic acid

2,4-Dimethyl phenol

2,4-Dinitrophenol

2,4-Dinitrotoluene

2,6-Dichlorophenol

2,6-Dinitrotoluene

2-Acetylaminofluorene

2-Chloronaphthalene

2-Chlorophenol

2-Hexanone

2-Methyl-4,6-dinitrophenol

2-Methylnaphthalene

2-Naphthylamine

2-Nitrophenol

2-Picoline

2-sec-Butyl-4,6-dinitrophenol

3,3"-Dichlorobenzidine

3,3"-Dimethylbenzidine

3-Methylcholanthrene

4-Aminobiphenyl

4-Bromophenyl phenyl ether

4-Chloro-m-cresol

4-Chloroaniline

4-Chlorophenyl phenyl ether

4-Nitrophenol

4-Nitroquinoline-1-oxide

5-Nitro-o-toluidine

7,12-Dimethylbenz(a)anthracene

Acenaphthene

Acenaphthylene

Acetone

Acetonitrile (Methyl cyanide)

Acetophenone

Acrolein

Acrylonitrile

Aldrin

Allyl chloride

Aluminum, total recoverable

Aniline

Anthracene

Antimony, total recoverable

Aramite
101998 Mod 201998 Mod 301998 Mod 401998 Mod Unit

$\begin{array}{lllllll}<10 & \mathrm{U} & <10 & \text { JU } & \mathrm{Q} & \mathrm{ug} / \mathrm{L}\end{array}$

$<.2$ U ug/

$<10 \quad \mathrm{U} \quad<10 \quad$ JU $Q \quad$ ug/L

$<25 \quad \mathrm{U} \quad<25 \quad$ JU $Q \quad \mathrm{ug} / \mathrm{L}$

$<10 \quad \mathrm{O} \quad<10$ JU $Q \quad$ ug/L

$<10 \quad \mathrm{U} \quad \mathrm{ug} / \mathrm{L}$

$<10 \quad \mathrm{U} \quad<10 \quad$ JU $Q \quad \mathrm{ug} / \mathrm{L}$

$<10 \quad \mathrm{U} \quad<10 \quad$ JU $\mathrm{Q} \quad$ ug/L

$<10 \quad \mathrm{U} \quad<10 \quad$ JU $Q \quad$ ug/L

$<50$ U ug/L

$<25 \quad \mathrm{U} \quad<25 \quad$ JU $\mathrm{Q} \quad \mathrm{ug} / \mathrm{L}$

$<10 \quad \mathrm{U} \quad<10 \quad \mathrm{JU} Q \quad \mathrm{ug} / \mathrm{L}$

$<10 \quad \mathrm{U} \quad$ ug/L

$<10 \quad \mathrm{U} \quad<10 \quad$ JU $Q \quad \mathrm{ug} / \mathrm{L}$

$<10 \quad \mathrm{U} \quad$ ug/L

$<10$ U ug/L

$<10 \mathrm{U} \quad<10$ JU $\mathrm{Q}$ ug $/$

$<20 \quad \mathrm{U} \quad \mathrm{ug} / \mathrm{L}$

$<10 \quad \mathrm{U} \quad u g / \mathrm{L}$

$<10 \quad \mathrm{U} \cdot \mathrm{ug} / \mathrm{L}$

$<10 \quad$ U $\quad<10 \quad$ JU $Q \quad$ ug/L

$<10 \quad \mathrm{U} \quad<10 \quad \pi \quad \mathrm{Q} \quad \mathrm{ug} / \mathrm{L}$

$<10 \quad \mathrm{U} \quad<10 \quad$ JU $\mathrm{Q} \quad$ ug/L

$<10 \quad \mathrm{U} \quad<10 \quad \mathrm{JU} \quad \mathrm{Q} \quad \mathrm{ug} / \mathrm{L}$

$<25 \mathrm{U} \quad<25 \quad \pi \mathrm{U} \mathrm{Q} / \mathrm{L}$

$<50 \quad \mathrm{U} \quad \mathrm{ug} / \mathrm{L}$

$<10$ U ug/L

$<10 \quad \mathrm{U} \quad$ ug/L

$<10 \quad$ U $\quad<10 \quad$ JU $Q \quad$ ug $/ \mathrm{L}$

$<10 \quad \mathrm{U} \quad<10$ JU $\mathrm{Q} \quad \mathrm{ug} / \mathrm{L}$

$<200$ U $u g / L$

$<5000$ U ug/L

$<10 \quad \mathrm{U} \quad \mathrm{ug} / \mathrm{L}$

$<500 \quad \mathrm{U} \quad \mathrm{ug} / \mathrm{L}$

$<500$ U ug/

$<1 \quad \mathrm{U} \quad \mathrm{ug} / \mathrm{L}$

$<100 \quad \mathrm{u} \quad \mathrm{ug} / \mathrm{L}$

211

$\begin{array}{llllll}186 & \mathrm{~J} & \mathrm{E} & 356 & & \mathrm{ug} / \mathrm{L} \\ <25 & \mathrm{U} & <25 & \mathrm{JU} \mathrm{Q} & \mathrm{ug} / \mathrm{L} \\ <10 & \mathrm{U} & <10 & \mathrm{JU} \mathrm{Q} & \mathrm{ug} / \mathrm{L} \\ <100 & \mathrm{U} & & & \mathrm{ug} / \mathrm{L} \\ & & <10 & \mathrm{U} & \mathrm{ug} / \mathrm{L}\end{array}$

Note: Concentrations ia bold exceed the Drinking Water Standards listed in Appendix A. Units are for all four quarters. 
WELL: TBG 3

ANALYTICAL DATA

\section{Analyte}

Arsenic, total recoverable

Barium, total recoverable

Benzene

Benzo(a)anthracene

Benzo(a)pyrene

Benzo(b)fluoranthene

Benzo(g,h,i)perylene

Benzo(k)fluoranthene

Benzyl alcohol

Beryllium, total recoverable

Bis(2-chloroethoxy) methane

Bis(2-chloroethyl) ether

Bis(2-ethylhexyl) phthalate

Bromodichloromethane

Bromoform

Bromomethane (Methyl bromide)

Butylbenzyl phthalate

Cadmium, total recoverable

Carbazole

Carbon disulfide

Chlordane

Chlorobenzene

Chlorobenzilate

Chloroethane

Chloroethene (Vinyl chloride)

Chloromethane (Methyl chloride)

Chloroprene

Chromium, total recoverable

Chrysene

Cobalt, total recoverable

Copper, total recoverable

Cyanide

Di-n-butyl phthalate

Di-n-octyl phthalate

Diallate

Dibenz(a,h)anthracene

Dibenzofuran

Dibromochloromethane

Dibromomethane (Methylene bromide),

Dichlorodifluoromethane

Dichloromethane (Methylene chloride)

Dieldrin

Diethyl phthalate
101998 Mod 201998 Mod 301998 Mod 401998 Mad Unit

$<10$ U ug/L

$105 \mathrm{ug} / \mathrm{L}$

$<50 \quad \mathrm{U} \quad \mathrm{ug} / \mathrm{L}$

$<10 \quad \mathrm{U} \quad<10 \quad$ JU $\mathrm{Q} \quad \mathrm{ug} / \mathrm{L}$

$<10 \quad \mathrm{U} \quad<10 \quad$ JU $Q \quad$ ug/L

$<10 \quad \mathrm{U} \quad<10 \quad$ JU Q $\quad$ ug/L

$<10 \quad \mathrm{U} \quad<10 \quad$ JU $\mathrm{Q}$ ug/L

$<10 \quad \mathrm{C} \quad<10$ JU $Q$ ug/L

$\begin{array}{llllll}<10 & \mathrm{U} & <10 & \mathrm{JU} & \mathrm{Q} & \mathrm{ug} / \mathrm{L}\end{array}$

3.45 J E $\mathrm{ug} / \mathrm{L}$

$<10 \quad \mathrm{U} \quad<10 \quad$ JU $\mathrm{Q} \quad \mathrm{ug} / \mathrm{L}$

$<10 \quad \mathrm{l} \quad<10 \quad \pi \quad \mathrm{Q} \quad$ ug/L

$4.65 \quad J \quad E \quad:<10 \quad J U \quad Q \quad u g / L$

$<50$ U $\quad \mathrm{ug} / \mathrm{L}$

$<50$ U ug/L

$<50$ U ug/L

$\begin{array}{lllllll}<10 & \mathrm{U} & <2.48 & \mathrm{~J} & \text { IQ } & \mathrm{ug} / \mathrm{L}\end{array}$

$<10$ U ug/L

$<20 \quad \mathrm{u} \quad \mathrm{ug} / \mathrm{L}$

$<50$ U ug/L

$<.2$ U ug/L

$<50$. U ug/L

$<10 \quad U \quad u g / L$

$<100 \mathrm{U}$ ug/L

$<50$ U ug/L

$<50 \quad \mathrm{u} \quad \mathrm{ug} / \mathrm{L}$

$<500 \quad \mathrm{u}$ ug/L

$<10$ U ug/L

$<10 \quad \mathrm{U} \quad<10 \quad \mathrm{JU} Q \quad \mathrm{ug} / \mathrm{L}$

4.3 J E $\mathrm{ug} / \mathrm{L}$

$<20$ U ug/L

$<10$ U ug/L

$<10 \quad$ U $\quad<10 \quad$ JU $Q \quad$ ug/L

$<10 \quad \mathrm{U} \quad<10$ JU $\mathrm{Q} \quad \mathrm{ug} / \mathrm{L}$

$<10 \quad \mathrm{U} . \mathrm{ug} / \mathrm{L}$

$<20 \quad \mathrm{U} \quad<10 \quad$ JU $Q \quad$ ug/L

$<10 \quad \mathrm{U} \quad<10 \quad \mathrm{JU} \mathrm{Q} \quad \mathrm{ug} / \mathrm{L}$

$<50 \quad U \quad u g / L$

$<50$ U ug/L

$<50$ U ug/L

57.4 J EV\& ug/L

$<.2$ U $u g / L$

$<10 \quad \mathrm{U} \quad<10 \quad$ JU $\mathrm{Q} \quad \mathrm{ug} / \mathrm{L}$

Note: Concentrations in bold exceed the Drinking Water Standards listed in Appendix A. Units are for all four quarters. 
WELL: TBG 3

ANALYTICAL DATA

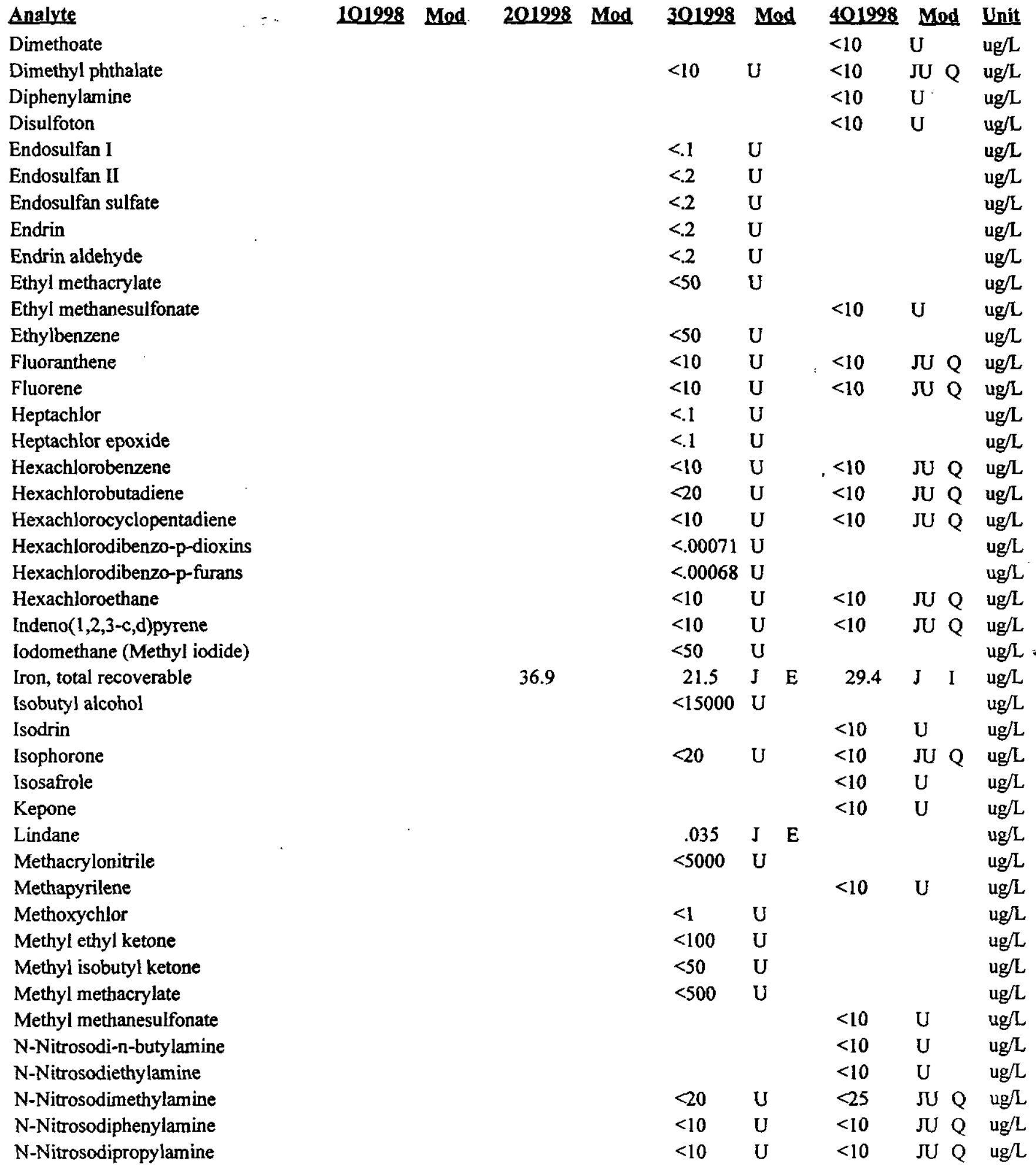

Note: Concentrations in bold exceed the Drinking Water Standards listed in Appendix A. Units are for all four quarters. 
WELL: TBG 3

ANALYTICAL DATA

\begin{tabular}{|c|c|c|c|c|c|c|c|}
\hline Analyte & 101998 Mod & $201998 \mathrm{Mod}$ & 301998 & Mod & $\underline{401998}$ & Mod & Unit \\
\hline N-Nitrosomethylethylamine & & & & & $<10$ & $\mathbf{U}$ & $\mathrm{ug} / \mathrm{L}$ \\
\hline $\mathrm{N}$-Nitrosomorpholine & & & & & $<10$ & $\mathbf{U}$ & $\mathrm{ug} / \mathrm{L}$ \\
\hline N-Nitrosopiperidine & & & & & $<10$ & $\mathrm{U}$ & $\mathrm{ug} / \mathrm{L}$ \\
\hline N-Nitrosopyrrolidine & & & & & $<10$ & $\mathrm{U}$ & $\mathrm{ug} / \mathrm{L}$ \\
\hline Naphthalene & & & $<20$ & $\mathrm{U}$ & $<10$ & JU $\mathrm{Q}$ & $\mathrm{ug} / \mathrm{L}$ \\
\hline Nickel, total recoverable & & & $<50$ & $\mathrm{U}$ & & & $\mathrm{ug} / \mathrm{L}$ \\
\hline Nitrobenzene & & & $<10$ & $\mathrm{U}$ & $<10$ & J Q & ug/L \\
\hline 0,O,O-Triethyl phosphorothioate & & & & & $<10$ & $\mathrm{U}$ & $\mathrm{ug} / \mathrm{L}$ \\
\hline РCB 1016 & & & $<2$ & $\mathrm{U}$ & & & $\mathrm{ug} / \mathrm{L}$ \\
\hline PCB 1221 & . & & $<2$ & $\mathrm{U}$ & & & $\mathrm{ug} / \mathrm{L}$ \\
\hline PCB 1232 & & & $<1$ & $\mathrm{U}$ & & & $\mathrm{ug} / \mathrm{L}$ \\
\hline РСB 1242 & & & $<1$ & $\mathrm{U}$ & & & $\mathrm{ug} / \mathrm{L}$ \\
\hline РCB 1248 & & & $<1$ & $U$ & & & $. \mathrm{ug} / \mathrm{L}$ \\
\hline PCB 1254 & & & $<1$ & $\mathbf{U}$ & & & $\mathrm{ug} / \mathrm{L}$ \\
\hline РСВ 1260 & & & $<1$ & $\mathrm{U}$ & & & $\mathrm{ug} / \mathrm{L}$ \\
\hline Parathion & & & & & $<10$ & $\mathrm{U}$ & ug/L \\
\hline Parathion methyl & & & & & $<10$ & $\mathbf{U}$ & $\mathrm{ug} / \mathrm{L}$ \\
\hline Pentachlorodibenzo-p-dioxins & & & $<.00066$ & $\mathrm{U}$ & & & $\mathrm{ug} / \mathrm{L}$ \\
\hline Pentachlorodibenzo-p-furans & & & $<.00056$ & $\mathrm{U}$ & & & $\mathrm{ug} / \mathrm{L}$ \\
\hline Pentachloroethane & & & $<2000$ & $\mathrm{U}$ & & & $\mathrm{ug} / \mathrm{L}$ \\
\hline Pentachloronitrobenzene & & & & & $<10$ & $\mathrm{U}$ & $\mathrm{ug} / \mathrm{L}$ \\
\hline Pentachlorophenol & & & $<25$ & $\mathrm{U}$ & $<25$ & JU $Q$ & $\mathrm{ug} / \mathrm{L}$ \\
\hline Phenacetin & & & & & $<10$ & $\mathbf{U}$ & $\mathrm{ug} / \mathrm{L}$ \\
\hline Phenanthrene & & & $<10$ & $\mathrm{U}$ & $<10$ & JU $Q$ & ug $/ \mathrm{L}=$ \\
\hline Phenol & & & $<10$ & $\mathrm{U}$ & $<10$ & JU Q & $\mathrm{ug} / \mathrm{L}$ \\
\hline Phorate & & & & & $<10$ & $\mathrm{U}$ & $\mathrm{ug} / \mathrm{L}$ \\
\hline Pronamid & & & & & $<10$ & $\mathrm{U}$ & ug/L \\
\hline Propionitrile & & & $<5000$ & $\mathrm{U}$ & & & $\mathrm{ug} / \mathrm{L}$ \\
\hline Pyrene & & & $<10$ & $\mathrm{U}$ & $<10$ & JU Q & $\mathrm{ug} / \mathrm{L}$ \\
\hline Pyridine & & & & & $<25$ & JU Q & $\mathrm{ug} / \mathrm{L}$ \\
\hline Safrole & & & & & $<10$ & $\mathrm{U}$ & ug/L \\
\hline Selenium, total recoverable & & & 4.57 & $\mathrm{~J} \quad \mathrm{E}$ & & & $\mathrm{ug} / \mathrm{L}$ \\
\hline Silver, total recoverable & & & $<20$ & $\mathrm{U}$ & & & $\mathrm{ug} / \mathrm{L}$ \\
\hline Styrene & & & $<50$ & $\mathrm{U}$ & & & $\mathrm{ug} / \mathrm{L}$ \\
\hline Sulfide & & & $<1000$ & $\mathrm{U}$ & & & $\mathrm{ug} / \mathrm{L}$ \\
\hline Sulfotepp & & & & & $<10$ & $\mathrm{U}$ & $\mathrm{ug} / \mathrm{L}$ \\
\hline Tetrachlorodibenzo-p-dioxins & & & $<.00048$ & $\mathrm{U}$ & & & $\mathrm{ug} / \mathrm{L}$ \\
\hline Tetrachlorodibenzo-p-furans & & & $<.00044$ & $\mathrm{U}$ & & & $\mathrm{ug} / \mathrm{L}$ \\
\hline Thallium, total recoverable & & & $<10$ & $\mathrm{U}$ & & & $\lg / \mathrm{L}$ \\
\hline Thionazin & & & & & $<10$ & $\mathrm{U}$ & $\mathrm{ug} / \mathrm{L}$ \\
\hline Tin, total recoverable & & & $<200$ & $\mathbf{U}$ & & & $\mathrm{ug} / \mathrm{L}$ \\
\hline Toluene & & & $<50$ & $\mathrm{U}$ & & & $\mathrm{ug} / \mathrm{L}$ \\
\hline Toxaphene & & & $<1$ & $\mathrm{U}$ & & & ug/L \\
\hline
\end{tabular}

Note: Concentrations in bold exceed the Drinking Water Standards listed in Appendix A. Units are for all four quarters. 
WELL: TBG 3

\section{ANALYTICAL DATA}

Analvte

Trichlorofluoromethane

Vanadium, total recoverable

Vinyl acetate

Xylenes

Zinc, total recoverable

a,a-Dimethylphenethylamine

alpha-Benzene hexachloride

beta-Benzene hexachloride

cis-1,2-Dichloroethylene

cis-1,3-Dichloropropene

delta-Benzene hexachloride

gamma-Chlordane

$\mathrm{m}$-Nitroaniline

$\mathrm{m} / \mathrm{p}$-Cresol

o-Cresol (2-Methylphenol)

o-Nitroaniline

o-Toluidine

p,p"-DDD

p,p"-DDE

p,p"-DDT

p-Cresol (4-Methylphenol)

p-Dimethylaminoazobenzene

p-Nitroaniline

p-Phenylenediamine

trans-1,2-Dichloroethylene

trans-1,3-Dichloropropene

trans-1,4-Dichloro-2-butene
101998 Mod 201998 Mod 301998 Mod 401998 Mod Unit

$<50 \quad \mathrm{U} \quad \mathrm{ug} / \mathrm{L}$

$<10$ U ug/L

$<200$ U ug/L

$<100 \quad \mathrm{u} \quad \mathrm{ug} / \mathrm{L}$

$26.3 \quad$ ug/L

$<.1 \quad \mathrm{U}$

111

$<50 \quad \mathrm{U}$

73.5

$<.1 \quad \mathrm{U}$

$<.1 \quad \mathrm{U}$

$<25 \quad \mathrm{U}$

$<10 \quad \mathrm{U}$

$<25 \quad \mathrm{U}$

$<.2 \quad \mathrm{U}$

$<.2 \quad \mathrm{U}$

$<.2 \quad \mathrm{U}$

$<10 \quad \mathrm{U}$

$<10$

$<10$

$<10$

$<10$

$<25$

$<20$

U

ug/L

$\mathrm{ug} / \mathrm{L}$

ug/L

$\mathrm{ug} / \mathrm{L}$

$\mathrm{ug} / \mathrm{L}$

ug/L

$\mathrm{ug} / \mathrm{L}$

J Q ug/L

U ug/L

$<10$ JU Q ug/L

$<25$ JU Q ug/L

$<10 \quad \mathrm{U} \quad \mathrm{ug} / \mathrm{L}$

$\mathrm{ug} / \mathrm{L}$

$\mathrm{ug} / \mathrm{L}$

$\mathrm{ug} / \mathrm{L}$

JU Q ug/L

JU $Q \quad u g / L$

$<50 \quad \mathrm{U}$

$<50 \quad \mathrm{U}$

$<200 \quad$ U

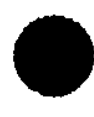


WSRC-RP-99-4003

Unclassified

WELL: TBG 4

SRS Coord Lat/Longitude Screen Zone Elevation Top of Standpipe Top of Casing Casing Pump Screen Zone N 71267.1 33.211 Deg N 109.3-89.3 ft msl $151.6 \mathrm{ft} \mathrm{msl} 151.3 \mathrm{ft} \mathrm{msl} 4$ " STL V Unconfined E 17177.781 .760 Deg W

$\begin{array}{llll}\text { SAMPLE DATE } & 03 / 05 / 98 & 05 / 11 / 98 & 08 / 04 / 98\end{array}$

FIELD DATA

\begin{tabular}{|c|c|c|c|c|c|}
\hline Analyte & 101998 & $\underline{201998}$ & 301998 & $\underline{401998}$ & Unit \\
\hline $\begin{array}{l}\text { Depth to water } \\
\mathrm{pH}\end{array}$ & $\begin{array}{l}46.2 \\
3.8\end{array}$ & $\begin{array}{l}44.4 \\
4.2\end{array}$ & $\begin{array}{l}47 \\
4.3\end{array}$ & & ft BTOS \\
\hline Sp. Conductance & 260 & 220 & 220 & & $\mathrm{uS} / \mathrm{cm}$ \\
\hline Water temperature & 17 & 18 & 24.1 & & deg. C \\
\hline Alkalinity as $\mathrm{CaCO} 3$ & 0 & 0 & 0 & & $\mathrm{mg} / \mathrm{L}$ \\
\hline Phenolpthalein Alkalinity & 0 & 0 & 0 & & $\mathrm{mg} / \mathrm{L}$ \\
\hline Turbidity & 3.4 & 1.5 & 1.7 & & NTU \\
\hline Volumes purged & 2.31553 & 2.42517 & 3.35366 & & gallons \\
\hline
\end{tabular}

\section{ANALYTICAL DATA}

Analyte

1,1,1,2-Tetrachloroethane

1,1,1-Trichloroethane

1,1,2,2-Tetrachloroethane

1,1,2-Trichloroethane

1,1-Dichloroethane

1,1-Dichloroethylene

1,2,3-Trichloropropane

1,2,4-Trichlorobenzene

1,2-Dibromo-3-chloropropane

1,2-Dibromoethane

1,2-Dichlorobenzene

1,2-Dichloroethane

1,2-Dichloropropane

1,3-Dichlorobenzene

1,4-Dichlorobenzene

1,4-Dioxane

2,2-Oxybis(1-chloropropane)

2,3,7,8-TCDD

2,4,5-T

2,4,5-TP (Silvex)

2,4,5-Trichlorophenol

2,4,6-Trichlorophenol

2,4-Dichlorophenol

2,4-Dichlorophenoxyacetic acid

2,4-Dimethyl phenol

2,4-Dinitrophenol

2,4-Dinitrotoluene

\begin{tabular}{|c|c|c|c|c|c|c|c|}
\hline 101998 & Mod & $\underline{201998}$ & Mod & 301998 & Mod & 401998 Mod & Unit \\
\hline & & & & $<25$ & $\mathbf{U}$ & & ug $/ \mathrm{L}$ \\
\hline$<4.62$ & $\mathbf{U}$ & $<.462$ & $\mathbf{U}$ & $<25$ & $\mathrm{U}$ & & $u g / L$ \\
\hline & & & & $<25$ & $\mathrm{U}$ & & $\mathrm{ug} / \mathrm{L}$ \\
\hline & & & & $<25$ & $\mathbf{U}$ & & $\mathrm{ug} / \mathrm{L}$ \\
\hline & & & & $<25$ & $\mathrm{U}$ & & $\mathrm{ug} / \mathrm{L}$ \\
\hline & & & & $<25$ & $\mathbf{U}$ & & $\mathrm{ug} / \mathrm{L}$ \\
\hline & & & & $<25$ & U & & $\mathrm{ug} / \mathrm{L}$ \\
\hline & & & & $<10$ & $\mathrm{U}$ & & $\mathrm{ug} / \mathrm{L}$ \\
\hline & & & & $<25$ & U & & $\mathrm{ug} / \mathrm{L}$ \\
\hline & & & & $<25$ & $\mathrm{U}$ & & $\mathrm{ug} / \mathrm{L}$ \\
\hline & & & & $<25$ & $\mathrm{U}$ & & $\mathrm{ug} / \mathrm{L}$ \\
\hline & & & & $<25$ & $\mathbf{U}$ & & $\mathrm{ug} / \mathrm{L}$ \\
\hline & & & & $<25$ & U & & $\mathrm{ug} / \mathrm{L}$ \\
\hline & & & & $<25$ & U & & $\mathrm{ug} / \mathrm{L}$ \\
\hline & & & & $<25$ & $\mathrm{U}$ & & $\mathrm{ug} / \mathrm{L}$ \\
\hline & & & & $<5000$ & $\mathrm{U}$ & & $\mathrm{ug} / \mathrm{L}$ \\
\hline & & & & $<10$ & U & & $\mathrm{ug} / \mathrm{L}$ \\
\hline & & & & $<.00078$ & U & & $\mathrm{ug} / \mathrm{L}$ \\
\hline & & & & $<.2$ & $\mathrm{U}$ & & $\mathrm{ug} / \mathrm{L}$ \\
\hline & & & & $<.2$ & $\mathrm{U}$ & & $\mathrm{ug} / \mathrm{L}$ \\
\hline & & & & $<10$ & U & & $\mathrm{ug} / \mathrm{L}$ \\
\hline & & & & $<25$ & $\mathrm{U}$ & & $\mathrm{ug} / \mathrm{L}$ \\
\hline & & & & $<10$ & U & & $\mathrm{ug} / \mathrm{L}$ \\
\hline & & & & $<.2$ & U & & $\mathrm{ug} / \mathrm{L}$ \\
\hline & & & & $<10$ & $\mathrm{U}$ & & ug/L \\
\hline & & & & $<25$ & U & & $\mathrm{ug} / \mathrm{L}$ \\
\hline & & & & $<10$ & U & & $\mathrm{ug} / \mathrm{L}$ \\
\hline
\end{tabular}

Note: Concentrations in bold exceed the Drinking Water Standards listed in Appendix A. Units are for all four quarters. 
WELL: TBG 4

\section{ANALYTICAL DATA}

\section{Analyte}

2,6-Dinitrotoluene

2-Chloronaphthalene

2-Chlorophenol

2-Hexanone

2-Methyl-4,6-dinitrophenol

2-Methylnaphthalene

2-Nitrophenol

3,3"-Dichlorobenzidine

4-Bromophenyl phenyl ether

4-Chloro-m-cresol

4-Chloroaniline

4-Chlorophenyl phenyl ether

4-Nitrophenol

Acenaphthene

Acenaphthylene-

Acetone

Acetonitrile (Methyl cyanide)

Acrolein

Acrylonitrile

Aldrin

Allyl chloride

Aluminum, total recoverable

Aniline

Anthracene

Antimony, total recoverable

Arsenic, total recoverable

Barium, total recoverable

Benzene

Benzo(a)anthracene

Benzo(a)pyrene

Benzo(b)fluoranthene

Benzo(g,h,i)perylene

Benzo(k)fluoranthene

Benzyl alcohol

Beryllium, total recoverable

Bis(2-chloroethoxy) methane

Bis(2-chloroethyl) ether

Bis(2-ethylhexyl) phthalate

Bromodichloromethane

Bromoform

Bromomethane (Methyl bromide)

Butylbenzyl phthalate

Cadmium, total recoverable
101998 Mod 201998 Mod

301998 Mod

401998 Mod Unit

$<10 \quad \mathrm{U}$

$<10 \quad \mathrm{U}$

$<10 \quad \mathrm{U}$

$<25 \quad \mathrm{U}$

$<25 \quad U$

$<10 \quad \mathrm{U}$

$<10 \quad \mathrm{U}$

$<10 \quad \mathrm{U}$

$<10 \quad \mathrm{U}$

$<10 \quad \mathrm{U}$

$<10 \quad \mathrm{U}$

$<10 \quad \mathrm{U}$

$<25$ U

$<10 \quad \mathrm{U}$

$<10 \quad \mathrm{U}$

$<100 \quad \mathrm{U}$

$<2500$ U

$<250 \quad \mathrm{U}$

$<250$ U

$<1$ U

$<50$ U

574

759

$<25$

$<10$

$<100 \quad \mathrm{U}$

$<10 \quad \mathrm{U}$

186

$<25 \mathrm{U}$

$<10 \quad \mathrm{U}$

$<10 \quad \mathrm{U}$

$<10 \quad \mathrm{U}$

$<10 \quad \mathrm{U}$

$<10 \quad \mathrm{U}$

$<10 \quad \mathrm{U}$

4.08 J $\quad E$

$<10 \quad \mathrm{U}$

$<10 \quad \mathrm{U}$

$<10 \quad \mathrm{U}$

$<25 \quad \mathrm{U}$

$<25 \quad \mathrm{U}$

$<25 \quad \mathrm{U}$

$<10 \quad \mathrm{U}$

$<10 \quad \mathrm{U}$
ug/L

$\mathrm{ug} / \mathrm{L}$

ug/L

ug/L

ug/L

ug/L

ug/L

ug/L

ug $/ \mathrm{L}$

$u g / L$

$u g / L$

$\mathrm{ug} / \mathrm{L}$

ug/L

ug/L

ug/L

ug/L

$u g / L$

ug/L

$\mathrm{ug} / \mathrm{L}$

$\mathrm{ug} / \mathrm{L}$

$\mathrm{ug} / \mathrm{L}$

$\mathrm{ug} / \mathrm{L}$

$\mathrm{ug} / \mathrm{L}$

ug/L =

ug/L

ug/L

ug/L

ug/L

ug/L

ug/L

$\mathrm{ug} / \mathrm{L}$

$\mathrm{ug} / \mathrm{L}$

$\mathrm{ug} / \mathrm{L}$

$\mathrm{ug} / \mathrm{L}$

ug/L

ug/L

ug/L

$\operatorname{ug} / \mathrm{L}$

$\mathrm{ug} / \mathrm{L}$

ug/L

ug/L

$\mathrm{ug} / \mathrm{L}$

ug/L

Note: Concentrations in bold exceed the Drinking Water Standards listed in Appendix A. Units are for all four quarters. 
WELL: TBG 4

ANALYTICAL DATA

Analvte

Carbazole

Carbon disulfide

Chlordane

Chlorobenzene

Chloroethane

Chloroethene (Vinyl chloride)

Chloromethane (Methyl chloride)

Chloroprene

Chromium, total recoverable

Chrysene

Cobalt, total recoverable

Copper, total recoverable

Cyanide

Di-n-butyl phthalate

Di-n-octyl phthalate

Dibenz(a,h)anthracene

Dibenzofuran

Dibromochloromethane

Dibromomethane (Methylene bromide',

Dichlorodifluoromethane

Dichloromethane (Methylene chloride)

Dieldrin

Diethyl phthalate

Dimethyl phthalate

Endosulfan I

Endosulfan II

Endosulfan sulfate

Endrin

Endrin aldehyde

Ethyl methacrylate

Ethylbenzene

Fluoranthene

Fluorene

Heptachlor

Heptachlor epoxide

Hexachlorobenzene

Hexachlorobutadiene

Hexachlorocyclopentadiene

Hexachlorodibenzo-p-dioxins

Hexachlorodibenzo-p-furans

Hexachloroethane

Indeno( $1,2,3-c, d)$ pyrene

Iodomethane (Methyl iodide)
101998 Mod 201998 Mod 301998 Mod 401998 Mod Unit

$<20$ U

$<25 \quad \mathrm{U}$

$<.2 \quad \mathrm{U}$

$<25 \quad U$

$<50 \quad \mathrm{U}$

$<25 \mathrm{U}$

$<25 \mathrm{U}$

$<250 \quad \mathrm{U}$

$<10 \quad \mathrm{U}$

$<10 \quad \mathrm{U}$

14.9 J E

$<20$ U

$<10 \quad \mathrm{U}$

$<10 \quad \mathrm{U}$

$<10 \quad \mathrm{U}$

$<20 \quad$ U

$<10 \quad$ U

$<25$ U

$<25 \quad \mathrm{U}$

$<25 \quad U$

$<25.1$ U V

$<.2 \quad \mathrm{U}$

$<10 \quad \mathrm{U}$

$<10 \quad \mathrm{U}$

$<.1$ U

$<.2 \quad \mathrm{U}$

$<.2 \quad \mathrm{U}$

$<.2$ U

$<.2 \quad \mathrm{U}$

$<25$ U

$<25$ U

$<10 \quad \mathrm{U}$

$<10 \quad$ U

$<.1 \quad \mathrm{U}$

$<.1 \quad \mathrm{U}$

$<10 \quad U$

$<20$ U

$<10 \quad \mathrm{U}$

$<.0011 \mathrm{U}$

$<.00072 \mathrm{U}$

$<10 \quad \mathrm{U}$

$<10 \quad \mathrm{U}$

$<25$ U $\mathrm{ug} / \mathrm{L}$

ug/L

$\mathrm{ug} / \mathrm{L}$

ug/L

$\mathrm{ug} / \mathrm{L}$

$\mathrm{ug} / \mathrm{L}$

ug/L

ug/L

ug $/ \mathrm{L}$

ug/L

ug/L

ug/L

ug/L

ug/L

$\mathrm{ug} / \mathrm{L}$

ug/L

$\mathrm{ug} / \mathrm{L}$

ug/L

ug/L

$u g / L$

ug/L

ug/L

ug $/ \mathrm{L}$

$\mathrm{ug} / \mathrm{L}$

$\mathrm{ug} / \mathrm{L}$

ug/L

ug/L

ug/L

$u g / L$

ug/L

$\mathrm{ug} / \mathrm{L}$

ug/L

$\mathrm{ug} / \mathrm{L}$

ug/L

ug/L

$u g / L$

ug/L

$u g / L$

$\mathrm{ug} / \mathrm{L}$

ug/L

$u g / L$

ug/L

ug/L

Note: Concentrations in bold exceed the Drinking Water Standards listed in Appendix A. Units are for all four quarters. 
WELL: TBG 4

\section{ANALYTICAL DATA}

Analvte

Iron, total recoverable

Isobutyl alcohol

Isophorone

Lindane

Methacrylonitrile

Methoxychlor

Methyl ethyl ketone

Methyl isobutyl ketone

Methyl methacrylate

$\mathrm{N}$-Nitrosodimethylamine

$\mathrm{N}$-Nitrosodiphenylamine

N-Nitrosodipropylamine

Naphthalene

Nickel, total recoverable

Nitrobenzene

PCB 1016

PCB 1221

PCB 1232

PCB 1242

PCB 1248

PCB 1254

PCB 1260

Pentachlorodibenzo-p-dioxins

Pentachlorodibenzo-p-furans

Pentachloroethane

Pentachlorophenol

Phenanthrene

Phenol

Propionitrile

Pyrene

Selenium, total recoverable

Silver, total recoverable

Styrene

Sulfide

Tetrachlorodibenzo-p-dioxins

Tetrachlorodibenzo-p-furans

Thallium, total recoverable

Tin, total recoverable

Toluene

Toxaphene

Trichlorofluoromethane

Vanadium, total recoverable

Vinyl acetate

\section{Mod $\frac{2019}{125}$}

\begin{tabular}{|c|c|c|c|c|c|}
\hline 301998 & $\mathbf{M}$ & & 401998 & Mod & Uni \\
\hline 51.4 & $\mathbf{J}$ & E & & & ug/l \\
\hline$<7500$ & $\mathrm{U}$ & & & & Ig 1 \\
\hline$<20$ & U & & & & $\mathrm{ug} / \mathrm{l}$ \\
\hline .019 & J & E & & & \\
\hline$<2500$ & $\mathbf{U}$ & & & & \\
\hline$<1$ & $\mathrm{U}$ & & & & \\
\hline
\end{tabular}

$<50 \quad \mathrm{U}$

$<25 \quad \mathrm{U}$

$<250 \quad \mathrm{U}$

$<20$ U

$<10 \quad \mathrm{U}$

$<10$ U

$<20$ U

$14.8 \quad \mathrm{~J} \quad \mathrm{E}$

$<10 \quad \mathrm{U}$

$<2 \quad \mathrm{U}$

$<\quad \mathrm{U}$

$<1 \quad U$

$<1 \quad$ U

$<1 \quad U$

$<$ U

$<1 \quad$ U

$<.0012 \mathrm{U}$

$<.00078 \mathrm{U}$

$<1000 \mathrm{U}$

$<25$ U

$<10 \quad \mathrm{U}$

$<10 \quad \mathrm{U}$

$<2500$ U

$<10 \quad \mathrm{U}$

$<10 \quad \mathrm{U}$

$<20$ U

$<25$ U

$<1000 \quad \mathrm{U}$

$<.00078 \mathrm{U}$

$<.00037 \mathrm{U}$

$<10 \quad \mathrm{U}$

$<200 \quad \mathrm{U}$

$<25$ U

$<$ U

$<25$ U

$<10 \quad \mathrm{U}$

$<100 \quad \mathrm{U}$
ug/L

$\mathrm{ug} / \mathrm{L}$

ug/L

$\mathrm{ug} / \mathrm{L}$

ug/L

$\mathrm{ug} / \mathrm{L}$

ug/L

ug/L

$\mathrm{ug} / \mathrm{L}$

ug/L

$\mathrm{ug} / \mathrm{L}$

ug/L

ug/L

ug/L

ug/L

ug/L

ug/L

ug/L

ug/L

ug/L

ug/L

$\mathrm{ug} / \mathrm{L}$

$u g / L$

ug/L

ug/L

ug/L

ug/L

ug/L

ug/L

$\mathrm{ug} / \mathrm{L}$

ug/L

$\mathrm{ug} / \mathrm{L}$

$\mathrm{ug} / \mathrm{L}$

$\mathrm{ug} / \mathrm{L}$

$\mathrm{ug} / \mathrm{L}$

ug/L

$\mathrm{ug} / \mathrm{L}$

Note: Concentrations in bold exceed the Drinking Water Standards listed in Appendix A. Units are for all four quarters. 
WELL: TBG 4

ANALYTICAL DATA

Analyte

101998 Mod 201998 Mod

Xylenes

Zinc, total recoverable

alpha-Benzene hexachloride

beta-Benzene hexachloride

cis-1,2-Dichloroethylene

cis-1,3-Dichloropropene

delta-Benzene hexachloride

gamma-Chlordane

m-Nitroaniline

o-Cresol (2-Methylphenol)

o-Nitroaniline

p,p"-DDD

p,p"-DDE

$p, p "-D D T$

p-Cresol (4-Methylphenol)

p-Nitroaniline

trans-1,2-Dichloroethylene

trans-1,3-Dichloropropene

trans-1,4-Dichloro-2-butene

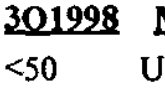

39.5

$<.1 \quad \mathrm{U}$

$<.1 \quad$ U

157

$<25$ U

$<.1 \quad \mathrm{U}$

$<.1 \quad \mathrm{U}$

$<25$ U

$<10 \quad U$

$<25 \quad \mathrm{U}$

$<.2 \quad \mathrm{U}$

$<.2 \quad \mathrm{U}$

$<.2$ U

$<10 \quad \mathrm{U}$

$<10 \quad \mathrm{U}$

$<25$ U

$<25 \quad \mathrm{U}$

$<100 \quad \mathrm{U}$
401998 Mod Unit

ug/L

$\mathrm{ug} / \mathrm{L}$

ug/L

$\mathrm{ug} / \mathrm{L}$

$\mathrm{ug} / \mathrm{L}$

$\mathrm{ug} / \mathrm{L}$

$\mathrm{ug} / \mathrm{L}$

$\mathrm{ug} / \mathrm{L}$

$\mathrm{ug} / \mathrm{L}$

$\mathrm{ug} / \mathrm{L}$

ug/L

$\mathrm{ug} / \mathrm{L}$

ug/L

ug/L

$u g / L$

$u g / L$

$\mathrm{ug} / \mathrm{L}$

ug/L

$\mathrm{ug} / \mathrm{L}$

Note: Concentrations in bold exceed the Drinking Water Standards listed in Appendix A. Units are for all tour quarters. 
WELL: TBG 5

SRS Coord, Lat/Longitude Screen Zone Elevation Top of Standpipe Top of Casing Casing Pump Screen Zone N 71226.5 33.212 Deg N $112.4-92.4 \mathrm{ft} \mathrm{msl}$ $149.6 \mathrm{ft} \mathrm{msl}$ $149.4 \mathrm{ft} \mathrm{msl}$

4 " STL

B

Unconfined E 17354.5 81.759 Deg W :

SAMPLE DATE

03/05/98

$05 / 11 / 98$

$08 / 04 / 98$

$12 / 04 / 98$

FIELD DATA

Analyte

Depth to water

$\mathrm{pH}$

Sp. Conductance

Water temperature

Alkalinity as $\mathrm{CaCO} 3$

Phenolpthalein Alkalinity

Turbidity

Volumes purged

Sampling codes
101998

44.15

5

76

17

3

0 .

4.1

1.42356

\section{8}

41.8

5

60

17

1

0

1.8

2.70780
301998

45.1

5.1

64

26.4

1

0

1.1

2.43390
401998

47.8

4.8

61

23.4

0

0

2.1

4.30806
Unit

ft BTOS

$\mathrm{uS} / \mathrm{cm}$

deg. C

$\mathrm{mg} / \mathrm{L}$

$\mathrm{mg} / \mathrm{L}$

NTU

gallons

\section{ANALYTICAL DATA}

\section{Analvte}

1,1,1,2-Tetrachloroethane

1,1,1-Trichloroethane

1,1,2,2-Tetrachloroethane

1,1,2-Trichloroethane

1,1-Dichloroethane

1,1-Dichloroethylene

1,2,3-Trichloropropane

1,2,4-Trichlorobenzene

1,2-Dibromo-3-chloropropane

1,2-Dibromoethane

1,2-Dichlorobenzene

1,2-Dichloroethane

1,2-Dichloropropane

1,3,5-Trinitrobenzene

1,3-Dichlorobenzene

1,3-Dinitrobenzene

1,4-Dichlorobenzene

1,4-Dioxane

1,4-Naphthoquinone

1-Naphthylamine

2,2-Oxybis(1-chloropropane)

2,3,4,6-Tetrachlorophenol

2,3,7,8-TCDD

2,4,5-T

2,4,5-TP (Silvex)

2,4,5-Trichlorophenol

2,4,6-Trichlorophenol
$101998 \mathrm{Mod}$

$<46.2 \quad \mathrm{U}$

U

201998 Mod

$<.462 \quad \mathrm{U}$

$<50$

$<50$

$<50$

$<50$

$<50$

$<50$

$<50$

$<10 \quad \mathrm{U}$

$<50$

$<50$

$<50$

$<50$

$<50$

$<50$

$<50$

$<10000$

$<10000$

$\begin{array}{ll}<10 & \mathrm{U} \\ & \\ <.0003 & \mathrm{U} \\ <.2 & \mathrm{U} \\ <.2 & \mathrm{U} \\ <10 & \mathrm{U} \\ <25 & \mathrm{U}\end{array}$

U

U

\section{$\mathrm{M}$}

U

U

U

U

U

U

U

U

U

U

U

U

U

U

$\mathrm{U}$

$<10$

$<10$

$<10$

$<10$.

$\mathrm{U}$

U

U ug/L

JU $Q \quad u g / L$

$\mathrm{ug} / \mathrm{L}$

ug/L

ug/L

ug $/$

$<10$ JU Q ug/L

$<25$ JU Q ug/L

Note: Concentrations in bold exceed the Drinking Water Standards listed in Appendix A. Units are for all four quarters. 
WELL: TBG 5

ANALYTICAL DATA

Analyte

2,4-Dichlorophenol

2,4-Dichlorophenoxyacetic acid

2,4-Dimethyl phenol

2,4-Dinitrophenol

2,4-Dinitrotoluene

2,6-Dichlorophenol

2,6-Dinitrotoluene

2-Acetylaminofluorene

2-Chloronaphthalene

2-Chlorophenol

2-Hexanone

2-Methyl-4,6-dinitrophenol

2-Methylnaphthalene

2-Naphthylamine

2-Nitrophenol

2-Picoline

2-sec-Butyl-4,6-dinitrophenol

3,3"-Dichlorobenzidine

3,3"-Dimethylbenzidine

3-Methylcholanthrene

4-Aminobiphenyl

4-Bromophenyl phenyl ether

4-Chloro-tn-cresol

4-Chloroaniline

4-Chlorophenyl phenyl ether

4-Nitrophenol

4-Nitroquinoline-1-oxide

5-Nitro-o-toluidine

7,12-Dimethylbenz(a)anthracene

Acenaphthene

Acenaphthylene

Acetone

Acetonitrile (Methyl cyanide)

Acetophenone

Acrolein

Acrylonitrile

Aldrin

Allyl chloride

Aluminum, total recoverable

Aniline

Anthracene

Antimony, total recoverable

Aramite

\section{Mod}

\section{8}

Mod

$\begin{array}{ll}\begin{array}{l}\text { 301998 } \\ <10\end{array} & \text { Mod } \\ <.2 & \mathrm{U} \\ <10 & \mathrm{U} \\ <25 & \mathrm{U} \\ <10 & \mathrm{U}\end{array}$

401998 Mod Unit

$<10$

JU Q

ug/L

$\mathrm{ug} / \mathrm{L}$

$<10$

$<25$

$<10$

$<10$

$<10 \quad \mathrm{U}$

$<10$

$<10$

$<10$

$<10$

$<10$

$<10$

$<50$

$<25$

$<10$

$<10$

$<10$

U

$<10$

$<10 \quad \mathrm{U}$

U

$<10$

$<10$

$<10$

$<10$

$<25$

$<25$

$<10$

$<10$

$<10$

$<10$

$<10$

$<10$

$<20$

$<10$

$<10$

JU Q ug/L

JU Q ug/L

JU $Q \quad u g / L$

$\mathrm{U}$

ug/L

J Q ug/L

U

ug/L

JU Q ug/L

JU Q ug/L

ug/L

JU $Q \quad u g / L$

JU $Q$ ug/L

- U ug/L

JU $Q \quad u g / L$

U ug/L

U ug/L

J $Q \quad$ ug/L

U ug/L

U

$\mathrm{ug} / \mathrm{L}$

$<10$

U

ug/L

$<10$

JU $Q$ ug/L

$<10$

JU $Q$ ug/L

$<10$

JU $Q$

ug/ $\mathrm{L}$ :

$<25$

JU $Q$ ug/L

$<50$

JU

$\mathrm{ug} / \mathrm{L}$

$<10$

$<10$

$<10 \quad \mathrm{U}$

$<10$

$<10$

$<10$

$<200$

U

$<5000$

U

U

ug $/ \mathrm{L}$

U ug/L

U ug/L

JU Q ug/L

JU $Q$

ug/L

ug/L

$\mathrm{ug} / \mathrm{L}$

$<10 \quad \mathrm{U} \quad \mathrm{ug} / \mathrm{L}$

$<500 \quad \mathrm{U}$

$<500 \quad \mathrm{U}$

$<.1 \quad \mathrm{U}$

$<100 \quad \mathrm{U}$

$<200 \quad \mathrm{U}$

$<25 \quad \mathrm{U}$

$<10 \quad \mathrm{U}$

$<100$

$$
\text { }
$$$$
\text { ug/L }
$$$$
\text { ug/L }
$$$$
\text { ug/L }
$$$$
\mathrm{ug} / \mathrm{L}
$$$$
145 \text { J I ug/L }
$$$$
<25 \text { JU Q ug/L }
$$$$
<10 \quad \pi \quad \text { Q } u g / L
$$$$
<10 \quad U \quad \text { u }
$$

Note: Concentrations in bold exceed the Drinking Water Standards listed in Appendix A. Units are for all four quarters. 
WELL: TBG 5

\section{ANALYTICAL DATA}

\section{Analyte}

Arsenic, total recoverable

Barium, total recoverable

Benzene

Benzo(a)anthracene

Benzo(a)pyrene

Benzo(b)fluoranthene

Benzo(g,h,i)perylene

Benzo(k)fluoranthene

Benzyl alcohol

Beryllium, total recoverable

Bis(2-chloroethoxy) methane

Bis(2-chloroethyl) ether

Bis(2-ethylhexyl) phthalate

Bromodichloromethane

Bromoform

Bromomethane (Methyl bromide)

Butylbenzyl phthalate

Cadmium, total recoverable

Carbazole

Carbon disulfide

Chlordane

Chlorobenzene

Chlorobenzilate

Chloroethane

Chloroethene (Vinyl chloride)

Chloromethane (Methyl chloride)

Chloroprene

Chromium, total recoverable

Chrysene

Cobalt, total recoverable

Copper, total recoverable

Cyanide

Di-n-butyl phthalate

Di-n-octyl phthalate

Diallate

Dibenz(a,h)anthracene.

Dibenzofuran

Dibromochloromethane

Dibromomethane (Methylene bromide',

Dichlorodifluoromethane

Dichloromethane (Methylene chloride)

Dieldrin

Diethyl phthalate

\section{Mod 201998 Mod}

$301998 \mathrm{Mad}$

$<10 \quad \mathrm{U}$

7.85 J $E$

401998 Mod Unit

$<50 \quad \mathrm{U}$

$<10 \quad U$

$<10 \quad \mathrm{U}$

$<10 \quad \mathrm{U}$

$<10 \quad \mathrm{U}$

$<10 \quad \mathrm{U}$

$<10 \quad \mathrm{U}$

$<10 \quad \mathrm{U}$

$<10$ U

$<10 \cdot \mathrm{U}$

$<10 \quad \mathrm{U}$

$<50$. U

$<50 \quad \mathrm{U}$

$<50 \quad \mathrm{U}$

$<10 \quad \mathrm{U}$

$<10 \quad \mathrm{U}$

$<20 \quad$ U

$<50 \quad \mathrm{U}$

$<.2 \quad \mathrm{U}$

$<50 \quad \mathrm{U}$

$<100 \quad$ U

$<50 \quad \mathrm{U}$

$<50 \quad \mathrm{U}$

$<500 \quad \mathrm{U}$

$<10 \quad \mathrm{U}$

$<10 \quad \mathrm{U}$

$<20$ U

$<20 \mathrm{U}$

$<10 \quad \mathrm{U}$

$<10 \quad \mathrm{U}$

$<10 \quad \mathrm{U}$

$<10$

$<10$

$<10$

$<10$

$<10$

$<10$

$<10$

$<10$

$<10$

$<10$

JU $Q \quad u g / L$

JU $Q \quad u g / L$

ug/L

ug/L

$u g / L$

$<55 \quad J \quad Q \quad u g / L$

$u g / L$

ug/L

ug/L

$\mathrm{ug} / \mathrm{L}$

$\mathrm{ug} / \mathrm{L}$

$<10 \quad \mathrm{U} \quad \mathrm{ug} / \mathrm{L}$

$\mathrm{ug} / \mathrm{L}$.

$\mathrm{ug} / \mathrm{L}$

$\mathrm{ug} / \mathrm{L}$

$\mathrm{ug} / \mathrm{L}$

$\mathrm{ug} / \mathrm{L}$

$<10 \quad$ JU $Q \quad$ ug/L

ug/L

ug/L

$\mathrm{ug} / \mathrm{L}$

$\pi U \quad \mathrm{Q} \quad \mathrm{ug} / \mathrm{L}$

JU $Q \quad$ ug/L

$<10 \quad \mathrm{U}$ ug/L

$<20 \quad \mathrm{U} \quad<10 \quad$ JU $Q \quad$ ug/L

$<10 \quad \mathrm{U}$

$<10$

JU $Q$

$\mathrm{ug} / \mathrm{L}$

ug/L

$\mathrm{ug} / \mathrm{L}$

$\mathrm{ug} / \mathrm{L}$

$\mathrm{ug} / \mathrm{L}$

$\mathrm{ug} / \mathrm{L}$

$<.2 \quad \mathrm{U}$

$<10 \quad U$ 
WELL: TBG 5

\section{ANALYTICAL DATA}

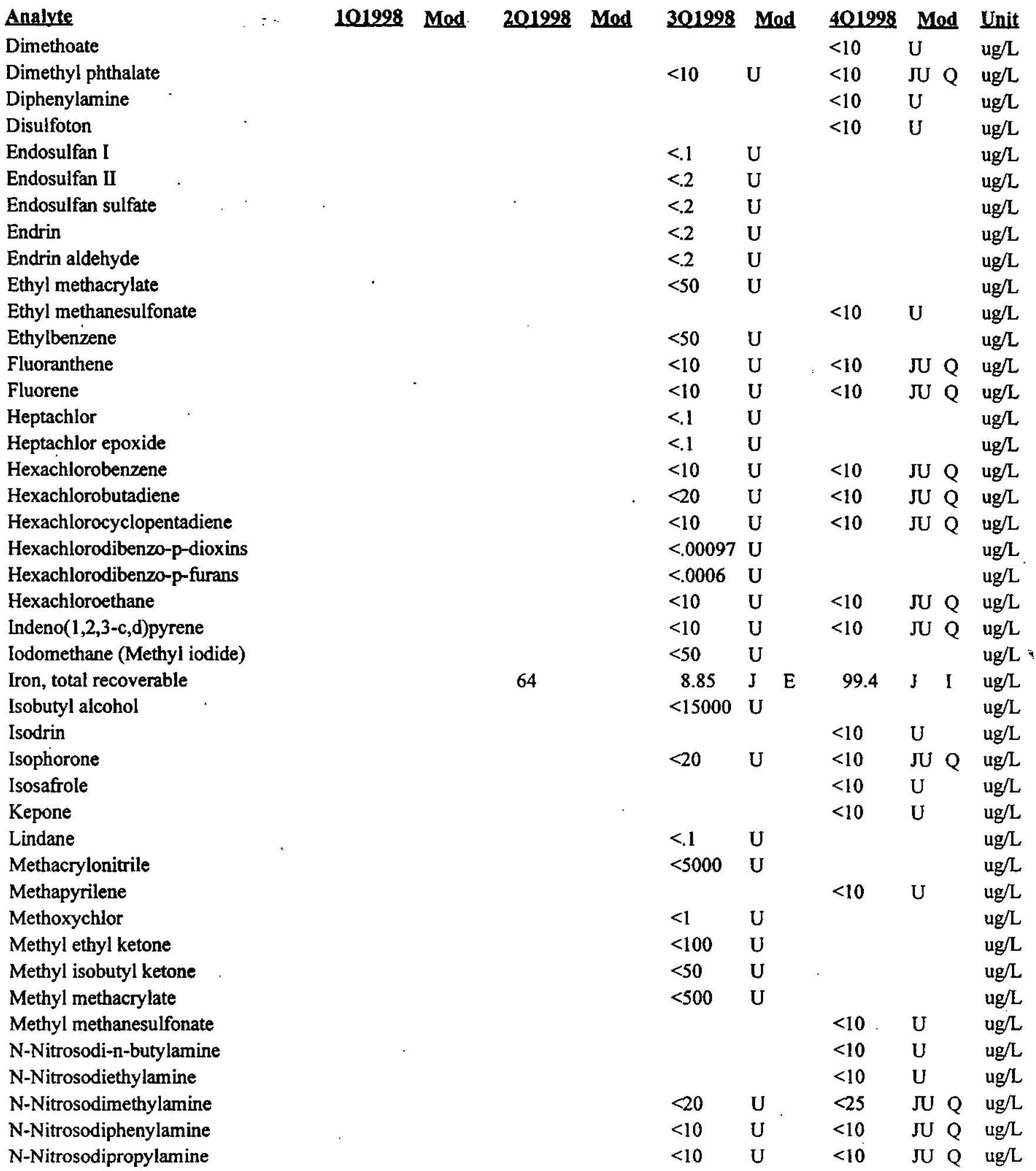

Note: Concentrations in bold exceed the Drinking Water Standards listed in Appendix A. Units are for all four quarters. 
WELL: TBG 5

\section{ANALYTICAL DATA}

\section{Analyte}

$\mathrm{N}$-Nitrosomethylethylamine

$\mathrm{N}$-Nitrosomorpholine

$\mathrm{N}$-Nitrosopiperidine

$\mathrm{N}$-Nitrosopyrrolidine

Naphthalene

Nickel, total recoverable

Nitrobenzene

O,O,O-Triethyl phosphorothioate

PCB 1016

PCB 1221

PCB 1232

PCB 1242

PCB 1248

PCB 1254

PCB 1260

Parathion

Parathion methyl

Pentachlorodibenzo-p-dioxins

Pentachlorodibenzo-p-furans

Pentachloroethane

Pentachloronitrobenzene

Pentachlorophenol

Phenacetin

Phenanthrene

Phenol

Phorate

Pronamid

Propionitrile

Pyrene

Pyridine

Safrole

Selenium, total recoverable

Silver, total recoverable

Styrene

Sulfide

Sulfotepp

Tetrachlorodibenzo-p-dioxins

Tetrachlorodibenzo-p-furans

Thallium, total recoverable

Thionazin

Tin, total recoverable

Toluene

Total organic halogens

\section{Mod 201998 Mod 301998 Mod \\ 101998 Mod 201998 Mod 301998 Mod}

401998 Mod Unit

$<10$ U ug/L

$<10 \quad \mathrm{U} \quad \mathrm{ug} / \mathrm{L}$

$<10 \quad$ U ug/L

$<10 \quad$ U $\quad$ ug/L

$<20 \quad \mathrm{U} \quad<10 \quad$ JU $Q \quad$ ug $/ \mathrm{L}$

$<50$ U ug/L

$<10 \quad \mathrm{U} \quad<10 \quad \mathrm{JU} Q \quad \mathrm{ug} / \mathrm{L}$

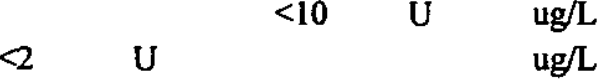

$<2$ U

$<1 \quad$ U

$<1 \quad$ U

$<1 \quad$ U

$<1 \quad \mathrm{U}$

$<1 \quad \mathrm{U}$

ug/L

ug/L

ug/L

ug/L

$\mathrm{ug} / \mathrm{L}$

$<10 \quad \mathrm{U} \quad \mathrm{ug} / \mathrm{L}$

$<.00094 \mathrm{U}$

$<10$

U

$<.0006 \mathrm{U}$

$<2000$ U

$<25 \quad \mathrm{U}$

$<10 \quad \mathrm{U}$

$<10 \quad \mathrm{U}$

$$
<10
$$

$<25$

$<10$

J

JU Q

$\mathrm{U}$

$<10$

JU

$<10$

JU $\mathrm{Q}$

$<10$

$\mathrm{U}$

$<10$

U

$<5000 \quad \mathrm{U}$

$<10 \quad \mathrm{U}$

$<10$

$<25$

$<10$

JU $Q$

U

$<10 \quad \mathrm{U}$

$<20 \quad \mathrm{U}$

$<50 \quad \mathrm{U}$

$<1000 \quad \mathrm{U}$

$u g / \mathrm{L}$

ug/L

ug/L

ug/L

$<10 \quad \mathrm{U} \quad$ ug/L

$<.0003 \mathrm{U}$

$<.00043 \mathrm{U}$

$<10 \quad \mathrm{U}$

$<200 \quad$ U

$<50 \quad \mathrm{U}$

ug/L

$\mathrm{ug} / \mathrm{L}$

$u g / L$

$<10 \quad \mathrm{U} \quad \mathrm{ug} / \mathrm{L}$

ug $/ \mathrm{L}$

ug $/ \mathrm{L}$

747

$\mathrm{ug} / \mathrm{L}$

Note: Concentrations in bold exceed the Drinking Water Standards listed in Appendix A. Units are for all four quarters. 
WSRC-RP-99-4003

Unclassified

WELL: TBG 5

ANALYTICAL DATA

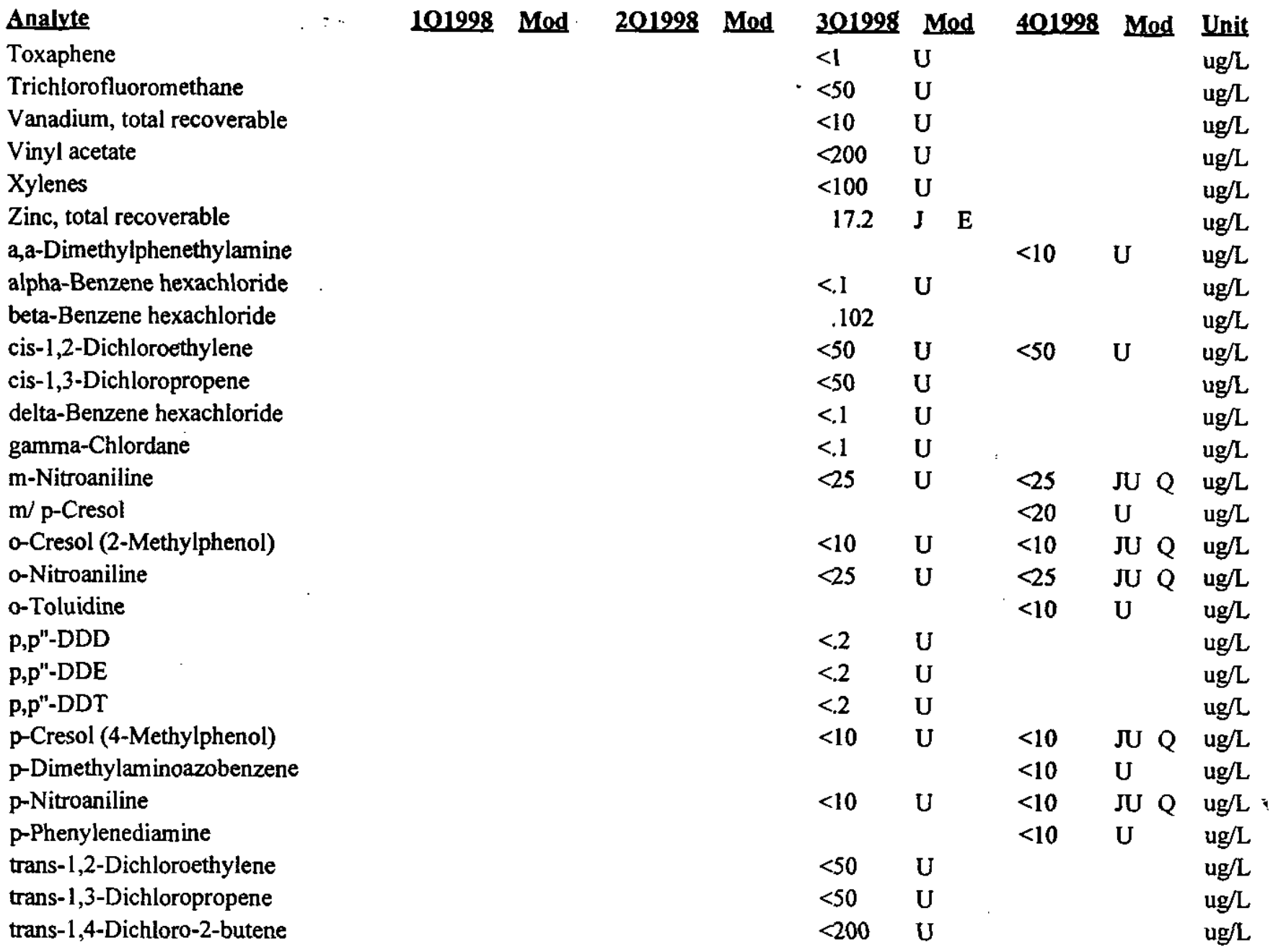

Note: Concentrations in bold exceed the Drinking Water Standards listed in Appendix A. Units are for all four quarters. 
WELL: TBG 5A

SRS Coord Lat/Longitude Screen Zone Elevation Top of Standpipe Top of Casing Casing Pump Screen Zone N $71206.8 \quad 33.212$ Deg N $80.0-70.0 \mathrm{ft} \mathrm{msl} \quad 150.2 \mathrm{ft} \mathrm{msl}$ $150.0 \mathrm{ft} \mathrm{ms}$ 4 " STL $\mathrm{S}$ Unconfined E 17348.8

SAMPLE DATE

03/02/98

$05 / 13 / 98$

$08 / 05 / 98$

$12 / 04 / 98$

\section{FIELD DATA}

\section{Analyte}

Depth to water

$\mathrm{pH}$

Sp. Conductance

Water temperature

Alkalinity as $\mathrm{CaCO} 3$

Phenolpthalein Alkalinity

Turbidity

Volumes purged

Sampling codes

$\begin{array}{ll}101998 & 201998 \\ 44.83 & 43.45 \\ 5.5 & 5.2 \\ 29 & 28 \\ 22 & 17 \\ 4 & 3 \\ 0 & 0 \\ .3 & 2.5 \\ 2.55727 & 4.04558\end{array}$

301998
46.7
5.4
28
22
5
0
.8
5.72219

\section{$\underline{401998}$}

48.9

4.9

29

22

2

0

.9

3.48012
Unit ft BTOS

$\mathrm{uS} / \mathrm{cm}$

deg. C

$\mathrm{mg} / \mathrm{L}$

$\mathrm{mg} / \mathrm{L}$

NTU

gallons

\section{ANALYTICAL DATA}

\section{Analyte}

1,1,1,2-Tetrachloroethane

1,1,1-Trichloroethane

1,1,2,2-Tetrachloroethane

1,1,2-Trichloroethane

1,1-Dichloroethane

1,1-Dichloroethylene

1,2,3-Trichloropropane

1,2,4-Trichlorobenzene

1,2-Dibromo-3-chloropropane

1,2-Dibromoethane

1,2-Dichlorobenzene

1,2-Dichloroethane

1,2-Dichloropropane

1,3,5-Trinitrobenzene

1,3-Dichlorobenzene

1,3-Dinitrobenzene

1,4-Dichlorobenzene

1,4-Dioxane

1,4-Naphthoquinone

1-Naphthylamine

2,2-Oxybis(1-chloropropane)

2,3,4,6-Tetrachlorophenol

2,3,7,8-TCDD

2,4,5-T

2,4,5-TP (Silvex)

2,4,5-Trichlorophenol

2,4,6-Trichlorophenol

\begin{tabular}{|c|c|c|c|c|c|c|c|c|}
\hline 101998 & Mod & 201998 & Mod & 301998 & Mod & 401998 & Mod & Unit \\
\hline & & & & $<5$ & $\mathrm{U}$ & & & $\mathrm{ug} / \mathrm{L}$ \\
\hline$<.462$ & UJ $O$ & $<.462$ & $\mathrm{U}$ & $<5$ & $\mathrm{U}$ & $<1$ & $\mathrm{U}$ & $\mathrm{ug} / \mathrm{L}$ \\
\hline & & & & $<5$ & $\mathrm{U}$ & & & $\mathrm{ug} / \mathrm{L}$ \\
\hline & & & & $<5$ & $\mathrm{U}$ & & & $\mathrm{ug} / \mathrm{L}$ \\
\hline & & & & $<5$ & $\mathrm{U}$ & & & ug/L \\
\hline & & & & $<5$ & $\mathrm{U}$ & & & $\mathrm{ug} / \mathrm{L}$ \\
\hline & & & & $<5$ & U & & & $\mathrm{ug} / \mathrm{L}$ \\
\hline & & & & $<10$ & $\mathrm{U}$ & $<10$ & JU Q & $\mathrm{ug} / \mathrm{L}$ \\
\hline & & & & $<5$ & $\mathrm{U}$ & & & $\mathrm{ug} / \mathrm{L}$ \\
\hline & & & & $<5$ & $\mathbf{U}$ & & & ug/L \\
\hline & & & & $<5$ & $\mathrm{U}$ & & & $\mathrm{ug} / \mathrm{L}$ \\
\hline & & & & $<5$ & $\mathbf{U}$ & & & $\mathrm{ug} / \mathrm{L}$ \\
\hline & & & & $<5$ & $\mathrm{U}$ & & & ug/L \\
\hline & & & & & & $<10$ & U & $\mathrm{ug} / \mathrm{L}$ \\
\hline & & & & $<5$ & $\mathrm{U}$ & & & $\mathrm{ug} / \mathrm{L}$ \\
\hline & & & & & & $<10$ & U & $\mathrm{ug} / \mathrm{L}$ \\
\hline & & & & $<5$ & $\mathbf{U}$ & & & $\mathrm{ug} / \mathrm{L}$ \\
\hline & & & & $<1000$ & $\mathrm{U}$ & & & $\mathrm{ug} / \mathrm{L}$ \\
\hline & & & & & & $<10$ & $U$ & $\mathrm{ug} / \mathrm{L}$ \\
\hline & & & & & & $<10$ & $\mathbf{U}$ & $\mathrm{ug} / \mathrm{L}$ \\
\hline & & & & $<10$ & $\mathrm{U}$ & $<10$ & JU Q & $\mathrm{ug} / \mathrm{L}$ \\
\hline & & & & & & $<10$ & $\mathrm{U}$ & $\mathrm{ug} / \mathrm{L}$ \\
\hline & & & & $<.00036$ & $\mathrm{U}$ & & & $\mathrm{ug} / \mathrm{L}$ \\
\hline & & & & $<.2$ & UJ O & & & $\mathrm{ug} / \mathrm{L}$ \\
\hline & & & & $<.2$ & UJ $\mathrm{O}$ & & & $\mathrm{ug} / \mathrm{L}$ \\
\hline & & & & $<10$ & $\mathrm{U}$ & $<10$ & JU Q & $\mathrm{ug} / \mathrm{L}$ \\
\hline & & & & $<25$ & $\mathrm{U}$ & $<25$ & $\mathrm{JU} Q$ & $\mathrm{ug} / \mathrm{L}$ \\
\hline
\end{tabular}

Note: Concentrations in boid exceed the Drinking Water Standards listed in Appendix A. Units are for all four quarters. 
WELL: TBG 5A

ANALYTICAL DATA

Analyte

2,4-Dichlorophenol

2,4-Dichlorophenoxyacetic acid

2,4-Dimethyl phenol

2,4-Dinitrophenol

2,4-Dinitrotoluene

2,6-Dichlorophenol

2,6-Dinitrotoluene

2-Acetylaminofluorene

2-Chloronaphthalene

2-Chlorophenol

2-Hexanone

2-Methyl-4,6-dinitrophenol

2-Methylnaphthalene

2-Naphthylamine

2-Nitrophenol

2-Picoline

2-sec-Butyl-4,6-dinitrophenol

3,3"-Dichlorobenzidine

3,3"-Dimethylbenzidine

3-Methylcholanthrene

4-Aminobiphenyl

4-Bromophenyl phenyl ether

4-Chloro-m-cresol

4-Chloroaniline

4-Chlorophenyl phenyl ether

4-Nitrophenol

4-Nitroquinoline-1-oxide

5-Nitro-o-toluidine

7,12-Dimethylbenz(a)anthracene

Acenaphthene

Acenaphthylene

Acetone

Acetonitrile (Methyl cyanide)

Acetophenone

Acrolein

Acrylonitrile

Aldrin

Allyl chloride

Aluminum, total recoverable

Aniline

Anthracene

Antimony, total recoverable

Aramite
101998 Mod 201998 Mod 301998 Mod 401998 Mod Unit

$\begin{array}{llllll}<10 & \mathrm{U} & <10 & \text { JU } & \mathrm{Q} & \mathrm{ug} / \mathrm{L}\end{array}$

$<.2$ UJ $\mathrm{O} \quad \mathrm{ug} / \mathrm{L}$

$<10 \quad \mathrm{U} \quad<10 \quad$ JU $\mathrm{Q} \quad$ ug/L

$<25 \quad \mathrm{U} \quad<25 \quad$ J $\mathrm{Q} \quad \mathrm{ug} / \mathrm{L}$

$<10 \quad \mathrm{U} \quad<10 \quad$ JU $Q \quad$ ug/L

$<10 \quad \mathrm{U} \quad \mathrm{ug} / \mathrm{L}$

$<10 \quad \mathrm{U} \quad<10 \quad \mathrm{JU} \quad \mathrm{Q} \quad \mathrm{ug} / \mathrm{L}$

$<10 \quad \mathrm{U} \quad$ ug/L

$\begin{array}{llllll}<10 & \mathrm{U} & <10 & \mathrm{JU} & \mathrm{Q} & \mathrm{ug} / \mathrm{L}\end{array}$

$<10 \quad \mathrm{U} \quad<10 \quad$ JU $\mathrm{Q} \quad \mathrm{ug} / \mathrm{L}$

$<5 \quad \mathrm{U} \quad$ ug/L

$<25 \quad$ U $\quad<25 \quad$ JU $Q \quad$ ug/L

$<10 \quad \mathrm{U}:<10 \quad$ JU $\mathrm{Q} \quad \mathrm{ug} / \mathrm{L}$

$<10 \quad \mathrm{U} \quad$ ug/L

$<10 \quad \mathrm{U} \quad<10 \quad$ JU $Q \quad$ ug/L

$<10 \quad \mathrm{U} \quad \mathrm{ug} / \mathrm{L}$

$<10 \quad \mathrm{U} \quad \mathrm{ug} / \mathrm{L}$

$<10 \quad \mathrm{U} \quad<10 \quad \mathrm{JU} Q \quad \mathrm{ug} / \mathrm{L}$

$<20$ U ug/L

$<10 \quad \mathrm{U} \quad \mathrm{ug} / \mathrm{L}$

$<10 \quad \mathrm{U} \quad \mathrm{ug} / \mathrm{L}$

$<10 \quad$ U $\quad<10 \quad$ JU Q $\quad$ ug/L

$<10 \quad \mathrm{U} \quad<10 \quad \mathrm{JU} \quad \mathrm{Q} \quad \mathrm{ug} / \mathrm{L}$

$<10 \quad \mathrm{U} \quad<10 \quad$ JU $\mathrm{Q} \quad \mathrm{ug} / \mathrm{L}$

$<10 \quad \mathrm{U} \quad<10 \quad \pi \mathrm{U} Q \quad \mathrm{ug} / \mathrm{L}$

$<25$ U $<25$ JU $Q \quad$ ug/

$<50$ U ug/L

$<10 \quad \mathrm{U} \quad$ ug/L

$<10 \quad \mathrm{U} \quad \mathrm{ug} / \mathrm{L}$

$<10 \quad \mathrm{U} \quad<10 \quad$ JU $Q \quad$ ug/L

$<10 \quad \mathrm{U} \quad<10 \quad$ JU $\mathrm{Q} \quad \mathrm{ug} / \mathrm{L}$

$<20$ U ug/L

$<500$ U ' ug/L

$<10 \quad \mathrm{U} \quad \mathrm{ug} / \mathrm{L}$

$<50$ U ug/L

$<50$ U ug/L

$<.1 \quad \mathrm{U}$ ug/L

$<10 \quad \mathrm{U} \quad \mathrm{ug} / \mathrm{L}$

$\begin{array}{llllllll}7.5 & \text { J } & \text { E } & <200 & \text { U } & <200 & \text { U } & \text { ug } / \mathrm{L}\end{array}$

$<25$ U $<25$ JU Q ug/L

$<10 \quad \mathrm{U} \quad<10 \quad \mathrm{JU} Q \quad \mathrm{ug} / \mathrm{L}$

$<100 \quad \mathrm{u} \quad \mathrm{ug} / \mathrm{L}$

$<10 \quad U \quad u g / L$

Note: Concentrations in bold exceed the Drinking Water Standards listed in Appendix A. Units are for all four quarters. 
WELL: TBG 5A

ANALYTICAL DATA

Analute

Arsenic, total recoverable

Barium, total recoverable

Benzene

Benzo(a)anthracene

Benzo(a)pyrene

Benzo(b)fluoranthene

Benzo(g,h,i)perylene

Benzo(k)fluoranthene

Benzyl alcohol

Beryllium, total recoverable

Bis(2-chloroethoxy) methane

Bis(2-chloroethyl) ether

Bis(2-ethylhexyl) phthalate

Bromodichloromethane

Bromoform

Bromomethane (Methyl bromide)

Butylbenzyl phthalate

Cadmium, total recoverable

Carbazole

Carbon disulfide

Chlorobenzene

Chlorobenzilate

Chloroethane

Chloroethene (Vinyl chloride)

Chloromethane (Methyl chloride)

Chloroprene

Chromium, total recoverable

Chrysene

Cobalt, total recoverable

Copper, total recoverable

Cyanide

Di-n-butyl phthalate

Di-n-octyl phthalate

Diallate

Dibenz(a,h)anthracene

Dibenzofuran

Dibromochloromethane

Dibromomethane (Methylene bromide),

Dichlorodifluoromethane

Dichloromethane (Methylene chloride)

Dieldrin

Diethyl phthalate

Dimethoate

\section{Mod 201998 Mod}

$\frac{301998}{<10} \mathrm{M}$

$<10 \quad \mathrm{U}$

$<5 \quad \mathrm{U}$

$<10$ U

$<10$ U

$<10$ U

$<10 \quad \mathrm{U}$

$<25 \quad$ U

$<10$ U

$<10$ U

$<10 \quad \mathrm{U}$

$<10 \quad$ U

$<10$ U

$<5$

$<5$

$<5$

$<20$

3.11

$<10$

$<5$

$<5$

$<10$

$<5$

$<5$

$<50$

$<10$

$<10$

7.09

8.07

$<10$

$<10$

$<20$

$<10$

$<10$

$<5$

$<5$

$<5$

$<10$

$<.2$

$<10$
401998 Mod Unit

ug/L

ug/L

$u g / L$

$<10$

$<10$

$<10$

$<10$

$<10$

$<10$

J $Q \quad$ ug/L

JU $Q$ ug/L

JU $Q \quad u g / L$

JU $Q \quad u g / L$

JU $Q \quad u g / L$

J̦ $Q \quad u g / L$

$u g / L$

$<10 \quad J U$ Q ug/L

$<10$ JU Q ug/L

$<10$ JU Q ug/L

ug/L

$\mathrm{ug} / \mathrm{L}$

ug/L

$<10$

JU $Q \quad$ ug/L

ug/L

$\mathrm{ug} / \mathrm{L}$

$\mathrm{ug} / \mathrm{L}$

ug/L

$<10 \mathrm{U} \quad \mathrm{ug} / \mathrm{L}$

ug/L

$\mathrm{ug} / \mathrm{L}$

$u g / L$

$\mathrm{ug} / \mathrm{L}$

$\mathrm{ug} / \mathrm{L}$

$<10 \quad$ JU Q ug/L

$\mathrm{ug} / \mathrm{L}$

$\mathrm{ug} / \mathrm{L}$

ug/L

$<10$ JU $Q \quad$ ug/L

$<10$ JU Q ug/L

$<10$ U ug/L

$<10$ JU $Q$ ug/L

$<10$

JU. $Q$

ug/L

ug/L

$\mathrm{ug} / \mathrm{L}$

$\mathrm{ug} / \mathrm{L}$

$\mathrm{ug} / \mathrm{L}$

$\mathrm{ug} / \mathrm{L}$

$<10$ JU Q ug/L

$<10$ U ug/L

Note: Concentrations in bold exceed the Drinking Water Standards listed in Appendix A. Units are for all four quarters. 
WELL: TBG 5A

\section{ANALYTICAL DATA}

\section{Analyte}

Dimethyl phthalate

Diphenylamine

Disulfoton

Endosulfan I

Endosulfan II

Endosulfan sulfate

Endrin

Endrin aldehyde

Ethyl methacrylate

Ethyl methanesulfonate

Ethylbenzene

Fluoranthene

Fluorene

Heptachlor

Heptachlor epoxide

Hexachlorobenzene

Hexachlorobutadiene

Hexachlorocyclopentadiene

Hexachlorodibenzo-p-dioxins

Hexachlorodibenzo-p-furans

Hexachloroethane

Indeno(1,2,3-c,d)pyrene

Iodomethane (Methyl iodide)

Iron, total recoverable

Isobutyl alcohol

Isodrin

Isophorone

Isosafrole

Kepone

Lindane

Methacrylonitrile

Methapyrilene

Methoxychlor

Methyl ethyl ketone

Methyl isobutyl ketone

Methyl methacrylate

Methyl methanesulfonate

$\mathrm{N}$-Nitrosodi-n-butylamine

$\mathrm{N}$-Nitrosodiethylamine

$\mathrm{N}$-Nitrosodimethylamine

$\mathrm{N}-\mathrm{Nitrosodiphenylamine}$

$\mathrm{N}$-Nitrosodipropylamine

$\mathrm{N}$-Nitrosomethylethylamine
101998 Mod 201998 Mod 301998 Mod 401998 Mod Unit $<10 \quad \mathrm{U} \quad<10 \quad$ JU $\mathrm{Q} \quad \mathrm{ug} / \mathrm{L}$

$<10 \quad \mathrm{U} \quad \mathrm{ug} / \mathrm{L}$

$<10 \quad \mathrm{U} \quad \mathrm{ug} / \mathrm{L}$

$<.1 \quad \mathrm{U}$

$<.2 \quad \mathrm{U}$

$<.2 \quad \mathrm{U}$

$<.2 \quad \mathrm{U}$

$<.2 \quad \mathrm{U}$

$<5 \quad \mathrm{U}$

$<10 \quad U$

$<5 \quad \mathrm{U}$

$<10$ U

$<10 \quad \mathrm{U}$

$<.1 \quad \mathrm{U}$

$<.1 \quad$ U

$<20$ U

$<10 \quad \mathrm{U}$

$<10 \quad \mathrm{U}$

$<.00103 \mathrm{U}$

$<.00055 \mathrm{U}$

$<10 \quad \mathrm{U}$

$<20$ U

$<5 \quad \mathrm{U}$

43.2

$23.8 \mathrm{~J}$

$<1500 \quad \mathrm{U}$

$<10 \quad \mathrm{U}$

U

$<.1 \quad \mathrm{U}$

$<500 \quad \mathrm{U}$

$<1 \quad$ U

$<10 \quad \mathrm{U}$

$<5 \quad U$

$<50^{\circ}$

U

$<10$

$<20$

$<20$
ug/L

$<10 \quad$ JU Q ug/L

$<10 \quad$ JU $Q \quad$ ug/L

$\mathrm{ug} / \mathrm{L}$

$\mathrm{ug} / \mathrm{L}$

$<10 \quad$ U $Q \quad$ ug/L

$<10 \quad$ JU $Q \quad$ ug/L

$<10 \quad$ JU $Q \quad$ ug/L

ug/L

$\mathrm{ug} / \mathrm{L}$

$<10$ JU Q ug/L

$<10 \quad$ JU $Q \quad$ ug/L

$\mathrm{ug} / \mathrm{L}$

$<22.1$ U ug/L :

ug/L

$<10 \quad$ U $\quad$ ug/L

$<10$ JU Q ug/L

$<10 \quad \mathrm{U} \quad \mathrm{ug} / \mathrm{L}$

$<10$ U ug/L

ug/L

$\mathrm{ug} / \mathrm{L}$

$<10 \quad \mathrm{U} \quad \mathrm{ug} / \mathrm{L}$

$u g / L$

$\mathrm{ug} / \mathrm{L}$

ug/L

ug/L

$<10$ U ug/L

$<10 \quad$ U ug/L

$<10$ U ug/L

$<25$ JU Q ug/L

$<10 \quad$ JU $Q$ ug/L

$<10 \quad$ JU $Q \quad$ ug/L

$<10 \quad \mathrm{U} \quad \mathrm{ug} / \mathrm{L}$

Note: Concentrations in bold exceed the Driaking Water Standards listed in Appendix A. Units are for all four quarters. 
WELL: TBG 5A

ANALYTICAL DATA

\section{Analyte}

$\mathrm{N}$-Nitrosomorpholine

$\mathrm{N}$-Nitrosopiperidine

N-Nitrosopyrrolidine

Naphthalene

Nickel, total recoverable

Nitrobenzene

O,O,O-Triethyl phosphorothioate

PCB 1016

PCB 1221

PCB 1232

PCB 1242

PCB 1248

PCB 1254

PCB 1260

Parathion

Parathion methyl

Pentachlorodibenzo-p-dioxins

Pentachlorodibenzo-p-furans

Pentachloroethane

Pentachloronitrobenzene

Pentachlorophenol

Phenacetin

Phenanthrene

Phenol

Phorate

Pronamid

Propionitrile

Pyrene

Pyridine

Safrole

Selenium, total recoverable

Silver, total recoverable

Styrene

Sulfide

Sulfotepp

Tetrachlorodibenzo-p-dioxins

Tetrachlorodibenzo-p-furans

Thallium, total recoverable

Thionazin

Tin, total recoverable

Toluene

Toxaphene

Trichlorofluoromethane

\section{Mod \\ 201998 Mod \\ 301998 Mod}

401998 Mod Unit

$<10 \quad \mathrm{U} \quad \mathrm{ug} / \mathrm{L}$

$<10$ U ug/L

$<10 \quad \mathrm{u} \quad \mathrm{ug} / \mathrm{L}$

$<10 \quad \mathrm{U}$

$<10$

JU $Q$

ug/L

$<50 \quad \mathrm{U}$

$<25 \quad$ U

$<10$

ug/L

$<10$ U ug/L

$<\quad U$

$<$ U

$<1 \quad$ U

$<1 \quad$ U

$<1 \quad$ U

$<1 \quad U$

$<1 \quad \mathrm{U}$

$<.0009 \mathrm{U}$

$<.00057 \mathrm{U}$

$<200$

$\mathrm{U}$

$<10 \quad \mathrm{U}$

$<10$

$<10$

U

U

ug/L

ug $/ \mathrm{L}$

$\mathrm{ug} / \mathrm{L}$

ug/L

$\mathrm{ug} / \mathrm{L}$

ug/L

$\mathrm{ug} / \mathrm{L}$

ug/L

ug/L

ug/L

ug/L

ug/L

$<10 \quad 0$

$<10 \quad U$

$<10 \quad \mathrm{U}$

$<10$

$<10$

$<10$

$<10$

$<10$

$<500 \quad \mathrm{U}$

$<25 \quad U$

$<10$

$<25$

$<10$

$<10$

U

$<20$ U

$<5 \quad U$

$<1000 \mathrm{U}$

$<10 \quad U$

$<.00036 \mathrm{U}$

$<.00047 \mathrm{U}$

$<10$

U

$<200 \quad$ U

$<5 \quad$ U

$<1 \quad$ U

$<5 \quad U$

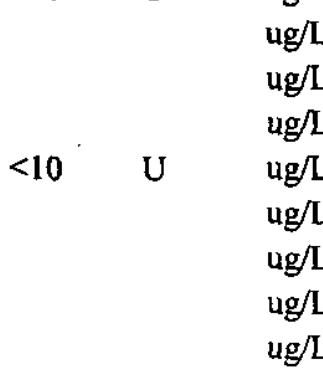

Note: Concentrations in bold exceed the Drinking Water Standards listed in Appendix A. Units are for all four quarters, 
WELL: TBG 5A

ANALYTICAL DATA

Analvte

Vanadium, total recoverable

Vinyl acetate

Xylenes

Zinc, total recoverable

a,a-Dimethylphenethylamine

alpha-Benzene hexachloride

alpha-Chlordane

beta-Benzene hexachloride

cis-1,2-Dichloroethylene

cis-1,3-Dichloropropene

delta-Benzene hexachloride

gamma-Chlordane

m-Nitroaniline

$\mathrm{m} / \mathrm{p}$-Cresol

o-Cresol (2-Methylphenol)

o-Nitroaniline

o-Toluidine

p,p"-DDD

P,P"-DDE

p,p"-DDT

p-Cresol (4-Methylphenol)

p-Dimethylaminoazobenzene

p-Nitroaniline

p-Phenylenediamine

trans-1,2-Dichloroethylene

trans-1,3-Dichloropropene

trans-1,4-Dichloro-2-butene
101998 Mod 201998 Mod

301998 Mod

$<10$

U

$<20$ U

$<10 \quad \mathrm{U}$

$10.6 \mathrm{~J} \quad \mathrm{E}$

$<.1 \quad$ U

$<.1 \quad \mathrm{U}$

$<.1 \quad \mathrm{U}$

$<5 \quad$ U

$<5 \quad$ U

$<.1 \quad \mathrm{U}$

$<.1 \quad \mathrm{U}$

$<25 \quad \mathrm{U}$

U $<25$

$<20$

$<10 \quad \mathrm{U}$

$<25$ U

$<10$

$<25$

$<10$

$<.2 \quad \mathrm{U}$

$<.2 \quad \mathrm{U}$

$<.2 \quad \mathrm{U}$

$<10 \quad \mathrm{U}$

$<10$

$<10$

$<10$

$<10$

$<5 \quad \mathrm{U}$

$<5 \quad U$

$<20 \quad \mathrm{U}$
Mod Unit

ug/L

$\mathrm{ug} / \mathrm{L}$

ug/L

$\mathrm{ug} / \mathrm{L}$

ug/L

ug/L

ug/L

ug/L

$\mathrm{ug} / \mathrm{L}$

$u g / L$

$\mathrm{ug} / \mathrm{L}$

$\mathrm{ug} / \mathrm{L}$

JU $Q \quad u g / L$

U ug/L

$\pi \mathrm{Q}$ ug/

JU $Q \quad u g / L$

U ug/L

$\mathrm{ug} / \mathrm{L}$

$\mathrm{ug} / \mathrm{L}$

$\mathrm{ug} / \mathrm{L}$

JU Q ug/L

U ug/L

JU $Q \quad u g / L$

U ug/L :

ug/L

$\mathrm{ug} / \mathrm{L}$

$\mathrm{ug} / \mathrm{L}$

Note: Concentrations in bold exceed the Drinking Water Standards listed in Appendix A. Units are for all four quarters. 
WELL: TBG 5B

SRS Coord. Lat/Longitude Screen Zone Elevation Top of Standpipe Top of Casing Casing Pump Screen Zone N $71216.8 \quad 33.212$ Deg N 56.2 - 46.2 ft msl $149.6 \mathrm{ft} \mathrm{msl}$

$149.4 \mathrm{ft} \mathrm{msl} 4$ " STL

E 17354.8 81.759 Deg W.

$03 / 03 / 98 \quad 05 / 15 / 98 \quad 08 / 05 / 98$

$12 / 04 / 98$

SAMPLE DATE

$03 / 03 / 98$

$05 / 15 / 98$

$\begin{array}{ll}\mathbf{2 0 1 9 9 8} & \mathbf{3 0 1 9 9 8} \\ 32.5 & 38.2 \\ 4.9 & 5 \\ 35 & 32 \\ 18.9 & 22.9 \\ 0 & 3 \\ 0 & 0 \\ 2.5 & 1.3 \\ 2.75986 & 3.37711\end{array}$

\begin{tabular}{|c|c|}
\hline 401998 & Unit \\
\hline $\begin{array}{l}37.55 \\
4.6\end{array}$ & ft BTOS \\
\hline 32 & $\mathrm{uS} / \mathrm{cm}$ \\
\hline 22 & deg. C \\
\hline 1 & $\mathrm{mg} / \mathrm{L}$ \\
\hline 0 & $\mathrm{mg} / \mathrm{L}$ \\
\hline 2.2 & NTU \\
\hline 2.64708 & gallons \\
\hline
\end{tabular}

Analyte

Depth to water

$\mathrm{pH}$

Sp. Conductance

Water temperature

Alkalinity as $\mathrm{CaCO} 3$

Phenolpthalein Alkalinity

Turbidity

Volumes purged

Sampling codes

101998
32.58
6.4
32
20.8
4
0
.8
2.50395

\section{ANALYTICAL DATA}

\section{Analyte}

1,1,1,2-Tetrachloroethane

1,1,1-Trichloroethane

1,1,2,2-Tetrachloroethane

1,1,2-Trichloroethane

1,1-Dichloroethane

1,1-Dichloroethylene

1,2,3-Trichloropropane

1,2,4-Trichlorobenzene

1,2-Dibromo-3-chloropropane

1,2-Dibromoethane

1,2-Dichlorobenzene

1,2-Dichloroethane

1,2-Dichloropropane

1,3,5-Trinitrobenzene

1,3-Dichlorobenzene

1,3-Dinitrobenzene

1,4-Dichlorobenzene

1,4-Dioxane

1,4-Naphthoquinone

1-Naphthylamine

2,2-Oxybis(1-chloropropane)

2,3,4,6-Tetrachlorophenol

2,3,7,8-TCDD

$2,4,5-\mathrm{T}$

2,4,5-TP (Silvex)

2,4,5-Trichlorophenol

2,4,6-Trichlorophenol
101998 Mod 201998 Mod 301998 Mod 401998 Mod Unit

$<5 \quad \mathrm{U} \quad \mathrm{ug} / \mathrm{L}$

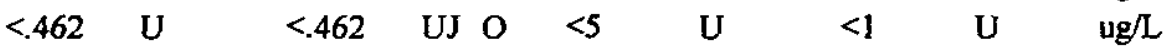

$<5 \quad \mathrm{U}$

$<5 \quad \mathrm{U}$

$<5 \quad \mathrm{U}$

$<5 \quad U$

$<5 \quad U$

$<10 \quad \mathrm{U}$

$<5 \quad \mathrm{U}$

$<5 \quad \mathrm{U}$

$<5 \quad \mathrm{U}$

$<5 \quad U$

$<5 \quad U$

$<5 \quad U$

$<5 \quad U$

$<1000 \mathrm{U}$

$<10$

JU Q

ug $/ \mathrm{L}$

$\mathrm{ug} / \mathrm{L}$

$\mathrm{ug} / \mathrm{L}$

$\mathrm{ug} / \mathrm{L}$

$\mathrm{ug} / \mathrm{L}$

ug/

$\mathrm{ug} / \mathrm{L}$

$\mathrm{ug} / \mathrm{L}$

$\mathrm{ug} / \mathrm{L}$

$\mathrm{ug} / \mathrm{L}$

ug/L

$<10 \quad \mathrm{U} \quad$ ug/L

ug/L

$<10 \quad \mathrm{U} \quad \mathrm{ug} / \mathrm{L}$

$\mathrm{ug} / \mathrm{L}$

$\mathrm{ug} / \mathrm{L}$

$<10 \quad \mathrm{U} \quad \mathrm{ug} / \mathrm{L}$

$<10 \quad \mathrm{U} \quad u g / \mathrm{L}$

$\begin{array}{llllll}<10 & \mathrm{U} & <10 & \mathrm{JU} & \mathrm{Q} & \mathrm{ug} / \mathrm{L}\end{array}$

$<10 \quad \mathrm{U} \quad \mathrm{ug} / \mathrm{L}$

$<.00053 \mathrm{U} \quad \mathrm{ug} / \mathrm{L}$

$<.2$ UJ $\mathrm{O} \quad \mathrm{ug} / \mathrm{L}$

$<.2$ UJ $\mathrm{O} \quad \mathrm{ug} / \mathrm{L}$

$<10 \quad \mathrm{U} \quad<10 \quad \mathrm{JU} \quad \mathrm{Q} \quad \mathrm{ug} / \mathrm{L}$

$<25 \quad \mathrm{U} \quad<25 \quad$ JU $Q \quad \mathrm{ug} / \mathrm{L}$

Note: Concentrations in bold exceed the Drinking Water Standards listed in Appendix A. Units are for all four quarters. 
WELL: TBG 5B

ANALYTICAL DATA

\section{Analyte}

2,4-Dichlorophenol

2,4-Dichlorophenoxyacetic acid

2,4-Dimethyl phenol

2,4-Dinitrophenol

2,4-Dinitrotoluene

2,6-Dichlorophenol

2,6-Dinitrotoluene

2-Acetylaminofluorene

2-Chloronaphthalene

2-Chlorophenol

2-Hexanone

2-Methyl-4,6-dinitrophenol

2-Methylnaphthalene

2-Naphthylamine

2-Nitrophenol

2-Picoline

2-sec-Butyl-4,6-dinitrophenol

3,3"-Dichlorobenzidine

3,3"-Dimethylbenzidine

3-Methylcholanthrene

4-Aminobiphenyl

4-Bromophenyl phenyl ether

4-Chloro-m-cresol

4-Chloroaniline

4-Chlorophenyl phenyl ether

4-Nitrophenol

4-Nitroquinoline-1-oxide

5-Nitro-o-toluidine

7,12-Dimethylbenz(a)anthracene

Acenaphthene

Acenaphthylene

Acetone

Acetonitrile (Methyl cyanide)

Acetophenone

Acrolein

Acrylonitrile

Aldrin

Allyl chloride

Aluminum, total recoverable

Aniline

Anthracene

Antimony, total recoverable

Aramite

\section{$201998 \mathrm{Mod}$}

$301998 \mathrm{Mo}$

$<10$

$$
<.2
$$$$
<10
$$$$
<25
$$$$
<10
$$$$
<1
$$$$
<10
$$$$
<10
$$$$
<5
$$$$
<2
$$$$
<10
$$$$
<
$$$$
\text { U }
$$$$
<10 \quad U
$$

401998 Mod Unit

$<10 \quad \mathrm{JU}$ Q ug/L

ug/L

$<10 \quad$ JU $Q \quad u g / L$

$<25$ JU Q ug/L

$<10$ JU $Q \quad$ ugh

$<10 \quad \mathrm{u} \quad \mathrm{ug} / \mathrm{L}$

$<10$ JU Q ug/L

$<10$ U ug/L

$<10$ JU Q ug/L

$<10$ JU Q ug/L

ug/L

$<25$ JU Q ug/L

$<10 \quad$ JU $Q \quad$ ug/L

$<10 \quad \mathrm{U} \quad \mathrm{ug} / \mathrm{L}$

$<10$ JU Q ug/L

$<10$ U ug/L

$<10$ U ug/L

$<10$ JU Q ug/L

$<20 \quad$ U $\quad u g / L$

$<10$ U ug/L

$<10$ U ug/L

$<10$ U $\quad<10 \quad$ J $Q \quad$ ug/L

$<10 \quad \mathrm{U} \quad<10 \quad \mathrm{JU} \mathrm{Q} \quad \mathrm{ug} / \mathrm{L}$

$<10 \quad U \quad<10 \quad \pi \quad Q \quad$ ug $/ \mathrm{L}$;

$<10$ U $<10$ JU Q ug/L

$<25 \quad \mathrm{U} \quad<25 \quad \pi \mathrm{U} \quad \mathrm{ug} / \mathrm{L}$

$<50$ U ug/L

$<10$ U ug/L

$<10$ U ug/L

$<10 \quad \mathrm{U} \quad<10 \quad$ J Q $\mathrm{ug} / \mathrm{L}$

$<10 \quad U \quad<10 \quad J U \quad Q \quad$ ug/L

$<20$ U ug/L

$<500 \quad U$

$<10$ U $\quad$ ug/L

$<50$ U ug/L

$<50$ U ug/L

$<.1 \quad \mathrm{U} \quad \mathrm{ug} / \mathrm{L}$

$<10$ U ug/h

$\begin{array}{llllllll}<100 & \mathrm{U} & <200 & \mathrm{U} & 119 & \mathrm{~J} & \mathrm{I} & \mathrm{ug} / \mathrm{L}\end{array}$

$<25$ U $<25$ JU Q ug/L

$<10 \quad \mathrm{U} . \quad<10 \quad$ JU $\mathrm{Q} \quad \mathrm{ug} / \mathrm{L}$

$<100 \quad \mathrm{u} \quad \mathrm{ug} / \mathrm{L}$

$<10 \quad U \quad$ ug $/ \mathrm{L}$

Note: Concentrations in bold exceed the Drinking Water Standards listed in Appendix A. Units are for all four quarters. 
WELL: TBG 5B

ANALYTICAL DATA

Analyte

Arsenic, total recoverable

Barium, total recoverable

Benzene

Benzo(a)anthracene

Benzo(a)pyrene

Benzorb)fluoranthene

$\operatorname{Benzo}(\mathrm{g}, \mathrm{h}, \mathrm{i})$ perylene

Benzo(k)fluoranthene

Benzyl alcohol

Beryllium, total recoverable

Bis(2-chloroethoxy) methane

Bis(2-chloroethyl) ether

Bis(2-ethylhexyl) phthalate

Bromodichloromethane

Bromoform

Bromomethane (Methyl bromide)

Butylbenzyl phthalate

Cadmium, total recoverable

Carbazole

Carbon disulfide

Chlorobenzene

Chlorobenzilate

Chloroethane

Chloroethene (Vinyl chloride)

Chloromethane (Methyl chloride)

Chloroprene

Chromium, total recoverable

Chrysene

Cobalt, total recoverable

Copper, total recoverable

Cyanide

Di-n-butyl phthalate

Di-n-octyl phthalate

Diallate

Dibenz(a,h)anthracene

Dibenzofuran

Dibromochloromethane

Dibromomethane (Methylene bromide)

Dichlorodifluoromethane

Dichloromethane (Methylene chloride)

Dieldrin

Diethyl phthalate

Dimethoate
101998 Mod 201998 Mod 301998 Mod 401998 Mod Unit $<10 \quad \mathrm{U}$

$\mathrm{ug} / \mathrm{L}$

16.2

$<5 \quad U$

$<10 \quad U$

$<10 \quad U$

$<10 \quad U$

$<10$ U

$<25$ U

$<10 \quad U$

$<10 \quad \mathrm{U}$

$<10 \quad U$

$<10 \quad \mathrm{U}$

$<10 \quad \mathrm{U}$

$<5 \quad \mathrm{U}$

$<5 \quad U$

$<5 \quad$ U

$<20 \quad U$

$<10 \quad \mathrm{U}$

$<10 \quad \mathrm{U}$

$<5 \quad \mathrm{U}$

$<5$

$<10 \quad \mathrm{U}$

$<5 \quad U$

$<5 \quad U$

$<50 \quad \mathrm{U}$

$<10 \quad \mathrm{U}$

$<10 \quad \mathrm{U}$

$<20$ U

$<20$ U

$<10 \quad \mathrm{U}$

$<10 \quad U$

$<20$ U

$<10 \quad \mathrm{U}$

$<10 \quad U$

$<5$

$<5$

$<5$ U

$<10 \quad U$

$<.2$ U

$<10 \quad U$
$<10$

$$
<10
$$

$<10$

$<10$

$<10$

$<10$

$<10$

$<10$

$<10$

$<10$

$<10$

$<10$

$<10$

$<10$

$<10$

(

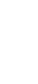

$<10$

JU $Q$

$\pi \quad \mathrm{Q} / \mathrm{L}$

$\pi \quad$ Q

JU $Q \quad u g / L$

$\pi$ Q ug/L

J $Q \quad u g / L$

JU $Q \quad u g / L$

$\mathrm{ug} / \mathrm{L}$

JU Q ug/L

JU Q ug/

JU $Q \quad u g / L$

ug/L

$\mathrm{ug} / \mathrm{L}$

ug/L

JU $Q \quad u g / L$

$\mathrm{ug} / \mathrm{L}$

ug/L

$u g / L$

ug/L

$u g / L$

ug/L

$\mathrm{ug} / \mathrm{L}$

$\mathrm{ug} / \mathrm{L}$

ug/L

ug/L

ug/L

$\mathrm{ug} / \mathrm{L}$

$\mathrm{ug} / \mathrm{L}$

ug/L

$\pi \quad \mathrm{Q} \quad \mathrm{ug} / \mathrm{L}$

JU $Q \quad u g / L$

U ug/L

JU $Q \quad$ ug/L

JU Q ug/L

ug/

$\operatorname{ug} / \mathrm{L}$

$\mathrm{ug} / \mathrm{L}$

ug/L

$\mathrm{ug} / \mathrm{L}$

$<10 \quad$ J $Q \quad$ ug/L

$<10$ U ug/L

Note: Concentrations in bold exceed the Drinking Water Standards listed in Appendix A. Units are for all four quarters.

TNX Area 
WELL: TBG 5B

ANALYTICAL DATA

\begin{tabular}{|c|c|c|c|c|c|c|c|c|}
\hline Analyte & 101998 & Mod 201998 & Mod & $\underline{\mathbf{3 0 1 9 9 8}}$ & Mod & 401998 & Mod & Unit \\
\hline Dimethyl phthalate & & & & $<10$ & $\mathrm{U}$ & $<10$ & JU Q & $\mathrm{ug} / \mathrm{L}$ \\
\hline Diphenylamine & & & & & & $<10$ & $\mathrm{U}$ & $\mathrm{ug} / \mathrm{L}$ \\
\hline Disulfoton & & & & & & $<10$ & $\mathrm{U}$ & $\mathrm{ug} / \mathrm{L}$ \\
\hline Endosulfan I & & & & $<.1$ & $U$ & & & $u g / L$ \\
\hline Endosulfan II & & & & $<.2$ & $\mathbf{U}$ & & & $\mathrm{ug} / \mathrm{L}$ \\
\hline Endosulfan sulfate & & & & $<.2$ & $\mathrm{U}$ & & & ug/L \\
\hline Endrin & & & & $<.2$ & $\mathbf{U}$ & & & $\mathrm{ug} / \mathrm{L}$ \\
\hline Endrin aldehyde & & & & $<.2$ & $\mathbf{U}$ & & & $u g / L$ \\
\hline Ethyl methacrylate & & & & $<5$ & $\mathbf{U}$ & . & & $\mathrm{ug} / \mathrm{L}$ \\
\hline Ethyl methanesulfonate & & & & & & $<10$ & $U$ & $\mathrm{ug} / \mathrm{L}$ \\
\hline Ethylbenzene & & & & $<5$ & $\mathrm{U}$ & & & ug/L \\
\hline Fluoranthene & & & & $<10$ & $\mathrm{U}$ & $<10$ & JU Q & $\mathrm{ug} / \mathrm{L}$ \\
\hline Fluorene & & & & $<10$ & $\mathrm{U}$ & $<10$ & $\mathrm{JU} Q$ & $\cdot u g / L$ \\
\hline Heptachlor & & & & $<.1$ & $\mathbf{U}$ & & & $\mathrm{ug} / \mathrm{L}$ \\
\hline Heptachlor epoxide & & & & $<.1$ & $\mathrm{U}$ & & & $\mathrm{ug} / \mathrm{L}$ \\
\hline Hexachlorobenzene & & & & $<20$ & $\mathrm{U}$ & $<10$ & JU $Q$ & $\mathrm{ug} / \mathrm{L}$ \\
\hline Hexachlorobutadiene & & & & $<10$ & $\mathrm{U}$ & $<10$ & JU $Q$ & $\mathrm{ug} / \mathrm{L}$ \\
\hline Hexachlorocyclopentadiene & & & & $<10$ & $\mathrm{U}$ & $<10$ & JU Q & $\mathrm{ug} / \mathrm{L}$ \\
\hline Hexachlorodibenzo-p-dioxins & & & & $<.00087$ & $\mathrm{U}$ & & & $\mathrm{ug} / \mathrm{L}$ \\
\hline Hexachlorodibenzo-p-furans & & & & $<.00079$ & $\mathrm{U}$ & & & $\mathrm{ug} / \mathrm{L}$ \\
\hline Hexachloroethane & & & & $<10$ & $\mathbf{U}$ & $<10$ & JU Q & $\mathrm{ug} / \mathrm{L}$ \\
\hline Indeno(1,2,3-c,d)pyrene & & & & $<20$ & $\mathbf{U}$ & $<10$ & JU Q & ug/L \\
\hline Iodomethane (Methyl iodide) & & & & $<5$ & $\mathbf{U}$ & & & ug/L \\
\hline Iron, total recoverable & & 683 & 6 & 687 & & 994 & & $\mathrm{ug} / \mathrm{L}$ \\
\hline Isobutyl alcohol & & & & $<1500$ & $\mathrm{U}$ & & & $\mathrm{ug} / \mathrm{L}$ \\
\hline Isodrin. & & & & & & $<10$ & $\mathrm{U}$ & ug/L \\
\hline Isophorone & & & & $<10$ & $\mathrm{U}$ & $<10$ & $\mathrm{JU} Q$ & ug/L \\
\hline Isosafrole & & & & & & $<10$ & $\mathbf{U}$ & $\mathrm{ug} / \mathrm{L}$ \\
\hline Kepone & & & & & & $<10$ & $\mathrm{U}$ & ug/L \\
\hline Lindane & & & & $<.1$ & $\mathbf{U}$ & & & $\mathrm{ug} / \mathrm{L}$ \\
\hline Methacrylonitrile & . & & & $<500$ & $\mathrm{U}$ & & & $\mathrm{ug} / \mathrm{L}$ \\
\hline Methapyrilene & & & & & & $<10$ & $\mathrm{U}$ & $\mathrm{ug} / \mathrm{L}$ \\
\hline Methoxychlor & & & & $<1$ & $\mathrm{U}$ & & & $\mathrm{ug} / \mathrm{L}$ \\
\hline Methyl ethyl ketone & & & & $<10$ & $\mathrm{U}$ & & & $\mathrm{ug} / \mathrm{L}$ \\
\hline Methyl isobutyl ketone & & & & $<5$ & $\mathrm{U}$ & & & $\mathrm{ug} / \mathrm{L}$ \\
\hline Methyl methacrylate & & & & $<50$ & $\mathrm{U}$ & & & $u g / L$ \\
\hline Methyl methanesulfonate & & & & & & $<10$ & $\mathbf{U}$ & $\mathrm{ug} / \mathrm{L}$ \\
\hline N-Nitrosodi-n-butylamine & & & & & & $<10$ & $\mathrm{U}$ & $\mathrm{ug} / \mathrm{L}$ \\
\hline N-Nitrosodiethylamine & & & & & & $<10$ & $U$ & $\mathrm{ug} / \mathrm{L}$ \\
\hline N-Nitrosodimethylamine & & & & $<10$ & $\mathrm{U}$ & $<25$ & J Q & $\mathrm{ug} / \mathrm{L}$ \\
\hline N-Nitrosodiphenylamine & & & & $<20$ & $\mathbf{U}$ & $<10$ & J Q & $\mathrm{ug} / \mathrm{L}$ \\
\hline N-Nitrosodipropylamine & & & & $<20$ & $\mathrm{U}$ & $<10$ & JU Q & $\mathrm{ug} / \mathrm{L}$ \\
\hline N-Nitrosomethylethylamine & & & & & & $<10$ & $\mathbf{U}$ & $\mathrm{ug} / \mathrm{L}$ \\
\hline
\end{tabular}

Note: Concentrations in bold exceed the Drinking Water Standards listed in Appendix A. Units are for all four quarters. 
WELL: TBG 5B

ANALYTICAL DATA

Analyte

$\mathrm{N}$-Nitrosomorpholine

$\mathrm{N}$-Nitrosopiperidine

N-Nitrosopyrrolidine

Naphthalene

Nickel, total recoverable

Nitrobenzene

O,O,O-Triethyl phosphorothioate

PCB 1016.

PCB 1221

PCB 1232

PCB 1242

PCB 1248

PCB 1254

PCB 1260

Parathion

Parathion methyl

Pentachlorodibenzo-p-dioxins

Pentachlorodibenzo-p-furans

Pentachloroethane

Pentachloronitrobenzene

Pentachlorophenol

Phenacetin

Phenanthrene

Phenol

Phorate

Pronamid

Propionitrile

Pyrene

Pyridine

Safrole

Selenium, total recoverable

Silver, total recoverable

Styrene

Sulfide

Sulfotepp

Tetrachlorodibenzo-p-dioxins

Tetrachlorodibenzo-p-furans

Thallium, total recoverable

Thionazin

Tin, total recoverable

Toluene

Toxaphene

Trichlorofluoromethane
101998 Mod 201998 Mod 301998 Mod 401998 Mod Unit $<10 \quad \mathrm{U} \quad \mathrm{ug} / \mathrm{L}$

$<10 \quad \mathrm{U} \quad$ ug/L

$<10 \quad \mathrm{U} \quad \mathrm{ug} / \mathrm{L}$

$<10 \quad \mathrm{U} \quad<10 \quad$ JU $\mathrm{Q} \quad \mathrm{ug} / \mathrm{L}$

$<50 \quad \mathrm{U} \quad \mathrm{ug} / \mathrm{L}$

$<25$ U $\quad<10 \quad$ JU $Q \quad$ ug $/ \mathrm{L}$

$<$ U ug/L

$<2$ U ug/L

$<1 \quad U \quad$ ug/L

$<$ U ug/L

$<1 \quad$ U ug/L

$<$ U : ug/L

$<$ U ug/L

$<10 \quad \mathrm{U} \quad$ ug/L

$<10 \quad \mathrm{U} \quad$ ug/L

$<.00082 \mathrm{U} \quad \mathrm{ug} / \mathrm{L}$

$<.00069 \mathrm{U} \quad$ ug/L

$<200$ U ug/L

$<10 \quad \mathrm{U} \quad$ ug/L

$<10 \quad \mathrm{U} \quad<25 \quad$ J $\mathrm{Q} \quad \mathrm{ug} / \mathrm{L}$

$<10$ U ug/L

$<10 \quad \mathrm{U} \quad<10 \quad$ JU $\mathrm{Q} \quad$ ug/

$<10 \quad \mathrm{U} \quad<10$ JU Q ug/ .

$<10$ U ug/L

$<10 \mathrm{U} \cdot \mathrm{ug} / \mathrm{L}$

$<500$ U

$<25$ U $\quad<10 \quad \pi \quad$ Q $u g / L$

$<25$ JU Q ug/L

$<10$ U ug/L

$<10 \quad \mathrm{U} \quad \mathrm{ug} / \mathrm{L}$

$<20$ U ug/

$<5 \quad \mathrm{U} \quad \mathrm{ug} / \mathrm{L}$

$<1000 \mathrm{U} \quad \mathrm{ug} / \mathrm{L}$

$<00053 \mathrm{U}<10 \quad \mathrm{u}$

$<.00053 \mathrm{U} \quad \mathrm{ug} / \mathrm{L}$

$<.00032 \mathrm{U}$ ug/L

$<10 \quad \mathrm{U} \quad \mathrm{ug} / \mathrm{L}$

$<10 \quad \mathrm{U} \quad$ ug/L

$<200$ U ug/L

$<5$ U ug/L

$<$ U ug/L

$<5 \quad$ U $\quad u g / L$

Note: Concentrations in bold exceed the Drinking Water Standards listed in Appendix A. Units are for all four quarters. 
WELL: TBG $\cdot 5 B$

\section{ANALYTICAL DATA}

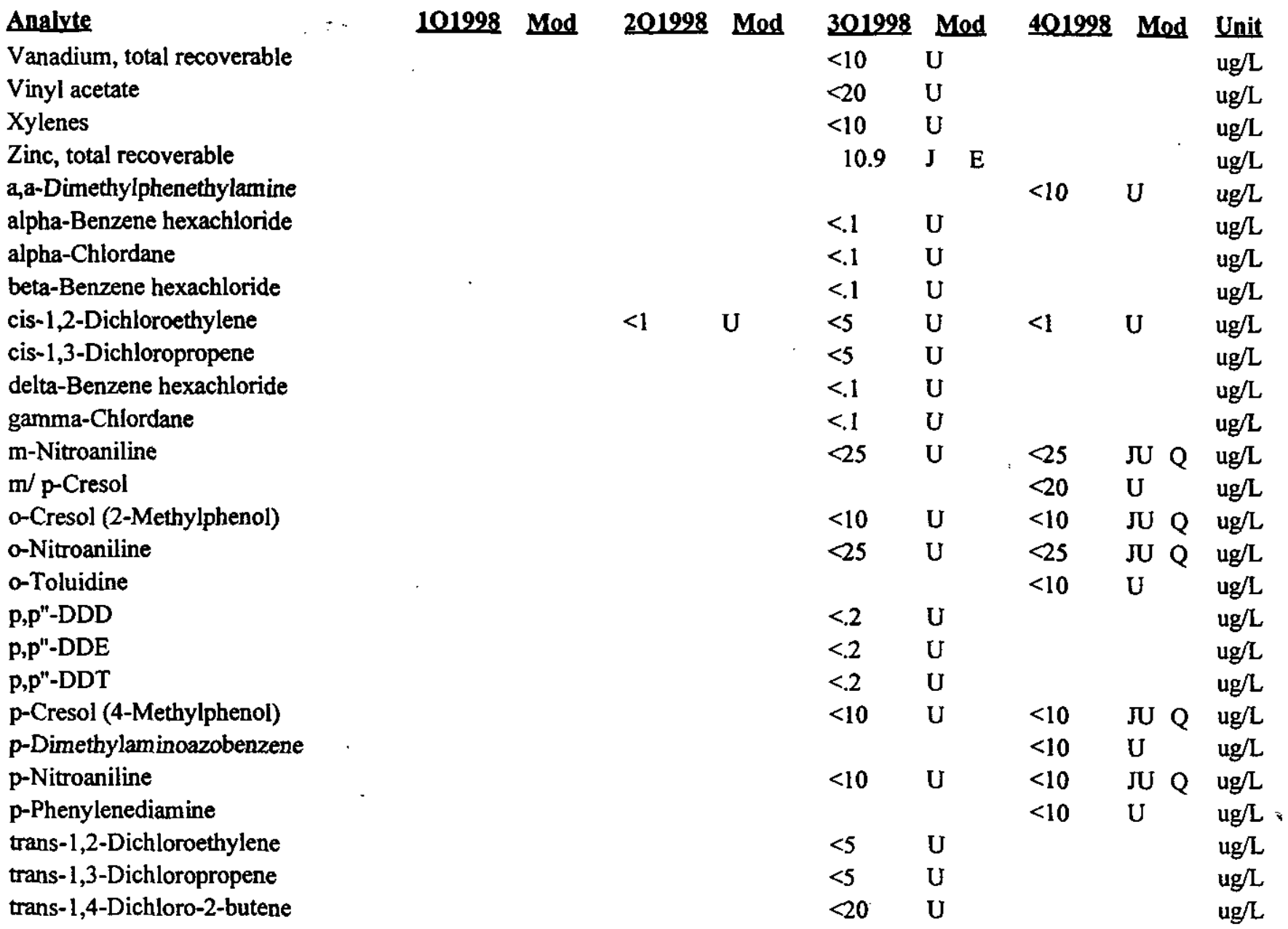


WELL: TBG 6

SRS Coord, Lat/Longitude Screen Zone Elevation Top of Standpipe Top of Casing Casing Pump Screen Zone N 71482.3 33.212 Deg N $109.1-89.1$ ft msl $148.3 \mathrm{ft} \mathrm{msl}$ $148.1 \mathrm{ft} \mathrm{msl}$ $4 " \mathrm{STL}$ $\mathrm{S}$ Unconfined

\section{FIELD DATA}

\section{Analyte}

Depth to water $\mathrm{pH}$

Sp. Conductance

Water temperature

Alkalinity as $\mathrm{CaCO} 3$

Phenolpthalein Alkalinity

Turbidity

Volumes purged

Sampling codes

$\begin{array}{ll}101998 & 201998 \\ 43.45 & 42 \\ 4.2 & 4.8 \\ 180 & 120 \\ 16 & 17 \\ 0 & 0 \\ 0 & 0 \\ 5 & 4.2 \\ 2.35276 & 3.13845\end{array}$

Analve

1,1,1,2-Tetrachloroethane

1,1,1-Trichloroethane

1,1,2,2-Tetrachloroethane

1,1,2-Trichloroethane

1,1-Dichloroethane

1,1-Dichloroethylene

1,2,3-Trichloropropane

1,2,4-Trichlorobenzene

1,2-Dibromo-3-chloropropane

1,2-Dibromoethane

1,2-Dichlorobenzene

1,2-Dichloroethane

1,2-Dichloropropane

1,3,5-Trinitrobenzene

1,3-Dichlorobenzene

1,3-Dinitrobenzene

1,4-Dichlorobenzene

1,4-Dioxane

1,4-Naphthoquinone

1-Naphthylamine

2,2-Oxybis(1-chloropropane)

2,3,4,6-Tetrachlorophenol

2,3,7,8-TCDD

2,4,5-T

2,4,5-TP (Silvex)

2,4,5-Trichlorophenol

2,4,6-Trichlorophenol
101998 Mod 201998 Mod

$\begin{array}{llll}101998 & \text { Mod } & 201998 & \text { Mod } \\ <4.62 & \text { UJ } O & <.462 \quad \text { U }\end{array}$

301998

301998

44.75

4.7

100

23.6

0

0

5.3

3.74412

\section{$<50 \quad \mathrm{U}$}

$<50 \quad U$

$<50$

$<50$

$<50$

$<50$

$<50$

$<10$

$<50$

$<50$

$<50$

$<50$

$<50$

$<50$

$<50$

$$
<
$$

$<10000$

$<10 \quad \mathrm{U}$

$<.00069 \mathrm{U}$

$<.2 \quad \mathrm{U}$

$<.2$ U

$<10 \quad$ U

$<25 \mathrm{U}$
401998

Mod

Unit

ug/L

ug/L

ug/L

$\mathrm{ug} / \mathrm{L}$

ug/L

ug/L

ug/L

$<10$ JU Q ug/L

$\mathrm{ug} / \mathrm{L}$

ug/L

$\mathrm{ug} / \mathrm{L}$

$\mathrm{ug} / \mathrm{L}$

$\mathrm{ug} / \mathrm{L}$

$\mathrm{ug} / \mathrm{L}$

ug/L

$\mathrm{ug} / \mathrm{L}$

$\mathrm{ug} / \mathrm{L}$

ug/L

$<10 \quad$ U $\quad$ ug/L

$<10 \quad$ U $\quad$ ug/L

$<10$ JU Q ug/L

$<10 \quad \mathrm{U} \quad \mathrm{ug} / \mathrm{L}$

ug/L

$\mathrm{ug} / \mathrm{L}$

ug/L

$<10 \quad \pi \quad Q \quad u g / L$

$<25$ JU Q ug/L

Note: Concentrations in bold exceed the Drinking Water Standards listed in Appendix A. Units are for all four quarters. 
WELL: TBG 6

ANALYTICAL DATA

Analvte

2,4-Dichlorophenol

2,4-Dichlorophenoxyacetic acid

2,4-Dimethyl phenol

2,4-Dinitrophenol

2,4-Dinitrotoluene

2,6-Dichlorophenol

2,6-Dinitrotoluene

2-Acetylaminofluorene

2-Chloronaphthalene

2-Chlorophenol

2-Hexanone

2-Methyl-4,6-dinitrophenol

2-Methylnaphthalene

2-Naphthylamine

2-Nitrophenol

2-Picoline

2-sec-Butyl-4,6-dinitrophenol

3,3"-Dichlorobenzidine

3,3"-Dimethylbenzidine

3-Methylcholanthrene

4-Aminobiphenyl

4-Bromophenyl phenyl ether

4-Chloro-m-cresol

4-Chloroaniline

4-Chlorophenyl phenyl ether

4-Nitrophenol

4-Nitroquinoline-1-oxide

5-Nitro-o-toluidine

7,12-Dimethylbenz(a)anthracene

Acenaphthene

Acenaphthylene

Acetone

Acetonitrile (Methyl cyanide)

Acetophenone

Acrolein

Acrylonitrile

Aldrin

Allyl chloride

Aluminum, total recoverable

Aniline

Anthracene

Antimony, total recoverable

Aramite

\section{Mod 201998 Mod}

301998 Mod

$<10 \quad \mathrm{U}$

$<.2 \quad \mathrm{U}$

$<10 \quad \mathrm{U}$

$<25 \mathrm{U}$

$<10 \quad \mathrm{U}$

$<10 \quad \mathrm{U}$

$<10 \quad \mathrm{U}$

$<10$

$<50$

$<25 \quad \mathrm{U}$

$<10$

$<10 \quad \mathrm{U}$

U

$<10$

U

$<10$

$<10$

$<10$

$<10$

$<25$

175

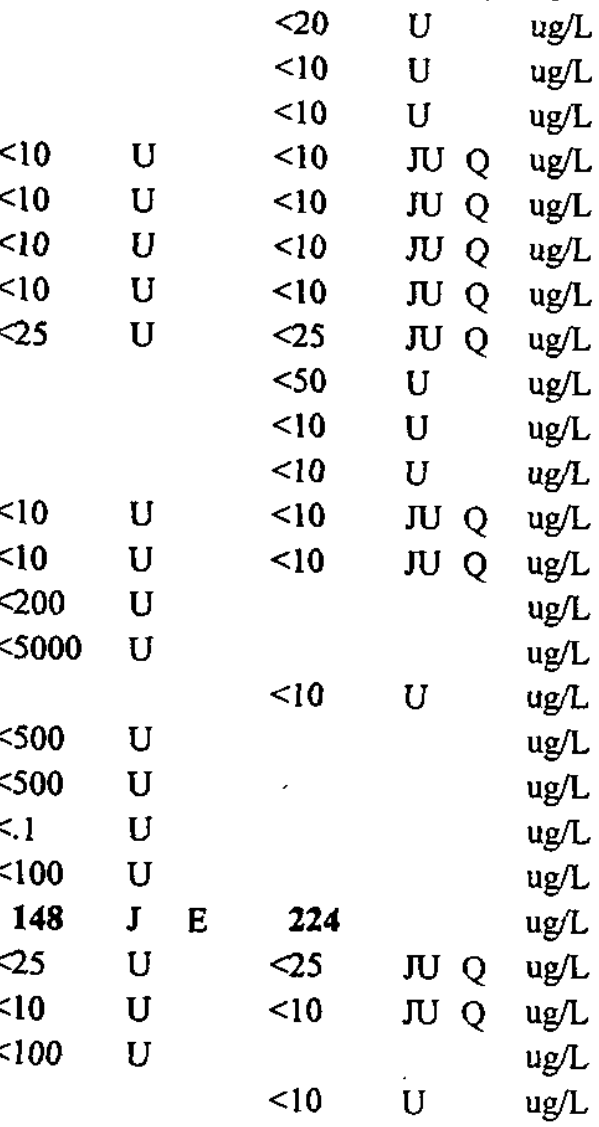

Note: Concentrations in bold exceed the Drinking Water Standards listed in Appendix A. Units are for all four quarters. 
WELL: TBG 6

ANALYTICAL DATA

\begin{tabular}{|c|c|c|c|c|c|c|c|c|c|c|}
\hline Analyte & 101998 & Mod 201998 & Mod & 301998 & Mo & & 401998 & Mo & & Unit \\
\hline Arsenic, total recoverable & & & & $<10$ & $\mathrm{U}$ & & & & & $\mathrm{ug} / \mathrm{L}$ \\
\hline Barium, total recoverable & & & & 46.7 & & & & & & $\mathrm{ug} / \mathrm{L}$ \\
\hline Benzene & & & & $<50$ & $\mathrm{U}$ & & & & & $\mathrm{ug} / \mathrm{L}$ \\
\hline Benzo(a)anthracene & & & & $<10$ & $\mathbf{U}$ & & $<10$ & JU & $\mathbf{Q}$ & $\mathrm{ug} / \mathrm{L}$ \\
\hline Benzo(a)pyrene & & & & $<10$ & $\mathbf{U}$ & & $<10$ & 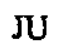 & $Q$ & ug/L \\
\hline Benzo(b)fluoranthene & & & & $<10$ & $\mathrm{U}$ & & $<10$ & JU & $\mathrm{Q}$ & $\mathrm{ug} / \mathrm{L}$ \\
\hline Benzo( $(g, h, i)$ perylene & & . & & $<10$ & $\mathrm{U}$ & & $<10$ & J & Q & ug/L \\
\hline Benzo(k)fluoranthene & & & & $<10$ & $\mathrm{U}$ & & $<10$ & $J U$ & $\mathbf{Q}$ & $\mathrm{ug} / \mathrm{L}$ \\
\hline Benzyl alcohol & & & & $<10$ & $\mathrm{U}$ & & $<10$ & JU & $\mathbf{Q}$ & $u g / L$ \\
\hline Beryllium, total recoverable & . & & & 2.72 & $J$ & $\mathbf{E}$ & & & & $\mathrm{ug} / \mathrm{L}$ \\
\hline Bis(2-chloroethoxy) methane & & & & $<10$ & $\mathrm{U}$ & & $<10$ & JU & Q & $u g / L$ \\
\hline Bis(2-chloroethyl) ether & & & & $<10$ & $\mathrm{U}$ & & $<10$ & $\mathrm{JU}$ & Q & $\mathrm{ug} / \mathrm{L}$ \\
\hline Bis(2-ethylhexyl) phthalate & & & & $<10$ & $\mathbf{U}$ & & $<10$ & JU & Q & $\mathrm{ug} / \mathrm{L}$ \\
\hline Bromodichloromethane & & & & $<50$ & $\mathrm{U}$ & & & & & $\mathrm{ug} / \mathrm{L}$ \\
\hline Bromoform & & & & $<50$ & $\mathrm{U}$ & & & & & $\mathrm{ug} / \mathrm{L}$ \\
\hline Bromomethane (Methyl bromide) & & & & $<50$ & $\mathbf{U}$ & & & & & $u g / L$ \\
\hline Butylbenzyl phthalate & & & & $<10$ & $\mathbf{U}$ & & $<10$ & JU & Q & $u g / L$ \\
\hline Cadmium, total recoverable & & & & $<10$ & $\mathrm{U}$ & & & & . & $\mathrm{ug} / \mathrm{L}$ \\
\hline Carbazole & & & & $<20$ & $\mathbf{U}$ & & & & & $\mathrm{ug} / \mathrm{L}$ \\
\hline Carbon disulfide & & & & $<50$ & $\mathrm{U}$ & & & & & $\mathrm{ug} / \mathrm{L}$ \\
\hline Chlordane & & & & $<.2$ & $\mathrm{U}$ & & & & & $\mathrm{ug} / \mathrm{L}$ \\
\hline Chlorobenzene & & & & $<50$ & $\mathbf{U}$ & & & & & ugl \\
\hline Chlorobenzilate & & & & & & & $<10$ & $\mathbf{U}$ & & $\mathrm{ug} / \mathrm{L}$ \\
\hline Chloroethane & & & & $<100$ & $\mathrm{U}$ & & & & & $u g / L$ \\
\hline Chloroethene (Vinyl chloride) & & & & $<50$ & $\mathrm{U}$ & & & & & $\mathrm{ug} / \mathrm{L}$ \\
\hline Chloromethane (Methyl chloride) & & & & $<50$ & $\mathrm{U}$ & & & & & $\mathrm{ug} / \mathrm{L}$ \\
\hline Chloroprene & & & & $<500$ & $\mathrm{U}$ & & & & & $\mathrm{ug} / \mathrm{L}$ \\
\hline Chromium, total recoverable & & & & $<10$ & $\mathbf{U}$ & & & & & ug/L \\
\hline Chrysene & & & & $<10$ & $\mathrm{U}$ & & $<10$ & JU & $\mathrm{Q}$ & ug/L \\
\hline Cobalt, total recoverable & & & & $<20$ & $\mathbf{U}$ & & & & & $\mathrm{ug} / \mathrm{L}$ \\
\hline Copper, total recoverable & & & & $<20$ & $\mathrm{U}$ & & & & & $\mathrm{ug} / \mathrm{L}$ \\
\hline Cyanide & & & & $<10$ & $\mathrm{U}$ & & & & & $\mathrm{ug} / \mathrm{L}$ \\
\hline Di-n-butyl phthalate & & & & $<10$ & $\mathrm{U}$ & & $<10$ & JU & Q & $\mathrm{ug} / \mathrm{L}$ \\
\hline Di-n-octyl phthalate & & & & $<10$ & $\mathrm{U}$ & & $<10$ & JU & Q & ug/L \\
\hline Diallate & & & & & & & $<10$ & $\mathrm{U}$ & & ug/L \\
\hline Dibenz(a,h)anthracene & & & & $<20$ & $\mathbf{U}$ & & $<10$ & JU & Q & $\mathrm{ug} / \mathrm{L}$ \\
\hline Dibenzofuran & & & & $<10$ & $\mathrm{U}$ & & $<10$ & JU & Q & $\operatorname{ug} / \mathrm{L}$ \\
\hline Dibromochloromethane & & & & $<50$ & $\mathrm{U}$ & & & & & ug/L \\
\hline Dibromomethane (Methylene bromide) & & & & $<50$ & $\mathbf{U}$ & & & & & $\mathrm{ug} / \mathrm{L}$ \\
\hline Dichlorodifluoromethane & & & & $<50$ & $\mathrm{U}$ & & & & & ug/L \\
\hline Dichloromethane (Methylene chloride) & & $\cdot$ & & 44.2 & $\mathbf{J}$ & EVE & & & & $\mathrm{ug} / \mathrm{L}$ \\
\hline Dieldrin & & & & $<.2$ & $\mathrm{U}$ & & & & & $\mathrm{ug} / \mathrm{L}$ \\
\hline Diethyl phthalate & & & & $<10$ & $\mathrm{U}$ & & $<10$ & JU & Q & ug/L \\
\hline
\end{tabular}

Note: Concentrations in bold exceed the Drinking Water Standards listed in Appendix A. Units are for all four quarters. 
WELL: TBG 6

ANALYTICAL DATA

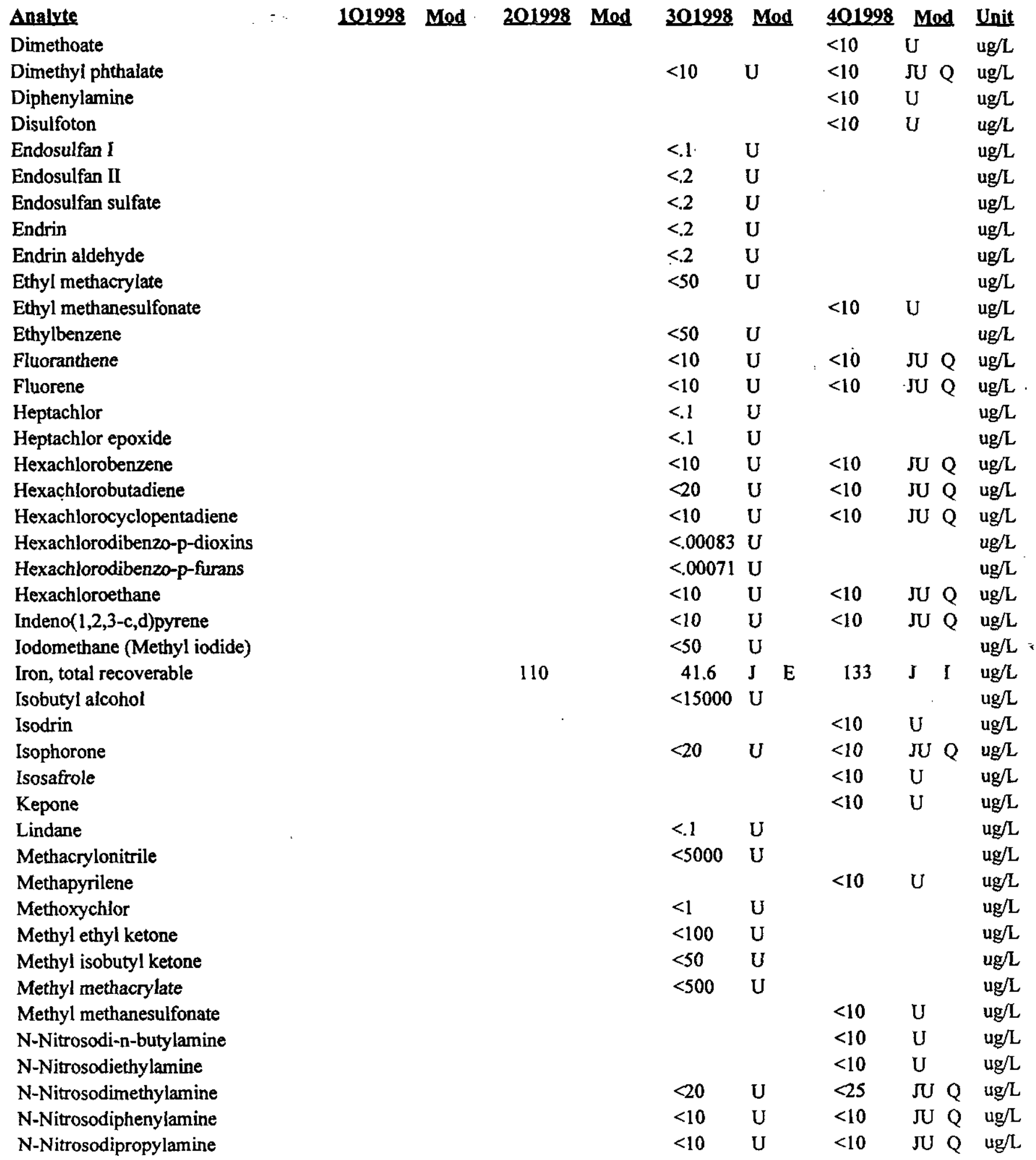

Note: Concentrations in bold exceed the Drinking Water Standards listed in Appendix A. Units are for all four quarters. 
WELL: TBG 6

ANALYTICAL DATA

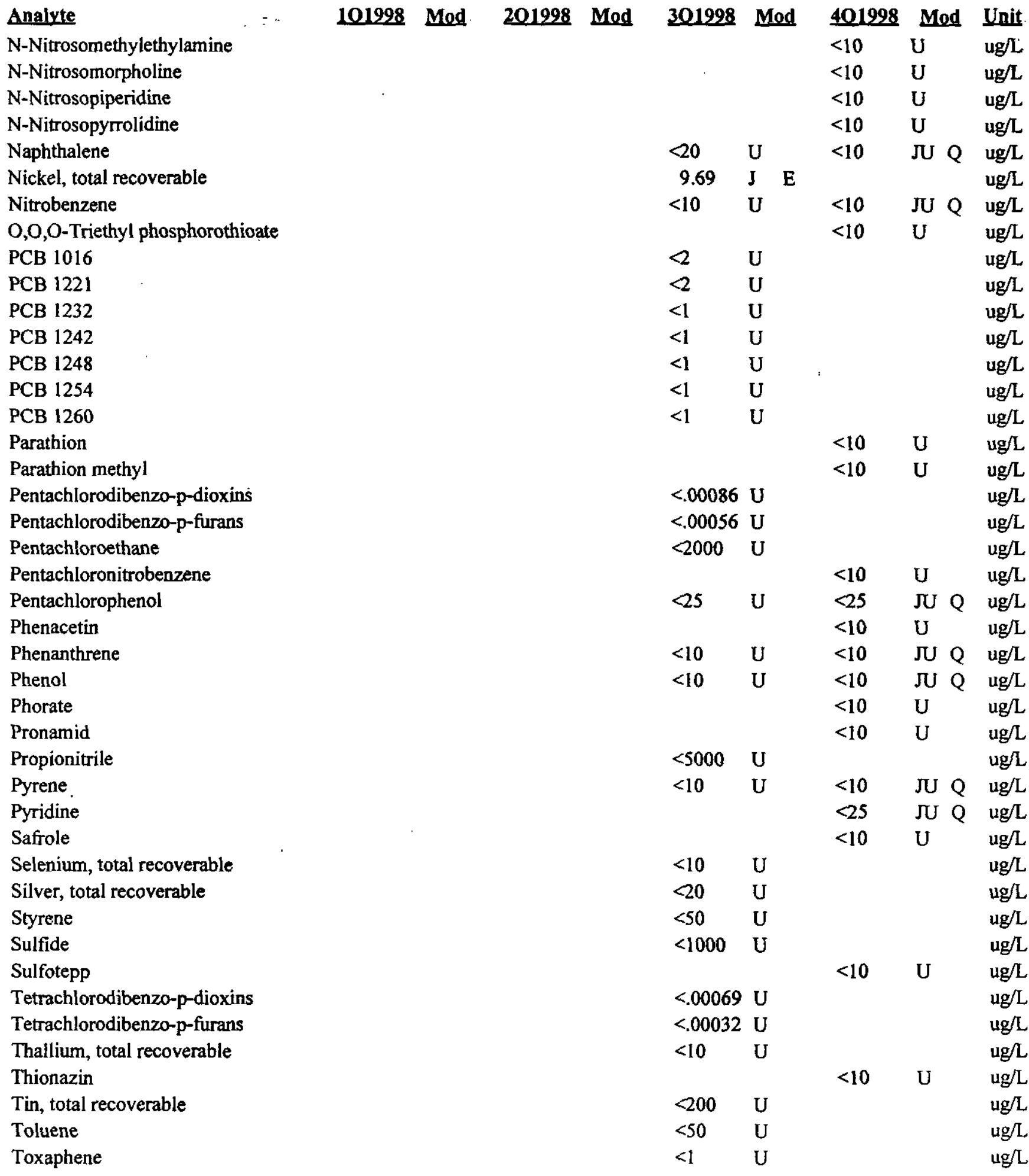

Note: Concentrations in bold exceed the Drinking Water Standards listed in Appendix A. Units are for all four quartery. 
WSRC-RP-99-4003

Unclassified

WELL: TBG 6

ANALYTICAL DATA

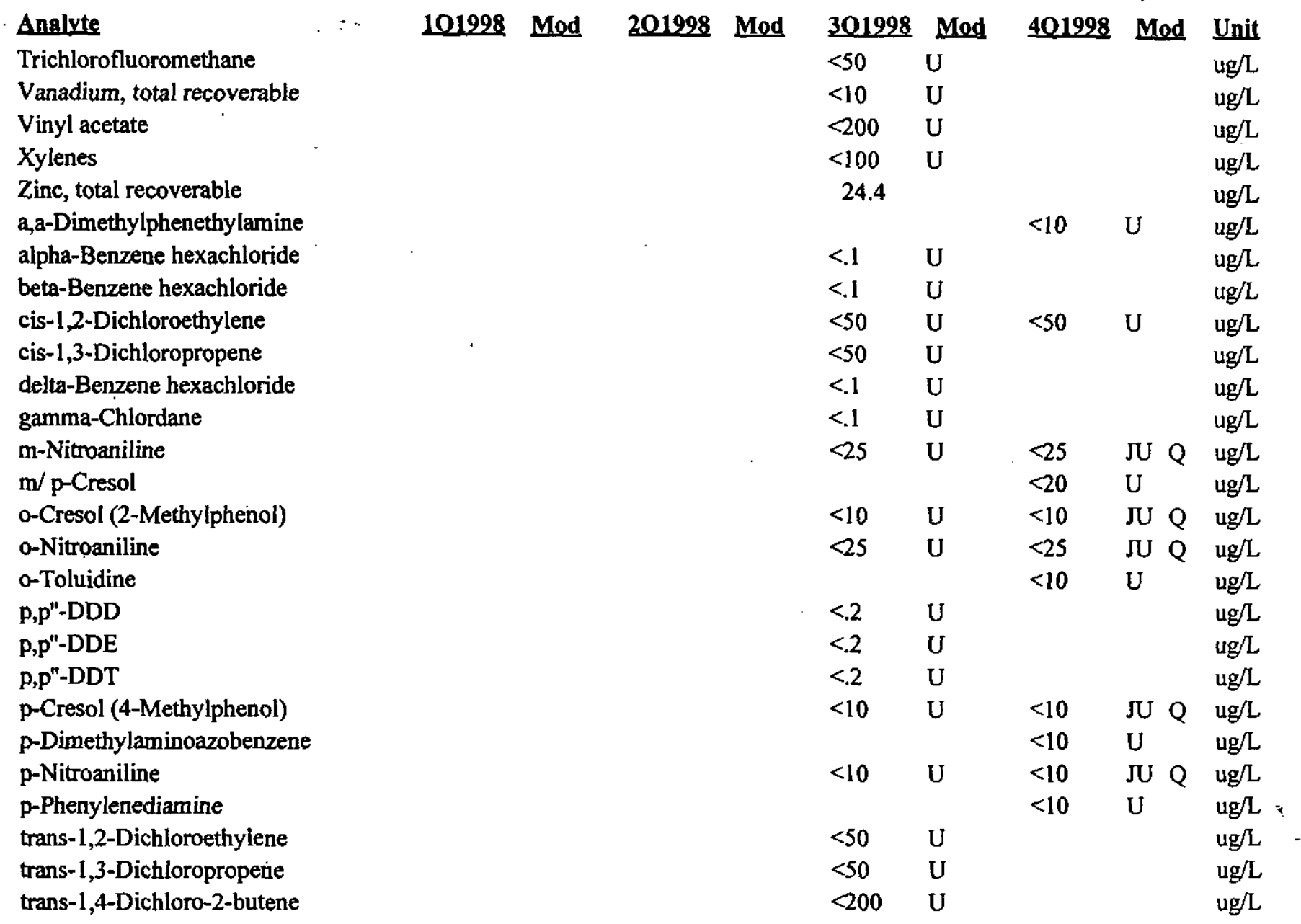

Note: Concentrations in bold exceed the Drinking Water Standards listed in Appendix A. Units are for all four quarters. 
WELL: TNX 1D

SRS Coord. Lat/Longitude Screen Zone Elevation Top of Standpipe Top of Casing Casing Pump Screen Zone N 71613.5 33.211 Deg N $99.6-79.6 \mathrm{ft} \mathrm{msl}$

$156.7 \mathrm{ft} \mathrm{msl}$

$156.5 \mathrm{ft} \mathrm{msl}$

4 "STL

$S$ Unconfined E 16699.681 .762 Deg W. :

SAMPLE DATE

03/02/98

05/13/98

08/05/98

$12 / 02 / 98$

FIELD DATA

Analyte

Depth to water

$\mathrm{pH}$

Sp. Conductance

Water temperature

Alkalinity as $\mathrm{CaCO} 3$

Phenolpthalein Alkalinity

Turbidity

Volumes purged

Sampling codes

101998
59.69
5.8
41
19.4
4
0
.6
3.54303

\section{ANALYTICAL DATA}

\section{Analyte}

1,1,1,2-Tetrachloroethane

1,1,1-Trichloroethane

1,1,2,2-Tetrachloroethane

1,1,2-Trichloroethane

1,1-Dichloroethane

1,1-Dichloroethylene

1,2,3-Trichloropropane

1,2,4-Trichlorobenzene

1,2-Dibromo-3-chloropropane

1,2-Dibromoethane

1,2-Dichlorobenzene

1,2-Dichloroethane

1,2-Dichloropropane

1,3,5-Trinitrobenzene

1,3-Dichlorobenzene

1,3-Dinitrobenzene

1,4-Dichlorobenzene

1,4-Dioxane

1,4-Naphthoquinone

1-Naphthylamine

2,2-Oxybis(1-chloropropane)

2,3,4,6-Tetrachlorophenol

2,3,7,8-TCDD

2,4,5-T

2,4,5-TP (Silvex)

2,4,5-Trichlorophenol

2,4,6-Trichlorophenol
101998 Mod 201998 Mod 301998 Mod

$<5 \quad U$

$\begin{array}{llllll}<.462 & \mathrm{U} & <.462 & \mathrm{U} & <5 & \mathrm{U}\end{array}$

$<5 \quad U$

$<5 \quad \mathrm{U}$

$<5 \quad U$

$<5 \quad \mathrm{U}$

$<5 \quad U$

$<10 \quad \mathrm{U}$

$<5 \quad \mathrm{U}$

$<5 \quad U$

$<5 \quad U$

$<5 \quad U$

$<5 \quad$ U

$<5 \quad$ U

$<5 \quad \mathrm{U}$

$<1000 \quad \mathrm{U}$

$<10 \quad \mathrm{U}$

$<.00052 \mathrm{U}$

$<.2$ UJ O

$<.2 \quad$ UJ $\mathrm{O}$

$<10 \quad \mathrm{U}$

$<25$ U
401998

59.7

5.1

41 uS $/ \mathrm{cm}$

18.2 deg. C

$5 \mathrm{mg} / \mathrm{L}$

$0 \quad \mathrm{mg} / \mathrm{L}$

$1 \quad$ NTU

4.87450 gallons
Unit

ft BTOS

Note: Concentrations in bold exceed the Drinking Water Standards listed in Appendix A. Units are for all four quarters. 
WELL: TNX 1D

ANALYTICAL DATA

Analyte

2,4-Dichlorophenol

2,4-Dichlorophenoxyacetic acid

2,4-Dimethyl phenol

2,4-Dinitrophenol

2,4-Dinitrotoluene

2,6-Dichlorophenol

2,6-Dinitrotoluene

2-Acetylaminofluorene

2-Chloronaphthalene

2-Chlorophenol

2-Hexanone

2-Methyl-4,6-dinitrophenol

2-Methylnaphthalene

2-Naphthylamine

2-Nitrophenol

2-Picoline

2-sec-Butyl-4,6-dinitrophenol

3,3"-Dichlorobenzidine

3,3"-Dimethylbenzidine

3-Methylcholanthrene

4-Aminobiphenyl

4-Bromophenyl phenyl ether

4-Chloro-m-cresol

4-Chloroaniline

4-Chlorophenyl phenyl ether

4-Nitrophenol

4-Nitroquinoline-1-oxide

5-Nitro-o-toluidine

7,12-Dimethylbenz(a)anthracene

Acenaphthene

Acenaphthylene

Acetone

Acetonitrile (Methyl cyanide)

Acetophenone

Acrolein

Acrylonitrile

Aldrin

Allyl chloride

Aluminum, total recoverable

Aniline

Anthracene

Antimony, total recoverable

Aramite
101998 Mod 201998 Mod

(a)

$<$

$$
<.2
$$$$
<10
$$$$
<25
$$$$
<10
$$

01998 Mod

401998 Mod Unit

$<10$

$U$

$<10$

JU $Q \quad u g / L$

UJ 0

$\mathrm{ug} / \mathrm{L}$

U $<25$

$<10$
$<25$

JU $Q \quad u g / L$

JU $Q \quad$ ug/L

JU $Q \quad$ ug/L

$<10 \quad \mathrm{U} \quad \mathrm{ug} / \mathrm{L}$

$<10 \quad U \quad<10 \quad \pi \quad Q \quad$ ug/L

$<10 \quad \mathrm{U} \quad \mathrm{ug} / \mathrm{L}$

$<10 \quad \mathrm{U} \quad<10 \quad$ JU $\mathrm{Q} \quad \mathrm{ug} / \mathrm{L}$

$<10 \quad \mathrm{U} \quad<10 \quad$ JU $Q \quad$ ug/L

$<5 \quad U$

$u g / L$

$<25 \quad \mathrm{U} \quad<25$

$<10 \quad \mathrm{U} \quad<10$

JU $Q \quad u g / L$

JU $Q \quad u g / L$

$<10$ U ug/L

$<10 \quad \mathrm{U} \quad<10$ JU $\mathrm{Q} \quad \mathrm{ug} / \mathrm{L}$

$<10$ U ug/L

$<10$ U ug/L

$<10 \quad U \quad<10 \quad$ JU $Q \quad$ ug/

$<20$ U ug/L

$<10 \quad \mathrm{U} \quad \mathrm{ug} / \mathrm{L}$

$<10$ U ug/L

$<10 \quad U \quad<10 \quad J U$ Q $\quad u g / L$

$<10 \quad \mathrm{U} \quad<10 \quad$ JU $\mathrm{Q} \quad \mathrm{ug} / \mathrm{L}$

$<10 \quad \mathrm{U}$

$<10 \quad \mathrm{U}$

$<25 \quad \mathrm{U}$

$<10$

JU Q ug/L *

$<10 \quad$ JU $\quad$ Q $\quad$ ug/L

$<25 \quad \pi \quad \mathrm{Q} \quad \mathrm{ug} / \mathrm{L}$

$<50$ U ug/L

$<10 \quad \mathrm{U} \quad \mathrm{ug} / \mathrm{L}$

$<10 \quad U \quad$ ug/L

$<10 \quad \mathrm{U} \quad<10 \quad$ JU $Q \quad \mathrm{ug} / \mathrm{L}$

$<10 \quad \mathrm{U} \quad<10 \quad \mathrm{JU} Q \mathrm{ug} / \mathrm{L}$

$<20 \quad U$

$<500 \quad U$

$\mathrm{ug} / \mathrm{L}$

$u g / L$

$<10$ U ug/L

$<50$ U ug/L

$<50 \quad U$

$<.1 \quad U$

$<10 \quad \mathrm{U}$

$<20 \quad$ U

$<200 \quad$ U

$<25 \quad \mathrm{U}$

$<10 \quad \mathrm{U}$

$<100 \quad \mathrm{U}$ $\mathrm{ug} / \mathrm{L}$

ug/L

$\mathrm{ug} / \mathrm{L}$

$<200$ U ug/L

$<25$. JU Q ug/L

$<10$ JU Q ug/L

$\mathrm{ug} / \mathrm{L}$

$<10 \quad \mathrm{U} \quad$ ug/L

Note: Concentrations in bold exceed the Drinking Water Standards listed in Appendix A. Units are for all four quarters. 
WELL: TNX 1D

\section{ANALYTICAL DATA}

\section{Analyte}

Arsenic, total recoverable

Barium, total recoverable

Benzene

Benzo(a)anthracene

Benzo(a)pyrene

Benzo(b)fluoranthene

Benzo(g,h,i)perylene

Benzo(k)fluoranthene

Benzyl alcohol

Beryllium, total recoverable

Bis(2-chloroethoxy) methane

Bis(2-chloroethyl) ether

Bis(2-ethylhexyl) phthalate

Bromodichloromethane

Bromoform

Bromomethane (Methyl bromide)

Butylbenzyl phthalate

Cadmium, total recoverable

Carbazole

Carbon disulfide

Chlorobenzene

Chlorobenzilate

Chloroethane

Chloroethene (Vinyl chloride)

Chloromethane (Methyl chloride)

Chloroprene

Chromium, total recoverable

Chrysene

Cobalt, total recoverable

Copper, total recoverable

Cyanide

Di-n-butyl phthalate

Di-n-octyl phthalate

Diallate

Dibenz(a,h)anthracene

Dibenzofuran

Dibromochloromethane

Dibromomethane (Methylene bromide)

Dichlorodifluoromethane

Dichloromethane (Methylene chloride)

Dieldrin

Diethyl phthalate

Dimethoate
101998 Mod 201998 Mod 301998 Mod 401998 Mod Unit

$<10 \quad \mathrm{U}$

14.9

$<5 \quad \mathrm{U}$

$<10 \quad \mathrm{U}$

$<10 \quad \mathrm{U}$

$<10 \quad \mathrm{U}$

$<10 \quad \mathrm{U}$

$<25$ U

$<10 \quad \mathrm{U}$

$<10 \quad \mathrm{U}$

$<10 \quad \mathrm{U}$

$<10 \quad \mathrm{U}$

$<10 \quad \mathrm{U}$

$<5 \quad \mathrm{U}$

$<5$ U

$<5$ U

$<2$ U

$<10 \quad \mathrm{U}$

$<10$ U

$<5 \quad$ U

$<5 \quad \mathrm{U}$

$<10 \quad \mathrm{U}$

$<5$ U

$<5 \quad \mathrm{U}$

$<50 \quad \mathrm{U}$

$<10 \quad \mathrm{U}$

$<10 \quad \mathrm{U}$

$<20$ U

$<20$ U

$<10 \quad$ U

$<10$ U

$<20$ U

$<10 \quad \mathrm{U}$

$<10$ U

$<5 \quad$ U

$<5 \quad U$

$<5 \quad$ U

$<4.26 \quad \mathrm{U} \quad \mathrm{V}$

$<.2 \quad \mathrm{U}$

$<10 \quad \mathrm{U}$

ug/L

ug/ $/$

ug/L

ug/L

$<10$ U ug/L

ug/L

ug/L

$\mathrm{ug} / \mathrm{L}$

ug/L

$\mathrm{ug} / \mathrm{L}$

$<10$ JU Q ug/

ug/L

$\mathrm{ug} / \mathrm{L}$

ug $/$

$<10 \quad$ JU $Q \quad$ ug/L

$<10$ JU Q ug/L

$<10$ U ug/L

$<10 \quad$ JU $Q \quad$ ug/L

$<10 \quad$ J $Q \quad$ ug/L

$\mathrm{ug} / \mathrm{L}$

$u g / L$

ug $/ \mathrm{L}$

ug/L

$\mathrm{ug} / \mathrm{L}$

$<$ i0 JU Q ug/L

$<10$ U ug/L

Note: Concentrations in bold exceed the Drinking Water Standards listed in Appendix A. Units are for all four quarters. 
WELL: TNX 1D

ANALYTICAL DATA

\section{Analyte}

Dimethyl phthalate

Diphenylamine

Disulfoton

Endosulfan I

Endosulfan II

Endosulfan sulfate

Endrin

Endrin aldehyde

Ethyl methacrylate

Ethyl methanesulfonate

Ethylbenzene

Fluoranthene

Fluorene

Heptachlor

Heptachlor epoxide

Hexachlorobenzene

Hexachlorobutadiene

Hexachlorocyclopentadiene

Hexachlorodibenzo-p-dioxins

Hexachlorodibenzo-p-furans

Hexachloroethane

Indeno(1,2,3-c,d)pyrene

Iodomethane (Methyl iodide)

Iron, total recoverable

Isobutyl alcohol

Isodrin

Isophorone

Isosafrole

Kepone

Lindane

Methacrylonitrile

Methapyrilene

Methoxychlor

Methyl ethyl ketone

Methyl isobutyl ketone

Methyl methacrylate

Methyl methanesulfonate

$\mathrm{N}$-Nitrosodi-n-butylamine

$\mathrm{N}$-Nitrosodiethylamine

N-Nitrosodimethylamine

$\mathrm{N}$-Nitrosodiphenylamine

$\mathrm{N}$-Nitrosodipropylamine

$\mathrm{N}$-Nitrosomethylethylamine
101998 Mod. 201998 Mod 301998 Mod 401998 Mod Unit

$<10 \quad \mathrm{U} \quad<10 \quad$ JU $\mathrm{Q} \quad \mathrm{ug} / \mathrm{L}$

$<10 \quad$ U ug/L

$<10 \quad U \quad$ ug/L

$<.1 \quad \mathrm{U} \quad \mathrm{ug} / \mathrm{L}$

$<.2$ U $\quad$ ug/L

$<.2 \quad \mathrm{U} \quad \mathrm{ug} / \mathrm{L}$

$<.2$ U $\quad \mathrm{ug} / \mathrm{L}$

$<.2$ U $\quad$ ug/ $/$

$<5 \quad \mathrm{U} \quad \mathrm{ug} / \mathrm{L}$

$<10 \quad \mathrm{U} \quad \mathrm{ug} / \mathrm{L}$

$<5 \quad U \quad u g / L$

$<10 \quad$ U $\quad<10 \quad$ JU $Q \quad$ ug/L

$<10 \quad \mathrm{U} \quad<10 \quad$ JU $Q \quad \mathrm{ug} / \mathrm{L}$

$<.1$ U $\mathrm{ug} / \mathrm{L}$

$<.1 \quad \mathrm{U} \quad \mathrm{ug} / \mathrm{L}$

$<20 \quad \mathrm{U} \quad<10 \quad$ JU $\mathrm{Q}$ ugh

$<10 \quad \mathrm{U} \quad<10$ JU $\mathrm{Q}$ ug/L

$<10 \quad \mathrm{U} \quad<10 \quad$ JU $\mathrm{Q} \quad \mathrm{ug} / \mathrm{L}$

$<.00078 \mathrm{U} \quad \mathrm{ug} / \mathrm{L}$

$<.001 \mathrm{U} \quad \mathrm{ug} / \mathrm{L}$

$<10 \quad \mathrm{U} \quad<10 \quad J U \mathrm{Q} \quad \mathrm{ug} / \mathrm{L}$

$<20 \quad \mathrm{U} \quad<10 \quad \mathrm{JU} \mathrm{Q} \quad \mathrm{ug} / \mathrm{L}$

$<5 \quad \mathrm{U} \quad \mathrm{ug} / \mathrm{L}$

720

61.3 J $\quad E \quad 75.6 \quad J \quad I \quad u g / L$;

$<1500 \mathrm{U} \quad$ ug/L

$<10 \quad U \quad u g / L$

$<10 \quad \mathrm{U} \quad<10 \quad$ JU $Q \quad$ ug/L

$<10$ U ug/L

$<10$ U ug/L

$<.1 \quad \mathrm{U} \quad \mathrm{ug} / \mathrm{L}$

$<500$ U ug/L

$<10 \quad \mathrm{U} \quad \mathrm{ug} / \mathrm{L}$

$<1 \quad \mathrm{U} \quad$ ug/L

$<10 \quad \mathrm{U} \quad \mathrm{ug} / \mathrm{L}$

$<5 \quad \mathrm{U} \quad \mathrm{ug} / \mathrm{L}$

$<50$ U ug/L

$<10 \quad \mathrm{U} \quad \mathrm{ug} / \mathrm{L}$

$<10$ U ug/L

$<10 \quad \mathrm{U} \quad \mathrm{ug} / \mathrm{L}$

$<10 \quad$ U $\quad<25 \quad$ J $Q \quad$ ug/L

$<20 \quad \mathrm{U} \quad<10 \quad \mathrm{JU} \quad \mathrm{Q} \quad \mathrm{ug} / \mathrm{L}$

$<20 \quad \mathrm{U} \quad<10 \quad \pi \mathrm{Q} \quad \mathrm{ug} / \mathrm{L}$

$<10 \quad \mathrm{U} \cdot \mathrm{ug} / \mathrm{L}$

Note: Concentrations in bold exceed the Drinking Water Standards listed in Appendix A. Units are for all four quarters. 
WELL: TNX ID

ANALYTICAL DATA

Analyte

N-Nitrosomorpholine

N-Nitrosopiperidine

$\mathrm{N}$-Nitrosopyrrolidine

Naphthalene

Nickel, total recoverable

Nitrobenzene

O,O,O-Triethyl phosphorothioate

PCB 1016

PCB 1221

PCB 1232

PCB 1242

PCB 1248

PCB 1254

PCB 1260

Parathion

Parathion methyl

Pentachlorodibenzo-p-dioxins

Pentachlorodibenzo-p-furans

Pentachloroethane

Pentachloronitrobenzene

Pentachlorophenol

Phenacetin

Phenanthrene

Phenol

Phorate

Pronamid

Propionitrile

Pyrene

Pyridine

Safrole

Selenium, total recoverable

Silver, total recoverable

Styrene

Sulfide

Sulfotepp

Tetrachlorodibenzo-p-dioxins

Tetrachlorodibenzo-p-furans

Thallium, total recoverable

Thionazin

Tin, total recoverable

Toluene

Toxaphene

Trichlorofluoromethane
101998 Mod 201998 Mod 301998 Mod 401998 Mod Unit

$<10 \quad \mathrm{U} \quad$ ug/L

$<10 \quad \mathrm{U} \quad \mathrm{ug} / \mathrm{L}$

$<10 \quad \mathrm{U} \quad \mathrm{ug} / \mathrm{L}$

$<10 \quad \mathrm{U} \quad<10 \quad$ JU $\mathrm{Q} \quad \mathrm{ug} / \mathrm{L}$

$<50$ U ug/L

$<25 \quad \mathrm{U} \quad<10 \quad$ JU $Q \quad$ ug/L

$<10$ U ug/L

$<\quad$ U $\quad u g / L$

$<$ U ug/L

$<1 \quad$ U ugh

$<1 \quad$ U ug/L

$<1 \quad$ U $\quad u g / L$

$<$ U ug/L

$<1 \quad \mathrm{U} \quad \mathrm{ug} / \mathrm{L}$

$<10 \quad \mathrm{U} \quad \mathrm{ug} / \mathrm{L}$

$<10 \quad \mathrm{U} \quad \mathrm{ug} / \mathrm{L}$

$<.0011 \mathrm{U} \quad \mathrm{ug} / \mathrm{L}$

$<.00076 \mathrm{U}$. $\mathrm{ug} / \mathrm{L}$

$<200$ U ug/L

$<10 \quad \mathrm{u} \quad \mathrm{ug} / \mathrm{L}$

$<10 \quad \mathrm{U} \quad<25 \quad$ JU Q $\quad \mathrm{ug} / \mathrm{L}$

$<10 \quad \mathrm{U} \quad$ ug/L

$<10 \quad \mathrm{U} \quad<10 \quad$ J $Q \quad$ ug/L

$<10 \quad \mathrm{U} \quad<10 \quad \mathrm{JU} \mathrm{Q} \quad \mathrm{ug} / \mathrm{L}$

$<10 \quad \mathrm{U} \quad \mathrm{ug} / \mathrm{L}$

$<10$ U ug/L

$<500$ U ug/L

$<25 \quad \mathrm{U} \quad<10 \quad$ JU $Q \quad \mathrm{ug} / \mathrm{L}$

$<25$ NU Q ug/L

$<10$ U ug/L

$<10$ U ug/L

$<20$ U ug/L

$<5 \quad$ U ug/L

$<1000 \quad \mathrm{u} \quad \mathrm{ug} / \mathrm{L}$

$\begin{array}{lll}<0 & \text { U } & \text { ug/L }\end{array}$

$<.00052 \mathrm{U} \quad \mathrm{ug} / \mathrm{L}$

$<.00045 \mathrm{U} \quad \mathrm{ug} / \mathrm{L}$

$<10 \quad \mathrm{U} \quad \mathrm{ug} / \mathrm{L}$

$<10 \quad \mathrm{U} \quad$ ug/L

$<200$ U ug/L

$<5 \quad \mathrm{U} \quad \mathrm{ug} / \mathrm{L}$

$<1 \quad$ U ug/L

$<5 \quad U \quad$ ug/L

Note: Concentrations in bold exceed the Drinking Water Standards listed in Appendix A. Units are for all four quarters. 
WELL: TNX $1 D$

\section{ANALYTICAL DATA}

\section{Analvte}

101998 Mod 201998 Mod

Vanadium, total recoverable

Vinyl acetate

Xylenes

Zinc, total recoverable

a,a-Dimethylphenethylamine alpha-Benzene hexachloride alpha-Chlordane beta-Benzene hexachloride cis-1,2-Dichloroethylene cis-1,3-Dichloropropene delta-Benzene hexachloride gamma-Chlordane m-Nitroaniline $\mathrm{m} / \mathrm{p}$-Cresol o-Cresol (2-Methylphenol) o-Nitroaniline

o-Toluidine

$\mathrm{p}, \mathrm{p}$ "-DDD

$p, p^{\prime \prime}-D D E$

p,p"-DDT

p-Cresol (4-Methylphenol)

p-Dimethylaminoazobenzene p-Nitroaniline p-Phenylenediamine trans-1,2-Dichloroethylene trans-1,3-Dichloropropene trans-1,4-Dichloro-2-butene

$\begin{aligned} & \mathbf{3 0 1 9 9 8} \\ & <10\end{aligned}$
$<20$
$<10$
$<20$

401998
$<.1 \quad$ U

$<.1 \quad \mathrm{U}$

$<.1 \quad \mathrm{U}$

$<5 \quad$ U

$<.1 \quad \mathrm{U}$

$<.1 \quad \mathrm{U}$

$<25 \quad \mathrm{U}$

$<10$

$<25$

$$
\text { U }
$$

$$
\text { U }
$$$$
<2
$$$$
<.2
$$$$
<, 2
$$$$
\text { U }
$$$$
<.2
$$$$
<10
$$$$
<10
$$

$<5$

$<5$

$<20$

$<10 \quad U$

\section{Mod}

U

U

U

J

$$
<10
$$$$
10
$$

U

U

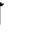

$<1 \quad$ U

Mod

ug/L

ug/L

$\mathrm{ug} / \mathrm{L}$

ug/L

ug/L

$\operatorname{ug} / \mathrm{L}$

$\operatorname{ug} / \mathrm{L}$

$\operatorname{ug} / \mathrm{L}$

ug/L

ug/L

ug/L

ugl

$<25$ JU Q ug/L

$<20$ U ug/L

$<10$ JU Q ug/L

$<25$ JU $Q \quad$ ug/L

$<10$ U ug/L

ug/L

$\mathrm{ug} / \mathrm{L}$

$\mathrm{ug} / \mathrm{L}$

$<10 \quad$ JU Q ug/L

$<10$ U ug/L

$<10$

JU $Q \quad u g / L$

$<10$

U

$\mathrm{ug} / \mathrm{L}$; ug/L $\mathrm{ug} / \mathrm{L}$ ug/L 
WELL: TNX 2D

SRS Coord. Lat/longitude Screen Zone Elevation Top of Standpipe Top of Casing Casing Pump Screen Zone N 71452.0 33.211 Deg N $102.8-82.8 \mathrm{ft} \mathrm{msl}$ E 16788.281 .761 Deg W. -

SAMPLE DATE

FIELD DATA

\section{Analyte}

Depth to water

$\mathrm{pH}$

Sp. Conductance

Water temperature

Alkalinity as $\mathrm{CaCO} 3$

Phenolpthalein Alkalinity

Turbidity

Volumes purged

Sampling codes
03/02/98

$155.3 \mathrm{ft} \mathrm{msl}$

$05 / 13 / 98$

$155.1 \mathrm{ft} \mathrm{msl}$

4" STL S

08/05/98

Unconfined

$12 / 02 / 98$

201998
54.97
5.7
41
22.9
4
0
1.9
4.39813

$\mathbf{3 0 1 9 9 8}$
57.6
5.6
50
21.8
6
0
1.9
4.77020

401998

58.97

5.6

48

21.8

20.3

6

0 .

3.4

4.56352

4.39813

4.77020

5

0

.7

3.65945

Unit
ft BTOS
uS/cm
deg. C
$\mathrm{mg} / \mathrm{L}$
$\mathrm{mg} / \mathrm{L}$
$\mathrm{NTU}$
gallons

ANALYTICAL DATA

Analyte

1,1,1,2-Tetrachloroethane

1,1,1-Trichloroethane

1,1,2,2-Tetrachloroethane

1,1,2-Trichloroethane

1,1-Dichloroethane

1,1-Dichloroethylene

1,2,3-Trichloropropane

1,2,4-Trichlorobenzene

1,2-Dibromo-3-chloropropane

1,2-Dibromoethane

1,2-Dichlorobenzene

1,2-Dichloroethane

1,2-Dichloropropane

1,3,5-Trinitrobenzene

1,3-Dichlorobenzene

1,3-Dinitrobenzene

1,4-Dichlorobenzene

1,4-Dioxane

1,4-Naphthoquinone

1-Naphthylamine

2,2-Oxybis(1-chloropropane)

2,3,4,6-Tetrachlorophenol

2,3,7,8-TCDD

2,4,5-T

2,4,5-TP (Silvex)

2,4,5-Trichlorophenol

2,4,6-Trichlorophenol
101998 Mod 201998 Mod 301998 Mod 401998 Mod Unit

$<5$ U ug/L

$\begin{array}{lllllllll}<.462 & \mathrm{U} & <.462 & \mathrm{U} & <5 & \mathrm{U} & <1 & \mathrm{U} & \mathrm{ug} / \mathrm{L}\end{array}$

$<5 \quad U$

$<5 \quad \mathrm{U}$

$<5 \quad U$

$<5 \quad U$

$<5 \quad \mathrm{U}$

$<10 \quad \mathrm{U}$

$<5 \quad \mathrm{U}$

$<5 \quad U$

$<5 \quad \mathrm{U}$

$<5 \quad \mathrm{U}$

$<5 \quad U$

$<5 \quad U$

$<\quad \mathrm{U}$

$<5 \quad \mathrm{U}$

$<1000 \quad U$

$$
<
$$$$
<10
$$$$
<.00053 \mathrm{U}
$$$$
<.2 \text { UJ } \mathrm{O}
$$$$
<2 \text { UJ } \mathrm{O}
$$$$
<10 \quad \mathrm{U}
$$$$
<25 \quad \mathrm{U} \quad<25
$$

$<10$
$<25$

ug/L

ug/L

$u g / L$

$u g / L$

ug/L

$<10$ JU Q ug/L ?

$\mathrm{ug} / \mathrm{L}$

ug/L

ug/L

$\mathrm{ug} / \mathrm{L}$

$\mathrm{ug} / \mathrm{L}$

$\mathrm{ug} / \mathrm{L}$

ug/L

$<10 \quad \mathrm{U} \quad \mathrm{ug} / \mathrm{L}$

ug/L

$u g / L$

$<10$ U ug/L

$<10 \quad U \quad u g / L$

$<10 \quad$ JU $Q \quad u g / L$

$<10$ U ug/L

$\mathrm{ug} / \mathrm{L}$

ug/L

$\mathrm{ug} / \mathrm{L}$

JU $Q \quad u g / L$

JU $Q \quad u g / L$

Note: Concentrations in bold exceed the Drinking Water Standards listed in Appendix A. Units are for all four quarters. 
WELL: TNX 2D

ANALYTICAL DATA

Analyte

2,4-Dichlorophenol

2,4-Dichlorophenoxyacetic acid

2,4-Dimethyl phenol

2,4-Dinitrophenol

2,4-Dinitrotoluene

2,6-Dichlorophenol

2,6-Dinitrotoluene

2-Acetylaminofluorene

2-Chloronaphthalene

2-Chlorophenol

2-Hexanone

2-Methyl-4,6-dinitrophenol

2-Methylnaphthalene

2-Naphthylamine

2-Nitrophenol

2-Picoline

2-sec-Butyl-4,6-dinitrophenol

3,3"-Dichlorobenzidine

3,3"-Dimethylbenzidine

3-Methylcholanthrene

4-Aminobiphenyl

4-Bromophenyl phenyl ether

4-Chloro-m-cresol

4-Chloroaniline

4-Chlorophenyl phenyl ether

4-Nitrophenol

4-Nitroquinoline-1-oxide

5-Nitro-o-toluidine

7,12-Dimethylbenz(a)anthracene Acenaphthene

Acenaphthylene

Acetone

Acetonitrile (Methyl cyanide)

Acetophenone

Acrolein

Acrylonitrile

Aldrin

Allyl chloride

Aluminum, total recoverable

Aniline

Anthracene

Antimony, total recoverable

Aramite
101998 Mod

201998 Mod

$\begin{array}{ll}\mathbf{3 0 1 9 9 8} & \text { Mod } \\ <10 & \mathrm{U} \\ <2 & \mathrm{UJ} O \\ <10 & \mathrm{U} \\ <25 & \mathrm{U} \\ <10 & \mathrm{U}\end{array}$

401998 Mod Unit

$<10$ JU Q ug/L

$\mathrm{ug} / \mathrm{L}$

$<10 \quad$ JU Q $\quad$ ug/L

$<25$ JU $Q$ ug/L

$<10$ JU Q ug/L

$<10 \quad \mathrm{U} \quad<10 \quad \pi \mathrm{Q} \quad \mathrm{ug} / \mathrm{L}$

$<10 \quad \mathrm{U} \quad<10 \quad$ JU $Q \quad \mathrm{ug} / \mathrm{L}$

$<10 \quad \mathrm{U} \quad<10 \quad \mathrm{JU} \mathrm{Q} \quad \mathrm{ug} / \mathrm{L}$

$<5 \quad \mathrm{U} \quad u g / \mathrm{L}$

$<25 \quad \mathrm{U} \quad<25 \quad \pi \quad \mathrm{Q} \quad \mathrm{ug} / \mathrm{L}$

$<10 \quad \mathrm{U}, \quad<10 \quad$ JU $Q \quad \mathrm{ug} / \mathrm{L}$

$<10 \cdot \mathrm{U} \quad \mathrm{ug} / \mathrm{L}$

$<10 \quad \mathrm{U} \quad<10 \quad \pi \quad \mathrm{Q} \quad \mathrm{ug} / \mathrm{L}$

$<10$ U ug/L

$<10$ U ug/L

$<10 \quad \mathrm{U} \quad<10 \quad$ J $Q \quad \mathrm{ug} / \mathrm{L}$

$<20 \quad \mathrm{U} \quad \mathrm{ug} / \mathrm{L}$

$<10$ U ug/L

$<10 \quad$ U ug/L

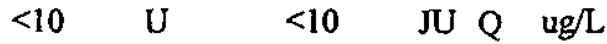

$<10 \quad$ U $\quad<10 \quad$ JU $\quad$ Q ug/L

$<10$ U $<10$ JU $Q$ ug/L:

$<10 \quad \mathrm{U} \quad<10 \quad$ JU $\mathrm{Q} \quad \mathrm{ug} / \mathrm{L}$

$<25$ U $\quad<25 \quad$ JU $Q \quad$ ug/L

$<50$ U ug/L

$<10$ U ug/L

$<10$ U ug/L

$<10 \quad \mathrm{U} \quad<10 \quad \pi \quad \mathrm{Q} \quad \mathrm{ug} / \mathrm{L}$

$<10 \quad \mathrm{U} \quad<10 \quad \pi \mathrm{Q} \quad \mathrm{ug} / \mathrm{L}$

$<20$ U ug/L

$<500 \quad \mathrm{U} \quad \mathrm{ug} / \mathrm{L}$

$<10 \quad \mathrm{U} \quad \mathrm{ug} / \mathrm{L}$

$<50$ U ug/L

$<50 \quad \mathrm{U} \quad \mathrm{ug} / \mathrm{L}$

$<.1 \quad \mathrm{U} \quad \mathrm{ug} / \mathrm{L}$

$<10$ U ug/L

24.8

$<200 \quad \mathrm{U}$

$<200$

U ug/L

$<25$ U $<25$ JU $Q \quad$ ug $/ \mathrm{L}$

$<10$ U $<10$ JU $Q$ ug/L

$<100 \quad \mathrm{U}$

$\mathrm{ug} / \mathrm{L}$

$<10 \quad \mathrm{U} \quad \mathrm{ug} / \mathrm{L}$

Note: Concentrations in bold exceed the Drinking Water Standards tisted in Appendix A. Units are for all four quarters. 
WELL: TNX 2D

\section{ANALYTICAL DATA}

\section{Analvte}

Arsenic, total recoverable

Barium, total recoverable

Benzene

Benzo(a)anthracene

Benzo(a)pyrene

Benzo(b)fluoranthene

Benzo(g,h,i)perylene

Benzo(k)fluoranthene

Benzyl alcohol

Beryllium, total recoverable

Bis(2-chloroethoxy) methane

$\mathrm{Bis}$ (2-chloroethyl) ether

Bis(2-ethylhexyl) phthalate

Bromodichloromethane

Bromoform

Bromomethane (Methyl bromide)

Butylbenzyl phthalate

Cadmium, total recoverable

Carbazole

Carbon disulfide

Chlorobenzene

Chlorobenzilate

Chloroethane

Chloroethene (Vinyl chloride)

Chloromethane (Methyl chloride)

Chloroprene

Chromium, total recoverable

Chrysene

Cobalt, total recoverable

Copper, total recoverable

Cyanide

Di-n-butyl phthalate

Di-n-octyl phthalate

Diallate

Dibenz(a,h)anthracene

Dibenzofuran

Dibromochioromethane

Dibromomethane (Methylene bromide)

Dichlorodifluoromethane

Dichloromethane (Methylene chloride)

Dieldrin

Diethyl phthalate

Dimethoate

\section{Mod $201998 \mathrm{Mad}$}

$301928 \mathrm{Mod}$

401998

$<10 \quad \mathrm{U}$

26.7

$<5 \quad \mathrm{U}$

$<10 \quad \mathrm{U}$

$<10 \quad \mathrm{U}$

$<10 \quad \mathrm{U}$

$<10 \quad \mathrm{U}$

$<25 \quad \mathrm{U}$

$<10 \quad \mathrm{U}$

$<10$ U

$<10 \quad \mathrm{U}$

$<10 \quad U$

$<10 \quad$ U

$<5 \quad$ U

$<5 \quad U$

$<5 \quad \mathrm{U}$

$<20$ U

$<10 \quad U$

$<10 \quad \mathrm{U}$

$<5 \quad$ U

$<5 \quad U$

$<10 \quad \mathrm{U}$

$<5 \quad U$

$<5 \quad \mathrm{U}$

$<50$ U

$<10 \quad U$

$<10 \quad \mathrm{U}$

$<20$ U

$<20$ U

$<10 \quad \mathrm{U}$

$<10 \quad U$

$<20$

U

$<10 \quad U$

$<10 \quad \mathrm{U}$

$<5 \quad$ U

$<5 \quad$ U

$<5 \quad U$

$<4.68$ U V

.245

$<10$

U
$<10$

$<10$

$<10$

$<10$

$$
<10
$$

$<10$

$<10$

$<10$

$<10$

JU $Q$

JU Q

JU Q

JU Q

JU Q

厅U $Q$

JU Q

JU $Q$

JU Q

$\mathrm{ug} / \mathrm{L}$

$\mathrm{ug} / \mathrm{L}$

ug/L

$\mathrm{ug} / \mathrm{L}$

ug/L

$\mathrm{ug} / \mathrm{L}$

$u g / L$

$\mathrm{ug} / \mathrm{L}$

$<10$ JU Q ug/L

$\mathrm{ug} / \mathrm{L}$

$\mathrm{ug} / \mathrm{L}$

ug/L

$u g / L$

$<10 \quad \mathrm{U} \quad \mathrm{ug} / \mathrm{L}$

$\mathrm{ug} / \mathrm{L}$

ug $/ \mathrm{L}$;

$\mathrm{ug} / \mathrm{L}$

$\mathrm{ug} / \mathrm{L}$

$\mathrm{ug} / \mathrm{L}$

$<10 \quad$ JU $Q \quad$ ug/L

$\mathrm{ug} / \mathrm{L}$

$\mathrm{ug} / \mathrm{L}$

ug/L

$<3.43 \quad \mathrm{~J} \quad$ IQ $\quad \mathrm{ug} / \mathrm{L}$

$<10$ JU Q ug/L

$<10$ U ug/L

$<10$ JU Q ug/L

$<10 \quad$ JU $Q \quad u g / L$

$\mathrm{ug} / \mathrm{L}$

$\mathrm{ug} / \mathrm{L}$

$\mathrm{ug} / \mathrm{L}$

ug/L

$\mathrm{ug} / \mathrm{L}$

$<10 \quad$ JU $Q \quad u g / L$

$<10 \quad \mathrm{U}$

$\mathrm{ug} / \mathrm{L}$

Note: Concentrations in bold exceed the Drinking Water Standards listed in Appendix A. Units are for all four quarters. 
WELL: TNX 2D

ANALYTICAL DATA

Analyte

Dimethyl phthalate

Diphenylamine

Disulfoton

Endosulfan I

Endosulfan II

Endosulfan sulfate

Endrin

Endrin aldehyde

Ethyl methacrylate

Ethyl methanesulfonate

Ethylbenzene

Fluoranthene

Fluorene

Heptachlor

Heptachlor epoxide

Hexachlorobenzene

Hexachlorobutadiene

Hexachlorocyclopentadiene

Hexachlorodibenzo-p-dioxins

Hexachlorodibenzo-p-furans

Hexachloroethane

Indeno(1,2,3-c,d)pyrene

Iodomethane (Methyl iodide)

Iron, total recoverable

Isobutyl alcohol

Isodrin

Isophorone

Isosafrole

Kepone

Lindane

Methacrylonitrile

Methapyrilene

Methoxychlor

Methyl ethyl ketone

Methyl isobutyl ketone

Methyl methacrylate

Methyl methanesulfonate

N-Nitrosodi-n-butylamine

$\mathrm{N}$-Nitrosodiethylamine

$\mathrm{N}$-Nitrosodimethylamine

$\mathrm{N}$-Nitrosodiphenylamine

$\mathrm{N}$-Nitrosodipropylamine

$\mathrm{N}$-Nitrosomethylethylamine

\section{Mod 201998 Mod}

$<10$

$\mathrm{U}$

$<$

$$
<
$$$$
<.2
$$$$
<
$$$$
<.2 \quad \mathrm{U}
$$$$
<.2 \quad \mathrm{U}
$$$$
<5 \quad \text { U }
$$$$
<5 \quad U
$$$$
<10 \quad \mathrm{U}
$$$$
<10 \quad U
$$$$
<.1 \quad \mathrm{U}
$$$$
<.1 \quad \mathrm{U}
$$$$
<20 \text { U }
$$$$
<10 \quad U
$$$$
<10 \quad \mathrm{U}
$$$$
<.0011 \mathrm{U}
$$$$
<.00074 \mathrm{U}
$$$$
<10 \quad U
$$$$
<20 \text { U }
$$$$
<5 \quad \mathrm{U}
$$

65

$<1500 \quad U$

J E

$<10 \quad \mathrm{U}$

$<.1 \quad U$

$<500 \quad \mathrm{U}$

$<1 \quad$ U

$<10$ U

$<5 \quad \mathrm{U}$

$<50 \quad \mathrm{U}$

$<10$

$<10$

$<10$

$<10$

U

$<10$

JU Q ug/L

$<10$

JU $Q$ ug/L

ug $/$

$\mathrm{ug} / \mathrm{L}$

JU Q ug/L

J $Q$ ug/

J Q ug/L

$\mathrm{ug} / \mathrm{L}$

ug/L

$<10$

JU $Q \quad u g / L$

$<10$

JU $Q \quad u g / L$

ug/L

372

$\mathrm{ug} / \mathrm{L}$; ug/L

ug/L

$<10$ U

$<10$

$<10$

$<10$

JU

U

ug/L

ug/L

ug/L

ug/L

$\mathrm{ug} / \mathrm{L}$

$<10$ U ug/L

$\mathrm{ug} / \mathrm{L}$

ug/L

ug $/ \mathrm{L}$

ug/L

$<10 \quad \mathrm{U} \quad \mathrm{ug} / \mathrm{L}$

$<10$ U ug/L

$<10$ U ug/L

$<10 \quad U$

$<20 \quad \mathrm{U}$

$<25$

$<10$

JU $Q \quad u g / L$

$<20$
$<10$

$<10$
$J U$ Q ug/L

JU Q ug/L

$\mathrm{U} \quad \mathrm{ug} / \mathrm{L}$

Note: Concentrations in bold exceed the Drinking Water Standards listed in Appendix A. Units are for sll four quarters. 
WELL: TNX 2D

ANALYTICAL DATA

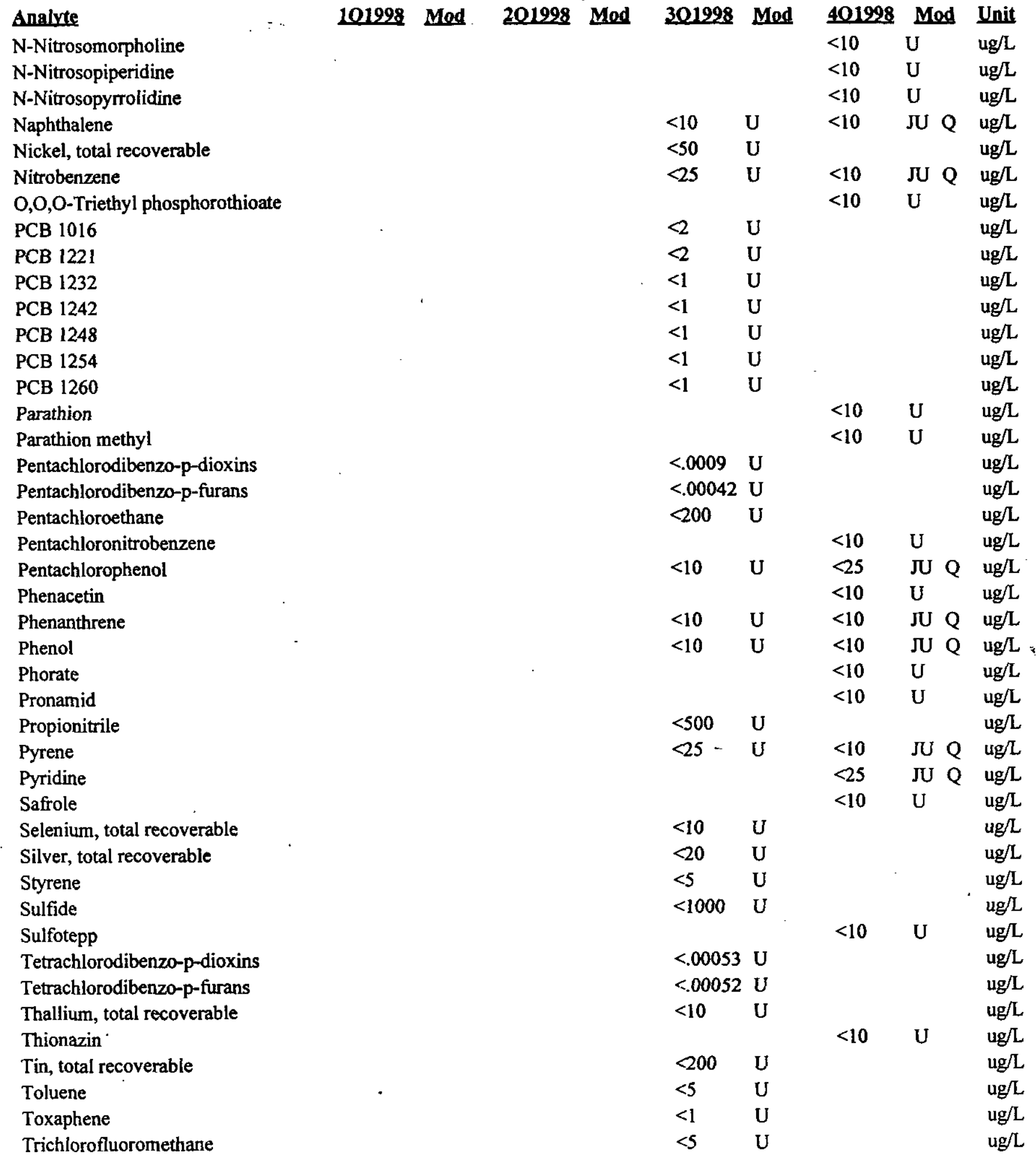

Note: Concentrations in bold exceed the Drinking Water Standards listed in Appendix A. Units are for all four quarters. 
WELL: TNX 2D

\section{ANALYTICAL DATA}

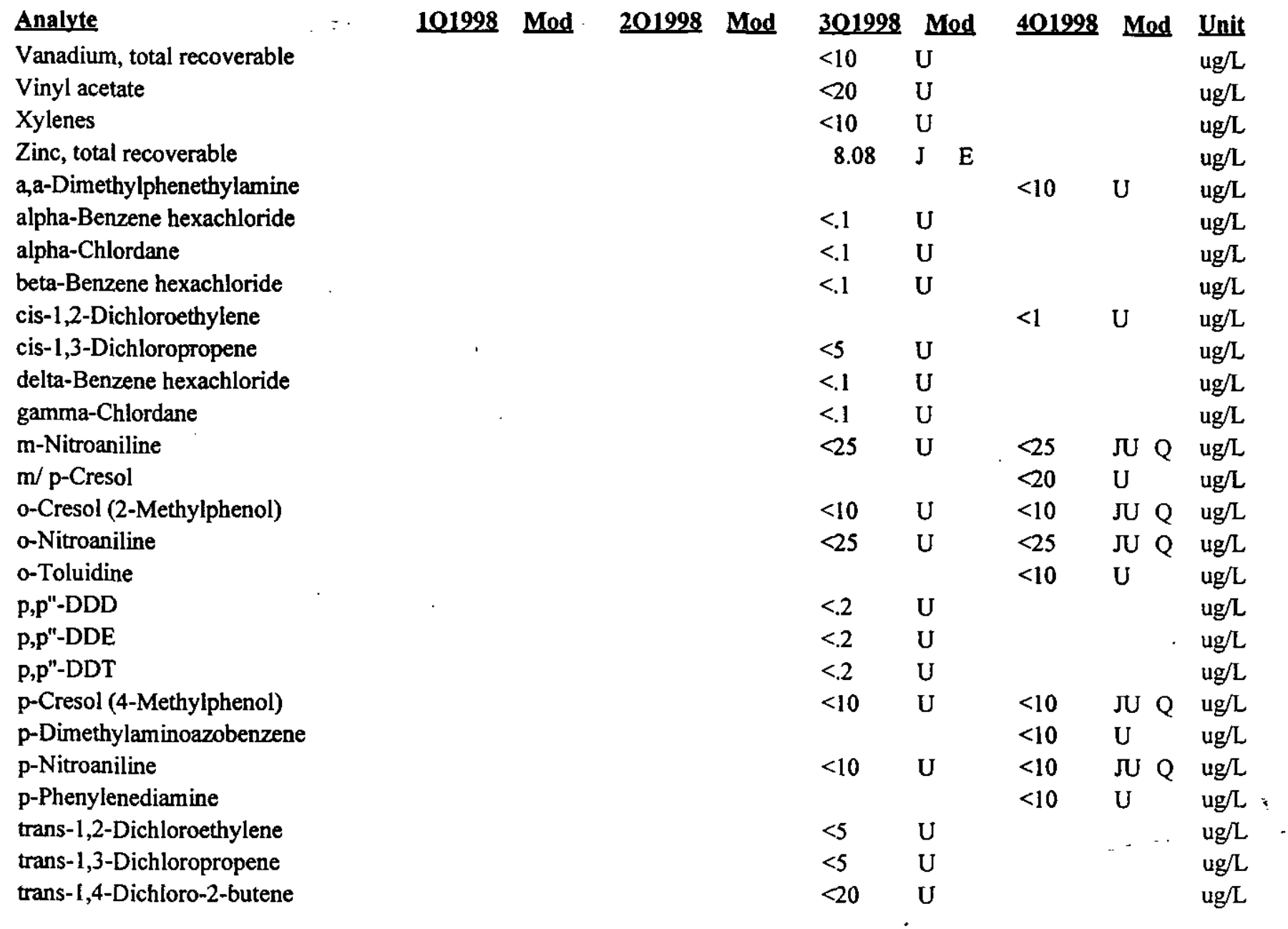


WELL: TNX 3D

SRS Coord. Lat/Longitude Screen Zone Elevation Top of Standpipe Top of Casing Casing Pump Screen Zone N $71236.733 .211 \mathrm{Deg} \mathrm{N} 104.9-84.9 \mathrm{ftmsl} 154.5 \mathrm{ftmsl} 154.3 \mathrm{ft} \mathrm{msl} 4$ " STL $\mathrm{S}$ Unconfined E 17043.1 81.760 Deg W.

$104.9-84.9 \mathrm{ft} \mathrm{msl} \quad 154.5 \mathrm{ft} \mathrm{msl} 154.3 \mathrm{ft} \mathrm{msl} 4$ " STL S Unconfined

SAMPLE DATE

FIELD DATA

\section{Analvte}

Depth to water

$\mathrm{pH}$

Sp. Conductance

Water temperature

Alkalinity as $\mathrm{CaCO} 3$

Phenolpthalein Alkalinity

Turbidity

Volumes purged

Sampling codes

\section{ANALYTICAL DATA}

\section{Analvte}

1,1,1,2-Tetrachloroethane

1,1,1-Trichloroethane

1,1,2,2-Tetrachloroethane

1,1,2-Trichloroethane

1,1-Dichloroethane

1,1-Dichloroethylene

1,2,3-Trichloropropane

1,2,4-Trichlorobenzene

1,2-Dibromo-3-chloropropane

1,2-Dibromoethane

1,2-Dichlorobenzene

1,2-Dichloroethane

1,2-Dichloropropane

1,3,5-Trinitrobenzene

1,3-Dichlorobenzene

1,3-Dinitrobenzene

1,4-Dichlorobenzene

1,4-Dioxane

1,4-Naphthoquinone

1-Naphthylamine

2,2-Oxybis(1-chloropropane)

2,3,4,6-Tetrachlorophenol

2,3,7,8-TCDD

$2,4,5-\mathrm{T}$

2,4,5-TP (Silvex)

2,4,5-Trichlorophenol

2,4,6-Trichlorophenol $\begin{array}{lll}03 / 05 / 98 & 05 / 11 / 98 & 08 / 04 / 98\end{array}$

$12 / 04 / 98$

201998

53.2

301998

56.45

.752785

LNS

.706281

LNS

\section{Mod 201998 Mod}

301998 Mod

401998 Mod Unit

$<5 \quad \mathrm{U}$

$<.924 \mathrm{U}$

$<.462 \mathrm{U}$

$<25$

$<25$

$<25$

$<25$

$<25$

$<25$

$<10$

$<25$

$<25$

$<25$

$<25$

$<25$

$<25 \quad$ U

(

$401998 \quad$ Unit

58.02

4.9

$110 \mathrm{uS} / \mathrm{cm}$

23.4 deg. C

$4 \mathrm{mg} / \mathrm{L}$

$0 \mathrm{mg} / \mathrm{L}$

4.2

.133953

NX

NTU

gallons

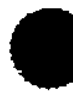


WELL: TNX 3D

\section{ANALYTICAL DATA}

\section{Analyte}

2,4-Dichlorophenol

2,4-Dichlorophenoxyacetic acid

2,4-Dimethyl phenol

2,4-Dinitrophenol

2,4-Dinitrotoluene

2,6-Dichlorophenol

2,6-Dinitrotoluene

2-Acetylaminofluorene

2-Chloronaphthalene

2-Chlorophenol

2-Hexanone

2-Methyl-4,6-dinitrophenol

2-Methylnaphthalene

2-Naphthylamine

2-Nitrophenol

2-Picoline

2-sec-Butyl-4,6-dinitrophenol

3,3"-Dichlorobenzidine

3,3"-Dimethylbenzidine

3-Methylcholanthrene

4-Aminobiphenyl

4-Bromophenyl phenyl ether

4-Chloro-m-cresol

4-Chloroaniline

4-Chlorophenyl phenyl ether

4-Nitrophenol

4-Nitroquinoline-1-oxide

5-Nitro-o-toluidine

7,12-Dimethylbenz(a)anthracene

Acenaphthene

Acenaphthylene

Acetone

Acetonitrile (Methyl cyanide)

Acetophenone

Acrolein

Acrylonitrile

Aldrin

Allyl chloride

Aluminum, total recoverable

Aniline

Anthracene

Antimony, total recoverable

Aramite
101998 Mod. 201998 Mod 301998 Mod 401998 Mod Unit

$<10 \quad$ U $\quad<10 \quad$ JU $Q \quad$ ug/L

$<.2$ UJ $\mathrm{O} \quad \mathrm{ug} / \mathrm{L}$

$\begin{array}{llllll}<10 & \mathrm{U} & <10 & \mathrm{JU} & \mathrm{Q} & \mathrm{ug} / \mathrm{L}\end{array}$

$<25 \quad \mathrm{U} \quad<25 \quad \mathrm{JU} \quad \mathrm{Q} \quad \mathrm{ug} / \mathrm{L}$

$\begin{array}{llllll}<10 & \mathrm{U} & <10 & \mathrm{U} & \mathrm{Q} & \mathrm{ug} / \mathrm{L}\end{array}$

$\begin{array}{lllll}<10 & \mathrm{U} & <10 & \mathrm{U} & \mathrm{ug} / \mathrm{L} \\ & <10 & \mathrm{JU} Q & \mathrm{ug} / \mathrm{L}\end{array}$

$<10 \quad \mathrm{U} \quad \mathrm{ug} / \mathrm{L}$

$<10 \quad \mathrm{U} \quad<10 \quad$ JU $\mathrm{Q} \quad$ ug/L

$\begin{array}{llllll}<10 & \mathrm{U} & <10 \quad \text { JU } & \mathrm{Q} & \mathrm{ug} / \mathrm{L}\end{array}$

$<25$ U ug/L

$<25 \quad$ U $\quad<25 \quad$ JU $Q \quad$ ug/L

$<10 \quad \mathrm{U} \quad<10 \quad \mathrm{JU} Q \quad \mathrm{Qg} / \mathrm{L}$

$<10 \quad \mathrm{U} \quad \mathrm{ug} / \mathrm{L}$

$<10 \quad \mathrm{U} \quad<10 \quad \pi \mathrm{Q} \quad \mathrm{ug} / \mathrm{L}$

$<10 \quad \mathrm{U} \quad \mathrm{ug} / \mathrm{L}$

$<10 \quad \mathrm{U} \quad$ ug/L

$<10 \quad \mathrm{U} \quad<10 \quad \mathrm{JU} \mathrm{Q} \quad \mathrm{ug} / \mathrm{L}$

$<20 \quad$ U ug/L

$<10 \quad \mathrm{U} \quad \mathrm{ug} / \mathrm{L}$

$<10 \quad \mathrm{U} \quad \mathrm{ug} / \mathrm{L}$

$<10 \quad \mathrm{U} \quad<10 \quad$ JU $\mathrm{Q} \quad \mathrm{ug} / \mathrm{L}$

$<10 \quad \mathrm{U} \quad<10 \quad$ J $Q \quad$ ug/L

$<10 \quad \mathrm{U} \quad<10 \quad \pi \mathrm{U}$ Q $\mathrm{ug} / \mathrm{L}$

$\begin{array}{llllll}<10 & \mathrm{U} & <10 & \mathrm{~J} & \mathrm{Q} & \mathrm{ug} / \mathrm{L}\end{array}$

$\begin{array}{llllll}<5 & \mathrm{U} & <25 & \mathrm{U} & \mathrm{Q} & \mathrm{ug} / \mathrm{L}\end{array}$

$<50 \quad \mathrm{U} \quad \mathrm{ug} / \mathrm{L}$

$<10$ U ug/L

$<10$ U ug/L

$<10 \quad \mathrm{U} \quad<10 \quad \mathrm{JU} \quad \mathrm{Q} \quad \mathrm{ug} / \mathrm{L}$

$<10 \quad \mathrm{U} \quad<10 \quad$ JU $Q \quad$ ug/L

$<100 \quad \mathrm{U} \quad \mathrm{ug} / \mathrm{L}$

$<2500 \mathrm{U} \quad \mathrm{ug} / \mathrm{L}$

$<10 \quad \mathrm{U} \quad \mathrm{ug} / \mathrm{L}$

$<250 \quad \mathrm{u} \quad u g / \mathrm{L}$

$<250 \quad \mathrm{U} \quad \mathrm{ug} / \mathrm{L}$

$<.1 \quad \mathrm{U} \quad \mathrm{ug} / \mathrm{L}$

$<50$ U ug/L

$755 \quad 296 \quad 717 \quad$ ug/L

$<25 \quad \mathrm{U} \quad<25 \quad \mathrm{JU} Q \quad \mathrm{ug} / \mathrm{L}$

$<10 \quad \mathrm{U} \quad<10 \quad \mathrm{U} \quad \mathrm{Q} \quad \mathrm{ug} / \mathrm{L}$

$<100 \quad \mathrm{U} \quad \mathrm{ug} / \mathrm{L}$

$<10 \quad$ U ug/L

Note: Concentrations in bold exceed the Drinking Water Standards listed in Appendix A. Units are for all four quarters. 
WELL: TNX 3D

ANALYTICAL DATA

Analyte

Arsenic, total recoverable

Barium, total recoverable

Benzene

Benzo(a)anthracene

Benzo(a)pyrene

Benzo(b)fluoranthene

Benzo(g,h,i)perylene

Benzo(k)fluoranthene

Benzyl alcohol

Beryllium, total recoverable

Bis(2-chloroethoxy) methane

Bis(2-chloroethyl) ether

Bis(2-ethylhexyl) phthalate

Bromodichloromethane

Bromoform

Bromomethane (Methyl bromide)

Butylbenzyl phthalate

Cadmium, total recoverable

Carbazole

Carbon disulfide

Chlorobenzene

Chlorobenzilate

Chloroethane

Chloroethene (Vinyl chloride)

Chloromethane (Methyl chloride)

Chloroprene

Chromium, total recoverable

Chrysene

Cobalt, total recoverable

Copper, total recoverable

Cyanide

Di-n-butyl phthalate

Di-n-octyl phthalate

Diallate

Dibenz(a,h)anthracene

Dibenzofuran

Dibromochloromethane

Dibromomethane (Methylene bromide)

Dichlorodifluoromethane

Dichloromethane (Methylene chloride)

Dieldrin

Diethyl phthalate

Dimethoate
101998 Mod . 201998 Mod

301998

Mod

$<10 \quad \mathrm{U}$

60.6

$<25$

$<10$

$<10$

$<10$

$<10$

$<25$

$<10$

$<10$

$<10$

4.05

$<10$

$<25$

$<25$

$<25$

$<20$

3

$<10$

$<25$

$<25$

$<50 \quad U$

$<25 \quad$ U

$<25$ U

$<250 \quad \mathrm{U}$

$<10 \quad \mathrm{U}$

$<10$.

8.69

$<20 \quad U$

$<10 \quad \mathrm{U}$

$<10 \quad \mathrm{U}$

$<20 \quad$ U

$<10 \quad \mathrm{U}$

$<10 \quad \mathrm{U}$

$<25 \quad \mathrm{U}$

$<25$ U

$<25 \quad U$

$<50 \quad \mathrm{U}$

$<.2 \quad \mathrm{U}$

$<10 \quad \mathrm{U}$
401998 Mod Unit

ug/L

$\mathrm{ug} / \mathrm{L}$

$\mathrm{ug} / \mathrm{L}$

$<10 \quad$ JU Q ug/L

$<10 \quad$ J $Q$ ug/L

$<10 \quad$ JU $Q \quad$ ug/L

$<10$ JU $Q \quad$ ug/L

$<10$ JU $Q \quad u g / L$

$<10$ JU Q ug/L

$\mathrm{ug} / \mathrm{L}$

JU $Q \quad$ ug/L

$\begin{array}{ccccc}\text { E } & <10 & J U & \mathrm{Q} & \mathrm{ug} / \mathrm{L} \\ & 1.95 & \mathrm{~J} & \mathrm{IQ} & \mathrm{ug} / \mathrm{L}\end{array}$

ug $/ \mathrm{L}$

$\operatorname{ug} / \mathrm{L}$

$\mathrm{ug} / \mathrm{L}$

$<10$ JU Q ug/L

ug/L

$\mathrm{ug} / \mathrm{L}$

$\mathrm{ug} / \mathrm{L}$

$\mathrm{ug} / \mathrm{L}$

$<10 \quad U \quad u g / L$

$\mathrm{ug} / \mathrm{L}$

$\mathrm{ug} / \mathrm{L}$ :

ug/L

ug/L

ug/L

$<10 \quad$ JU $Q \quad$ ug/L

$u g / L$

$u g / L$

ug/L

$<10 \quad$ JU $Q \quad$ ug/L

$<10$ JU Q ug/L

$<10$ U ug/L

$<10 \quad$ JU $Q \quad$ ug/L

$<10 \quad$ JU $\mathrm{Q} \quad$ ug/L

$\mathrm{ug} / \mathrm{L}$

ug/L

ug/L

$\mathrm{ug} / \mathrm{L}$

ug/L

$<10 \quad$ JU Q ug/L

$<10$ U ug/L

Note: Concentrations in bold exceed the Drinking Water Standards listed in Appendix A. Units are for all four quarters. 
WELL: TNX 3D

\section{ANALYTICAL DATA}

\section{Analyte}

Dimethyl phthalate

Diphenylamine

Disulfoton

Endosulfan I

Endosulfan II

Endosulfan sulfate

Endrin

Endrin aldehyde

Ethyl methacrylate

Ethyl methanesulfonate

Ethylbenzene

Fluoranthene

Fluorene

Heptachlor

Heptachlor epoxíde

Hexachlorobenzene

Hexachlorobutadiene

Hexachlorocyclopentadiene

Hexachlorodibenzo-p-dioxins

Hexachlorodibenzo-p-furans

Hexachloroethane

Indeno(1,2,3-c,d)pyrene

Iodomethane (Methyl iodide)

Iron, total recoverable

Isobutyl alcohol

Isodrin

Isophorone

Isosafrole

Kepone

Lindane

Methacrylonitrile

Methapyrilene

Methoxychlor

Methyl ethyl ketone

Methyl isobutyl ketone

Methyl methacrylate

Methyl methanesulfonate

$\mathrm{N}$-Nitrosodi-n-butylamine

N-Nitrosodiethylamine

$\mathrm{N}$-Nitrosodimethylamine

$\mathrm{N}-\mathrm{Nitrosodiphenylamine}$

$\mathrm{N}$-Nitrosodipropylamine

$\mathrm{N}$-Nitrosomethylethylamine

\section{Mod 201998 Mod 301998 Mod 401998 Mod Unit}

$<10 \quad \mathrm{U} \quad<10 \quad \pi \quad \mathrm{Q} \quad \mathrm{ug} / \mathrm{L}$

$<10 \quad \mathrm{U} \quad \mathrm{ug} / \mathrm{L}$

$<10 \quad \mathrm{U} \quad \mathrm{ug} / \mathrm{L}$

$<.1 \quad \mathrm{U} \quad \mathrm{ug} / \mathrm{L}$

$<.2$ U $\mathrm{ug} / \mathrm{L}$

$<.2$ U $\quad$ ug/L

$<.2$ U $\quad \mathrm{ug} / \mathrm{L}$

$<.2$ U ug/L

$<25$ U ug/L

$<10 . \quad \mathrm{U} \quad \mathrm{ug} / \mathrm{L}$

$<25$ U $\quad$ ug/L

$<10 \quad \mathrm{U} \quad<10 \quad \pi \mathrm{U} Q \quad \mathrm{Qg} / \mathrm{L}$

$<10 \quad \mathrm{U},<10$ JU $\mathrm{Q} \quad \mathrm{ug} / \mathrm{L}$

$<.1$ U $\mathrm{ug} / \mathrm{L}$

$<.1 \quad \mathrm{U} \quad \mathrm{ug} / \mathrm{L}$

$<20 \quad \mathrm{U} \quad<10 \quad$ JU $\mathrm{Q} \quad \mathrm{ug} / \mathrm{L}$

$<10 \quad$ U $\quad<10 \quad$ JU $Q \quad$ ug $/ \mathrm{L}$

$<10 \quad$ U $\quad<10$ JU $Q \quad$ ug/L

$<.00094 \mathrm{U} \quad \mathrm{ug} / \mathrm{L}$

$<.00062 \mathrm{U} \quad \mathrm{ug} / \mathrm{L}$

$<10 \quad \mathrm{U} \quad<10 \quad$ JU $Q \quad$ ug/L

$<20 \quad \mathrm{U} \quad<10 \quad \pi \quad \mathrm{Q} \quad \mathrm{ug} / \mathrm{L}$

$<25 \mathrm{U} \quad \mathrm{ug} / \mathrm{L}$

120

$\begin{array}{llll}79.3 & J & E & 443\end{array}$

ug/L :

$\begin{array}{lllll}<500 \quad \mathrm{U} & <10 \quad \mathrm{U} & \mathrm{ug} / \mathrm{L} \\ & \mathrm{ug} / \mathrm{L}\end{array}$

$<10 \quad \mathrm{U} \quad<10 \quad$ JU $Q \quad$ ug/L

$<10 \quad \mathrm{U} \quad \mathrm{ug} / \mathrm{L}$

$<10$ U ug/L

$<.1$ U $\quad$ ug $/ \mathrm{L}$

$<2500$ U ug/L

. $<10 \quad \mathrm{U} \quad \mathrm{ug} / \mathrm{L}$

$<1 \quad$ U $\quad u g / L$

$<50$ U ug/L

$<25$ U $\mathrm{ug} / \mathrm{L}$

$<250$ U ug/L

$<10 \quad$ U ug/L

$<10 \quad$ U $\quad \mathrm{ug} / \mathrm{L}$

$<10 \quad \mathrm{U} \quad \mathrm{ug} / \mathrm{L}$

$<10 \quad$ U $\quad<25 \quad$ J $Q \quad$ ug/L

$<20 \quad$ U $\quad<10 \quad$ JU $Q \quad u g / L$

$<20 \quad \mathrm{U} \quad<10 \quad$ JU $\quad \mathrm{Q} \quad \mathrm{ug} / \mathrm{L}$

$<10 \quad \mathrm{U} \quad \mathrm{ug} / \mathrm{L}$

Note: Concentrations in bold exceed the Drinking Water Standards listed in Appendix A. Units are for all four quarters. 


\section{ANALYTICAL DATA}

\section{Analyte}

$\mathrm{N}$-Nitrosomorpholine

$\mathrm{N}$-Nitrosopiperidine

$\mathrm{N}-\mathrm{Nitrosopyrrolidine}$

Naphthalene

Nickel, total recoverable

Nitrobenzene

O,O,O-Triethyl phosphorothioate

PCB 1016

PCB 1221

PCB 1232

PCB 1242

PCB 1248

PCB 1254

PCB 1260

Parathion

Parathion methyl

Pentachlorodibenzo-p-dioxins

Pentachlorodibenzo-p-furans

Pentachloroethane

Pentachloronitrobenzene

Pentachlorophenol

Phenacetin

Phenanthrene

Phenol

Phorate

Pronamid

Propionitrile

Pyrene

Pyridine

Safrole

Selenium, total recoverable

Silver, total recoverable

Styrene

Sulfide

Sulfotepp

Tetrachlorodibenzo-p-dioxins

Tetrachlorodibenzo-p-furans

Thallium, total recoverable

Thionazin

Tin, total recoverable

Toluene

Toxaphene

Trichlorofluoromethane
101998 Mod 201998 Mod 301998 Mod 401998 Mod Unit

$<10 \quad \mathrm{u} \quad \mathrm{ug} / \mathrm{L}$

$<10 \quad \mathrm{U} \quad \mathrm{ug} / \mathrm{L}$

$<10 \quad U \quad$ ug/L

$<10 \quad \mathrm{U} \quad<10 \quad \mathrm{JU} \mathrm{Q} \quad \mathrm{ug} / \mathrm{L}$

$<50$ U ug/L

$<25 \quad \mathrm{U} \quad<10 \quad$ JU $Q \quad$ ug/L

$<10$ U ug/L

$<$ U ug/L

$<\quad \mathrm{U}$

$<1 \quad \mathrm{U}$

$<1 \quad \mathrm{U}$

$<1 \quad \mathrm{U}$

$<1 \quad$ U

$<1 \quad U$

$<, 00087 \mathrm{U}$

$<, 00083 \mathrm{U}$

$<1000 \mathrm{U}$

$<10 \quad \mathrm{U}$

$<10$

$<25$

$<10$

$<10 \quad U$

$<10$

$<10$

$<10$

$\mathrm{ug} / \mathrm{L}$

$\mathrm{ug} / \mathrm{L}$

$\mathrm{ug} / \mathrm{L}$

$\mathrm{ug} / \mathrm{L}$

$\mathrm{ug} / \mathrm{L}$

ug/L

$<10 \quad \mathrm{U} \quad$ ug/L

$<10 \quad U \quad u g / L$

$\mathrm{ug} / \mathrm{L}$

ug/L

ug/L

$<10 \quad \mathrm{U}$

$<10$

$<2500 \quad \mathrm{U}$

$<25 \quad \mathrm{U}$

$<10$

$<25$

$<10$

U

ug/L

JU $Q \quad$ ug/L

U ug/L

JU Q ug/L

J $Q$ ug/L ;

$\mathrm{U}$

ug/L

$\mathrm{U} \quad u g / \mathrm{L}$ $\mathrm{ug} / \mathrm{L}$

JU Q ug/L

JU $Q$ ug/L

$<10 \quad U$

U

$\mathrm{ug} / \mathrm{L}$

$u g / L$

$<20 \quad$ U

$<25 \quad \mathrm{U}$

$\mathrm{ug} / \mathrm{L}$

$u g / L$

$<1000 \mathrm{U}$

ug/L

$<10 \quad$ U $\quad$ ug/L

$<.00051 \mathrm{U}$

$<.0005 \mathrm{U}$

$<10 \quad \mathrm{U}$

$<10$

$<200 \quad \mathrm{U}$

$<25 \quad \mathrm{U}$

$<1 \quad U$

$<25 \quad \mathrm{U}$
ug/L

ug/L

ug/L

ug/L

ug/L

$\mathrm{ug} / \mathrm{L}$

$u g / L$

ug/L

Note: Concentrations in bold exceed the Drinking Water Standards listed in Appendix A. Units are for all four quarters. 
WELL: TNX 3D

ANALYTICAL DATA

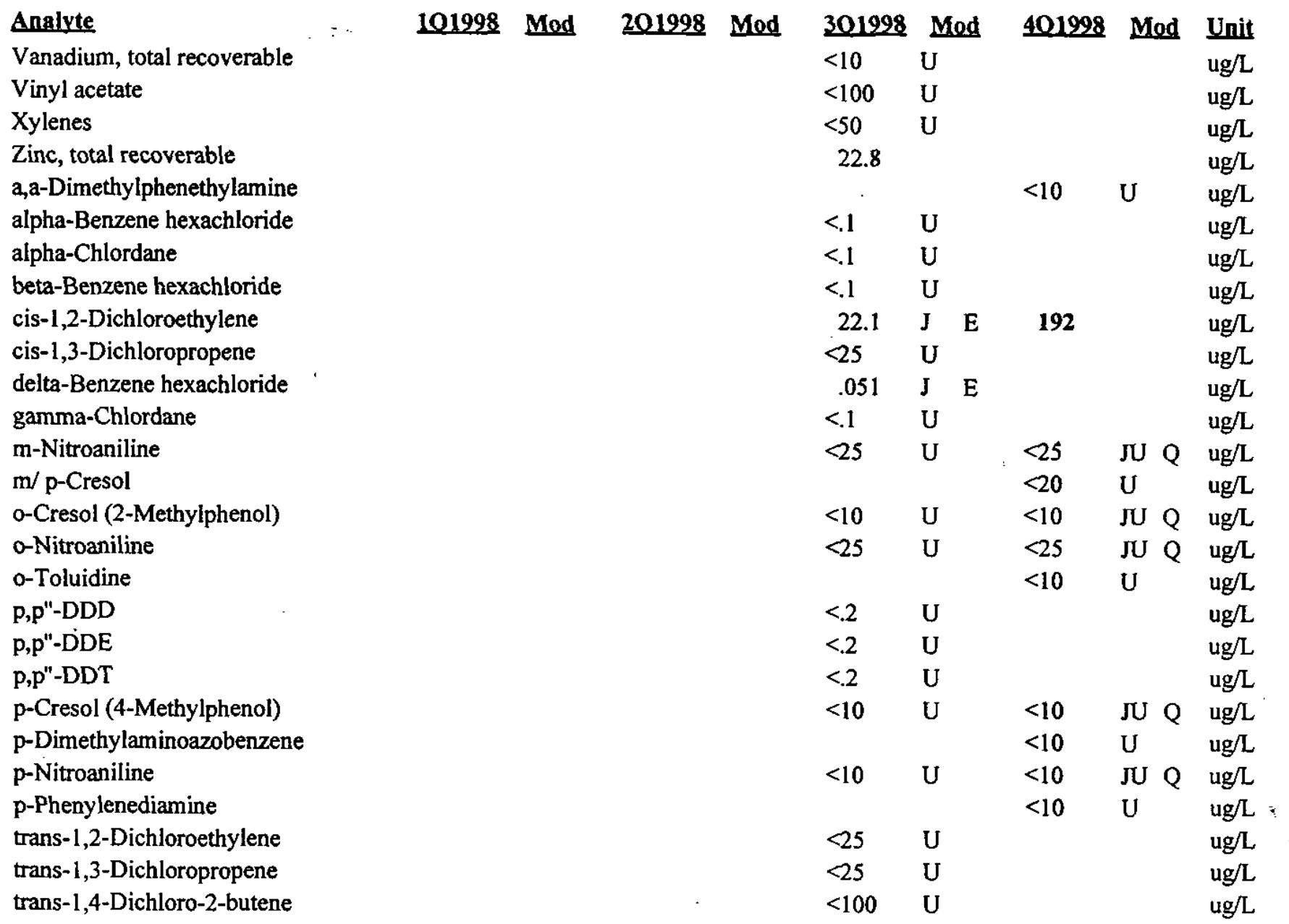

Note: Concentrations in bold exceed the Drinking Water Standards listed in Appendix A. Units are for all four quarters. 
WELL: TNX 4D

\begin{tabular}{|c|c|c|c|c|c|c|c|}
\hline SRS Coord. & Lat/Longitude & Screen Zone Elevation & Top of Standpipe & Top of Casing & Casing & Punp & Screen Zone \\
\hline $\begin{array}{l}\text { N } 71002.7 \\
\text { E } 17223.0\end{array}$ & $\begin{array}{l}33.211 \text { Deg N } \\
81.759 \text { Deg W }\end{array}$ & $105.5-85.5 \mathrm{ft} \mathrm{msl}$ & $150.0 \mathrm{ft} \mathrm{msl}$ & $149.8 \mathrm{ft} \mathrm{msl}$ & $4^{\prime \prime} \mathrm{STL}$ & $\mathrm{s}$ & Unconfined \\
\hline SAMPLE D & ATE & $03 / 02 / 98$ & $05 / 13 / 98$ & $08 / 06 / 98$ & & $12 / 02 / 98$ & \\
\hline
\end{tabular}

\section{FIELD DATA}

\begin{tabular}{|c|c|c|c|c|c|}
\hline Analyte & 101998 & 201998 & 301998 & 401998 & Unit \\
\hline Depth to water & 45.02 & 42.9 & 45.88 & 48.57 & ft BTOS \\
\hline $\mathrm{pH}$ & 4.9 & & 4.9 & 5.2 & \\
\hline Sp. Conductance & 67 & & 45 & 55 & $\mathrm{uS} / \mathrm{cm}$ \\
\hline Water temperature & 19.8 & & 21.4 & 21.9 & deg. C \\
\hline Alkalinity as $\mathrm{CaCO} 3$ & 1 & & 0 & 0 & $\mathrm{mg} / \mathrm{L}$ \\
\hline Phenolpthalein Alkalinity & 0 & & 0 & 0 & $\mathrm{mg} / \mathrm{L}$ \\
\hline Turbidity & 1.8 & & 3.8 & 12.8 & NTU \\
\hline Volumes purged & 3.08357 & $\begin{array}{l}.783565 \\
\text { LNS }\end{array}$ & 2.64823 & .387639 & gallons \\
\hline
\end{tabular}

\section{ANALYTICAL DATA}

\section{Analyte}

1,1,1,2-Tetrachloroethane

1,1,1-Trichloroethane

1,1,2,2-Tetrachloroethane

1,1,2-Trichloroethane

1,1-Dichloroethane

1,1-Dichloroethylene

1,2,3-Trichloropropane

1,2,4-Trichlorobenzene

1,2-Dibromo-3-chloropropane

1,2-Dibromoethane

1,2-Dichlorobenzene

1,2-Dichloroethane

1,2-Dichloropropane

1,3,5-Trinitrobenzene

1,3-Dichlorobenzene

1,3-Dinitrobenzene

1,4-Dichlorobenzene

1,4-Dioxane

1,4-Naphthoquinone

1-Naphthylamine

2,2-Oxybis(1-chloropropane)

2,3,4,6-Tetrachlorophenol

2,3,7,8-TCDD .

2,4,5-T

2,4,5-TP (Silvex)

2,4,5-Trichlorophenol

2,4,6-Trichlorophenol
101998 Mod 201998 Mod 301998 Mod 401998 Mod Unit

$<5 \quad \mathrm{U}$

$\begin{array}{llll}<.462 & \mathrm{U} & <.462 \quad \mathrm{U}\end{array}$

$<5$

$<5 \quad U$

$<5 \quad \mathrm{U}$

$<5 \quad \mathrm{U}$

$<5 \quad$ U

$<5 \quad \mathrm{U}$

$<5 \quad \mathrm{U}$

$<10 \quad \mathrm{U}$

$<5 \quad \mathrm{U}$

$<5 \quad \mathrm{U}$

$<5 \quad U$

$<5 \quad \mathrm{U}$

$<5 \quad \mathrm{U}$

$<5 \quad \mathrm{U}$

$<5 \quad \mathrm{U}$

$<1000 \mathrm{U}$

$<10 \quad$ U

$<.00054 \mathrm{U}$

$<2$ UJ $\mathrm{O}$

$<2$ UJ $\mathrm{O}$

$<10$

$\mathrm{U}$

U

U
U
U
U
U
U
U
U
U
U

U
U
UJ O
U O
U

$<1$

U

ug/L

$\mathrm{ug} / \mathrm{L}$

ug/L

ug/L

ug/L

ug/L

ug/L

$<10$ JU Q ug/L :

ug/L

ug/L

$\mathrm{ug} / \mathrm{L}$

ug/L

$\mathrm{ug} / \mathrm{L}$

$<10 \quad$ U ug/L

$\mathrm{ug} / \mathrm{L}$

$<10 \quad \mathrm{U} \quad$ ug/L

$\mathrm{ug} / \mathrm{L}$

$\mathrm{ug} / \mathrm{L}$

$<10 \quad \mathrm{U} \quad \mathrm{ug} / \mathrm{L}$

$<10 \quad$ U $\quad$ ug/L

$<10$ JU Q ug/L

$<10$ U ug/L

ug/L

$\mathrm{ug} / \mathrm{L}$

$\mathrm{ug} / \mathrm{L}$

Note: Concentrations in bold exceed the Drinking Water Standards listed in Appendix A. Units are for all four quarters. 
WELL: TNX 4D

ANALYTICAL DATA

Analyte

2,4-Dichlorophenol

2,4-Dichlorophenoxyacetic acid

2,4-Dimethyl phenol

2,4-Dinitrophenol

2,4-Dinitrotoluene

2,6-Dichlorophenol

2,6-Dinitrotoluene

2-Acetylaminofluorene

2-Chloronaphthalene

2-Chlorophenol

2-Hexanone

2-Methyl-4,6-dinitrophenol

2-Methylnaphthalene

2-Naphthylamine

2-Nitrophenol

2-Picoline

2-sec-Butyl-4,6-dinitrophenol

3,3"-Dichlorobenzidine

3,3"-Dimethylbenzidine

3-Methylcholanthrene

4-Aminobiphenyl

4-Bromophenyl phenyl ether

4-Chloro-m-cresol

4-Chloroaniline

4-Chlorophenyl phenyl ether

4-Nitrophenol

4-Nitroquinoline-1-oxide

5-Nitro-o-toluidine

7,12-Dimethylbenz(a)anthracene

Acenaphthene

Acenaphthylene

Acetone

Acetonitrile (Methyl cyanide)

Acetophenone

Acrolein

Acrylonitrile

Aldrin

Allyl chloride

Aluminum, total recoverable

Aniline

Anthracene

Antimony, total recoverable

Aramite
101998 Mod 201998 Mod

301998 Mod 401998 Mod Unit

$<10 \quad$ U $\quad<10 \quad$ JU $Q \quad$ ug/L

$<.2$ UJ $\mathrm{O} \quad \mathrm{ug} / \mathrm{L}$

$<10$ U $\quad<10$ JU Q ug/L

$<25 \quad$ U $\quad<25 \quad$ JU $Q \quad$ ug/L

$<10 \quad \mathrm{U} \quad<10 \quad \mathrm{JU} \mathrm{Q} \quad \mathrm{ug} / \mathrm{L}$

$\begin{array}{lllll}<10 & \mathrm{U} & <10 & \mathrm{U} & \mathrm{ug} / \mathrm{L} \\ & <10 & \mathrm{JU} \mathrm{Q} & \mathrm{ug} / \mathrm{L}\end{array}$

$<10 \quad \mathrm{U} \quad \mathrm{ug} / \mathrm{L}$

$<10 \quad \mathrm{U} \quad<10 \quad$ JU $Q$

$<10 \quad \mathrm{U} \quad<10 \quad \pi \quad \mathrm{Q} \quad \mathrm{ug} / \mathrm{L}$

$<5 \quad \mathrm{U} \quad \mathrm{ug} / \mathrm{L}$

$<25 \quad \mathrm{U} \quad<25 \quad$ U $\mathrm{Q} \quad$ ug/L

$<10 \quad \mathrm{U}:<10$ JU $\mathrm{Q} \quad \mathrm{ug} / \mathrm{L}$

$<10$ U ug/L

$<10 \quad \mathrm{U} \quad<10 \quad$ JU $Q \quad$ ug/L

$<10 \quad U \quad$ ug/L

$<10 \quad$ U $\quad u g / L$

$<10 \quad \mathrm{U} \quad<10 \quad \pi \quad \mathrm{Q} \quad \mathrm{ug} / \mathrm{L}$

$<20 \quad \mathrm{U} \quad \mathrm{ug} / \mathrm{L}$

$<10 \quad \mathrm{U} \quad \mathrm{ug} / \mathrm{L}$

$<10 \quad$ U ug/L

$\begin{array}{llllll}<10 & \mathrm{U} & <10 & \mathrm{NU} & \mathrm{Q} & \mathrm{ug} / \mathrm{L}\end{array}$

$<10 \quad \mathrm{U} \quad<10 \quad$ JU $\mathrm{Q} \quad \mathrm{ug} / \mathrm{L}$

$<10 \quad \mathrm{U} \quad<10 \quad \pi \mathrm{U} \quad \mathrm{ug} / \mathrm{L}$

$<10 \quad \mathrm{U} \quad<10 \quad$ JU $Q \quad \mathrm{ug} / \mathrm{L}$

$<25$ U $<25 \quad \mathrm{JU} Q \quad u g / \mathrm{L}$

$<50 \quad$ U $\quad u g / L$

$<10 \quad \mathrm{U} \quad \mathrm{ug} / \mathrm{L}$

$<10 \quad \mathrm{U} \quad \mathrm{ug} / \mathrm{L}$

$<10 \quad \mathrm{U} \quad<10 \quad$ JU $Q \quad$ ug $/ \mathrm{L}$

$<10 \quad$ U $\quad<10 \quad$ JU $Q \quad$ ug/L

$<20$ U ug/L

$<500 \quad \mathrm{U} \quad \mathrm{ug} / \mathrm{L}$

$<10 \quad \mathrm{U} \quad \mathrm{ug} / \mathrm{L}$

$<50$ U ug/L

$<50$ U ug/L

$<.1$ U $\quad$ ug/L

$<10$ U $\quad$ ug/L

137

$<200 \quad \mathrm{U} \quad 276 \quad \mathrm{ug} / \mathrm{L}$

$<25$ U $<25$ JU Q ug/L

$<10 \quad \mathrm{U} \quad<10 . \quad \pi \mathrm{Q} u g / \mathrm{L}$

$<100 \quad \mathrm{U}$

$\mathrm{ug} / \mathrm{L}$

$<10 \quad \mathrm{U} \quad \mathrm{ug} / \mathrm{L}$

Note: Concentrations in bold exceed the Drinking Water Standards listed in Appendix A. Units are for all four quarters. 
WELL: TNX 4D

ANALYTICAL DATA

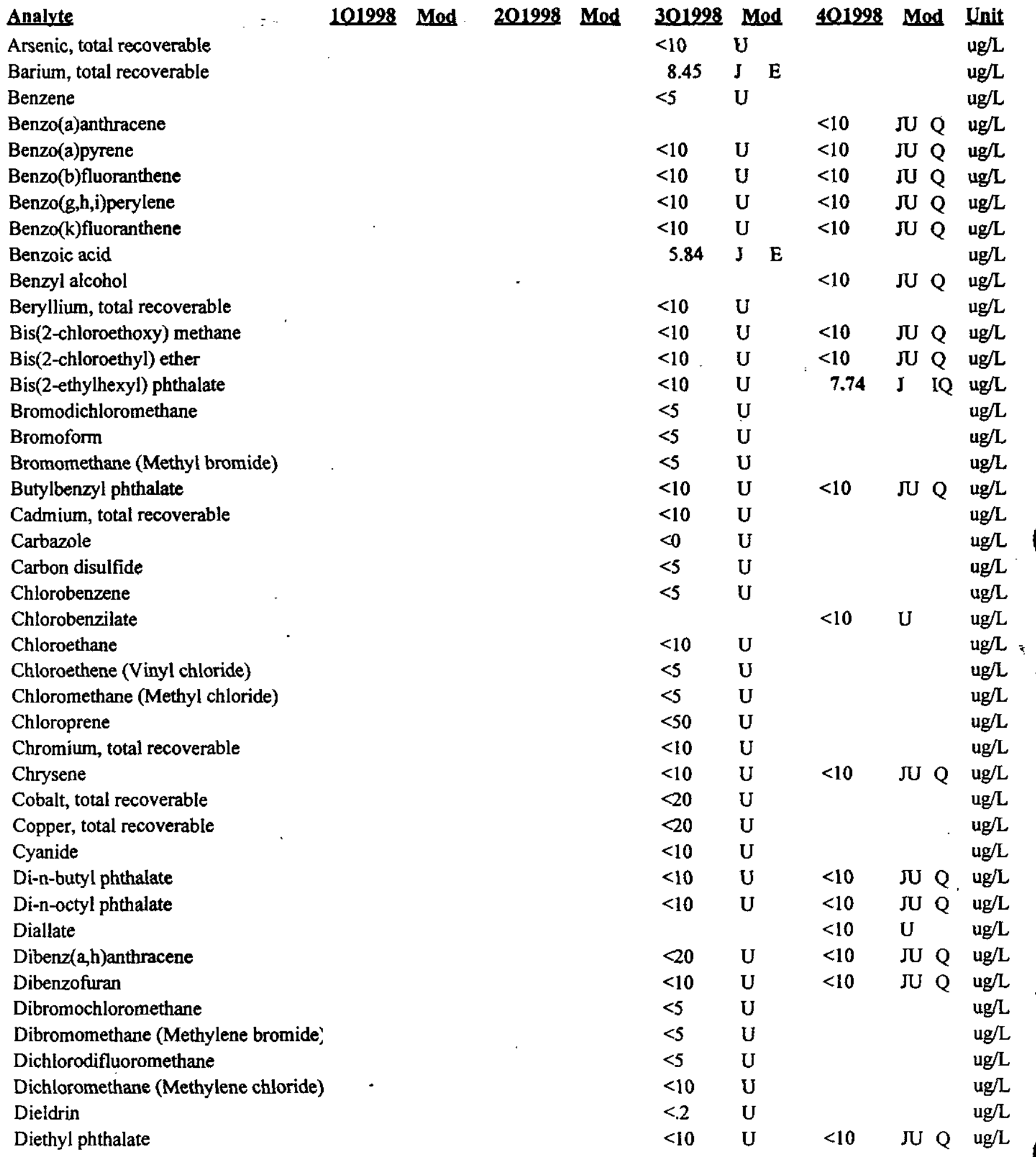

Note: Concentrations in bold exceed the Drinking Water Standards listed in Appendix A. Units are for all four quarters. 
WELL: TNX 4D

ANALYTICAL DATA

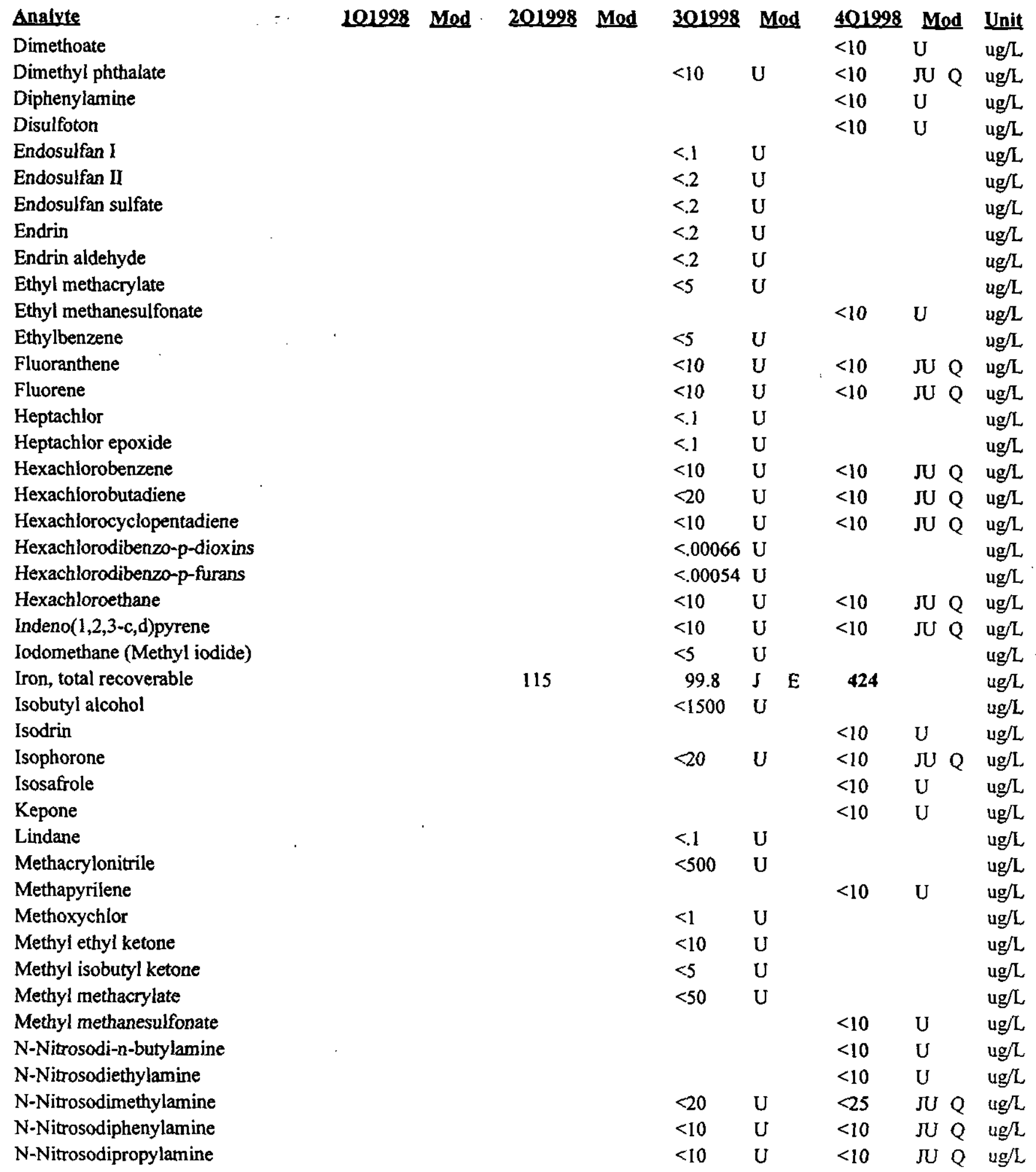

Note: Concentrations in bold exceed the Drinking Water Standards listed in Appendix A. Units are for all four quarters. 
WELL: TNX 4D

\section{ANALYTICAL DATA}

\begin{tabular}{|c|c|c|c|c|c|c|c|c|}
\hline Analyte & 101998 & Mod & $201998 \mathrm{Med}$ & 301998 & Mod & 401998 & Mod & Unit \\
\hline N-Nitrosomethylethylamine & & & & & & $<10$ & $\mathrm{U}$ & $\mathrm{ug} / \mathrm{L}$ \\
\hline N-Nitrosomorpholine & & & & & & $<10$ & $\mathrm{U}$ & $\mathrm{ug} / \mathrm{L}$ \\
\hline N-Nitrosopiperidine & & & & & & $<10$ & $\mathbf{U}$ & $u g / L$ \\
\hline N-Nitrosopyrrolidine & & & & & & $<10$ & $\mathrm{U}$ & $\mathrm{ug} / \mathrm{L}$ \\
\hline Naphthalene & & & & $<20$ & $\mathbf{U}$ & $<10$ & JU $Q$ & ug/L \\
\hline Nickel, total recoverable & & & & $<50$ & U & & & $\mathrm{ug} / \mathrm{L}$ \\
\hline Nitrobenzene & & & & $<10$ & $\mathrm{U}$ & $<10$ & JU $Q$ & $\mathrm{ug} / \mathrm{L}$ \\
\hline O,O,O-Triethyl phosphorothioate & & & & & & $<10$ & $\mathrm{U}$ & $\mathrm{ug} / \mathrm{L}$ \\
\hline PCB 1016 & & & & $<2$ & U & & & $\mathrm{ug} / \mathrm{L}$ \\
\hline PCB 1221 & & & & $<2$ & $\mathrm{U}$ & & & $\mathrm{ug} / \mathrm{L}$ \\
\hline PCB 1232 & & & & $<1$ & $\mathrm{U}$ & & & $\mathrm{ug} / \mathrm{L}$ \\
\hline PCB 1242 & & & & $<1$ & $\mathrm{U}$ & & & $\mathrm{ug} / \mathrm{L}$ \\
\hline PCB 1248 & & & & $<1$ & U & & & ug/L \\
\hline PCB 1254 & & & & $<1$ & $\mathrm{U}$ & & & $\mathrm{ug} / \mathrm{L}$ \\
\hline PCB 1260 & & & & $<1$ & $\mathrm{U}$ & & & ug/L \\
\hline Parathion & & & & & & $<10$ & $\mathbf{U}$ & ug/L \\
\hline Parathion methyl & & & & & & $<10$ & $\mathbf{U}$ & ug/L \\
\hline Pentachlorodibenzo-p-dioxins & & & & $<.00082$ & U & & & ug/L \\
\hline Pentachlorodibenzo-p-furans & & & & $<.00063$ & $\mathrm{U}$ & & & $\mathrm{ug} / \mathrm{L}$ \\
\hline Pentachloroethane & & & & $<200$ & $\mathrm{U}$ & & & $\mathrm{ug} / \mathrm{L}$ \\
\hline Pentachloronitrobenzene & & & & & & $<10$ & $\mathrm{U}$ & $\mathrm{ug} / \mathrm{L}$ \\
\hline Pentachlorophenol & & & & $<25$ & $\mathrm{U}$ & $<25$ & $\mathrm{JU} Q$ & $\mathrm{ug} / \mathrm{L}$ \\
\hline Phenacetin & & & & & & $<10$ & $\mathrm{U}$ & ug/L \\
\hline Phenanthrene & & & & $<10$ & $\mathbf{U}$ & $<10$ & JU Q & $\operatorname{ug} / L$ \\
\hline Phenol & & & & $<10$ & U & $<10$ & $\mathrm{JU} Q$ & $\operatorname{ug} / \mathrm{L}$ \\
\hline Phorate . & & & & & & $<10$ & $\mathrm{U}$ & $\mathrm{ug} / \mathrm{L}$ \\
\hline Pronamid & & & & & & $<10$ & $\mathrm{U}$ & $\mathrm{ug} / \mathrm{L}$ \\
\hline Propionitrile & & & & $<500$ & U & & & ug/L \\
\hline Pyrene & & & & $<10$ & U & $<10$ & JU Q & $\mathrm{ug} / \mathrm{L}$ \\
\hline Pyridine & . & & & & & $<25$ & $\pi \mathrm{Q}$ & $\mathrm{ug} / \mathrm{L}$ \\
\hline Safrole & & & & & & $<10$ & $\mathrm{U}$ & $\mathrm{ug} / \mathrm{L}$ \\
\hline Selenium, total recoverable & & & & $<10$ & $\mathrm{U}$ & & & ug $/ \mathrm{L}$ \\
\hline Silver, total recoverable & & & & $<20$ & $\mathrm{U}$ & & & $\operatorname{ug} / \mathrm{L}$ \\
\hline Styrene & & & & $<5$ & $\mathrm{U}$ & & & $\mathrm{ug} / \mathrm{L}$ \\
\hline Sulfide & & & & $<1000$ & $U$ & & & $\mathrm{ug} / \mathrm{L}$ \\
\hline Sulfotepp & & & & & & $<10$ & $U$ & $\mathrm{ug} / \mathrm{L}$ \\
\hline Tetrachlorodibenzo-p-dioxins & & & & $<.00054$ & $\mathrm{U}$ & & & $\operatorname{ug} / \mathrm{L}$ \\
\hline Tetrachlorodibenzo-p-furans & & & & $<.00036$ & $U$ & & & $\mathrm{ug} / \mathrm{L}$ \\
\hline Thallium, total recoverable & ${ }^{\prime}$ & & & $<10$ & U & & & ug/L \\
\hline Thionazin & & & & & & $<10$ & $\mathrm{U}$ & $\mathrm{ug} / \mathrm{L}$ \\
\hline Tin, total recoverable & & & & $<200$ & $\mathrm{U}$ & & & $\operatorname{ug} / \mathrm{L}$ \\
\hline Toluene & & & & $<5$ & $\mathrm{U}$ & & & $\operatorname{ug} / \mathrm{L}$ \\
\hline Toxaphene & & & & $<1$ & $\mathrm{U}$ & & & $\operatorname{ug} / \mathrm{L}$ \\
\hline
\end{tabular}

Note: Concentrations in bold exceed the Drinking Water Standards listed in Appendix A. Units are for all four quarters. 
WELL: TNX 4D

ANALYTICAL DATA

\section{Analyte}

Trichlorofluoromethane

Vanadium, total recoverable

Vinyl acetate

Xylenes

Zinc, total recoverable

a, a-Dimethylphenethylamine

alpha-Benzene hexachloride

alpha-Chlordane

beta-Benzene hexachloride

cis-1,2-Dichloroethylene

cis-1,3-Dichloropropene

delta-Benzene hexachloride

gamma-Chlordane

m-Nitroaniline

$\mathrm{m} / \mathrm{p}$-Cresol

o-Cresol (2-Methylphenol)

o-Nitroaniline

o-Toluidine

$\mathrm{p}, \mathrm{p}$ "-DDD

$p, p "-D D E$

p,p"-DDT

p-Cresol (4-Methylphenol)

p-Dimethylaminoazobenzene

p-Nitroaniline

p-Phenylenediamine

trans-1,2-Dichloroethylene

trans-1,3-Dichloropropene

trans-1,4-Dichloro-2-butene
101998 Mod 201998 Mod 301998 Mod 401998 Mod Unit

$<5 \quad \mathrm{U}$

$<10$ U

$<20$ U

$<10 \quad U$

44

$<.1 \quad \mathrm{U}$

$<.1 \quad$ U

$<1 \quad$ U

$<5 \quad$ U

$<5 \quad U$

$<.1$ U

$<1 \quad \mathrm{U}$

$<25 \quad$ U

$<10 \quad U$

$<25 \quad \mathrm{U}$

$<.2 \quad \mathrm{U}$

$<.2 \quad \mathrm{U}$

$<2$ U

$<10 \quad U$

$<10 \quad \mathrm{U}$

$<5 \quad$ U

$<5 \quad \mathrm{U}$

$<20 \quad U$
$<10$

$<10$

$<10$

$<10$

ug/L

ug/L

ug/L

ug/

ug/

$u g / L$

ug/L

ug/ $\mathrm{L}$

ug/L

ug/L

$\mathrm{ug} / \mathrm{L}$

ug/L

$\mathrm{ug} / \mathrm{L}$

$<25$ JU Q ug/L

$<20 \quad U \quad$ ug/L

$<10$ JU Q ug/L

$<25$ JU $Q \quad$ ug/L

$<10$ U ug/L

$\mathrm{ug} / \mathrm{L}$

$\mathrm{ug} / \mathrm{L}$

ug/L

JU $Q \quad u g / L$

U ug/L

JU Q ug/L :

$\mathrm{U}$ $\mathrm{ug} / \mathrm{L}$

ug/L

ug/h

ug $几$

Note: Concentrations in bold exceed the Drinking Water Standards listed in Appendix A. Units are for all four quarters. 
SRS Coord. Lat/Longitude ScreenZone Elevation Top of Standpipe Top of Casing Casing Pump Screen Zone $\begin{array}{lllll}\text { N 71738.1 } & 33.212 & \mathrm{Deg} \mathrm{N} & 103.6-83.6 \mathrm{ft} \mathrm{msl} & 151.1 \mathrm{ft} \mathrm{msl} \\ \text { E } 17080.6 & 81.761 & \mathrm{Deg} \mathrm{W}\end{array}$

SAMPLE DATE

FIELD DATA

Analyte

Depth to water

$\mathrm{pH}$

Sp. Conductance

Water temperature

Alkalinity as $\mathrm{CaCO} 3$

Phenolpthalein Alkalinity

Turbidity

Volumes purged

Sampling codes
03/02/98

$05 / 13 / 98$

$150.9 \mathrm{ft} \mathrm{msl}$

08/05/98

$4 "$ STL

$\mathrm{S}$

Unconfined

$12 / 02 / 98$

201998

48.5

48.13

7.3

25

19.7

10

0

1.4

2.86270

1.05410

LNS

$\mathbf{3 0 1 9 9 8}$
51.14
5.4
32
20.6
3
0
1.2
2.45261

401998

52.57

5.4

50

20.2

6

0

5.1

.310466

NX

\section{ANALYTICAL DATA}

\section{Analyte}

1,1,1,2-Tetrachloroethane

1,1,1-Trichloroethane

1,1,2,2-Tetrachloroethane

1,1,2-Trichloroethane

1,1-Dichloroethane

1,1-Dichloroethylene

1,2,3-Trichloropropane

1,2,4-Trichlorobenzene

1,2-Dibromo-3-chloropropane

1,2-Dibromoethane

1,2-Dichlorobenzene

1,2-Dichloroethane

1,2-Dichloropropane

1,3,5-Trinitrobenzene

1,3-Dichlorobenzene

1,3-Dinitrobenzene

1,4-Dichlorobenzene

1,4-Dioxane

1,4-Naphthoquinone

1-Naphthylamine

2,2-Oxybis(1-chloropropane)

2,3,4,6-Tetrachlorophenol

2,3,7,8-TCDD

2,4,5-T

2,4,5-TP (Silvex)

2,4,5-Trichlorophenol

2,4,6-Trichlorophenol
101998 Mod 201998 Mod 301998 Mod 401998 Mod Unit

$<5 \quad \mathrm{U}$

$<.462$ U $\quad<.462 \quad \mathrm{U} \quad<$

$<5$

$<5$

$<5$

$<5$

$<5$

$<5$

$<10$

$<5$

$<5$

$<5$

$<5$

$<5$

$<5 \quad U$

$<5 \quad \mathrm{U}$

$<1000$

$\mathrm{U}$

$<10 \quad \mathrm{U}$

$<.00042 \mathrm{U}$

$<.2$ UJ $\mathrm{O}$

$<.2$ UJ $\mathrm{O}$

$<10 \quad \mathrm{U}$

$<25$ $\mathrm{ug} / \mathrm{L}$

$u g / L$

$\mathrm{ug} / \mathrm{L}$

$\mathrm{ug} / \mathrm{L}$

ug/L

$\mathrm{ug} / \mathrm{L}$

ug/L

$<10 \quad$ JU Q ug/L :

$\mathrm{ug} / \mathrm{L}$

ug/L

ug/L

ug/L

ug $/$

$\mathrm{ug} / \mathrm{L}$

ug/L

ug/L

$\mathrm{ug} / \mathrm{L}$

$\mathrm{ug} / \mathrm{L}$

$<10 \quad \mathrm{U}, \quad \mathrm{ug} / \mathrm{L}$

$<10 \quad \mathrm{U} \quad \mathrm{ug} / \mathrm{L}$

$<10$ J Q ug/L

$<10$ U ug/L

$\mathrm{ug} / \mathrm{L}$

$\mathrm{ug} / \mathrm{L}$

ug/L

$<10 \quad \pi \quad Q \quad u g / L$

$<25 \quad J U^{\circ} \mathrm{Q} \quad \mathrm{ug} / \mathrm{L}$

Note: Concentrations in bold exceed the Drinking Water Standards listed in Appendix A. Units are for all four quarters. 
WELL: TNX 7D

ANALYTICAL DATA

\section{Analyte}

2,4-Dichlorophenol

2,4-Dichlorophenoxyacetic acid

2,4-Dimethyl phenol

2,4-Dinitrophenol

2,4-Dinitrotoluene

2,6-Dichlorophenol

2,6-Dinitrotoluene

2-Acetylaminofluorene

2-Chloronaphthalene

2-Chlorophenol

2-Hexanone

2-Methyl-4,6-dinitrophenol

2-Methylnaphthalene

2-Naphthylamine

2-Nitrophenol

2-Picoline

2-sec-Butyl-4,6-dinitrophenol

3,3"-Dichlorobenzidine

3,3"-Dimethylbenzidine

3-Methylcholanthrene

4-Aminobiphenyl

4-Bromophenyl phenyl ether

4-Chloro-m-cresol

4-Chloroaniline

4-Chlorophenyl phenyl ether

4-Nitrophenol

4-Nitroquinoline-1-oxide

5-Nitro-o-toluidine

7,12-Dimethylbenz(a)anthracene

Acenaphthene

Acenaphthylene

Acetone

Acetonitrile (Methyl cyanide)

Acetophenone

Acrolein

Acrylonitrile

Aldrin

Allyl chloride

Aluminum, total recoverable

Aniline

Anthracene

Antimony, total recoverable

Aramite
101998 Mod 201998 Mod 301998 Mod 401998 Mod Unit

$<10 \quad \mathrm{U} \quad<10 \quad J U \mathrm{Q} \quad \mathrm{ug} / \mathrm{L}$

$<.2$ UJ $O \quad \mathrm{ug} / \mathrm{L}$

$<10 \quad \mathrm{U} \quad<10 \quad$ JU $Q \quad$ ug/L

$<25 \quad$ U $\quad<25$ JU $Q \quad$ ug/L

$<10 \quad \mathrm{U} \quad<10 \quad$ JU $Q \quad$ ug/L

$<10 \quad \mathrm{U} \quad<10 \quad$ JU $\mathrm{Q} \quad \mathrm{ug} / \mathrm{L}$

$<10 \quad \mathrm{U} \quad<10 \quad \mathrm{JU} Q \mathrm{ug} / \mathrm{L}$

$\begin{array}{llllll}<10 & \mathrm{U} & <10 \quad \mathrm{~J} \mathrm{Q} & \mathrm{ug} / \mathrm{L}\end{array}$

$<5 \quad U \quad \therefore \quad$ ug/L

$<25 \quad U \quad<25$ JU $Q$ ug/L

$<10 \quad U \quad<10 \quad \mathrm{JU} Q \quad \mathrm{Qg} / \mathrm{L}$

$<10 \quad \mathrm{U} \quad \mathrm{ug} / \mathrm{L}$

$\begin{array}{llllll}<10 & U & <10 \quad \pi \quad Q & \mathrm{ug} / \mathrm{L}\end{array}$

$<10 \quad U \quad$ ug/L

$<10 \quad U \quad u g / L$

$<10 \quad \mathrm{U} \quad<10 \quad \pi \quad \mathrm{Q} \quad$ ug/

$<20 \quad U \quad u g / L$

$<10 \quad U \quad u g / L$

$<10 \quad$ U $\quad$ ug/L

$<10 \quad \mathrm{U} \quad<10 \quad$ JU $Q \quad$ ug $/ \mathrm{L}$

$<10 \quad \mathrm{U} \quad<10 \quad$ JU $Q \quad$ ug/L

$<10 \quad \mathrm{U} \quad<10 \quad$ JU $\mathrm{Q} \quad \mathrm{ug} / \mathrm{L}$

$<10 \quad \therefore U_{-}, \quad<10 \quad$ JU $Q \quad$ ug/L

$<25 \quad \mathrm{U} \quad<25 \quad \pi \mathrm{U} \quad \mathrm{Q} \quad \mathrm{ug} / \mathrm{L}$

$<50$ U ug/L

$<10 \quad \mathrm{U} \quad \mathrm{ug} / \mathrm{L}$

$<10 \quad \mathrm{U} \quad \mathrm{ug} / \mathrm{L}$

$<10 \quad \mathrm{U} \quad<10 \quad \pi \quad \mathrm{Q} \quad \mathrm{ug} / \mathrm{L}$

$<10 \quad \mathrm{U} \quad<10 \quad$ JU $\mathrm{Q} \quad$ ug/L

$<20$ U ug/L

$<500 \quad \mathrm{U} \cdot \mathrm{ug} / \mathrm{L}$

$\begin{array}{lllll}<50 \quad \mathrm{U} & <10 \quad \mathrm{U} & \mathrm{ug} / \mathrm{L} \\ \mathrm{ug} / \mathrm{L}\end{array}$

$<50$ U ug/L

$<.1 \quad \mathrm{U} \quad \mathrm{ug} / \mathrm{L}$

$<10 \quad \mathrm{U} \quad$ ug/L

$\begin{array}{lllllll}64.8 & <200 & U & 110 & \text { J } & \text { I } & \mathrm{ug} / \mathrm{L}\end{array}$

$<25 \quad U \quad<25 \quad \pi \quad$ Q $\quad$ ug/L

$<10 \quad \mathrm{U} \quad<10$ JU $Q \quad$ ug/L

$<100 \quad \mathrm{u} * \mathrm{ug} / \mathrm{L}$

$<10 \quad U \quad$ ug/L

Note: Concentrations in bold exceed the Drinking Water Standards listed in Appendix A. Units are for all four quarters. 
WELL: TNX 7D

ANALYTICAL DATA

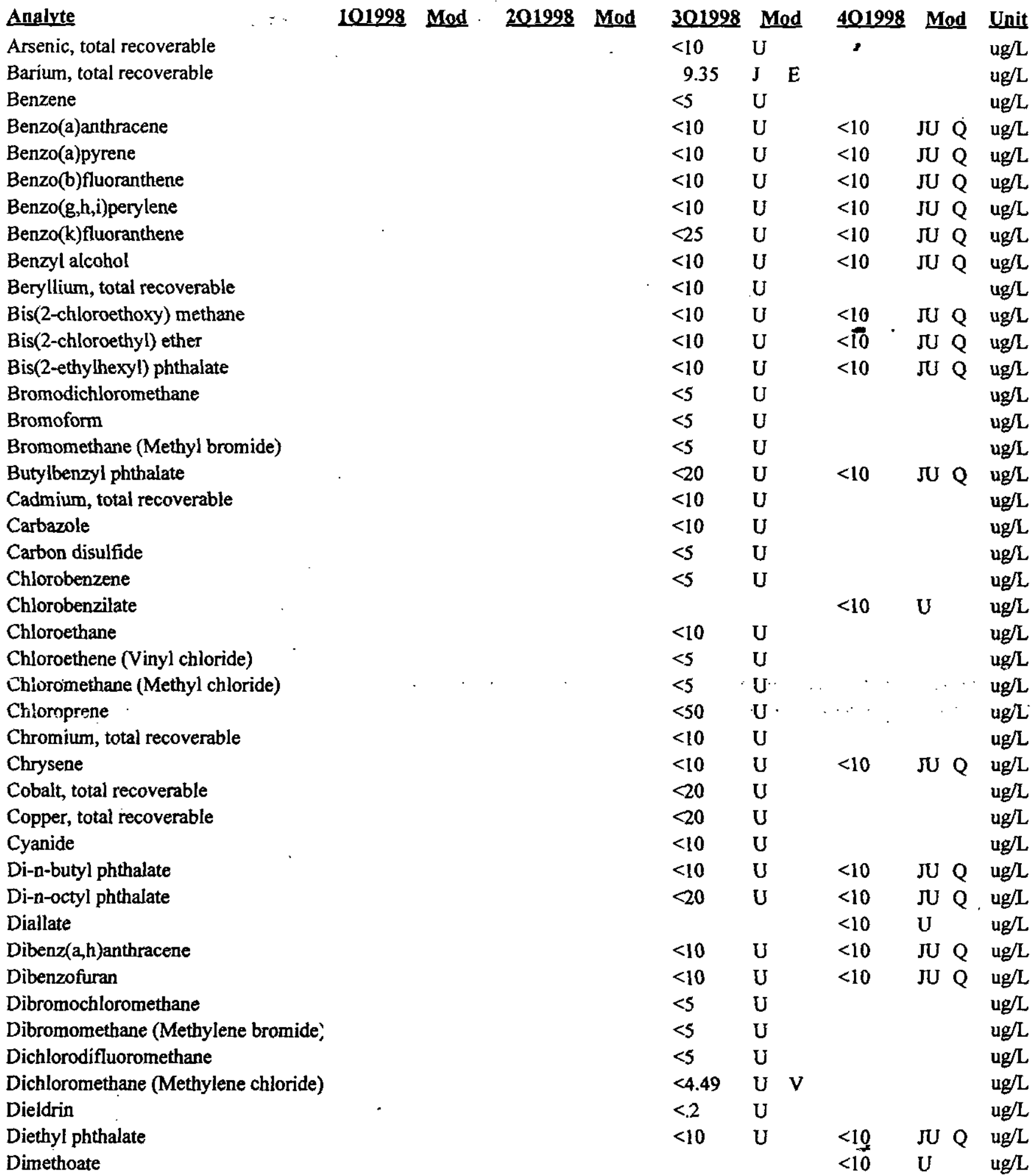

Note: Concentrations in bold exceed the Drinking Water Standards listed in Appendix A. Units are for all four quarters. 
WELL: TNX 7D

ANALYTICAL DATA

\begin{tabular}{|c|c|c|c|c|c|c|c|c|c|}
\hline Analyte , & 101998 & Mod & 201998 & Mod & 301998 & Mod & 401998 & Mod & Unit \\
\hline Dimethyl phthalate & & & & & $<10$ & $\mathbf{U}$ & $<10$ & $\overline{J U Q}$ & $\overline{\mathrm{ug} / \mathrm{L}}$ \\
\hline Diphenylamine & & & & & & & $<10$ & $\mathrm{U}$ & $\mathrm{ug} / \mathrm{L}$ \\
\hline Disulfoton & & & & & & & $<10$ & $\mathrm{U}$ & $\mathrm{ug} / \mathrm{L}$ \\
\hline Endosulfan I & & & & & $<.1$ & $\mathrm{U}$ & & & $\pi$ \\
\hline Endosulfan II & & & & & $<.2$ & $\mathrm{U}$ & & & $3 / \mathrm{L}$ \\
\hline Endosulfan sulfate & & & & & $<.2$ & $\mathbf{U}$ & & & $g / L$ \\
\hline Endrin & & & & & $<.2$ & $\mathbf{U}$ & & & $g / L$ \\
\hline Endrin aldehyde & & & & & $<.2$ & $\mathrm{U}$ & & & $g / L$ \\
\hline Ethyl methacrylate & & & & & $<5$ & $U$ & & & $g / \mathrm{L}$ \\
\hline Ethyl methanesulfonate & & & & & & & $<10$ & $\mathbf{U}$ & $\lg / \mathrm{L}$ \\
\hline Ethylbenzene & & & & & $<5$ & $\mathrm{U}$ & $\therefore$ & & $\operatorname{gg} / \mathrm{L}$ \\
\hline Fluoranthene & & & & & $<10$ & $\mathrm{U}$ & $<10$ & $\mathrm{JU} Q$ & $\mathrm{ug} / \mathrm{L}$ \\
\hline Fluorene & & & & & $<10$ & $\mathrm{U}$ & $<10$ & $\mathrm{JU} Q$ & $g / L$ \\
\hline Heptachlor & & & & & $<.1$ & $\mathrm{U}$ & & & $\mathrm{g} / \mathrm{L}$ \\
\hline Heptachlor epoxide & & & & & $<.1$ & $\mathbf{U}$ & & & $g / L$ \\
\hline Hexachlorobenzene & & & & & $<20$ & $\mathrm{U}$ & $<10$ & $J U Q$ & $g / L$ \\
\hline Hexachlorobutadiene & & & & & $<10$ & $\mathbf{U}$ & $<10$ & $\mathrm{JU} Q$ & $o / \mathrm{L}$ \\
\hline Hexachlorocyclopentadiene & & & & & $<10$ & $\mathbf{U}$ & $<10$ & $\mathrm{JU} \mathrm{Q}$ & $g / \mathrm{L}$ \\
\hline Hexachlorodibenzo-p-dioxins & & & & & $<.00083$ & $\mathbf{U}$ & & & $g / L$ \\
\hline Hexachlorodibenzo-p-furans & & & & & $<.00071$ & $\mathrm{U}$ & & & $g / L$ \\
\hline Hexachloroethane & & & & & $<10$ & $\mathrm{U}$ & $<10$ & $\mathrm{JU} \mathrm{Q}$ & $\mathrm{g} / \mathrm{L}$ \\
\hline Indeno(1,2,3-c,d)pyrene & & & & & $<20$ & $\mathbf{U}$ & $<10$ & $\mathrm{JU} \mathrm{Q}$ & $\lg / \mathrm{L}$ \\
\hline Iodomethane (Methyl iodide) & & & & & $<5$ & $\mathrm{U}$ & & & $g / L$ \\
\hline Iron, total recoverable & & & 136 & & 150 & $\mathrm{~J} \quad \mathrm{E}$ & 253 & & $g / L$ \\
\hline Isobutyl alcohol & & & & .. & $<1500$ & $\mathrm{U}$ & & & $g$ \\
\hline Isodrin & & & & & & & $<10$ & U & $\lg /$ \\
\hline Isophorone & & & & & $<10$ & $\mathrm{U}$ & $<10$ & $J U Q$ & $\mathrm{Ig}$ \\
\hline Isosafrole & & & & & & & $<10$ & $\mathrm{U}$ & $g / L$ \\
\hline Kepone & 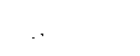 & & & & & & $<10$ & $U$ & $g / L$ \\
\hline Lindane & & & & & $<.1$ & $\mathrm{U}$ & & & $y$ \\
\hline Methacrylonitrile & & & & & $<500$ & $\mathrm{U}$ & & & $\lg / \mathrm{L}$ \\
\hline Methapyrilene & & & & & & & $<10$ & $U$ & $\mathrm{Ig} / \mathrm{L}$ \\
\hline Methoxychlor & & & & & $<1$ & $\mathrm{U}$ & & & $1 g / L$ \\
\hline Methyl ethyl ketone & & & & & $<10$ & $\mathrm{U}$ & & & $\mathrm{g} / \mathrm{L}$ \\
\hline Methyl isobutyl ketone & & & & & $<5$ & $\mathrm{U}$ & & & $g / L$ \\
\hline Methyl methacrylate & & & & & $<50$ & $\mathrm{U}$ & & & $1 \mathrm{~g} / \mathrm{L}$ \\
\hline Methyl methanesulfonate & & & & & & & $<10$ & $U$ & $\mathrm{ug} / \mathrm{L}$ \\
\hline N-Nitrosodi-n-butylamine & . & & & & & & $<10$ & $U$ & $\mathrm{ug}$ \\
\hline N-Nitrosodiethylamine & & & & & & & $<10$ & $\mathbf{U}$ & $u g / L$ \\
\hline N-Nitrosodimethylamine & & & & & $<10$ & $\mathrm{U}$ & $<25$ & JU Q & $1 \mathrm{~g}$ \\
\hline N-Nitrosodiphenylamine & & & & & $<20$ & $U$ & $<10$ & $\mathrm{JU} Q$ & ug/ \\
\hline N-Nitrosodipropylamine & & & & & $<20$ & $\mathbf{U}$ & $<10$ & $\mathrm{JU} \mathrm{Q}$ & $u_{E}$ \\
\hline N-Nitrosomethylethylamine & & & & & & & $<10$ & $\mathrm{U}$ & \\
\hline
\end{tabular}

Note: Concentrations in bold exceed the Drinking Water Standards listed in Appendix A. Units are for all four quarters. 
WELL: TNX 7D

\section{ANALYTICAL DATA}

\section{Analyte}

$\mathrm{N}$-Nitrosomorpholine

$\mathrm{N}$-Nitrosopiperidine

N-Nitrosopyrrolidine

Naphthalene

Nickel, total recoverable

Nitrobenzene

O,O,O-Triethyl phosphorothioate

PCB 1016

PCB 122I

PCB 1232

PCB 1242

PCB 1248

PCB 1254

PCB 1260

Parathion

Parathion methyl

Pentachlorodibenzo-p-dioxins

Pentachlorodibenzo-p-furans

Pentachloroethane

Pentachloronitrobenzene

Pentachlorophenol

Phenacetin

Phenanthrene

Phenol

Phorate

Pronamid

Propionitrile

Pyrene

Pyridine

Safrole

Selenium, total recoverable

Silver, total recoverable

Styrene

Sulfide

Sulfotepp

Tetrachlorodibenzo-p-dioxins

Tetrachlorodibenzo-p-furans

Thallium, total recoverable

Thionazin

Tin, total recoverable

Toluene

Toxaphene

Trichlorofluoromethane
101998 Mod 201998 Mod 301998 Mod 401998 Mod Unit

$<10$ U ug $/ \mathrm{L}$

$<10 \quad \mathrm{U} \quad \mathrm{ug} / \mathrm{L}$

$<10 \quad \mathrm{U} \quad \mathrm{ug} / \mathrm{L}$

$<10 \quad \mathrm{U} \quad<10 \quad$ JU $\mathrm{Q} \quad \mathrm{ug} / \mathrm{L}$

$<50 \quad \mathrm{U} \quad \mathrm{ug} / \mathrm{L}$

$<25 \quad \mathrm{U} \quad<10 \quad$ JU $Q \quad$ ug/L

$<10 \quad \mathrm{U} \quad \mathrm{ug} / \mathrm{L}$

$<2 \quad \mathrm{U} \quad$ ug/L

$<2 \quad \mathrm{U} \quad \mathrm{ug} / \mathrm{L}$

$<1 \quad$ U ug/L

$<1 \quad$ U $\quad \therefore \quad$ ug/L

$<1 \quad \mathrm{U} \quad-\cdots \quad$ ug/L

$<1 \quad$ U ug/L

$<1 \quad \mathrm{U} \quad \mathrm{ug} / \mathrm{L}$

$<10 \quad \mathrm{U} \quad \mathrm{ug} / \mathrm{L}$

$<.0009 \mathrm{U} \quad \mathrm{ug} / \mathrm{L}$

$<.00065 \mathrm{U} \quad \mathrm{ug} / \mathrm{L}$

$<200$ U ug/L

$<10 \quad \mathrm{U} \quad \mathrm{ug} / \mathrm{L}$

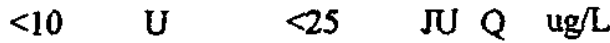

$<10$ U ug/L

$<10 \quad \mathrm{U} \quad<10 \quad$ JU $Q \quad$ ug/L

$<10 \quad \mathrm{U} \quad<10 \quad \mathrm{JU} \mathrm{Q} \quad \mathrm{ug} / \mathrm{L}$

$<10$ U ug/L

$<10$ U $\mathrm{ig} / \mathrm{L}$

$<500 \quad \mathrm{U} \quad$ ug/ $\mathrm{L}$

$<25 \quad \mathrm{U} \quad<10 \quad \pi \mathrm{Q} \quad \mathrm{ug} / \mathrm{L}$

$<25 \quad \pi \quad Q \quad u g / L$

$<10 \quad \mathrm{U} \quad \mathrm{ug} / \mathrm{L}$

$<10 \quad \mathrm{U} \quad$ ug/L

$<20$ U ug/L

$<5$ U ug/L

$<1000 \mathrm{U} \quad \mathrm{ug} / \mathrm{L}$

$<10 \quad U \quad$ ug/L

$<.00042 \mathrm{U} \quad \mathrm{ug} / \mathrm{L}$

$<.00027 \mathrm{U}$ ug/L

$<10$ U ug/L

$<10$ U ug/L

$<200$ U ug/L

$<5$ U ug/L

$<$ U $\quad$ U ug/L

$<$ U $\quad$ ug/L

Note: Concentrations in bold exceed the Drinking Water Standards listed in Appendix A. Units are for all four querters. 
WELL: TNX 7D

ANALYTICAL DATA

Analyte

Vanadium, total recoverable

Vinyl acetate

Xylenes

Zinc, total recoverable

a,a-Dimethylphenethylamine

alpha-Benzene hexachloride

alpha-Chlordane

beta-Benzene hexachloride

cis-1,2-Dichloroethylene

cis-1,3-Dichloropropene

delta-Benzene hexachloride

gamma-Chlordane

m-Nitroaniline

$\mathrm{m} / \mathrm{p}$-Cresol

o-Cresol (2-Methylphenol)

o-Nitroaniline

o-Toluidine

p,p"-DDD

p,p"-DDE

p,p"-DDT

p-Cresol (4-Methylphenol)

p-Dimethylaminoazobenzene

p-Nitroaniline

p-Phenylenediamine

trans-1,2-Dichloroethylene

trans-1,3-Dichloropropise

trans-1,4-Dichloro-2-butene

\section{$101998 \mathrm{Mo}$}

\section{Mod \\ 401998}

$<10$

U

$<20$ U

$<10$ U

6.7 J E

$<.1 \quad$ U

$<.1 \quad$ U

$<.1 \quad U$

$<5 \quad \mathrm{U}$

$<.1$ U

$<.1$ U

$<25 \quad$ U

$<10 \quad 4$

$<25$

$<.2 \cdot \mathrm{U}$

$<.2 \quad \mathrm{U}$

$<2$ U

$<10 \quad U$

$<10$

U

$<5$

$<5$

$<20$
Mod

Unit

ug/L

ug/L

$\mathrm{ug} / \mathrm{L}$

ug/L

$<10$ U ug/L

$\mathrm{ug} / \mathrm{L}$

$\mathrm{ug} / \mathrm{L}$

ug/

$<1 \quad$ U $\quad$ ug/L

$\mathrm{ug} / \mathrm{L}$

ug/L

$\mathrm{ug} / \mathrm{L}$

$\therefore$

$<25$

$<20$

$<10$

$<25$

$<10$

JU Q

$\mathrm{ug} / \mathrm{L}$

ug/L

JU Q ug/L

JU $Q \quad u g / L$

U

ug/L

$\mathrm{ug} / \mathrm{L}$

$\mathrm{ug} / \mathrm{L}$

$\mathrm{ug} / \mathrm{L}$

$<10$

$<10$

Л

$<10$

$<10$

JU

ug/L

ug/L

$\mathrm{ug} / \mathrm{L}$

ug/L

ug/L

ug/L

ug/L

Note: Concentrations in bold exceed the Drinking Water Standards listed in Appendix A. Units are for all four quarters. 
WELL: TNX 8D

SRS Coord. Latlongitude Screen Zone Elevation Top of Standpipe Top of Casing Casing Pump Screen Zone N 70591.9 33.208 Deg N E 16168.3 81.761 Deg W $94.0-74.0$ ft msI

$03 / 02 / 98$ $00.5 \mathrm{ft} \mathrm{msl}$

$05 / 12 / 98$ $100.3 \mathrm{ft} \mathrm{msl}$

$08 / 04 / 98$ , $12 / 01 / 98$
SAMPLE DATE

FIELD DATA

$\begin{array}{ll}\text { Analyte } & \mathbf{1 0 1 9 9 8} \\ \text { Depth to water } & 4.18 \\ \text { pH } & 5.3 \\ \text { Sp. Conductance } & 140 \\ \text { Water temperature } & 16 \\ \text { Alkalinity as CaCO3 } & 10 \\ \text { Phenolpthalein Alkalinity } & 0 \\ \text { Turbidity } & 3.4 \\ \text { Volumes purged } & 3.37681\end{array}$

\section{ANALYTICAL DATA}

\section{Analyte}

1,1,1,2-Tetrachloroethane

1,1,1-Trichloroethane

1,1,2,2-Tetrachloroethane

1,1,2-Trichloroethane

1,1-Dichloroethane

1,1-Dichloroethylene

1,2,3-Trichloropropane

1,2,4-Trichlorobenzene

1,2-Dibromo-3-chloropropane

1,2-Dibromoethane

1,2-Dichlorobenzene

1,2-Dichloroethane

1,2-Dichloropropane

1,3,5-Trinitrobenzene

1,3-Dichlorobenzene

1,3-Dinitrobenzene

1,4-Dichlorobenzene

1,4-Dioxane

1,4-Naphthoquinone

1-Naphthylamine

2,2-Oxybis(1-chloropropane)

2,3,4,6-Tetrachlorophenol

2,3,7,8-TCDD

$2,4,5-\mathrm{T}$

2,4,5-TP (Silvex)

2,4,5-Trichlorophenol

2,4,6-Trichlorophenol

\section{$101998 \mathrm{Mod}$ \\ 201998 Mod}

$<.462 \quad \mathrm{U}$

$<.462 \quad U$

$\frac{201998}{5.66}$
4.6
100
19
5
0
2
3.17581

$\mathbf{3 0 1 9 9 8}$
6.85
5
110
21.7
7
0
1.2

5.17274
4 " STL

$S$

Unconfined

\begin{tabular}{|c|c|}
\hline 401998 & Unit \\
\hline $\begin{array}{l}7.74 \\
5.1\end{array}$ & ft BTOS \\
\hline $\begin{array}{l}110 \\
19.1\end{array}$ & $\begin{array}{l}\text { uS/cm } \\
\text { deg. C }\end{array}$ \\
\hline 7 & $\mathrm{mg} / \mathrm{L}$ \\
\hline 0 & $\mathrm{mg} / \mathrm{L}$ \\
\hline $\begin{array}{l}1.4 \\
4.27092\end{array}$ & $\begin{array}{l}\text { NTU } \\
\text { gallons }\end{array}$ \\
\hline
\end{tabular}

301998 Mod 401998 Mod Unit

$<$ U ug/L

$\begin{array}{lllll}< & \mathrm{U} & <1 & \mathrm{U} & \mathrm{ug} / \mathrm{L}\end{array}$

$<5 \quad U$

$<5 \quad U$

$<5 \quad \mathrm{U}$

$<5 \quad \mathrm{U}$

$<5 \quad U$

$\div 10 . \quad \mathrm{U}$

$<5 \quad \mathrm{U}$

$<5 \quad \mathrm{U}$

$<5 \quad \mathrm{U}$

$<5 \quad \mathrm{U}$

$<5 \quad \mathrm{U}$

$<5 \quad \mathrm{U}$

$<5 \quad \mathrm{U}$

$<1000 \mathrm{U}$

$\begin{array}{llll}<10 & \mathrm{U} & \mathrm{ug} / \mathrm{L} \\ <10 & \mathrm{U} & \mathrm{ug} / \mathrm{L}\end{array}$

$<10 \quad \mathrm{U} \quad<10 \quad \pi \mathrm{U} Q \quad \mathrm{ug} / \mathrm{L}$

$<10 \quad \mathrm{U} \quad \mathrm{ug} / \mathrm{L}$

$<.00059 \mathrm{U}$

$<2 \quad \mathrm{U}$

$<.2 \quad \mathrm{U}$

$<10 \quad \mathrm{U}$

$<25 \quad$ U

ug/L

ug/L

ug/L

$\mathrm{ug} / \mathrm{L}$

ug/L

$=10$ JU Q ug/L

$\mathrm{ug} / \mathrm{L}$

$\mathrm{ug} / \mathrm{L}$

$\mathrm{ug} / \mathrm{L}$

$\mathrm{ug} / \mathrm{L}$

$\mathrm{ug} / \mathrm{L}$

$<10$ U ugh

$u g / L$

$<10 \quad \mathrm{u} \quad u g / \mathrm{L}$

$\mathrm{ug} / \mathrm{L}$

$\mathrm{ug} / \mathrm{L}$

$\mathrm{ug} / \mathrm{L}$

$\mathrm{ug} / \mathrm{L}$

$<10 \quad$ U $Q \quad u g / L$

$<25$ JU Q ug/L

Note: Concentrations in bold exceed the Drinking Water Standards listed in Appendix A. Units are for all four quarters. 
WELL: TNX 8D

ANALYTICAL DATA

Analyte

2,4-Dichlorophenol

2,4-Dichlorophenoxyacetic acid

2,4-Dimethyl phenol

2,4-Dinitrophenol

2,4-Dinitrotoluene

2,6-Dichlorophenol

2,6-Dinitrotoluene

2-Acetylaminofluorene

2-Chloronaphthalene

2-Chlorophenol

2-Hexanone

2-Methyl-4,6-dinitrophenol

2-Methylnaphthalene

2-Naphthylamine

2-Nitrophenol

2-Picoline

2-sec-Butyl-4,6-dinitrophenol

3,3"-Dichlorobenzidine

3,3"-Dimethylbenzidine

3-Methylcholanthrene

4-Aminobiphenyl

4-Bromophenyl phenyl ether

4-Chloro-m-cresol

4-Chloroaniline

4-Chlorophenyl phenyl ether

4-Nitrophenol

4-Nitroquinoline-1-oxide

5-Nitro-o-toluidine

7,12-Dimethylbenz(a)anthracene

Acenaphthene

Acenaphthylene

Acetone

Acetonitrile (Methyl cyanide)

Acetophenone

Acrolein

Acrylonitrile

Aldrin

Allyl chloride

Aluminum, total recoverable

Aniline

Anthracene

Antimony, total recoverable

Aramite

\section{Mod 201998 Mod}

$\begin{array}{ll}\begin{array}{l}301998 \\ <10\end{array} & \text { Mod } \\ <.2 & \mathrm{U} \\ <10 & \mathrm{U} \\ <25 & \mathrm{U} \\ <10 & \mathrm{U}\end{array}$

401998 Mod Unit

$<10 \quad$ J $Q$ ug/L

ug/L

$<10 \quad$ JU Q ug/L

$<25$ JU Q ug/L

$<10$ JU Q ug/L

$<10 \quad \mathrm{U} \quad \mathrm{ug} / \mathrm{L}$

$<10 \quad \mathrm{U} \quad<10 \quad$ JU $Q \quad$ ug/L

$<10$ U ug/L

$<10 \quad \mathrm{U} \quad<10 \quad$ JU $\mathrm{Q} \quad \mathrm{ug} / \mathrm{L}$

$<10$ U $<10$ JU $Q \quad$ ug/L

$<5 \quad \mathrm{U} \quad \therefore \quad . \quad \mathrm{ug} / \mathrm{L}$

$<25$ U $\quad<25 \quad J \quad$ Q $\quad$ ug/L

$<10 \quad \mathrm{U} \quad<10 \quad$ J $Q \quad$ ug/L

$<10$ U ug/L

$<10 \quad \mathrm{U} \quad<10 \quad \mathrm{JU} \quad \mathrm{Q} \quad \mathrm{ug} / \mathrm{L}$

$<10$ U $\quad \mathrm{ug} / \mathrm{L}$

$<10 \quad$ U $\quad$ ug/L

$<10 \quad \mathrm{U} \quad<10 \quad \mathrm{JU} \quad \mathrm{Q} \quad \mathrm{ug} / \mathrm{L}$

$<20$ U ug/L

$<10$ U ug/L

$<10$ U ug/L

$<10 \quad \mathrm{U} \quad<10 \quad \mathrm{JU} Q \quad \mathrm{ug} / \mathrm{L}$

$<10 \quad \mathrm{U} \quad<10 \quad$ JU $Q \quad$ ug/L

$<10 \quad$ U $\quad<10 \quad$ JU $Q \quad$ ug/L

$<10 . \quad \mathrm{U} \quad<10$ JU $Q \quad$ ug/L

$<25 \quad U \quad<25 \quad$ JU $Q \quad$ ug/L

$<50$ U ug/L

$<10 \quad$ U ug/L

$<10$ U ug/L

$\begin{array}{llllll}<10 & \mathrm{U} & <10 & \mathrm{JU} & \mathrm{Q} & \mathrm{ug} / \mathrm{L}\end{array}$

$<10 \quad \mathrm{U} \quad<10 \quad \mathrm{JU} \quad \mathrm{Q} \quad \mathrm{ug} / \mathrm{L}$

$<20$ U ug/L

$<500$ U ug/L

$<10 \quad \mathrm{U} \quad \mathrm{ug} / \mathrm{L}$

$<50$ U ug/L

$<50$ U ug/L

$<.1$ U $\quad$ ug/L

$<10$ U ug/L

$29.6<200 \quad$ U $\quad<200 \quad$ U $\quad$ ug/L

$<25 \quad \mathrm{U} \quad<25 \quad$ JU $Q \quad$ ug/L

$<10 \quad \mathrm{U} \cdot \quad<10 \quad$ JU $Q \quad \mathrm{ug} / \mathrm{L}$

$<100 \quad \mathrm{U} \quad * \quad \mathrm{ug} / \mathrm{L}$

$<10 \quad \mathrm{U} \quad \mathrm{ug} / \mathrm{L}$

Note: Concentrations in bold exceed the Drinking Water Standards listed in Appendix A. Units are for all four quarters. 
WELL: TNX 8D

\section{ANALYTICAL DATA}

Analyte

Arsenic, total recoverable

Barium, total recoverable

Benzene

Benzo(a)anthracene

Benzo(a)pyrene

Benzo(b)fluoranthene

Benzo(g,h,i)perylene

Benzo(k)fluoranthene

Benzyl alcohol

Beryllium, total recoverable

Bis(2-chloroethoxy) methane

Bis(2-chloroethyl) ether

Bis(2-ethylhexyl) phthalate

Bromodichloromethane

Bromoform

Bromomethane (Methyl bromide)

Butylbenzyl phthalate

Cadmium, total recoverable

Carbazole

Carbon disulfide

Chlordane

Chlorobenzene

Chlorobenzilate

Chloroethane

Chloroethene (Vinyl chloride)

Chloromethane (Methyl chloride)

Chloroprene

Chromium, total recoverable

Chrysene

Cobalt, total recoverable

Copper, total recoverable

Cyanide

Di-n-butyl phthalate

Di-n-octyl phthalate

Diallate

Dibenz(a,h)anthracene

Dibenzofuran

Dibromochloromethane

Dibromomethane (Methylene bromide',

Dichlorodifluoromethane

Dichloromethane (Methylene chloride)

Dieldrin

Diethyl phthalate
101998 Mod 201998 Mod

$<10$

70.5

$<5$

$<10 \quad \mathrm{U}$

$<10$

$<10$

$<10$

$<10$

$<10$

$<10$

$<10$

$<10$

$<10$

$<5$

$<5$

$<5$

$<10$

$<10$

$<20$

$<5$

$<.2$

$<5$

$<10$

$<5$

$<5$

$<50$

$<10$

$<10$

3.36

$<20$

$<10$

$<10$.

$<10$

$<20$

$<10$

$<5$

$<5$

$<5$

$<4.85$

$<.2$

$<10$
401998

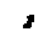

64.3

Mod Unit

$\mathrm{ug} / \mathrm{L}$ $\mathrm{ug} / \mathrm{L}$ ug/L

$<10 \quad$ JU Q ug/L

$<10$ JU Q ug/L

$<10$ JU Q ug/L

$<10 \quad$ JU Q $\quad$ ug/L

$<10 \quad$ JU Q ug/L

$<$ I0 JU Q ug/L

$\mathrm{ug} / \mathrm{L}$

$<10$ JU $Q$ ug/L

$<\widehat{10}$ JU Q ug/L

$<10$ JU Q ug/L

$\mathrm{ug} / \mathrm{L}$

$\mathrm{ug} / \mathrm{L}$

$\mathrm{ug} / \mathrm{L}$

$<10$ JU Q ug/L

$\mathrm{ug} / \mathrm{L}$

$\mathrm{ug} / \mathrm{L}$

$\mathrm{ug} / \mathrm{L}$

$\mathrm{ug} / \mathrm{L}$

$\mathrm{ug} / \mathrm{L}$

$<10$ U ug/L

ug/L

ug/L

$\mathrm{ug} / \mathrm{L}$

$\mathrm{ug} / \mathrm{L}$

ug/L

U $\quad$ Q ug/L

$\mathrm{ug} / \mathrm{L}$

$\mathrm{ug} / \mathrm{L}$

$\mathrm{ug} / \mathrm{L}$

$<10 \quad$ JU $Q \quad$ ug/L

$<10$ JU Q ug/L

$<10$ U ug/L

$<10$ JU Q ug/L

$<10$ JU Q ug/L

ug $/ \mathrm{L}$

$\mathrm{ug} / \mathrm{L}$

ug/L

$\mathrm{ug} / \mathrm{L}$

$\operatorname{ug} / \mathrm{L}$

JU Q ug/L

Note: Concentrations in bold exceed the Drinking Water Standards listed in Appendix A. Units are for all four quarters. 
WELL: TNX 8D

\section{ANALYTICAL DATA}

Analyte

Dimethoate

Dimethyl phthalate

Diphenylamine

Disulfoton

Endosulfan I

Endosulfan II

Endosulfan sulfate

Endrin

Endrin aldehyde

Ethyl methacrylate

Ethyl methanesulfonate

Ethylbenzene

Fluoranthene

Fluorene

Heptachlor

Heptachlor epoxide

Hexachlorobenzene

Hexachlorobutadiene

Hexachlorocyclopentadiene

Hexachlorodibenzo-p-dioxins

Hexachlorodibenzo-p-furans

Hexachloroethane

Indeno(1,2,3-c,d)pyrene

Iodomethane (Methyl indide)

Iron, total recoverable

Isobutyl alunhoi

Isodrin

Isophorone

Isosafrole

Kepone

Lindane

Methacrylonitrile

Methapyrilene

Methoxychlor

Methyl ethyl ketone

Methyl isobutyl ketone

Methyl methacrylate

Methyl methanesulfonate

$\mathrm{N}$-Nitrosodi-n-butylamine

$\mathrm{N}-\mathrm{Nitrosodiethylamine}$

$\mathrm{N}$-Nitrosodimethylamine

$\mathrm{N}$-Nitrosodiphenylamine

$\mathrm{N}$-Nitrosodipropylamine
101998 Mod 201998 Mod 301998 Mod 401998 Mod Unit

$<10 \quad \mathrm{U} \quad$ ug/L

$<10 \quad \mathrm{U} \quad<10 \quad$ JU $\mathrm{Q} \quad \mathrm{ug} / \mathrm{L}$

$<10 \quad \mathrm{U} \quad \mathrm{ug} / \mathrm{L}$

$<10 \quad \mathrm{U} \quad \mathrm{ug} / \mathrm{L}$

$<.1 \quad \mathrm{U} \quad \mathrm{ug} / \mathrm{L}$

$<.2 \quad \mathrm{U} \quad \mathrm{ug} / \mathrm{L}$

$<2$ U $\quad \mathrm{ug} / \mathrm{L}$

$<.2$ U ug/L

$<.2 \quad \mathrm{U} \quad \mathrm{ug} / \mathrm{L}$

$<5 \quad \mathrm{U} \quad \mathrm{ug} / \mathrm{L}$

$<10$. U ug/L

$<5 \quad \mathrm{U} \quad \mathrm{ug} / \mathrm{L}$

$<10 \quad \mathrm{U} \quad<10 \quad$ J $Q \quad$ ug/L

$<10 \quad \mathrm{U} \quad<10 \quad$ JU $\mathrm{Q} \quad \mathrm{ug} / \mathrm{L}$

$<, 1$ U ug/L

$<.1 \quad \mathrm{U} \quad$ ug/L

$<10 \quad \mathrm{U} \quad<10 \quad$ JU $\mathrm{Q} \quad \mathrm{ug} / \mathrm{L}$

$<20 \quad \mathrm{U} \quad<10 \quad$ JU $\mathrm{Q} \quad \mathrm{ug} / \mathrm{L}$

$<10 \quad \mathrm{U} \quad<10 \quad$ JU $Q \quad \mathrm{ug} / \mathrm{L}$

$<.0011 \mathrm{U}$ ug/L

$<.00075 \mathrm{U}$ ug/L

$<10 \quad \mathrm{U} \quad<10 \quad \mathrm{JU} \mathrm{Q} \quad \mathrm{ug} / \mathrm{L}$

$<10 \quad U \quad<10 \quad \pi \quad Q \quad u g / L$

$<5 \quad \mathrm{U} \quad \mathrm{ug} / \mathrm{L}$

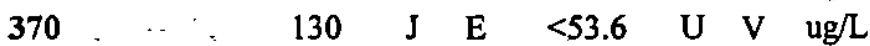

$<1500 \quad \mathrm{U} \quad \mathrm{ug} / \mathrm{L}$

$<10 \quad U \quad u g / L$

$<20 \quad \mathrm{U} \quad<10 \quad$ JU $Q \quad$ ug/L

$<10 \quad \mathrm{U} \quad \mathrm{ug} / \mathrm{L}$

$<10 \quad \mathrm{U} \quad$ ug/L

$<.1 \quad$ U $\quad$ ug/L

$<500$ U ug/L

$<10 \quad$ U ug $/ \mathrm{L}$

$<1 \quad$ U

$<10 \quad U$

$<5 \quad \mathrm{U}$

$<50 \quad \mathrm{U}$

ug/L

ug/L

$\mathrm{ug} / \mathrm{L}$

$\mathrm{ug} / \mathrm{L}$

$<10 \quad \mathrm{U} \quad \mathrm{ug} / \mathrm{L}$

$<10 \quad \mathrm{U} \quad \mathrm{ug} / \mathrm{L}$

$<10 \quad$ U ug/L

$<20 \quad \mathrm{U} \quad<25 \quad$ JU $Q \quad \mathrm{ug} / \mathrm{L}$

$<10 \quad$ U $\quad<1 \theta \quad$ JU $Q$

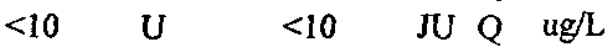

Note: Concentrations in bold exceed the Drinking Water Standards listed in Appendix A. Units are for all four quarters. 
WELL: TNX 8D

\section{ANALYTICAL DATA}

\section{Analyte}

$\mathrm{N}$-Nitrosomethylethylamine

$\mathrm{N}$-Nitrosomorpholine

N-Nitrosopiperidine

$\mathrm{N}-\mathrm{Nitrosopyrrolidine}$

Naphthalene

Nickel, total recoverable

Nitrobenzene

O,O,O-Triethyl phosphorothioate

PCB 1016

PCB 1221

PCB 1232

PCB 1242

PCB 1248

PCB 1254

PCB 1260

Parathion

Parathion methyl

Pentachlorodibenzo-p-furans

Pentachloroethane

Pentachloronitrobenzene

Pentachlorophenol

Phenacetin

Phenanthrene

Phenol

Phorate

Pronamid

Propionitrile

Pyrene

Pyridine

Safrole

Selenium, total recoverable

Silver, total recoverable

Styrene

Sulfide

Sulfotepp

Tetrachlorodibenzo-p-dioxins

Tetrachlorodibenzo-p-furans

Thallium, total recoverable

Thionazin

Tin, total recoverable

Toluene

Total organic halogens

Toxaphene
101998 Mod 201998 Mod 301998 Mod 401998 Mod Unit

$<10 \quad \mathrm{U} \quad \mathrm{ug} / \mathrm{L}$

$<10$ U ug/L

$<10 \quad \mathrm{U} \quad \mathrm{ug} / \mathrm{L}$

$<10 \quad \mathrm{U} \quad \mathrm{ug} / \mathrm{L}$

$<20 \quad \mathrm{U} \quad<10 \quad \mathrm{JU} \quad \mathrm{Q} \quad \mathrm{ug} / \mathrm{L}$

$<50 \quad \mathrm{U}$

$<10$ U $<10$ J $Q \quad$ ug/L

$<10$ U ug/L

$<$ U ug/L

$<2 \quad$ U $\quad$ ug/L

$<1 \quad$ U $\quad-\quad$ ug/L

$<1$ U ug/L

$<$ U ug/L

$<$ U $\quad u g / L$

$<1 \quad$ U $\quad$ ug/L

$<10 \quad \mathrm{U} \quad \mathrm{ug} / \mathrm{L}$

$<10 \quad \mathrm{U} \quad \mathrm{ug} / \mathrm{L}$

$<.00066 \mathrm{U} u g / \mathrm{L}$

$<200$ U ug/L

ug/L

$<25$ JU Q ug/L

$<10$ U ug/L

$<10$ U $\quad<10 \quad$ JU $Q \quad u g / L$

$<10$ U $<10$ JU $Q \quad u g / L$

$<10$ U ug/L

$<10$ U ug/L

$<500$ U ug/L

$<10 \quad \mathrm{U} \quad<10 \quad \pi \mathrm{Q} \quad \mathrm{ug} / \mathrm{L}$

$<25$ JU Q ug/L

$<10$ U ug/L

$<10 \quad \mathrm{U}$

$<20 \quad$ U

$<5 \quad U$

$<1000 \quad U$

$<.00059 \mathrm{U}$

$\mathrm{ug} / \mathrm{L}$

ug/L

$\mathrm{ug} / \mathrm{L}$

$\mathrm{ug} / \mathrm{L}$

$<10 \quad \mathrm{u} \quad \mathrm{ug} / \mathrm{L}$

$<.00043 \mathrm{U}$

$\mathrm{ug} / \mathrm{L}$

ug/L

$<10 \quad \mathrm{U}$

$\mathrm{ug} / \mathrm{L}$

$<200 \quad U$

$<10$

U

ug/L

$\mathrm{ug} / \mathrm{L}$

ug/

$<5 \quad$ U

$<120$

U

$\mathrm{ug} / \mathrm{L}$

$\mathrm{ug} / \mathrm{L}$

Note: Concentrations in bold exceed the Drinking Water Standards listed in Appendix A. Units are for all four quarters. 
WSRC-RP-99-4003

Unclassified

WELL: TNX 8D

ANALYTICAL DATA

\section{Analyte}

101998 Mod

201998 Mod

301998 Mod

401998 Mod Unit

Trichlorofluoromethane $<5 \quad \mathrm{U}$

$<10 \quad \mathrm{U}$

ug/L

Vanadium, total recoverable

$<20 \quad \mathrm{U}$

$\mathrm{ug} / \mathrm{L}$

Vinyl acetate

$<10 \quad \mathrm{U}$

Xylenes

Zinc, total recoverable

4.25

a, a-Dimethylphenethylamine alpha-Benzene hexachloride beta-Benzene hexachloride cis-1,2-Dichloroethylene cis-1,3-Dichloropropene delta-Benzene hexachloride gamma-Chlordane m-Nitroaniline

$\mathrm{m} / \mathrm{p}$-Cresol o-Cresol (2-Methylphenol)

o-Nitroaniline

o-Toluidine

p,p"-DDD

$p, p^{\prime \prime}-D D E$

p,p"-DDT

p-Cresol (4-Methylphenol)

p-Dimethylaminoazobenzene

p-Nitroaniline

p-Phenylenediamine trans-1,2-Dichloroethylene trans-1,3 Dichlot opropene trans-1,4-Dichloro-2-butene

$\begin{array}{lllll}<.1 & \mathrm{U} & <10 & \mathrm{U} & \mathrm{ug} / \mathrm{L} \\ <.1 & \mathrm{U} & & & \mathrm{ug} / \mathrm{L} \\ <5 & \mathrm{U} & <1 & \mathrm{U} & \mathrm{ug} / \mathrm{L} \\ <5 & \mathrm{U} & & & \mathrm{ug} / \mathrm{L} \\ <.1 & \mathrm{U} & - & & \mathrm{ug} / \mathrm{L} \\ <.1 & \mathrm{U} & & & \mathrm{ug} / \mathrm{L} \\ <25 & \mathrm{U} & <25 & \mathrm{JU} \mathrm{Q} & \mathrm{ug} / \mathrm{L} \\ & & <20 & \mathrm{U} & \mathrm{ug} / \mathrm{L} \\ <10 & \mathrm{U} & <10 & \mathrm{JU} \mathrm{Q} & \mathrm{ug} / \mathrm{L} \\ <25 & \mathrm{U} & <25 & \mathrm{JU} \mathrm{Q} & \mathrm{ug} / \mathrm{L} \\ & & <10 & \mathrm{U} & \mathrm{ug} / \mathrm{L} \\ <.2 & \mathrm{U} & & & \mathrm{ug} / \mathrm{L} \\ <.2 & \mathrm{U} & & & \mathrm{ug} / \mathrm{L} \\ <.2 & \mathrm{U} & & & \mathrm{ug} / \mathrm{L} \\ <10 & \mathrm{U} & <10 & \mathrm{JU} \mathrm{Q} & \mathrm{ug} / \mathrm{L} \\ & & <10 & \mathrm{U} & \mathrm{ug} / \mathrm{L} \\ <10 & \mathrm{U} & <10 & \mathrm{JU} \mathrm{Q} & \mathrm{ug} / \mathrm{L} \\ & & <10 & \mathrm{U} & \mathrm{ug} / \mathrm{L} \\ <5 & \mathrm{U} & & & \mathrm{ug} / \mathrm{L} \\ <5 & \mathrm{U} & . & & \mathrm{ug} / \mathrm{L} \\ <20 & \mathrm{U} & & & \mathrm{ug} / \mathrm{L}\end{array}$

Note: Concentrations in bold exceed the Drinking Water Standards listed in Appendix A. Units are for all four quarters. 
WSRC-RP-99-4003

Unclassified

WELL: TNX 9D

SRS Coord. Lat/Longitude Screen Zone Elevation Top of Standpipe Top of Casing Casing Pump Screen Zone N 70791.4 33.209 Deg N E 16145.8 81.762 Deg W $95.4-75.4 \mathrm{ft} \mathrm{msl} \quad 101.9 \mathrm{ft} \mathrm{msl}$

SAMPLE DATE

$03 / 03 / 98$

$05 / 12 / 98$

$101.7 \mathrm{ft} \mathrm{msl}$ 4 "STL $\mathrm{S}$ Unconfined

FIELD DATA

Analyte

Depth to water

pH

Sp. Conductance

Water temperature

Alkalinity as $\mathrm{CaCO} 3$

Phenolpthalein Alkalinity

Turbidity

Volumes purged

Sampling codes

\section{ANALYTICAL DATA}

\section{Analyte}

1,1,1,2-Tetrachloroethane

1,1,1-Trichloroethane

1,1,2,2-Tetrachloroethane

1,1,2-Trichloroethane

1,1-Dichloroethane

1,1-Dichloroethylene

1,2,3-Trichloropropane

1,2,4-Trichlorobenzene

1,2-Dibromo-3-chloropropane

1,2-Dibromoethane

1,2-Dichlorobenzene

1,2-Dichloroethane

1,2-Dichloropropane

1,3-Dichlorobenzene

1,4-Dichlorobenzene

1,4-Dioxane

2,2-Oxybis(1-chloropropane)

2,3,7,8-TCDD

2,4,5-T

2,4,5-TP (Silvex)

2,4,5-Trichlorophenol

2,4,6-Trichlorophenol

2,4-Dichlorophenol

2,4-Dichlorophenoxyacetic acid

2,4-Dimethyl phenol

2,4-Dinitrophenol

2,4-Dinitrotoluene

\begin{tabular}{|c|c|c|c|c|}
\hline 101998 & 201998 & 301998 & 401998 & Unit \\
\hline 5.72 & 6.25 & 8.75 & & ft BTOS \\
\hline 3.9 & 5.8 & & & \\
\hline $\begin{array}{l}90 \\
16.8\end{array}$ & 96 & 170 & & $\mathrm{uS} / \mathrm{cm}$ \\
\hline 16.8 & 19 & 20.5 & & deg. C \\
\hline 10 & 11 & 3 & & $\mathrm{mg} / \mathrm{L}$ \\
\hline 0 & 0 & 0 & & $\mathrm{mg} / \mathrm{L}$ \\
\hline 1.8 & 1.3 & 3 & . & NTU \\
\hline 6.59236 & 7.07074 & 2.69266 & - & gallons \\
\hline
\end{tabular}

$101998 \mathrm{Mod}$

201998 Mod

301998 Mod 401998 Mod Unit $<5 \quad \mathrm{U}$

$<5 \quad U$

ug/L

ug/L

$<5 \quad \mathrm{U}$

$<5$ U

$<5$ U

$<5$ U

$<5 \quad \mathrm{U}$

$<10 \quad \mathrm{U}$

$<5$ U

$<5 \quad \mathrm{U}$

$<5 \quad \mathrm{U}$

$<5 \quad$ U

$<5 \quad \mathrm{U}$

$<5 \quad \mathrm{U}$

$<5 \quad$ U

$<1000 \quad \mathrm{U}$

$<10 \quad \mathrm{U}$

$<.00046 \mathrm{U}$

$<.2 \quad \mathrm{U}$

$<2$ U

$<10 \quad U$

ug/L

ug/L

$\mathrm{ug} / \mathrm{L}$

$\mathrm{ug} / \mathrm{L}$

ug/L

ug/L

$\mathrm{ug} / \mathrm{L}$

$\mathrm{ug} / \mathrm{L}$

ug/L

ug/L

$\mathrm{ug} / \mathrm{L}$

ug/L

ug/L

ug/L

$u g / L$

$u g / L$

ug/L

$\mathrm{ug} / \mathrm{L}$

ug/L

$\mathrm{ug} / \mathrm{L}$

$\mathrm{ug} / \mathrm{L}$

ug/L

ug/L

$u g / L$

$\mathrm{ug} / \mathrm{L}$

Note: Concentrations in bold exceed the Driaking Water Standards listed in Appendix A. Units are for all four quarters. 
WELL: TNX 9D

\section{ANALYTICAL DATA}

\section{Analyte}

2,6-Dinitrotoluene

2-Chloronaphthalene

2-Chlorophenol

2-Hexanone

2-Methyl-4,6-dinitrophenol

2-Methylnaphthalene

2-Nitrophenol

3,3"-Dichlorobenzidine

4-Bromophenyl phenyl ether

4-Chloro-m-cresol

4-Chloroaniline

4-Chlorophenyl phenyl ether

4-Nitrophenol

Acenaphthene

Acenaphthylene

Acetone

Acetonitrile (Methyl cyanide)

Acrolein

Acrylonitrile

Aldrin

Allyl chloride

Aluminum, total recoverable

Aniline

Anthracene

Antimony, total recoverable

Arsenic, total recoverable

Barium, total recoverable

Benzene

Benzo(a)anthracene

Benzo(a)pyrene

Benzo(b)fluoranthene

Benzo(g,h,i)perylene

Benzo(k)fluoranthene

Benzyl alcohol

Beryllium, total recoverable

Bis(2-chloroethoxy) methane

Bis (2-chloroethyl) ether

Bis(2-ethylhexyl) phthalate

Bromodichloromethane

Bromoform

Bromomethane (Methyl bromide)

Butylbenzyl phthalate

Cadmium, total recoverable
101998 Mod

201998 Mod

301998 Mod

$<10 \quad \mathrm{U}$

$<10 \quad \mathrm{U}$

$<10 \quad \mathrm{U}$

$<5 \quad \mathrm{U}$

$<25 \quad \mathrm{U}$

$<10 \quad \mathrm{U}$

$<10 \quad U$

$<10 \quad \mathrm{U}$

$<10$ U

$<10 \quad \mathrm{U}$

$<10 \quad \mathrm{U}$

$<10 \quad \mathrm{U}$

$<25$ U

$<10 \quad \mathrm{U}$

$<10 \quad \mathrm{U}$

$<20$ U

$<500 \quad \mathrm{U}$

$<50 \quad \mathrm{U}$

$<50 \quad \mathrm{U}$

$<.1 \quad U$

$<10 \quad \mathrm{U}$

79.6

74.3 J $E$

$<25 \mathrm{U}$

$<10$ U

$<100 \quad \mathrm{U}$

$4.83 \quad \mathrm{~J} \quad \mathrm{E}$

60.7

$<5 \quad$ U

$<10 \quad \mathrm{U}$

$<10 \quad \mathrm{U}$

$<10 \quad \mathrm{U}$

$<10 \quad \mathrm{U}$

$<10$ U

$<10 \quad \mathrm{U}$

2.61 J $E$

$<10$ U

$<10 \quad \mathrm{U}$

$<10 \quad U$

$<5 \quad \mathrm{U}$

$<5 \quad \mathrm{U}$

$<5 \quad \mathrm{U}$

$<10 \quad \mathrm{U}$

3.42 J E
401998 Mod Unit

ug/L

ug/L

ug/L

ug/L

ug/L

ug/L

$u g / L$

ug/L

ug/L

ug/L

ug/L

ug/L

ug/L

ug/L

ug/L

ug/L

$\mathrm{ug} / \mathrm{L}$

ug/L

ug/L

$\mathrm{ug} / \mathrm{L}$

$\mathrm{ug} / \mathrm{L}$

ug/L

$u g / L$

$\mathrm{ug} / \mathrm{L}$

ug/L

$\mathrm{ug} / \mathrm{L}$

ug/L

ug $/ \mathrm{L}$

$\mathrm{ug} / \mathrm{L}$

ug/L

$\mathrm{ug} / \mathrm{L}$

$\mathrm{ug} / \mathrm{L}$

$\mathrm{ug} / \mathrm{L}$

$\mathrm{ug} / \mathrm{L}$

$\mathrm{ug} / \mathrm{L}$

$u g / L$

$\mathrm{ug} / \mathrm{L}$

ug/L

$\mathrm{ug} / \mathrm{L}$

ug/L

ug/L

$\mathrm{ug} / \mathrm{L}$

$\mathrm{ug} / \mathrm{L}$

Note: Concentrations in bold exceed the Drinking Water Standards listed in Appendix A. Units are for all four quarters. 
WELL: TNX 9D

\section{ANALYTICAL DATA}

\begin{tabular}{|c|c|c|c|c|c|c|c|c|}
\hline Analyte & 101998 & $\underline{201998}$ & Mod & 301998 & Mod & $\underline{401998}$ & Mod & Unit \\
\hline Carbazole & & & & $<20$ & $\bar{U}$ & , & & \\
\hline Carbon disulfide & & & & $<5$ & $\mathrm{U}$ & & & \\
\hline Chlordane & & & & $<.2$ & $\mathrm{U}$ & & & \\
\hline Chlorobenzene & & · & . & $<5$ & $\mathrm{U}$ & & & \\
\hline Chloroethane & & & & $<10$ & $\mathrm{U}$ & & & \\
\hline Chloroethene (Vinyl chloride) & & & & $<5$ & $\mathbf{U}$ & & & \\
\hline Chloromethane (Methyl chloride) & & & & $<5$ & $\mathrm{U}$ & & & \\
\hline Chloroprene & & & & $<50$ & $\mathrm{U}$ & & & \\
\hline Chromium, total recoverable & & & & $<10$ & $\mathrm{U}$ & & & \\
\hline Chrysene & & & & $<10$ & $\mathrm{U}$ & & & \\
\hline Cobalt, total recoverable & & & & 4.37 & J $\quad \mathbf{E}$ & $\therefore$ & & \\
\hline Copper, total recoverable & & & & $<20$ & U & & & \\
\hline Cyanide & & & & $<10$ & $\mathrm{U}$ & & & \\
\hline Di-n-butyl phthalate & & & & $<10$ & $\mathrm{U}$ & & & \\
\hline Di-n-octyl phthaiate & & & & $<10$ & $\mathbf{U}$ & & & \\
\hline Dibenz(a,h)anthracene & & & & $<20$ & $\mathrm{U}$ & & & \\
\hline Dibenzofuran & & & & $<10$ & $\mathbf{U}$ & & & \\
\hline Dibromochloromethane & & & & $<5$ & $\mathrm{U}$ & & & \\
\hline Dibromomethane (Methylene bromide) & & & & $<5$ & $\mathrm{U}$ & & & \\
\hline Dichlorodifluoromethane & & & & $<5$ & $\mathrm{U}$ & & & \\
\hline Dichloromethane (Methylene chloride) & & & & $<4.11$ & U V & & & \\
\hline Dieldrin & & & & $<.2$ & $\mathrm{U}$ & & & \\
\hline Diethyl phthalate & & & & $<10$ & $\mathbf{U}$ & & & \\
\hline Dimethyl phthalate & & & & $<10$ & U & . & & \\
\hline Endosulfan I & & & & $<.1$ & $\mathrm{U}$ & & & \\
\hline Endosulfan $\mathbf{I I}$ & & &. & $<2$ & $\mathbf{U}$ & & & \\
\hline Endosulfan sulfate & & & & $<.2$ & $\mathrm{U}$ & & & \\
\hline Endrin & & & & $<.2$ & $\mathrm{U}$ & & & \\
\hline Endrin aldehyde & & & & $<.2$ & $U$ & & & \\
\hline Ethyl methacrylate & & & & $<5$ & $\mathbf{U}$ & & & \\
\hline Ethylbenzene & & & & $<5$ & $U$ & & & \\
\hline Fluoranthene & & & & $<10$ & $\mathbf{U}$ & & & \\
\hline Fluorene & & & & $<10$ & $\mathbf{U}$ & & & \\
\hline Heptachlor & & & & $<.1$ & $\mathbf{U}$ & & & \\
\hline Heptachlor epoxide & & & & $<.1$ & $\mathbf{U}$ & & & \\
\hline Hexachlorobenzene & & & & $<10$. & $\mathrm{U}$ & & & \\
\hline Hexachlorobutadiene & & & & $<20$ & $\mathbf{U}$ & & & \\
\hline Hexachlorocyclopentadiene & & & & $<10$ & $\mathbf{U}$ & & & \\
\hline Hexachlorodibenzo-p-dioxins & . & & & $<.0015$ & $\mathrm{U}$ & & & \\
\hline Hexachlorodibenzo-p-furans & & & & $<.00087$ & $\mathbf{U}$ & & & \\
\hline Hexachloroethane & & & & $<10$ & $\mathbf{U}$ & & & \\
\hline Indeno( $1,2,3-c, d)$ pyrene & & & & $<10$ & $\mathrm{U}$ & - & & \\
\hline Iodomethane (Methyl iodide) & & & & $<5$ & $\mathbf{U}$ & & & \\
\hline
\end{tabular}

Note: Concentrations in bold exceed the Drinking Water Standards listed in Appendix A. Units are for all four quarters. 
WELL: TNX 9D

ANALYTICAL DATA

Analyte

Iron, total recoverable

Isobutyl alcohol

Isophorone

Lindane

Methacrylonitrile

Methoxychlor

Methyl ethyl ketone

Methyl isobutyl ketone

Methyl methacrylate

$\mathrm{N}$-Nitrosodimethylamine

$\mathrm{N}$-Nitrosodiphenylamine

$\mathrm{N}$-Nitrosodipropylamine

Naphthalene

Nickel, total recoverable

Nitrobenzene

PCB 1016

PCB 1221

PCB 1232

PCB 1242

PCB 1248

PCB 1254

PCB 1260

Pentachlorodibenzo-p-dioxins

Pentachlorodibenzo-p-furans

Pentachloroethane

Pentachlorophenol

Phenanthrene

Phenol

Propionitrile

Pyrene

Selenium, total recoverable

Silver, total recoverable

Styrene

Sulfide

Tetrachlorodibenzo-p-dioxins

Tetrachlorodibenzo-p-furans

Thallium, total recoverable

Tin, total recoverable

Toluene

Toxaphene

Trichlorofluoromethane

Vanadium, total recoverable

Vinyl acetate
$101998 \mathrm{Mod}$

$\frac{20199}{3400}$

2590

$<1500$

$<20$

$<.1$

$<500$

$<1$

$<10 \quad \mathrm{U}$

$<5 \quad \mathrm{U}$

$<50 \quad \mathrm{U}$

$<20 \quad \mathrm{U}$

$<10 \quad \mathrm{U}$

$<10 \quad U$

$<20 \quad \mathrm{U}$

$7.97 \mathrm{~J}$

$<10 \quad \mathrm{U}$

$<2 \quad$ U

$<2$ U

$<1 \quad U$

$<1 \quad$ U

$<1 \quad$ U

$<1 \quad$ U

$<1 \quad$ U

$<.00081 \mathrm{U}$

$<00074 \mathrm{U}$

$<200 \quad \mathrm{U}$

$<25 \quad \mathrm{U}$

$<10 \quad \mathrm{U}$

$<10 \quad \mathrm{U}$

$<500 \quad U$

$<10 \quad \mathrm{U}$

$<10 \quad \mathrm{U}$

$<20 \quad U$

$<5 \quad$ U

$<1000 \mathrm{U}$

$<.00046 \mathrm{U}$

$<.00038 \mathrm{U}$

$<10 \quad \mathrm{U}$

$<200 \quad$ U

$<5 \quad \mathrm{U}$

$<1 \quad U$

$<5 \quad \mathrm{U}$

$<10 \quad \mathrm{U}$

$<20$ U
401998 Mod Unit

ug/L

ug $/ \mathrm{L}$

ug/L

$u g / \mathrm{L}$

ug/L

$\mathrm{ug} / \mathrm{L}$

$\mathrm{ug} / \mathrm{L}$

ug/L

$u g / L$

ug/L

ug/L

ug/L

$\mathrm{ug} / \mathrm{L}$

ug/L

ug/L

ug/L

ug/L

ug/L

ug/L

ug/L

ug/L

ug/L

ug/L

ug/L

ug/L

ug/

ug/L

$\mathrm{ug} / \mathrm{L}$

ug/L

ug/L

ug/L

ug/L

$\mathrm{ug} / \mathrm{L}$

ug/L

ug/L

ug/L

$\mathrm{ug} / \mathrm{h}$

ug/ $\mathrm{L}$

ug/

ug/L

$u g / L$

$\mathrm{ug} / \mathrm{L}$

ug/L

Note: Concentrations in bold exceed the Drinking Water Standards listed in Appendix A. Units are for all four quarters. 
WELL: TNX 9D

\section{ANALYTICAL DATA}

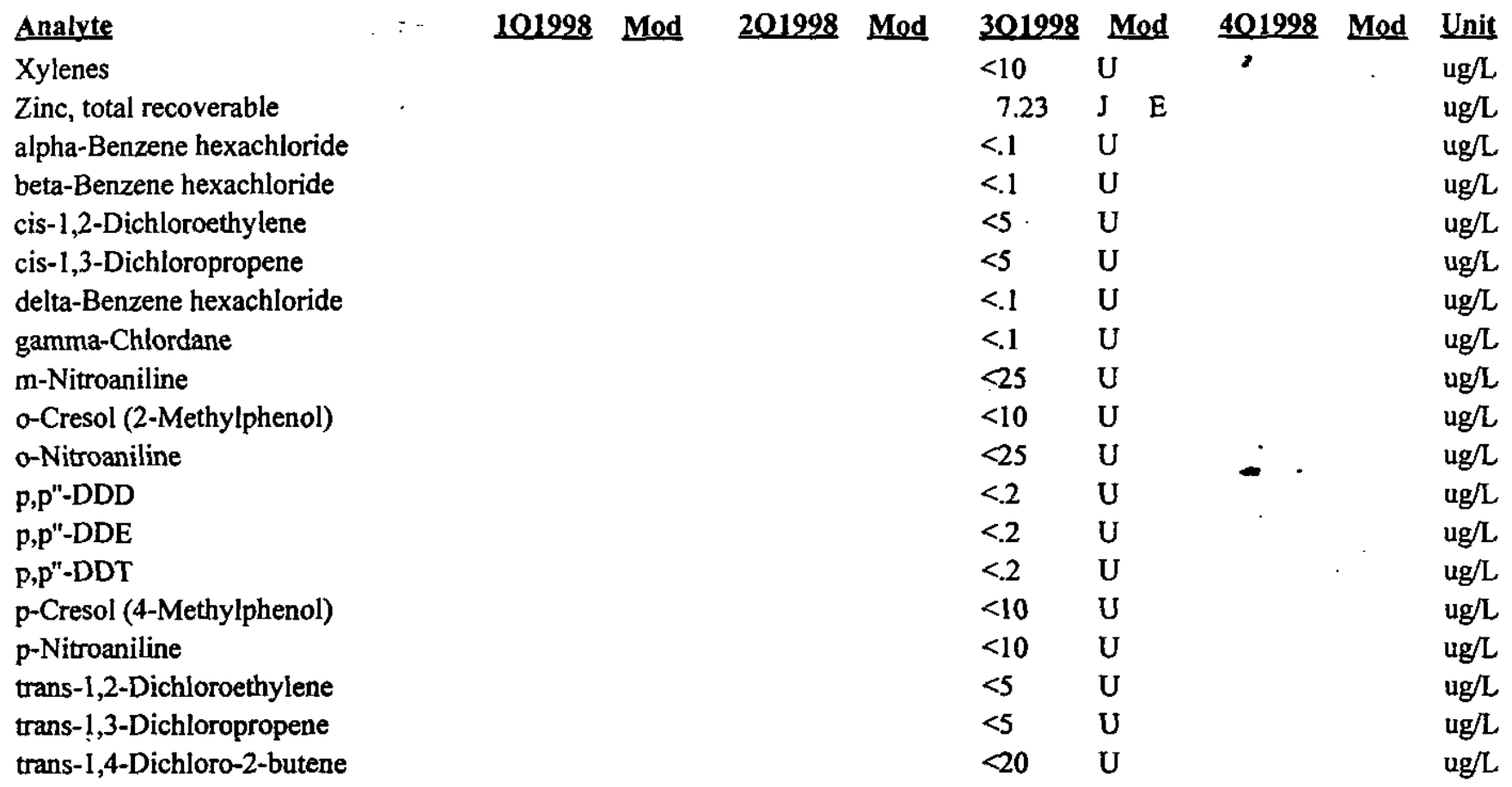

Note: Concentrations in bold exceed the Drinking Water Standards listed in Appendix A. Units are for all four quarters. 
WSRC-RP-99-4003

Unclassified

WELL: TNX 10D

SRS Coord, Lat/Longitude Screen Zone Elevation Top of Standpipe Top of Casing Casing Pump Screen Zone N 70999.3 $33.209 \operatorname{Deg} N$ E 16166.781 .762 Deg W

$97.0-77.0 \mathrm{ft} \mathrm{msl}$ $102.5 \mathrm{ft} \mathrm{msl}$

SAMPLE DATE

03/05/98

$05 / 12 / 98$

$102.3 \mathrm{ft} \mathrm{msl}$

4" PVC

$\mathrm{S}$

Unconfined

08/05/98

\section{FIELD DATA}

$\begin{array}{ll}\text { Analyte } & \mathbf{1 0 1 9 9 8} \\ \text { Depth to water } & 6 \\ \text { pH } & 5 \\ \text { Sp. Conductance } & 140 \\ \text { Water temperature } & 13 \\ \text { Alkalinity as CaCO3 } & 14 . \\ \text { Phenolpthalein Alkalinity } & 0 \\ \text { Turbidity } & 14.4 \\ \text { Volumes purged } & 3.94920\end{array}$

201998

6.75

301998

9.56

5.2

140

20.6

2

0

14.5

NPS

Sampling codes

3.00229

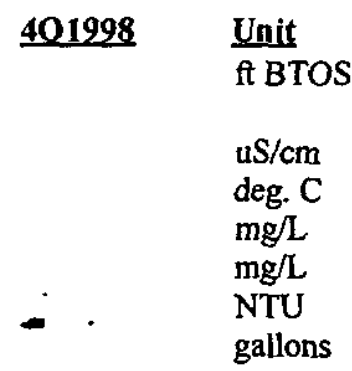

\section{ANALYTICAL DATA}

\section{Analvite}

1,1,1,2-Tetrachloroethane

1,1,1-Trichloroethane

1,1,2,2-Tetrachloroethane

1,1,2-Trichloroethane

1,1-Dichloroethane

1,1-Dichloroethylene

1,2,3-Trichloropropane

1,2,4-Trichlorobenzene

1,2-Dibromo-3-chloropropane

1,2-Dibromoethane

1,2-Dichlorobenzene

1,2-Dichloroethane

1,2-Dichloropropane

1,3-Dichlorobenzene

1,4-Dichlorobenzene

1,4-Dioxane

2,2-Oxybis(1-chloropropane)

2,3,7,8-TCDD

2,4,5-T

2,4,5-TP (Silvex).

2,4,5-Trichlorophenol

2,4,6-Trichlorophenol

2,4-Dichlorophenol

2,4-Dichlorophenoxyacetic acid

2,4-Dimethyl phenol

2,4-Dinitrophenol

2,4-Dinitrotoluene
101998 Mod 201998 Mod 301998 Mod 401998 Mod Unit

$<5 \quad \mathrm{U}$

$<5 \quad U$

$<5 \quad U$

$<5 \quad U$

$<5 \quad \mathrm{U}$

$<5 \quad \mathrm{U}$

$<5 \quad \mathrm{U}$

$<10 \quad$ U

$<5 \quad \mathrm{U}$

$<5 \quad \mathrm{U}$

$<5 \quad \mathrm{U}$

$<5 \quad U$

$<5 \quad \mathrm{U}$

$<5 \quad U$

$<5 \quad U$

$<1000 \quad U$

$<10 \quad U$

$<.00057 \mathrm{U}$

$<.2$ UJ $\mathrm{O}$

$<.2$ UJ $\mathrm{O}$

$<10$ U

$<25 \quad \mathrm{U}$

$<10 \quad \mathrm{U}$

$<2$ UJ $\mathrm{O}$

$<10 \quad \mathrm{U}$

$<25 \quad$ U

$<10 \quad \mathrm{U}$
ug/L

ug/L

ug/L

ug/L

ug $/ \mathrm{L}$

ugh

ug/L

$\mathrm{ug} / \mathrm{L}$

ug/L

ug/L

ug/L

ug/L

ug/L

ug/L

$\mathrm{ug} / \mathrm{L}$

ug/L

$u g / L$

ug/L

$u g / L$

ug/L

ug/L

ug/L

$\mathrm{ug} / \mathrm{L}$

$\mathrm{ug} / \mathrm{L}$

ug/L

ug/L

ug/L

Note: Concentrations in bold exceed the Drinking Water Standards listed in Appendix A. Units are for all four quarters. 
WELL: TNX 10D

ANALYTICAL DATA

\begin{tabular}{|c|c|c|c|c|c|c|c|c|}
\hline Analyte & 101998 & Mod. & 201998 Mod & 301998 & Mod & 401998 & Mod & Unit \\
\hline 2,6-Dinitrotoluene & & & & $<10$ & $\mathrm{U}$ & $=$ & & $\mathrm{ug} / \mathrm{L}$ \\
\hline 2-Chloronaphthalene & & & & $<10$ & $\mathrm{U}$ & & & $\mathrm{ug} / \mathrm{L}$ \\
\hline 2-Chlorophenol & & & & $<10$ & $\mathrm{U}$ & & & $\mathrm{ug} / \mathrm{L}$ \\
\hline 2-Hexanone & & & & $<5$ & $\mathrm{U}$ & & & $\operatorname{gg} / \mathrm{L}$ \\
\hline 2-Methyl-4,6-dinitrophenol & & & & $<25$ & $\mathrm{U}$ & & & $\operatorname{gg} /$ \\
\hline 2-Methylnaphthalene & & & & $<10$ & $\mathrm{U}$ & & & $\mathrm{ug} / \mathrm{L}$ \\
\hline 2-Nitrophenol & & & & $<10$ & $\mathrm{U}$ & & & $\mathrm{ug} / \mathrm{L}$ \\
\hline 3,3"-Dichlorobenzidine & & & & $<10$ & $\mathbf{U}$ & & & $\lg / \mathrm{L}$ \\
\hline 4-Bromophenyl phenyl ether & & & & $<10$ & $\mathrm{U}$ & & & $1 \mathrm{~g} / \mathrm{L}$ \\
\hline 4-Chloro-m-cresol & · & & & $<10$ & $\mathbf{U}$ & & & $\operatorname{gg} / \mathrm{L}$ \\
\hline 4-Chloroaniline & & & & $<10$ & $\mathbf{U}$ & $\therefore$ & & $\operatorname{ug} / \mathrm{L}$ \\
\hline 4-Chlorophenyl phenyl ether & & & & $<10$ & $\mathrm{U}$ & & & $\lg / \mathrm{L}$ \\
\hline 4-Nitrophenol & & & & $<25$ & U & & & $\mathrm{ug} / \mathrm{L}$ \\
\hline Acenaphthene & & & & $<10$ & $\mathrm{U}$ & & & $g / L$ \\
\hline Acenaphthylene & & & & $<10$ & $\mathrm{U}$ & & & $\lg / \mathrm{L}$ \\
\hline Acetone & & & & $<20$ & $\mathrm{U}$ & & & $\lg / \mathrm{L}$ \\
\hline Acetonitrile (Methyl cyanide) & & & & $<500$ & $\mathrm{U}$ & & & $\mathrm{ug} / \mathrm{L}$ \\
\hline Acrolein & & & & $<50$ & $\mathrm{U}$ & & & $\mathrm{ug} / \mathrm{L}$ \\
\hline Acrylonitrile & & & & $<50$ & $U$ & & & $g / L$ \\
\hline Aldrin & & & & $<.1$ & $\mathbf{U}$ & & & $\lg / \mathrm{L}$ \\
\hline Allyl chloride & & & & $<10$ & $\mathrm{U}$ & & & $\mathrm{ug} / \mathrm{L}$ \\
\hline Aluminum, total recoverable & & & 305 & 1340 & & & & $\mathrm{ug} / \mathrm{L}$ \\
\hline Aniline & & & & $<25$ & $\mathrm{U}$ & & & $\mathrm{ug} / \mathrm{L}$ \\
\hline Anthracene & 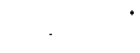 & & & $<10$ & $\mathbf{U}$ & & & $\lg / \mathrm{L}$ \\
\hline Antimony, total recoverable & . & $\because$ & - & $<100$ & $\mathbf{U}$ & & & $\mathrm{ug} / \mathrm{L}$ \\
\hline Arsenic, total recoverabie & . & & & $<10$ & $\mathrm{U}$ & $\cdot$ & & $u g / L$ \\
\hline Barium, total recoverable & & & & 67.5 & & & & $u g / L$ \\
\hline Benzene & & & & $<5$ & $\mathrm{U}$ & & & $\mathrm{ug} / \mathrm{L}$ \\
\hline Benzo(a)anthracene & & & & $<10$ & $\mathrm{U}$ & & & $1 g / \mathrm{L}$ \\
\hline Benzo(a)pyrene & & & & $<10$ & $\mathrm{U}$ & & & $g / L$ \\
\hline Benzo(b)fluoranthene & & & & $<10$ & $\mathbf{U}$ & & & $u g / L$ \\
\hline Benzo(g,h,i)perylene & & & & $<10$ & $\mathrm{U}$ & & & $\mathrm{ug} / \mathrm{L}$ \\
\hline Benzo(k)fluoranthene & & & & $<25$ & $\mathrm{U}$ & & & $\mathrm{g} / \mathrm{L}$ \\
\hline Benzyl alcohol & & & & $<10$ & $\mathrm{U}$ & & & $\mathrm{ug} / \mathrm{L}$ \\
\hline Beryllium, total recoverable & & & & $<10$ & $\mathrm{U}$ & . & & $\mathrm{ug} / \mathrm{L}$ \\
\hline Bis(2-chloroethoxy) methane & & & & $<10$ & $\mathrm{U}$ & & & $\mathrm{ug} / \mathrm{L}$ \\
\hline Bis(2-chloroethyl) ether & & & & $<10$ & $\mathrm{U}$ & & & $\mathrm{ug} / \mathrm{L}$ \\
\hline Bis(2-ethylhexyl) phthalate & & & & $<10$ & $\mathbf{U}$ & . & & ug $/ \mathrm{L}$ \\
\hline Bromodichloromethane & & & & $<5$ & $\mathrm{U}$ & & & ug/L \\
\hline Bromoform & & & & $<5$ & $\mathrm{U}$ & & & $\mathrm{ug} / \mathrm{L}$ \\
\hline Bromomethane (Methyl bromide) & & & & $<5$ & $\mathrm{U}$ & & & $\lg / \mathrm{L}$ \\
\hline Butylbenzyl phthalate & & & & $<20$ & $\mathrm{U}$ & $\approx$ & & $\lg / \mathrm{L}$ \\
\hline Cadmium, total recoverable & & & & $<10$ & $\mathrm{U}$ & & & $g / L$ \\
\hline
\end{tabular}

Note: Concentrations in bold exceed the Drinking Water Standards listed in Appendix A. Units are for all four quarters. 
WELL: TNX 10D

ANALYTICAL DATA

\section{Analyte}

Carbazole

Carbon disulfide

Chlorobenzene

Chloroethane

Chloroethene (Vinyl chloride)

Chloromethane (Methyl chloride)

Chloroprene

Chromium, total recoverable

Chrysene

Cobalt, total recoverable

Copper, total recoverable

Cyanide

Di-n-butyl phthalate

Di-n-octyl phthalate

Dibenz(a,h)anthracene

Dibenzofuran

Dibromochloromethane

Dibromomethane (Methylene bromide',

Dichlorodifluoromethane

Dichloromethane (Methylene chloride)

Dieldrin

Diethyl phthalate

Dimethyl phthalate

Endosulfan I

Endosulfan II

Eviusilfan sulfate

Endrin

Endrin aldehyde

Ethyl methacrylate

Ethylbenzene

Fluoranthene

Fluorene

Heptachlor

Heptachlor epoxide

Hexachlorobenzene

Hexachlorobutadiene

Hexachlorocyclopentadiene

Hexachlorodibenzo-p-dioxins

Hexachlorodibenzo-p-furans

Hexachloroethane

Indeno(1,2,3-c,d)pyrene

Iodomethane (Methyl iodide)

Iron, total recoverable

\section{Mod 201998 Mod}

301998 Med

$<10 \quad U$

9.37

$<5 \quad U$

$<10$ U

$<5 \quad \mathrm{U}$

$<5 \quad U$

$<50 \quad \mathrm{U}$

$<10 \quad \mathrm{U}$

$<10 \quad U$

$<20$ U

$<20$ U

$<10 \quad$ U

$<10$ U

$<20 \quad \mathrm{U}$

$<10$ U

$<10 \quad \mathrm{U}$

$<5 \quad \mathrm{U}$

$<5 \quad$ U

$<5 \quad \mathrm{U}$

$<4.17$ U V

$<2$ U

$<10 \quad \mathrm{U}$

$<10 \quad \mathrm{U}$

$<.1 \quad$ U

$<.2$ U

$<2$ U

$<.2 \quad \mathrm{U}$

$<.2 \quad \mathrm{U}$

$<5 \quad U$

$<5 \quad \mathrm{U}$

$<10 \quad \mathrm{U}$

$<10 \quad \mathrm{U}$

$<.1 \quad$ U

$<1 \quad \mathrm{U}$

$<20$ U

$<10 \quad \mathrm{U}$

$<10 \quad U$

$<.0012 \mathrm{U}$

$<.0007 \mathrm{U}$

$<10 \quad U$

$<20$ U

$<5$

401998 Mod Unit

$u g / L$

$\mathrm{ug} / \mathrm{L}$

$u g / L$

$u g / L$

ug/L

ug $/ \mathrm{L}$

ug $/$

$\mathrm{ug} / \mathrm{L}$

$\mathrm{ug} / \mathrm{L}$

$u g / L$

ug/L

ug/L

ug $/ \mathrm{L}$

ug $/ \mathrm{L}$

$u g / L$

$u g / L$

ug/L

$u g / L$

ug/L

ug/L

$u g / L$

ug $/ \mathrm{L}$

ug $/ \mathrm{L}$

ug/L

ug/L

ugit

$u g / L$

$\mathrm{ug} / \mathrm{L}$

ug/L

ug/L

ug/L

$u g / L$

ug $/ \mathrm{L}$

ug/L

$u g / L$

$\mathrm{ug} / \mathrm{L}$

$\mathrm{ug} / \mathrm{L}$

ug/L

$\mathrm{ug} / \mathrm{L}$

ug/L

$u g / L$

$\mathrm{ug} / \mathrm{L}$

ug/L

Note: Concentrations in bold exceed the Drinking Water Standards listed in Appendix A. Units are for all four quarters. 
WELL: TNX 10D

\section{ANALYTICAL DATA}

\section{Analyte}

Isobutyl alcohol

Isophorone

Lindane

Methacrylonitrile

Methoxychlor

Methyl ethyl ketone

Methyl isobutyl ketone

Methyl methacrylate

$\mathrm{N}$-Nitrosodimethylamine

$\mathrm{N}$-Nitrosodiphenylamine

N-Nitrosodipropylamine

Naphthalene

Nickel, total recoverable

Nitrobenzene

PCB 1016

PCB 1221

PCB 1232

PCB 1242

PCB 1248

PCB 1254

PCB 1260

Pentachlorodibenzo-p-dioxins

Pentachlorodibenzo-p-furans

Pentachloroethane

Pentachlorophenol

Phenantbrene

Phenol

Propionitrile

Pyrene

Selenium, total recoverable

Silver, total recoverable

Styrene

Sulfide

Tetrachlorodibenzo-p-dioxins

Tetrachlorodibenzo-p-furans

Thallium, total recoverable

Tin, total recoverable

Toluene

Toxaphene

Trichlorofluoromethane

Vanadium, total recoverable

Vinyl acetate

Xylenes
101998 Mod 201998 Mod 301998 Mod 401998 Mod Unit

$<1500 \mathrm{U}$

$<10 \quad \mathrm{U}$

$<.1 \quad \mathrm{U}$

$<500 \quad \mathrm{U}$

$<1 \quad U$

$<10 \quad \mathrm{U}$

$<5 \quad \mathrm{U}$

$<50 \quad \mathrm{U}$

$<10 \quad \mathrm{U}$

$<20$ U

$<20$ U

$<10 \quad \mathrm{U}$

7.9 J E

$<25 \mathrm{U}$

$<$ U

$<$ U

$<1 \quad$ U

$<$ U

$<1 \quad$ U

$<1 \quad$ U

$<1 \quad$ U

$<.00095 \mathrm{U}$

$<.00071 \mathrm{U}$

$<200 \quad U$

$<10 \quad \mathrm{U}$

$<10 \quad \mathrm{U}$

$<10$ U

$<500 \quad \mathrm{U}$

$<25 \mathrm{U}$

$<10$ U

$<20$ U

$<5 \quad U$

$<1000 \mathrm{U}$

$<.00057 \mathrm{U}$

$<.00068 \mathrm{U}$

$<10 \quad \mathrm{U}$

$<200 \quad$ U

$<5 \quad \mathrm{U}$

$<1 \quad U$

$<5 \quad \mathrm{U}$

$<10 \quad \mathrm{U}$

$<2$ U

$<10 \quad \mathrm{U}$
ug/L

ug/L

ug/L

ug $/$

$u g / L$

$\mathrm{ug} / \mathrm{L}$

$\mathrm{ug} / \mathrm{L}$

$\operatorname{ug} / \mathrm{L}$

ug/L

$\mathrm{ug} / \mathrm{L}$

ug $/$

ug/L

$\mathrm{ug} / \mathrm{L}$

$u g / \mathrm{L}$

ug $/$

ug/L

ug/

$\mathrm{ug} / \mathrm{L}$

ug/L

$\operatorname{ug} / \mathrm{L}$

$\operatorname{ug} / \mathrm{L}$

ug $\mathcal{L}$

ug/L

ug/

ug/L

$\mathrm{ug} / \mathrm{L}$

ug $/ \mathrm{L}$

$\operatorname{ug} / \mathrm{L}$

ug $/ L$

ug/L

ug/L

ug $/ \mathrm{L}$

ug/

ug/L

$\operatorname{ug} / 2$

ug/L

ugh

$\mathrm{ug} / \mathrm{L}$

ug/L

ug $\mathcal{L}$

$\operatorname{ug} / \mathrm{L}$

$\mathrm{ug} / \mathrm{L}$

ug/L

Note: Concentrations in bold exceed the Drinking Water Standards listed in Appendix A. Units are for all four quarters. 
WSRC-RP-99-4003

Unclassified

WELL: TNX 10D

ANALYTICAL DATA

Analvte

101998 Mod 201998 Mod

301998 Mod

401998 Mod Unit

21.4

$<.1 \quad$ U

$<.1 \quad \mathrm{U}$

$<.1 \quad U$

$<5 \quad \mathrm{U}$

$<5 \quad U$

$<.1 \quad$ U

$<.1 \quad$ U

$<25 \quad U$

$<10 \quad \mathrm{U}$

$<25 \quad \mathrm{U}$

$<.2 \quad \mathrm{U}$

- ug/L

alpha-Benzene hexachloride

ug/L

beta-Benzene hexachloride

cis-1,3-Dichloropropene

delta-Benzene hexachloride

gamma-Chlordane

$\mathrm{m}$-Nitroaniline

o-Cresol (2-Methylphenol)

o-Nitroaniline

$<.2 \quad \mathrm{U}$

$p, p^{\prime \prime}-D D E$

p,p"-DDT

p-Cresol (4-Methylphenol)

$<.2$ U

$<10 \quad U$

$<10$ U

p-Nitroaniline

trans-1,2-Dichloroethylene

trans-1,3-Dichloropropene

$<5 \quad \mathrm{U}$

$<5 \quad \mathrm{U}$

ug/L

$u g / L$

ug/

ug/L

$\mathrm{ug} / \mathrm{L}$

ug/L

$\mathrm{ug} / \mathrm{L}$

$u g / L$

$\mathrm{ug} / \mathrm{L}$

ug/L

$\mathrm{ug} / \mathrm{L}$

ug/L

ug/L

ug/L

$\mathrm{ug} / \mathrm{L}$

ug/L

trans-1,4-Dichloro-2-butene

$<20 \quad \mathrm{U}$

ug/L

Note: Concentrations in bold exceed the Drinking Water Standards listed in Appendix A. Units are for all four quarters. 
WELL: TNX 11D

SRS Coord, Lat/Longitude Screen Zone Elevation Top of Standpipe Top of Casing Casing Pump Screen Zone N 71199.3 33.210 Deg N $\quad 93.2-73.2 \mathrm{ft} \mathrm{msl} 100.3 \mathrm{ft} \mathrm{msl} 99.8 \mathrm{ft} \mathrm{msl} 4$ "PVC $\mathrm{S}$ Unconfined E 16165.581 .762 Deg W. - -
SAMPLE DATE
$03 / 03 / 98$
$05 / 12 / 98$
$08 / 06 / 98$

FIELD DATA

\begin{tabular}{|c|c|c|c|c|c|}
\hline Analyte & 101998 & $\underline{201998}$ & 301998 & 401998 & Unit \\
\hline Depth to water & 4.06 & 5.5 & 7.45 & & ft BTOS \\
\hline $\mathrm{pH}^{*}$ & 7.1 & 5 & 5 & & \\
\hline Sp. Conductance & 53 & 56 & 50 & & $\mathrm{uS} / \mathrm{cm}$ \\
\hline Water temperature & 19.6 & 23 & 21.2 & & deg. C \\
\hline Alkalinity as $\mathrm{CaCO} 3$ & 3 & 2 & 1 & & $\mathrm{mg} / \mathrm{L}$ \\
\hline Phenolpthalein Alkalinity & 0 & 0 & 0 & & $\mathrm{mg} / \mathrm{L}$ \\
\hline Turbidity & 2.4 & 2.9 & 10.2 & - & NTU \\
\hline $\begin{array}{l}\text { Volumes purged } \\
\text { Sampling codes }\end{array}$ & 3.99020 & 3.25107 & 4.05973 & & gallons \\
\hline
\end{tabular}

\section{ANALYTICAL DATA}

\section{Analyte}

1,1,1,2-Tetrachloroethane

1,1,1-Trichloroethane

1,1,2,2-Tetrachloroethane

1,1,2-Trichloroethane

1,1-Dichloroethane

1,1-Dichloroethylene

1,2,3-Trichloropropane

1,2,4-Trichlorobenzene

1,2-Dibromo-3-chloropropane

1,2-Dibromoethane

1,2-Dichlorobenzene

1,2-Dichloroethane

1,2-Dichloropropane

1,3-Dichlorobenzene

1,4-Dichlorobenzene

1,4-Dioxane

2,2-Oxybis(1-chloropropane)

2,3,7,8-TCDD

2,4,5-T

2,4,5-TP (Silvex)

2,4,5-Trichlorophenol

2,4,6-Trichlorophenol

2,4-Dichlorophenol

2,4-Dichlorophenoxyacetic acid

2,4-Dimethyl phenol

2,4-Dinitrophenol

2,4-Dinitrotoluene

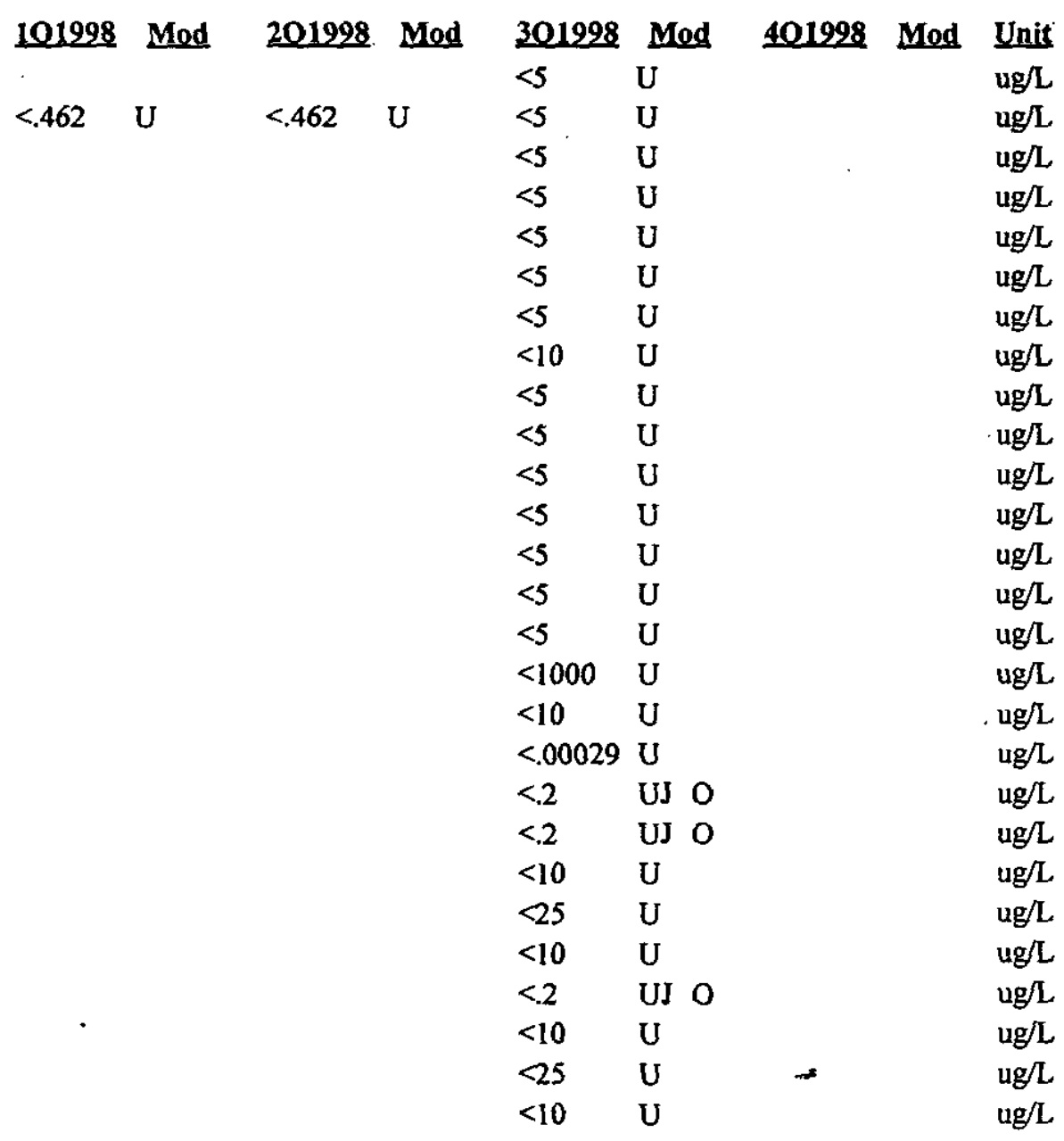

Note: Concentrations in bold exceed the Drinking Water Standards listed in Appendix A. Units are for all four quarters. 
WELL: TNX 11D

ANALYTICAL DATA

Analyte

2,6-Dinitrotoluene

2-Chloronaphthalene

2-Chlorophenol

2-Hexanone

2-Methyl-4,6-dinitrophenol

2-Methylnaphthalene

2-Nitrophenol

3,3"-Dichlorobenzidine

4-Bromophenyl phenyl ether

4-Chloro-m-cresol

4-Chloroaniline

4-Chlorophenyl phenyl ether

4-Nitrophenol

Acenaphthene

Acenaphthylene

Acetone

Acetonitrile (Methyl cyanide)

Acrolein

Acrylonitrile

Aldrin

Allyl chloride

Aluminum, total recoverable

Aniline

Anthracene

Antimony, total recoverable

Assenic, total recoverable

Barium, total recoverable

Benzene

Benzo(a)pyrene

Benzo(b)fluoranthene

Benzo(g,h,i)perylene

Benzo(k)fluoranthene

Benzoic acid

Beryllium, total recoverable

Bis(2-chloroethoxy) methane

Bis(2-chloroethyl) ether

Bis(2-ethylhexyl) phthalate

Bromodichloromethane

Bromoform

Bromomethane (Methyl bromide)

Butylbenzyl phthalate

Cadmium, total recoverable

Carbazole

\section{Mod 201998 Mod}

\begin{tabular}{|c|c|c|}
\hline 301998 & Mod & 401998 \\
\hline$<10$ & $U$ & \\
\hline$<10$ & $U$ & \\
\hline$<10$ & $U$ & \\
\hline$<5$ & $U$ & \\
\hline$<25$ & $U$ & \\
\hline
\end{tabular}

Unit

ug/L

ug/L

ug/L

ug/L

$\mathrm{ug} / \mathrm{L}$

$\mathrm{ug} / \mathrm{L}$

$\mathrm{ug} / \mathrm{L}$

$\mathrm{ug} / \mathrm{L}$

$\mathrm{ug} / \mathrm{L}$

ug/L

ug/L

ug/L

ug/L

ug/L

$\mathrm{ug} / \mathrm{L}$

$\mathrm{ug} / \mathrm{L}$

ug/L

ug/L

ug/L

$\mathrm{ug} / \mathrm{L}$

ug/L

ug/L

$\mathrm{ug} / \mathrm{L}$

$\mathrm{ug} / \mathrm{L}$

ug/L

ug/ $\mathrm{L}$

ug/L

$\mathrm{ug} / \mathrm{L}$

ug/L

ug/L

ug/L

ug $/$

ug/L

ug/L

$\mathrm{ug} / \mathrm{L}$

$\mathrm{ug} / \mathrm{L}$

$\mathrm{ug} / \mathrm{L}$

ug/L

ug/L

ug/L

ug/L

ug/L

$\mathrm{ug} / \mathrm{L}$

Note: Concentrations in bold exceed the Drinking Water Standards listed in Appendix A. Units are for all four quarters. 
WELL: TNX 11D

\section{ANALYTICAL DATA}

\section{Analyte}

Carbon disulfide

Chlorobenzene

Chloroethane

Chloroethene (Vinyl chloride)

Chloromethane (Methyl chloride)

Chloroprene

Chromium, total recoverable

Chrysene

Cobalt, total recoverable

Copper, total recoverable

Cyanide

Di-n-butyl phthalate

Di-n-octyl phthalate

Dibenz(a,h)anthracene

Dibenzofuran

Dibromochloromethane

Dibromomethane (Methylene bromide',

Dichlorodifluoromethane

Dichloromethane (Methylene chloride)

Dieldrin

Diethyl phthalate

Dimethyl phthalate

Endosulfan I

Endosulfan II

Endosulfan sulfate

Endrin

Endrin aldehyde

Ethyl methacrylate

Ethylbenzene

Fluoranthene

Fluorene

Heptachlor

Heptachlor epoxide

Hexachlorobenzene

Hexachlorobutadiene

Hexachlorocyclopentadiene

Hexachlorodibenzo-p-dioxins

Hexachlorodibenzo-p-furans

Hexachloroethane

Indeno(1,2,3-c,d)pyrene

Iodomethane (Methyl iodide)

Iron, total recoverable

Isobutyl alcohol
101998 Mod 201998 Mod 301998 Mod 401998 Mod Unit

$<5 \quad \mathrm{U}$

$<5 \quad \mathrm{U}$

$<10 \quad U$

$<5 \quad$ U

$<5 \quad \mathrm{U}$

$<50 \quad \mathrm{U}$

$<10 \quad \mathrm{U}$

$<10 \quad U$

$<20$ U

$<20$ U

$<10 \quad$ U

$<10 \quad \mathrm{U}$

$<10$ U

$<20$ U

$<10$ U

$<5 \quad$ U

$<5 \quad \mathrm{U}$

$<5 \quad$ U

$<10 \quad \mathrm{U}$

$<.2 \quad \mathrm{U}$

$<10$ U

$<10 \quad U$

$<.1$ U

$<2$ U

$<2$ U

$<.2$ U

$<.2$ U

$<$ U

$<5 \quad U$

$<10 \quad \mathrm{U}$

$<10$ U

$<1$ U

$<.1 \quad$ U

$<10 \quad$ U

$<20$ U

$<10$ U

$<.00089 \mathrm{U}$

$<.00042 \mathrm{U}$

$<10 \quad U$

$<10 \quad U$

$<5 \quad \mathrm{U}$

1100

$<1500 \quad \mathrm{U}$ ug/

ug/L

$u g / L$

ug/L

ug/L

$\mathrm{ug} / \mathrm{L}$

ug/L

$\mathrm{ug} / \mathrm{L}$

ug $/ \mathrm{L}$

ug/L

ug/L

ug $/$

ug $/ \mathrm{L}$

ug/L

ug/L

ug/L

ug/L

$\mathrm{ug} / \mathrm{L}$

ug/L

ug/L

ug/L

$u g / L$

$\mathrm{ug} / \mathrm{L}$

$u g / L$

ug/L

$\mathrm{ug} / \mathrm{L}$

$u g / L$

ug/L

ug $/$

ug/L

ug/

ug/L

ug $/$

$u g / L$

ug $/ \mathrm{L}$

$\mathrm{ug} / \mathrm{L}$

$\mathrm{ug} / \mathrm{L}$

ug/L

$\mathrm{ug} / \mathrm{L}$

$\mathrm{ug} / \mathrm{L}$

$\mathrm{ug} / \mathrm{L}$

$\mathrm{ug} / \mathrm{L}$

ug/L

Note: Concentrations in bold exceed the Drinking Water Standards listed in Appendix A. Units are for all four quarters. 
WELL: TNX 11D

ANALYTICAL DATA

Analyte

Isophorone

Lindane

Methacrylonitrile

Methoxychlor

Methyl ethyl ketone

Methyl isobutyl ketone

Methyl methacrylate

$\mathrm{N}$-Nitrosodimethylamine

$\mathrm{N}$-Nitrosodiphenylamine

N-Nitrosodipropylamine

Naphthalene

Nickel, total recoverable

Nitrobenzene

PCB 1016

PCB 1221

PCB 1232

PCB 1242

PCB 1248

PCB 1254

PCB 1260

Pentachlorodibenzo-p-dioxins

Pentachlorodibenzo-p-furans

Pentachloroethane

Pentachlorophenol

Phenanthrene

Phe

Propionitrile

Pyrene

Selenium, total recoverable

Silver, total recoverable

Styrene

Sulfide

Tetrachlorodibenzo-p-dioxins

Tetrachlorodibenzo-p-furans

Thallium, total recoverable

Tin, total recoverable

Toluene

Toxaphene

Trichlorofluoromethane

Vanadium, total recoverable

Vinyl acetate

Xylenes

Zinc, total recoverable
101998 Mod 201998 Mod 301998 Mod 401998 Mod Unit

$<20 \quad$ U

$<.1 \quad \mathrm{U}$

$<500 \quad U$

$<$ I. U

$<10 \quad U$

$<5 \quad$ U

$<50 \quad$ U

$<20 \quad$ U

$<10 \quad$ U

$<10 \quad$ U

$<20 \quad \mathrm{U}$

$<50$ U

$<10$ U

$<2$ U

$<$ U

$<$ U

$<1$ U

$<1 \quad$ U

$<1 \quad U$

$<$ U

$<.00063 \mathrm{U}$

$<.00045 \mathrm{U}$

$<200$ U

$<25$. U

$<10 \quad \mathrm{U}$

$<10$ U

$<500$ U

$<10 \quad U$

$<10$ U

$<20$ U

$<5 \quad \mathrm{U}$

$<1000$ U

$<.00029 \mathrm{U}$

$<.00039 \mathrm{U}$

$<10 \quad \mathrm{U}$

$<200$ U

$<5 \quad U$

$<$ U

$<$ U

$<10$ U

$<20$ U

$<10 \quad \mathrm{U}$

$<20$ U $u g / \mathrm{L}$

ug/L

ug/L

$u g / L$

$u g / L$

$u g / L$

ug/L

ug/L

$\mathrm{ug} / \mathrm{L}$

ug/L

ug/L

$\mathrm{ug} / \mathrm{L}$

$u g / L$

ug/L

$\mathrm{ug} / \mathrm{L}$

ug/L

ug/L

ug/L

$u g / L$

$u g / L$

ug/L

$u g / L$

ug/L

ugh

$u g / L$

$\mathrm{ug} / \mathrm{L}$

$\mathrm{ug} / \mathrm{L}$

$\mathrm{ug} / \mathrm{L}$

$\mathrm{ug} / \mathrm{L}$

ug/L

$\mathrm{ug} / \mathrm{L}$

ug/L

$u g / \mathrm{L}$

ug/L

$\mathrm{ug} / \mathrm{L}$

$\mathrm{ug} / \mathrm{L}$

$u g / \mathrm{L}$

ug/L

$u g / L$

$u g / L$

$u g / L$

ug/L

$\mathrm{ug} / \mathrm{L}$

Note: Concentrations in bold exceed the Drinking Water Stendards listed in Appendix A. Units are for all four quarters. 
WELL: TNX 11D

ANALYTICAL DATA

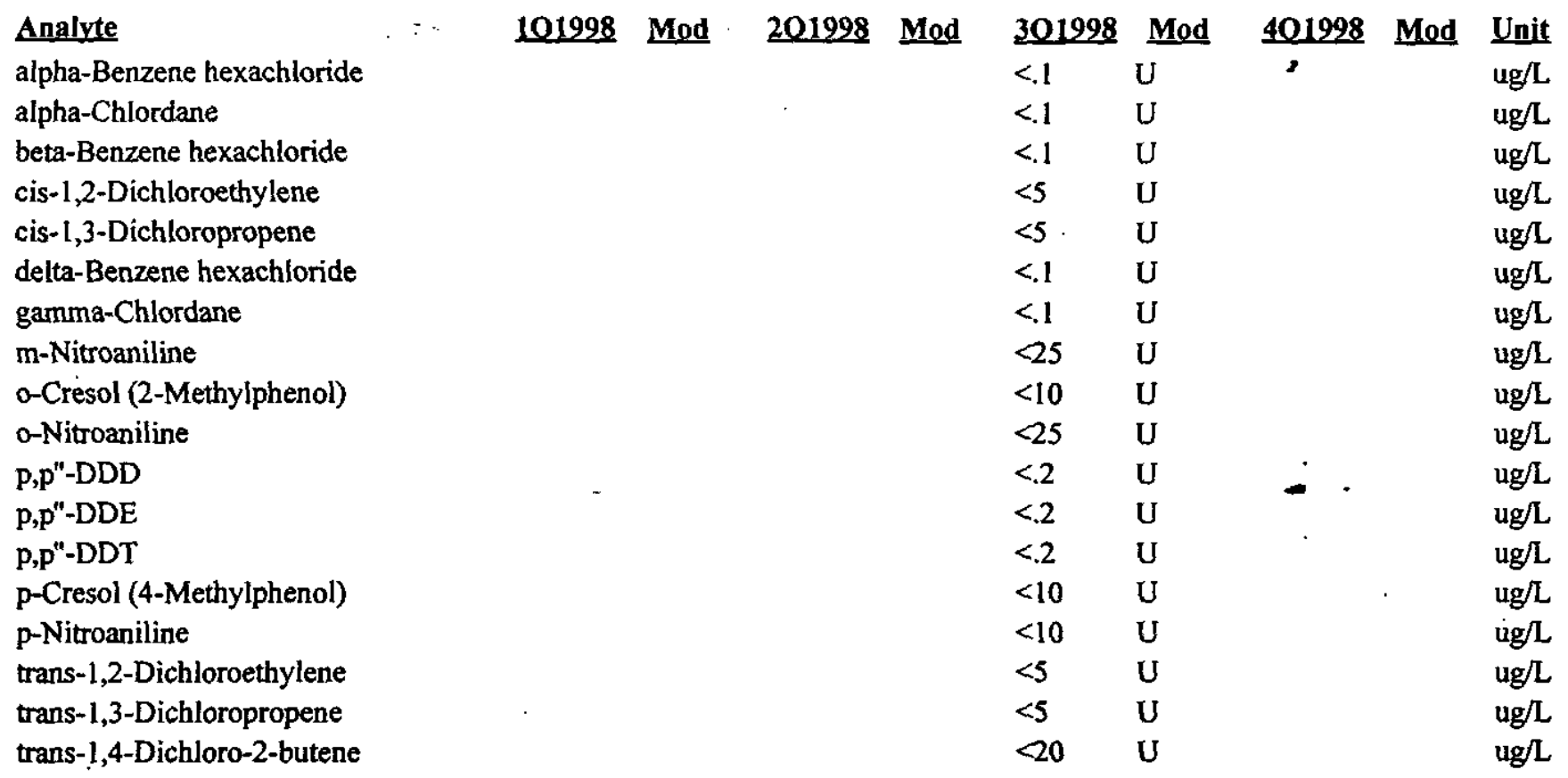

Note: Concentrations in bold exceed the Drinking Water Standards listed in Appendix A. Units are for all four quarters. 
WSRC-RP-99-4003

Unclassified

WELL: TNX 12D

SRS Coord, Lat/Longitude Screen Zone Elevation Top of Standoipe Top of Casing Casing Pump Screen Zone

N 71598.3 33.210 Deg N . - -93.1 - 73.1 ft msl E $16176.3 \quad 81.763$ Deg W

SAMPLE DATE

$03 / 02 / 98$

$99.4 \mathrm{ft} \mathrm{ms}$

$05 / 12 / 98$

8/06/98

4 " PVC

,

$12 / 01 / 98$

FIELD DATA

Analyte

Depth to water

$\mathrm{pH}$

Sp. Conductance

Water temperature

Alkalinity as $\mathrm{CaCO} 3$

Phenolpthalein Alkalinity

Turbidity

Volumes purged

Sampling codes

\section{ANALYTICAL DATA}

\section{Analyite}

1,1,1,2-Tetrachloroethane

1,1;1-Trichloroethane

1,1,2,2-Tetrachloroethane

1,1,2-Trichloroethane

1,1-Dichloroethane

1,1-Dichloroethylene

1,2,3-Trichloropropane

1,2,4-Trichiorobenzene

1,2-Dibromo-3-chloropropane

1,2-Liorumuethane

1,2-Dichlorobenzene

1,2-Dichloroethane

1,2-Dichloropropane

1,3,5-Trinitrobenzene

1,3-Dichlorobenzene

1,3-Dinitrobenzene

1,4-Dichlorobenzene

1,4-Dioxane

1,4-Naphthoquinone

1-Naphthylamine

2,2-Oxybis(1-chloropropane)

2,3,4,6-Tetrachlorophenol

2,3,7,8-TCDD

2,4,5-T

2,4,5-TP (Silvex)

2,4,5-Trichlorophenol

2,4,6-Trichlorophenol

\begin{tabular}{|c|c|}
\hline 101998 & 201998 \\
\hline 2.7 & 3.93 \\
\hline 7.2 & 6.3 \\
\hline 59 & 55 \\
\hline 18.5 & 19.6 \\
\hline 15. & 16 \\
\hline 0 & 0 \\
\hline .4 & \\
\hline 2.93152 & 3.02540 \\
\hline
\end{tabular}

301998

5.34

6

58

20.2

14

0

.4

2.42316

$\begin{array}{ll}\frac{401998}{5.71} & \text { Unit } \\ 5.7 & \text { ft BTOS } \\ 57 & \\ 20.2 & \text { uS/cm } \\ 12 & \text { deg. C } \\ 0 & \mathrm{mg} / \mathrm{L} \\ .7 & \mathrm{mg} / \mathrm{L} \\ 4.41094 & \text { NTU } \\ & \text { gallons }\end{array}$

101998 Mod

$<.462 \quad \mathrm{U}$

$<.462$
301998

$<5$

$<5 \quad U$

$<5 \quad U$

$<5 \quad U$

$<5 \quad U$

$<5 \quad U$

$<5 \quad U$

$<10 \quad U$

$<5$

$<5$

$<5$

$<5$

$<5$

$<5$

$<5$

$<1000$

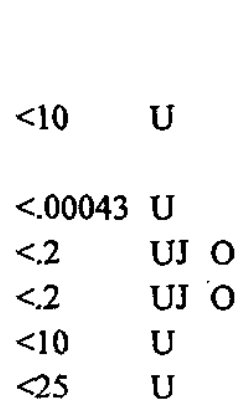

401998

Mor

Unit

ug/L

ug/L

$\mathrm{ug} / \mathrm{L}$

ug/L

ug/L

ug/L

ug/L

$<10 \quad J U Q \cdot u g / L$

$u g / L$

ug/L

ug/L

ug/L

ug/L

ug/L

ug/L

ug/L

ug/L

ug/L

$<10$ U $\mathrm{ug} / \mathrm{L}$

$<10$ U ug/L

$<10$ JU Q ug/L

$<10$ U ug/L

$\mathrm{ug} / \mathrm{L}$

$\mathrm{ug} / \mathrm{L}$

ug/L

$<$ to JU Q ug/L

$<25$ JU Q ug/L

Note: Concentrations in bold exceed the Driaking Water Standards listed in Appendix A. Units are for all four quarters. 
WELL: TNX 12D

ANALYTICAL DATA

Analyte

2,4-Dichlorophenol

2,4-Dichlorophenoxyacetic acid

2,4-Dimethyl phenol

2,4-Dinitrophenol

2,4-Dinitrotoluene

2,6-Dichlorophenol

2,6-Dinitrotoluene

2-Acetylaminofluorene

2-Chloronaphthalene

2-Chlorophenol

2-Hexanone

2-Methyl-4,6-dinitrophenol

2-Methylnaphthalene

2-Naphthylamine

2-Nitrophenol

2-Picoline

2-sec-Butyl-4,6-dinitrophenol

3,3"-Dichlorobenzidine

3,3"-Dimethylbenzidine

3-Methylcholanthrene

4-Aminobiphenyl

4-Bromophenyl phenyl ether

4-Chloro-m-cresol

4-Chloroaniline

4-Chlorophenyl phenyl ether

4-Nitrophenol

4-Nitroquinoline-1-oxide

5-Nitro-0-toluidine

7,12-Dimethylbenz(a)anthracene

Acenaphthene

Acenaphthylene.

Acetone

Acetonitrile (Methyl cyanide)

Acetophenone

Acrolein

Acrylonitrile

Aldrin

Allyl chloride

Aluminum, total recoverable

Aniline

Anthracene

Antimony, total recoverable

Aramite
101998 Mod 201998 Mod 301998 Mod 401998 Mod Unit

$<10$ U $<10 \quad J \mathrm{Q}$ ug/L

$<.2$ UJ $\mathrm{O}$ ug/L

$<10 \quad \mathrm{U} \quad<10$ JU Q ug/L

$<25$ U $<25$ J $Q \quad$ ug/

$<10 \quad \mathrm{U} \quad<10$ JU $Q \quad$ ug $/ \mathrm{L}$

$<10 \quad U \quad$ ug/L

$<10 \quad \mathrm{U} \quad<10 \quad \mathrm{U} Q \mathrm{Qg} / \mathrm{L}$

$<10 \quad U \quad u g / L$

$<10$ U $<10$ J $Q \quad$ ug/

$<10 \quad \mathrm{C} \quad<10 \quad$ J $Q \quad$ ug/L

$<$ U $\doteq$ ug/

$<25 \mathrm{U}<25$ JU Q ug/L

$<10 \quad \mathrm{U} \quad<10$ JU $\mathrm{Q}$ ug/L

$<\quad<10$ U ug/L

$<10 \quad \mathrm{U} \quad<10 \quad$ JU $Q \quad$ ug/L

$<10$ U ug/

$<10$ U ug/

$<10$ U $<10$ JU Q ug

$<20$ U ug/L

$<10 \quad \mathrm{U} \quad \mathrm{ug} / \mathrm{L}$

$<10$ U ug/L

$<10 \quad$ U. $\quad<10 \quad$ JU $Q \quad$ ug/L

$<10 \quad \mathrm{U} \quad<10 \quad$ JU $\mathrm{Q} \quad$ ug $/ \mathrm{L}$

$<10$ IJ $\quad<10$ JU Q ugh

$<10 \quad$ U $\quad<10 \quad$ JU $Q \quad$ ug/L

$<25$ U $<25$ JU Q ug/L

$<50$ U ug/L

$<10$ U ug/L

$<10$ U ugl

$<10 \quad \mathrm{U} \quad<10 \quad$ JU $Q \quad$ ug/

$<10$ U $<10$ JU $Q$ ugh

$<20$ U ug/L

$<500$ U ug/L

$<10 \quad \mathrm{U} \quad$ ug/L

$<50$ U ug/L

$<50$ U . ug/L

$<.1 \quad \mathrm{U} \quad \mathrm{ug} / \mathrm{L}$

$<10 \quad \mathrm{U} \quad \mathrm{ug} / \mathrm{L}$

$<20 \quad \mathrm{U} \quad<200 \quad \mathrm{U} \quad<200 \quad \mathrm{U} \quad \mathrm{ug} / \mathrm{L}$

$<25 \quad \mathrm{U} \quad<25$ JU Q ug/L

$<10 \quad \mathrm{U} \quad<10 \quad$ JU $Q$ ugh

$<100 \mathrm{U}+\mathrm{ug} / \mathrm{L}$

$<10$ U $\quad$ ug/L

Note: Concentrations in bold exceed the Drinking Water Standards listed in Appendix A. Units are for all four quarters. 
WSRC-RP-99-4003

Unclassified

WELL: TNX 12D

ANALYTICAL DATA

\begin{tabular}{|c|c|c|c|c|c|c|c|c|c|}
\hline Analyte & 101998 & Mod & $\underline{201998}$ & Mod & 301998 & Mod & $\underline{401998}$ & Mod & Unit \\
\hline Arsenic, total recoverable & & & & & $<10$ & $\mathrm{U}$ & & & $\mathrm{ug} / \mathrm{L}$ \\
\hline Barium, total recoverable & & & & & 17.1 & & 18.8 & & ug/L \\
\hline Benzene & & & & & $<5$ & $U$ & & & $\mathrm{ug} / \mathrm{L}$ \\
\hline Benzo(a)anthracene & & & & & & & $<10$ & JU Q & $u g / L$ \\
\hline Benzo(a)pyrene & & & & & $<10$ & $\mathrm{U}$ & $<10$ & JU C & $\mathrm{ug} / \mathrm{L}$ \\
\hline Benzo(b)fluoranthene & & & & & $<10$ & $\mathrm{U}$ & $<10$ & J $\mathrm{C}$ & $\mathrm{ug} / \mathrm{L}$ \\
\hline Benzo(g,h,i)perylene & & & & & $<10$ & $\mathrm{U}$ & $<10$ & $\mathrm{JU} C$ & $\mathrm{ug} / \mathrm{L}$ \\
\hline Benzo(k)fluoranthene & & & & & $<10$ & $\mathbf{U}$ & $<10$ & JU Q & $u g / L$ \\
\hline Benzoic acid & & & & & $<25$ & $\mathbf{U}$ & & & $\mathrm{ug} / \mathrm{L}$ \\
\hline Benzyl alcohol & & & & & & & $<10$ & Л $Q$ & $u g / L$ \\
\hline Beryllium, total recoverable & & & & & $<10$ & $U$ & $\therefore$ & & $\mathrm{ug} / \mathrm{L}$ \\
\hline Bis(2-chloroethoxy) methane & & & & & $<10$ & $\mathrm{U}$ & $<10$ & J $Q$ & ug/L \\
\hline Bis(2-chloroethyl) ether & & & & & $<10$ & $\mathrm{U}$ & $<10$ & J $\mathrm{C}$ & $\mathrm{ug} / \mathrm{L}$ \\
\hline Bis(2-ethylhexyl) phthalate & & & & & $<10$ & $\mathrm{U}$ & $<10$ & 厅 $\mathrm{C}$ & $\mathrm{ug} / \mathrm{L}$ \\
\hline Bromodichloromethane & & & & & $<5$ & $\mathrm{U}$ & & & ug/L \\
\hline Bromoform & & & & & $<5$ & $\mathrm{U}$ & & & $u g / L$ \\
\hline Bromomethane (Methyl bromide) & & & & & $<5$ & $\mathrm{U}$ & & & $\mathrm{ug} / \mathrm{L}$ \\
\hline Butylbenzyl phthalate & & & & & $<10$ & $\mathbf{U}$ & $<10$ & JU C & ug/L \\
\hline Cadmium, total recoverable & & & & & $<10$ & $U$ & & & $\mathrm{ug} / \mathrm{L}$ \\
\hline Carbazole & & & & & $<0$ & $\mathrm{U}$ & & & $u g / L$ \\
\hline Carbon disulfide & & & & & $<5$ & $U$ & & & ug/L \\
\hline Chlorobenzene & & & & & $<5$ & $\mathrm{U}$ & & & $\mathrm{ug} / \mathrm{L}$ \\
\hline Chlorobenzilate & & & & & & & $<10$ & $\mathrm{U}$ & $\mathrm{ug} / \mathrm{L}$ \\
\hline Chloroethane & & & & & $<10$ & $\mathrm{U}$ & & & $\mathrm{ug} / \mathrm{L}$ \\
\hline Chloroethene (Vinyl chloride) & & & & & $<5$ & $\mathrm{U}$ & & & ug/L \\
\hline Chloromethase (Methyi chlorici) & & & & & $<5$ & 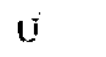 & & & ug/L \\
\hline Chloroprene & & & & & $<50$ & $\mathrm{U}$ & & & $\mathrm{ug} / \mathrm{L}$ \\
\hline Chromium, total recoverable & & & & & $<10$ & $\mathrm{U}$ & & & $\mathrm{ug} / \mathrm{L}$ \\
\hline Chrysene & & & & & $<10$ & $\mathrm{U}$ & $<10$ & Ju $\mathrm{C}$ & $\mathrm{ug} / \mathrm{L}$ \\
\hline Cobalt, total recoverable & & & & & $<20$ & $\mathrm{U}$ & & & $\mathrm{ug} / \mathrm{L}$ \\
\hline Copper, total recoverable & & & & & $<20$ & $\mathrm{U}$ & & & $\mathrm{ug} / \mathrm{L}$ \\
\hline Cyanide & & & & & $<10$ & $\mathrm{U}$ & & & $u g / L$ \\
\hline Di-n-butyl phthalate & & & & & $<10$ & $\mathrm{U}$ & $<10$ & $J C$ & ug/L \\
\hline Di-n-octyl phthalate & & & & & $<10$ & $\mathrm{U}$ & $<10$ & $\mathrm{JU} C$ & $\mathrm{ug} / \mathrm{L}$ \\
\hline Diallate & & & & & & & $<10$ & $\mathrm{U}$ & $\mathrm{ug} / \mathrm{L}$ \\
\hline Dibenz(a,h)anthracene & & & & & $<20$ & $U$ & $<10$ & JU C & ug/L \\
\hline Dibenzofuran & & & & & $<10$ & $\mathrm{U}$ & $<10$ & $\pi$ & $u g / L$ \\
\hline Dibromochloromethane & & & & & $<5$ & $\mathrm{U}$ & & & $\mathrm{ug} / \mathrm{L}$ \\
\hline Dibromomethane (Methylene bromide', & & & & & $<5$ & $\mathrm{U}$ & & & $\mathrm{ug} / \mathrm{L}$ \\
\hline Dichlorodifluoromethane & & & & & $<5$ & $U$ & & & ug/L \\
\hline Dichloromethane (Methylene chloride) & & & & & $<10$ & $\mathrm{U}$ & & & $\mathrm{ug} / \mathrm{L}$ \\
\hline Dieldrin & & & & & $<.2$ & $\mathrm{U}$ & $\star$ & & $\mathrm{ug} / \mathrm{L}$ \\
\hline Diethyl phthalate & & & & & $<10$ & $U$ & $<10$ & $\pi \mathrm{C}$ & $\mathrm{ug} / \mathrm{L}$ \\
\hline
\end{tabular}

Note: Concentrations in bold exceed the Drinking Water Standards listed in Appendix A. Units are for all four quarters. 
WELL: TNX 12D

\section{ANALYTICAL DATA}

\section{Analyte}

Dimethoate

Dimethyl phthalate

Diphenylamine

Disulfoton

Endosulfan I

Endosulfan II

Endosulfan sulfate

Endrin

Endrin aldehyde

Ethyl methacrylate

Ethyl methanesulfonate

Ethylbenzene

Fluoranthene

Fluorene

Heptachlor

Heptachlor epoxide

Hexachlorobenzene

Hexachlorobutadiene

Hexachlorocyclopentadiene

Hexachlorodibenzo-p-dioxins

Hexachlorodibenzo-p-furans

Hexachloroethane

Indeno(1,2,3-c,d)pyrene

Iodomethane (Methyl iodide)

Iron, total recoverable

Isoiutyl aicohol

Isodrin

Isophorone

Isosafrole

Kepone

Lindane

Methacrylonitrile

Methapyrilene

Methoxychlor

Methyl ethyl ketone

Methyl isobutyl ketone

Methyl methacrylate

Methyl methanesulfonate

N-Nitrosodi-n-butylamine

$\mathrm{N}$-Nitrosodiethylamine

N-Nitrosodimethylamine

N-Nițosodiphenylamine

N-Nitrosodipropylamine
101998 Mod 201998 Mod 301998 Mod 401998 Mod Unit

$<$ U U ug/L

$<10 \quad \mathrm{U} \quad<10 \quad \mathrm{JU} Q \quad \mathrm{ug} / \mathrm{L}$

$<10 \quad \mathrm{U} \quad \mathrm{ug} / \mathrm{L}$

$<10 \quad \mathrm{U} \quad$ ug $\Omega$

$<.1 \quad$ U $\quad$ ug/L

$<.2$ U

$<.2$ U

$<.2 \quad$ U

$<.2 \quad$ U

$<5 \quad U$

$<5 \quad \mathrm{U}$

$<10 \quad \mathrm{U}$

$<10 \quad \mathrm{U}$

$<.1 \quad$ U

$<.1 \quad \mathrm{U}$

$<10 \quad \mathrm{U}$

$<20$ U

$<10 \quad \mathrm{U}$

$<.00065 \mathrm{U}$

$<.00062 \mathrm{U}$

$<10 \quad \mathrm{U}$

$<10 \quad \mathrm{U}$

$<5 \quad$ UJ

24.9

$23.2 \mathrm{I}$

$<1500 \mathrm{U}$

$<20 \quad$ U

$<.1 \quad \mathrm{U}$

$<500 \quad$ U

$<1 \quad$ U

$<10 \quad \mathrm{U}$

$<5 \quad \mathrm{U}$

$<50 \quad \mathrm{U}$

$<10$ U ug/L

$u g / L$

$u g / L$

$u g / \mathrm{L}$

ug $/ L$

$\mathrm{ug} / \mathrm{L}$

$\mathrm{ug} / \mathrm{L}$

$<10 \quad$ JU $Q \quad$ ug/L

$<10$ J $Q$ ug/L

ug/L

$\mathrm{ug} / \mathrm{L}$

$<10 \quad \pi \quad Q \quad u g / L$

$<10$ JU $Q \quad \operatorname{ug} / \mathrm{L}$

$<10$ J $Q$ ug/L

$\mathrm{ug} / \mathrm{L}$

$\mathrm{ug} / \mathrm{L}$

$<10 \quad$ JU $Q \quad$ ug/L

$<10 \quad$ JU $Q \quad u g / L$

ug/L

$<13.3 \quad$ U $\quad V \quad$ ug/L

ug/l.

$<10 \quad \mathrm{U} \quad$ ug/L

$<10 \quad$ JU Q ug/L

$<10 \quad \mathrm{U} \quad \mathrm{ug} / \mathrm{L}$

$<10$ U ug/L

$\mathrm{ug} / \mathrm{L}$

ug/L

$<10 \quad \mathrm{U} \quad u g / \mathrm{L}$

$u g / L$

ug/L

$\mathrm{ug} / \mathrm{L}$

ug/L

$<10 \quad \mathrm{U} \quad \mathrm{ug} / \mathrm{L}$

$<10 \quad \mathrm{U} \quad \mathrm{ug} / \mathrm{L}$

$<10 \quad \mathrm{U} \quad$ ug/L

$<20 \quad \mathrm{U} \quad<25 \quad \pi \quad \mathrm{Q} \quad \mathrm{ug} / \mathrm{L}$

$<10 \quad \mathrm{U} \quad<10 \quad$ J $Q \quad \mathrm{Qg} / \mathrm{L}$

$<10 \quad \mathrm{U} \quad<10 \quad$ JU $Q \quad$ ug/L

Note: Concentrations in bold exceed the Drinking Water Standards listed in Appendix A. Units are for all four quarters. 
WELL: TNX 12D

ANALYTICAL DATA

\begin{tabular}{|c|c|c|c|c|c|c|c|c|}
\hline Analyte & 101998 & 201998 & Mod & 301998 & Mod & 401998 & Mod & Unit \\
\hline $\mathrm{N}$-Nitrosomethylethylamine & & & & & & $<10$ & $\mathrm{U}$ & $\mathrm{ug} / \mathrm{L}$ \\
\hline $\mathrm{N}$-Nitrosomorpholine & & & & & & $<10$ & $U$ & $\mathrm{ug} / \mathrm{L}$ \\
\hline $\mathrm{N}$-Nitrosopiperidine & & & & & & $<10$ & $U$ & $\mathrm{ug} / \mathrm{L}$ \\
\hline N-Nitrosopyrrolidine & & & & & & $<10$ & $U$ & $\mathrm{ug} / \mathrm{L}$ \\
\hline Naphthalene & & & & $<20$ & $\mathrm{U}$ & $<10$ & $\mathrm{JU} Q$ & $\mathrm{ug} / \mathrm{L}$ \\
\hline Nickel, total recoverable & & & & 7.69 & $\mathbf{J} \quad \mathrm{E}$ & & & $\mathrm{ug} / \mathrm{L}$ \\
\hline Nitrobenzene & . & & & $<10$ & $\mathbf{U}$ & $<10$ & JU Q & $\mathrm{ug} / \mathrm{L}$ \\
\hline O,O,O-Triethyl phosphorothioate & & & & & & $<10$ & $\mathrm{U}$ & $\mathrm{ug} / \mathrm{L}$ \\
\hline PCB 1016 & & & & $<2$ & $\mathrm{U}$ & & & $\mathrm{ug} / \mathrm{L}$ \\
\hline PCB 1221 & & & & $<2$ & $\mathrm{U}$ & & & ug/L \\
\hline PCB 1232 & & & & $<1$ & $U$ & $\therefore$ & & $\mathrm{ug} / \mathrm{L}$ \\
\hline PCB 1242 & & & • & $<1$ & $\mathrm{U}$ & & & $\mathrm{ug} / \mathrm{L}$ \\
\hline PCB 1248 & & & & $<1$ & $\mathbf{U}$ & & & $\mathrm{ug} / \mathrm{L}$ \\
\hline PCB 1254 & & & & $<1$ & $\mathrm{U}$ & & & $\mathrm{ug} / \mathrm{L}$ \\
\hline PCB 1260 & & & & $<1$ & $U$ & & & $\mathrm{ug} / \mathrm{L}$ \\
\hline Parathion & & & & & & $<10$ & $\mathrm{U}$ & $\mathrm{ug} / \mathrm{L}$ \\
\hline Parathion methyl & & & & & & $<10$ & $\mathbf{U}$ & $\mathrm{ug} / \mathrm{L}$ \\
\hline Pentachlorodibenzo-p-dioxins & & & & $<.00077$ & $\mathrm{U}$ & & & $\mathrm{ug} / \mathrm{L}$ \\
\hline Pentachlorodibenzo-p-furans & & & & $<.0005$ & $\mathbf{U}$ & & & $\mathrm{ug} / \mathrm{L}$ \\
\hline Pentachloroethane & & & & $<200$ & $\mathbf{U}$ & & & $\mathrm{ug} / \mathrm{L}$ \\
\hline Pentachloronitrobenzene & & & & & & $<10$ & $U$ & ug/L \\
\hline Pentachlorophenol & & & & $<25$ & $\mathrm{U}$ & $<25$ & JU $Q$ & $\mathrm{ug} / \mathrm{L}$ \\
\hline Phenacetin & & & & & & $<10$ & $\mathrm{U}$ & $\mathrm{ug} / \mathrm{L}$ \\
\hline Phenanthrene & & & & $<10$ & $\mathbf{U}$ & $<10$ & $\pi U Q$ & ug/L \\
\hline Phenol & . & & & $<10$ & U & $<10$ & JU $Q$ & ig/L \\
\hline Phorate & & & . & & & $<10$ & $\mathrm{U}$ & $u g / L$ \\
\hline Pronamid & & & & & & $<10$ & $U$ & $u g / L$ \\
\hline Propionitrile & - & & & $<500$ & $\mathrm{U}$ & & & $\mathrm{ug} / \mathrm{L}$ \\
\hline Pyrene & & & & $<10$ & $\mathbf{U}$ & $<10$ & JU $Q$ & $\mathrm{ug} / \mathrm{L}$ \\
\hline Pyridine & & & & & & $<25$ & $\mathrm{JU} Q$ & $\mathrm{ug} / \mathrm{L}$ \\
\hline Safrole & & & & & & $<10$ & $\mathrm{U}$ & $\mathrm{ug} / \mathrm{L}$ \\
\hline Selenium, total recoverable & & & & $<10$ & U & & & ug/L \\
\hline Silver, total recoverable & & & & $<20$ & $U$ & & & $\mathrm{ug} / \mathrm{L}$ \\
\hline Styrene & & & & $<5$ & $\mathrm{U}$ & & & $\mathrm{ug} / \mathrm{L}$ \\
\hline Sulfide & & & & $<1000$ & $\mathrm{U}$ & & & $\mathrm{ug} / \mathrm{L}$ \\
\hline Sulfotepp & & & & & & $<10$ & $\mathrm{U}$ & $\mathrm{ug} / \mathrm{L}$ \\
\hline Tetrachlorodibenzo-p-dioxins & & & & $<.00043$ & $\mathbf{U}$ & & & $\mathrm{ug} / \mathrm{L}$ \\
\hline Tetrachlorodibenzo-p-furans & & & & $<.00035$ & $U$ & & & $\mathrm{ug} / \mathrm{L}$ \\
\hline Thallium, total recoverable & & & & $<10$ & $\mathrm{U}$ & & & $\mathrm{ug} / \mathrm{L}$ \\
\hline Thionazin & . & & & & & $<10$ & $\mathrm{U}$ & $\mathrm{ug} / \mathrm{L}$ \\
\hline Tin, total recoverable & & & & $<200$ & $U$ & & & $\mathrm{ug} / \mathrm{L}$ \\
\hline Toluene & & & & $<5$ & $\mathrm{U}$ & - & & $\mathrm{ug} / \mathrm{L}$ \\
\hline Total organic halogens & & & & & & $<120$ & $\mathrm{U}$ & $\mathrm{ug} / \mathrm{L}$ \\
\hline
\end{tabular}

Note: Concentrations in bold exceed the Drinking Water Standards listed in Appendix A. Units are for all four quarters. 
WELL: TNX 12D

\section{ANALYTICAL DATA}

\section{Analyte}

Toxaphene

Trichlorofluoromethane

Vanadium, total recoverable

Vinyl acetate

Xylenes

Zinc, total recoverable

a,a-Dimethylphenethylamine

alpha-Benzene hexachloride

alpha-Chlordane

beta-Benzene hexachloride

cis-1,2-Dichloroethylene

cis-1,3-Dichloropropene

delta-Benzene hexachloride

gamma-Chlordane

m-Nitroaniline

$\mathrm{m} / \mathrm{p}$-Cresol

o-Cresol (2-Methylphenol)

o-Nitroaniline

o-Toluidine

$p, p^{i-}-D D D$

$p, p^{n}-D D E$

p,p"-DDT

p-Cresol (4-Methylphenol)

p-Dimethylaminoazobenzene

p-Nitroaniline

p-Phenylenediarnine

trans-1,2-Dichloroethylene

trans-1,3-Dichloropropene

trans-1,4-Dichloro-2-butene

101998 Mod 201998 Mod

301998

$<1$

$<5 \quad$ U

$<10 \quad \mathrm{U}$

$<20 \quad$ U

$<10 \quad U$

4.52 J E

$<.1 \quad$ U

$<.1$ U

$<1 \quad \mathrm{U}$

$<5 \quad \mathrm{U}$

$<1 \quad$ U

$<.1 \quad U$

$<25 \quad \mathrm{U}$

$<10 \quad \mathrm{U}$

$<25 \quad \mathrm{U}$

$<.2 \quad \mathrm{U}$

$<.2 \quad \mathrm{U}$

$<.2$ U

$<10$ U

$<10 \quad \mathrm{U}$

U

$<5 \quad \mathrm{U}$

$<5 \quad \mathrm{U}$

$<20 \quad \mathrm{U}$
401998 Mod Unit

ug/L

$\mathrm{ug} / \mathrm{L}$

$\mathrm{ug} / \mathrm{L}$

$u g / L$

ug/L

ug/L

ug/L

ug/L

ug/L

ug/L

$<1$ U U ug/L

ug/L

ug/L

$\mathrm{ug} / \mathrm{L}$

$<25$ JU Q ug/L

$<20$ U ug/L

$<10$ JU Q ug/L

$<25$ JU Q ug/L

$<10$ U ug/L

$\mathrm{ug} / \mathrm{L}$

ug/L

ug/L

$<10$ JU Q ug/L

$<10$ U $\cdot u g / L$

$<10$ JU Q ug/L

$<10$ U ug/L

$\mathrm{ug} / \mathrm{L}$

ug/L

ug/L 
WSRC-RP-99-4003

- Unclassified

WELL: TNX 27D

SRS Coord. Lat/Longitude Screen Zone Elevation Top of Standpipe Top of Casing Casing Pump Screen Zone N 71180.133 .210 Deg N . : $101.3-81.3 \mathrm{ft} \mathrm{msl} 110.6 \mathrm{fm}$ E 16609.181 .761 Deg W

SAMPLE DATE

FIELD DATA

Analyte

Depth to water

$\mathrm{pH}$

Sp. Conductance

Water temperature

Alkalinity as $\mathrm{CaCO} 3$

Phenolpthalein Alkalinity

Turbidity

Volumes purged

Sampling codes

\section{ANALYTICAL DATA}

\section{Analyte}

1,1,1,2-Tetrachloroethane

1,1,1-Trichloroethane

1,1,2,2-Tetrachloroethane

1,1,2-Trichloroethane

1,1-Dichloroethane

1,1-Dichloroethylene

1,2,3-Trichloropropane

1,2,4-Trichlorobenzene

1,2-Dibromo-3-chloropropane

1,2-Dibromoethane

1,2-Dichlorobenzene

1,2-Dichloroethane

1,2-Dichloropropane

1,3,5-Trinitrobenzene

1,3-Dichlorobenzene

1,3-Dinitrobenzene

1,4-Dichlorobenzene

1,4-Dioxane

1,4-Naphthoquinone

1-Naphthylamine

2,2-Oxybis(1-chloropropane)

2,3,4,6-Tetrachlorophenol

2,3,7,8-TCDD

2,4,5-T

2,4,5-TP (Silvex)

2,4,5-Trichlorophenol

2,4,6-Trichlorophenol
$03 / 04 / 98$

$05 / 15 / 98$

$110.6 \mathrm{ft} \mathrm{msl}$

2 " PVC

08/05/98

$12 / 04 / 98$

Unconfined

201998

12.69

5.9

100

20.1

23

0

1.9

8.05200

$\frac{301998}{14.39}$
6
140
20.7
24
0
1.9
3.66832

401998

15.8

5.4

220

20.7

4

0

32

4.05004
Unit

ft BTOS

$\mathrm{uS} / \mathrm{cm}$

deg. C

$\mathrm{mg} / \mathrm{L}$

$\mathrm{mg} / \mathrm{L}$

NTU

gallons
101998 Mod 201998 Mod 301998 Mod 401998 Mod Unit

$<5 \quad \mathrm{U}$

$<5 \quad U$

$<5 \quad \mathrm{U}$

$<5 \quad U$

$<5 \quad \mathrm{U}$

$<5 \quad U$

$<5 \quad \mathrm{U}$

$<10$ U

$<5 \quad v$

$<5 \quad \mathrm{U}$

$<5 \quad \mathrm{U}$

$<5 \quad \mathrm{U}$

$<5 \quad \mathrm{U}$

$<5 \quad$ U

$<5 \quad U$

$<1000 \mathrm{U}$

$<10$

$<10$

$<10 \quad \mathrm{U}$

$<10$

$<.00029 \mathrm{U}$

$<.2$ UJ O

$<2$ UJ $\mathrm{O}$

$<10$ U

$<25 \quad U$
$<10$

ug/L

$<1 \quad$ U ug/L

$\mathrm{ug} / \mathrm{L}$

ug $/ \mathrm{L}$

ug/L

ug/L

$\mathrm{ug} / \mathrm{L}$

$<10$ JU $Q \quad$ ug/L

ug $/ \mathrm{L}$

ug/L

ug/L

ug/L

ug/L

ug/L

ug/L

$<10 \quad \mathrm{U} \quad$ ug/L

ug/L

ug/L

U ug/L

U ug/L

JU $Q \quad$ ug/L

U ug/L

ug/L

$\mathrm{ug} / \mathrm{L}$

ug $/ \mathrm{L}$

JU Q ug/L

JU $Q \quad u g / L$

Note: Concentrations in bold exeed the Drinking Water Standards listed in Appendix A. Units are for alf four quarters. 
WELL: TNX 27D

\section{ANALYTICAL DATA}

\section{Analyte}

2,4-Dichlorophenol

2,4-Dichlorophenoxyacetic acid

2,4-Dimethyl phenol

2,4-Dinitrophenol

2,4-Dinitrotoluene

2,6-Dichlorophenol

2,6-Dinitrotoluene

2-Acetylaminofluorene

2-Chloronaphthalene

2-Chlorophenol

2-Hexanone

2-Methyl-4,6-dinitrophenol

2-Methylnaphthalene

2-Naphthylamine

2-Nitrophenol

2-Picoline

2-sec-Butyl-4,6-dinitrophenol

3,3"-Dichlorobenzidine

3,3"-Dimethylbenzidine

3-Methylcholanthrene

4-Aminobiphenyl

4-Bromophenyl phenyl ether

4-Chloro-m-cresol

4-Chloroaniline

4-Chlorophenyl phenyl ether

4-Nitrophenol

4-Nitroquinoline-1-oxide

5-Nitro-o-toluidine

7,12-Dimethylbenz(a)anthracene

Acenaphthene

Acenaphthylene

Acetone

Acetonitrile (Methyl cyanide)

Acetophenone

Acrolein

Acrylonitrile

Aldrin

Allyl chloride

Aluminum, total recoverable

Aniline

Anthracene

Antimony, total recoverable

Aramite
101998 Mod 201998 Mod 301998 Mod 401998 Mod Unit

$<10 \quad \mathrm{U} \quad<$ To JU $Q \quad$ ug/L

$<.2$ UJ $O$ ug/L

$<10$ U $\quad<10$ J $Q \quad$ ug/L

$<25 \mathrm{U} \quad<25 \quad$ U Q ug/L

$<10 \quad \mathrm{U} \quad<10 \quad \pi \quad \mathrm{Q} \quad$ ug/L

$<10$ U ug/L

$<10 \quad \mathrm{U} \quad<10$ JU Q ug/L

$<10 \quad U \quad \mathrm{ug} / \mathrm{L}$

$<10 \quad \mathrm{U} \quad<10 \quad$ JU Q $\quad$ ug/L

$<10$ U $<10$ JU $Q \quad$ ug/L

$<5 \quad \mathrm{U} \quad \sim \quad \mathrm{ug} / \mathrm{L}$

$<25 \quad \mathrm{U} \quad<25 \quad \pi \mathrm{U} Q \quad \mathrm{Qg} / \mathrm{L}$

$<10 \quad \mathrm{U} \quad<10 \quad$ JU $\mathrm{Q} \quad \mathrm{ug} / \mathrm{L}$

$<10 \quad<10 \quad U \quad u g / L$

$<10 \quad \mathrm{U} \quad<10 \quad \mathrm{JU} \mathrm{Q} \quad \mathrm{ug} / \mathrm{L}$

- $\quad<10 \quad \mathrm{U} \quad \mathrm{ug} / \mathrm{L}$

$<10 \quad \mathrm{U} \quad \mathrm{ug} / \mathrm{L}$

$<10$ U $\quad<10$ J Q ug/L

$<20$ U ug/L

$<10 \quad \mathrm{U} \quad$ ug/L

$<10$ U ug/L

$<10 \quad \mathrm{U} \quad<10 \quad \mathrm{JU} Q \quad \mathrm{Qg} / \mathrm{L}$

$<10 \quad \mathrm{U} \quad<10 \quad$ JU $Q \quad$ ug/L

$<10 \quad \mathrm{U} \quad<10 \quad \pi \mathrm{Q} \quad \mathrm{ug} / \mathrm{L}$

$<10 \quad \mathrm{U} \cdot<10$ JU $\mathrm{Q} \quad \mathrm{ug} / \mathrm{L}$

$<25$ U $\quad<25 \quad J U Q \quad$ ug/L

$<50$ U ug/L

$<10$ U ug/L

$<10 \quad \mathrm{U} \quad \mathrm{ug} / \mathrm{L}$

$\begin{array}{lllll}<10 & \mathrm{U} & <10 & \text { J } Q & \text { ug/ } \\ <10 & \mathrm{U} & <10 & \text { JU Q } & \text { ug/L }\end{array}$

$<20 \mathrm{U} \quad \mathrm{ug} / \mathrm{L}$

$<500 \quad \mathrm{U} \quad u g / \mathrm{L}$

$<50 \quad \mathrm{U} \quad \mathrm{ug} / \mathrm{L}$

$<50$ U ug/L

$<.1 \quad$ U $u g / L$

$<10$ U ug/L

$<20 \quad \mathrm{U} \cdot<200$ UJ $\quad 237 \quad \mathrm{ug} / \mathrm{L}$

$<25 \quad \mathrm{U} \quad<25 \quad \pi U \mathrm{Q} \quad \mathrm{ug} / \mathrm{L}$

$<10 \quad \mathrm{U} \quad<10 \quad \pi \quad \mathrm{Q} \quad \mathrm{ug} / \mathrm{L}$

$<100 \quad \mathrm{U}$

Note: Concentrations ia bold exceed the Drinking Water Standards listed in Appendix A. Units are for all four quarters. 
WELL: TNX 27D

\section{ANALYTICAL DATA}

Analyte

Arsenic, total recoverable

Barium, total recoverable

Benzene

Benzo(a)anthracene

Benzo(a)pyrene

Benzo(b)fluoranthene

Benzo(g,h,i)perylene

Benzo(k)fluoranthene

Benzyl alcohol

Beryllium, total recoverable

Bis(2-chloroethoxy) methane

Bis(2-chloroethyl) ether

Bis(2-ethylhexyl) phthalate

Bromodichloromethane

Bromoform

Bromomethane (Methyl bromide)

Butylbenzyl phthalate

Cadmium, total recoverable

Calcium, total recoverable

Carbazole

Carbon disulfide

Chloride

Chlorobenzene

Chlorobenzilate

Chloroethane

Chiuroethene (Vinyl chloride)

Chloromethane (Methyl chloride)

Chloroprene

Chromium, total recoverable

Chrysene

Cobalt, total recoverable

Copper, total recoverable

Cyanide

Di-n-butyl phthalate

Di-n-octyl phthalate

Diallate

Dibenz(a,h)anthracene

Dibenzofuran

Dibromochloromethane

Dibromomethane (Methylene bromide),

Dichlorodifluoromethane

Dichloromethane (Methylene chloride)

Dieldrin
101998 Mod 201998 Mod 301998 Mod 401998 Mod Unit

$<10 \quad \mathrm{U}$

70

$<5 \quad \mathrm{U}$

$<10 \quad \mathrm{U}$

$<10 \quad U$

$<10 \quad U$

$<10 \quad U$

$<25 \quad \mathrm{U}$

$<10 \quad \mathrm{U}$

$<10 \quad \mathrm{U}$

$<10 \quad \mathrm{U}$

$<10 \quad \mathrm{U}$

$<10$ U

$<5 \quad$ U

$<5$ U

$<5 \quad U$

$<20$ U

$<10 \quad U$

$<17500$

$<10 \quad \mathrm{U}$

$<5$

7160

$<5$

$<10 \quad U$

$<5 \quad U$

$<5 \quad U$

$<50 \quad \mathrm{U}$

$3.31 \mathrm{~J}$

$<10 \quad \mathrm{U}$

3.78 J $\quad \mathrm{E}$

$11.4 \quad J \quad E$

$<10$ U

$<10 \quad \mathrm{U}$

$<20 \quad U$

$<10 \quad U$

$<10 \quad \mathrm{U}$

$<5 \quad U$

$<5 \quad U$

$<5 \quad$ U

$<4.11$ U V

$<.2$ U
ug/L

ug/L

ug/L

$<10 \quad$ JU Q $\quad$ ug/L

$<10 \quad$ JU $Q \quad$ ag/L

$<10$ JU $Q \quad$ ug/L

$<10$ JU $Q \quad u g / L$

$<10 \quad$ JU $Q \quad$ ug/L

$<10 \quad$ JU $Q \quad$ ug/L.

$\mathrm{ug} / \mathrm{L}$

$<\dot{\omega}$. $\pi \mathrm{Q}$ ug/L

$<10$ JU $Q$ ug/L.

$<10$ JU Q ug/L

$\mathrm{ug} / \mathrm{L}$

$u g /$

$\mathrm{ug} / \mathrm{L}$

$<10$ JU Q ug/L

ug $/ \mathrm{L}$

$\mathrm{ug} / \mathrm{L}$

$\mathrm{ug} / \mathrm{L}$

$\mathrm{ug} / \mathrm{L}$

$\mathrm{ug} / \mathrm{L}$

$u g / L$

$<10 \quad U$ ug $/ \mathrm{L}$

$u g / L$

$u g / L$.

ug/L

ug/L

$\mathrm{ug} / \mathrm{L}$

$<10 \quad$ JU Q ug/L

$\mathrm{ug} / \mathrm{L}$

$\mathrm{ug} / \mathrm{L}$

$\mathrm{ug} / \mathrm{L}$

$<10 \quad$ JU $Q \quad$ ug/L

$<10 \quad$ JU. Q ug/L

$<10$ U ug/L

$<10$ JU Q ug/L

$<10$ JU Q ug/L

ug/L

$\mathrm{ug} / \mathrm{L}$

$\mathrm{ug} / \mathrm{L}$

ug/L

$\mathrm{ug} / \mathrm{L}$

Note: Concentrations in bold exceed the Drinking Water Standards listed in Appendix A. Units are for all four quarters. 
WELL: TNX 27D

ANALYTICAL DATA

\begin{tabular}{|c|c|c|c|c|c|c|c|c|c|c|c|c|}
\hline Analyte & $\therefore$ & 101998 & Mod & 201998 & & Lod & 301998 & Mod & $\underline{401998}$ & Mo & & Unit \\
\hline Diethyl phthalate & & & & & & & $<10$ & $\mathrm{U}$ & $<10$ & $\mathrm{JU}$ & $\mathrm{Q}$ & $\overline{u g} / \mathrm{L}$ \\
\hline Dimethoate & & & & & & & & & $<10$ & $\mathrm{U}$ & & $\mathrm{ug} / \mathrm{L}$ \\
\hline Dimethyl phthalate & & & & & & & $<10$ & $\mathrm{U}$ & $<10$ & JU & $Q$ & ug/L \\
\hline Diphenylamine & & & & & & & & & $<10$ & $\mathbf{U}$ & & $\mathrm{ug} / \mathrm{L}$ \\
\hline Disulfoton & & & & & & & . & & $<10$ & $\mathrm{U}$ & & $g / L$ \\
\hline Endosulfan I & & & & & & & $<.1$ & $\mathrm{U}$ & & & & $\mathrm{g} / \mathrm{L}$ \\
\hline Endosulfan II & & & & & & & $<.2$ & $\mathrm{U}$ & & & & $\mathrm{g} / \mathrm{L}$ \\
\hline Endosulfan sulfate & & & & & & & $<.2$ & $\mathrm{U}$ & & & & $g / L$ \\
\hline Endrin & & & & & & & $<.2$ & $\mathrm{U}$ & & & & $g / L$ \\
\hline Endrin aldehyde & & & & & & & $<.2$ & $\mathrm{U}$ & & & & $\mathrm{g} / \mathrm{L}$ \\
\hline Ethyl methacrylate & & & & & & & $<5$ & $U$ & i & & & $\mathrm{g} / \mathrm{L}$ \\
\hline Ethyl methanesulfonate & & & & & & & & & $<10$ & $\mathrm{U}$ & & $\mathrm{g} / \mathrm{L}$ \\
\hline Ethylbenzene & & & & v & & & $<5$ & $\mathrm{U}$ & & & & $g / L$ \\
\hline Fluoranthene & & & & & & & $<10$ & $\mathrm{U}$ & $<10$ & Л & $Q$ & $\mathrm{~g} / \mathrm{L}$ \\
\hline Fluorene & & & & & & & $<10$ & $\mathbf{U}$ & $<10$ & JU & $\mathrm{Q}$ & $\lg / \mathrm{L}$ \\
\hline Fluoride & & & & & & & $<69.4$ & $\mathrm{U}$ & & & & $g / L$ \\
\hline Heptachlor & & & & & & & $<.1$ & $\mathbf{U}$ & & & & $g / L$ \\
\hline Heptachlor epoxide & & & & & & & $<.1$ & $\mathbf{U}$ & & & & $g / L$ \\
\hline Hexachlorobenzene & & & & & & & $<20$ & $\mathbf{U}$ & $<10$ & $\mathrm{JU}$ & $Q$ & $g /$ \\
\hline Hexachlorobutadiene & & & & & & & $<10$ & $\mathrm{U}$ & $<10$ & $\boldsymbol{U}$ & $Q$ & $\lg / \mathrm{L}$ \\
\hline Hexachlorocyclopentadiene & & & & & & & $<10$ & $U$ & $<10$ & $\pi$ & $Q$ & $g / L$ \\
\hline Hexachlorodibenzo-p-dioxins & & & & & & & $<.0011$ & $\mathbf{U}$ & & & & $g / L$ \\
\hline Hexachlorodibenzo-p-furans & & & & & & & $<.00061$ & $\mathrm{U}$ & & & & $/ \mathrm{L}$ \\
\hline Hexachloroethane & & & & & & & $<10$ & $\mathrm{U}$ & $<10$ & JU & $Q$ & $g / L$ \\
\hline Indeno(1,2,3-c,d)pyrene . & & & & & & & $<20$ & $\mathrm{U}$ & $<10^{\circ}$ & $\pi$ & $Q$ & g/ \\
\hline Iodomethane (Meihyi iodide) & & & & & & & $<5$ & $\mathbf{U}$ & & & & $g / L$ \\
\hline Iron, total recoverable & & & & 8.6 & $\mathrm{~J}$ & $E$ & $<94.2$ & $U E$ & 108 & $\mathrm{~J}$ & I & $\mathrm{g} / \mathrm{L}$ \\
\hline Isobutyl alcohol & & & & & & & $<1500$ & $U$ & & & & $g / L$ \\
\hline Isodrin & & & & & & & & & $<10$ & U & & $g / L$ \\
\hline Isophorone & & & & & & & $<10$ & $U$ & $<10$ & JU & $Q$ & $g / L$ \\
\hline Isosafrole & & & & & & & & & $<10$ & $\mathrm{U}$ & & $g / L$ \\
\hline Kepone & & & & & & & & & $<10$ & $u$ & & $g / L$ \\
\hline Lindane & & & & & & & $<.1$ & $\mathrm{U}$ & & & & \\
\hline Lithium, total recoverable & & & & & & & $<.91$ & $\mathrm{U}$ & & & & $g / \mathrm{L}$ \\
\hline Magnesium, total recoverable & & & & & & & $<1920$ & & & & & 几 \\
\hline Methacrylonitrile & & & & & & & $<500$ & $\mathrm{U}$ & & & & $g$ \\
\hline Methapyrilene . & & & & & & & & & $<10$ & $\mathbf{U}$ & & \\
\hline Methoxychlor & & & & & & & $<1$ & $\mathrm{U}$ & & & & \\
\hline Methyl ethyl ketone & & & & & & & $<10$ & $\mathrm{U}$ & & & & L \\
\hline Methyl isobutyl ketone & & & & & & & $<5$ & $\mathrm{U}$ & & & & $L$ \\
\hline Methyl methacrylate & & & & & & & $<50$ & $\mathrm{U}$ & & & & \\
\hline Methyl methanesulfonate & & & & & & & & & $<10$ & $\mathrm{U}$ & & $\Lambda$ \\
\hline N-Nitrosodi-n-butylamine & & & & & & & & & $<10$ & U & & \\
\hline
\end{tabular}

Note: Concentrations in bold exceed the Driaking Water Standards listed in Appendix A. Units are for all four quarters. 
WSRC-RP-99-4003

Unclassified

WELL: TNX 27D

ANALYTICAL DATA

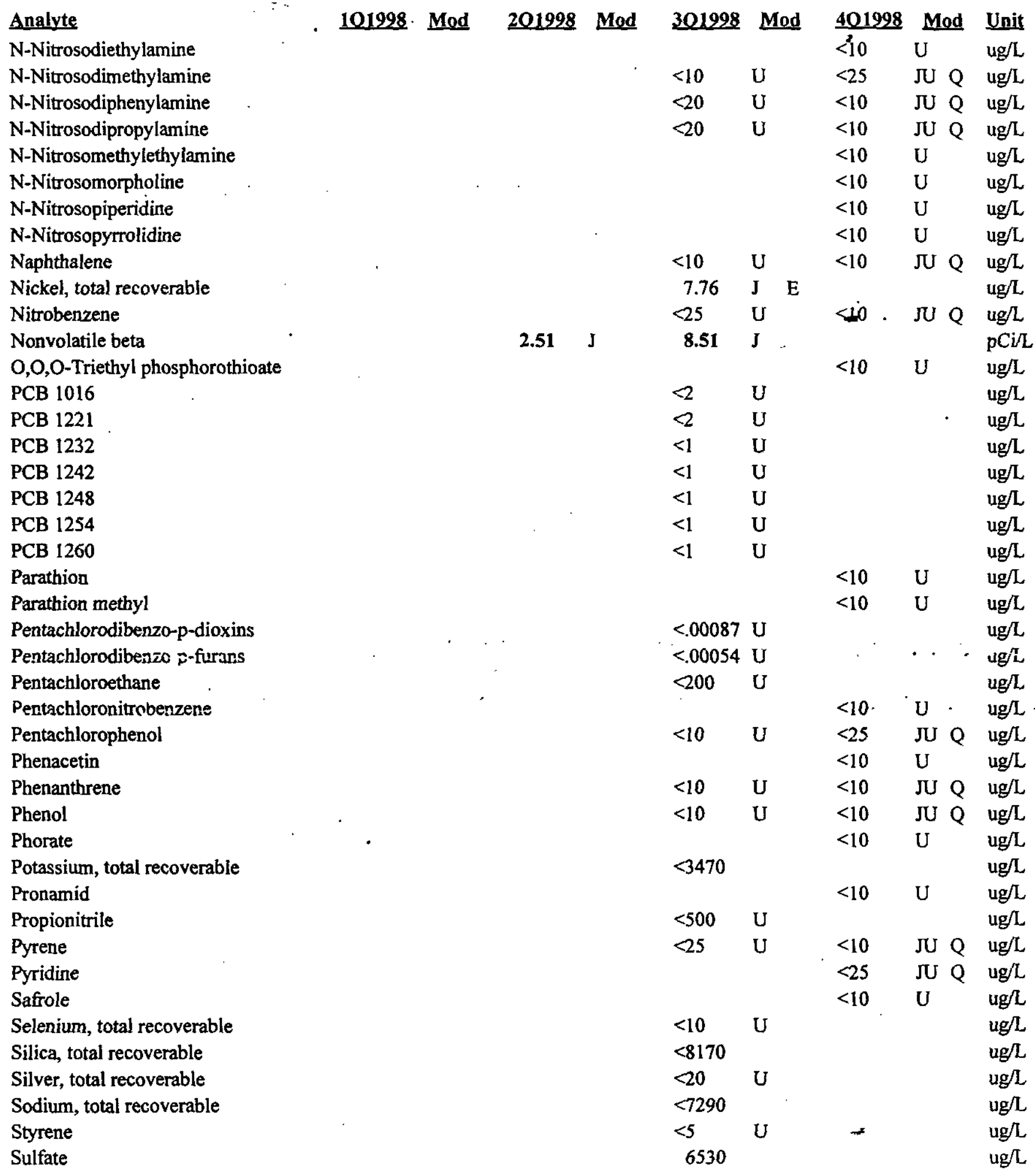

Note: Concentrations in bold exceed the Drinking Water Standards listed in Appendix A. Units are for all four quarters. 
WELL: TNX 27D

ANALYTICAL DATA

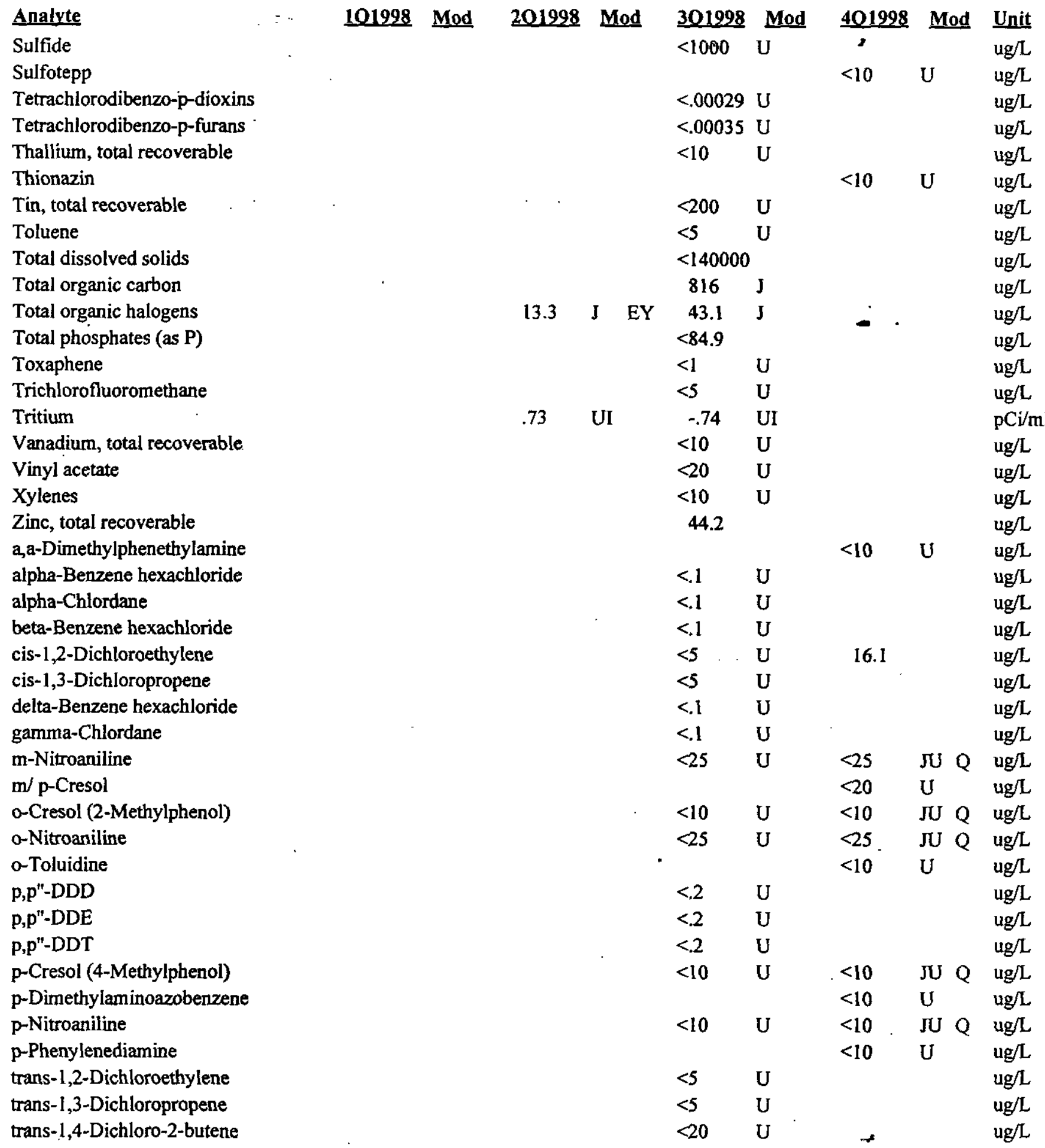

Note: Concentrations in bold exceed the Drinking Water Standards listed in Appendix A. Units are for all four quarters. 
WSRC-RP-99-4003

Unclassified

WELL: XSB 1A

SRS Coord. Lat/Longitude Screen Zone Elevation Top of Standpipe Top of Casing Casing Pump Screen Zone N 71105.4 33.211 Deg N E $16883.0 \quad 81.760 \mathrm{Deg} \mathrm{W}$

\section{$53.6-43.6 \mathrm{ft} \mathrm{msl}$ \\ $156.2 \mathrm{ft} \mathrm{msl}$}

$03 / 03 / 98$

$05 / 15 / 98$

08/06/98
$\mathrm{S}$

$12 / 03 / 98$

\section{FIELD DATA}

\section{Analyte}

Depth to water

$\mathrm{pH}$

Sp. Conductance

Water temperature

Alkalinity as $\mathrm{CaCO} 3$

Phenolpthalein Alkalinity

Turbidity

Volumes purged

Sampling codes

$\begin{array}{lll}\mathbf{1 0 1 9 9 8} & \underline{\mathbf{2 0 1 9 9 8}} & \mathbf{3 0 1 9 9 8} \\ 56.97 & 56.75 & 59.31 \\ 5.2 & 5.2 & 5.2 \\ 84 & 70 & 71 \\ 20.9 & 19.2 & 22.3 \\ 5 & 1 & 4 \\ 0 & 0 & 0 \\ .3 & 1.7 & .5 \\ 2.39260 & 2.87621 & 2.95747\end{array}$

$\begin{array}{ll}\frac{401998}{60.6} & \text { Unit } \\ 4.7 & \text { ft BTOS } \\ 65 & \\ 19.8 & \text { uS/cm } \\ 4 & \text { deg. C } \\ 0 & \text { mg/L } \\ 5 & \text { mg/L } \\ 2.73684 & \text { NTU } \\ & \text { gallons }\end{array}$

\section{ANALYTICAL DATA}

\section{Analyte}

1,1,1,2-Tetrachloroethane

1,1,1-Trichloroethane

1,1,2,2-Tetrachloroethane

1,1,2-Trichloroethane

1,1-Dichloroethane

1,1-Dichloroethylene

1,2,3-Trichloropropane

1,2,4-Trichlorobenzeite

1,2-Dibromo-3-chloropropane

1,2-Dibromoethane

1,2-Dichlorobenzene

1,2-Dichloroethane

1,2-Dichloropropane

1,3,5-Trinitrobenzene

1,3-Dichlorobenzene

1,3-Dinitrobenzene

1,4-Dichlorobenzene

1,4-Dioxane

1,4-Naphthoquinone

1-Naphthylamine

2,2-Oxybis(1-chloropropane)

2,3,4,6-Tetrachlorophenol

2,3,7,8-TCDD

$2,4,5-T$

2,4,5-TP (Silvex)

2,4,5-Trichlorophenol

2,4,6-Trichlorophenol
101998 Mod 201998 Mod 301998 Mod 401998 Mod Unit

$<5 \quad U$

$<5 \quad \mathrm{U}$

$<5 \quad \mathrm{U}$

$<5 \quad U$

$<5 \quad \mathrm{U}$

$<5 \quad \mathrm{U}$

$<5 \quad \mathrm{U}$

$<10 \quad U$

$<5 \quad \mathrm{U}$

$<5 \quad \mathrm{U}$

$<5 \quad U$

$<5 \quad \mathrm{U}$

$<5 \quad \mathrm{U}$

$<5 \quad U$

$<5 \quad \mathrm{U}$

$<1000$

U

$<10 \quad \mathrm{U}$

$<.00047 \mathrm{U}$

$<.2$ UJ $\mathrm{O}$

$<.2$ UJ $\mathrm{O}$

$<10$ U

$<25$

U

$<$

U

$\mathrm{ug} / \mathrm{L}$

ug/L

ug/L

$u g / L$

ug/L

ug/L

ug/L

$<10 \quad \pi \quad \mathrm{Q} \quad \mathrm{ug} / \mathrm{L}$

ug/L

ug/L

$u g / L$

$\mathrm{ug} / \mathrm{L}$

$\mathrm{ug} / \mathrm{L}$

$\mathrm{ug} / \mathrm{L}$

ug/L

$\mathrm{ug} / \mathrm{L}$

ug/L

$\mathrm{ug} / \mathrm{L}$

$<10 \quad$ U $\quad u g / L$

$<10 \quad$ U ug/L

$<10 \quad$ JU Q ug/L

$<10 \quad \mathrm{U} \quad \mathrm{ug} / \mathrm{L}$

$\mathrm{ug} / \mathrm{L}$

ug/L

ug $/$

$<10$ JU Q ug/L

$<25$ JU Q ug/L

Note: Concentrations in bold exceed the Drinking Water Standards fisted in Appendix A. Units are for all four quarters. 
WELL: XSB 1A

\section{ANALYTICAL DATA}

Analyte

2,4-Dichlorophenol

2,4-Dichlorophenoxyacetic acid

2,4-Dimethyl phenol

2,4-Dinitrophenol

2,4-Dinitrotoluene

2,6-Dichlorophenol

2,6-Dinitrotoluene

2-Acetylaminofluorene

2-Chloronaphthalene

2-Chlorophenol

2-Hexanone

2-Methyl-4,6-dinitrophenol

2-Methyinaphthalene

2-Naphthylamine

2-Nitrophenol

2-Picoline

2-sec-Butyl-4,6-dinitrophenol

3,3"-Dichlorobenzidine

3,3"-Dimethylbenzidine

3-Methylcholanthrene

4-Aminobiphenyl

4-Bromophenyl phenyl ether

4-Chloro-m-cresol

4-Chloroaniline

4-Chlorophenyl phenyl ether

A-Nitrophenol

4-Nitroquinoline-1-oxide

5-Nitro-0-toluidine

7,12-Dimethylbenz(a)anthracene

Acenaphthene

Acenaphthylene

Acetone

Acetonitrile (Methyl cyanide)

Acetophenone

Acrolein

Acrylonitrile

Aldrin

Allyl chloride

Aluminum, total recoverable

Aniline

Anthracene

Antimony, total recoverable

Aramite

201998 Mod

\begin{tabular}{|c|c|c|c|c|}
\hline 301998 & Mod & $\underline{401998}$ & Mod & Unit \\
\hline$<10$ & $U$ & $<10$ & JU Q & $\mathrm{ug} / \mathrm{L}$ \\
\hline$<.2$ & UJ $O$ & & & $\mathrm{ug} / \mathrm{L}$ \\
\hline$<10$ & $U$ & $<10$ & $J \mathrm{Q}$ & $u g / L$ \\
\hline$<25$ & $U$ & $<25$ & $\mathrm{JU} \mathrm{Q}$ & $\mathrm{ug} / \mathrm{L}$ \\
\hline \multirow[t]{2}{*}{$<10$} & $\mathrm{U}$ & $<10$ & $\mathrm{JU} \mathrm{Q}$ & $\mathrm{ug} / \mathrm{L}$ \\
\hline & & $<10$ & $\mathrm{U}$ & $\mathrm{ug} / \mathrm{L}$ \\
\hline \multirow[t]{2}{*}{$<10$} & $\mathrm{U}$ & $<10$ & $\mathrm{JU} \mathrm{Q}$ & ug/L \\
\hline & & $<10$ & $U$ & $\mathrm{ug} / \mathrm{L}$ \\
\hline 10 & $\mathbf{U}$ & $<10$ & $\mathrm{JU} Q$ & $\mathrm{~g} / \mathrm{L}$ \\
\hline 10 & U & $<10$ & $\mathrm{JU} Q$ & $\mathrm{~L}$ \\
\hline & $\mathbf{U}$ & & & \\
\hline
\end{tabular}

$<25 \quad \mathrm{U} \quad<\overrightarrow{25}$ JU Q ug/L

$<10 \quad \mathrm{U} \quad<10 \quad \mathrm{JU} Q \quad \mathrm{ug} / \mathrm{L}$

$<10$ U ug/L

$<10 \quad \mathrm{U} \quad<10 \quad \pi \quad \mathrm{Q} \quad$ ug/L

$<10 \quad \mathrm{U} \quad \mathrm{ug} / \mathrm{L}$

$<10$ U ug/L

$\begin{array}{llllll}<10 & \mathrm{U} & <10 & \mathrm{JU} & \mathrm{Q} & \mathrm{ug} / \mathrm{L}\end{array}$

$<20$ U ug/L

$<10 \quad \mathrm{U} \quad \mathrm{ug} / \mathrm{L}$

$<10 \quad \mathrm{U} \quad \mathrm{ug} / \mathrm{L}$

$\begin{array}{lllllll}<10 & \mathrm{U} & <10 & \mathrm{JU} & \mathrm{Q} & \mathrm{ug} / \mathrm{L}\end{array}$

$<10 \quad$ U $\quad<10 \quad$ J $Q \quad$ ug/L

$<10 \quad$ U $\quad<10 . J U, Q \quad u g / L$

$<10 \quad \mathrm{U} \quad<10 \quad \mathrm{JU} \mathrm{J}^{\circ} \mathrm{Q} \quad \mathrm{ug} / \mathrm{L}$

$<25 \quad \mathrm{U} \quad<25 \quad \mathrm{JU} \cdot \mathrm{Q} \quad \mathrm{ug} / \mathrm{L}$

$<50$ U ug/L

$<10 . \quad$ U ug/L

$<10 \quad$ U $\quad$ ug/L

$<10 \quad \mathrm{U} \quad<10 \quad$ JU $Q \quad$ ug/L

$<10 \quad \mathrm{U} \quad<10 \quad \pi \quad \mathrm{Q} \quad \mathrm{ug} / \mathrm{L}$

$<20 \mathrm{U} \quad \mathrm{ug} / \mathrm{L}$

$<500 \quad \mathrm{u} \quad \mathrm{ug} / \mathrm{L}$

$<10 \quad \mathrm{U} \quad \mathrm{ug} / \mathrm{L}$

$<50$ U ug/L

$<50$ U ug/L

$<.1$ U ug/L

$<10$ U ug/L

$<100 \quad \mathrm{U} \quad<200 \quad \mathrm{U} \quad<200 \quad \mathrm{U} \quad \mathrm{ug} / \mathrm{L}$

$<25 \quad \mathrm{U} \quad<25 \quad \mathrm{JU} \quad \mathrm{Q} \quad \mathrm{ug} / \mathrm{L}$

$<10 \quad \mathrm{U} \quad<10 \quad$ JU $Q \quad$ ug/L

$<100 \quad \mathrm{U} \quad \mathrm{ug} / \mathrm{L}$

$<10$ U ug/L

Note: Concentrations in bold exceed the Drinking Water Standards listed in Appendix A. Units are for all four quarters. 
WELL: XSB 1A

\section{ANALYTICAL DATA}

\section{Analyte}

Arsenic, total recoverable

Barium, total recoverable

Benzene

Benzo(a)anthracene

Benzo(a)pyrene

Benzo(b)fluoranthene

Benzo(g,h,i)perylene

Benzo(k)fluoranthene

Benzoic acid

Benzyl alcohol

Beryllium, total recoverable

Bis(2-chloroethoxy) methane

Bis(2-chloroethyl) ether

Bis(2-ethylhexyl) phthalate

Bromodichloromethane

Bromoform

Bromomethane (Methyl bromide)

Butylbenzyl phthalate

Cadmium, total recoverable

Carbazole

Carboni disulfide

Chlorobenzene

Chlorobenzilate

Chloroethane

Chloroethene (Vinyl chloride)

Chloromethane (Methyl chloride)

Chloroprene

Chromium, total recoverable

Chrysene

Cobalt, total recoverable

Copper, total recoverable

Cyanide

Di-n-butyl phthalate

Di-n-octyl phthalate

Diallate

Dibenz(a,h)anthracene

Dibenzofuran

Dibromochloromethane

Dibromomethane (Methylene bromide)

Dichlorodifluoromethane

Dichloromethane (Methylene chloride)

Dieldrin

Diethyl phthalate
101998 Mod 201998 Mod 301998 Mod 401998 Mod Unit

$<10 \quad \mathrm{U}$

$<10 \quad \mathrm{U}$

$<5 \quad \mathrm{U}$

$<10 \quad \mathrm{U}$

$<10 \quad \mathrm{U}$

$<10 \quad \mathrm{U}$

$<10 \quad \mathrm{U}$

$<25$ U

$<10 \quad U$

$<10 \quad U$

$<10$ U

$<10$ U

$<5 \quad \mathrm{U}$

$<5 \quad \mathrm{U}$

$<5 \quad \mathrm{U}$

$<10 \quad U$

$<10 \quad \mathrm{U}$

$<0 \quad \mathrm{U}$

$<5 \quad \mathrm{U}$

$<5 \quad \mathrm{U}$

$<10 . \mathrm{U}$

$<5 \quad U$

$<5 \quad U$

$<50$ U

$<10 \quad \mathrm{U}$

$<10 \quad$ U

$<20 \quad U$

$<20$ U

$<10 \quad \mathrm{U}$

$<10 \quad \mathrm{U}$

$<10$

U

$<20$

$<10$

$$
U
$$

U

$<5$

$<5$

$<5$

$<10$

$<.2$

$<10$

U

$<10$

$<10$

$<10$

$<10$

$<10$

$<10$

$<10$

$<-$

$<10$

$<10$

$<10$

$75 \mathrm{~J}$ I

ug/L

$\mathrm{ug} / \mathrm{L}$

ug/L

JU $Q \quad u g / L$

$J U \mathrm{Q} \quad u g / \mathrm{L}$

$\pi \quad Q \quad u g / L$

JU $Q \quad u g / L$

JU $Q$ ug/L

ug/L

JU $Q$ ug/L

ug/L

JU Q ug/L

JU $Q \quad$ ug/L

JU $Q \quad$ ug/L

ug/L

ug/L

$u g / L$

$<10$ JU $Q \quad u g / L$

ug/L

$\mathrm{ug} / \mathrm{L}$

ug/L

ug/L

$<$ I0 U ug/L

$u g / L$

ug/L

$\mathrm{ug} / \mathrm{L}$

$\mathrm{ug} / \mathrm{L}$

$\mathrm{ug} / \mathrm{L}$

$<10 \quad \pi \quad Q \quad u g / L$

$\mathrm{ug} / \mathrm{L}$

$\mathrm{ug} / \mathrm{L}$

$\mathrm{ug} / \mathrm{L}$

$<10$ JU Q ug/L

$<10$ JU Q ug/L

$<10 \quad U$

$\mathrm{ug} / \mathrm{L}$

$<10$ JU Q $\mathrm{ug} / \mathrm{L}$

$<10$ JU $Q$ ug/L

$\mathrm{ug} / \mathrm{L}$

ug/L

$\mathrm{ug} / \mathrm{L}$

$\mathrm{ug} / \mathrm{L}$

$u g / L$

$<10$ JU $Q \quad u g / L$

Note: Concentrations in bold exceed the Drinking Water Standards listed in Appendix A. Units are for all four quarters. 
WELL: XSB 1A

ANALYTICAL DATA

Analyte

$101998 \mathrm{Mod}$

Dimethoate

Dimethyl phthalate

Diphenylamine

Disulfoton

Endosulfan I

Endosulfan II

Endosulfan sulfate

Endrin

Endrin aldehyde

Ethyl methacrylate

Ethyl methanesulfonate

Ethylbenzene

Fluoranthene

Fluorene

Heptachlor

Heptachlor epoxide

Hexachlorobenzene

Hexachlorobutadiene

Hexachlorocyclopentadiene

Hexachlorodibenzo-p-dioxins

Hexachlorodibenzo-p-furans

Hexachloroethane

Indeno(1,2,3-c,d)pyrene

lodomethane (Methyl iodide)

Iron, total recoverable

Isobutyl alcoho!

Isodrin

Isophorone

Isosafrole

Kepone

Lindane

Methacrylonitrile

Methapyrilene

Methoxychlor

Methyl ethyl ketone

Methyl isobutyl ketone

Methyl methacrylate

Methyl methanesulfonate

$\mathrm{N}$-Nitrosodi-n-butylamine

$\mathrm{N}$-Nitrosodiethylamine

$\mathrm{N}$-Nitrosodimethylamine

$\mathrm{N}$-Nitrosodiphenylamine

$\mathrm{N}$-Nitrosodipropylamine

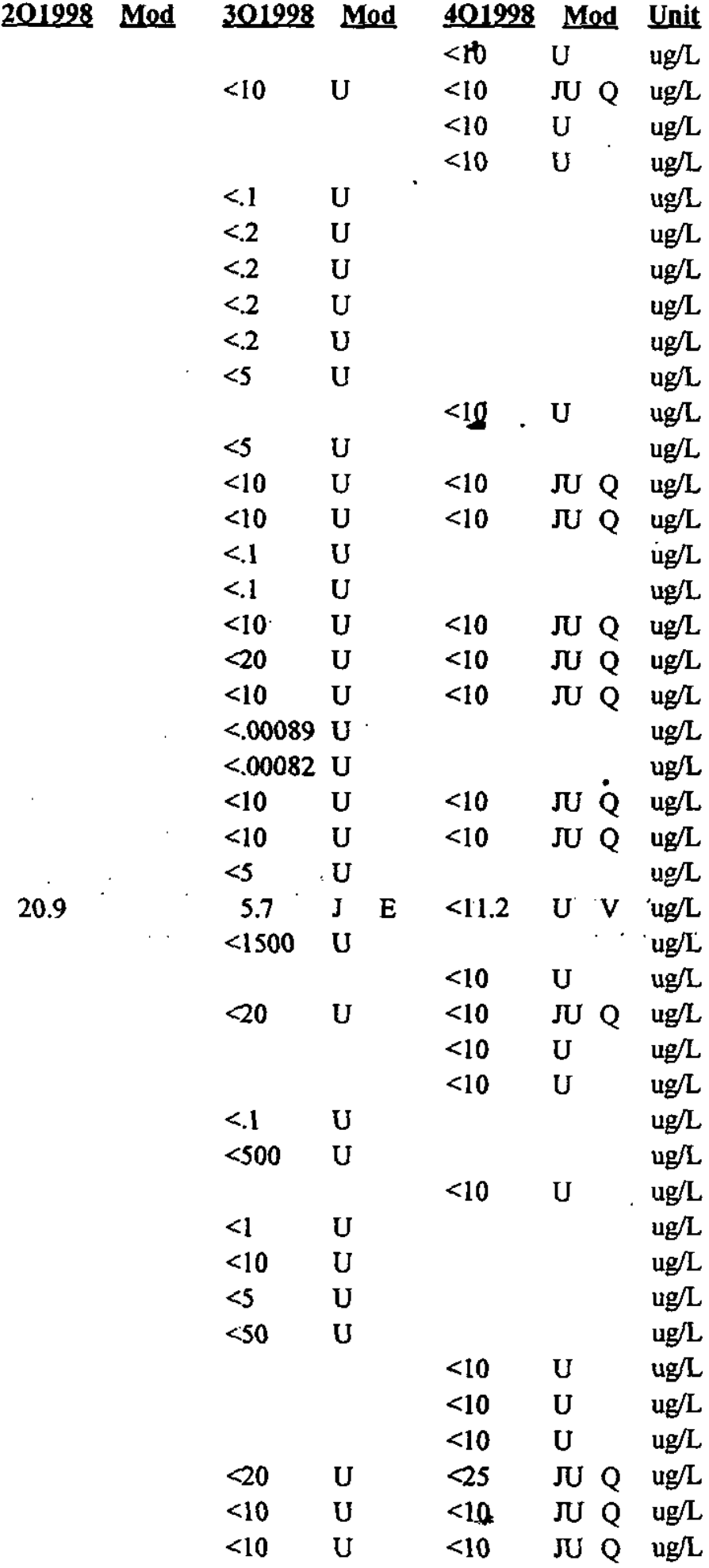

Note: Concentrations in bold exceed the Drinking Water Standards listed in Appendix A. Units are for all four quarters. 
WSRC-RP-99-4003

Unclassified

WELL: XSB 1A

ANALYTICAL DATA

\section{Analyte}

$\mathrm{N}$-Nitrosomethylethylamine

$\mathrm{N}$-Nitrosomorpholine

$\mathrm{N}-\mathrm{Nitrosopiperidine}$

N-Nitrosopyrrolidine

Naphthalene

Nickel, total recoverable

Nitrobenzene

O,O,O-Triethyl phosphorothioate

PCB 1016

PCB 1221

PCB 1232

PCB 1242

PCB 1248

PCB 1254

PCB 1260

Parathion

Parathion methyl

Pentachlorodibenzo-p-dioxins

Pentachlorodibenzo-p-furans

Pentachloroethane

Pentachloronitrobenzene

Pentachlorophenol

Phenacetin

Phenanthrene

Phenol

Phorate

Pronamid

Propionitrile

Pyrene

Pyridine

Safrole

Selenium, total recoverable

Silver, total recoverable

Styrene

Sulfide

Sulfotepp

Tetrachlorodibenzo-p-dioxins

Tetrachlorodibenzo-p-furans

Thallium, total recoverable

Thionazin

Tin, total recoverable

Toluene

Toxaphene
101998 Mod 201998 Mod 301998 Mod 401998 Mod Unit $<10$ U ug/L

$<10$ U ug/L

$<10$ U ug/L

$<10 \quad \mathrm{U} \quad \mathrm{ug} / \mathrm{L}$

$<20 \quad \mathrm{U} \quad<10 \quad$ JU $\mathrm{Q} \quad \mathrm{ug} / \mathrm{L}$

$<50 \quad \mathrm{u} \quad \mathrm{ug} / \mathrm{L}$

$<10 \quad \mathrm{U} \quad<10 \quad \mathrm{JU} Q \quad \mathrm{ug} / \mathrm{L}$

$<10$ U ug/L

$<$ U ug/L

$<2$ U ug/L

$<1 \quad U \quad \therefore \quad . \quad u g / L$

$<$ U ug/L

$<1 \quad$ U $\quad$ ug/L

$<1 \quad$ U $\quad$ ug/L

$<1 \quad$ U $\quad$ ug $/ \mathrm{L}$

$<10 \quad \mathrm{U} \quad \mathrm{ug} / \mathrm{L}$

$<10 \quad \mathrm{U} \quad$ ug/L

$<.00091 \mathrm{U} \quad \mathrm{ug} / \mathrm{L}$

$<.00049 \mathrm{U} \quad \mathrm{ug} / \mathrm{L}$

$<200$ U ug/L

$<10 \quad \mathrm{U} \quad \mathrm{ug} / \mathrm{L}$

$<25 \quad \mathrm{U} \quad<25 \quad \mathrm{JU} \quad \mathrm{Q} \quad \mathrm{ug} / \mathrm{L}$

$<10 \quad \mathrm{U} \quad \mathrm{ug} / \mathrm{L}$

$<10 \cdot \mathrm{U} \quad<10$ JU $\mathrm{Q} \quad \mathrm{ug} / \mathrm{i}$

$<10 \quad U \quad<10 \quad \pi \quad Q \quad$ ug/L

$<10$ U ug/L

$<10 \quad \mathrm{U} \quad \mathrm{ug} / \mathrm{L}$

$<500 \quad \mathrm{U} \quad \mathrm{ug} / \mathrm{L}$

$<10 \quad \mathrm{U} \quad<10 \quad J U \quad \mathrm{Q} \quad \mathrm{ug} / \mathrm{L}$

$<25$ JU $Q \quad u g / L$

$<10 \quad \mathrm{U} \quad \mathrm{ug} / \mathrm{L}$

$<10 \quad \mathrm{U} \quad \mathrm{ug} / \mathrm{L}$

$<20$ U ug/L

$<5 \quad \mathrm{U} \quad \mathrm{ug} / \mathrm{L}$

$<1000 \mathrm{U} \quad \mathrm{ug} / \mathrm{L}$

$<.00047 \mathrm{U} \quad$ ug/L

$<.00038 \mathrm{U} \quad \mathrm{ug} / \mathrm{L}$

$<10$ U ug/L

$<10 \quad \mathrm{U} \quad \mathrm{ug} / \mathrm{L}$

$<200 \quad \mathrm{U} \quad \mathrm{ug} / \mathrm{L}$

$<5 \quad$ U $\quad * \quad$ ug/L

$<1 \quad \mathrm{U} \quad \mathrm{ug} / \mathrm{L}$

Note: Concentrations in bold exceed the Drinking Water Standards listed in Appendix A. Units are for all four quarters. 
WELL: XSB 1A

ANALYTICAL DATA

\section{Analyte}

Trichlorofluoromethane

Vanadium, total recoverable

Vinyl acetate

Xylenes

Zinc, total recoverable

a,a-Dimethylphenethylamine

alpha-Benzene hexachloride

alpha-Chlordane

beta-Benzene hexachloride

cis-1,2-Dichloroethylene

cis-1,3-Dichloropropene

delta-Benzene hexachloride

gamma-Chlordane

m-Nitroaniline

$\mathrm{m} / \mathrm{p}$-Cresol

o-Cresol (2-Methylphenol)

o-Nitroaniline

o-Toluidine

p,p"-DDD

$p, p^{\prime \prime}-D D E$

p,p"-DDT

p-Cresol (4-Methylphenol)

p-Dimethylaminoazobenzene

p-Nitroaniline

p-Phenylenediamine

trans-1,2-Dich!nroethylene

trans-1,3-Dichloropropene

trans-1,4-Dichloro-2-butene
101998 Mod 201998 Mod 301998 Mod 401998 Mod Unit $<5 \quad \mathrm{U}$

$<10 \quad \mathrm{U}$

$<20 \quad \mathrm{U}$

$<10 \quad \mathrm{U}$

$<20 \quad \mathrm{U}$

$<.1 \quad \mathrm{U}$

$<.1$ U

$<1 \quad \mathrm{U}$

$<1$

$<5$ U

$<5 \quad \mathrm{U}$

$<.1 \quad \mathrm{U}$

$<1 \quad$ U

$<25 \quad \mathrm{U}$

$<10 \quad \mathrm{U}$

$<25 \quad \mathrm{U}$

$<.2 \quad \mathrm{U}$

$<.2 \quad \mathrm{U}$

$<.2 \quad \mathrm{U}$

$<10 \quad \mathrm{U}$

$<10 \quad U$

$<5 \quad U$

$<5 \quad U$

$<20$ U $u g / L$

$\mathrm{ug} / \mathrm{L}$

$\mathrm{ug} / \mathrm{L}$

$\mathrm{ug} / \mathrm{L}$

ug/L

$<10 \quad \mathrm{U} \quad \mathrm{ug} / \mathrm{L}$

$\mathrm{ug} / \mathrm{L}$

$\mathrm{ug} / \mathrm{L}$

$\mathrm{ug} / \mathrm{L}$

$<1 \quad$ U ug/L

$\therefore$ ug/L

ug/L

$\mathrm{ug} / \mathrm{L}$

$<25$ JU Q ug/

$<20 \quad \mathrm{U} \quad$ ug/L

$<10 \quad$ JU Q ug/L

$<25 \quad$ JU Q ug/L

$<10$ U ug/L

$\mathrm{ug} / \mathrm{L}$

$\mathrm{ug} / \mathrm{L}$

ug/L

$<10$ JU Q ug/L

$<10$ U ug/L

$<10 \quad$ JU $Q \quad$ ug/L

$<10$ U ug/L

ug/L

$\mathrm{ug} / \mathrm{L}$

$\mathrm{ug} / \mathrm{L}$

Note: Concentrations in bold exceed the Drinking Water Standards listed in Appendix A. Units are for all four quarters. 
WSRC-RP-99-4003

Unclassified

WELL: XSB 1B

SRS Coord, Lat/Longitude Screen Zone Elevation Top of Standpige Top of Casing Casing Pump Screen Zone N 71105.0 33.211 Deg N * $74.6-64.6 \mathrm{ft} \mathrm{msl}$ E $16872.9 \quad 81.760$ Deg W

SAMPLE DATE

$156.2 \mathrm{ft} \mathrm{msl}$

$155.9 \mathrm{ft} \mathrm{msl}$

4 "STL

$\mathrm{S}$

Semiconfined

FIELD DATA

Analyte

Depth to water

$\mathrm{pH}$

Sp. Conductance

Water temperature

Alkalinity as $\mathrm{CaCO} 3$

Phenolpthalein Alkalinity

Turbidity

Volumes purged

Sampling codes

\section{ANALYTICAL DATA}

Analyte

1,1,1,2-Tetrachloroethane

1,1,1-Trichloroethane

1,1,2,2-Tetrachloroethane

1,1,2-Trichloroethane

1,1-Dichloroethane

1,1-Dichloroethylene

1,2,3-Trichloropropane

1,2.4-Trichlorobenzene

1,2-Dibromo-3-chloropropane

i,2-Dibromoethane

1,2-Dichlorobenzene

1,2-Dichloroethane

1,2-Dichloropropane

1,3,5-Trinitrobenzene

1,3-Dichlorobenzene

1,3-Dinitrobenzene

1,4-Dichlorobenzene

1,4-Dioxane

1,4-Naphthoquinone

1-Naphthylamine

2,2-Oxybis(1-chloropropane)

2,3,4,6-Tetrachlorophenol

2,3,7,8-TCDD

2,4,5-T

2,4,5-TP (Silvex)

2,4,5-Trichlorophenol

2,4,6-Trichlorophenol
03/03/98

\begin{tabular}{ll}
$\frac{101998}{49.24}$ & $\underline{201998}$ \\
5.3 & 50.4 \\
35 & 5.2 \\
20.4 & 34 \\
4 & 16 \\
0 & 4 \\
5.4 & 0 \\
2.71824 & 4.2 \\
\hline
\end{tabular}

$08 / 06 / 98$

$12 / 02 / 98$

\author{
$08 / 06 / 98$
}

301998
54.58
4.9
33
23.2
2
0
4.3
4.69107

$\begin{array}{ll}\frac{401998}{55.35} & \text { Unit } \\ 5.2 & \text { ft BTOS } \\ 31 & \\ 21.6 & \text { uS/cm } \\ 2 & \text { deg. C } \\ 0 & \mathrm{mg} / \mathrm{L} \\ 7.4 & \mathrm{mg} / \mathrm{L} \\ 6.02124 & \text { NTU } \\ & \text { gallons }\end{array}$

\begin{tabular}{|c|c|c|c|c|c|c|c|c|}
\hline 101998 & Mod & 201998 & Mod & 301998 & Mod & 401998 & Mod & Unit \\
\hline & & & & $<5$ & $\mathrm{U}$ & & & $\mathrm{ug} / \mathrm{L}$ \\
\hline$<.462$ & UJ 0 & $<.462$ & UJ $O$ & $<5$ & U & $<1$ & U & $\mathrm{ug} / \mathrm{L}$ \\
\hline & & & & $<5$ & $\mathrm{U}$ & & & $\mathrm{ug} / \mathrm{L}$ \\
\hline & & & & $<5$ & U & & & $g / L$ \\
\hline & & & & $<5$ & $\mathrm{U}$ & & & $g / L$ \\
\hline & & & & $<5$ & $\mathrm{U}$ & & & $\lg / \mathrm{L}$ \\
\hline & & & & $<5$ & $U$ & & & ug/L \\
\hline & & & & $<10$ & $U$ & $<10$ & $\pi \mathrm{Q}$ & $\mu \mathrm{g} / \mathrm{i}$. \\
\hline & . & $\therefore$ & & $<5$ & U & & & ug/L \\
\hline & & & & $<5$ & $\mathbf{U}$ & . & & $\mathrm{ug} / \mathrm{L}$ \\
\hline & & & & $<5$ & $\mathrm{U}$ & & & $\operatorname{ug} / \mathrm{L}$ \\
\hline & & & & $<5$ & $\mathrm{U}$ & & & ug $/ \mathrm{L}$ \\
\hline & & & & $<5$ & $\mathrm{U}$ & & & $\mathrm{ug} / \mathrm{L}$ \\
\hline & & & & & & $<10$ & $\mathrm{U}$ & $\mathrm{ug} / \mathrm{L}$ \\
\hline & & & & $<5$ & $\mathrm{U}$ & & & $\mathrm{ug} / \mathrm{L}$ \\
\hline & & & & & & $<10$ & U & $\mathrm{ug} / \mathrm{L}$ \\
\hline & & & & $<5$ & $U$ & & & $\mathrm{ug} / \mathrm{L}$. \\
\hline & & & & $<1000$ & $U$ & & & $\mathrm{ug} / \mathrm{L}$ \\
\hline & & & & & & $<10$ & $U$ & $\mathrm{ug} / \mathrm{L}$ \\
\hline & & & & & & $<10$ & $U$ & $u g / L$ \\
\hline & & & & $<10$ & $\mathrm{U}$ & $<10$ & $J U Q$ & $\operatorname{ug} / \mathrm{L}$ \\
\hline & & & & & & $<10$ & $\mathrm{U}$ & $\mathrm{ug} / \mathrm{L}$ \\
\hline & & & & $<.00058$ & $U$ & & & $g / L$ \\
\hline & & & & $<.2$ & UJ $O$ & & & $\mathrm{ug} / \mathrm{L}$ \\
\hline & & & & $<.2$ & UJ $O$ & & & ug/L \\
\hline & & & & $<10$ & $U$ & $<10$ & $\pi \mathrm{Q}$ & $\mathrm{ug} / \mathrm{L}$ \\
\hline & & & & $<25$ & U & $<25$ & $\pi \cup Q$ & $\mathrm{~g} / \mathrm{L}$ \\
\hline
\end{tabular}

Note: Concentrations in bold exceed the Drinking Water Standards listed in Appendix A. Units are for all four quarters. 
WELL: XSB 1B

ANALYTICAL DATA

Analvte

2,4-Dichlorophenol

2,4-Dichlorophenoxyacetic acid

2,4-Dimethyl phenol

2,4-Dinitrophenol

2,4-Dinitrotoluene

2,6-Dichlorophenol

2,6-Dinitrotoluene

2-Acetylaminofluorene

2-Chloronaphthalene

2-Chlorophenol

2-Hexanone

2-Methyl-4,6-dinitrophenol

2-Methyinaphthalene

2-Naphthylamine

2-Nitrophenol

2-Picoline

2-sec-Butyl-4,6-dinitrophenol

3,3"-Dichlorobenzidine

3,3"-Dimethylbenzidine

3-Methylcholanthrene

4-Aminobiphenyl

4-Bromophenyl phenyl ether

4-Chloro-m-cresol

4-Chloroaniline

4-Chlorophenyl phenyl ether

4-Nitrophenol

4-Nitroquinoline-1-oxide

5-Nitro-o-toluidine

7,12-Dimethylbenz(a)anthracene

Acenaphthene

Acenaphthylene

Acetone

Acetonitrile (Methyl cyanide)

Acetophenone

Acrolein

Acrylonitrile

Aldrin

Allyl chloride

Aluminum, total recoverable

Aniline

Anthracene

Antimony, total recoverable

Aramite
101998 Mod 201998 Mod 301998 Mod 401998 Mod Unit

$<10 \quad$ U. $<10 \quad J U$ Q ug/L

$<.2$ UJ $\mathrm{U} \quad \mathrm{ug} / \mathrm{L}$

$<10 \quad \mathrm{U} \quad<10 \quad \mathrm{JU} \quad \mathrm{Q} \quad \mathrm{ug} / \mathrm{L}$

$<25 \quad$ U $\quad<25 \quad$ JU $Q \quad$ ug/L

$<10 \quad \mathrm{U} \quad<10 \quad$ JU $Q \quad \mathrm{ug} / \mathrm{L}$

$<10 \quad \mathrm{U} \quad \mathrm{ug} / \mathrm{L}$

$<10 \quad \mathrm{U} \quad<10 \quad$ JU $\mathrm{Q} \quad \mathrm{ug} / \mathrm{L}$

$<10 \quad \mathrm{U} \quad \mathrm{ug} / \mathrm{L}$

$<10 \quad$ U $\quad<10 \quad$ JU $Q \quad \mathrm{ug} / \mathrm{L}$

$<10 \quad \mathrm{U} \quad<10 \quad$ JU $Q \quad$ ug/L

$<5 \quad \mathrm{O} \quad{ }^{\circ} \quad$ ugh

$<5$ U $<25$ JU Q ugl

$<10$ U $\quad<10$ JU Q ig/L

$<10 \cdot \mathrm{U} \quad \mathrm{ug} / \mathrm{L}$

$<10 \quad \mathrm{U} \quad<10 \quad$ JU $Q \quad \mathrm{ug} / \mathrm{L}$

$<10$ U ug/L

$<10$ U ug/L

$<10 \quad \mathrm{U} \quad<10 \quad$ JU $Q \quad \mathrm{ug} / \mathrm{L}$

$<20$ U ug/L

$<10 \quad \mathrm{U} \quad \mathrm{ug} / \mathrm{L}$

$<10$ U ug/L

$\begin{array}{llllll}<10 & \mathrm{U} & <10 & \mathrm{JU} & \mathrm{Q} & \mathrm{ug} / \mathrm{L}\end{array}$

$<10 \quad$ U $\quad<10 \quad$ J $Q \quad$ ug/L

$<10 \quad \mathrm{U} \quad<10 \quad$ J $Q \quad$ ug/L

$<10 \quad \mathrm{U} \cdot \quad<10 \quad$ J $Q \quad$ ug $/ \mathrm{L}$

$<25$ U $<25$ JU $Q \quad \mathrm{ug} / \mathrm{L}$

$<50$ U ug/L

$<10$ U ug/L

$<10$ U ug/L

$<10 \quad \mathrm{U} \quad<10 \quad$ JU $Q \quad$ ug/L

$<10$ U $\quad<10$ JU Q ug/L

$<20$ U ug/L

$<500 \quad \mathrm{u} \quad \mathrm{ug} / \mathrm{L}$

$<10 \quad \mathrm{U} \quad \mathrm{ug} / \mathrm{L}$

$<50 \quad \mathrm{U} \quad \mathrm{ug} / \mathrm{L}$

$<50 \quad U$

$<.1 \quad \mathrm{U}$

$\mathrm{ug} / \mathrm{L}$

ug/L

$<10$ U ug/L

15.5 J $\quad$ E $\quad<200 \quad$ U $\quad<200 \quad$ U $\quad$ I $\quad u g / L$

$<25 \quad \mathrm{U} \quad<25 \quad$ JU $Q \quad \mathrm{ug} / \mathrm{L}$

$<10 \quad \mathrm{U} \quad<10 \quad$ JU $Q \quad \mathrm{ug} / \mathrm{L}$

$<100 \quad \mathrm{U} \quad \mathrm{ug} / \mathrm{L}$

$<10 \quad$ U $\quad$ ug/L

Note: Concentrations in bold exceed the Drinking Water Standards listed in Appendix $\mathbf{A}$. Units are for all four quarters. 
WELL: XSB 1B

ANALYTICAL DATA

\section{Analyte}

Arsenic, total recoverable

Barium, total recoverable

Benzene

Benzo(a)anthracene

Benzo(a)pyrene

Benzo(b)fluoranthene

Benzo(g,h,i)perylene

Benzo(k)fluoranthene

Benzoic acid

Benzyl alcohol

Beryllium, total recoverable

Bis(2-chloroethoxy) methane

Bis(2-chloroethyl) ether

Bis(2-ethylhexyl) phthalate

Bromodichloromethane

Bromoform

Bromomethane (Methyl bromide)

Butylbenzyl phthalate

Cadmium, total recoverable

Carbazole

Carbon disulfide

Chlorobenzene

Chlorobenzilate

Chloruethane

Chloroethene (Vinyl chloride)

Chloromethane (Methy! cnloride)

Chloroprene

Chromium, total recoverable

Chrysene

Cobalt, total recoverable

Copper, total recoverable

Cyanide

Di-n-butyl phthalate

Di-n-octyl phthalate

Diallate

Dibenz(a,h)anthracene

Dibenzofuran

Dibromochloromethane

Dibromomethane (Methylene bromide)

Dichlorodifluoromethane

Dichloromethane (Methylene chloride)

Dieldrin

Diethyl phthalate
$101998 \mathrm{Mad} 201998 \mathrm{Mad}$

\begin{tabular}{|c|c|c|c|c|}
\hline 301998 & Mod & $\underline{401998}$ & Mod & Unit \\
\hline$<10$ & $\mathrm{U}$ & & & $\mathrm{ug} / \mathrm{L}$ \\
\hline 20.5 & & 22.4 & & $\mathrm{ug} / \mathrm{L}$ \\
\hline$<5$ & $U$ & & & $\mathrm{ug} / \mathrm{L}$ \\
\hline & & $<10$ & $J U Q$ & $\mathrm{ug} / \mathrm{L}$ \\
\hline$<10$ & $\mathrm{U}$ & $<10$ & JU Q & $\mathrm{ug} / \mathrm{L}$ \\
\hline$<10$ & $\mathbf{U}$ & $<10$ & $\pi \mathrm{Q}$ & $\mathrm{ug} / \mathrm{L}$ \\
\hline$<10$ & $\mathrm{U}$ & $<10$ & JU $Q$ & $\mathrm{ug} / \mathrm{L}$ \\
\hline$<10$ & $\mathrm{U}$ & $<10$ & U Q & $\mathrm{ug} / \mathrm{L}$ \\
\hline$<25$ & $\mathrm{U}$ & & & $\mathrm{ug} / \mathrm{L}$ \\
\hline & & $<10$ & $J U Q$ & $\mathrm{ug} / \mathrm{L}$ \\
\hline$<10$ & $\mathrm{U}$ & $\therefore$ & & $u g / L$ \\
\hline$<10$ & $\mathrm{U}$ & $<10$ & J Q & $\mathrm{ug} / \mathrm{L}$ \\
\hline$<10$ & $\mathbf{U}$ & $<10$ & JU $Q$ & $\mathrm{ug} / \mathrm{L}$ \\
\hline$<10$ & $\mathrm{U}$ & $<10$ & $\pi Q$ & $\mathrm{ug} / \mathrm{L}$ \\
\hline$<5$ & $\mathrm{U}$ & & & $\mathrm{ug} / \mathrm{L}$ \\
\hline$<5$ & $\mathrm{U}$ & & & $\mathrm{ug} / \mathrm{L}$ \\
\hline$<5$ & $\mathrm{U}$ & & & $\mathrm{ug} / \mathrm{L}$ \\
\hline$<10$ & $\mathrm{U}$ & $<10$ & J Q & $\mathrm{ug} / \mathrm{L}$ \\
\hline$<10$ & $\mathbf{U}$ & & & ug/L \\
\hline$<0$ & $\mathrm{U}$ & & & $\mathrm{ug} / \mathrm{L}$ \\
\hline$<5$ & $\mathbf{U}$ & & & ug/L \\
\hline$<5$ & $\mathbf{U}$ & & & $\mathrm{ug} / \mathrm{L}$ \\
\hline & & $<10$ & $\mathrm{U}$ & $\mathrm{ug} / \mathrm{L}$ \\
\hline$<10$ & $\mathbf{U}$ & & & $\mathrm{ug} / \mathrm{L}$ \\
\hline$<5$ & $U$ & & & ug/L \\
\hline$<5$ & $\mathrm{U}^{\circ}$ & & & $\mathrm{ug} / \mathrm{L}$ \\
\hline$<50$ & $\mathbf{U}$ & & & $\mathrm{ug} / \mathrm{L}$ \\
\hline$<10$ & $\mathrm{U}$ & & & $\mathrm{ug} / \mathrm{L}$ \\
\hline$<10$ & U & $<10$ & 厅 Q & $\mathrm{ug} / \mathrm{L}$ \\
\hline 3.15 & $\mathrm{~J} \quad \mathrm{E}$ & & & $\mathrm{ug} / \mathrm{L}$ \\
\hline$<20$ & $\mathrm{U}$ & & & $\mathrm{ug} / \mathrm{L}$ \\
\hline$<10$ & $\mathrm{U}$ & & & $\mathrm{ug} / \mathrm{L}$ \\
\hline$<10$ & $\mathbf{U}$ & $<10$ & JU Q & $\mathrm{ug} / \mathrm{L}$ \\
\hline$<10$ & $\mathrm{U}$ & $<10$ & JU $Q$ & $\mathrm{ug} / \mathrm{L}$ \\
\hline & & $<10$ & $\mathrm{U}$ & $\mathrm{ug} / \mathrm{L}$ \\
\hline$<20$ & $\mathrm{U}$ & $<10$ & J Q & $\mathrm{ug} / \mathrm{L}$ \\
\hline$<10$ & $\mathrm{U}$ & $<10$ & JU Q & $\mathrm{ug} / \mathrm{L}$ \\
\hline$<5$ & $\mathrm{U}$ & & & $\operatorname{ug} / \mathrm{L}$ \\
\hline$<5$ & $\mathbf{U}$ & & & $\mathrm{ug} / \mathrm{L}$ \\
\hline$<5$ & $\mathrm{U}$ & & & $\mathrm{ug} / \mathrm{L}$ \\
\hline$<10$ & $\mathbf{U}$ & & & $\mathrm{ug} / \mathrm{L}$ \\
\hline$<.2$ & $\mathbf{U}$ & + & & $u g / L$ \\
\hline$<10$ & $\mathrm{U}$ & $<10$ & $\mathrm{JU} Q$ & $\mathrm{ug} / \mathrm{L}$ \\
\hline
\end{tabular}

Note: Concentrations in bold exceed the Drinking Water Standards listed in Appendix A. Units are for all four quarters. 
WELL: XSB 1B

ANALYTICAL DATA

Analyte

101998 Mod 201998 Mod

Dimethoate

Dimethyl phthalate

$\begin{array}{lll} & <10 & \mathrm{U} \\ & & \\ & <.1 & \mathrm{U} \\ <.2 & \mathrm{U} \\ <.2 & \mathrm{U} \\ <.2 & \mathrm{U} \\ <.2 & \mathrm{U} \\ <5 & \mathrm{U}\end{array}$

Diphenylamine

Disulfoton

Endosulfan I

Endosulfan II

Endosulfan sulfate

Endrin

Endrin aldehyde

Ethyl methacrylate

Ethyl methanesulfonate

Ethylbenzene

Fluoranthene

Fluorene

Heptachlor

Heptachlor epoxide

Hexachlorobenzene

Hexachlorobutadiene

Hexachlorocyclopentadiene

Hexachlorodibenzo-p-dioxins

Hexachlorodibenzo-p-furans

Hexachloroethane

Indeno(1,2,3-c,d)pyrene

Iodomethane (Methyl iodide)

Iron, total recoverable

Isobutyl alcohol

Isodrin

Isophorone

Isosafrole

Kepone

Lindane

Methacrylonitrile

Methapyrilene

Methoxychlor

Methyl ethyl ketone

Methyl isobutyl ketone

Methyl methacrylate

Methyl methanesulfonate

N-Nitrosodi-n-butylamine

$\mathrm{N}$-Nitrosodiethylamine

$\mathrm{N}$-Nitrosodimethylamine

N-Nitrosodiphenylamine

$<5 \quad \mathrm{U}$

$<10 \quad \mathrm{U}$

$<10 \quad \mathrm{U}$

$<.1 \quad \mathrm{U}$

$<1 \quad \mathrm{U}$

$<10 \quad \mathrm{U}$

$<20$ U

$<10 \quad \mathrm{U}$

$<.00065 \mathrm{U}$

$<.00074 \mathrm{U}$

$<10 \quad \mathrm{U}$

$<10 \quad \mathrm{U}$

$<5 \quad$ U

788

6

813

$<1500$

U

$<20 \quad \mathrm{U}$

$<10$. U ug/L

$<10$ JU Q ug/L

4019

Mod Unit

$<$ P0 U ug/L

$<10$ JU Q ug/L

$<10$ U ug/L

$<10$ U ug/L

ug $/$

ug $/$

ug/L

$\mathrm{ug} / \mathrm{L}$

ug/L

ug/L

$<10 \quad$ JU Q $\quad$ ug/L

$\mathrm{ug} / \mathrm{L}$

ug/L

$<10$ JU $Q \quad$ ug/L

$<10$ JU Q ug/L

$<10$ JU Q ug/L

ug/L

$\mathrm{ug} / \mathrm{L}$

$<10$ JU Q ug/L

$<10$ JU Q ug/L

1470 ug $/$

$1470 \quad u g / 2$

$<10 \quad U \cdot \begin{aligned} & \operatorname{ug} / \mathrm{L} \\ & \operatorname{ug} / \mathrm{L}\end{aligned}$

$<10$ JU Q ug/L

$<10$ U ug/L

$<10$ U ug/L

$<.1 \quad \mathrm{U}$

$<500$ U ug/L

ug/L

$<10 \quad \mathrm{U}$

ug/L

$<5 \quad \mathrm{U}$

$u g / L$

$<50 \quad U$

$\mathrm{ug} / \mathrm{L}$

$\mathrm{ug} / \mathrm{L}$

$<10$ U ug/L

$<10$ U ug/L

$<10 \quad \mathrm{U} \quad \mathrm{ug} / \mathrm{L}$

$<20 \quad$ U $. \quad<25 \quad \pi \quad$ Q $\quad$ ug/L

$<10 \quad \mathrm{U} \quad<10 \quad J U \mathrm{Q} \quad \mathrm{ug} / \mathrm{L}$

$\mathrm{N}$-Nitrosodipropylamine.

$<10 \quad U$

$<10$ JU Q ug/L

Note: Concentrations in bold exced the Drinking Water Standards listed in Appendix A. Units are for all four quarters. 
WSRC-RP-99-4003

Unclassified

WELL: XSB $1 B$

\section{ANALYTICAL DATA}

\begin{tabular}{|c|c|c|c|c|c|c|c|c|c|c|}
\hline \multirow{2}{*}{ Analyte } & \multirow[t]{2}{*}{101998} & \multirow[t]{2}{*}{ Mod } & \multirow[t]{2}{*}{201998} & \multirow[t]{2}{*}{ Mod } & \multirow[t]{2}{*}{$\underline{301998}$} & Mod & \multirow{2}{*}{$\frac{401998}{<10}$} & \multicolumn{2}{|c|}{ Mod } & \multirow{2}{*}{$\frac{\text { Unit }}{\text { ug/L }}$} \\
\hline & & & & & & & & $\mathrm{U}$ & & \\
\hline N-Nitrosomorpholine & & & & & & & $<10$ & $\mathrm{U}$ & & $\mathrm{ug} / \mathrm{L}$ \\
\hline N-Nitrosopiperidine & & & & & & & $<10$ & $\mathrm{U}$ & & $\mathrm{ug} / \mathrm{L}$ \\
\hline N-Nitrosopyrrolidine & & & & & & & $<10$ & $\mathrm{U}$ & & ug \\
\hline Naphthalene & & & & & $<20$ & $U$ & $<10$ & JU & $Q$ & $\mathrm{ug} / \mathrm{L}$ \\
\hline Nickel, total recoverable & & & & & 13.8 & $J \quad E$ & & & & $u g / L$ \\
\hline Nitrobenzene & & & & & $<10$ & $\mathrm{U}$ & $<10$ & JU & Q & $\mathrm{ug} / \mathrm{L}$ \\
\hline O,O,O-Triethyl phosphorothioate & & & & & & & $<10$ & $\mathrm{U}$ & & $\mathrm{ug} / \mathrm{L}$ \\
\hline PCB 1016 & & & & & $<2$ & $\mathrm{U}$ & & & & $\mathrm{ug} / \mathrm{L}$ \\
\hline PCB 1221 & & & & & $<2$ & $\mathrm{U}$ & & & & $\mathrm{ug} / \mathrm{L}$ \\
\hline PCB 1232 & & & & & $<1$ & $\mathrm{U}$ & $\therefore$ & & & $\mathrm{ug} / \mathrm{L}$ \\
\hline PCB 1242 & & & & & $<1$ & $\mathrm{U}$ & & & & $\mathrm{ug} / \mathrm{L}$ \\
\hline PCB 1248 & & & & & $<1$ & $\mathbf{U}$ & & & & $u g / L$ \\
\hline PCB 1254 & & & & & $<1$ & $\mathbf{U}$ & & & & $\mathrm{ug} / \mathrm{L}$ \\
\hline PCB 1260 & & & & & $<1$ & $\mathrm{U}$ & & & & $\mathrm{ug} / \mathrm{L}$ \\
\hline Parathion & & & & & & & $<10$ & $\mathrm{U}$ & & ug/L \\
\hline Parathion methyl & & & & & & & $<10$ & $\mathbf{U}$ & & ug/L \\
\hline Pentachlorodibenzo-p-dioxins & & & & & $<.00072$ & $U$ & & & & $\mathrm{ug} / \mathrm{L}$ \\
\hline Pentachlorodibenzo-p-furans & & & & & $<.0006$ & $\mathrm{U}$ & & & & $\mathrm{ug} / \mathrm{L}$ \\
\hline Pentachloroethane & & & & & $<200$ & $\mathrm{U}$ & & & & $\mathrm{ug} / \mathrm{L}$ \\
\hline Pentachloronitrobenzene & & & & & & & $<10$ & $\mathrm{U}$ & & $\mathrm{ug} / \mathrm{L}$ \\
\hline Pentachlorophenol & & & & & $<25$ & $\mathbf{U}$ & $<25$ & $\pi$ & Q & $\mathrm{ug} / \mathrm{L}$ \\
\hline Phenacetin & & & & & & & $<10$ & $\mathbf{U}$ & & $\mathrm{ug} / \mathrm{L}$ \\
\hline Phenanthrene & $\therefore$ & . & & & $<10$ & $\mathrm{U}$ & $<10$ & $\mathrm{JU}$ & $\mathrm{Q}$ & $\mathrm{ug} / \mathrm{L}$ \\
\hline Phenol & & & . & & $<10$ & $\mathrm{U}$ & $<10 \%$ & $J$ & $Q$ & $u g / 1$ \\
\hline Phorate & & & & & & & $<10$ & $\mathbf{U}$ & & $\mathrm{ug} / 1$ \\
\hline Pronamid & & & & & & & $<10$ & $\mathrm{U}$ & & $\mathrm{ug} / \mathrm{l}$ \\
\hline Propionitrile & & & & & $<500$ & $\mathrm{U}$ & & & & $u g / L$ \\
\hline Pyrene & & & & & $<10$ & $\mathbf{U}$ & $<10$ & JU & $\mathrm{Q}$ & $\mathrm{ug} / \mathrm{L}$ \\
\hline Pyridine & & & & & & & $<25$ & $\mathrm{JU}$ & & $\mathrm{ug} / \mathrm{L}$ \\
\hline Safrole & & & & & & & $<10$ & $\mathrm{U}$ & & $\mathrm{ug} / \mathrm{L}$ \\
\hline Selenium, total recoverable & & & & & $<10$ & $U$ & & & & $\mathrm{ug} /$ \\
\hline Silver, total recoverable & & & & & $<20$ & $\mathrm{U}$ & & & & $\mathrm{ug} / \mathrm{l}$ \\
\hline Styrene & & & & & $<5$ & $\mathbf{U}$ & & & & $\mathrm{ug} /$ \\
\hline Sulfide & & & & & $<1000$ & $\mathrm{U}$ & & & & $\mathrm{ug} / \mathrm{l}$ \\
\hline Sulfotepp & & & & & & & $<10$ & $\mathrm{U}$ & & $\mathrm{ug}$ \\
\hline Tetrachlorodibenzo-p-dioxins & & & & & $<.00058$ & $\mathrm{U}$ & & & & ug \\
\hline Tetrachlorodibenzo-p-furans & & & & & $<.00055$ & $\mathbf{U}$ & & & & $\mathrm{ug} /$ \\
\hline Thallium, total recoverable & & & & & $<10$ & $\mathrm{U}$ & & & & ug/ \\
\hline Thionazin & & & & & & & $<10$ & $\mathbf{U}$ & & $\mathrm{ug}$ \\
\hline Tin, total recoverable & & & & & $<200$ & $\mathrm{U}$ & & & & dg \\
\hline Toluene & & & & & $<5$ & $\mathbf{U}$ & $=$ & & & ug \\
\hline Toxaphene & & & & & $<1$ & $\mathrm{U}$ & & & & \\
\hline
\end{tabular}

Note: Concentrations in bold exceed the Drinking Water Standards listed in Appendix A. Units are for all four quarters. 
WELL: XSB 1B

\section{ANALYTICAL DATA}

\section{Analyte}

101998 Mod

201998 Mod

Trichlorofluoromethane

Vanadium, total recoverable

Vinyl acetate

Xylenes

Zinc, total recoverable a,a-Dimethylphenethylamine alpha-Benzene hexachloride alpha-Chlordane beta-Benzene hexachloride cis-1,2-Dichloroethylene cis-1,3-Dichloropropene delta-Benzene hexachloride gamma-Chlordane $\mathrm{m}$-Nitroaniline $\mathrm{m} / \mathrm{p}$-Cresol o-Cresol (2-Methylphenol)

o-Nitroaniline

o-Toluidine

p,p"-DDD

$p, p "-D D E$

p,p"-DDT

p-Cresol (4-Methylphenol)

p-Dimethylaminoazobenzene p-Nitroaniline p-Phenylenediamine trans-1,2-Dichioruetrylene trans-1,3-Dichloropropene trans-1,4-Dichloro-2-butene

\begin{tabular}{|c|c|c|c|c|}
\hline 301998 & Mod & $\underline{401998}$ & Mod & Unit \\
\hline 5 & $\mathrm{U}$ & 2 & & $\mathrm{ug} / \mathrm{L}$ \\
\hline 10 & U & & & $\mathrm{ug} / \mathrm{L}$ \\
\hline$<20$ & $\mathrm{U}$ & & & $u g / L$ \\
\hline$<10$ & $\mathrm{U}$ & & & $u g / L$ \\
\hline 10.4 & J E & & & $\mathrm{ug} / \mathrm{L}$ \\
\hline & & $<10$ & $\mathrm{U}$ & $\mathrm{ug} / \mathrm{L}$ \\
\hline .1 & U & & & $\mathrm{ug} / \mathrm{L}$ \\
\hline 1 & U & & & $\mathrm{ug} / \mathrm{L}$ \\
\hline 1 & U & & & $u g / L$ \\
\hline & $\mathbf{U}$ & $<1$ & U & $\mathrm{ug} / \mathrm{L}$ \\
\hline 65 & $\mathrm{U}$ & - & & ug/L \\
\hline .1 & U & & & $\mathrm{ug} / \mathrm{L}$ \\
\hline. .1 & U & & & $\mathrm{ug} / \mathrm{L}$ \\
\hline 25 & U & $<25$ & JU $Q$ & $\mathrm{ug} / \mathrm{L}$ \\
\hline & & $<20$ & U & $\mathrm{ug} / \mathrm{L}$ \\
\hline 10 & U & $<10$ & JU $Q$ & $\mathrm{ug} / \mathrm{L}$ \\
\hline 25 & U & $<25$ & $J U Q$ & $\mathrm{ug} / \mathrm{L}$ \\
\hline & & $<10$ & $\mathbf{U}$ & $\mathrm{ug} / \mathrm{L}$ \\
\hline & $\mathrm{U}$ & & & $\mathrm{ug} / \mathrm{L}$ \\
\hline 2 & $\mathrm{U}$ & & & $\mathrm{ug} / \mathrm{L}$ \\
\hline 6.2 & U & & & $\mathrm{ug} / \mathrm{L}$ \\
\hline 10 & $\mathrm{U}$ & $<10$ & $\pi \mathrm{Q}$ & $\mathrm{ug} / \mathrm{L}$ \\
\hline & & $<10$ & $\mathrm{U}$ & $\mathrm{ug} / \mathrm{L}$ \\
\hline & U & $<10$ & $\mathrm{JU} Q$ & $\mathrm{ug} / \mathrm{L}$ \\
\hline & & $<10$ & $\mathrm{U}$ & $\mathrm{ug} / \mathrm{L}$ \\
\hline 55 & $\mathrm{U}$ & & $\therefore$ & $\mathrm{ug} / \mathrm{L}$ \\
\hline$<5$ & U & & & $\mathrm{ug} / \mathrm{L}$ \\
\hline 20 & $\mathrm{U}$ & & & $u g / L$ \\
\hline
\end{tabular}


WSRC-RP-99-4003

Unclassified

WELL: XSB 1D

SRS Coord Lat/Longitude Screen Zone Elevation Top of Standipipe Top of Casing Casing Pump Screen Zone N 71104.8 33.211 Deg N $\cdot 107.9-87.9 \mathrm{ftmsl} 156.2 \mathrm{ftmsl} 156.0 \mathrm{ftmsl} 4$ "STL $\mathrm{S} \quad$ Unconfined E 16893.581 .760 Deg W

$\begin{array}{llll}03 / 05 / 98 & 05 / 13 / 98 & 08 / 06 / 98 & 12 / 03 / 98\end{array}$

SAMPLE DATE

$03 / 05 / 98$

$05 / 13 / 98$

$08 / 06 / 98$

$2 / 03 / 98$

\section{FIELD DATA}

Analyte
Depth to water
pH
Sp. Conductance
Water temperature
Alkalinity as CaCO3
Phenolpthalein Alkalinity
Turbidity
Volumes purged
Sampling codes

ANALYTICAL DATA

Analyte

1,1,1,2-Tetrachloroethane

1,1,1-Trichloroethane

1,1,2,2-Tetrachloroethane

1,1,2-Trichloroethane

1,1-Dichloroethane

1,1-Dichloroethylene

1,2,3-Trichloropropane

1,2,4-Trichlorobenzene

1,2-Dibromo-3-chloropropane

1,2-Dibromoethane

1,2-Dichlorobenzene

1,2-Dichloroethane

1,2-Dichloropropane

1,3,5-Trinitrobenzene

1,3-Dichlorobenzene

1,3-Dinitrobenzene

1,4-Dichlorobenzene

1,4-Dioxane

1,4-Naphthoquinone

1-Naphthylamine

2,2-Oxybis(1-chloropropane)

2,3,4,6-Tetrachlorophenol

2,3,7,8-TCDD

2,4,5-T

2,4,5-TP (Silvex)

2,4,5-Trichlorophenol

2,4,6-Trichlorophenol

\begin{tabular}{lll}
101998 & $\mathbf{2 0 1 9 9 8}$ & $\mathbf{3 0 1 9 9 8}$ \\
\hline 57 & 56.45 & 59.1 \\
4.6 & 4.2 & 4.6 \\
62 & 660 & 80 \\
17 & 16 & 23.1 \\
0 & 0 & 0 \\
0 & 0 & 0 \\
.7 & .9 & .3 \\
9.61327 & 18.8422 & 7.96070
\end{tabular}

401998

60.57

4.3

74

21.1

0

0

$\frac{1}{6.27571}$

Unit

ft BTOS

$\mathrm{uS} / \mathrm{cm}$

deg. C

$\mathrm{mg} / \mathrm{L}$

$\mathrm{mg} / \mathrm{L}$

NTU

gallons
101998 Mod 201998 Mod

$<.462 \mathrm{U} \quad<.462 \mathrm{U}$$$
<
$$$$
<5
$$$$
<5
$$$$
<5
$$$$
<5
$$$$
<5
$$$$
<10 \quad \mathrm{U}
$$$$
<5 \quad U
$$$$
<5 \quad \text { U }
$$$$
<5 \quad \mathrm{U}
$$$$
<5 \quad U
$$$$
<5 \quad \mathrm{U}
$$$$
<5 \quad U
$$$$
<5 \quad \mathrm{U}
$$$$
<1000 \mathrm{U}
$$$$
<10 \quad \mathrm{U} \quad \mathrm{ug} / \mathrm{L}
$$$$
\begin{array}{lllll}
<10 \quad \mathrm{U} & <10 & \mathrm{U} & \mathrm{ug} / \mathrm{L} \\
& <10 & \mathrm{JU} & \mathrm{ug} / \mathrm{L}
\end{array}
$$$$
<10 \quad \mathrm{U} \quad \mathrm{ug} / \mathrm{L}
$$$$
<.00076 \mathrm{U} \quad \mathrm{ug} / \mathrm{L}
$$$$
<.2 \text { UJ } \mathrm{O} \quad \mathrm{ug} / \mathrm{L}
$$$$
<.2 \text { UJ } \mathrm{O} \quad \mathrm{ug} / \mathrm{L}
$$$$
<10 \quad U \quad<\text { TH JU Q ug/L }
$$$$
<25 \text { U }<25 \quad \text { JU } Q \quad \text { ug/L }
$$

Note: Concentrations in boid exceed the Drinking Water Standards listed in Appendix A. Units are for all four quarters. 
WELL: XSB 1D

ANALYTICAL DATA

Analvte

2,4-Dichlorophenol

2,4-Dichlorophenoxyacetic acid

2,4-Dimethyl phenol

2,4-Dinitrophenol

2,4-Dinitrotoluene

2,6-Dichlorophenol

2,6-Dinitrotoluene

2-Acetylaminofluorene

2-Chloronaphthalene

2-Chlorophenol

2-Hexanone

2-Methyl-4,6-dinitrophenol

2-Methylnaphthalene

2-Naphthylamine

2-Nitrophenol

2-Picoline

2-sec-Butyl-4,6-dinitrophenol

3,3"-Dichlorobenzidine

3,3"-Dimethylbenzidine

3-Methylcholanthrene

4-Aminobiphenyl

4-Bromophenyl phenyl ether

4-Chloro-m-cresol

4-Chloroaniline

4-Chlorophenyi phenyl ether

4-Nitrophenol

4-Nitroquinoline-1-oxide

5-Nitro-o-toluidine

7,12-Dimethylbenz(a)anthracene

Acenaphthene

Acenaphthylene

Acetone

Acetonitrile (Methyl cyanide)

Acetophenone

Acrolein

Acrylonitrile

Aldrin

Allyl chloride

Aluminum, total recoverable

Aniline

Anthracene

Antimony, total recoverable

Aramite
101998 Mod 201998 Mod 301998 Mod 401998 Mod Unit

$<10 \quad \mathrm{U} \quad<$ P0 JU $\mathrm{Q} \quad$ ug/L

$<2$ UJ $\mathrm{O} \quad \mathrm{ug} / \mathrm{L}$

$\begin{array}{llllll}<10 & \mathrm{U} & <10 & \text { JU } & \mathrm{Q} & \mathrm{ug} / \mathrm{L}\end{array}$

$<25 \quad \mathrm{U} \quad<25 \quad$ JU $Q \quad \mathrm{ug} / \mathrm{L}$

$<10 \quad \mathrm{U} \quad<10 \quad$ JU $\mathrm{Q} \quad$ ug/L

$<10$ U ug/L

$<10 \quad \mathrm{U} \quad<10 \quad$ JU $\mathrm{Q} \quad \mathrm{ug} / \mathrm{L}$

$<10 \quad \mathrm{U} \quad \mathrm{ug} / \mathrm{L}$

$\begin{array}{llllll}<10 & \mathrm{U} & <10 & \mathrm{JU} & \mathrm{Q} & \mathrm{ug} / \mathrm{L}\end{array}$

$<10 \quad \mathrm{U} \quad<10 \quad \pi \quad \mathrm{Q} \quad \mathrm{ug} / \mathrm{L}$

$<5 \quad \mathrm{O} \quad-\quad . \quad \mathrm{ug} / \mathrm{L}$

$<25$ U $<25$ JU $Q$ ug/L

$<10 \quad \mathrm{U} \quad<10 \quad \mathrm{U} Q \mathrm{Q} \quad \mathrm{ug} / \mathrm{L}$

$<10 \quad \mathrm{U} \quad \mathrm{U} \quad \mathrm{ug} / \mathrm{L}$

$<10 \quad \mathrm{U} \quad<10 \quad$ JU $\mathrm{Q} \quad \mathrm{ug} / \mathrm{L}$

$<10 \quad \mathrm{U} \quad$ ug/L

$<10 \quad \mathrm{U} \quad \mathrm{ug} / \mathrm{L}$

$\begin{array}{llllll}<10 & \mathrm{U} & <10 & \mathrm{JU} & \mathrm{Q} & \mathrm{ug} / \mathrm{L}\end{array}$

$<20$ U ug/L

$<10$ U ug/L

$<10$ U ug/L

$\begin{array}{llllll}<10 & \mathrm{U} & <10 & \mathrm{JU} & \mathrm{Q} & \mathrm{ug} / \mathrm{L}\end{array}$

$<10 \quad \mathrm{U} \quad<10 \quad$ JU $\mathrm{Q} \quad \mathrm{ug} / \mathrm{L}$

$<10 \quad \mathrm{U} \quad<10 \quad \pi \mathrm{Q} \quad$ ug/L

$<10 \quad \mathrm{U} \quad<10 \quad$ JU $Q \quad$ ug/L

$<25 \quad \mathrm{U} \quad<25 \quad$ JU $Q \quad$ ug/L

$<50 \quad \mathrm{U} \quad \mathrm{ug} / \mathrm{L}$

$<10 \quad \mathrm{U} \quad \mathrm{ug} / \mathrm{L}$

$<10 \quad \mathrm{U} \quad$ ug/L

$\begin{array}{llllll}<10 & \mathrm{U} & <10 & \mathrm{JU} & \mathrm{Q} & \mathrm{ug} / \mathrm{L}\end{array}$

$<10$ U $\quad<10 \quad$ JU $Q \quad$ ug/L

$<20$ U ug/L

$<500$ U

$<10 \quad \mathrm{U} \quad \mathrm{ug} / \mathrm{L}$

$<50 \quad \mathrm{U} \quad \mathrm{ug} / \mathrm{L}$

$<50 \quad \mathrm{U} \quad \mathrm{ug} / \mathrm{L}$

$<.1 \quad \mathrm{U} \quad \mathrm{ug} / \mathrm{L}$

$<10 \quad \mathrm{U} \quad u g / \mathrm{L}$

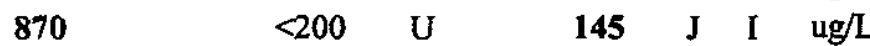

$<25 \quad \mathrm{U} \quad<25 \quad \mathrm{JU} \mathrm{Q} \quad \mathrm{ug} / \mathrm{L}$

$<10 \quad \mathrm{U} \quad<10 \quad$ JU $\mathrm{Q} \quad \mathrm{ug} / \mathrm{L}$

$<100 \quad \mathrm{U}+\mathrm{ug} / \mathrm{L}$

$<10$ U ug/L

Note: Concentrations in bold exceed the Drinking Water Standards listed in Appendix A. Units are for all four quarters. 
WELL: XSB 1D

\section{ANALYTICAL DATA}

\begin{tabular}{|c|c|c|c|c|c|c|c|c|c|}
\hline Analyte & 101998 & Mod & 201998 Mod & 301998 & Mod & $\underline{401998}$ & Mod & & Unit \\
\hline Arsenic, total recoverable & & & & $<10$ & $\mathrm{U}$ & & & & $\mathrm{ug} / \mathrm{L}$ \\
\hline Barium, total recoverable & & & & 17.8 & & 15.3 & & & $\mathrm{ug} / \mathrm{L}$ \\
\hline Benzene & & & & $<5$ & $\mathbf{U}$ & & & & $u g / L$ \\
\hline Benzo(a)anthracene & & & & & & $<10$ & $\mathrm{JU} \mathrm{C}$ & & $\mathrm{ug} / \mathrm{L}$ \\
\hline Benzo(a)pyrene & & . & & $<10$ & U & $<10$ & $\pi \mathrm{C}$ & Q & $\mathrm{ug} / \mathrm{L}$ \\
\hline Benzo(b)fluoranthene & & & & $<10$ & $\mathrm{U}$ & $<10$ & JU C & $Q$ & $\mathrm{ug} / \mathrm{L}$ \\
\hline Benzo(g,h,i)perylene & & & & $<10$ & $\mathrm{U}$ & $<10$ & JU C & $Q$ & ug/L \\
\hline Benzo(k)fluoranthene & & & & $<10$ & $U$ & $<10$ & $\pi C$ & $Q$ & ug/L \\
\hline Benzoic acid & & & & $<25$ & $\mathrm{U}$ & & & & $\mathrm{ug} / \mathrm{L}$ \\
\hline Benzyl alcohol & & & & & & $<10$ & JU C & Q & $\mathrm{ug} / \mathrm{L}$ \\
\hline Beryllium, total recoverable & & & & $<10$ & $\mathrm{U}$ & - & & & $\mathrm{ug} / \mathrm{L}$ \\
\hline Bis(2-chloroethoxy) methane & & & & $<10$ & $U$ & $<10$ & $J U$ & $Q$ & $u g / L$ \\
\hline Bis(2-chloroethyl) ether & & & & $<10$ & $\mathrm{U}$ & $<10$ & Ju C & Q & $\mathrm{ug} / \mathrm{L}$ \\
\hline Bis(2-ethylhexyl) phthalate & & & & $<10$ & $\mathrm{U}$ & 8.68 & $\mathrm{~J}$ & IQ & ug/L \\
\hline Bromodichloromethane & & & & $<5$ & $\mathrm{U}$ & & & & $\mathrm{ug} / \mathrm{L}$ \\
\hline Bromoform & & & & $<5$ & $U$ & & & & $\mathrm{ug} / \mathrm{L}$ \\
\hline Bromomethane (Methyl bromide) & & & & $<5$ & $\mathrm{U}$ & & & & $\mathrm{ug} / \mathrm{L}$ \\
\hline Butylbenzyl phthalate & & & & $<10$ & $\mathrm{U}$ & $<10$ & Ju $\mathrm{C}$ & Q & $\mathrm{ug} / \mathrm{L}$ \\
\hline Cadmium, total recoverable & & & & $<10$ & $\mathbf{U}$ & & & & $\mathrm{ug} / \mathrm{L}$ \\
\hline Carbazole & & & & $<0$ & $\mathbf{U}$ & & & & $\mathrm{ug} / \mathrm{L}$ \\
\hline Carbon disulfide & & & & $<5$ & $\mathrm{U}$ & & & & $\mathrm{ug} / \mathrm{L}$ \\
\hline Chlorobenzene & & & & $<5$ & $\mathrm{U}$ & & & & $\mathrm{ug} / \mathrm{L}$ \\
\hline Chlorobenzilate & & & & & & $<10$ & $\mathrm{U}$ & & $\mathrm{ug} / \mathrm{L}$ \\
\hline Chlöroethane & & & & $<10$ & $\mathbf{U}$ & & & & $\mathrm{ug} / \mathrm{L}$ \\
\hline Chloroethene (Vinyl chloride) & & & & $<5$ & $\mathbf{U}$ & & & & $u g / L$ \\
\hline Chloromerhane (Niethyl chloriae) & . & & & $<5$ & $\mathrm{U}$ & & & & $\mathrm{ug} / \mathrm{L}$ \\
\hline Chloroprene & & & & $<50$ & $\mathrm{U}$ & & & & $\mathrm{ug} / \mathrm{L}$ \\
\hline Chromium, total recoverable & & & & $<10$ & $\mathrm{U}$ & & & & $\mathrm{ug} / \mathrm{L}$ \\
\hline Chrysene & $\because$ & & & $<10$ & $U$ & $<10$ & $\pi c$ & Q & $\mathrm{ug} / \mathrm{L}$ \\
\hline Cobalt, total recoverable & & & & 5.24 & $\mathrm{~J} \quad \mathrm{E}$ & & & & $\mathrm{ug} / \mathrm{L}$ \\
\hline Copper, total recoverable & & & & 6.87 & $\mathrm{~J} \quad \mathrm{E}$ & & & & $\mathrm{ug} / \mathrm{L}$ \\
\hline Cyanide & & & & $<10$ & $\mathrm{U}$ & & & & $\mathrm{ug} / \mathrm{L}$ \\
\hline Di-n-butyl phthalate & & & & $<10$ & $U$ & $<10$ & $\pi U$ & $Q$ & $u g / L$ \\
\hline Di-n-octyl phthalate & & & & $<10$ & $\mathrm{U}$ & $<10$ & $\mathrm{JUC}$ & $\mathrm{Q}$ & $\mathrm{ug} / \mathrm{L}$ \\
\hline Diallate & & & & & & $<10$ & $\mathrm{U}$ & & $\mathrm{ug} / \mathrm{L}$ \\
\hline Dibenz(a,h)anthracene & & & & $<20$ & $\mathrm{U}$ & $<10$ & $\mathrm{JU}$ & Q & $\mathrm{ug} / \mathrm{L}$ \\
\hline Dibenzofuran & & & & $<10$ & U & $<10$ & $\pi U$ & Q & $u g / L$ \\
\hline Dibromochloromethane & & & & $<5$ & $\mathrm{U}$ & & & & $\mathrm{ug} / \mathrm{L}$ \\
\hline Dibromomethane (Methylene bromide') & & & & $<5$ & $\mathrm{U}$ & & & & $\mathrm{ug} / \mathrm{L}$ \\
\hline Dichlorodifluoromethane & & & & $<5$ & $\mathrm{U}$ & & & & $\mathrm{ug} / \mathrm{L}$ \\
\hline Dichloromethane (Methylene chloride) & & & & $<10$ & $\mathrm{U}$ & . & & & $\mathrm{ug} / \mathrm{L}$ \\
\hline Dieldrin & & & & $<.2$ & $U$ & $=$ & & & $\mathrm{ug} / \mathrm{L}$ \\
\hline Diethyl phthalate & & & & $<10$ & $\mathbf{U}$ & $<10$ & $\mathrm{JU} \mathrm{C}$ & $\mathrm{Q}$ & $\mathrm{ug} / \mathrm{L}$ \\
\hline
\end{tabular}

Note: Concentrations in bold exceed the Drinking Water Standards listed in Appendix A. Units are for all four quarters. 
WELL: XSB 1D

ANALYTICAL DATA

Analyte

101998 Mod 201998 Mod 301998 Mod 401998 Mod Unit

Dimethoate

Dimethyl phthalate

Diphenylamine

Disulfoton

Endosulfan I

Endosulfan II

Endosulfan sulfate

Endrin

Endrin aldehyde

Ethyl methacrylate

Ethyl methanesulfonate

Ethylbenzene

Fluoranthene

Fluorene

Heptachlor

Heptachlor epoxide

Hexachlorobenzene

Hexachlorobutadiene

Hexachlorocyclopentadiene

Hexachlorodibenzo-p-dioxins

Hexachlorodibenzo-p-furans

Hexachloroethane

Indeno(1,2,3-c,d)pyrene

Iodomethane (Methyl iodide)

Iron, total recoverable

Isobutyl alcohol

Isodrin

Isophorone

Isosafrole

Kepone

Lindane

Methacrylonitrile

Methapyrilene

Methoxychlor

Methyl ethyl ketone

Methyl isobutyl ketone

Methyl methacrylate

Methyl methanesulfonate

$\mathrm{N}$-Nitrosodi-n-butylamine

N-Nitrosodiethylamine

N-Nitrosodimethylamine

112

\begin{tabular}{|c|c|c|c|c|c|c|c|}
\hline & & & & $<10$ & $U$ & & $\mathrm{ug} / \mathrm{L}$ \\
\hline & $<10$ & $\mathrm{U}$ & & $<10$ & JU & $\mathrm{Q}$ & $\mathrm{ug} / \mathrm{L}$ \\
\hline & & & & $<10$ & $\mathrm{U}$ & & $\mathrm{ug} / \mathrm{L}$ \\
\hline & & & & $<10$ & $\mathrm{U}$ & & $\mathrm{ug} / \mathrm{L}$ \\
\hline & $<.1$ & $\mathrm{U}$ & & & & & ug/L \\
\hline & $<.2$ & $\mathrm{U}$ & & & & & $\mathrm{ug} / \mathrm{L}$ \\
\hline & $<.2$ & $\mathbf{U}$ & & & & & $\mathrm{ug} / \mathrm{L}$ \\
\hline & $<.2$ & $\mathrm{U}$ & & & & & ug $/ \mathrm{L}$ \\
\hline & $<.2$ & U & & & & & $\mathrm{ug} / \mathrm{L}$ \\
\hline & $<5$ & $\mathbf{U}$ & & & & & $\mathrm{ug} / \mathrm{L}$ \\
\hline & & & & $<10$ & $\mathbf{U}$ & & $\mathrm{ug} / \mathrm{L}$ \\
\hline & $<5$ & $\mathrm{U}$ & & & & & $\mathrm{ug} / \mathrm{L}$ \\
\hline & $<10$ & $\mathbf{U}$ & & $<10$ & JU & $\mathrm{Q}$ & $\mathrm{ug} / \mathrm{L}$ \\
\hline & $<10$ & U & & $<10$ & JU & $\mathrm{Q}$ & $\mathrm{ug} / \mathrm{L}$ \\
\hline & $<.1$ & $\mathrm{U}$ & & & & & ug/ \\
\hline & $<.1$ & $\mathbf{U}$ & & & & & $\mathrm{ug} / \mathrm{L}$ \\
\hline & $<10$ & $\mathrm{U}$ & & $<10$ & JU & $Q$ & ug $/ \mathrm{L}$ \\
\hline & $<20$ & U & & $<10$ & JU & $\mathrm{Q}$ & $\mathrm{ug} / \mathrm{L}$ \\
\hline & $<10$ & $\mathrm{U}$ & & $<10$ & JU & $Q$ & $\mathrm{ug} / \mathrm{L}$ \\
\hline & $<.0011$ & $\mathrm{U}$ & & & & & $\mathrm{ug} / \mathrm{L}$ \\
\hline & $<.0006$ & $\mathbf{U}$ & & & & & $\mathrm{ug} / \mathrm{L}$ \\
\hline & $<10$ & $\mathbf{U}$ & & $<10$ & J & $Q$ & ug/L \\
\hline & $<10$ & $\mathrm{U}$ & & $<10$ & $\mathrm{JU}$ & $\mathrm{Q}$ & $\mathrm{ug} / \mathrm{L}$ \\
\hline & $<5$ & $\mathrm{U}$ & & & & & $\mathrm{ug} / \mathrm{L}$ \\
\hline 112 & 25.6 & $\mathrm{~J}$ & $\mathrm{E}$ & 84.2 & J & I & $\mathrm{ug} / \mathrm{L}$ \\
\hline & $<1500$ & $\mathbf{U}$ & & & & & $\mathrm{ug} / \mathrm{t}$ \\
\hline & & & & $<10$ & $\mathrm{U}$ & & $\mathrm{ag} / \mathrm{L}$ \\
\hline & $<20$ & $\mathbf{U}$ & & $<10$ & JU & $\mathrm{Q}$ & $\mathrm{ug} / \mathrm{L}$ \\
\hline & & & & $<10$ & $\mathrm{U}$ & & $\mathrm{ug} / \mathrm{L}$ \\
\hline & & & & $<10$ & $\mathrm{U}$ & & $\mathrm{ug} / \mathrm{L}$ \\
\hline & $<.1$ & $\mathbf{U}$ & & & & & $\mathrm{ug} / \mathrm{L}$ \\
\hline & $<500$ & $U$ & & & & & $\mathrm{ug} / \mathrm{L}$ \\
\hline & & & & $<10$ & $\mathrm{U}$ & & $\mathrm{ug} / \mathrm{L}$ \\
\hline & $<1$ & $\mathbf{U}$ & & & & & ug $/ \mathrm{L}$ \\
\hline & $<10$ & $\mathrm{U}$ & & & & & $\mathrm{ug} / \mathrm{L}$ \\
\hline & $<5$. & $U$ & & & & & ug/L \\
\hline & $<50$ & $\mathrm{U}$ & & & & & $\mathrm{ug} / \mathrm{L}$ \\
\hline & & & & $<10$ & $\mathrm{U}$ & & $\mathrm{ug} / \mathrm{L}$ \\
\hline & & & & $<10$ & $\mathrm{U}$ & & $\mathrm{ug} / \mathrm{L}$ \\
\hline & & & & $<10$ & $\mathrm{U}$ & & $\mathrm{ug} / \mathrm{L}$ \\
\hline & $<20$ & $\mathbf{U}$ & & $<25$ & JU & $Q$ & $\mathrm{ug} / \mathrm{L}$ \\
\hline & $<10$ & $\mathrm{U}$ & & $<10$ & JU & $\mathrm{Q}$ & $\mathrm{ug} / \mathrm{L}$ \\
\hline & $<10$ & $\mathrm{U}$ & & $<10$ & $\pi$ & $Q$ & ug $/ \mathrm{L}$ \\
\hline
\end{tabular}

N-Nitrosodiphenylamine

$<10 \quad \mathrm{U}$

$<10$

Note: Concentrations in bold exceed the Drinking Water Standards listed in Appendix A. Units are for all four quarters. 
WELL: XSB 1D

ANALYTICAL DATA

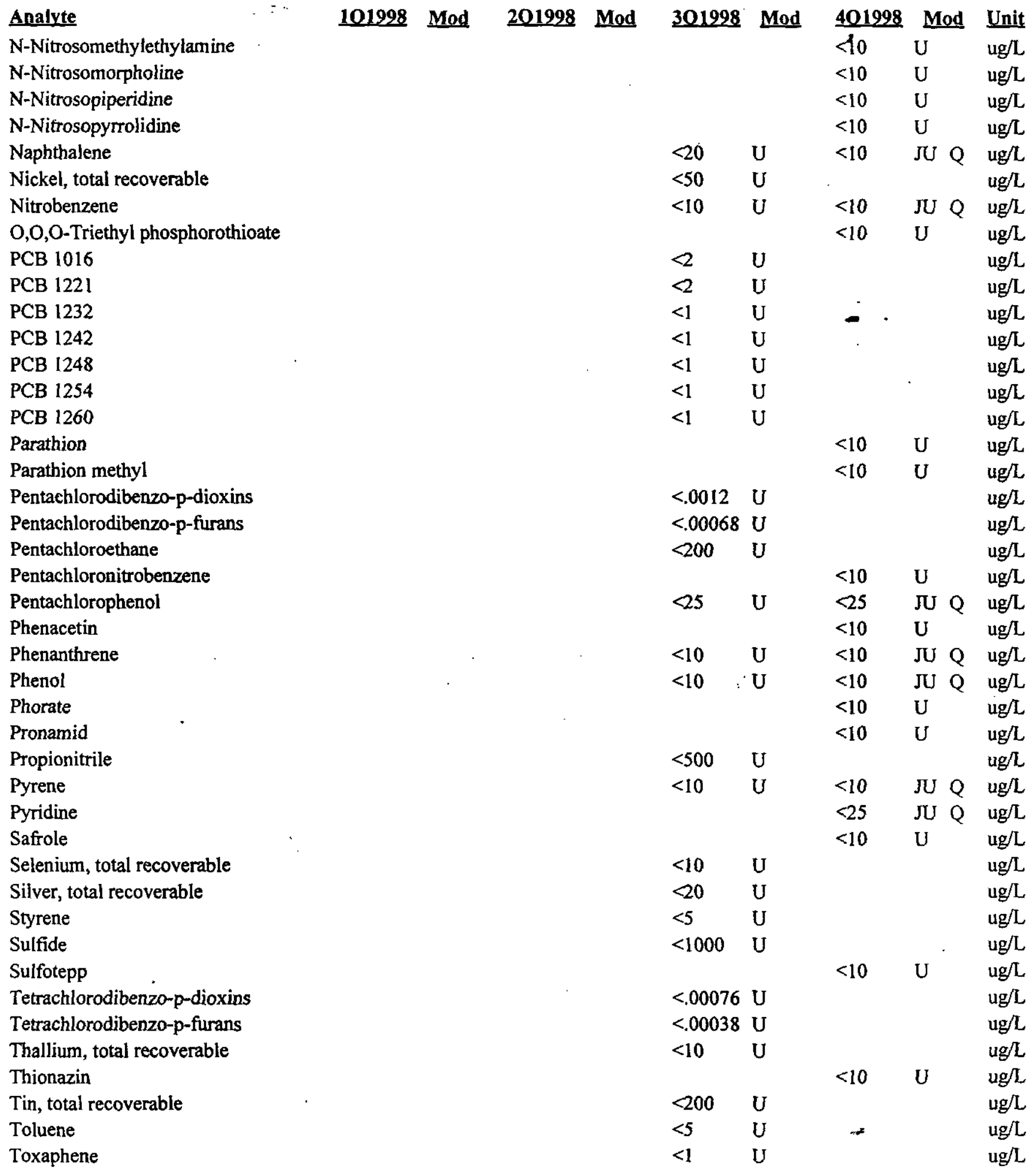

Note: Concentrations in bold exceed the Drinking Water Standards listed in Appendix A. Units are for all four quarters. 
WELL: XSB 1D

ANALYTICAL DATA

\section{Analyte}

Trichlorofluoromethane

Vanadium, total recoverable

Vinyl acetate

Xylenes

Zinc, total recoverable

a, a-Dimethylphenethylamine

alpha-Benzene hexachloride

alpha-Chlordane

beta-Benzene hexachloride

cis-1,2-Dichloroethylene

cis-1,3-Dichloropropene

delta-Benzene hexachloride

gamma-Chlordane

m-Nitroaniline

$\mathrm{m} / \mathrm{p}$-Cresol

o-Cresol (2-Methylphenol)

o-Nitroaniline

o-Toluidine

p,p"-DDD

$p, p^{n}-\mathrm{DDE}$

p,p"-DDT

p-Cresol (4-Methylphenol)

p-Dimethylaminoazobenzene

p-Nitroaniline

p-Phenylenediamine

trans-1,2-Dichloioethylune

trans-1,3-Dichloropropene

trans-1,4-Dichloro-2-butene

\section{Mod 201998 Mod}

\begin{tabular}{|c|c|}
\hline 301998 & M \\
\hline$<5$ & $\mathrm{U}$ \\
\hline$<10$ & $\mathbf{U}$ \\
\hline$<20$ & $\mathrm{U}$ \\
\hline$<10$ & $U$ \\
\hline $9: 21$ & J \\
\hline
\end{tabular}

401998 Mod Unit

3

ug/L

ug/L

ug/L

ug/L

$\mathrm{ug} / \mathrm{L}$

$<10 \quad \mathrm{U} \quad \mathrm{ug} / \mathrm{L}$

$<.1 \quad \mathrm{U}$

$\mathrm{ug} / \mathrm{L}$

$<.1 \quad$ U

$\mathrm{ug} / \mathrm{L}$

$<.1 \quad$ U

$<5 \quad U$

$<5 \quad U$

$<.1 \quad \mathrm{U}$

$<.1 \quad \mathrm{U}$

$<25 \quad$ U

U

$<$

$\mathrm{ug} / \mathrm{L}$

$\mathrm{ug} / \mathrm{L}$

$\mathrm{ug} / \mathrm{L}$

$\mathrm{ug} / \mathrm{L}$

ug $/ \mathrm{L}$

$<25$ JU Q ugl

$<20$ U ug/L

$<10 \quad \mathrm{U}$

$<10$

JU $Q \quad u g / L$

$<25$

J $Q$

$<10$

U

ug/L

$\mathrm{ug} / \mathrm{L}$

$<.2 \quad \mathrm{U}$

$<2$ U

$u g / L$

ug $/$

$\mathrm{ug} / \mathrm{L}$

$<.2 \quad \mathrm{U}$

$<10 \quad \mathrm{U}$

$<10$

$<10$

JU Q ug/L

$<10 \quad U$

$<10$

$<10$

$\mathrm{U}$

$\mathrm{ug} / \mathrm{L}$

JU Q ug/L

$\mathrm{U}$

$\mathrm{ug} / \mathrm{L}$

$<5 \quad U$

$<5 \quad \mathrm{U}$

$<20 \quad \mathrm{U}$ ug $/ \mathrm{L}$

ug/L

ug $/ \mathrm{L}$

Note: Concentrations in bold exceed the Drinking Water Standards listed in Appendix A. Units are for alt four quarters. 
WSRC-RP-99-4003

Unclassified

WELL: XSB 2D

SRS Coord. Lat/Longitude Screen Zone Elevation Ton of Standpipe Top of Casing Casing Pump Screen Zone N 71086.0 33.210 Deg N . $=104.0-84.0 \mathrm{ftmsl} \cdot 155.0 \mathrm{ft}$ msl $154.8 \mathrm{ft} \mathrm{msl} 4$ "STL S Unconfined E 16823.181 .761 Deg W

$\begin{array}{lllll}\text { SAMPLE DATE } & 03 / 05 / 98 & 05 / 11 / 98 & 08 / 04 / 98 & 12 / 04 / 98\end{array}$

FIELD DATA

\begin{tabular}{|c|c|c|c|c|c|}
\hline Analvte & 101998 & 201998 & 301998 & 401998 & Unit \\
\hline Depth to water & 55.8 & 55.15 & 58 & 59.44 & ft BTOS \\
\hline $\mathrm{pH}$ & 5.6 & 6 & 5.8 & 4.8 & \\
\hline Sp. Conductance & 160 & 145 & 120 & 120 & $\mathrm{uS} / \mathrm{cm}$ \\
\hline Water temperature & 17 & 18 & 24.6 & 20.2 & deg. C \\
\hline Alkalinity as $\mathrm{CaCO} 3$ & 16 & 23 & 12 & 9 & $\mathrm{mg} / \mathrm{L}$ \\
\hline Phenolpthalein Alkalinity & 0 & 0 & 0 & 0 & $\mathrm{mg} / \mathrm{L}$ \\
\hline Turbidity & .7 & 1.4 & .6 & & NTU \\
\hline Volumes purged & 10.4675 & 4.48064 & 11.1947 & 3.62311 & gallons \\
\hline
\end{tabular}

\section{ANALYTICAL DATA}

\section{Analvite}

1,1,1,2-Tetrachloroethane

1,1,1-Trichloroethane

1,1,2,2-Tetrachloroethane

1,1,2-Trichloroethane

1,1-Dichloroethane

1,1-Dichloroethylene

1,2,3-Trichloropropane

1,2,4-Trichlorobenzene

1,2-Dibromo-3-chloropropane

1,2-Dibromoethane

1,2-Dichlorobenzene

1,2-Dichloroethane

1,2-Dichloropropane

1,3,5-Trinitrobenzene

1,3-Dichlorobenzene

1,3-Dinitrobenzene

1,4-Dichlorobenzene

1,4-Dioxane

1,4-Naphthoquinone

1-Naphthylamine

2,2-Oxybis(1-chloropropane)

2,3,4,6-Tetrachlorophenol

2,3,7,8-TCDD

2,4,5-T

2,4,5-TP (Silvex)

2,4,5-Trichlorophenol

2,4,6-Trichlorophenol

\begin{tabular}{|c|c|c|c|c|c|c|c|c|}
\hline 101998 & Mod & 201998 & Mod & $\underline{301998}$ & Mod & 401998 & Mod & Unit \\
\hline & & & & $<5$ & $\mathrm{U}$ & & & $\mathrm{ug} / \mathrm{L}$ \\
\hline$<.462$ & $\mathbf{U}$ & $<.462$ & $\mathbf{U}$ & $<5$ & $\mathbf{U}$ & $<1$ & $U$ & ug/L \\
\hline . & & & & $<5$ & U & & & $u g / L$ \\
\hline & & & & $<5$ & $U$ & & & ug/L \\
\hline & & & & $<5$ & $\mathrm{U}$ & & & ug/L \\
\hline & & & & $<5$ & $\mathbf{U}$ & & & ug $/ \mathrm{L}$ \\
\hline & & $\therefore$ & & $<5$ & $\mathrm{U}$ & & & $\mathrm{ug} / \mathrm{L}$ \\
\hline & & & & $<10$ & $\mathrm{U}$ & $<10$ & JU Q & $\mathrm{ug} / \mathrm{L}$ \\
\hline & & & & $<5$ & U & & & $u g /$, \\
\hline 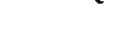 & & & & $<5$ & $U$ & & · & $u g / L$ \\
\hline & & & & $<5$ & $\mathrm{U}$ & & & $\mathrm{ug} / \mathrm{L}$ \\
\hline & & & & $<5$ & $\mathbf{U}$ & & & $\mathrm{ug} / \mathrm{L}$ \\
\hline & & & & $<5$ & $\mathrm{U}$ & & & $\mathrm{ug} / \mathrm{L}$ \\
\hline & & & & & & $<10$ & $\mathrm{U}$ & $\mathrm{ug} / \mathrm{L}$ \\
\hline & & & & $<5$ & $\mathrm{U}$ & & & $\mathrm{ug} / \mathrm{L}$ \\
\hline & & & & & & $<10$ & $U$ & $\mathrm{ug} / \mathrm{L}$ \\
\hline & & & & $<5$ & $\mathrm{U}$ & & & $\mathrm{ug} / \mathrm{L}$ \\
\hline & & & & $<1000$ & $\mathrm{U}$ & & & $\mathrm{ug} / \mathrm{L}$ \\
\hline & & & & & & $<10$ & $\mathbf{U}$ & $\mathrm{ug} / \mathrm{L}$ \\
\hline & & & & & & $<10$ & $\mathrm{U}$ & ug/L \\
\hline & & & & $<10$ & $\mathrm{U}$ & $<10$ & $J U Q$ & $\mathrm{ug} / \mathrm{L}$ \\
\hline & & & & & & $<10$ & $U$ & $u g / L$ \\
\hline & & & & $<.00044$ & $\mathrm{U}$ & & & $\mathrm{ug} / \mathrm{L}$ \\
\hline & & & & $<.2$ & $\mathrm{U}$ & & & $\mathrm{ug} / \mathrm{L}$ \\
\hline & & & & $<.2$ & $\mathrm{U}$ & & & $\mathrm{ug} / \mathrm{L}$ \\
\hline & & & & $<10$ & $\mathrm{U}$ & $<100$ & JU Q & $\mathrm{ug} / \mathrm{L}$ \\
\hline & & & & $<25$ & $\mathbf{U}$ & $<25$ & $\mathrm{JU} Q$ & $\mathrm{ug} / \mathrm{L}$ \\
\hline
\end{tabular}

Note: Concentrations in bold exceed the Drinking Water Standards listed in Appendix A. Units are for all four quarters. 
WELL: XSB 2D

\section{ANALYTICAL DATA}

\section{Analyte}

2,4-Dichlorophenol

2,4-Dichlorophenoxyacetic acid

2,4-Dimethyl phenol

2,4-Dinitrophenol

2,4-Dinitrotoluene

2,6-Dichlorophenol

2,6-Dinitrotoluene

2-Acetylaminofluorene

2-Chloronaphthalene

2-Chlorophenol

2-Hexanone

2-Methyl-4,6-dinitrophenol

2-Methylnaphthalene

2-Naphthylamine

2-Nitrophenol

2-Picoline

2-sec-Butyl-4,6-dinitrophenol

3,3"-Dichlorobenzidine

3,3"-Dimethylbenzidine

3-Methylcholanthrene

4-Aminobiphenyl

4-Bromophenyl phenyl ether

4-Chloro-m-cresol

4-Chloroaniline

4-Chlorophenyl phenyl ether

4-Nitrophenol

4-Nitroquinoline-1-oxide

5-Nitro-o-toluidine

7,12-Dimethylbenz(a)anthracene

Acenaphthene

Acenaphthylene

Acetone

Acetonitrile (Methyl cyanide)

Acetophenone

Acrolein

Acrylonitrile

Aldrin

Allyl chloride

Aluminum, total recoverable

Aniline

Anthracene

Antimony, total recoverable

Aramite
101998 Mod 201998 Mod 301998 Mod 401998 Mod Unit

$<10 \quad \mathrm{U} \cdot<1$ JU $\mathrm{Q}$ ug/L

$<.2$ U ug/L

$<10 \quad \mathrm{U} \quad<10 \quad \mathrm{JU} Q \quad \mathrm{ug} / \mathrm{L}$

$<25$ U $<25$ JU $Q \quad$ ug/L

$<10 \quad \mathrm{U} \quad<10 \quad$ JU $\mathrm{Q} \quad \mathrm{ug} / \mathrm{L}$

$<10$ U ug/L

$<10 \quad \mathrm{U} \quad<10 \quad$ JU $Q \quad$ ug/L

$<10$ U $\quad$ ug/L

$<10 \quad \mathrm{U} \quad<10 \quad$ U $Q \quad$ ug/L

$<10 \quad \mathrm{U} \quad<10 \quad$ JU $Q \quad$ ug $/ \mathrm{L}$

$<5 \quad \mathrm{U} \quad * \quad . \quad \mathrm{ug} / \mathrm{L}$

$<25 \quad$ U $\quad<25 \quad$ JU Q $\quad$ ug/L

$<10 \quad \mathrm{U} \quad<10 \quad$ JU $Q \quad$ ug/L

$<10$ JU $Q \quad$ ug/L

$<10 \quad$ U $\quad u g / L$

$<10 \quad \mathrm{U} \quad \mathrm{ug} / \mathrm{L}$

$<10 \quad \mathrm{U} \quad<10 \quad$ JU $Q \quad$ ug/L

20 U ug/L

$<10 \quad \mathrm{U} \quad \mathrm{ug} / \mathrm{L}$

$<10 \quad \mathrm{U} \quad$ ug/L

$<10 \quad \mathrm{U} \quad<10 \quad$ JU $\mathrm{Q} \quad$ ug/L

$<10 \quad \mathrm{U} \quad<10 \quad \pi \mathrm{Q} Q \quad \mathrm{ug} / \mathrm{L}$

$<10 \quad \mathrm{U} \quad<10 \quad \pi \quad \mathrm{Q} \quad \mathrm{ug} / \mathrm{L}$

$<10 \quad \mathrm{U} \quad<10 \quad$ JU $\mathrm{Q} \quad$ ug $/ \mathrm{L}$

$<25 \quad \mathrm{U} \quad<25$ JU $\mathrm{Q} \quad \mathrm{ug} / \mathrm{L}$

$<50$ U ug/L

$<10 \quad \mathrm{U} \quad \mathrm{ug} / \mathrm{L}$

$<10 \quad \mathrm{U} \quad \mathrm{ug} / \mathrm{L}$

$<10 \quad \mathrm{U} \quad<10 \quad$ JU $Q \quad$ ug/L

$<10 \quad \mathrm{U} \quad<10 \quad$ JU $\mathrm{Q} \quad \mathrm{ug} / \mathrm{L}$

$<20$ U ug/L

$<500 \quad \mathrm{u} \quad \mathrm{ug} / \mathrm{L}$

$<10 \quad \mathrm{U} \quad$ ug/L

$\mathrm{ug} / \mathrm{L}$

$\mathrm{ug} / \mathrm{L}$

$\mathrm{ug} / \mathrm{L}$

ug $/ \mathrm{L}$

$13 \quad J \quad E \quad<200 \quad \mathrm{U} \quad<200 \quad \mathrm{U} \quad \mathrm{ug} / \mathrm{L}$

$<25 \quad \mathrm{U} \quad<25 \quad$ JU $\mathrm{Q} \quad \mathrm{ug} / \mathrm{L}$

$<10 \quad \mathrm{U}, \quad<10$ JU $\mathrm{Q} \quad \mathrm{ug} / \mathrm{L}$

$<100 \mathrm{U}+\mathrm{ug} / \mathrm{L}$

$<10 \quad \mathrm{U} \quad \mathrm{ug} / \mathrm{L}$

Note: Concentrations in bold exceed the Driaking Water Standards listed in Appendix A. Units are for all four quarters. 
WELL: XSB 2D

ANALYTICAL DATA

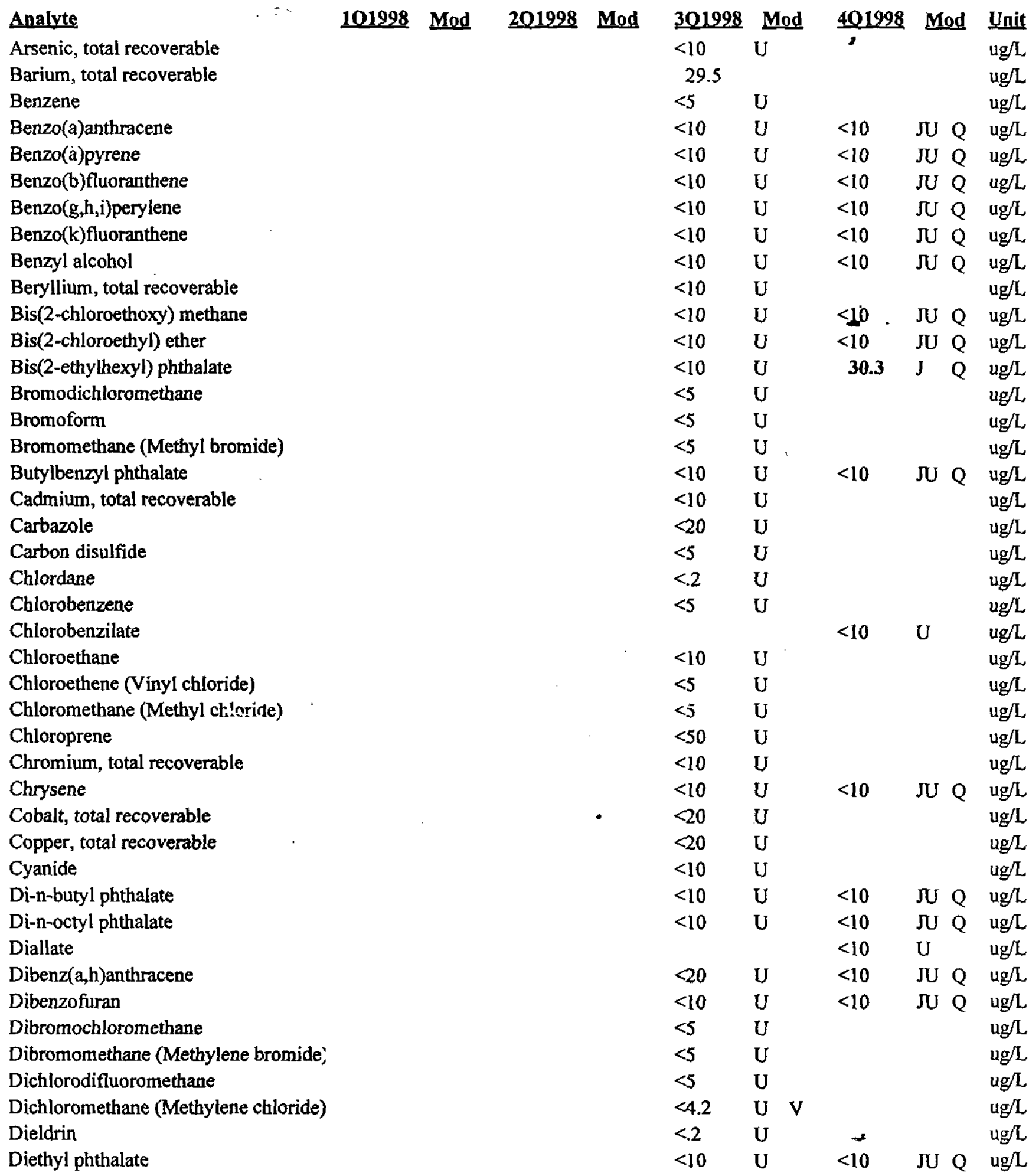

Note: Concentrations in bold exceed the Drinking Water Standards listed in Appendix A. Units are for all four quarters. 
WELL: XSB 2D

ANALYTICAL DATA

Analyte

Dimethoate

Dimethyl phthalate

Diphenylamine

Disulfoton

Endosulfan I

Endosulfan II

Endosulfan sulfate

Endrin

Endrin aldehyde

Ethyl methacrylate

Ethyl methanesulfonate

Ethylbenzene

Fluoranthene

Fluorene

Heptachlor

Heptachlor epoxide

Hexachlorobenzene

Hexachlorobutadiene

Hexachlorocyclopentadiene

Hexachlorodibenzo-p-dioxins

Hexachlorodibenzo-p-furans

Hexachloroethane

Indeno(1,2,3-c,d)pyrene

Iodomethane (Methyl iodide)

Iron, totai recoverable

Isobuiyl alcoliol

Isodrin

Isophorone

Isosafrole

Kepone

Lindane

Methacrylonitrile

Methapyrilene

Methoxychlor

Methyl ethyl ketone

Methyl isobutyl ketone

Methyl methacrylate

Methyl methanesulfonate

$\mathrm{N}$-Nitrosodi-n-butylamine

$\mathrm{N}$-Nitrosodiethylamine

$\mathrm{N}$-Nitrosodimethylamine

N-Nitrosodiphenylamine

N-Nitrosodipropylamine
101998 Mod 201998 Mod 301998 Mod 401998 Mod Unit

\begin{tabular}{|c|c|c|c|c|c|c|c|c|}
\hline & & & & & $<10$ & U & & $\mathrm{ug} / \mathrm{L}$ \\
\hline & & & $<10$ & $\mathrm{U}$ & $<10$ & JU & $\mathrm{Q}$ & $\mathrm{ug} / \mathrm{L}$ \\
\hline & & & & & $<10$ & U & & $\mathrm{ug} / \mathrm{L}$ \\
\hline & & & & & $<10$ & $U$ & & $u g / L$ \\
\hline & & & $<.1$ & $\mathrm{U}$ & & & & $\mathrm{ug} / \mathrm{L}$ \\
\hline & & & $<.2$ & $U$ & & & & $\mathrm{ug} / \mathrm{L}$ \\
\hline & & & $<.2$ & $\mathrm{U}$ & & & & $\mathrm{ug} / \mathrm{L}$ \\
\hline & & & $<.2$ & $\mathrm{U}$ & & & & $\mathrm{ug} / \mathrm{L}$ \\
\hline & & & $<.2$ & $U$ & & & & $u g / L$ \\
\hline & & & $<5$ & $\mathrm{U}$ & & & & $\mathrm{ug} / \mathrm{L}$ \\
\hline & & & & & $<10$ & $\mathrm{U}$ & & ugh \\
\hline & & & $<5$ & $\mathrm{U}$ & & & & $\mathrm{ug} / \mathrm{L}$ \\
\hline & & & $<10$ & $\mathrm{U}$ & $<10$ & $\mathrm{JU}$ & $Q$ & ug/L \\
\hline & & & $<10$ & $\mathrm{U}$ & $<10$ & $\pi$ & $Q$ & $\mathrm{ug} / \mathrm{L}$ \\
\hline & & & $<.1$ & $\mathrm{U}$ & & & & $\mathrm{ug} / \mathrm{L}$ \\
\hline & & & $<.1$ & $\mathrm{U}$ & & & & $\mathrm{ug} / \mathrm{L}$ \\
\hline & & & $<10$ & $\mathrm{U}$ & $<10$ & $\mathrm{JU}$ & $\mathrm{Q}$ & $\mathrm{ug} / \mathrm{L}$ \\
\hline & & & $<20$ & $\mathrm{U}$ & $<10$ & $\mathrm{JU}$ & $\mathbf{Q}$ & ug/L \\
\hline & & & $<10$ & $\mathrm{U}$ & $<10$ & $\mathrm{JU}$ & $\mathrm{Q}$ & ug $/ \mathrm{L}$ \\
\hline & & & $<.00083$ & $U$ & & & & $\mathrm{ug} / \mathrm{L}$ \\
\hline & & & $<.00068$ & $U$ & & & & ug/L \\
\hline & & & $<10$ & $U$ & $<10$ & JU & $\mathrm{Q}$ & $\mathrm{ug} / \mathrm{L}$ \\
\hline & & & $<10$ & $U$ & $<10$ & $\mathrm{JU}$ & $Q$ & $\mathrm{ug} / \mathrm{L}$ \\
\hline & & & $<5$ & $\mathrm{U}$ & & & & $\mathrm{ug} / \mathrm{L}$ \\
\hline 3 & $\mathbf{J}$ & $\mathrm{E}$ & 83.7 & $J \quad E$ & $<200$ & $U$ & & $\mathrm{ug} / \mathrm{L}$ \\
\hline & & & $<1500$ & $\mathrm{U}$ & & & & ug $/$ \\
\hline & & & & & $<10$ & $\mathrm{U}$ & & $\mathrm{ug} / \mathrm{L}$ \\
\hline & & & $<20$ & $\mathrm{U}$ & $<10$ & J & $Q$ & $\mathrm{ug} / \mathrm{L}$ \\
\hline & & & & & $<10$ & $\mathrm{U}$ & & $u g / L$ \\
\hline & & & & & $<10$ & $U$ & & $\mathrm{ug} / \mathrm{L}$ \\
\hline & & & $<.1$ & $\mathrm{U}$ & & & & $\mathrm{ug} / \mathrm{L}$ \\
\hline & & & $<500$ & $\mathrm{U}$ & & & & $\mathrm{ug} / \mathrm{L}$ \\
\hline & & & & & $<10$ & $\mathrm{U}$ & & $\mathrm{ug} / \mathrm{L}$ \\
\hline & & & $<1$ & $\mathrm{U}$ & & & & $\mathrm{ug} / \mathrm{L}$ \\
\hline & & & $<10$ & $\mathrm{U}$ & & & & $\mathrm{ug} / \mathrm{L}$ \\
\hline & & & $<5$ & $\mathrm{U}$ & & & & $u g / L$ \\
\hline & & & $<50$ & $U$ & & & & $\mathrm{ug} / \mathrm{L}$ \\
\hline & & & & & $<10$ & $U$ & & $\mathrm{ug} / \mathrm{L}$ \\
\hline & & & & & $<10$ & $\mathbf{U}$ & & $\mathrm{ug} / \mathrm{L}$ \\
\hline & & & & & $<10$ & $U$ & & $u g / L$ \\
\hline & & & $<20$ & U & $<25$ & $\mathrm{JU}$ & $Q$ & $\lg / \mathrm{L}$ \\
\hline & & & $<10$ & $\mathrm{U}$ & $<10$ & $\pi U$ & $Q$ & $u g / L$ \\
\hline & & & $<10$ & $\mathrm{U}$ & $<10$ & $\mathrm{JU}$ & $\mathrm{Q}$ & /L \\
\hline
\end{tabular}

Note: Concentrations in bold exceed the Drinking Water Standards listed in Appendix A. Units are for all four querters. 
WELL: XSB 2D

ANALYTICAL DATA

\begin{tabular}{|c|c|c|c|c|c|c|c|c|c|}
\hline Analyte & 101998 & 201998 & Mod & 301998 & Mod & 401998 & $\mathbf{M}$ & od & Unit \\
\hline N-Nitrosomethylethylamine & & & & & & $<10$ & $\mathrm{U}$ & & ug/L \\
\hline N-Nitrosomorpholine & & & & . & & $<10$ & $U$ & & ug/L \\
\hline N-Nitrosopiperidine & & & & & & $<10$ & $\mathrm{U}$ & & $\mathrm{ug} / \mathrm{L}$ \\
\hline N-Nitrosopyrrolidine & & & & & & $<10$ & $\mathrm{U}$ & & $g / L$ \\
\hline Naphthalene & & & & $<20$ & $U$ & $<10$ & $\pi$ & $Q$ & $g / L$ \\
\hline Nickel, total recoverable & & & & $<50$ & $\mathrm{U}$ & & & & $g / L$ \\
\hline Nitrobenzene & & & & $<10$ & $\mathrm{U}$ & $<10$ & JU & $\mathrm{Q}$ & $\mathrm{lg} / \mathrm{L}$ \\
\hline 0,0,0-Triethyl phosphorothioate & & & & & & $<10$ & U & & $g / L$ \\
\hline PCB 1016 & & & & $<2$ & $\mathbf{U}$ & & & & $g / L$ \\
\hline PCB 1221 & & & & $<2$ & $\mathrm{U}$ & & & & $\mathrm{g} / \mathrm{L}$ \\
\hline PCB 1232 & & & & $<1$ & $U$ & $\therefore$ & & & $\mathrm{lg} / \mathrm{L}$ \\
\hline PCB 1242 & & & & $<1$ & $U$ & & & & $g / L$ \\
\hline PCB 1248 & & & & $<1$ & $\mathrm{U}$ & & & & $g / L$ \\
\hline PCB 1254 & & & & $<1$ & $\mathrm{U}$ & & & & $/ \mathrm{L}$ \\
\hline РCB 1260 & & & & $<1$ & $U$ & & & & $g / L$ \\
\hline Parathion & & & & & & $<10$ & $U$ & & $g / L$ \\
\hline Parathion methyl & & & & & & $<10$ & $\mathrm{U}$ & & $g / L$ \\
\hline Pentachlorodibenzo-p-dioxins & & & & $<.00087$ & $U$ & & & & $\sqrt{L}$ \\
\hline Pentachlorodibenzo-p-furans & & & & $<.00076$ & $\mathrm{U}$ & & & & $g / L$ \\
\hline Pentachloroethane & & & & $<200$ & $\mathbf{U}$ & & & & $g / L$ \\
\hline Pentachloronitrobenzene & & & & & & $<10$ & $U$ & & $g / L$ \\
\hline Pentachlorophenol & & & & $<25$ & $U$ & $<25$ & $\pi$ & $Q$ & $g / L$ \\
\hline Phenacetin & & & & & & $<10$ & $U$ & & $g / L$ \\
\hline Phenanthrene & & & & $<10$ & $\mathrm{U}$ & $<10$ & JU & $Q$ & $g / L$ \\
\hline Phenol & & & & $<10$ & $U$ & $<10$ & JU & $\mathrm{Q}$ & $g / L$ \\
\hline Phor ate & & & & & & $<10$ & $\mathrm{U}$ & & g/n. \\
\hline Pronamid & & & & & & $<10$ & U & & $g / L$ \\
\hline Propionitrile & & & & $<500$ & $\mathrm{U}$ & & & & $g / L$ \\
\hline Pyrene & & & & $<10$ & $U$ & $<10$ & JU & $Q$ & $g / L$ \\
\hline Pyridine & & & & & & $<25$ & JU & $Q$ & $g / L$ \\
\hline Safrole & & & & & & $<10$ & $\mathrm{U}$ & & $g / L$ \\
\hline Selenium, total recoverable & & & & $<10$ & $U$ & & & & \\
\hline Silver, total recoverable & & & & $<20$ & $U$ & & & & $g / L$ \\
\hline Styrene & & & & $<5$ & $\mathrm{U}$ & & & & $g / L$ \\
\hline Sulfide & & & & $<1000$ & $\mathrm{U}$ & & & & $\lg / \mathrm{L}$ \\
\hline Sulfotepp & & & & & & $<10$ & $\mathrm{U}$ & & $/ \mathrm{L}$ \\
\hline Tetrachlorodibenzo-p-dioxins & & & & $<.00044$ & $\mathrm{U}$ & & & & \\
\hline Tetrachlorodibenzo-p-furans & & & & $<.00039$ & $\mathrm{U}$ & & & & $g / L$ \\
\hline Thallium, total recoverable & & & & $<10$ & $\mathrm{U}$ & & & & $/ \mathrm{L}$ \\
\hline Thionazin & & & & & & $<10$ & $U$ & & $/ \mathrm{L}$ \\
\hline Tin, total recoverable & & & & $<200$ & $\mathrm{U}$ & & & & \\
\hline Toluene & & & & $<5$ & $\mathbf{U}$ & - & & & \\
\hline Total organic halogens & & & & & & 18.7 & & I & \\
\hline
\end{tabular}

Note: Concentrations in bold exceed the Drinking Water Standards listed in Appendix A. Units are for all four quarters. 
WELL: XSB 2D

ANALYTICAL DATA

Analyte

Toxaphene

Trichlorofluoromethane

Vanadium, total recoverable

Vinyl acetate

Xylenes

Zinc, total recoverable

a,a-Dimethylphenethylamine

alpha-Benzene hexachloride

beta-Benzene hexachloride

cis-1,2-Dichloroethylene

cis-1,3-Dichloropropene

delta-Benzene hexachloride

gamma-Chlordane

m-Nitroaniline

$\mathrm{m} / \mathrm{p}-$ Cresol

o-Cresol (2-Methylphenol)

o-Nitroaniline

o-Toluidine

p,p"-DDD

p,p"-DDE

p,p"-DDT

p-Cresol (4-Methylphenol)

p-Dimethylaminoazobenzene

p-Nitroaniline

p-Phenylenediamine

trans-1,2-Dichloroethylene

trans-1,3-Dichloropropene

trans-1,4-Dichloro-2-butene
101998 Mod 201998 Mod 301998 Mod 401998 Mod Unit

$<1 \quad \mathrm{U}$

$<5 \quad \mathrm{U}$

$<10 \quad U$

$<20 \quad \mathrm{U}$

$<10 \quad \mathrm{U}$

27.4

$<1 \quad$ U

$<1 \quad$ U

$<5 \quad U$

$<5 \quad \mathrm{U}$

$<\mathrm{I} \quad \mathrm{U}$

$<1 \quad$ U

$<25 \quad \mathrm{U}$

$<10 \quad \mathrm{U}$

$<25 \quad \mathrm{U}$

$<.2, \mathrm{U}$

$<2 \quad \mathrm{U}$

$<.2 \quad \mathrm{U}$

$<10 \quad \mathrm{U}$

$<10 \quad \mathbf{U}$

$<5 \quad \mathrm{U}$

$<5 \quad$ U

$<20$ U $\operatorname{ug} / \mathrm{L}$

$\mathrm{ug} / \mathrm{L}$

ug/

ug/L

ug/L

ug/L

$<10 \quad$ U ug/L

$\mathrm{ug} / \mathrm{L}$

ug/L

$<1 \quad$ U ug/L

$\therefore \quad$ ug/L

$\mathrm{ug} / \mathrm{L}$

$\mathrm{ug} / \mathrm{L}$

$<25 \quad$ JU Q ug/L

$<20$ U ug/L

$<10 \quad \pi \mathrm{Q} \quad$ ug/L

$<25$ J $Q \quad$ ug/L

$\operatorname{ug} / \mathrm{L}$

ug/L

ug/L

$<10 \quad$ JU $Q \quad \mathrm{ug} / \mathrm{L}$

$<10$ U ug/L

$<10$ U $Q$ ug/L

$<10 \quad U \quad$ ug/L

$\mathrm{ug} / \mathrm{L}$

ug/L

ug/L
$<10$ U ug/L

Note: Concentrations in bold exceed the Drinking Water Standards listed in Appendix A. Units are for all four quarters. 
WELL: XSB 3A

SRS Coord. Lat/Longitude Screen Zone Elevation Top of Standpipe Top of Casing Casing Pump Screen Zone

N 70915.3 33.210 Deg N E $16901.381 .760 \mathrm{Deg} \mathrm{W}$

SAMPLE DATE - $103.2-87.4 \mathrm{ft} \mathrm{msl}$

$157.3 \mathrm{ft} \mathrm{msl}$

$157.0 \mathrm{ft} \mathrm{msl}$

$4 " \mathrm{STL}$

$S$

Unconfined

FIELD DATA

Analyte

Depth to water

$\mathrm{pH}$

Sp. Conductance

Water temperature

Alkalinity as $\mathrm{CaCO} 3$

Phenolpthalein Alkalinity

Turbidity

Volumes purged

Sampling codes

\section{ANALYTICAL DATA}

Analyte

1,1,1,2-Tetrachloroethane

1,1,1-Trichloroethane

1,1,2,2-Tetrachloroethane

1,1,2-Trichloroethane

1,1-Dichloroethane

1,1-Dichloroethylene

1,2,3-Trichloropropane

1,2,4-Trichlorobenzene

1,2-Dibromo-3-chloropropane

1,2-Dibromoethane

1,2-Dichlorobenzene

1,2-Dichloroethane

1,2-Dichloropropane

1,3,5-Trinitrobenzene

1,3-Dichlorobenzene

1,3-Dinitrobenzene

1,4-Dichlorobenzene

1,4-Dioxane

1,4-Naphthoquinone

1-Naphthylamine

2;2-Oxybis(1-chloropropane)

2,3,4,6-Tetrachlorophenol

2,3,7,8-TCDD

$2,4,5-T$

2,4,5-TP (Silvex)

2,4,5-Trichlorophenol

2,4,6-Trichlorophenol
03/03/98

$05 / 13 / 98$

\begin{tabular}{|c|c|c|c|c|}
\hline 101998 & 201998 & 301998 & $\underline{401998}$ & Unit \\
\hline 56.54 & 55.9 & 58.57 & 59.9 & ft BTOS \\
\hline 4.7 & 5 & 4.8 & 4.9 & \\
\hline 180 & 120 & 190 & 170 & $\mathrm{uS} / \mathrm{cm}$ \\
\hline 21.4 & 16 & 21.9 & 21.2 & deg. C \\
\hline 4 & 1 & 0 & 0 & $\mathrm{mg} / \mathrm{L}$ \\
\hline 0 & 0 & 0 & 0 & $\mathrm{mg} / \mathrm{L}$ \\
\hline .4 & 1.1 & .7 & & NTU \\
\hline 3.73511 & 7.56632 & 2.62588 & 5.81468 & gallons \\
\hline
\end{tabular}

$12 / 03 / 98$

$08 / 06 / 98$

\section{Mod 201998 Mod}

\section{Mod}

$<25 \quad \mathrm{U}$

$<.462 \mathrm{U} \quad<.462 \quad \mathrm{U}$

$<25$

$\mathrm{U}$

401998 Mod

Unit

$<25$

U

$<1$

ug/L

$<25 \quad U$

$<25 \quad \mathrm{U}$

$<25 \quad \mathrm{U}$

$<25 \quad \mathrm{U}$

$-10 \quad U$

$<25 \quad \mathrm{U}$

$<25 \quad \mathrm{U}$

$<25$ U

$<25 \quad \mathrm{U}$

$<25 \quad \mathrm{U}$

$<25 \quad U$

$<10$

U

$\mathrm{ug} / \mathrm{L}$

ug $/ \mathrm{L}$

ug/L

ug/L

ug/L

$\mathrm{ug} / \mathrm{L}$

$\mathrm{ug} / \mathrm{L}$

$\mathrm{ug} / \mathrm{L}$

ug $/ \mathrm{L}$

ug $/$

ug/L

ug/L

ug/L

$u g / L$

$<10 \quad \mathrm{U} \quad \mathrm{ug} / \mathrm{L}$

$<25 \quad \mathrm{U}$

ug/L

$<5000 \mathrm{U}$

$\mathrm{ug} / \mathrm{L}$

$<10 \quad \mathrm{U} \quad \mathrm{ug} / \mathrm{L}$

$<10$ U $\quad$ ug/L

$<10 \quad \mathrm{U} \quad<10 \quad$ JU $Q \quad$ ug/L

$<10$ U ug/

$<.00042 \mathrm{U}$

ug/L

$<.2$ UJ O

$\mathrm{ug} / \mathrm{L}$

$<.2$ UJ O

$<10 \quad U$

$<25 \mathrm{U}$

$<10 \pi \quad$ Q $u g / L$

$\mathrm{ug} / \mathrm{L}$

$<25$ JU $Q \quad$ ug/L

Note: Concentrations in bold exceed the Drinking Water Standards listed in Appendix A. Units are for all four quarters. 
WELL: XSB 3A

ANALYTICAL DATA

Analyte

2,4-Dichlorophenol

2,4-Dichlorophenoxyacetic acid

2,4-Dimethyl phenol

2,4-Dinitrophenol

2,4-Dinitrotoluene

2,6-Dichlorophenol

2,6-Dinitrotoluene

2-Acetylaminofluorene

2-Chloronaphthalene

2-Chlorophenol

2-Hexanone

2-Methyl-4,6-dinitrophenol

2-Methylnaphthalene

2-Naphthylamine

2-Nitrophenol

2-Picoline

2-sec-Butyl-4,6-dinitrophenol

3,3"-Dichlorobenzidine

3,3"-Dimethylbenzidine

3-Methylcholanthrene

4-Aminobiphenyl

4-Bromophenyl phenyl ether

4-Chloro-m-cresol

4-Chloroaniline

4-Chlorophenyl phenyl ether

4-Nitrophenui

4-Nitroquinoline-1-oxide

5-Nitro-0-toluidine

7,12-Dimethylbenz(a)anthracene

Acenaphthene

Acenaphthylene

Acetone

Acetonitrile (Methyl cyanide)

Acetophenone

Acrolein

Acrylonitrile

Aldrin

Allyl chloride

Aluminum, total recoverable

Aniline

Anthracene

Antimony, total recoverable

Aramite
101998 Mod 201998 Mod 301998 Mod 401998 Mod Unit

$<\mathrm{l} 0 \quad \mathrm{U} \quad<\mathrm{HO} \quad \mathrm{JU} \mathrm{Q} \quad \mathrm{ug} / \mathrm{L}$

$<.2$ UJ $\mathrm{U}$ ug/L

$<10$ U $\quad<10 \quad$ JU $Q \quad$ ug/L

$<25$ U $<25$ JU Q ugh

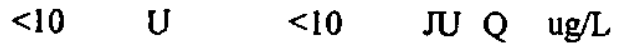

$<10 \quad \mathrm{u} \quad \mathrm{ug} / \mathrm{L}$

$<10 \quad U \quad<10$ " $\quad Q \quad$ ug $/ \mathrm{L}$

$<10 \quad \mathrm{U} \quad \mathrm{ug} / \mathrm{L}$

$<10 \quad \mathrm{U} \quad<10 \quad \mathrm{JU}$ Q $\quad$ ug/L

$<10 \quad \mathrm{U} \quad<10 \quad J U \mathrm{Q} \quad \mathrm{ug} / \mathrm{L}$

$<25 \quad \mathrm{U} \quad \therefore . \quad \mathrm{ug} / \mathrm{L}$

$<25 \quad \mathrm{U} \quad<25$ JU $Q \quad$ ug/L

$<10 \quad \mathrm{U} \quad<10 \quad \mathrm{JU} \mathrm{Q}$ ug/L

$<10 \quad \mathrm{U} \quad \mathrm{ug} / \mathrm{L}$

$\begin{array}{llllll}<10 & \mathrm{U} & <10 & \text { JU } & \mathrm{Q} & \mathrm{ug} / \mathrm{L}\end{array}$

$<$ I0 U ug/L

$<10$ U ug/L

$<10 \quad \mathrm{U} \quad<10 \quad \mathrm{JU} \mathrm{Q} \quad \mathrm{ug} / \mathrm{L}$

$<20$ U ug/L

$<10$ U ug/L

$<10$ U ug/L

$<10 \quad \mathrm{U} \quad<10 \quad$ JU $Q \quad$ ug/L

$<10 \quad \mathrm{U} \quad<10 \quad \mathrm{U} Q \mathrm{Q} / \mathrm{L}$

$<10$ U $<10$ JU Q ug/L

$<10 \quad U \quad<10 \quad$ JU $Q \quad$ ug/L

$<25$ U $<25$ JU Q ug/L

$<50$ U ug/L

$<10$ U ug/L

$<10 \quad$ U $\quad u g / L$

$<10 \quad \mathrm{U} \quad<10 \quad$ JU $Q \quad$ ug/L

$<10 \quad \mathrm{U} \quad<10 \quad \mathrm{JU} \mathrm{Q} \quad \mathrm{ug} / \mathrm{L}$

$<100 \quad \mathrm{u} \quad \mathrm{ug} / \mathrm{L}$

$<2500$ U ug/L

$<10$ U . ug/

$<250$ U ug/L

$<250$ U ug/L

$<.1 \quad U \quad u g / L$

$<50$ U ug/L

$\begin{array}{llllll}58.8 & <200 & \mathrm{U} & <200 & \mathrm{U} & \mathrm{ug} / \mathrm{L}\end{array}$

$<25 \quad U \quad<25 \quad$ U $Q \quad u g / L$

$<10$ U $<10 \quad \pi \quad Q \quad$ ug $/ \mathrm{L}$

$<100 \quad \mathrm{O} \quad \mathrm{ug} / \mathrm{L}$

$<10 \quad \dot{U} \quad$ ug/L

Note: Concentrations in bold exced the Drinking Water Standards listed in Appendix A. Units are for all four quarters. 
WELL: XSB 3A

ANALYTICAL DATA

\begin{tabular}{|c|c|c|c|c|c|c|c|c|c|}
\hline Analyte & 101998 & Mod & $\underline{201998}$ & Mod & 301998 & $\underline{\text { Mod }}$ & $\underline{401998}$ & Mod & Unit \\
\hline Arsenic, total recoverable & & & & & $<10$ & $\mathrm{U}$ & & & $u g / L$ \\
\hline Barium, total recoverable & & & & & 59.5 & & 55.5 & & $\mathrm{ug} / \mathrm{L}$ \\
\hline Benzene & & & & & $<25$ & $\mathrm{U}$ & & & $\mathrm{ug} / \mathrm{L}$ \\
\hline Benzo(a)anthracene & & & & & & & $<10$ & JU C & ug/L \\
\hline Benzo(a)pyrene & & & & & $<10^{\circ}$ & $\mathrm{U}$ & $<10$ & JU C & ug/L \\
\hline Benzo(b)fluoranthene & & & & & $<10$ & $\mathrm{U}$ & $<10$ & JU C & $\lg / \mathrm{L}$ \\
\hline Benzo $(g, h, i)$ perylene & & & & & $<10$ & $\mathrm{U}$ & $<10$ & JU $\mathrm{C}$ & $\lg / \mathrm{L}$ \\
\hline Benzo(k)fluoranthene & & & & & $<10$ & $\mathbf{U}$ & $<10$ & JU $Q$ & $\mathrm{ug} / \mathrm{L}$ \\
\hline Benzoic acid & & & & & $<25$ & $\mathrm{U}$ & & & $/ \mathrm{L}$ \\
\hline Benzyl alcohol & & & & & & & $<10$ & JU Q & ugl \\
\hline Beryllium, total recoverable & & & & & $<10$ & $\mathrm{U}$ & $\therefore$ & & $\mathrm{ug} / \mathrm{L}$ \\
\hline Bis(2-chloroethoxy) methane & & & & & $<10$ & $\mathrm{U}$ & $<10$ & JU Q & $\mathrm{ug} / \mathrm{L}$ \\
\hline Bis(2-chloroethyl) ether & & & & & $<10$ & $\mathrm{U}$ & $<10$ & JU Q & $\mathrm{ug} / \mathrm{L}$ \\
\hline Bis(2-ethylhexyl) phthalate & & & & & 2.63 & $\mathbf{J} \quad \mathrm{E}$ & $<10$ & JU Q & $\mathrm{ug} / \mathrm{L}$ \\
\hline Bromodichloromethane & & & & & $<25$ & $\mathrm{U}$ & & & $\mathrm{Lg} / \mathrm{L}$ \\
\hline Bromoform & & & & & $<25$ & $\mathrm{U}$ & & & $\mathrm{ug} / \mathrm{L}$ \\
\hline Bromomethane (Methyl bromide) & & & & & $<25$ & $\mathrm{U}$ & & & $u g / L$ \\
\hline Butylbenzyl phthalate & & & & & $<10$ & U & $<10$ & JU Q & $\mathrm{ug} / \mathrm{L}$ \\
\hline Cadmium, total recoverable & & & & & $<10$ & $\mathrm{U}$ & & & $\mathrm{ug} / \mathrm{L}$ \\
\hline Carbazole & & & & & $<0$ & $U$ & & & $u g / L$ \\
\hline Carbon disulfide & & & & & $<25$ & $U$ & & & $u g / L$ \\
\hline Chlorobenzene & & & & & $<25$ & $U$ & & & $u g / L$ \\
\hline Chlorobenzilate & & & & & & & $<10$ & $u$ & $u g / L$ \\
\hline Chloroethane & & & & & -50 & $U$ & $\ldots$ & & a \\
\hline Chloroethene (Vinyl chloride) & & & & & $<25$ & $U$ & & & $\mathrm{ug}$ \\
\hline Chloromethane (Methyl chloride) & & & & . & $<25$ & $\mathrm{U}$ & & & $\operatorname{ug} / \mathrm{L}$ \\
\hline Chloroprene & & & & & $<250$ & $\mathrm{U}$ & & & $\mathrm{ug} / \mathrm{L}$ \\
\hline Chromium, total recoverable & & & & & 3.96 & J E & & & ug \\
\hline Chrysene & & & & & $<10$ & $\mathrm{U}$ & $<10$ & JU $\mathrm{C}$ & $\mathrm{ug}$ \\
\hline Cobalt, total recoverable & & & & & 8.98 & J E & & & \\
\hline Copper, total recoverable & & & & & 88 & & & & $\mathrm{ug} / \mathrm{L}$ \\
\hline Cyanide & & & & & $<10$ & $\mathrm{U}$ & & & ug \\
\hline Di-n-butyl phthalate & & & . & & $<10$ & $\mathrm{U}$ & $<10$ & JU Q & $\mathrm{ug} / \mathrm{L}$ \\
\hline Di-n-octyl phthalate & & & & & $<10$ & $\mathrm{U}$ & $<10$ & JU Q & UgLL \\
\hline Diallate & & & & & & & $<10$ & & ug/L \\
\hline Dibenz(a,h)anthracene & & & & & $<20$ & $\mathrm{U}$ & $<10$ & $J U Q$ & ug \\
\hline Dibenzofuran & & & & & $<10$ & $\mathrm{U}$ & $<10$ & JU 0 & ug \\
\hline Dibromochloromethane & & & & & $<25$ & $\mathrm{U}$ & & & \\
\hline Dibromomethane (Methylene bromide' & & & & & $<25$ & $\mathrm{U}$ & & & \\
\hline Dichlorodifluoromethane & & & & & $<25$ & $\mathrm{U}$ & & & ug \\
\hline Dichloromethane (Methylene chloride) & & & & & $<50$ & $\mathbf{U}$ & & & $a_{y}$ \\
\hline Dieldrin & & & & & $<.2$ & $\mathrm{U}$ & $\neq$ & & $\mathrm{ug} / \mathrm{L}$ \\
\hline Diethyl phthalate & & & & & $<10$ & $\mathrm{U}$ & $<10$ & JU $Q$ & \\
\hline
\end{tabular}

Note: Concentrations in bold exceed the Drinking Water Standards listed in Appendix A. Units are for all four quarters. 
WELL: XSB 3A

ANALYTICAL DATA

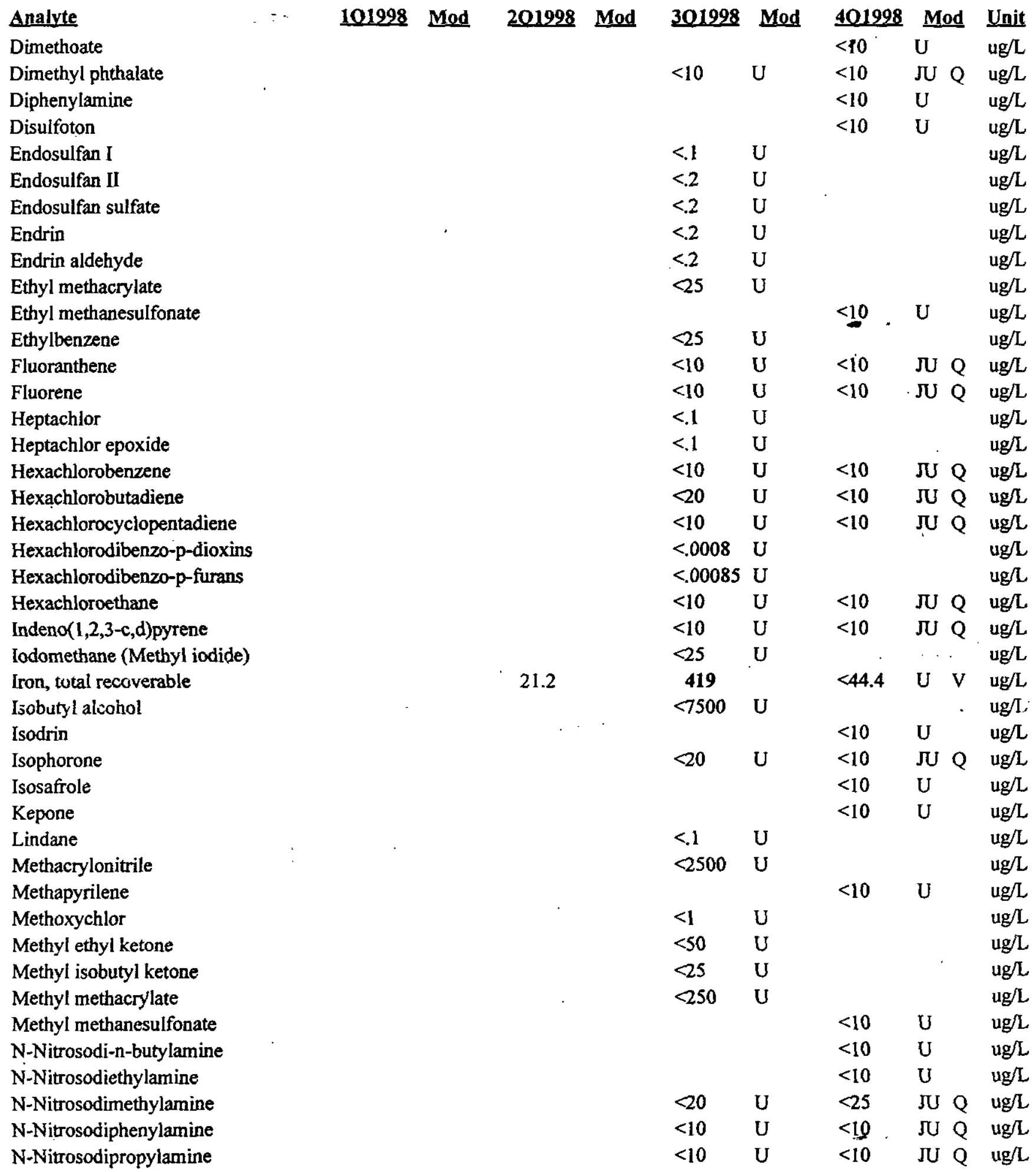

Note: Concentrations in bold exceed the Drinking Water Standards listed in Appendix A. Units are for all four quarters. 
WSRC-RP-99-4003

Unclassified

WELL: XSB 3A

ANALYTICAL DATA

Analyte

101998 Mod 201998 Mod 301998 Mod 401998 Mod Unit

N-Nitrosomethylethylamine

$\mathrm{N}$-Nitrosomorpholine

$\mathrm{N}$-Nitrosopiperidine

N-Nitrosopyrrolidine

Naphthalene

Nickel, total recoverable

Nitrobenzene

O,O,O-Triethyl phosphorothioate

PCB 1016

PCB 1221

PCB 1232

PCB 1242

PCB 1248

PCB 1254

PCB 1260

Parathion

Parathion methyl

Pentachlorodibenzo-p-dioxins

Pentachlorodibenzo-p-furans

Pentachloroethane

Pentachloronitrobenzene

Pentachlorophenol

Phenacetin

Phenanthrene

Phenol

Phorate

Pronamid

Propionitrile

Pyrene

Pyridine

Safrole

Selenium, total recoverable

Silver, total recoverable

Styrene

Sulfide

Sulfotepp

Tetrachlorodibenzo-p-dioxins

Tetrachlorodibenzo-p-furans

Thallium, total recoverable

Thionazin

Tin, total recoverable

Toluene

$<.0011 \mathrm{U}$

$<.00084 \mathrm{U}$

$<1000$ U ug/L

$<10$ U

ug/L

$<10$ U ug/L

$<10$ U ug/L

$<10$ U ug/L

$<20 \quad \mathrm{U}$

$<10$

JU

$u g / L$

$<50 \quad \mathrm{U}$

$<10 \quad \mathrm{U}$

ug/L

$<10 \quad \pi \mathrm{Q} Q \mathrm{ug} / \mathrm{L}$

$<10$ U ug/L

$<2 \quad \mathrm{U}$

$\mathrm{ug} / \mathrm{L}$

$<2 \quad U$

$u g / L$

$<1 \quad$ U

$<1 \quad \mathrm{U}$

$<1 \quad$ U

$<1 \quad$ U

$<1 \quad \mathrm{U}$

ug/L

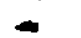

$\mathrm{ug} / \mathrm{L}$

ug/L

$\mathrm{ug} / \mathrm{L}$

ug/L

$<10$ U ug/L

$<10 \quad \mathrm{U} \quad \mathrm{ug} / \mathrm{L}$

ug/L

ug/L

$<10 \quad U \quad$ ug $/ \mathrm{L}$

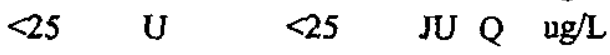

$<10 \quad \mathrm{U} \quad \mathrm{ug} / \mathrm{L}$

$<10 \quad$ U $\quad<10 \quad$ JU $Q \quad u g / L$

$<10 \quad \mathrm{U} \quad<10 \quad$ JU $Q \quad$ ug/L

$<10 \quad$ U $\quad u g / L$

$<10 \quad \mathrm{u} \quad \mathrm{ug} / \mathrm{L}$

$<2500 \quad \mathrm{u} \quad \mathrm{ug} / \mathrm{L}$

$\begin{array}{llllll}<10 & \mathrm{U} & <10 & \mathrm{JU} & \mathrm{Q} & \mathrm{ug} / \mathrm{L}\end{array}$

$<25 \quad$ JU $Q \quad$ ug/L

$<10 \quad \mathrm{U} \quad \mathrm{ug} / \mathrm{L}$

$<10 \quad \mathrm{U} \quad \mathrm{ug} / \mathrm{L}$

$<20 \quad \mathrm{u} \quad \mathrm{ug} / \mathrm{L}$

$<25$ U ug/L

$<1000 \mathrm{U}$ ug/L

$<10 \quad \mathrm{U}$

$<.00042 \mathrm{U} \quad \mathrm{ug} / \mathrm{L}$

$<.00046 \mathrm{U} \quad \mathrm{ug} / \mathrm{L}$

$<10 \quad \mathrm{U} \quad u g / \mathrm{L}$

$<10 \quad \mathrm{U} \quad$ ug/L

$<200 \mathrm{U}$ ug/L

$<25$ U $\approx-\quad u g / L$

Total organic halogens

$\begin{array}{llll}54.7 & \mathrm{~J} & \mathrm{I} & \mathrm{ug} / \mathrm{L}\end{array}$

Note: Concentrations in bold exceed the Drinking Water Standards listed in Appendix A. Units are for aff four quarters. 
WELL: XSB 3A

\section{ANALYTICAL DATA}

\section{Analyte}

\section{Mod 201998 Mod}

\begin{tabular}{|c|c|}
\hline 301998 & $\mathbf{M}$ \\
\hline$<1$ & $\mathrm{U}$ \\
\hline$<25$ & $\mathrm{U}$ \\
\hline$<10$ & U \\
\hline$<100$ & $\mathbf{U}$ \\
\hline$<50$ & $\mathrm{U}$ \\
\hline & \\
\hline
\end{tabular}

401998 Mod Unit

Toxaphene

Trichlorofluoromethane

Vanadium, total recoverable

Vinyl acetate

Xylenes

Zinc, total recoverable

a,a-Dimethylphenethylamine

alpha-Benzene hexachloride

alpha-Chlordane

beta-Benzene hexachloride

cis-1,2-Dichloroethylene

cis-1,3-Dichloropropene

delta-Benzene hexachloride

gamma-Chlordane

m-Nitroaniline

$\mathrm{m} / \mathrm{p}$-Cresol

o-Cresol (2-Methylphenol)

o-Nitroaniline

o-Toluidine

p,p"-DDD

$p, p "-D D E$

p,p"-DDT

p-Cresol (4-Methylphenol)

p-Dimethylaminoazobenzene

p-Nitroaniline

$13.6 \mathrm{~J}$

$<.1 \quad \mathrm{U}$

$<.1 \quad \mathrm{U}$

$<.1 \quad \mathrm{U}$

$<25 \quad \mathrm{U}$

$<25 \quad U$

$<, \mathrm{U} \quad \mathrm{U}$

$<.1 \quad U$

$<25 \quad$ U

$<10 \quad$

$<25 \quad$ U

$<.2 \quad \mathrm{U}$

$<.2 \quad \mathrm{U}$

$<.2 \quad \mathrm{U}$

$<10 \quad \mathrm{U}$

p-Phenylenediamine

trans-1,2-Dichloroethylene

trans-1,3-Dichloropropene

trans-1,4-Dichloro-2-butene

$\begin{array}{lllll}<10 & \mathrm{U} & <10 & \mathrm{U} & \mathrm{ug} / \mathrm{L} \\ & & <10 & \mathrm{JU}_{\mathrm{Q}} \mathrm{Q} & \mathrm{ug} / \mathrm{L} \\ <25 & \mathrm{U} & & \mathrm{U} & \mathrm{ug} / \mathrm{L} \\ <25 & \mathrm{U} & & & \mathrm{ug} / \mathrm{L} \\ <100 & \mathrm{U} & & & \mathrm{ug} / \mathrm{L} \\ & & & & \mathrm{ug} / \mathrm{L}\end{array}$

Note: Concentrations in bold exceed the Drinking Water Standards listed in Appendix A. Units are for all four quarters. 
WSRC-RP-99-4003

Unclassified

WELL: XSB 4D

SRS Coord. Lat/Longitude Screen Zone Elevation Top of Standpipe Top of Casing Casing Pump Screen Zone N $70997.9 \quad 33.210$ Deg N . : $103.9-83.9$ ft msl E 16826.281 .760 Deg W

$155.2 \mathrm{ft} \mathrm{msl}$

$05 / 12 / 98$

03/03/98

SAMPLE DATE

FIELD DATA

\section{Analyte}

Depth to water

$\mathrm{pH}$

Sp. Conductance

Water temperature

Alkalinity as $\mathrm{CaCO} 3$

Phenolpthalein Alkalinity

Turbidity

Volumes purged

Sampling codes

\section{ANALYTICAL DATA}

\section{Analyte}

1,1,1,2-Tetrachloroethane

1,1,1-Trichloroethane

1,1,2,2-Tetrachloroethane

1,1,2-Trichloroethane

1,1-Dichloroethane

1,1-Dichloroethylene

1,2,3-Trichloropropane

1,2,4-Trichlorobenzene

1,2-Dibromo-3-chloropropane

1,2-Dibromoethane

1,2-Dichlorobenzene

1,2-Dichloroethane

1,2-Dichloropropane

1,3,5-Trinitrobenzene

1,3-Dichlorobenzene

1,3-Dinitrobenzene

1,4-Dichlorobenzene

1,4-Dioxane

1,4-Naphthoquinone

I-Naphthylamine

2,2-Oxybis(1-chloropropane)

2,3,4,6-Tetrachlorophenol

2,3,7,8-TCDD

2,4,5-T

2,4,5-TP (Silvex)

2,4,5-Trichlorophenol

2,4,6-Trichlorophenol
201998

55

5.4

220

18

4

0

2.7

6.09756

101998
55.67
5.4
160
21
10
0
1
3.28147

$154.9 \mathrm{ft} \mathrm{msl}$

$08 / 05 / 98$
$\mathrm{S}$

,

$12 / 03 / 98$

Unconfined

$\underline{301998}$
57.72
5.2
140
22.3
6
0
.7
9.52744

$\begin{array}{ll}\frac{401998}{59.19} & \text { Unit } \\ 5.2 & \text { ft BTOS } \\ 130 & \\ 21.1 & \text { uS/cm } \\ 11 & \text { deg. C } \\ 0 & \mathrm{mg} / \mathrm{L} \\ 1.2 & \mathrm{mg} / \mathrm{L} \\ 6.84104 & \text { NTU } \\ & \text { gallons }\end{array}$

101998 Mod

$<.462 \quad \mathrm{U}$

201998 Mod

$\begin{array}{ll}301998 & M \\ <5 & \mathrm{U}\end{array}$

$\begin{array}{ll}<5 & \mathrm{U} \\ <5 & \mathrm{U}\end{array}$

$<5 \quad \mathrm{U}$

$<5 \quad U$

$<5 \quad U$

$<5 \quad U$

$<10 \quad \mathrm{U}$

$<5$.U

$<5 \quad \mathrm{U}$

$<5 \quad \mathrm{U}$

$<5 \quad \mathrm{U}$

$<5$

$<5 \quad \mathrm{U}$

$<5 \quad \mathrm{U}$

$<1000$

$\mathrm{U}$

$<10 \quad U$

$<.00048 \mathrm{U}$

$<.2$ UJ $\mathrm{O}$

$<.2$ UJ $\mathrm{O}$

$<10 \quad \mathrm{U}$

$<25$

Mod

401998

Mod

Unit

ug/L

ug/L

$\mathrm{ug} / \mathrm{L}$

$\mathrm{ug} / \mathrm{L}$

$\mathrm{ug} / \mathrm{L}$

$\mathrm{ug} / \mathrm{L}$

$\mathrm{ug} / \mathrm{L}$

$<10 \quad$ JU $Q \quad$ ug/L

$u g / L$

ug/L

$\mathrm{ug} / \mathrm{L}$

$\mathrm{ug} / \mathrm{L}$

ug/L

$<10 \quad U \quad u g / L$

$\mathrm{ug} / \mathrm{L}$

$<10 \quad \mathrm{U} \quad$ ug/L

$\mathrm{ug} / \mathrm{L}$

ug/L

$\begin{array}{lll}<10 & \mathrm{U} & \mathrm{ug} / \mathrm{L} \\ <10 & \mathrm{U} & \mathrm{ug} / \mathrm{L}\end{array}$

$<10$ JU Q ug/L

$<10 \quad$ U ug/L

$\mathrm{ug} / \mathrm{L}$

ug/L

ug/L

$<10$. JU $Q$ ug/L

$<25$ JU Q ug/L

Note: Concentrations in bold exceed the Drinking Water Standards listed in Appendix A. Units are for all four quarters. 
WELL: XSB 4D

\section{ANALYTICAL DATA}

Analyte

2,4-Dichlorophenol

2,4-Dichlorophenoxyacetic acid

2,4-Dimethyl phenol

2,4-Dinitrophenol

2,4-Dinitrotoluene

2,6-Dichlorophenol

2,6-Dinitrotoluene

2-Acetylaminofiuorene

2-Chloronaphthalene

2-Chlorophenol

2-Hexanone

2-Methyl-4,6-dinitrophenol

2-Methylnaphthalene

2-Naphthylamine

2-Nitrophenol

2-Picoline

2-sec-Butyl-4,6-dinitrophenol

3,3"-Dichlorobenzidine

3,3"-Dimethylbenzidine

3-Methylcholanthrene

4-Aminobiphenyl

4-Bromophenyl phenyl ether

4-Chloro-m-cresol

4-Chloroaniline

4-Chlorophenyl phenyl ether

4-Nitrophenol

4-Nitroquinoline-1-oxide

5-Nitro-o-toluidine

7,12-Dimethylbenz(a)anthracene

Acenaphthene

Acenaphthylene

Acetone

Acetonitrile (Methyl cyanide)

Acetophenone

Acrolein

Acrylonitrile

Aldrin

Allyl chloride

Aluminum, total recoverable

Aniline

Anthracene

Antimony, total recoverable

Aramite
101998 Mod 201998 Mod 301998 Mod 401998 Mod Unit

$<10 \quad \mathrm{U} \quad<10$ JU Q ug/L

$<.2$ UJ $O \quad$ ug/L

$<10 \quad \mathrm{U} \quad<10 \quad$ JU $Q \quad \mathrm{ug} / \mathrm{L}$

$<25 \quad$ U $\quad<25$ JU $Q \quad$ ug/L

$<10 \quad \mathrm{U} \quad<10 \quad \mathrm{JU} Q \quad \mathrm{ug} / \mathrm{L}$

$<10$ U ug/

$<10 \quad \mathrm{U} \quad<10 \quad$ JU $\mathrm{Q} \quad \mathrm{ug} / \mathrm{L}$

$<10$ U ug/L

$<10 \quad$ U $\quad<10 \quad$ JU $Q \quad$ ug/L

$<10 \quad \mathrm{U} \quad<10 \quad \mathrm{JU} \quad \mathrm{Q} \quad \mathrm{ug} / \mathrm{L}$

$<5 \quad \mathrm{U} \quad \mathrm{ug} / \mathrm{L}$

$<25 \quad \mathrm{U} \quad<25^{\circ} \mathrm{UU} \quad \mathrm{ug} / \mathrm{L}$

$<10 \quad \mathrm{U} \quad<10 \quad$ JU $Q \quad$ ug/L

$<10$ U ug/L

$\begin{array}{llllll}<10 & \mathrm{U} & <10 & \mathrm{JU} & \mathrm{Q} & \mathrm{ug} / \mathrm{L}\end{array}$

$<10$ U ug/L

$<10$ U ug/L

$<10 \quad$ U $\quad<10 \quad$ JU $Q \quad$ ug/L

$<20$ U ug/L

$<10$ U ug/L

$<10$ U ug/L

$<10 \quad \mathrm{U} \quad<10 \quad$ JU $Q \quad \mathrm{ug} / \mathrm{L}$

$<10 \quad \mathrm{U} \quad<10 \quad$ JU $Q \quad \mathrm{ug} / \mathrm{L}$

$<10 \quad \mathrm{U} \quad<10 \quad$ JU $Q \quad$ ug/L

$<10 \quad \mathrm{U} \quad<10 \quad$ JU $\mathrm{Q}$ ug/L

$<25 \mathrm{U} \quad<25 \cdot \pi \mathrm{Q}$ ug/l.

$<50 \quad \mathrm{u} \quad \mathrm{ug} / \mathrm{L}$

$<10 \quad U \quad$ ug/L

$<10$ U ug/L

$<10 \quad$ U $\quad<10 \quad$ J $Q \quad$ ug/L

$<10$ U $<10$ JU $Q$ ugh

$<2$ U ug/L

$<500$ U ug/L

$<10 \quad \mathrm{U} \quad \mathrm{ug} / \mathrm{L}$

$<50 \quad \mathrm{U} \quad \mathrm{ug} / \mathrm{L}$

$<50 \quad \mathrm{U} \quad \mathrm{ug} / \mathrm{L}$

$<. I \quad$ U ug/L

$<10 \quad \mathrm{U} \quad \mathrm{ug} / \mathrm{L}$

$49.1<200 \quad \mathrm{U} \quad<200 \quad \mathrm{U} \quad \mathrm{ug} / \mathrm{L}$

$<25$ U $<25$ JU $Q \quad$ ug/

$<10 \quad \mathrm{U} \quad<10 \quad$ JU $Q \quad$ ug/L

$<100 \quad \mathrm{*} \quad \mathrm{*} \quad \mathrm{ug} / \mathrm{L}$

$<10$ U ug/L

Note: Concentrations in bold exceed the Drinking Water Standards listed in Appendix A. Units are for all four quarters. 
WELL: XSB 4D

\section{ANALYTICAL DATA}

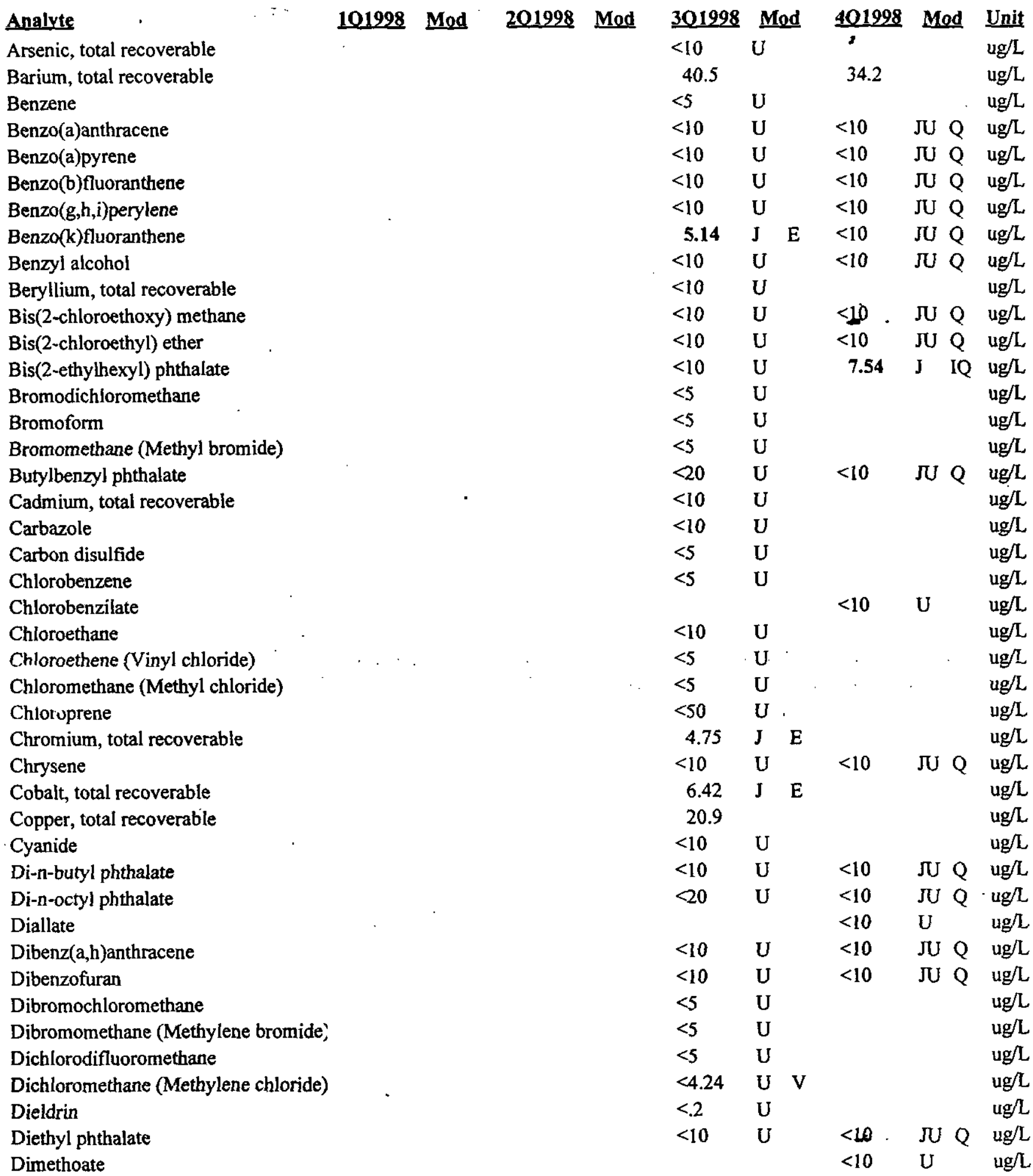

Note: Concentrations in bold exceed the Drinking Water Standards listed in Appendix A. Units are for all four quarters. 
WELL: XSB 4D

ANALYTICAL DATA

\section{Analvte}

Dimethyl phthalate

Diphenylamine

Disulfoton

Endosulfan 1

Endosulfan II

Endosulfan suifate

Endrin

Endrin aldehyde

Ethyl methacrylate

Ethyl methanesulfonate

Ethylbenzene

Fluoranthene

Fluorene

Heptachlor

Heptachlor epoxide

Hexachlorobenzene

Hexachlorobutadiene

Hexachlorocyclopentadiene

Hexachlorodibenzo-p-dioxins

Hexachlorodibenzo-p-furans

Hexachloroethane

Indeno(1,2,3-c,d)pyrene

Iodomethane (Methyl iodide)

Iron, total recoverable

Isobutyl alcohol

Isodrin

Isophorone

Isosafrole

Kepone

Lindane

Methacrylonitrile

Methapyrilene

Methoxychlor

Methyl ethyl ketone

Methyl isobutyl ketone

Methyl methacrylate

Methyl methanesulfonate

$\mathrm{N}$-Nitrosodi-n-butylamine

$\mathrm{N}$-Nitrosodiethylamine

$\mathrm{N}$-Nitrosodimethylamine

N-Nitrosodiphenylamine

$\mathrm{N}$-Nitrosodipropylamine

$\mathrm{N}$-Nitrosomethylethylamine
101998 Mod 201998 Mod

\author{
(a)
}

401998 Mod Unit

$<10$ JU Q ug/L

$<10$ U ug/L

$<10$ U . ug/L

$<.1 \quad \mathrm{U} \quad \mathrm{ug} / \mathrm{L}$

$<.2$ U

$<.2 \quad \mathrm{U}$

$<.2 \quad \mathrm{U}$

$<.2$ U

$<5 \quad U$

$<5 \quad \mathrm{U}$

$<10 \quad$ U

$<10 \quad \mathrm{U}$

$<.1 \quad \mathrm{U}$

$<.1 \quad \mathrm{U}$

$<20$ U

$<10 \quad \mathrm{U}$

$<10 \quad \mathrm{U}$

$<.0015 \mathrm{U}$

$<.0016 \mathrm{U}$

$<10 \quad \mathrm{U}$

$<20$ U

$<5 \quad \mathrm{U}$

91.4

68.2

$<1500 \quad \mathrm{U}$

$<10 \quad \mathrm{U}$

$<10$

$<10$

$<10$

$<10$

$<10$

$<10$

$<10$

$<10$

$<10$

ug $/ \mathrm{L}$

$\mathrm{ug} / \mathrm{L}$

$\mathrm{ug} / \mathrm{L}$

$\mathrm{ug} / \mathrm{L}$

ug/L

U ug/L

$<10$

J $Q$

$\mathrm{ug} / \mathrm{L}$

ug/L

JU $Q \quad u g / L$

$\mathrm{ug} / \mathrm{L}$

$\mathrm{ug} / \mathrm{L}$

U $Q \quad$ ug $L$

$\pi \mathrm{Q} u g / \mathrm{L}$

JU $Q$ ug/L

ug/L

ug/L

$\begin{array}{lll}J U & Q & u g / L \\ \pi & Q & u g / L\end{array}$

$<10$. J $Q$ ug/L

ug/L

$<10.9 \quad$ U $\quad V \quad u g / L$

ug/L

U ug/L

JU $Q \quad u g / L$

U ug/L

$<.1 \quad \mathrm{U}$

$<500 \quad$ U

U

ug/L

ug/L

$\mathrm{ug} / \mathrm{L}$

$<10 \quad \mathrm{ug} / \mathrm{L}$

$<1 \quad$ U $\quad . u g / L$

$<10 \quad \mathrm{U}$

$<5 \quad$ U

$<50 \quad$ U

$<10$

$<10$

$<10$

$<10 \quad \mathrm{U}$

$<20$ U

$<20$ U

$<25$ JU Q ug/L

$<10 \quad$ JU $Q \quad$ ug/L

$<10$. U $Q$ ug/L

$<10$ U ug/L

Note: Concentrations in bold exceed the Drinking Water Standards listed in Appendix A. Units are for all four quarters. 
WELL: XSB 4D

\section{ANALYTICAL DATA}

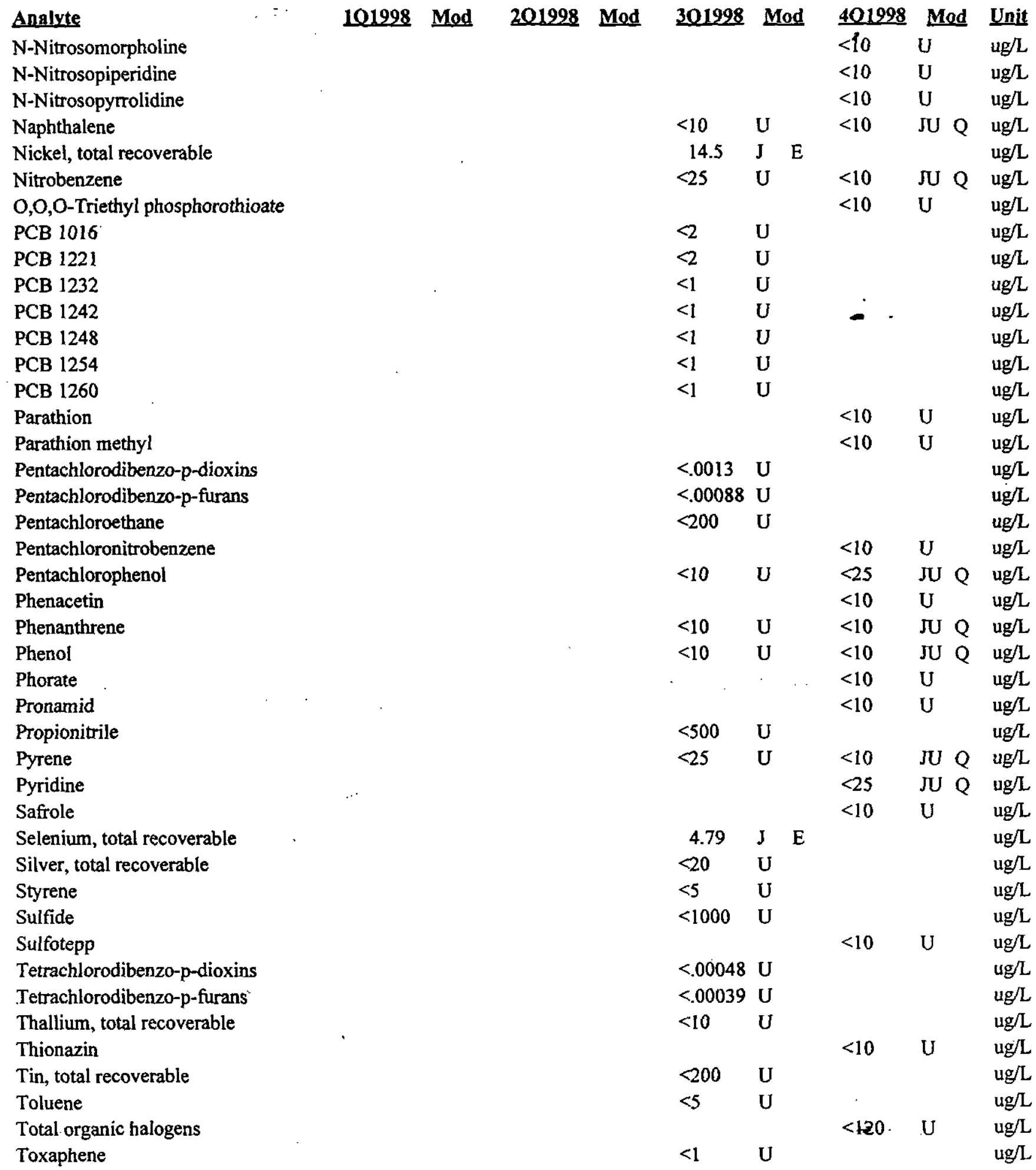

Note: Concentrations in bold exceed the Drinking Water Standards listed in Appendix A. Units are for all four quarters. 
WELL: XSB 4D

ANALYTICAL DATA

Analyte

Trichlorofluoromethane

Vanadium, total recoverable

Vinyl acetate

Xylenes

Zinc, total recoverable

a,a-Dimethylphenethylamine

alpha-Benzene hexachloride

alpha-Chlordane

beta-Benzene hexachloride

cis-1,2-Dichloroethylene

cis-1,3-Dichloropropene

delta-Benzene hexachloride

gamma-Chlordane

m-Nitroaniline

$\mathrm{m} / \mathrm{p}$-Cresol

o-Cresol (2-Methylphenol)

o-Nitroaniline

o-Toluidine

p,p"-DDD

p,p"-DDE

p,p"-DDT

p-Cresol (4-Methylphenol)

p-Dimethylaminoazobenzene

p-Nitroaniline

p-Phenylenediamine

trans-1,2-Dichloroeihylene

trans-1,3-Dichloropropene

trans-1,4-Dichloro-2-butene

\section{Mod 201998 Mod}

\begin{tabular}{|c|c|c|c|c|}
\hline 301998 & Mod & 401998 & Mod & Unit \\
\hline$<5$ & $\mathrm{U}$ & 2 & & ug/L \\
\hline$<10$ & $\mathrm{U}$ & & & $\mathrm{ug} / \mathrm{L}$ \\
\hline$<20$ & U & & & $\mathrm{ug} / \mathrm{L}$ \\
\hline$<10$ & $\mathrm{U}$ & & & $\mathrm{ug} / \mathrm{L}$ \\
\hline 16 & J & & & $\mathrm{ug} / \mathrm{L}$ \\
\hline & & $<10$ & $\mathrm{U}$ & $\mathrm{ug} / \mathrm{L}$ \\
\hline$<.1$ & U & & & $\mathrm{ug} / \mathrm{L}$ \\
\hline$<.1$ & $\mathbf{U}$ & & & $\mathrm{ug} / \mathrm{L}$ \\
\hline$<.1$ & U & & & $\mathrm{ug} / \mathrm{L}$ \\
\hline$<5$ & 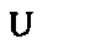 & $<1$ & $\mathbf{U}$ & $\mathrm{ug} / \mathrm{L}$ \\
\hline$<5$ & $\mathrm{U}$ & $\therefore$ & & $\mathrm{ug} / \mathrm{L}$ \\
\hline$<.1$ & U & & & $\mathrm{ug} / \mathrm{L}$ \\
\hline$<.1$ & U & & & $\mathrm{ug} / \mathrm{L}$ \\
\hline$<25$ & $\mathrm{U}$ & $<25$ & $J U$ & ug/L \\
\hline & & $<20$ & $\mathrm{U}$ & $\mathrm{ug} / \mathrm{L}$ \\
\hline$<10$ & $\mathrm{U}$ & $<10$ & $\mathrm{JU} Q$ & $\mathrm{ug} / \mathrm{L}$ \\
\hline$<25$ & $\mathbf{U}$ & $<25$ & JU Q & $\mathrm{ug} / \mathrm{L}$ \\
\hline & & $<10$ & $\mathrm{U}$ & ug $/ \mathrm{L}$ \\
\hline$<.2$ & $\mathrm{U}$ & & & $\mathrm{ug} / \mathrm{L}$ \\
\hline$<.2$ & $\mathrm{U}$ & & & $\operatorname{ug} / \mathrm{L}$ \\
\hline$<.2$ & $\mathrm{U}$ & & & $\mathrm{ug} / \mathrm{L}$ \\
\hline$<10$ & $\mathrm{U}$ & $<10$ & JU Q & $\mathrm{ug} / \mathrm{L}$ \\
\hline & & $<10$ & $\mathbf{U}$ & $\mathrm{ug} / \mathrm{L}$ \\
\hline$<10$ & $\mathbf{U}$ & $<10$ & $\pi U Q$ & $\mathrm{ug} / \mathrm{L}$ \\
\hline & & $<10$ & & $\mathrm{ug} / \mathrm{L}$ \\
\hline$<5$ & $\mathrm{U}$ & & & $\mathrm{ug} / \mathrm{L}$ \\
\hline$<5$ & $\mathrm{U}$ & & & ug/L \\
\hline$<20$ & $\mathbf{U}$ & & & $\mathrm{ug} / \mathrm{L}$ \\
\hline
\end{tabular}


WSRC-RP-99-4003

Unclassified

WELL: XSB 5A

SRS Coord. Lat/Longitude Screen Zone Elevation Top of Standpipe Top of Casing Casing Pump Screen Zone

N 70956.3 33.210 Deg N

E 16703.7 81.76I Deg W

$-108.9-108.9 \mathrm{ft} \mathrm{msl}$

SAMPLE DATE

FIELD DATA

Analyte

Depth to water

$\mathrm{pH}$

Sp. Conductance

Water temperature

Alkalinity as $\mathrm{CaCO} 3$

Phenolpthalein Alkalinity

Turbidity

Volumes purged

Sampling codes

ANALYTICAL DATA

Analyte

1,1,1,2-Tetrachloroethane

1,1,1-Trichloroethane

1,1,2,2-Tetrachloroethane

1,1,2-Trichloroethane

1,1-Dichloroethane

1,1-Dichloroethylene

1,2,3-Trichloropropane

$i, 2,4$-Trichlorobenzene

1,2-Dibromo-3-chloropropane

1,2-Dibromoethane

1,2-Dichlorobenzene

1,2-Dichloroethane

1,2-Dichloropropane

1,3,5-Trinitrobenzene

1,3-Dichlorobenzene

1,3-Dinitrobenzene

1,4-Dichlorobenzene

1,4-Dioxane

1,4-Naphthoquinone

1-Naphthylamine .

2,2-Oxybis(l-chloropropane)

2,3,4,6-Tetrachlorophenol

2,3,7,8-TCDD

2,4,5-T

2,4,5-TP (Silvex)

2,4,5-Trichlorophenol

2,4,6-Trichlorophenol
$112.2 \mathrm{f} \mathrm{msl}$

$05 / 15 / 98$

$03 / 04 / 98$

101998

13.06

5.7

230

19.3

7

0

2.7

$-5.5098$
$112.0 \mathrm{ft} \mathrm{msl}$

$08 / 10 / 98$

301998

14.92

5.3

200

20.9

11

0

1.6

$-6.1904$
4 " STL

$S$

$12 / 07 / 98$

$\begin{array}{ll}\frac{401998}{16.7} & \text { Unit } \\ 5.1 & \text { ft BTOS } \\ 170 & \\ 20.2 & \text { uS/cm } \\ 6 & \text { deg. C } \\ 0 & \mathrm{mg} / \mathrm{L} \\ 1.9 & \mathrm{mg} / \mathrm{L} \\ -5.8286 & \text { NTU } \\ & \text { gallons }\end{array}$

101998 Mod 201998 Mod 301998 Mod 401998 Mod Unit

$<5 \quad U \quad \mathrm{ugh}$

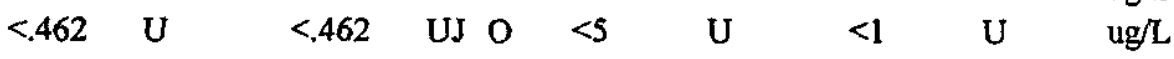

$<5 \quad \mathrm{u}$ ug/L

$<5 \quad U \quad$ ug $/ \mathrm{L}$

$<5 \quad U$. ug/L

$<5 \quad U \quad$ ugh

$<5 \quad \mathrm{u} \quad \mathrm{ug} / \mathrm{L}$

$<10$ U $<10 \quad J U$ Q

$<5 \quad U$ ug/L

$<5 \quad \mathrm{U} \quad \mathrm{ug} / \mathrm{L}$

$<5 \quad U \quad u g / L$

$<5 \quad U \quad u g / L$

$<5 \quad U \quad u g / L$

$<10 \quad U \quad u g / L$

$<5 \quad U \quad \mathrm{ug} / \mathrm{L}$

$<10 \quad \mathrm{U} \quad$ ug/L

$<5 \quad U \quad u g / L$

$<1000 \quad U \quad \mathrm{ug} / \mathrm{L}$

$<10 \quad \mathrm{U} \cdot \quad \mathrm{ug} / \mathrm{L}$

$<10 \quad \mathrm{U} \quad \mathrm{ug} / \mathrm{L}$

$<10 \quad \mathrm{U} \quad<10 \quad$ JU $Q \quad$ ug/L

$<10 \quad \mathrm{U} \quad \mathrm{ug} / \mathrm{L}$

$<.00058 \mathrm{U} \quad$ ugh

$<, 2$ UJ $O \quad$ ug/L

$<.2$ UJ O $\mathrm{ug} / \mathrm{L}$

$<10 \quad U \quad<10 \cdot$ JU $Q$ ug/L

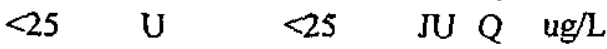

Note: Concentrations in bold exceed the Drinking Water Standards listed in Appendix A. Units are for all four quarters. 
WELL: XSB 5A

\section{ANALYTICAL DATA}

\section{Analyte}

2,4-Dichlorophenol

2,4-Dichlorophenoxyacetic acid

2,4-Dimethyl phenol

2,4-Dinitrophenol

2,4-Dinitrotoluene

2,6-Dichlorophenol

2,6-Dinitrotoluene

2-Acetylaminofluorene

2-Chloronaphthalene

2-Chlorophenol

2-Hexanone

2-Methyl-4,6-dinitrophenol

2-Methylnaphthalene

2-Naphthylamine

2-Nitrophenol

2-Picoline

2-sec-Butyl-4,6-dinitrophenol

3,3"-Dichlorobenzidine

3,3"-Dimethylbenzidine

3-Methylcholanthrene

4-Aminobiphenyl

4-Bromophenyl phenyl ether

4-Chloro-m-cresol

4-Chloroaniline

4-Chlorophenyl phenyl ether

4-Nitrophennl

4-Nitroquinoline-1-oxide

5-Nitro-o-toluidine

7,12-Dimethylbenz(a)anthracene

Acenaphthene

Acenaphthylene

Acetone

Acetonitrile (Methyl cyanide)

Acetophenone

Acrolein

Acrylonitrile

Aldrin

Allyl chloride

Aluminum, total recoverable

Aniline

Anthracene

Antimony, total recoverable

Aramite
101998 Mod 201998 Mod 301998 Mod 401998 Mod Unit

$<10 \quad U \quad<$ PO J $Q \quad$ ug $/ \mathrm{L}$

$<.2$ UJ $\mathrm{O} \quad \mathrm{ug} / \mathrm{L}$

$<10 \quad \mathrm{U} \quad<10 \quad J U \mathrm{Q} \quad \mathrm{ug} / \mathrm{L}$

$<25 \quad \mathrm{U} \quad<25 \quad$ JU $Q \quad \mathrm{ug} / \mathrm{L}$

$<10 \quad \mathrm{U} \quad<10$ JU $\mathrm{Q} \quad \mathrm{ug} / \mathrm{L}$

$<10$ U ug/L

$<10 \quad \mathrm{U} \quad<10 \quad$ JU $Q \quad$ ug/L

$<10$ U ug/L

$<10 \quad \mathrm{U} \quad<10 \quad$ JU $Q \quad$ ug/L

$<10 \quad \mathrm{U} \quad<10 \quad \mathrm{JU} Q \mathrm{ug} / \mathrm{L}$

$<$ U $\therefore$ ug $/$

$<25 \quad \mathrm{U} \quad<25 \quad \pi \quad \mathrm{Q} \quad \mathrm{ug} / \mathrm{L}$

$<10 \quad \mathrm{U} \quad<10 \quad \pi U \quad \mathrm{Q} \quad \mathrm{ug} / \mathrm{L}$

$<10 \cdot \mathrm{U} \quad$ ug/L

$<10 \quad \mathrm{U} \quad<10 \quad \pi \mathrm{U} \quad \mathrm{ug} / \mathrm{L}$

$<10 \quad \mathrm{U} \quad$ ug/L

$<10$ U ug/L

$<10 \quad \mathrm{U} \quad<10 \quad$ JU $Q \quad$ ug/L

$<20$ U ug/L

$<10$ U ug $/$

$<10$ U ug/L

$<10 \quad \mathrm{U} \quad<10 \quad$ JU $Q \quad$ ug/L

$<10 \quad \mathrm{U} \quad<10 \quad$ JU $\mathrm{Q} \quad \mathrm{ug} / \mathrm{L}$

$<10 \quad \mathrm{U} \quad<10 \quad$ J $Q \quad$ ug $/ \mathrm{L}$

$\begin{array}{llllll}<10 & \mathrm{U} & <10 \quad \mathrm{JU} & \mathrm{Q} & \mathrm{ug} / \mathrm{L}\end{array}$

$<25 \quad \mathrm{U} \quad<25 \quad J U \mathrm{Q} \quad \mathrm{ug} / \mathrm{L}$

$<50$ U ug/L

$<10$ U ug/

$<10$ U ug/L

$<10 \quad$ U $\quad<10 \quad$ N $Q \quad$ ug $/ \mathrm{L}$

$<10 \quad \mathrm{U} \quad<10 \quad J U \quad \mathrm{Q} \quad \mathrm{ug} / \mathrm{L}$

$<20$ U ug/L

$<500$ U ug/L

$<50$ U $\quad \mathrm{ug} / \mathrm{L}$

$<50$ U $\quad$ ug/L

$<.1 \quad \mathrm{U} \quad \mathrm{ug} / \mathrm{L}$

$<10$ U ug/L

26.9

$<200 \quad$ U

$<25 \quad U$

$<10 \quad \mathrm{U}$

$<100 \quad \mathrm{U}$

$\begin{array}{lll}<200 & \text { U } & \text { ug/L } \\ <25 & \text { JU Q } & \text { ug/L } \\ <10 & \text { J Q } & \text { ug/L } \\ < & & \text { ug/L } \\ <10 & \text { U } & \text { ug/L }\end{array}$

Note: Concentrations in bold exceed the Driaking Water Standards listed in Appendix A. Units are for all four quarters. 
WELL: XSB SA

ANALYTICAL DATA

\begin{tabular}{|c|c|c|c|c|c|c|c|c|c|c|}
\hline Analyte & 101998 & Mod & 201998 Mod & $\underline{301998}$ & $\mathbf{M}$ & od & 401998 & Mo & od & Unit \\
\hline Arsenic, total recoverable & & & & $<10$ & $\mathrm{U}$ & & & & & $\mathrm{ug} / \mathrm{L}$ \\
\hline Barium, total recoverable & & & & 4.78 & $\mathrm{~J}$ & $E$ & & & & $/ \mathrm{L}$ \\
\hline Benzene & & & & $<5$ & $\mathrm{U}$ & & & & & $/ \mathrm{L}$ \\
\hline Benzo(a)anthracene & & & & $<10$ & $\mathrm{U}$ & & $<10$ & JU & Q & $\sqrt{L}$ \\
\hline Benzo(a)pyrene & & & & $<10$ & $U$ & & $<10$ & $\pi$ & $Q$ & \\
\hline Benzo(b)fluoranthene & & & & $<10$ & $\mathrm{U}$ & & $<10$ & $\pi$ & $\mathrm{Q}$ & I l \\
\hline Benzo(g,h,i)perylene & & & & $<10$ & $\mathrm{U}$ & & $<10$ & Ju & Q & $\sqrt[3]{\mathrm{L}}$ \\
\hline Benzo(k)fluoranthene & & & & $<10$ & $\mathrm{U}$ & & $<10$ & JU & $Q$ & g/L \\
\hline Benzyl alcohol & & & & $<10$ & $\mathrm{U}$ & & $<10$ & JU & $Q$ & \\
\hline Beryllium, total recoverable & & & & $<10$ & $U$ & & & & & b \\
\hline Bis(2-chloroethoxy) methane & & & & $<10$ & $\mathrm{U}$ & & $<10$ & JU & Q & $3 / \mathrm{L}$ \\
\hline Bis(2-chloroethyl) ether & & & & $<10$ & $\mathrm{U}$ & & $<10$ & JU & $Q$ & $g / \mathrm{L}$ \\
\hline Bis(2-ethylhexyl) phthalate & & & & $<10$ & $\mathrm{U}$ & & $<10$ & JU & $\mathrm{Q}$ & \\
\hline Bromodichloromethane & & & & $<5$ & $\mathbf{U}$ & & & & & \\
\hline Bromoform & & & & $<5$ & $U$ & & & & & L \\
\hline Bromomethane (Methyl bromide) & & & & $<5$ & $\mathrm{U}$ & & & & & $/ \mathrm{L}$ \\
\hline Butylbenzyl phthalate & & & & $<10$ & $\mathrm{U}$ & & $<10$ & Ju & & $g / L$ \\
\hline Cadmium, total recoverable & & & & $<10$ & $\mathrm{U}$ & & & & & \\
\hline Carbon disulfide & & & & $<5$ & $\mathbf{U}$ & & & & & \\
\hline Chlorobenzene & & & & $<5$ & $U$ & & & & & $/ \mathrm{L}$ \\
\hline Chlorobenzilate & & & & & & & $<10$ & $\mathrm{U}$ & & J \\
\hline Chloroethane & & & & $<10$ & $\mathrm{U}$ & & & & & \\
\hline Chloroethene (Vinyl chloride) & & & & $<5$ & $\mathrm{U}$ & & & & & $\mathrm{g}$ \\
\hline Chloromethane (Methyl chloride) & & & & $<5$ & $U$ & & · & & & \\
\hline Chloroprene & & & & $<50$ & $\mathrm{U}$ & & & & & \\
\hline Chromium, total recoverable & & & & 3.77 & $\mathrm{~J}$ & $\mathrm{E}$ & & & & \\
\hline Chrysene & & & & $<10$ & $\mathrm{U}$ & & $<10$ & $\mathrm{JU}$ & & $\mathrm{L}$ \\
\hline Cobalt, total recoverable & & & & 3.11 & $\mathbf{J}$ & $E$ & & & & \\
\hline Copper, total recoverable & & & & 179 & & & & & & \\
\hline Cyanide & & & & $<10$ & $U$ & & & & & 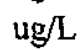 \\
\hline Di-n-butyl phthalate & & & & $<10$ & $\mathbf{U}$ & & $<10$ & & $Q$ & $\mathrm{~L}$ \\
\hline Di-n-octyl phthalate & & & & $<10$ & $\mathrm{U}$ & & $<10$ & & $Q$ & ug \\
\hline Diallate & & & & & & & $<10$ & $U$ & & \\
\hline Dibenz(a,h)anthracene & & & & $<20$ & $U$ & & $<10$ & JU & & \\
\hline Dibenzofuran & & & & $<10$ & $\mathrm{U}$ & & $<10$ & JU & Q & \\
\hline Dibromochloromethane & & & & $<5$ & $\mathrm{U}$ & & & & & \\
\hline Dibromomethane (Methylene bromide') & & & & $<5$ & $\mathrm{U}$ & & & & & \\
\hline Dichlorodifluoromethane & & & & $<5$ & U & & & & & \\
\hline Dichloromethane (Methylene chloride) & & & & $<10$ & $\mathrm{U}$ & & & & & \\
\hline Dieldrin & & & & $<.2$ & $\mathrm{U}$ & & & & & \\
\hline Diethyl phthalate. & & & & $<10$ & $U$ & & $<10$ & JU & Q & \\
\hline Dimethoate & & & & & & & $<1 \theta$ & $\mathrm{U}$ & & \\
\hline Dimethyl phthalate & & & & $<10$ & $U$ & & $<10$ & JU & $Q$ & \\
\hline
\end{tabular}

Note: Concentrations in bold exceed the Driaking Water Standards listed in Appendix A. Units are for all four quarters. 
WELL: XSB 5A

\section{ANALYTICAL DATA}

\section{Analyte}

Diphenylamine

Disulfoton

Endosulfan I

Endosulfan II

Endosulfan sulfate

Endrin

Endrin aldehyde

Ethyl methacrylate

Ethyl methanesulfonate

Ethylbenzene

Fluoranthene

Fluorene

Heptachlor

Heptachlor epoxide

Hexachlorobenzene

Hexachlorobutadiene

Hexachlorocyclopentadiene

Hexachlorodibenzo-p-dioxins

Hexachlorodibenzo-p-furans

Hexachloroethane

Indeno(1,2,3-c,d)pyrene

Iodomethane (Methyl iodide)

Iron, total recoverable

Isobutyl alcohol

Isodrin

Isophorone

Isosafrole

Kepone

Lindane

Methacrylonitrile

Methapyrilene

Methoxychlor

Methyl ethyl ketone

Methyl isobutyl ketone

Methyl methacrylate

Methyl methanesulfonate

$\mathrm{N}$-Nitrosodi-n-butylamine

$\mathrm{N}$-Nitrosodiethylamine

$\mathrm{N}$-Nitrosodimethylamine

$\mathrm{N}$-Nitrosodiphenylamine

$\mathrm{N}$-Nitrosodipropylamine

$\mathrm{N}$-Nitrosomethylethylamine

$\mathrm{N}$-Nitrosomorpholine
101998 Mod 201998 Mod 301998 Mod 401998 Mod Unit

$<$ f0 U ug/L

$<10$ U ug/L

$<.1 \quad \mathrm{U}$

$<.2 \quad \mathrm{U}$

$<.2 \quad \mathrm{U}$

$<.2 \quad \mathrm{U}$

$<.2 \quad \mathrm{U}$

$<5 \quad \mathrm{U}$

$<5 \quad$ U $\quad<10 \quad U \quad \begin{array}{lll}\mathrm{ug} / \mathrm{L} \\ \mathrm{ug} / \mathrm{L}\end{array}$

$<10$ U $<10$. JU Q ug/L

$<10 \quad \mathrm{U} \quad<10 \quad$ JU $Q \quad$ ug/L

$<.1 \quad \mathrm{U}$

$<.1 \quad \mathrm{U}$

$<10 \quad \mathrm{U}$

$<20 \quad U$

$<10 \quad \mathrm{U}$

$<.00072 \mathrm{U}$

$<.00057 \mathrm{U}$

$<10 \quad \mathrm{U}$

$<10 \quad \mathrm{U}$

$<5 \quad U$

532

$99.3 \mathrm{~J}$

$<1500$ II

$<20 \quad U$

$<10$

$<10$

$<10$

$<.1 \quad \mathrm{U}$

$<500 \quad \mathrm{U}$

$<10$

$<1 \quad \mathrm{U}$

$<10 \quad \mathrm{U}$

$<5 \quad \mathrm{U}$

$<50 \quad$ U

U

$<10 \quad U$

$<10 \quad \mathrm{U}$

ug/L

ug/L

$\mathrm{ug} / \mathrm{L}$

ug/L

$\mathrm{ug} / \mathrm{L}$

$\mathrm{ug} / \mathrm{L}$

ug/L

ug/L

$<10 \quad$ JU $Q \quad$ ug/L

$<10$ JU Q ug/L

$<10$ JU Q ug/L

$u g / L$

ug/L

$<10$ JU Q ug/L

$<10$ JU Q ug/

$\mathrm{ug} / \mathrm{L}$

$<100$ JU L ug/L

ug/

$\mathrm{ug} / \mathrm{L}$

JU $Q \quad u g / L$

U ug/L

U ug/L

ug/L

ug/L

ugh $\mathrm{L}$

$u g / \mathrm{L}$

ug/L

ug/L

ug/L

$<10 \quad \mathrm{u} \quad \mathrm{ug} / \mathrm{L}$

$<10 \quad$ U ug/L

$<10$. U ug/L

$<25$ JU Q ug/L

$<10 \quad J U$ Q ug/L

$<10 \quad J U$ Q ug/L

$<10$. U ug/L

$<10 \quad \mathrm{U} \quad \mathrm{ug} / \mathrm{L}$

Note: Concentrations in bold exceed the Drinking Water Standards listed in Appendix A. Units are for all four quarters. 
WELL: XSB 5A

ANALYTICAL DATA

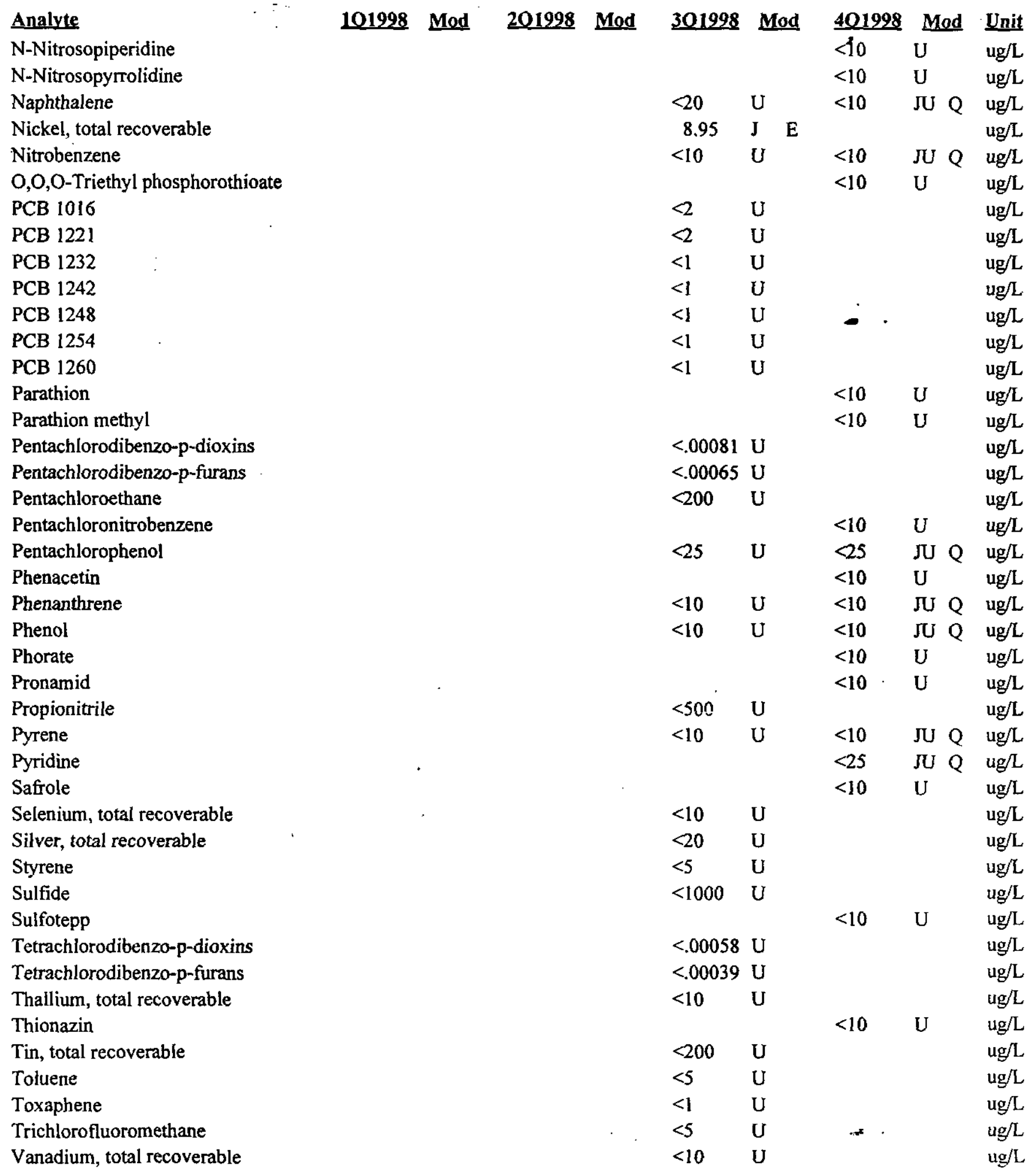

Note: Concentrations in bold exceed the Drinking Water Standards listed in Appendix A. Units are for all four quarters. 
WELL: XSB 5A

\section{ANALYTICAL DATA}

\section{Analyte}

Vinyl acetate

Xylenes

Zinc, total recoverable

a,a-Dimethylphenethylamine

alpha-Benzene hexachloride

alpha-Chlordane

beta-Benzene hexachloride

cis-1,2-Dichloroethylene

cis-1,3-Dichloropropene

delta-Benzene hexachloride

gamma-Chlordane

m-Nitroaniline

$\mathrm{m} / \mathrm{p}$-Cresol

o-Cresol (2-Methylphenol)

o-Nitroaniline

o-Toluidine

p,p"-DDD

$p, p "-D D E$

p,p"-DDT

p-Cresol (4-Methylphenol)

p-Dimethylaminoazobenzene

p-Nitroaniline

$\mathrm{p}$-Phenylenediamine

trans-1,2-Dichloroethylene

trans-1,3-Dichloropropene

101998 Mod 201998 Mod 301998 Mod 401998 Mod Unit

$<20$ U

$<10 \quad U$

ug/L

U

$\mathrm{ug} / \mathrm{L}$

$<.1 \quad \mathrm{U} \quad \mathrm{ug} / \mathrm{L}$

$<.1 \quad$ U $\quad$ ug/L

$<.1 \quad \mathrm{U} \quad$ ug/L

$<5 \quad \mathrm{U} \quad<\mathrm{I} \quad \mathrm{U} \quad \mathrm{ug} / \mathrm{L}$

$<5$ U ug/L

$<1 \quad \mathrm{U} \quad \mathrm{ug} / \mathrm{L}$

$<.1 \quad \mathrm{U} \quad 0^{\circ} \quad \mathrm{ug} / \mathrm{L}$

$<25 \mathrm{U} \quad<25$ JU Q ug/L

$<20$ U ug/

$\begin{array}{llllll}<10 & \mathrm{U} & <10 \quad \mathrm{JU} & \mathrm{Q} & \mathrm{ug} / \mathrm{L}\end{array}$

$<25 \quad \mathrm{U} \quad<25 \quad \pi \quad \mathrm{Q} \quad \mathrm{ug} / \mathrm{L}$

$<.2 \quad \mathrm{U} \quad \mathrm{ug} / \mathrm{L}$

$<.2$ U $\mathrm{ug} / \mathrm{L}$

$<10 \quad \mathrm{U} \quad<10 \quad$ JU $Q \quad$ ug/L

$\begin{array}{lllll} & <10 & < & \mathrm{U} & \mathrm{ug} / \mathrm{L}\end{array}$

$<10 \quad \mathrm{U} \quad \mathrm{ug} / \mathrm{L}$

$<5$ U . ug/L.

$<5 \quad \mathrm{u} \quad \mathrm{ug} / \mathrm{L}$

$<20$ U ug/L

trans-1,4-Dichloio-2-butene

Note: Concentrations in bold exceed the Drinking Water Standards listed in Appendix A. Units are for all four quarters. 
Table B-3. Groundwater Monitoring Results for Individual Recovery Wells, Monthly Sampling

WELL: TRW 1

\begin{tabular}{|c|c|c|c|c|c|c|c|}
\hline SRS Coord, & Lat/Longitude & Screen Zone Elevation & Top of Standpipe & Top of Casing & Casing & Pump & Screen Zone \\
\hline $\begin{array}{l}\text { N } 71162.8 \\
\text { E } 16947.0\end{array}$ & $\begin{array}{l}\text { 33.211 Deg N } \\
\text { 81.760 Deg W }\end{array}$ & $106.4-81.4 \mathrm{ft} \mathrm{msl}$ & $\mathrm{ft} \mathrm{msl}$ & $156.3 \mathrm{ft} \mathrm{msl}$ & $6 " \mathrm{STL}$ & & Unconfined \\
\hline SAMPLE D & ATE & $01 / 16 / 98$ & $02 / 27 / 98$ & $03 / 31 / 98$ & & $04 / 20 / 98$ & \\
\hline
\end{tabular}

FIELD DATA

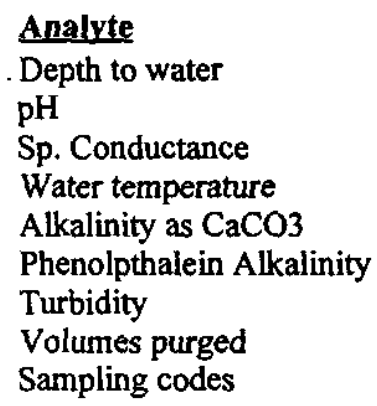

ANALYTICAL DATA

Analyte

Carbon tetrachloride

Chloroform

Gross alpha

Lead, total recoverable

Mercury, total recoverable

Tetrachloroethylene

Trichloroethylene

$\begin{array}{ll} & 63.27 \\ 5.4 & 5.1 \\ 120 & 70 \\ 18 & 20.6 \\ 7 & 1 \\ 0 & 0 \\ 2.1 & .5 \\ & \\ & \mathrm{C}\end{array}$

55

55.41

Unit

ft BTOS

$\mathrm{uS} / \mathrm{cm}$

deg. C

$\mathrm{mg} / \mathrm{L}$

$\mathrm{mg} / \mathrm{L}$

$\therefore \quad$ NTU

C

gallons
Mod

12.1

.709

1.31

20.4

3.55

.98

99.2

UI

UI

I

Mod

11.1

$<4.28$
$<5.69$

116
Mod Unit

ug/L $\mathrm{ug} / \mathrm{L}$ $\mathrm{pCi} / \mathrm{L}$ ug/L ug/L $\mathrm{ug} / \mathrm{L}$ ug/L

Note: Concentrations in bold exceed the Drinking Water Standards listed in Appendix A. Units are for all four quarters. 
WELL: TRW 1

SRS Coord, Lat/Longitude Screen Zone Elevation Top of Standoipe Top of Casing Casing Pump Screen Zone

N 71162.8 33.2ll Deg N 106.4 - $81.4 \mathrm{ft} \mathrm{msl}$ E $16947.0 \quad 81.760$ Deg W

ft msl

$05 / 13 / 98$

SAMPLE DATE

FIELD DATA

Analvte

Depth to water

$\mathrm{pH}$

Sp. Conductance

Water temperature

Alkalinity as $\mathrm{CaCO} 3$

Phenolpthalein Alkalinity

Turbidity

Volumes purged

Sampling codes

C

62.85

160

23

0

0

1.6

C

$06 / 03 / 98$

$07 / 15 / 98$

?

$08 / 11 / 98$

Unconfined

ANALYTICAL DATA

Analvte

Carbon tetrachloride

Chloroform

Gross alpha

Lead, total recoverable

Mercury, total recoverable

Tetrachloroethylene

Trichloroethylene
Mod

14

.971

3.54

$<5$

.84

1.39

170
Mod

\begin{tabular}{|c|c|c|c|c|c|c|}
\hline \multicolumn{4}{|c|}{ Med } & \multirow{2}{*}{\multicolumn{2}{|c|}{ Med }} & Unit \\
\hline 10.4 & & & 8.17 & & & $\mathrm{ug} / \mathrm{L}$ \\
\hline $\begin{array}{l}<1 \\
7.03\end{array}$ & $\mathbf{U}$ & & $\begin{array}{l}<1 \\
9.17\end{array}$ & $\mathbf{U}$ & & ug/L \\
\hline$<5$ & $\mathrm{U}$ & & $<10$ & $\mathrm{U}$ & & $\mathrm{ug} / \mathrm{L}$ \\
\hline .482 & & & .897 & & & $\mathrm{ug} / \mathrm{L}$ \\
\hline 2.29 & $\mathbf{J}$ & C & 1.43 & $\mathbf{J}$ & I & $\mathrm{ug} / \mathrm{L}$ \\
\hline 122 & L & & 85.9 & & & $\mathrm{ug} / \mathrm{L}$ \\
\hline
\end{tabular}


WSRC-RP-99-00119

- Unclassified

WELL: TRW 1

SRS Coord, Lat/Longitude Screen Zone Elevation Top of Standpipe Top of Casing Casing Pump Screen Zone N 71162.8 33.211 Deg N : : 106.4- $81.4 \mathrm{ft} \mathrm{msl}$ E $16947.0 \quad 81.760 \mathrm{Deg} \mathrm{W}$

ft msl

$09 / 15 / 98$

SAMPLE DATE

FIELD DATA

Analyte

Depth to water

$\mathrm{pH}$

Sp. Conductance

Water temperature

Alkalinity as $\mathrm{CaCO} 3$

Phenolpthalein Alkalinity

Turbidity

Volumes purged

Sampling codes

65.9
5.3
100
21.5
1
0
1
0
$\mathrm{CN}$

$10 / 15 / 98$

$156.3 \mathrm{ft} \mathrm{msl}$

$6 "$ STL

Unconfined

ANALYTICAL DATA

Analyte

Carbon tetrachloride

Chloroform

Gross alpha

Lead, total recoverable

Mercury, total recoverable

Tetrachloroethylene

Trichloroethylene

\begin{tabular}{|c|c|c|c|c|c|c|c|c|c|c|c|c|}
\hline \multicolumn{4}{|c|}{ Mod } & \multicolumn{2}{|c|}{ Mod } & \multicolumn{4}{|c|}{ Mod } & \multirow{2}{*}{\multicolumn{2}{|c|}{ Mod }} & Unit \\
\hline 8.99 & $\mathbf{J}$ & 0 & 10.8 & $\mathbf{J}$ & $\mathrm{K}$ & 11.3 & $J$ & I & 12.6 & & & $u g / L$ \\
\hline .957 & J & EO & 1.4 & & & $<5$ & $\mathrm{U}$ & & 2.06 & & & $g / L$ \\
\hline 5.92 & & & 3.5 & & & 2.24 & & & & & & $\mathrm{Ci} / \mathrm{L}$ \\
\hline$<100$ & $\mathbf{U}$ & & $<100$ & $\mathrm{U}$ & & $<47$ & $\mathrm{U}$ & & $<10$ & $\mathbf{U}$ & & $\mathrm{ug} / \mathrm{L}$ \\
\hline .579 & & & .64 & & & .829 & & & .291 & $\mathrm{~J}$ & I & $u g / L$ \\
\hline 1.76 & $\mathrm{~J}$ & $\mathrm{ClO}$ & 1.76 & $\mathbf{J}$ & K & $<5$ & $\mathrm{U}$ & & 1.54 & & & $u g / L$ \\
\hline 86,9 & $\mathbf{J}$ & $\mathrm{CO}$ & 97.9 & & & 91.8 & $\mathrm{~J}$ & K & 103 & & & $u g / \mathrm{L}$ \\
\hline
\end{tabular}

Note: Concentrations in bold exceed the Drinking Water Standards listed in Appendix A. Units are for all four quarters. 
WSRC-RP-99-00119

- Unclassified

WELL: TRW 2

SRS Coord. Lat/ongitude Screen Zone Elevation Top of Standpipe Top of Casing Casing Pump Screen Zone

N 71259.6 33.211 Deg N $\quad .12 .2-77.2 \mathrm{ft}$ msl

E 16803.8 81.761 Deg W

ft msl

SAMPLE DATE

$01 / 16 / 98$

$02 / 27 / 98$

$154.3 \mathrm{ft} \mathrm{msl} \mathrm{6"STL}$

$03 / 31 / 98$

$04 / 20 / 98$

\section{FIELD DATA}

Analyte

Depth to water

$\mathrm{pH}$

Sp. Conductance

Water temperature

Alkalinity as $\mathrm{CaCO} 3$

Phenolpthalein Alkalinity

Turbidity

Volumes purged

Sampling codes

\section{ANALYTICAL DATA}

Analyte

Carbon tetrachloride

Chloroform

Gross alpha

Lead, total recoverable

Mercury, total recoverable

Tetrachloroethylene

Trichloroethylene

$\begin{array}{ll} & \\ 5.4 & 60.65 \\ 96 & 4.8 \\ 17 & 57 \\ 7 & 20.1 \\ 0 & 1 \\ 1.6 & 0 \\ & .4 \\ & \\ & \end{array}$

$\begin{array}{clccc} & \text { Mod } & & \text { Mod } \\ 2.98 & & 2.8 & \\ <.428 & \mathrm{U} & <.856 & \mathrm{U} \\ 1.18 & & & \\ <5 & \mathrm{U} & & \\ <.2 & \text { UJ } \mathrm{I} & & \\ <.569 & \mathrm{U} & <1.14 & \mathrm{U} \\ 33.2 & & 24.2 & \end{array}$

Mod

57.57

4.4

$80 u S / \mathrm{cm}$

20.4 deg. C

$1 \mathrm{mg} / \mathrm{L}$

$0 \mathrm{mg} / \mathrm{L}$

.4. NTU

gallons

Unit

ft BTOS

$\mathrm{mg} / \mathrm{L}$

vTU

CNP

C

$\begin{array}{ccc} & \text { Mod } & \text { Unit } \\ 3.62 & & \mathrm{ug} / \mathrm{L} \\ .519 & & \mathrm{ug} / \mathrm{L} \\ & & \mathrm{pCi} / \mathrm{L} \\ <5 & \mathrm{U} & \mathrm{ug} / \mathrm{L} \\ .43 & & \mathrm{ug} / \mathrm{L} \\ .782 & & \mathrm{ug} / \mathrm{L} \\ 35.8 & & \mathrm{ug} / \mathrm{L}\end{array}$


WSRC-RP-99-00119

Unclassified

WELL: TRW 2

SRS Coord. Lat/Longitude Screen Zone Elevation Top of Standpipe Top of Casing Casing Pump Screen Zone N 71259.6 33.211 Deg N : $12.2-77.2$ ft msl E $16803.8 \quad 81.761$ Deg W $\mathrm{ftmsl} \quad 154.3 \mathrm{fmsl} 6$ "STL Unconfined

SAMPLE DATE

$05 / 13 / 98$

$06 / 03 / 98$

$07 / 15 / 98$

$08 / 11 / 98$

\section{FIELD DATA}

\section{Analyte}

Depth to water

pH

Sp. Conductance

Water temperature

Alkalinity as $\mathrm{CaCO} 3$

Phenolpthalein Alkalinity

Turbidity

Volumes purged

Sampling codes
60.4

ANALYTICAL DATA

\section{Analvte}

Carbon tetrachloride

Chloroform

Gross alpha

Lead, total recoverable

Mercury, total recoverable

Tetrachloroethylene

Trichloroethylene

$\begin{array}{ll}60.4 & 4.8 \\ & 110 \\ & 23.4 \\ & 0 \\ & 0 \\ & .2 \\ \mathrm{C} & \mathrm{C}\end{array}$

4.8

23.4

0

0

C

Mod

8.11

.526

5.82

1.09

.84

79

62.5
3.9
180
24.2
0
0
3.5
C

62.5

180

24.2

3.5

C

62.7

4.5

83

23.3

2

0

. 2 .

Unit

ft BTOS

uS/cm

deg. C

$\mathrm{mg} / \mathrm{L}$

$\mathrm{mg} / \mathrm{L}$

NTU

gallons

C

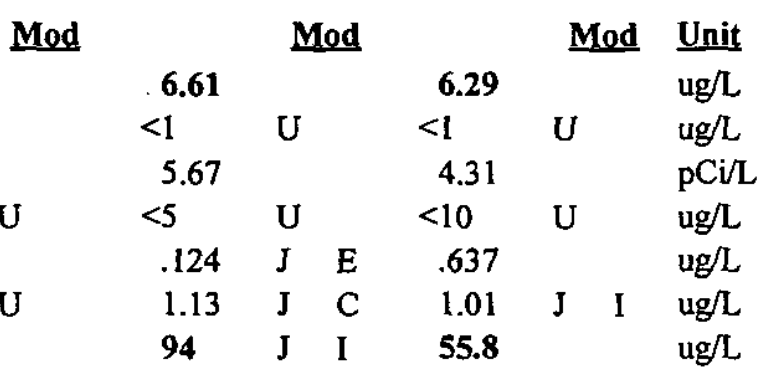

Note: Concentrations in bold exceed the Drinking Water Standards listed in Appendix A. Units are for all four quarters. 
WELL: TRW 2

SRS Coord, Lat/Longitude Screen Zone Elevation Top of Standpipe Top of Casing Casing Pump Screen Zone N $71259.6 \quad 33.211$ Deg N $\quad-112.2-77.2 \quad \mathrm{ft} \mathrm{msl}$ E 16803.8 81.761 Deg W

$09 / 15 / 98$

SAMPLE DATE

FIELD DATA

Analyte

Depth to water

pH

Sp. Conductance

Water temperature

Alkalinity as $\mathrm{CaCO} 3$

Phenolpthalein Alkalinity

Turbidity

Volumes purged

Sampling codes

$\begin{array}{ll}63.2 & 63 \\ 5.3 & 5 \\ 100 & 82 \\ 21.1 & 18.4 \\ 1 & 5 \\ 0 & 0 \\ .9 & 7 \\ 0 & 0 \\ \mathrm{CN} & \mathrm{CN}\end{array}$

ft msl

$154.3 \mathrm{ft} \mathrm{msl}$

6" STL

$10 / 15 / 98$

$11 / 30 / 98$

$12 / 16 / 98$
Unconfined

Unit

ft BTOS

$\mathrm{uS} / \mathrm{cm}$

deg. C

$\mathrm{mg} / \mathrm{L}$

$\mathrm{mg} / \mathrm{L}$

NTU

gallons

\section{ANALYTICAL DATA}

Analyte

Carbon tetrachloride

Chloroform

Gross alpha

Lead, total recoverable

Mercury, total recoverable

Tetrachloroethylene

Trichloroethylene

\begin{tabular}{|c|c|c|c|c|c|c|c|c|c|c|c|c|}
\hline \multicolumn{4}{|c|}{ Mod } & \multicolumn{2}{|c|}{ Mod } & \multicolumn{4}{|c|}{ Mod } & \multicolumn{2}{|c|}{ Mod } & \multirow{2}{*}{$\frac{\text { Unit }}{\mathrm{ug} / \mathrm{L}}$} \\
\hline 9.22 & & & 10.4 & $\mathrm{~J}$ & $\mathrm{~K}$ & 8.2 & & & 8.16 & & & \\
\hline$<1$ & $\mathbf{U}$ & & .699 & & & .559 & $\mathbf{J}$ & I & .807 & $\mathrm{~J}$ & I & $\mathrm{ug} / \mathrm{L}$ \\
\hline 3.94 & & & 3.02 & $\mathbf{J}$ & I & & & & & & & $\mathrm{pCi} / \mathrm{L}$ \\
\hline$<100$ & $U$ & & $<100$ & $\mathbf{U}$ & & $<10$ & $\mathrm{U}$ & & $<10$ & $\mathrm{U}$ & & ug/L \\
\hline$<.5$ & $\mathbf{U}$ & & $<.5$ & $U$ & & $<.5$ & $\mathrm{U}$ & & .251 & $\mathbf{J}$ & I & $\mathrm{ug} / \mathrm{L}$ \\
\hline 1.47 & j & CI & 1.46 & $J$ & $\mathrm{~K}$ & 1.09 & & & 1.06 & & & $\mathrm{ug} / \mathrm{L}$ \\
\hline 60.3 & $\mathrm{~J}$ & $\mathrm{C}$ & 64.1 & & & 57.6 & & & 61.2 & & & ug/L \\
\hline
\end{tabular}

Note: Concentrations in bold exceed the Drinking Water Standards listed in Appendix A. Units are for all four quarters. 
WELL: TRW 3

SRS Coord, Lat/Longitude Screen Zone Elevation Top of Standpipe Top of Casing

N 71334.4 33.211 Deg N : $112.3-77.4 \mathrm{ft} \mathrm{msl}$

ft $\mathrm{msl}$

E 17004.7 81.761 Deg W

SAMPLE DATE

$02 / 27 / 98$

$154.5 \mathrm{ft} \mathrm{msl}$

$\frac{\text { Casing }}{6 " \mathrm{STL}}$

Pump

Screen Zone

$03 / 31 / 98$

FIELD DATA

Analyte

Depth to water

54.02

52.74

$\mathrm{pH}$

Sp. Conductance

Water temperature

Alkalinity as $\mathrm{CaCO} 3$

Phenolpthalein Alkalinity

- Turbidity

Volumes purged

Sampling codes

NPW

Mod

Mod

Mod

Mod Unit
Analyte

ANALYTICAL DATA ft BTOS

$\mathrm{uS} / \mathrm{cm}$ deg. C $\mathrm{mg} / \mathrm{L}$ $\mathrm{mg} / \mathrm{L}$

NTU gallons
$04 / 20 / 98$

53.19

Unit

Unconfined

$\therefore$

C

Note: Concentrations in bold exceed the Drinking Water Standards listed in Appendix A. Units are for all four quarters. 
WELL: TRW 3

SRS Coord. Lat/Longitude Screen Zone Elevation Top of Standpipe Top of Casing Casing Pump Screen Zone N 71334.4 33.211 Deg N $112.3-77.4 \mathrm{ft} \mathrm{msl}$ E 17004.7 81.761 Deg W

$\mathrm{ft}$ msl

$05 / 13 / 98$

SAMPLE DATE

FIELD DATA

Analyte

Depth to water

$\mathrm{pH}$

Sp. Conductance

Water temperature

Alkalinity as $\mathrm{CaCO} 3$

Phenolpthalein Alkalinity

Turbidity

Volumes purged

Sampling codes
53.8

5.4
65
23
7
0
.6

C

5.4

\section{(2)}

(2)

6

C

C
$154.5 \mathrm{ft} \mathrm{msl} 6$ " STL

$07 / 15 / 98$

Unconfined

,

$08 / 11 / 98$

Unit

ft BTOS

5.1

63 uS $/ \mathrm{cm}$

24.5 deg. C

$8 \mathrm{mg} / \mathrm{L}$

$0 \quad \mathrm{mg} / \mathrm{L}$

5. NTU

CS

ANALYTICAL DATA

Analyte

Carbon tetrachloride

Chloroform

Gross aipha

Lead, total recoverable

Mercury, total recoverable

Tetrachloroethylene

Trichloroethylene

\begin{tabular}{|c|c|c|c|c|c|c|}
\hline & \multicolumn{2}{|c|}{ Mod } & \multicolumn{3}{|c|}{ Mod } & Mo \\
\hline 3.66 & & & 5.12 & & 9.57 & \\
\hline$<.428$ & $\mathrm{U}$ & & $<.428$ & $\mathrm{U}$ & $<1$ & $\mathbf{U}$ \\
\hline 4.97 & & & 1.88 & $\mathrm{~J}$ & 3.11 & \\
\hline 3.5 & $\mathbf{J}$ & $\mathrm{E}$ & $<5$ & $\mathbf{U}$ & $<5$ & $\mathrm{U}$ \\
\hline .05 & $\mathbf{J}$ & $\mathrm{E}$ & $<.2$ & $\mathbf{U}$ & $<.2$ & $\mathrm{U}$ \\
\hline$<.569$ & $U$ & & $<.569$ & $\mathrm{U}$ & $<1$ & $\mathrm{U}$ \\
\hline 93.4 & & & 108 & & 115 & $\mathbf{L}$ \\
\hline
\end{tabular}

$\begin{array}{cccc}8.25 & \text { Mod } & \text { Unit } \\ <1 & & & \text { ug/L } \\ 3.38 & & & \mathrm{ug} / \mathrm{L} \\ 8.92 & \mathrm{~J} & \mathrm{E} & \mathrm{ug} / \mathrm{L} \\ <.5 & \mathrm{U} & & \mathrm{ug} / \mathrm{L} \\ <1 & \mathrm{U} & & \mathrm{ug} / \mathrm{L} \\ 91.1 & & & \mathrm{ug} / \mathrm{L}\end{array}$

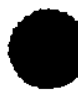

2.8

C

4.3

24.8

0 
WSRC-RP-99-00119

Unclassified

WELL: TRW 3

$\frac{\text { SRS Coord. }}{\text { N 71334.4 }} \frac{\text { Lat/Longitude }}{33.211 \mathrm{Deg} \mathrm{N}} \frac{\text { Screen Zone Elevation Top of Standpipe Top of Casing }}{-112.3-77.4 \mathrm{ft} \mathrm{msl}} \frac{\mathrm{ft} \mathrm{msl}}{154.5 \mathrm{ft} \mathrm{msl}} \frac{\text { Casing }}{6 T \mathrm{~L}}$ Pump $\frac{\text { Screen Zone }}{\text { Unconfined }}$ E 17004.7 81.761 Deg W

$$
\text { : } 112.3-77.4 \mathrm{ft} \mathrm{msl}
$$

$$
09 / 15 / 98
$$

$10 / 15 / 98$

$11 / 30 / 98$

$12 / 16 / 98$

\section{FIELD DATA}

\section{Analyte}

Depth to water

$\mathrm{pH}$

Sp. Conductance

Water temperature

Alkalinity as $\mathrm{CaCO} 3$

Phenolpthalein Alkalinity

Turbidity

Volumes purged

Sampling codes
5.9

62

23

1

0

1.8

CNS
4.8

68

16.5

1.4

CND
Unit ft BTOS

5.8

55

21.7

8

0

8

CNS
uS/cm

deg. C $\mathrm{mg} / \mathrm{L}$ $\mathrm{mg} / \mathrm{L}$ NTU gallons

\section{ANALYTICAL DATA}

\section{Analyte}

Carbon tetrachloride

Chloroform

Gross alpha

Lead, total recoverable

Mercury, total recoverable

Tetrachloroethylene

Trichloroethylene
Mod

$$
7.27
$$

$.828 \quad J \quad E$

1.69

$<100$

$<.5$

$<1$

88.6
Mod

$9.66 \mathrm{~J} \quad \mathrm{~K}$

844

$$
1.04
$$

$<100$

$<.5$.

.745

93.9

J I

U
Mod

6.77 .64

U $<5$

J $\mathrm{K}<\mathrm{l}$

92.4
Mod Unit ug/L ug/L $\mathrm{pCi} / \mathrm{L}$ ug/L ug/L ug/L $\mathrm{ug} / \mathrm{L}$

Note: Concentrations in bold exceed the Drinking Water Standards listed in Appendix A. Units are for all four quarters. 
WELL: TRW 4

SRS Coord. Lat/Longitude Screen Zone Elevation Top of Standpioe Top of Casing Casing Pump Screen Zone N 71454.2 33.212 Deg N $111.9+81.9 \mathrm{ft} \mathrm{msl}$ E 17144.681 .760 Deg W

SAMPLE DATE

FIELD DATA

Analyte

Depth to water

$\mathrm{pH}$

Sp. Conductance

Water temperature

Alkalinity as $\mathrm{CaCO} 3$

Phenolpthalein Alkalinity

Turbidity

Volumes purged

Sampling codes
$02 / 27 / 98$

49.1

Mod

Mod

Mod

48.46

Unit

48.05

ft BTOS

$\mathrm{uS} / \mathrm{cm}$

deg. C

$\mathrm{mg} / \mathrm{L}$

$\mathrm{mg} / \mathrm{L}$

$\therefore \quad$ NTU

Gallons

ANALYTICAL DATA

Analyte

CNP

C

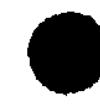

$04 / 20 / 98$

Mod

Med

Mod Unit

Note: Concentrations in bold exceed the Drinking Water Standards listed in Appendix A. Units are for all four quarters. 
WSRC-RP-99-00119

Unclassified

WELL: TRW 4

SRS Coord. Lat/Longitude Screen Zone Elevation Top of Standòipe Top of Casing Casing Pump Screen Zone N 71454.2 33.212 Deg N : : $111.9-81.9 \mathrm{ft} \mathrm{msl}$ E $17144.6 \quad 81.760 \mathrm{Deg} \mathrm{W}$

ft msl

$05 / 13 / 98$

$06 / 03 / 98$

$150.9 \mathrm{ft} \mathrm{msl}$

6" STL

,

SAMPLE DATE

FIELD DATA

Analyte

Depth to water

$\mathrm{pH}$

Sp. Conductance

Water temperature

Alkalinity as $\mathrm{CaCO} 3$

Phenolpthalein Alkalinity

Turbidity

Volumes purged

Sampling codes

\section{ANALYTICAL DATA}

\section{Analyte}

Carbon tetrachloride

Chloroform

Gross alpha

Lead, total recoverable

Mercury, total recoverable

Tetrachloroethylene

Trichloroethylene
48.95

5
66
23.3
0
0
.5
C

C

60.5

5.7

74

26

6

0

9.7

C

$\begin{array}{lll}\mathrm{C} & \mathrm{C} & \mathrm{C}\end{array}$
Mod

1.79

2.31

$<.428$

4.79

$<5$

.05

$<.569$

71
Mod

U

U

J

U

U

$E$

2.82

$<1$

1.49

$<5$

$<.2$

$<1$

$<89.2$
Mod

U

$<1$

3.69

$<5$

$<.2$

$<$
Unit

63.15

4.7

82

22.6

3

0

8 .

C

$\mathrm{uS} / \mathrm{cm}$

deg. C

$\mathrm{mg} / \mathrm{L}$

$\mathrm{mg} / \mathrm{L}$

NTU

gallons

$08 / 11 / 98$

ft BTOS

Mod Unit

J $O$ ug/L

$\begin{array}{llll}U & \mathrm{O} & \mathrm{ug} / \mathrm{L}\end{array}$

$\mathrm{pCi} / \mathrm{L}$

ug/L

$\begin{array}{lllll}<.5 & \text { U } & & \mathrm{ug} / \mathrm{L} \\ <262 & \text { U } & \text { IO } & \mathrm{ug} / \mathrm{L}\end{array}$

$130 \mathrm{~L} \quad u g / \mathrm{L}$

Note: Concentrations in bold exceed the Drinking Water Standards listed in Appendix A. Units are for all four quarters. 
WELL: TRW 4

SRS Coord. Lat/Longitude Screen Zone Elevation Top of Standpipe Top of Casing Casing N 71454.2 33.212 Deg N E 17144.6 81.760 Deg W

SAMPLE DATE

\section{FIELD DATA}

Analyte Depth to water $\mathrm{pH}$ Sp. Conductance Water temperature Alkalinity as $\mathrm{CaCO} 3$ Phenolpthalein Alkalinity Turbidity Volumes purged Sampling codes

$$
=111.9-81.9 \mathrm{ft} \mathrm{msl}
$$

$09 / 15 / 98$
$150.9 \mathrm{ft} \mathrm{msl}$

$10 / 15 / 98$

$11 / 30 / 98$
Pump Screen Zone Unconfined $12 / 16 / 98$

$\begin{array}{ll}61.7 & 65.82 \\ 5.1 & 5.3 \\ 84 & 95 \\ 19.5 & 18.2 \\ 6 & 4 \\ 0 & 0 \\ 2.6 & 3.7 \\ 0 & \\ \mathrm{CN} & \mathrm{CN}\end{array}$

Unit

62.55
5.6
74
23.1
2
0
3.5
0
$\mathrm{CN}$

5.3

95

18.2

0

3.7

$\mathrm{CN}$
5.4

21.8

4

1.8

$\mathrm{CN}$ ft BTOS

$\mathrm{uS} / \mathrm{cm}$

deg. C $\mathrm{mg} / \mathrm{L}$

$\mathrm{mg} / \mathrm{L}$

NTU

gallons

\section{ANALYTICAL DATA}

\section{Analvte}

Carbon tetrachloride

Chloroform

Gross alpha

Lead, total recoverable

Mercury, total recoverable

Tetrachloroethylene

Trichloroethylene

$\begin{array}{cc} & \text { Mod } \\ 2.36 & \\ <1 & \mathrm{U} \\ 3.18 & \\ <100 & \mathrm{U} \\ <.5 & \mathrm{U} \\ <1 & \mathrm{U} \\ 111 & \mathrm{~L}\end{array}$

Mod

\begin{tabular}{|c|c|c|c|c|}
\hline \multicolumn{4}{|c|}{ Mod } & \\
\hline$<10$ & $\mathrm{U}$ & K & $<10$ & $\mathbf{U}$ \\
\hline$<1$ & $\mathbf{U}$ & & $<10$ & $\mathrm{U}$ \\
\hline 2.39 & $\mathbf{J}$ & I & & \\
\hline$<100$ & $\mathrm{U}$ & & $<10$ & $\mathrm{U}$ \\
\hline .252 & $\mathrm{~J}$ & I & $<.5$ & $\mathrm{U}$ \\
\hline$<1$ & $\mathrm{U}$ & & $<1$ & $U$ \\
\hline 124 & & & 125 & \\
\hline
\end{tabular}

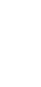


Table B-4. Field Data-for Secondary Wells

WELL: P 26B

SRS Coord, Lat/Longitude Screen Zone Elevation Top of Standpipe Top of Casing Casing Pump Screen Zone N 71996.2 33.214 Deg N = 82.4- $71.9 \mathrm{ft}$ msl E 18050.981 .759 Deg W $154.5 \mathrm{ft} \mathrm{msl}$ $154.1 \mathrm{ft}$ msl 4"PVC $\mathbf{S}$ Unconfined

SAMPLE DATE

$01 / 29 / 98$

$04 / 21 / 98$

08/11/98

$12 / 01 / 98$

FIELD DATA

Analyte

Depth to water

pH

Sp. Conductance

Water temperature

Alkalinity as $\mathrm{CaCO} 3$

Phenolpthalein Alkalinity

Turbidity

Volumes purged

Sampling code

\begin{tabular}{lll}
\hline 101998 & & 201998 \\
\hline 43.92 & & 38.85 \\
5.9 & 6.1 \\
80 & 81 \\
19.3 & 19.8 \\
30 & 32 \\
0 & 0 \\
1.1 & 1.9 \\
3.14595 & 2.28571
\end{tabular}

$\begin{array}{llll}\frac{301998}{42.34} & \frac{401998}{45.06} & & \text { Unit } \\ & 5.9 & & \\ & 69 & & \text { uS/cm } \\ & 19 & \text { deg. C } \\ & 17 & \text { mg/L } \\ & 0 & \text { mg/L } \\ & 5.8 & \text { NȚU } \\ & 3.28355 & \text { gallons } \\ \text { NP } & & \end{array}$


WSRC-RP-99-00119

- Unclassified

WELL: P 26D

SRS Coord, Lat/Longitude Screen Zone Elevation Top of Standpipe Ton of Casing Casing Pump ScreenZone N 71969.3 33.214 Deg N $121.9-101.8 \mathrm{ft} \mathrm{msl}$ E 18041.6 81.759 Deg W $154.5 \mathrm{ft}$ msl $153.9 \mathrm{ft} \mathrm{ms}$ 4"PVC $\mathbf{S}$ Unconfined

SAMPLE DATE

$\begin{array}{lll}04 / 21 / 98 & 08 / 10 / 98 & 12 / 01 / 98\end{array}$

FIELD DATA

Analyte

101998

201998
28.42
5.5
33
19.4
8
0
.3
4.05560

301998

401998

Unit

Depth to water

pH

31.7

Sp. Conductance

5.1

34.91

4.7

30

Water temperature

19.5

Alkalinity as $\mathrm{CaCO} 3$

6

Phenolpthalein Alkalinity

Turbidity

Volumes purged

Sampling code

.5

4.70768

31

17.7

2

0

1.2

4.87734 ,

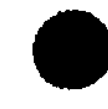

\section{(n)}


WSRC-RP-99-00119

Unclassified

WELL: TBG 7

SRS Coord, Lat/Longitude Screen Zone Elevation Top of Standpipe Top of Casing Casing Pump Screen Zone N 71298.5 33.212 Deg N E 17548.1 81.759 Deg W

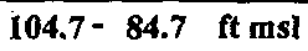

$01 / 29 / 98 \quad 04 / 21 / 98$

146.9 ft $\mathrm{ms}$

$146.8 \mathrm{ft} \mathrm{msl}$

4 "STL

$08 / 12 / 98$

$12 / 04 / 98$

S $\frac{\text { Screen Znconfined }}{\text { Uncong }}$

SAMPLE DATE

\section{FIELD DATA}

Analvte

Depth to water

pH

Sp. Conductance

Water temperature

Alkalinity as $\mathrm{CaCO} 3$

Phenolpthalein Alkalinity

Turbidity

Volumes purged

101998

41.07

5.1

51

22.3

10

0

.6

Sampling code

201998
36.78
5.4
49
23.1
11
0
.8
3.07045

\section{8}

40.05

5

50

23.5

4

0

1.1

$\mathbf{3 . 8 7 1 4 7}$
,

Unconfined

$\begin{array}{ll}\frac{401998}{42.74} & \text { Unit } \\ 5.2 & \text { ft msl } \\ 44 & \\ 22.8 & \text { uS/cm } \\ 5 & \text { deg. C } \\ 0 & \mathrm{mg} / \mathrm{L} \\ .9 & \mathrm{mg} / \mathrm{L} \\ 3.07083 & \text {. } \\ & \text { gallons }\end{array}$


WELL: TNX 5D

SRS Coord. Lat/Longitude Screen Zone Elevation Ton of Standpine Top of Casing Casing Pump Screen Zone $\begin{array}{ll}\text { N 70995.3 } & 33.211 \text { Deg N } \\ \text { E 17363.7 } & \text { 81.759 Deg W. }\end{array}$

SAMPLE DATE $108.5-88.5 \mathrm{ft} \mathrm{ms}$ $149.5 \mathrm{ft} \mathrm{msl}$ $149.3 \mathrm{ft}$ msl

4"STL $\mathbf{S}$ Unconfined

$01 / 29 / 98$

04/21/98

08/12/98

$12 / 02 / 98$

\section{FIELD DATA}

Analyte

Depth to water

pH

Sp. Conductance

Water temperature

Alkalinity as $\mathrm{CaCO} 3$

Phenolpthalein Alkalinity

Turbidity

Volumes purged

Sampling code

$\begin{array}{rlllll}101998 & \frac{201998}{44.45} & \frac{301998}{49.26} & 43.05 & \frac{401998}{45.95} & \text { Unit } \\ & & 4.3 & 5.3 & \text { ft msI } \\ & & 130 & 90 & \text { uS/cm } \\ & & 23.6 & 19.7 & \text { deg. C } \\ & & 2 & 6 & \text { mg/L } \\ & & 0 & 0 & \text { mg/L } \\ & & 10.3 & 12.9 & \text { - NTU } \\ \text {.652644 } & .778472 & 0 & .102653 & \text { gaillons } \\ \text { LNS } & \text { LNS } & \text { NX } & \text { NX } & \text {. }\end{array}$


WELL: TNX 6D

SRS Coord, Lat/Longitude Screen Zone Elevation Top of Standpipe Top of Casing Casing Pump Screen Zone

N 70717.6 33.211 Deg N . $109.8-89.8 \mathrm{ft} \mathrm{msI}$ $150.7 \mathrm{ft} \mathrm{msl}$ $150.5 \mathrm{ft}$ msl

E 17428.7 81.758 Deg W

$\begin{array}{llll}01 / 29 / 98 & 04 / 21 / 98 & 08 / 12 / 98 & 12 / 02 / 98\end{array}$

SAMPLE DATE

\section{FIELD DATA}

Analyte

101998

Depth to water

45.13

201998

301998

42.9

pH

37.81

4.5

Sp. Conductance

Water temperature

130

Alkalinity as $\mathrm{CaCO} 3$

28.1

Phenolpthalein Alkalinity

5

Turbidity

Volumes purged

Sampling code

4.2

.532771

LNS

LNS

NX

$\begin{array}{ll}\frac{401998}{46.31} & \text { Unit } \\ 5.5 & \text { ft msl } \\ 150 & \\ 20.7 & \text { uS/cm } \\ 2 & \text { deg. C } \\ 0 & \mathrm{mg} / \mathrm{L} \\ 11.6 & \mathrm{mg} / \mathrm{L} \\ .105934 & \text { NṬU } \\ \text { NX } & \text { gallons }\end{array}$

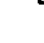

Unconfined , 
WSRC-RP-99-00119

Unclassified

WELL: YSB 1A

SRS Coord, Lat/Longitude Screen Zone Elevation Top of Standpipe Top of Casing Casing Pump Screen Zone N 71162.2 33.212 Deg N $\quad-128.4-98.4$ ft msl E 17808.8 81.758 Deg W

145.9 ft msl

$145.5 \mathrm{ft}$ msl 4 "STL

$01 / 29 / 98$

$04 / 23 / 98$

$08 / 11 / 98$

$12 / 03 / 98$

FIELD DATA

Analyte

Depth to water

$\mathrm{pH}$

Sp. Conductance

Water temperature

Alkalinity as $\mathrm{CaCO} 3$

Phenolpthalein Alkalinity

Turbidity

Volumes purged

Sampling code

$\begin{array}{lllll}101998 & 201998 & \frac{301998}{24.96} & \frac{401998}{44.39} & \text { Unit } \\ 24.9 & 19.5 & \text { ft msl } \\ 5.1 & 5.1 & 5.1 & 5.3 & \\ 55 & 38 & 39 & 30 & \text { uS/cm } \\ 21.9 & 19.8 & 22.8 & 22.9 & \text { deg. C } \\ 4 & 4 & 4 & 2 & \mathrm{mg} / \mathrm{L} \\ 0 & 0 & 0 & 0 & \mathrm{mg} / \mathrm{L} \\ 2.2 & .9 & 4.9 & 2.2 & \text { NTU } \\ 2.81532 & 4.36329 & 3.37376 & 29.8128 & \text { - gallons }\end{array}$


WSRC-RP-99-00119

Unclassified

WELL: YSB 2A

SRS Coord. Lathongitude Screen Zone Elevation Top of Standpipe Top of Casing Casing Pump Screen Zone N 71010.0 33.212 Deg N . $127.7-97.7 \mathrm{ft} \mathrm{msl} \cdot 144.8 \mathrm{ft}$ msl E 17850.2 81.758. Deg W $144.7 \mathrm{ft}$ msi 4 "STL Unconfined

SAMPLE DATE $\begin{array}{llll}01 / 29 / 98 & 04 / 21 / 98 & 08 / 12 / 98 & 12 / 01 / 98\end{array}$

\section{FIELD DATA}

Analyte

Depth to water

pH

Sp. Conductance

Water temperature

Alkalinity as $\mathrm{CaCO} 3$

Phenolpthalein Alkalinity

Turbidity

Volumes purged

Sampling code

\begin{tabular}{|c|c|c|c|c|}
\hline 101998 & 201998 & 301998 & 401998 & Unit \\
\hline $\begin{array}{l}22.3 \\
5.1\end{array}$ & $\begin{array}{l}17 \\
4.9\end{array}$ & $\begin{array}{l}25.31 \\
4.5\end{array}$ & $\begin{array}{l}45 \\
5.2\end{array}$ & ft msl \\
\hline 45 & 47 & 37 & 31 & uS/cm \\
\hline 20.1 & 19.4 & 20.4 & 20.7 & deg. $C$ \\
\hline 8 & 1 & 4 & 2 & $\mathrm{mg} / \mathrm{L}$ \\
\hline o & 0 & 0 & 0 & $\mathrm{mg} / \mathrm{L}$ \\
\hline .8 & 5.6 & & & NTUU \\
\hline 2.71551 & 2.79472 & 3.51404 & 40.3963 & gaillons \\
\hline
\end{tabular}


WELL: YSB 3A

SRS Coord, Lat/Longitude Screen Zone Elevation Top of Standpipe Top of Casing Casing Pump Screen Zone N 70859.0 33.211 Deg N 126.7 - $96.7 \mathrm{ft} \mathrm{msl}$ E 17755.2 81.758 Deg W $144.2 \mathrm{ft} \mathrm{msl}$

SAMPLE DATE

FIELD DATA

Analyte

Depth to water

pH

Sp. Conductance

Water temperature

Alkalinity as $\mathrm{CaCO} 3$

Phenolpthalein Alkalinity

Turbidity

Volumes purged

Sampling code $\begin{array}{llll}01 / 29 / 98 & 04 / 21 / 98 & 08 / 12 / 98 & 12 / 02 / 98\end{array}$

$\begin{array}{lllll}101998 & \underline{201998} & \underline{301998} & \underline{401998} & \text { Unit } \\ 20.65 & 15.54 & 27.56 & 25.95 & \text { ft msl } \\ 6.1 & 5.5 & 5.5 & 5.5 & \\ 71 & 78 & 110 & 160 & \text { uS/cm } \\ 16.7 & 17.1 & 22.7 & 19.3 & \text { deg. C } \\ 15 & 16 & 28 & 23 & \mathrm{mg} / \mathrm{L} \\ 0 & 0 & 0 & 0 & \mathrm{mg} / \mathrm{L} \\ 4.3 & 2.5 & 4.9 & 3.9 & \text { NTU } \\ 4.59327 & 4.52598 & 5.89886 & 5.95409 & \text { - gallons }\end{array}$


WELL: YSB 4A

SRS Coord. Lat/Longitude Screen Zone Elevation Top of Standpipe Top of Casing Casing Pump Screen Zone N 71020.7 33.212 Deg N $127.6-97.6 \mathrm{ft} \mathrm{msl}$. $144.8 \mathrm{ft} \mathrm{msl} 144.6 \mathrm{ft} \mathrm{msl} 4$ "STL . S Unconfined E 17739.8 81.758 Deg W.

$\begin{array}{lllll}\text { SAMPLE DATE } & 01 / 29 / 98 & 04 / 23 / 98 & 08 / 11 / 98 & 12 / 01 / 98\end{array}$

FIELD DATA

Analyte

101998

Depth to water

23.95

pH

5.3

Sp. Conductance

67

Water temperature

20

Alkalinity as $\mathrm{CaCO} 3$

9

Phenolpthalein Alkalinity

0

Turbidity

12.3

Volumes purged

Sampling code

201998

18.76

$\underline{301998}$

24.3

5

75

22.5

11

0

2.6

2.80695

NPSW
401998 Unit

45.36 ft msl

5.3

$54 \quad \mathrm{uS} / \mathrm{cm}$

21.3 deg. C

$5 \mathrm{mg} / \mathrm{L}$

o $\mathrm{mg} / \mathrm{L}$

3 NTU

36.2507 gallons 
Table B-5. Groundwater Monitoring Results for Other TNX Area Wells

WELL: TNX 13D

SRS Coord. Lat/Longitude Screen Zone Elevation Top of Standpipe Top of Casing Casing

N 70842.0 33.208 Deg N

$89.9-87.9 \mathrm{ft} \mathrm{msl}$

ft msl

$94.9 \mathrm{ft} \mathrm{msl}$

$2 " \mathrm{PVC}$

Pump Screen Zone E 15938.8 81.762 Deg W -.

$12 / 02 / 98$

SAMPLE DATE

FIELD DATA
Analyte
Depth to water .
$\mathrm{pH}$
Sp. Conductance
Water temperature
Alkalinity as $\mathrm{CaCO} 3$
Phenolpthalein Alkalinity
Turbidity
Volumes purged
Sampling codes

101998

201998

301998

401998

5.63

5.6

145

18.1

1

0

1.7

8.90155

\section{ANALYTICAL DATA}

\section{Analyte}

Aluminum, total recoverable

Boron, total recoverable

Iron, total recoverable

Lead, total recoverable

Manganese, total recoverable

Mercury, total recoverable

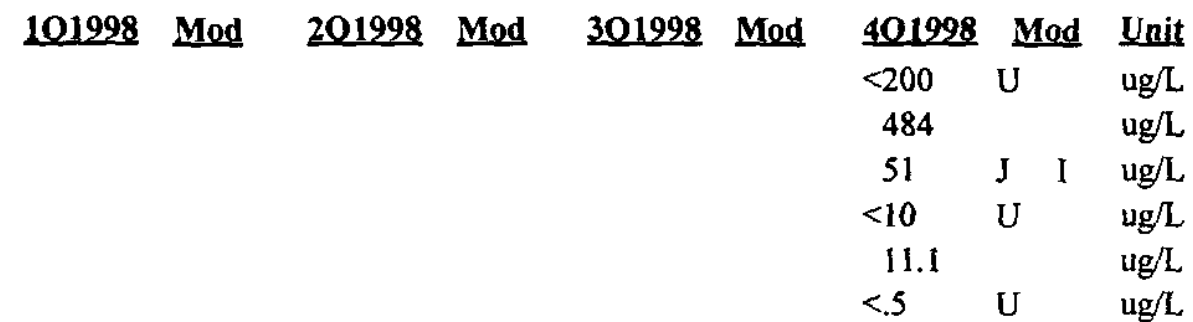

Note: Concentrations in bold exceed the Drinking Water Standards listed in Appendix A. Units are for all four quarters. 
WSRC-RP-99-00119

Unclassified

WELL: TNX 14D

SRS Coord. Lat/Longitude Sereen Zone Elevation Ton of Standpipe Top of Casing Casing Pump Screen Zone

N 70931.8 33.209 Deg N

$87.8-85.8 \mathrm{ft} \mathrm{msl}$

$\mathrm{ft} \mathrm{msl}$

$92.8 \mathrm{ft} \mathrm{msl}$

2 " PVC

Unconfined

E 15971.1 81.762 Deg W. :-

\section{SAMPLE DATE}

$12 / 02 / 98$

\section{FIELD DATA}

Analyte
Depth to water
pH
Sp. Conductance
Water temperature
Alkalinity as CaCO3
Phenolpthalein Alkalinity
Turbidity
Volumes purged
Sampling codes

101998

201998

301998

401998 Unit

3.34 ft BTOS

$\mathrm{pH}$

5.3

90 uS $/ \mathrm{cm}$

19.3 deg. $C$

Water temperature

0

0

3.5

4.99800

$\mathrm{mg} / \mathrm{L}$

$\mathrm{mg} / \mathrm{L}$

NTU

gallons

\section{ANALYTICAL DATA}

\section{Analyte}

Aluminum, total recoverable

Boron, total recoverable

Iron, total recoverable

Lead, total recoverable

Manganese, total recoverable

Mercury, total recoverable

\begin{tabular}{|c|c|c|c|c|c|c|c|c|c|}
\hline 101998 & Mod & 201998 & Mod & 301998 & Mod & 4019 & & ad & Unit \\
\hline & & & & & & 78.6 & $\mathrm{~J}$ & I & $\mathrm{ug} / \mathrm{L}$ \\
\hline & & & & & & 794 & & & $\mathrm{ug} / \mathrm{L}$ \\
\hline & & & & & & 401 & & & ug \\
\hline & & & & & & $<10$ & $\mathrm{U}$ & & g/ \\
\hline & & & & & & 18.8 & & & \\
\hline & & & & & & $<.5$ & U & & \\
\hline
\end{tabular}


$\frac{\text { SRS Coord. Lat/Longitude }}{\text { N 71021.1 }}$ E 16002.1 81.763 Deg W

SAMPLE DATE

FIELD DATA

\section{8}

Depth to water

$\mathrm{pH}$

Sp. Conductance

Water temperature

Alkalinity as $\mathrm{CaCO} 3$

Phenolpthalein Alkalinity

Turbidity

Volumes purged

Sampling codes

\section{$87.9-85.9 \mathrm{ft} \mathrm{msI}$}

$\mathrm{ft} \mathrm{msl}$

ft msl

$\frac{\text { Top of Casing }}{93.1 \mathrm{ft} \mathrm{msl}}$
Pump Screen Zone

$S$ Unconfined

$12 / 02 / 98$

ANALYTICAL DATA

\section{Analyte}

Aluminum, total recoverable

101998 Mod

201998

301998

401998

5.87

4.9

135

20.1

1

0

4.1

4.58463

Unit

ft BTOS

$\mathrm{uS} / \mathrm{cm}$

deg. C

$\mathrm{mg} / \mathrm{L}$

$\mathrm{mg} / \mathrm{L}$

NTU

gallons

Boron, total recoverable

Iron, total recoverable

Lead, total recoverable

Manganese, total recoverable

Mercury, total recoverable

\section{Mod 301998 Mod}

401998 Mod Unit

241

648

$\mathrm{ug} / \mathrm{L}$

$150 \mathrm{~J} \quad \mathrm{I} \quad \mathrm{ug} / \mathrm{L}$

$<10 \quad \mathrm{U} \quad \mathrm{ug} / \mathrm{L}$

$75.8 \quad$ ug/L

$<.5 \mathrm{U} \quad \mathrm{ug} / \mathrm{L}$

Note: Concentrations in bold exceed the Drinking Water Standards listed in Appendix A. Units are for all four quarters. 
WELL: TNX 17D

SRS Coord. Lat/Longitude Screen Zone Elevation Top of Standpipe Top of Casing Casing Pump Screen Zone N 71583.8 33.210 Deg N $91.7-89.7 \mathrm{ft} \mathrm{msl}$ ft $\mathrm{msi}$ $96.8 \mathrm{ft} \mathrm{msl} 2 " \mathrm{PVC}$

$P$

Unconfined E 16047.4 81.764 Deg W :

SAMPLE DATE

$12 / 02 / 98$

FIELD DATA

Analyte
Depth to water
pH
Sp. Conductance
Water temperature
Alkalinity as CaCO3
Phenolpthalein Alkalinity
Turbidity
Volumes purged
Sampling codes

101998

201998

301998

401998 Unit

6.75 ft BTOS

5.8

$98 \quad u S / c m$

20.5 deg. C

$29 \mathrm{mg} / \mathrm{L}$

$0 \quad \mathrm{mg} / \mathrm{L}$

2 NTU

Volumes purged

104.530 gallons

\section{ANALYTICAL DATA}

\section{Analyte}

Aluminum, total recoverable

Boron, total recoverable

Iron, total recoverable

Lead, total recoverable

Manganese, total recoverable

Mercury, total recoverable

\begin{tabular}{|c|c|c|c|c|c|c|c|c|c|}
\hline 101998 & Mod & 201998 & Mod & 301998 & Mod & 40199 & & od & Unit \\
\hline & & & & & & $<200$ & $U$ & & $\mathrm{ug} / \mathrm{L}$ \\
\hline & & & & & & 17.9 & $\mathrm{~J}$ & I & $u g / L$ \\
\hline & & & & & & 5080 & & & ug \\
\hline & & & & & & $<10$ & U & & $g / L$ \\
\hline & & & & & & 1110 & & & ug/ \\
\hline & & & & & & $<.5$ & $\mathrm{U}$ & & \\
\hline
\end{tabular}


WELL: TNX16D

SRS Coord. Lat/Longitude Screen Zone Elevation Top of Standpipe

N $71111.3 \quad 33.201913$

E $16012.2 \quad 81.762683$

SAMPLE DATE

Field Data

Analyte

Depth to water

$\mathrm{pH}$

Sp. Conductance

Water Temperature

Alkalinity as $\mathrm{CaCO} 3$

Phenolpthalein Alkalinity

Turbidity

Volumes Purged

Sampling Codes

\section{ANALYTICAL DATA}

Analyte

1,1,1-Trichloroethane

Carbon tetrachloride

Chloroform

Tetrachloroethylene

Trichloroethylene

cis-1,2-Dichloroethylene

Aluminum, total recoverable

Aluminum, total recoverable

Barium, total recoverable

Barium, total recoverable

Boron, total recoverable

Boron, total recoverable

Carbon tetrachloride

Iron, total recoverable

Iron, total recoverable

Lead, total recoverable

Lead, total recoverable

Manganese, total recoverable

Manganese, total recoverable

Mercury, total recoverable

Nitrate as nitrogen

Gross alpha
88.1-86.1 ft. msl

$93.4 \mathrm{ft} . \mathrm{msl}$

$4 / 21 / 98$

$7 / 17 / 98$

$12 / 3 / 98$

$\begin{array}{ccc}1 \text { Q1998 } & \text { Q1998 } & \text { 3Q1998 } \\ 2.05 & 4.85 \\ 4.4 & 4.4 \\ 140 & 170 \\ 16 & 20.7 \\ 0 & 0 \\ 0 & 0 \\ 0.2 & 0.3 \\ 3 & 2\end{array}$

$\begin{array}{rcc}\text { Casing } & \text { Pump Screen Zone } \\ \text { 2"SS } & \text { S Unconfined }\end{array}$ 
WELL: TNX 18D

SRS Coord Lat/Longitude Screen Zone Elevation Top of Standpipe Top of Casing Casing Pump Screen Zone N 70748.2 33.208 Deg N 86.9 - 84.9 ft msl ft msl $92.1 \mathrm{ft} \mathrm{msl}$ 2 "PVC $\mathrm{P}$ Unconfined

\section{FIELD DATA}

Analvte
Depth to water
pH
Sp. Conductance
Water temperature
Alkalinity as CaCO3
Phenolpthalein Alkalinity
Turbidity
Volumes purged
Sampling codes

101998

201998

301998

401998 Unit

.5 . ft BTOS

5.5

135

16.8

10

0

.8

2.73025

$\mathrm{uS} / \mathrm{cm}$

deg. C

$\mathrm{mg} / \mathrm{L}$

$\mathrm{mg} / \mathrm{L}$

NTU

Sampling codes

\section{ANALYTICAL DATA}

\section{Analyte}

101998 Mo

Aluminum, total recoverable

Barium, total recoverable

Boron, total recoverable

Iron, total recoverable

Lead, total recoverable

Manganese, total recoverable

Mercury, total recoverable

\begin{tabular}{|c|c|c|c|c|c|c|c|c|c|}
\hline 101998 & Mod & 201998 & Mod & 301998 & Mod & 401998 & & & Unit \\
\hline & & & & & & $<200$ & $\mathrm{U}$ & & $\mathrm{ug} / \mathrm{L}$ \\
\hline & & & & & & 55.8 & & & $\mathrm{ug} / \mathrm{L}$ \\
\hline & & & & & & 701 & & & $\mathrm{ug} / \mathrm{L}$ \\
\hline & & & & & & $<12.7$ & U & V & $\mathrm{ug} / \mathrm{L}$ \\
\hline & & & & & & $<10$ & $\mathrm{U}$ & & $\mathrm{ug} / \mathrm{L}$ \\
\hline & & & & & & 3.44 & $\mathrm{~J}$ & I & $\mathrm{ug} / \mathrm{L}$ \\
\hline & & & & & & $<.5$ & $\mathrm{U}$ & & $\mathrm{ug} / \mathrm{L}$ \\
\hline
\end{tabular}

Note: Concentrations in bold exceed the Drinking Water Standards listed in Appendix A. Units are for all four quarters. 
WELL: TNX 19D

$\frac{\text { SRS Coord, }}{\text { N 70626.7 }} \frac{\text { Lat/Longitude }}{33.208 \mathrm{Deg} \mathrm{N}} \frac{\text { Sereen Zone Elevation Top of Standpipe Top of Casing }}{86.9-84.9 \mathrm{ft} \mathrm{msl}} \underset{\mathrm{ft} \mathrm{msl}}{92.7 \mathrm{ft} \mathrm{msl}} \frac{\text { Casing }}{2 " \mathrm{PVC}} \frac{\text { Pump }}{\mathrm{P}} \frac{\text { Screen Zone }}{\text { Unconfined }}$ E 15848.4 81.762 Deg W :..

\section{SAMPLE DATE}

\section{FIELD DATA}

$\underline{101998}$

Depth to water

$\mathrm{pH}$

Sp. Conductance

Water temperature

Alkalinity as $\mathrm{CaCO} 3$

Phenolpthalein Alkalinity

Turbidity

Volumes purged

Sampling codes
•

201998

$\underline{301998}$

40199

3.3

5.3

130

18.5

12

0

.7

2.71003
Unit

ft BTOS

$\mathrm{uS} / \mathrm{cm}$

deg. C

$\mathrm{mg} / \mathrm{L}$

$\mathrm{mg} / \mathrm{L}$

NTU

gallons

\section{ANALYTICAL DATA}

\section{Analvte}

Aluminum, total recoverable

Boron, total recoverable

Iron, total recoverable

Lead, total recoverable

Manganese, total recoverable

Mercury, total recoverable

\section{Mod 201998 Mod 301998 Mod}

\begin{tabular}{|c|c|c|}
\hline 401998 & Mod & \\
\hline$<200$ & $\mathbf{U}$ & \\
\hline$<100$ & $\mathbf{U}$ & \\
\hline$<100$ & JU L & \\
\hline$<10$ & $U$ & \\
\hline 54.6 & & \\
\hline . & $\mathrm{U}$ & \\
\hline
\end{tabular}


WELL: TNX 20D

$\frac{\text { SRS Coord, }}{\mathrm{N} 70579.0} \frac{\text { Lat/Longitude }}{33.208 \mathrm{Deg} \mathrm{N}} \frac{\text { Sereen Zone Elevation Top of Standpipe Top of Casing }}{88.2-86.2 \mathrm{ft} \mathrm{msl}} \underset{\mathrm{ft} \mathrm{msl}}{\frac{\text { Casing }}{93.5 \mathrm{ft} \mathrm{msl}}} \frac{\text { Pump }}{\text { 2 PVC }} \frac{\text { Screen Zone }}{\text { Unconfined }}$ E 15826.1 81.762 Deg W ...

SAMPLE DATE

$12 / 07 / 98$

FIELD DATA

Analyte
Depth to water
pH
Sp. Conductance
Water temperature
Alkalinity as CaCO3
Phenolpthalein Alkalinity
Turbidity
Volumes purged
Sampling codes

101998

$\underline{201998}$

301998

4Q1998 Unit

3.88

ft BTOS

5.3

$100 \quad$ uS $/ \mathrm{cm}$

17.9 deg. C

$13 \mathrm{mg} / \mathrm{L}$

0 , $\mathrm{mg} / \mathrm{L}$

1 NTU

Sampling codes

3.56583 gallons

\section{ANALYTICAL DATA}

Analyte

Aluminum, total recoverable

Boron, total recoverable

Iron, total recoverable

Lead, total recoverable

Manganese, total recoverable

Mercury, total recoverable

\section{Mod 201998 Mod 301998 Mod}

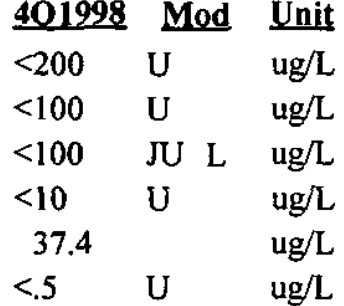

Note: Concentrations in bold exceed the Drinking Water Standards listed in Appendix A. Units are for all four quarters. 
WELL: TNX 21D

SRS Coord. Lat/Longitude Screen Zone Elevation Top of Standpipe Top of Casing Casing Pump Screen Zone N 70446.8 33.207 Deg N $88.9-86.9$ ft msl ft mst $94.4 \mathrm{ft} \mathrm{msl}$ 2 "PVC

$\frac{\text { ump }}{P} \frac{\text { Screen Zone }}{\text { Unconfined }}$ E 15833.581 .762 Deg W. :

SAMPLE DATE

$12 / 07 / 98$

\section{FIELD DATA}
Analyte
Depth to water
$\mathrm{pH}$
Sp. Conductance
Water temperature
Alkalinity as $\mathrm{CaCO} 3$
Phenolpthalein Alkalinity
Turbidity
Volumes purged
Sampling codes

\section{ANALYTICAL DATA}

Analyte

101998 Mod

Aluminum, total recoverable

Boron, total recoverable

Iron, total recoverable

Lead, total recoverable

Manganese, total recoverable

Mercury, total recoverable
101998

201998

301998
2.69

5.5

76

19.7

16

0

38.9

12.6768

$\mathrm{T}$

Unit
ft BTOS
uS/cm
deg. C
$\mathrm{mg} / \mathrm{L}$
$\mathrm{mg} / \mathrm{L}$
NTU
gallons

$\begin{array}{clll}\begin{array}{c}\text { 401998 } \\ \mathbf{3 8 7 0}\end{array} & \text { Mod } & \text { Unit } \\ <100 & \text { U } & \text { ug/L } \\ <100 & \text { JU } & \text { L } & \text { ug/L } \\ <10 & \text { U } & \text { ug/L } \\ 29.3 & & \text { ug/L } \\ <.5 & \text { U } & \text { ug/L }\end{array}$

Note: Concentrations in bold exceed the Drinking Water Standards listed in Appendix A. Units are for all four quarters. 
$\frac{\text { SRS Coord. }}{\mathrm{N} 70184.7} \frac{\text { Lat/Longitude }}{33.207 \mathrm{Deg} \mathrm{N}} \frac{\text { Sereen Zone Elevation Top of Standpipe Top of Casing }}{87.8-85.8 \mathrm{ftmsl}} \frac{\text { Casing }}{93.0 \mathrm{ft} \mathrm{msl}} \frac{\text { Pump }}{\mathrm{P} \text { Screen Zone }}$ E 15757.7 81.762 Deg W :

SAMPLE DATE

\section{FIELD DATA}

Analyte
Depth to water
pH
Sp. Conductance
Water temperature
Alkalinity as CaCO3
Phenolpthalein Alkalinity
Turbidity
Volumes purged
Sampling codes

101998

$\underline{201998}$

$\underline{301998}$

401998 Unit

4.31

ft BTOS

5.2

$60 u \mathrm{uS} / \mathrm{cm}$

18.9 deg. C

$10 \mathrm{mg} / \mathrm{L}$

$0 \mathrm{mg} / \mathrm{L}$

2.3 NTU

Sampling codes

4.21977 gallons

\section{ANALYTICAL DATA}

\section{Analyte}

\section{Mod 201998 Mod 301998 Mod}

\begin{tabular}{|c|c|c|c|c|c|c|c|c|}
\hline \multirow[t]{7}{*}{101998} & Mod & $\underline{201998}$ & Mod & 301998 & Mod & 401998 & Mod & Unit \\
\hline & & & & & & $<200$ & U & $\mathrm{ug} / \mathrm{L}$ \\
\hline & & & & & & $<100$ & $\mathrm{U}$ & $\mathrm{ug} / \mathrm{L}$ \\
\hline & & & & & & $<100$ & JU L & $\mathrm{ug} / \mathrm{L}$ \\
\hline & & & & & & $<10$ & $\mathrm{U}$ & $\mathrm{ug} / \mathrm{L}$ \\
\hline & & & & & & 49.5 & & $\mathrm{ug} / \mathrm{L}$ \\
\hline & & & & & & $<.5$ & $U$ & ug/ \\
\hline
\end{tabular}

Aluminum, total recoverable

Boron, total recoverable

Iron, total recoverable

Lead, total recoverable

Manganese, total recoverable

Mercury, total recoverable

Note: Concentrations in bold exceed the Drinking Water Standards listed in Appendix A. Units are for all four quarters. 
WELL: TNX 23D

SRS Coord, Lat/Longitude Sereen Zone Elevation Top of Standpipe Top of Casing Casing Pump Screen Zone N 71414.5 33.211 Deg N $104.8-84.8 \mathrm{ftmsl}$ 155.3 ft mst $155.1 \mathrm{ftmsl} 2$ " PVC V Unconfined E 16927.0 81.761 Deg W :-

SAMPLE DATE

FIELD DATA

Analyte

Depth to water

$\mathrm{pH}$

Sp. Conductance

Water temperature

Alkalinity as $\mathrm{CaCO} 3$

Phenolpthalein Alkalinity

Turbidity

Volumes purged

Sampling codes
$04 / 20 / 98$

201998

53.8

5.6

46

17

1

0

1.8

4.43459

$07 / 17 / 98$

$\mathbf{3 0 1 9 9 8}$
57.1
5.7
53
22.5
11
0
2.8
6.46711

$\underline{401998}$

Unit

ft BTOS

$\mathrm{uS} / \mathrm{cm}$

deg. C

$\mathrm{mg} / \mathrm{L}$

$\mathrm{mg} / \mathrm{L}$

NTU

gallons

\section{ANALYTICAL DATA}

\section{Analyte}

1,1,1-Trichloroethane

Aluminum, total recoverable

Carbon tetrachloride

Chloroform

Gross alpha

Iron, total recoverable

Lead, total recoverable

Mercury, total recoverable

Nitrate as nitrogen

Tetrachloroethylene

Trichloroethylene

cis-1,2-Dichloroethylene

\begin{tabular}{|c|c|c|c|c|c|}
\hline $101998 \mathrm{Mod}$ & 201998 & $\underline{\text { Mo }}$ & & 301998 & Mos \\
\hline & $<.462$ & UJ & 0 & $<1$ & $U$ \\
\hline & $<20$ & U & & $<100$ & $\mathrm{U}$ \\
\hline & $<.405$ & UJ & O & $<1$ & $\mathrm{U}$ \\
\hline & $<.428$ & UJ & $\mathrm{O}$ & $<1$ & U \\
\hline & .44 & UI & & .69 & \\
\hline & $<42.4$ & $\mathrm{U}$ & & 82.4 & $\mathrm{~J}$ \\
\hline & $<5$ & U & & $<5$ & U \\
\hline & $<.2$ & U & & $<.2$ & U \\
\hline & $<300$ & & V & 112 & \\
\hline & $<.569$ & UJ & $\mathrm{O}$ & $<1$ & $\mathrm{U}$ \\
\hline & $<.735$ & UJ & $\mathrm{O}$ & $<1$ & U \\
\hline & & & & $<1$ & U \\
\hline
\end{tabular}

401998 Mod Unit

$u g / L$

$\mathrm{ug} / \mathrm{L}$

$\mathrm{ug} / \mathrm{L}$

ug/L

$\mathrm{pCi} / \mathrm{L}$

ug/L

$\mathrm{ug} / \mathrm{L}$

ug/L

$\mathrm{ug} / \mathrm{L}$

ug/L

$u g / L$

ug/L 
WELL: TNX 24D

SRS Coord. Lat/Longitude Screen Zone Elevation Top of Standpipe Top of Casing Casing Pump Screen Zone N 71536.9 33.213 Deg N 114.8 - $99.8 \mathrm{ft} \mathrm{msl}$ $143.3 \mathrm{ft} \mathrm{msl}$ $142.9 \mathrm{ft} \mathrm{msl}$ $2^{\text {" PVC }}$

Unconfined
SAMPLE DATE

\section{FIELD DATA}

101998

Depth to water $\mathrm{pH}$

Sp. Conductance

Water temperature

Alkalinity as $\mathrm{CaCO} 3$

Phenolpthalein Alkalinity

Turbidity

Volumes purged

Sampling codes
$12 / 03 / 98$

$\underline{201998}$

$\underline{301998}$

$\begin{array}{ll}\frac{401998}{33.64} & \text { Unit } \\ 5.3 & \text { ft BTOS } \\ 91 & \text { uS/cm } \\ 21.6 & \text { deg. C } \\ 9 & \text { mg/L } \\ 0 & \text { mg/L } \\ 2.6 & \text { NTU } \\ 4.51194 & \text { gallons }\end{array}$

\section{ANALYTICAL DATA}

Analyte

Aluminum, total recoverable

Barium, total recoverable

Boron, total recoverable

Iron, total recoverable

Lead, total recoverable

Manganese, total recoverable

Mercury, total recoverable

\begin{tabular}{|c|c|c|c|c|c|c|c|c|c|}
\hline 101998 & Mod & 201998 & Mod & 301998 & Mod & 401998 & & od & Unit \\
\hline & & & & & & $<200$ & $U$ & & $\operatorname{ug} / \mathrm{L}$ \\
\hline & & & & & & 10.4 & & & $u g / L$ \\
\hline & & & & & $\theta^{\circ}$ & 14.3 & J & I & $u g / L$ \\
\hline & & & & & & $<68.9$ & U & V & ug $/ \mathrm{L}$ \\
\hline & & & & & & $<10$ & U & & ug/L \\
\hline & & & & & & 7.43 & J & I & $u g / L$ \\
\hline & & & & & & $<.5$ & U & & $g / 1$ \\
\hline
\end{tabular}

Note: Concentrations in bold exceed the Drinking Water Standards listed in Appendix A. Units are for all four quarters. 
WSRC-RP-99-00119

Unclassified

WELL: TNX 26D

SRS Coord. Lat/Longitude Sereen $Z_{0 n e}$ Elevation Top of Standpipe Top of Casing Casing Pump Screen Zone N 70424.4 33.208 Deg N $90.1-87.8 \mathrm{ft} \mathrm{msl}$

ft msl

$100.8 \mathrm{ft} \mathrm{msl}$

$2 " \mathrm{PVC}$

$P$

Unconfined

E 16251.081 .761 Deg W :-

SAMPLE DATE

\section{FIELD DATA}

\section{Analyte}

Depth to water

pH

Sp. Conductance

Water temperature

Alkalinity as $\mathrm{CaCO} 3$

Phenolpthalein Alkalinity

Turbidity

Volumes purged

Sampling codes
$04 / 20 / 98$

$07 / 17 / 98$

201998

4.7

101998

301998
6.98
5.8
98
22.8
18
0
15.1
2.02577

2.02577
$12 / 04 / 98$

$\begin{array}{lll}\frac{401998}{7.97} & & \text { Unit } \\ 5.9 & & \text { ft BTOS } \\ 80 & & \\ 19.6 & & \text { uS/cm } \\ 0 & & \text { deg. C } \\ 0 & \text { mg/L } \\ 14.4 & \text { NTU } \\ 9.69791 & \text { gallons }\end{array}$

\section{Mod}

201998 Mod

$<.462 \mathrm{U}$

$629 \mathrm{~J} \quad \mathrm{~L}$

301998 Mod

$<1$

$<8$

Arsenic, total recoverable

Barium, total recoverable

Boron, total recoverable

Cadmium, total recoverable

Calcium, total recoverable

Carbon tetrachloride

Chloride

Chloroform

Chromium, total recoverable

Fluoride

Gross alpha

Iron, total recoverable

Lead, total recoverable

Lithium, total recoverable

Magnesium, total recoverable

Manganese, total recoverable

Mercury, total recoverable

Nitrate as nitrogen

Nitrate-nitrite as nitrogen

Nonvolatile beta

Potassium, total recoverable

Selenium, total recoverable

Silica, total recoverable

Silver, total recoverable

Sodium, total recoverable
41.1

128

$<2 \quad \mathrm{U}$

$<3150$

$<.405 \quad \mathrm{U}$

10470

$<.428$

$1.1 \mathrm{~J} \quad \mathrm{E}$

$<100 \quad \mathrm{U}$

5.51

1290

$<5$

$1.4 \quad \mathrm{~J} \quad \mathrm{E}$

$<973$

85.4

258

$7.98 \quad J \quad X$

$<1030$.

$<5 \quad \mathrm{U}$

$<5240$

$<2$

$<14200$
$<.14 \quad \mathrm{U} \quad \mathrm{V}$
401998 Mod Unit

$<1 \quad$ U ug/L

$766 \quad u g / L$

$<40 \quad \mathrm{U} \quad$ ug/L

$35.4 \quad \mathrm{ug} / \mathrm{L}$

$219 \quad u g / L$

$<4.7$ U $\quad \mathrm{ug} / \mathrm{L}$

$<3710 \quad$ ug/L

$<1 \quad$ JU L $\quad$ ug/L

$7500 \quad$ ug/L

$<1 \quad \mathrm{U} \quad$ ug/L

$<2.5 \quad$ U $\quad \vee \quad u g / L$

$58.3 \quad \mathrm{ug} / \mathrm{L}$

$7.71 \quad \mathrm{pCi} / \mathrm{L}$

$338 \quad u g / L$

$<10 \quad U \quad u g / L$

$.43 \quad \mathrm{~J} \quad \mathrm{I} \quad \mathrm{ug} / \mathrm{L}$

$<1060 \quad$ ug/L

$106 u \mathrm{ug} / \mathrm{L}$

$<.5 \quad \mathrm{U} \quad \mathrm{ug} / \mathrm{L}$

$527 \mathrm{~J} \quad \mathrm{Q} \quad u g / \mathrm{L}$

$528 \quad u g / L$

$6.51 \quad \mathrm{pCi} / \mathrm{L}$

$<922 \quad \mathrm{ug} / \mathrm{L}$

$<66$ U ug/L

$<12400 \quad \mathrm{ug} / \mathrm{L}$

$<5 \quad$ U ug/L

$<11300$ ug/L

Note: Concentrations in bold exced the Drinking Water Standards listed in Appendix A. Units are for all four quarters. 
WELL: TNX 26D

ANALYTICAL DATA

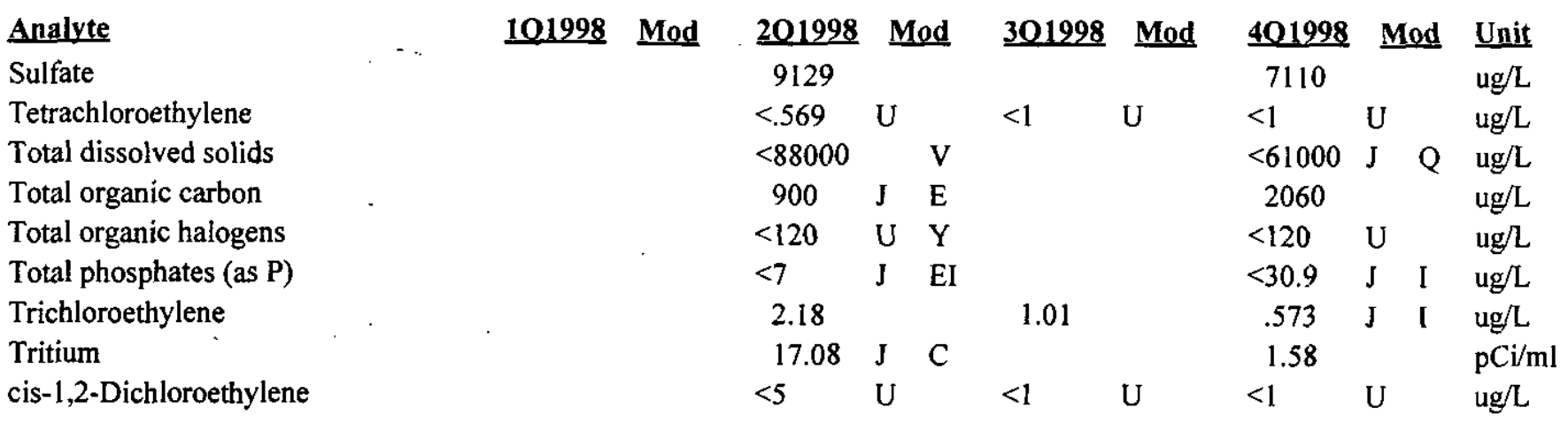

Note: Concentrations in bold exceed the Drinking Water Standards listed in Appendix A. Units are for all four quarters. 
1998 Comprehensive TNX Area Annual Groundwater and Effectiveness Monitoring Report (U)

WSRC-RP-99-4003

Savannah River Site

May 1999

Table B-6. Water Elevations for TNX-Area Wells from SRTC Measurements In 1998

\begin{tabular}{|c|c|c|c|c|c|c|c|c|c|c|c|}
\hline & Jan-98 & Feb-98 & Mar-98 & Apr-98 & May-98 & Jun-98 & Jul-98 & Aug-98 & Sep-98 & Oct-98 & Nov-98 \\
\hline P26A & 120.64 & 121.92 & 122.51 & 122.68 & 122.26 & 120.3 & 119.27 & 118.96 & 118.94 & 117.98 & 117.71 \\
\hline P26B & 110.26 & 112.27 & 115.24 & 115.65 & 115.53 & 114.35 & 112.77 & 112.03 & 111.42 & 110.5 & 109.7 \\
\hline P26D & 117.25 & 120.52 & 124.53 & 126.12 & 126.48 & 125.47 & 123.37 & 122.44 & 121.78 & 120.73 & 119.94 \\
\hline TBG 1 & 100.65 & 101.81 & 103.38 & 102.98 & 102.31 & 100.39 & 99.22 & 98.88 & 98.74 & 98.34 & 97.67 \\
\hline TBG 3 & 103.22 & 104.61 & 105.74 & 106.81 & 106.37 & 105.28 & 104.23 & 103.38 & 102.82 & 102.42 & 101.64 \\
\hline TBG 4 & 103.56 & 104.84 & 106.29 & 107.24 & 107.06 & 106.15 & 105.04 & capped & capped & capped & capped \\
\hline TBG 5A & 102.29 & 103.97 & 105.98 & 106.48 & 105.87 & 104.42 & 107 & 102.5 & 102.17 & 101.67 & 100.67 \\
\hline TBG 5B & 115.85 & 117.21 & 117.93 & 118.07 & 117.57 & 116.56 & 117.97 & 113.77 & 113.95 & 113.07 & $\uparrow 12.72$ \\
\hline TBG 5D & 102.94 & 104.72 & 106.81 & 107.99 & 107.69 & 106.55 & 104.92 & 104.13 & 103.71 & 103.28 & 102.23 \\
\hline TBG 6 & 103.36 & 104.55 & 105.94 & 106.47 & 106.22 & 105.19 & 104.04 & 103.36 & 102.99 & 102.47 & 101.72 \\
\hline TBG 7 & 105.14 & 107.27 & 109.39 & 110.48 & 110.52 & 108.68 & 107.27 & 106.66 & 106.4 & 105.71 & 104.42 \\
\hline TCM 1 & 95.67 & 96.34 & 96.58 & 96.48 & 96.17 & 93.33 & 93.23 & 92.9 & 92.84 & 92.45 & 92.35 \\
\hline TCM 2 & 95.31 & 95.94 & 96.24 & 96.18 & 95.71 & 93.65 & 93.7 & 93.22 & 93.34 & 92.96 & 92.86 \\
\hline TCM 3 & 95.38 & 96.07 & 96.19 & 96.19 & 95.71 & 93.66 & 93.71 & 93.31 & 93.34 & 92.92 & 92.84 \\
\hline TCM 4 & N/A & N/A & $N / A$ & N/A & N/A & N/A & $N / A$ & 92.72 & 92.83 & 92.54 & 92.4 \\
\hline TCM 5 & N/A & N/A & $N / A$ & N/A & N/A & N/A & N/A & 93 & 92.75 & 92.69 & 92.58 \\
\hline ТСM 6 & N/A & N/A & N/A & N/A & $N / A$ & N/A & N/A & $N / A$ & $N / A$ & 92.41 & 92.31 \\
\hline TCM 8 & N/A & $N / A$ & N/A & N/A & N/A & N/A & N/A & 92.64 & 92.79 & 92.36 & 92.21 \\
\hline TIR 1L & 95.61 & 96.18 & 96.42 & 96.38 & 95.97 & 93.48 & 93.13 & 92.88 & 92.78 & 92.39 & 92.31 \\
\hline TIR 1M & 95.38 & 95.85 & 96.01 & 96.03 & 95.54 & 93.36 & 93.26 & 93.06 & 93.05 & 92.72 & 92.57 \\
\hline TIR 1U & 95.28 & 95.75 & 96.04 & 96.03 & 95.41 & 93.34 & 93.24 & 93.01 & 93.05 & 92.59 & 92.57 \\
\hline TIR 2 & 94.71 & 95.35 & 95.54 & 95.61 & 95.08 & 92.55 & 95.49 & 92.33 & 92.35 & 92.05 & 91.99 \\
\hline TIR 3B & 97.11 & 97.28 & 97.65 & 97.49 & 97.12 & 96.27 & 95.66 & 95.66 & 95.45 & 95.01 & 94.73 \\
\hline TNX 10 & 100.24 & 100.93 & 101.84 & 100.97 & 100.42 & 99.23 & 98.33 & 98.3 & 98.14 & 97.59 & 96.99 \\
\hline TNX 2D & 99.69 & 100.59 & 101.65 & 101.08 & 100.34 & 98.97 & 98.09 & 97.96 & 97.86 & 97.39 & 96.75 \\
\hline TNX 3D & 99.36 & 100.39 & 102.35 & 102.15 & 101.07 & 99.38 & 98.41 & 98.01 & 97.84 & 97.46 & 96.78 \\
\hline TNX 40 & 102.36 & 104.25 & 106.87 & 107.53 & 107.15 & 106.01 & 104.7 & 103.87 & 103.54 & 103 & 101.97 \\
\hline TNX 5D & 104.34 & 106.63 & 108.87 & 110.53 & 110.25 & 108.89 & 107.01 & 106.17 & 105.78 & · $\quad 105.17$ & 104.04 \\
\hline TNX 60 & 104.72 & 107.54 & 111.47 & 113.75 & 112.34 & 110.67 & 108.26 & 107.39 & 106.87 & 106.36 & 104.65 \\
\hline TNX 7D & 101.64 & 102.49 & 103.56 & 102.69 & 101.95 & 100.68 & 99.64 & 99.58 & 99.48 & 98.92 & 98.15 \\
\hline TNX 8D & 146.28 & 146.74 & 146.36 & 146.96 & 146.18 & 144.26 & 144.05 & 144.09 & 144.01 & 143.53 & 143.46 \\
\hline TNX 9D & 94.08 & 94.55 & 94.65 & 94.75 & 94.05 & 92.05 & 91.84 & 91.86 & 91.78 & 91.41 & 91.25 \\
\hline TNX 100 & 95.88 & 96.33 & 96.01 & 96.6 & 95.04 & 92.72 & 92.62 & 92.26 & 92.33 & 91.98 & 91.75 \\
\hline TNX 11D & 97.57 & 98.27 & 98.49 & 98.47 & 98.07 & 95.55 & 95.49 & 95.14 & 95.17 & 94.74 & 94.73 \\
\hline TNX 12D & 96.29 & 96.59 & 96.72 & 96.62 & 96.24 & 94.4 & 94.39 & 94.26 & 94.16 & 93.72 & 93.7 \\
\hline TNX 13D & 91.73 & Uw & uw & uw & uw & 89.83 & 89.77 & 89.65 & 89.72 & 89.57 & 89.32 \\
\hline TNX 14D & 92.08 & Uw & uw & uw & uw & 89.96 & 89.86 & 89.85 & 89.91 & 89.65 & 89.57 \\
\hline TNX 15D & uw & Uw & uw & uw & uw & 87.88 & 87.48 & 87.57 & 87.65 & 87.54 & 87.28 \\
\hline TNX 16D & trans & Trans & trans & trans & 90.95 & 88.56 & 87.95 & 87.98 & 87.98 & 87.82 & 87.67 \\
\hline TNX 17D & 92.36 & 92.97 & 93.01 & 93.11 & 92.91 & 90.68 & 90.85 & 90.44 & 90.54 & 90.24 & 90.14 \\
\hline TNX 18D & uw & Uw & uw & uw & uw & water@tp & water@tp & water@tp & water@tp & water@tp & water@tp \\
\hline TNX 19D & uw & Uw & uw & uw & uw & 89.94 & 89.57 & 89.72 & 89.88 & 89.58 & 89.52 \\
\hline
\end{tabular}


1998 Comprehensive TNX Area Annual Groundwater and Effectiveness Monitoring Report (U)
WSRC-RP-99-4003

Unclassified

Savannah River Site

May 1999

\begin{tabular}{|c|c|c|c|c|c|c|c|c|c|c|c|}
\hline & Jan-98 & Feb-98 & Mar-98 & Apr-98 & May-98 & Jun-98 & Jul-98 & Aug-98 & Sep-98 & Oct-98 & Nov-98 \\
\hline TNX 20D & uw & Uw & uw & uw & uw & 90.27 & 89.91 & 90.17 & 90.12 & 89.87 & 89.77 \\
\hline TNX 21D & uw & Uw & uw & uw & uw & 92.36 & 91.92 & 92.32 & 92.32 & 91.9 & 91.85 \\
\hline TNX 23D & 99.71 & 100.56 & 102.07 & 101.55 & 100.69 & 99.11 & 98.15 & 97.92 & 97.78 & 97.39 & 96.64 \\
\hline TNX 24D & 120.32 & 121.26 & 122.55 & 123.51 & 123.55 & 122.78 & 121.75 & 121.24 & 120.98 & 120.32 & 119.87 \\
\hline TNX 26D & 95.75 & 96.26 & 96.01 & 96.42 & 95.62 & 93.94 & 93.38 & 93.88 & 94.16 & 93.08 & 93.03 \\
\hline TNX 27D & 97.8 & 98.05 & 98.74 & 98.4 & 97.86 & 96.71 & 95.98 & 95.96 & 95.88 & 95.57 & 94.93 \\
\hline TNX 610 & uw & Uw & uw & uw & uw & 87.3 & dnm & dnm & dnm & 85.65 & dnm \\
\hline TNX 61M & uw & Uw & uw & uw & uw & 87.04 & dnm & dnm & dnm & 85.92 & dnm \\
\hline TNX 61S & uw & Uw & uw & uw & uw & 86.88 & dnm & dnm & $\mathrm{dnm}$ & 86.88 & dnm \\
\hline TNX 65D & uw & Uw & uw & uw & uw & 85.12 & dinm & dnm & dnm & 82.87 & dnm \\
\hline TNX 65M & uw & Uw & uw & uw & uw & 85.63 & dnm & dnm & dnm & 84.04 & dnm \\
\hline TNX 65S & uw & Uw & uw & uw & uw & 85.72 & dnm & $\mathrm{dnm}$ & dnm & 85.62 & dnm \\
\hline TNX 66D & uw & Uw & uw & uw & uw & 87.47 & dnm & $\mathrm{dnm}$ & dnm & 84.38 & dnm \\
\hline TNX 66M & uw & Uw & uw & uw & uw & 85.96 & dnm & dnm & dnm & 84.7 & dnm \\
\hline TNX 665 & uw & Uw & uw & uw & uw & 86.45 & dnm & dnm & $\mathrm{dnm}$ & 86.46 & dnm \\
\hline TNX 72D & uw & Uw & uw & uw & uw & 89.18 & dnm & $\mathrm{dnm}$ & dnm & 85.48 & dnm \\
\hline TNX 72M & uw & Uw & uw & uw & uw & 86.62 & dnm & dnm & dnm & 85.45 & $\mathrm{dnm}$ \\
\hline$T N X 72 S$ & uw & Uw & uw & uw & uw & 87.91 & dnm & dnm & dnm & 87.93 & dnm \\
\hline TRW 1 & 91.66 & 92.64 & 101.2 & 100.85 & 93.27 & 92.37 & 91.61 & 90.82 & 90.47 & 90.08 & 89.03 \\
\hline TRW 2 & 91.95 & 93.03 & 100.68 & 96.81 & 93.7 & 92.61 & 92.06 & 91.32 & 91.27 & 91.21 & 90.28 \\
\hline TRW 3 & $\mathrm{nr}$ & $\mathrm{Nr}$ & 101.86 & 101.35 & 100.64 & $d r y$ & $d r y$ & dry & diry & dry & dry \\
\hline TRW 4 & $\mathrm{nr}$ & $\mathrm{Nr}$ & 102.84 & 102.54 & 101.81 & 96.28 & 90.08 & 86.89 & 88.78 & 86.16 & 86.08 \\
\hline XSB 1A & 98.05 & 98.84 & 100.6 & 100.24 & 99.11 & 97.9 & 96.04 & 96.66 & 96.76 & 96.23 & 95.57 \\
\hline XSB 1B & 105.54 & 106.54 & 107 & 106.91 & 106.47 & 102.53 & 101.52 & 101.44 & 101.62 & 100.71 & 100.78 \\
\hline XSB 1D & 98.02 & 99.03 & 101.24 & 101.05 & 99.64 & 98.39 & 97.46 & 97.11 & 96.91 & 96.59 & 95.84 \\
\hline XSB 2D & 98.14 & 98.98 & 100.92 & $100.61^{*}$ & 99.48 & 98.28 & 97.43 & 96.89 & 96.92 & 96.4 & 95.81 \\
\hline XSB 3A & 98.63 & 99.64 & 101.25 & 101.35 & 100.37 & 99.23 & 98.26 & 97.92 & 97.74 & 97.37 & 96.61 \\
\hline XSB 40 & 98.44 & 99.31 & 100.92 & 100.91 & 99.87 & 98.71 & 97.78 & 97.38 & 97.33 & 96.8 & 96.16 \\
\hline XSB 5A & 98.04 & 98.8 & 99.92 & 99.54 & 98.75 & 97.84 & 96.85 & 96.83 & 95.62 & 96.17 & 95.7 \\
\hline YSB IA & 119.64 & 123.08 & 125.8 & 126.47 & 126.92 & 124.39 & 122.35 & 122.55 & 122.43 & 121.36 & 120.23 \\
\hline YSB 2A & 121.48 & 124.41 & 127.31 & 127.93 & 127.47 & 125.68 & 124.46 & 123.59 & 123.76 & 122.26 & 121.01 \\
\hline YSE 3A & 122.22 & 125.02 & 128.54 & 128.61 & 128.45 & 125.22 & 123.7 & 123.24 & 123.61 & 121.42 & 119.88 \\
\hline YSB 4A & 119.86 & 122.58 & 125.36 & 126.03 & 125.89 & 124.05 & 122.24 & 121.91 & 122.03 & 120.64 & 119.55 \\
\hline
\end{tabular}

Notes:

$\begin{array}{ll}\text { N/A } & \text { - No samples taken } \\ \text { Uw - } & \text { - Well almost underwater, river too high } \\ \text { Capped } & \text { - Well was capped } \\ \text { Dry } & \text { - Well was dry. } \\ \text { DNM } & \text { - Did not measure } \\ \mathrm{Nr} & \text { - Not Running } \\ \text { Water@tp } & \text { - Water at top of casing } \\ \text { Trans } & \text { - Transducer in well, no way to measure }\end{array}$

